\title{
The deepwater megabenthic invertebrates on the western continental margin of Australia (100-1100 m depths): composition, distribution and novelty
}

\author{
F.R. McEnnulty ${ }^{1}$, K.L. Gowlett-Holmes ${ }^{1}$, A. Williams ${ }^{1}$, F. Althaus ${ }^{1}$, \\ J. Fromont ${ }^{2}$, G.C.B. Poore ${ }^{3}$, T.D. O'Hara ${ }^{3}$, L. Marsh², P. Kott', S. Slack-Smith², \\ P. Alderslade ${ }^{1}$ and M.V. Kitahara ${ }^{5}$
}

\author{
${ }^{1}$ CSIRO Wealth from Oceans Flagship, Marine \& Atmospheric Research, GPO Box 1538, Hobart, \\ Tasmania, 7001, Australia. Email: felicity.mcennulty@csiro.au \\ 2 Western Australian Museum, Welshpool, Western Australia, Australia. \\ ${ }^{3}$ Museum Victoria, Melbourne, Victoria, Australia. \\ ${ }^{4}$ Queensland Museum, Brisbane, Queensland, Australia. \\ ${ }^{5}$ Museum of Tropical Queensland/James Cook University, Townsville, Queensland, Australia.
}

\begin{abstract}
Taxonomic study of the first regional scale collection of deepwater invertebrate megabenthos from Australia's western continental margin reveals a previously undiscovered high species richness and novelty. Benthic samples were taken systematically at 19 sites from Albany to Barrow Island $\left(\sim 35^{\circ} \mathrm{S}\right.$ to $\left.21^{\circ} \mathrm{S}\right)$ on the deep continental shelf (100 $\mathrm{m}$ depth) and upper continental slope $(400 \mathrm{~m})$. At some sites, additional samples were taken across-shelf at depths of $200 \mathrm{~m}, 700 \mathrm{~m}$ and $1000 \mathrm{~m}$, with a couple as deep as $1200 \mathrm{~m}$ and $1500 \mathrm{~m}$. Multibeam (swath) mapping of complex seabed topography enabled targeted collection of the epifauna on hard and soft substrates and targetting of features including submarine canyons.
\end{abstract}

A total of 2001 species including 396 (20\%) confirmed new species, 876 (44\%) previously described species and 727 (36\%) uncertain species were identified within the taxa Porifera (Demospongiae), Cnidaria (Octocorallia, Antipatharia and Scleractinia), Mollusca, Echinodermata, Crustacea (Decapoda), Pycnogonida and Ascidiacea. The high proportion of new and uncertain species stemmed from poor knowledge of entire major groups (e.g. the Demospongiae), and from less well studied deep-sea taxa within otherwise well-known groups (e.g. the Bivalvia). Over half the species (1075) were collected only once. Distributional patterns of taxa show the western margin is an overlap zone of tropical and temperate fauna with a strong Indo-Pacific affinity (42\% of described species) but an endemic fauna also exists (17\% of described species).

This paper provides an annotated checklist of all these identified species, along with live colour images of over half the species. All species can be cross-referenced to images and other collection information via a unique eight-digit computer code maintained in the Codes for Australasian Aquatic Biota database.

The research provides valuable information for consideration in the regional marine planning process and all biological specimens are lodged in Australian museums to enable ongoing examination of the diverse and largely undescribed fauna.

KEYWORDS: taxonomy, megabenthos, biodiversity, new species, biogeography, continental slope 


\section{INTRODUCTION}

\section{SCOPE}

Knowledge of the benthic invertebrates on the western continental shelf and slope of Australia is limited. Collections by French and English explorers in the 18th and 19th centuries generally were from shallow coastal waters of considerably less than $100 \mathrm{~m}$ depth. This first account of composition, geographic range and novelty of deep megabenthic invertebrates from the vast western continental margin of Australia constitutes a significant advance in knowledge for this region. Our data are from the first systematic survey of the deep shelf and slope, and are based on authoritative taxonomic identifications for six dominant taxa of megabethos (i.e. animals large enough to be sorted without microscopy and easily caught in bottom trawls with $25 \mathrm{~mm}$ stretched-mesh net cod-ends) from the survey. The information will aid regional marine management planning by providing insights into areas of high species diversity and endemism, and the associations of fauna with their habitats and geological features affecting their distribution.

\section{BACKGROUND}

Deep continental margins, especially the deep continental shelf and continental slope to depths of about $1500 \mathrm{~m}$, are areas of expanding human use for fishing and for mineral, oil and gas extraction, and a priority target for conservation (Williams et al. 2009; Buhl-Mortensen et al. 2010). Knowledge of species distributions, and biogeographical patterns, are fundamental in planning for sustainable use and effective conservation of these often large marine areas. Australia's current ecosystembased, integrated and regional-scale planning initiatives depend on the identification of natural 'bioregions' as planning units. Such 'bioregions' initially only incorporated data on the distribution of fishes (Heap et al. 2005; Last et al. 2005) because benthic invertebrate biodiversity was insufficiently known or integrated to be included in the national scale analysis of biological distributions. Accordingly, it was uncertain to what extent the distributions of fishes represented the distributions of the larger benthic invertebrates in this national bioregionalisation. The gaps in knowledge, and the imperative for Australia to implement its national network of marine protected areas by 2012, were primary stimuli for additional surveys of benthic invertebrates in Australian waters. This paper, and a companion analytical paper (Williams et al. 2010), report results from one of those surveys.

Research on the benthic invertebrate fauna of continental margins (deep shelf and slope depths
$>100 \mathrm{~m}$ ) has been conducted in relatively few areas of the world oceans, including the northeast Atlantic Ocean and Gulf of Mexico (Howell et al. 2002; Macpherson 2002, 2003; Wicksten and Packard 2005; Escobar-Briones et al. 2008; Rowe and Kennicutt 2008; Narayanaswamy et al. 2010); the northern European continental margin (Gray et al. 1997; Gage et al. 2005) and the north-west Atlantic (Georges Bank - Thouzeau et al. 1991); the north-east Pacific (Ruhl 2007) and off New Zealand to 3,000 m depth (Fell 1958; Clark and McKnight 2000).

In Australia, information on the slope fauna comes from sporadic collections that have provided numerous specimens catalogued in most Australian and some overseas museums, and has resulted in many taxonomic publications. In southeastern Australia, the diversity of megabenthos has been documented on seamounts (Koslow et al. 2001; O'Hara 2007, 2008a), in submarine canyons (Schlacher et al. 2007) and for the temperate deep shelf (Whitelegge 1900; O'Hara and Poore 2000; Ward et al. 2006; O'Hara 2008b). An extensive survey of macrofauna and larger megabenthos was undertaken off south-eastern Australia by Museum Victoria (Poore et al. 1994). However, few syntheses of distributional patterns have been undertaken of slope invertebrate faunas over wide geographic ranges in Australian waters, and regional studies are lacking. Off south-west and central Western Australia, Williams et al. (2010) presented an analysis of the distribution patterns of the benthic invertebrate communities in relation to broad-scale environmental patterns, but details of the species and their distribution were not included. Such detail is the focus of the present paper.

The waters of the western continental margin of Australia contain geographical features such as canyons, seamounts, seeps, the largest western coastal fringing coral reef in the world and the unique southward flowing eastern boundary jet, the Leeuwin Current, from Ningaloo to Cape Leeuwin $\left(22^{\circ} \mathrm{S}\right.$ to $\left.34^{\circ} \mathrm{S}\right)$ (Waite et al. 2007). In the Indo-Australian basin north-west of Australia, shallow, warm, less saline, tropical Indian and West Pacific Ocean waters mix with subtropical Indian Ocean water before joining the Leeuwin current's southward flow along the shelf break $\sim 250 \mathrm{~m}$ (Domingues et al. 2007). Beneath this the more saline, Leeuwin Undercurrent flows northward along the upper continental slope $(\sim 250-400 \mathrm{~m})$, sourced from subtropical water Indian Ocean water, South Indian Ocean Central Water, Subantarctic Mode Water and Antarctic Intermediate Water (Ridgeway and Condie 2004; Waite et al. 2007). Lateral exchange with the offshore ocean plays a large role in the coastal boundary system, with the western edge of the 
Leeuwin Current and waters east of $100^{\circ} \mathrm{E}$ subjected to intense eddy activity, the strongest eddy kinetic field in all the eastern boundary currents globally (Domingues et al. 2007; Feng 2010). Once the Leeuwin Current reaches Cape Leeuwin the more dominant destination is offshore advection into the south-east Indian Subtropical Gyre (with the recognised subsequent loss of larvae). The second destination is rounding the south-west corner of Western Australian and combining with the South Indian Ocean Current (35-37' $\mathrm{S})$ to form the boundary jet moving along the southern Australian continental slope and then down the west coast of Tasmania (Domingues et al. 2007).

Waters off Western Australia are recognised as globally significant biodiversity hotspots for coral reefs and endemism (Roberts et al. 2002). The north-western continental margin also seems to be characterised by relatively high numbers of species at deep shelf and upper slope depths (e.g. decapods - Ward and Rainer 1988). That the same might be true for the south-west continental slope was suggested for benthic fishes (Williams et al. 2001).

The shallow water invertebrate fauna off Western Australia has been studied only in areas adjacent to urban centres and key environmental features where it is readily accessible. Taxonomic workshops have focused on the flora and fauna of shallow coastal waters at Albany, Rottnest Island, Abrolhos Islands, Dampier and Esperance (Wells et al. 1990, 1991, 1993, 2003, 2005; Wells 1997; Walker and Wells 1999). Shallow water surveys have been undertaken in the north-west, e.g. the Dampier Archipelago (Jones 2004), and the north-west atolls, e.g. Mermaid, Scott and Seringapatam Reefs (Bryce 2009).

Various publications on shallow Western Australian invertebrates include: crustaceans (Morgan and Jones 1991; Jones and Morgan 2002), molluscs (Wells and Bryce 1988, 1993; Norman and Reid 2000) and ascidians (Kott 1985, 1990, 1992, 2001, 2005a). Some more general works (Allen and Steene 1994; Edgar 1997; Gowlett-Holmes 2008) include descriptions of some species that extend into deeper waters; nevertheless, the benthic invertebrate fauna of Australia's western margin is little sampled and poorly known.

Australia's west coast was visited by scientific biological expeditions from as early as Dampier (1703). However, the sampling effort generally focused on land or waters (often considerably) shallower than $100 \mathrm{~m}$, and hence the deep water fauna remained completely unknown. The Baudin expedition (1801-1803) (Péron and de Freycinet 1824) collected in very shallow waters along the coast. Two German expeditions led by Michaelsen and Hartmeyer on the Meteor in 1900 and the Hamburg in 1905 sampled mainly off Shark Bay, but also in south-west Western Australian waters, the relevant taxonomic papers are as follows. The Echinodermata type material from that voyage, described by Koehler (1907), Erwe (1913) and Döderlein, (1914) is lodged at the Western Australian Museum, Perth (WAM). Additional material is described by A.H. Clark (1911) and H.L. Clark (1923). Broch (1910) and Kükenthal (1910) described the Alcyonacea and Pennatulacea. A few of Kükenthal's 1910 types are lodged in the WAM although most are in the Museum of Natural History, Berlin. The Mollusca were later described by Thiele (1930) and the brachyuran crabs from the Hamburg Museum expedition were described by Balss (1935). The Ascidiacea, described by Hartmeyer and Michaelsen (1928) are lodged in the Hamburg Museum. Some of these voyages also resulted in species descriptions for sponges (Lamarck 1813-1814; Hentschel 1909, 1911; Weltner 1910) and subsequent revisions (Topsent 1930, 1932, 1933).

In 1908, dredge samples of molluscs were taken off southern Western Australia and in the Great Australian Bight (Verco and Cotton 1935). In 19101912 the federal Fisheries Investigation Ship (FIS) Endeavour conducted two trawling cruises off the mid-west coast of Western Australia, from which the echinoderms were described by H.L. Clark (1916). Octocorals from Dr E. Mjöberg's Swedish scientific expeditions to north-western Australia in 1910-1913 are described by Broch (1916) and are lodged in the Swedish Museum of Natural History, Stockholm. The Ascidians from the same voyage were described by Hartmeyer (1919).

In 1929 and 1932, the Carnegie Australian Harvard (CAH) Expeditions targeted echinoderms from shallow waters to $\sim 100 \mathrm{~m}$ around Australia (H.L. Clark 1938). In the 1960s and 1970s other invertebrate collections were made offshore with the FV Davena, HMAS Diamantina, FRV Umitaka Maru and the MV Sprightly. The HMAS Diamantina sampled the west coast shelf systematically over five cruises from Cape Leeuwin to North West Cape. In 1979 and the 1980s, the RV Soela and more recently the RV Lady Basten sampled on the North West Shelf. Many specimens from these surveys are lodged at the WAM (Echinodermata - Marsh 1976; Marsh et al. 1999; Cnidaria - Griffith and Fromont 1998).

The more recent deep-water marine invertebrate surveys have mostly focused on studies contributing to the management of commercial species (rock lobster, crab, prawn and cephalopods) or areas of economic interest subject to exploration and Environmental Impact Studies for developments in the mining or oil and gas industries of the North West Shelf (George 1962, 1966; Ward and Rainer 1988; Wadley and Evans 1992; Smith et al. 2004; Melville-Smith et al. 2006, 2007; Davie et al. 2007). 


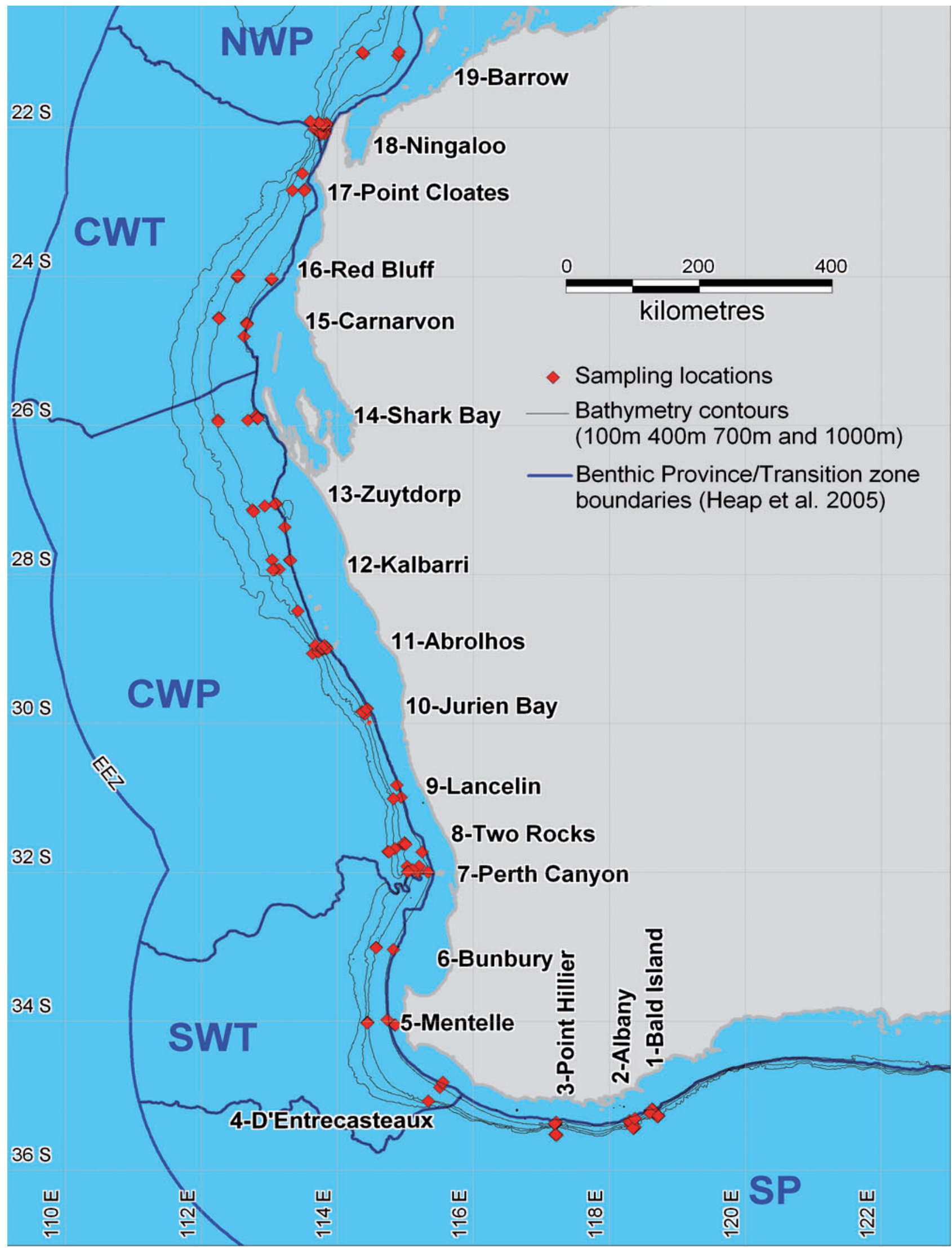

FIGURE $1 \quad$ Location of the sampling stations (red diamonds) at 19 sampling sites along the western continental margin of Australia. Also shown are the 100, 200, 400, 700 and $1000 \mathrm{~m}$ depth contours, the $200 \mathrm{nM}$ Exclusive Economic Zone (EEZ) boundary (thick blue line) and the province/transition zone boundaries of Australia's marine bioregionalisation: Southern Province (SP), Central Western Province (CWP), North Western Province (NWP), South Western Transition Zone (SWT) and Central Western Transition Zone (CWT). 


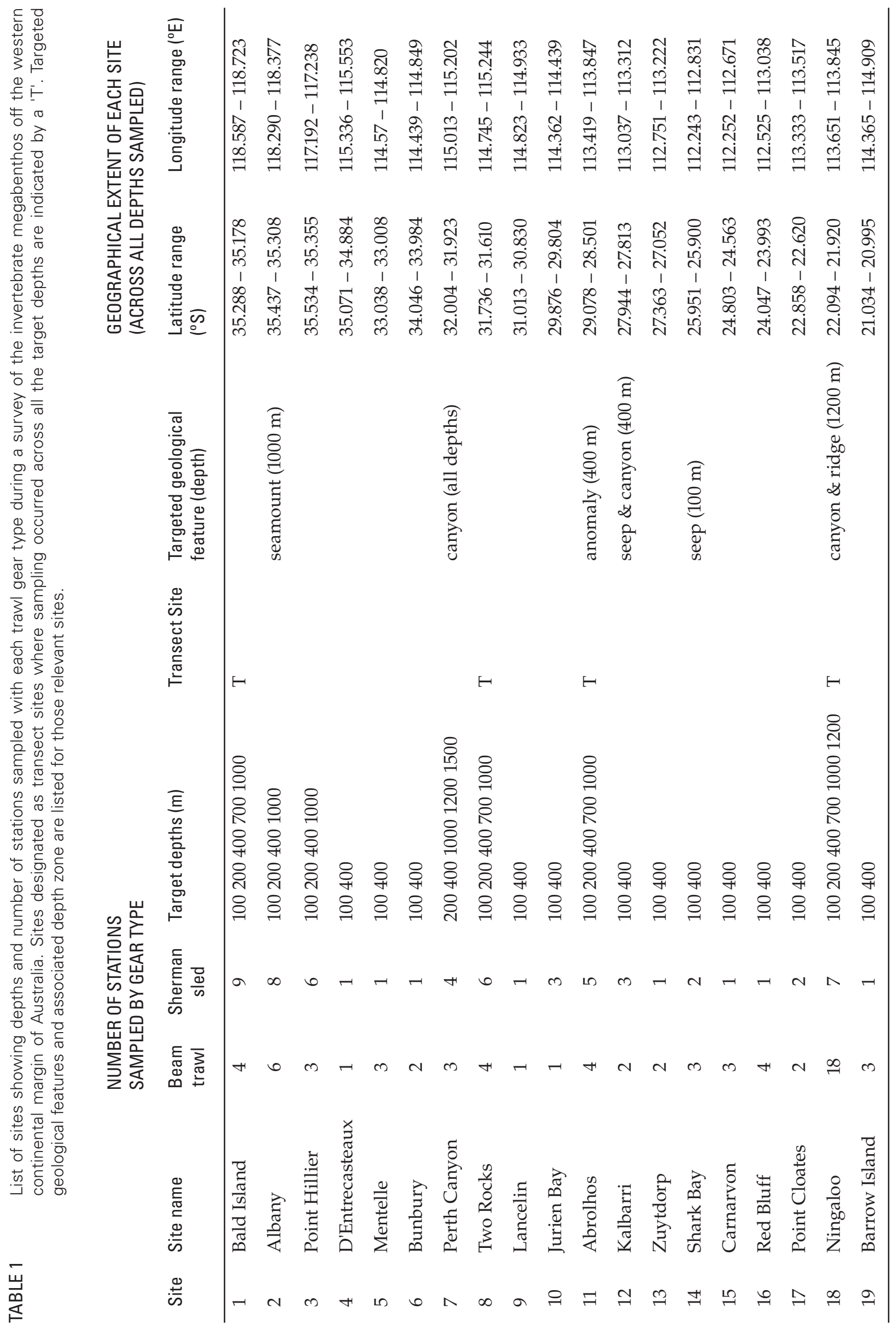



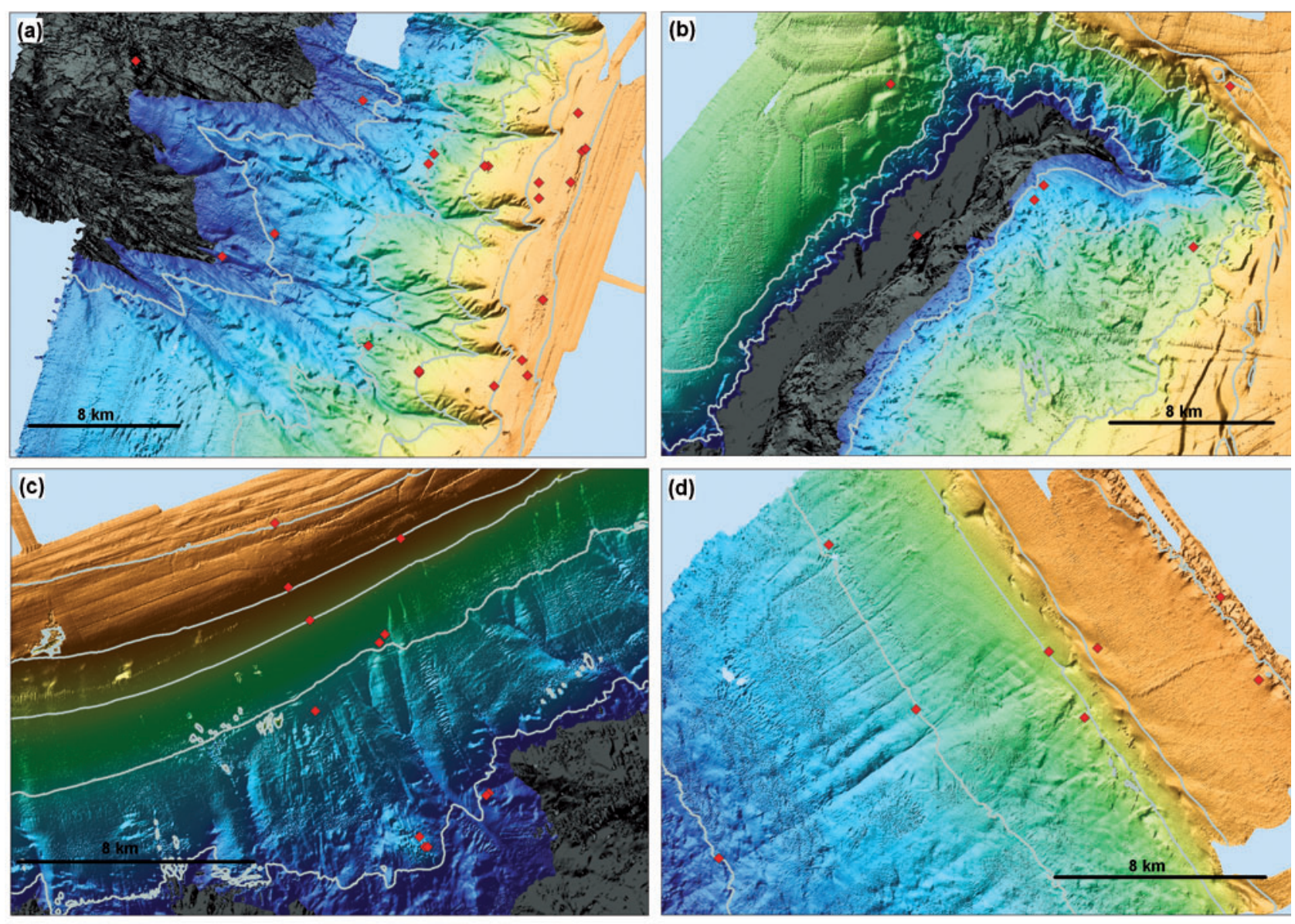

FIGURE 2

Examples of bottom topography and depths at four sites on the western continental margin of Australia mapped using multibeam acoustics: (a) site 18 - Ningaloo, (b) site 7 - Perth Canyon, (c) site 2 - Albany, (d) site 11 - Abrolhos. Colour shading shows depth from 100 m (orange) depth to $\sim 1000 \mathrm{~m}$ (dark blue) with depths >1100 m (black) Sampling stations (red diamonds); 100, 200, 400, 700 and $1000 \mathrm{~m}$ depth contours (grey lines); scale bar $=8 \mathrm{~km}$.

As with material taken elsewhere along Australia's continental shelf and slope, identifying and resolving the taxonomy of marine species collected from deepwater off Australia's western margin is difficult in regard to the availability of relevant information about known species. Many type specimens, including the shallow water material collected on historical exploratory voyages to Australia, are lodged in European museums (London, Paris, Hamburg and Amsterdam) rather than in Australia. Although this historic and irreplaceable information has been carefully conserved and the specimens and associated data are readily accessible to scholars, overseas visits add appreciably to the cost of identification (as much of the material is deemed too precious to be loaned). Further, biological collections and associated data, including voucher specimens, and other data from the more recent environmental assessment surveys for industry, may be commercial-in-confidence, not retained and/or not lodged in a museum and thus not readily available for study.

\section{STATEMENT OF CONTRIBUTION OF THIS PAPER}

This paper is an annotated checklist of the megabenthic invertebrate species collected on a systematic regional-scale survey of the deep shelf and upper slope of the western continental margin of Australia, the first of its kind in Australia. Samples were taken from $100 \mathrm{~m}$ and $400 \mathrm{~m}$ depths at 19 locations from Barrow Island to Albany $\left(\sim 21^{\circ} \mathrm{S}\right.$ to $\left.35^{\circ} \mathrm{S}\right)$, and at additional depths (200 m, $700 \mathrm{~m}$ and $1000 \mathrm{~m})$ at selected sites. Our checklist provides identifications along with biogeographical information and images of species from six target taxa - Demospongiae: Porifera, Octocorallia: Cnidaria, Mollusca, Echinodermata, Decapoda: Crustacea and Ascidiacea: Tunicata and for the additional taxa - Scleractinia: Cnidaria and Antipatharia: Cnidaria and Pycnogonida. Many species are new to science or represent new distribution records for Western Australia and Australia. 


\section{METHODS}

\section{SURVEY DESIGN}

In 2005 , benthic marine invertebrates were collected systematically at sites located at one degree longitudinal intervals off Australia's continental margin between Bald Island and Cape Leeuwin, and one degree latitudinal intervals from Cape Leeuwin to Barrow Island, from the FRV Southern Surveyor (survey SS200510). The sites ranged in latitude from $21^{\circ} \mathrm{S}$ to $35^{\circ} \mathrm{S}$ and longitude from $112^{\circ} \mathrm{E}$ to $118^{\circ} \mathrm{E}$ (over $2050 \mathrm{~km}$ of coastline). The Perth Canyon site was added into the sampling design as a feature of special interest resulting in 19 sites (Table 1, Figure 1). This sampling approach was designed to position sampling stations within each of the three bigeographical provinces and the two transition zones of the western region designated in the national bioregionalisation by Heap et al. (2005) (boundaries are shown in Figure 1). At each site, sampling stations were targeted at the two major bathomes (depth zones) the outer continental shelf (samples taken at $100 \mathrm{~m}$ depth) and upper continental slope (samples taken at $\sim 400 \mathrm{~m}$ ). At selected sites within each province, the sampling design was extended into across-shelf transects to include additional sampling stations within the following bathomes - continental shelf break (at $\sim 200 \mathrm{~m}$ ), deep upper continental slope (at $\sim 700 \mathrm{~m}$ ) and mid-continental slope (at $\sim 1000 \mathrm{~m}$ ). Two very deep sample stations on the mid-continental slope at $\sim 1200 \mathrm{~m}$ at Ningaloo and $\sim 1500 \mathrm{~m}$ in the Perth Canyon also included (Table 1).

Determining the exact locations of the sample sites was supported by detailed maps of the seafloor topography obtained in a prior sister survey of the region by the National Facility Vessel FRV Southern Surveyor in July/August 2005 (SS200507). On that survey, each site was mapped using multibeam acoustics (Kloser et al. 2007) (see mapped examples in Figure 2), the stations surveyed with the towed, high-resolution video and still image system described by Shortis et al. (2008) (see habitat examples in Figure 3). Each station was also sampled with a Smith-McIntyre grab (for sediment analysis and identification of the macrofauna (benthic organisms retained on a $1 \mathrm{~mm}$ sieve when elutriated) (lodged with R. Wilson, Museum Victoria). Based on the SS200507 survey acoustic results, geomorphic features - primarily canyons and a seamount, but also other interesting features such as rocky banks and a seep site - were added to the sampling design for this survey as specific targets (Table 1). The multibeam backscatter data from SS200507 allowed specific targeting of separate soft and hard terrains; these stations were nested with bathomes and features if both terrain types were present for the SS200510 survey. Examples of the various habitats sampled are shown in Figure 3 (from the towed, high-resolution video and still image system imagery taken during the mapping survey SS200507). The surveys and associated environmental data are described in more detail in the National Facility survey reports (2005a, 2005b) and by Williams et al. (2010).

A total of 172 stations at 19 sites were sampled along $2050 \mathrm{~km}$ of the western continental margin between $85 \mathrm{~m}$ to $1584 \mathrm{~m}$ depths, covering both soft and hard terrains (when present).

\section{INVERTEBRATE COLLECTION}

Megabenthic invertebrates (megabenthos: benthic organisms large enough to be sorted without microscopy and easily caught in bottom trawls with $25 \mathrm{~mm}$ stretched-mesh net cod-ends (as opposed to macrofauna: benthic organisms retained on a $0.5-1 \mathrm{~mm}$ sieve) were collected with two epibenthic samplers - a 'Sherman' sled and a beam-trawl. The Sherman sled (Lewis 1999, 2009) is a robust heavy design for use on rocky (hard) terrains and steep gradients. Its mouth dimensions are $1.2 \times 0.6 \mathrm{~m}$. The beam-trawl was a CSIRO modified version of the French IRD design light beam-sled (Forest 1981; Lewis 2010) that was used on soft sediment terrains. Its mouth dimensions are $4.0 \times 0.5 \mathrm{~m}$. Images and basic descriptions of both gears can be found at: http://www.cmar.csiro.au/research/ seamounts/epibenthic.htm. Tow distances along the seabed were measured using a USBL beacon attached to the sleds to determine the duration of bottom contact, and dynamic GIS mapping to plot the tow track. Tow direction and duration was dependent on the roughness and topography of the substrate. In general, tows were along depth contours and of about 20 minutes duration (but were less for stations when the sled was full of catch within 5-10 minutes).

The entire megabenthic invertebrate catch was sorted and identified at sea by taxonomists to the lowest taxonomic resolution possible; these nominal species are herein known as 'operational taxonomic units' (OTUs). Each OTU was counted, weighed (to the nearest gram) and representatives photographed prior to preservation to record live colours, and then included in a continually updated image reference catalogue for comparative purposes while at sea. Proof sheets of images of live specimens are shown in Appendix 2. Specimens were preserved in the standard medium for that phylum (e.g. 70\% ethanol - Porifera, Echinodermata, Octocorallia, Scleractinia, Antipatharia, and some Mollusca; 10\% formalin Decapoda, some Mollusca, Ascidiacea and other minor groups). Additional material from various 

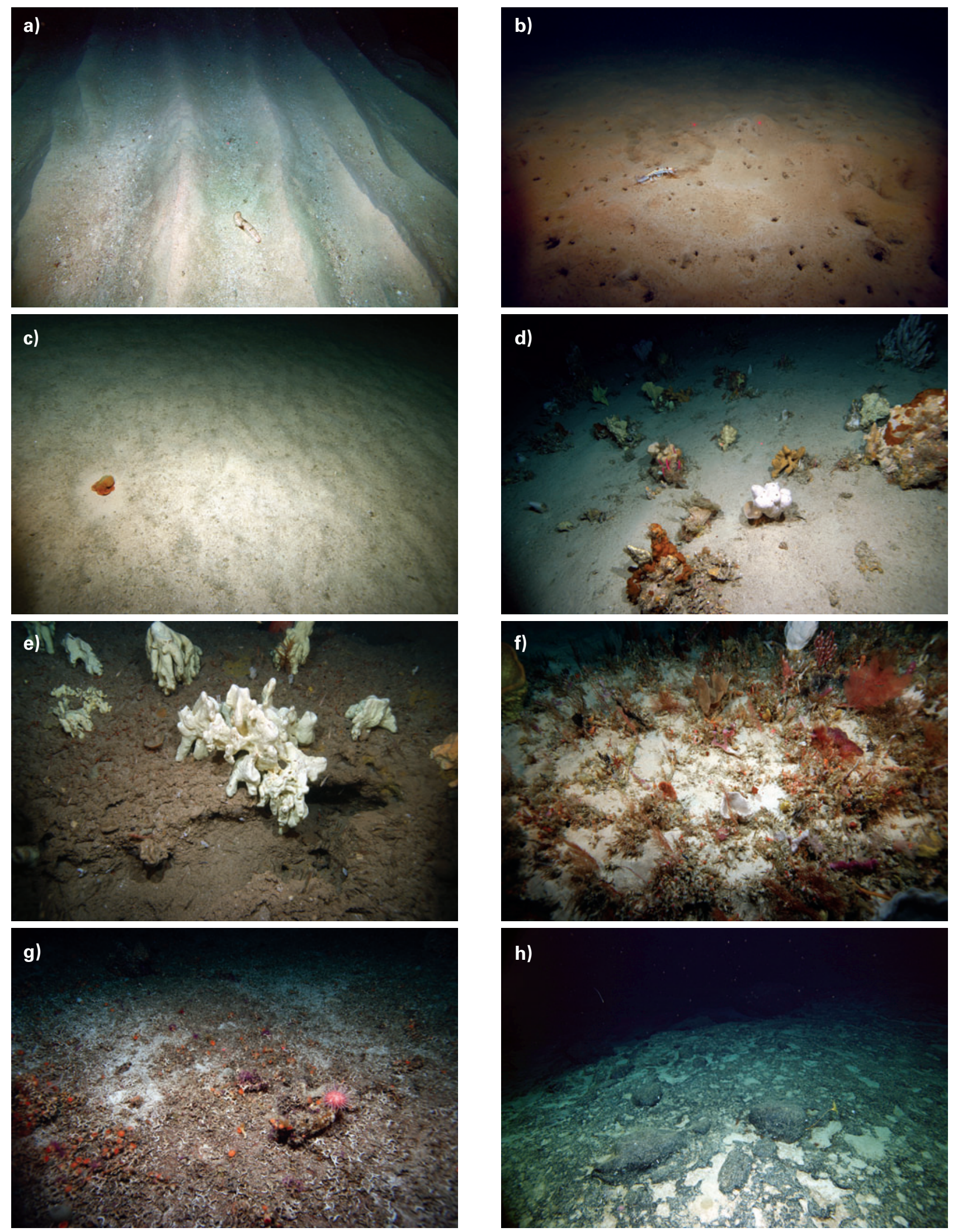

FIGURE 3

Example images of habitat types sampled along the western continental margin of Australia taken with the towed, high-resolution video and still image system on the SS200507 survey: (a) hard rippled sand (site 13 - Zuytdorp, 100 m depth); (b) Soft muddy sediments (site 18 - Ningaloo, 200 m depth); (c) sand (site 17 - Point Cloates, $400 \mathrm{~m}$ depth); (d) layer of sand over hard substrate (site 2 - Albany, $200 \mathrm{~m}$ depth); (e) reef (site 18 - Ningaloo, 100 m depth); (f) sandy reef (Site 13 - Zuytdorp, 100 m depth); (g) deep coral on seamount (site 2 - Albany, 1000 m); (h) deep bare rock (site 2 - Albany, 1000 m depth). 
specimens was preserved in $95 \%$ analytical-grade ethanol for potential genetic analyses.

Species taxonomy is tracked using a computerised Code for Australasian Aquatic Biota (CAAB). These numbers consist of eight digits and are assigned to each described species in Australian waters. The first two digits define the major group, e.g. phylum; the next three digits define the family or other higher group; the last three digits are the species within that family or group, e.g. CAAB number 28821004 is for decapod crustacean, Ibacus peronii Leach, 1815. A code starting with ' 99 ' is an OTU that has not been described or distinguished further. The second two digits in a '99' code are equivalent to the first two digits in a permanent CAAB number; '99' codes are issued sequentially and the order of the remaining digits do not have taxonomic significance. '99' codes are used until a species is described, when it is replaced by a permanent CAAB number. ' 99 ' codes can be accessed through the public $\mathrm{CAAB}$ database by ticking the project specific codes box on the search screen, e.g. CAAB number 99100454 is for the uncertain sponge species, Clathria (Thalysias) cf. juniperina. Updates to species names can be obtained by checking the $\mathrm{CAAB}$ number in the online database at http://www.marine.csiro.au/ caab. The following six major taxonomic groups were selected as target groups for identification to species-level: Porifera (Demospongiae), Cnidaria (Octocorallia), Mollusca, Echinodermata, Decapoda and Ascidiacea (Table 2a). Their selection was based on several factors including their prominence in field collection ( $80 \%$ of catch) and the availability of specialist taxonomists. The remaining nontarget invertebrate taxa were also distributed to museums for lodgement and curation (Table 2b). Identifications have been undertaken on some of these groups i.e. Pycnogonida, Antipatharia and Scleractinia, and these are also included in this manuscript.

Once identifications were completed by the taxonomists, each species was allocated a permanent $\mathrm{CAAB}$ number and species recorded in Australian waters for the first time were added to the $C A A B$ database. Published names of new species were upgraded for the associated images. Catch composition records were also upgraded as species taxonomy was reviewed; weights were adjusted for lots where the OTU has been split into multiple species.

This checklist uses the following terms to categorise the status of species identified. 'Described species' are those species whose description was in the published literature prior to this survey e.g. Dermechinus horridus (A. Agassiz, 1879). 'New species' are those species collected on this survey determined by the taxonomists to be undescribed e.g. Oparinisis n. sp. A or Ophiura sp. MoV2734 ('MoV number' is a unique code used by taxonomists at Museum Victoria). Some new species descriptions have been published subsequent to the survey e.g. Oxypleurodon wilsoni Richer de Forges and Poore, 2008. The remainder of the examined taxa are categorised as 'uncertain species'. These taxa were identified to the lowest OTU possible (in some cases only to genus or family level) but could not be confidently determined as previously described taxa, or recognised as new species either. The possibility exists that these species may belong to known species but taxonomic uncertainty precludes a definite identification. In addition, some species were identified as uncertain using the 'cf.' or '?' prefix. Examples of uncertain species include: Raspailia (Raspaxilla) sp. SS1, ?Hyotissa sp. 1, Bathycrinidae sp. 1, cf. Patagiaster sp. 1 and Dermomurex cf. antonius.

Data presented here are based on quality assured data confirmed against the original field collection station data and images. Unsuccessful (aborted) sampling events and specimen lots with incomplete location data have been removed from the summary analyses. Information on the biogeographical distributions of the previously described species and whether they were new records for Australian or Western Australian waters, or Australian endemics was collated from the literature and the taxonomists' knowledge.

The checklist (Appendix 1) summarises the geographical and depth ranges for each species or OTU recorded on the survey. The data have not been corrected for varying sampling intensity among sites. This list, and all summaries and analyses presented here, represent the upgrades and species descriptions as of December 2010.

The checklist (Appendix 1) summarises the geographical and depth ranges for each species or OTU identified on the survey. The species names and OTU designations in this list, and presented in the summaries within this publication, reflect the taxonomic work published and/or received by CSIRO as of December 2010.

\section{RESULTS}

\section{OVERVIEW OF FAUNAL COMPOSITION}

Porifera dominated the collection by biomass, comprising $86 \%$ of the total catch (wet weight). The other target groups in decreasing order, each at least an order of magnitude less by weight, were: Echinodermata, Decapoda, Mollusca, Cnidaria (Octocorals, Antipatharia and Scleractinia) and 
Ascidiacea. The largest specimen, belonging to the Demospongiae, was a single individual of Biemna sp. SS2 that weighed $37 \mathrm{~kg}$. Additional large individuals in this genus weighed 17, 6 and $5 \mathrm{~kg}$ (see Appendix 2: image 10084810). Another 126 individual Demospongiae specimens were over $1 \mathrm{~kg}$ wet weight. The heaviest non-sponge invertebrate specimens were of the Holothuroidea and Echinoidea and these weighed less than $1 \mathrm{~kg}$.
At sea, the sorting of the samples yielded 1,750 nominal 'species' (OTUs) across all the invertebrate taxa (see Table 2a, b), with the six major taxonomic groups accounting for $80 \%$ of these. Over 19,000 specimens comprising 2,001 species were subsequently identified in the laboratory by the taxonomists from the six major groups (a further 38 OTUs were only identified at higher taxonomic levels). Of the examined species, 876

TABLE 2 List of the lodgement locations and taxonomic experts working on specimens of the invertebrate megabenthos off the western continental margin of Australia. The number of lots of specimens from the field data are shown for each major taxon.

2A)

Specimen lodgement details for the major taxa targeted for identification.

Note: holotypes of newly described species are lodged at Western Australian Museum

TAXONOMIC GROUP
TAXONOMIST

AND TAXONOMIST

AFFILIATION
SAMPLE

LODGEMENT

LOCATION
NUMBER OF

SPECIMEN LOTS

RECORDED IN

FIELD DATA

\begin{tabular}{|c|c|c|c|}
\hline Porifera: Demospongiae & Jane Fromont & $\begin{array}{l}\text { Western Australian } \\
\text { Museum }\end{array}$ & 889 \\
\hline Cnidaria: Octocorallia & Phil Alderslade & $\begin{array}{l}\text { Museum \& Art } \\
\text { Gallery of the } \\
\text { Northern Territory }\end{array}$ & 150 \\
\hline Mollusca: Bivalvia & Shirley Slack-Smith & $\begin{array}{l}\text { Western Australian } \\
\text { Museum }\end{array}$ & 285 \\
\hline Mollusca: Scaphapoda & Shirley Slack-Smith & $\begin{array}{l}\text { Western Australian } \\
\text { Museum }\end{array}$ & 32 \\
\hline Mollusca: Gastropoda & Shirley Slack-Smith & $\begin{array}{l}\text { Western Australian } \\
\text { Museum }\end{array}$ & 357 \\
\hline Mollusca: Opisthobranchia & $\begin{array}{l}\text { Richard Willan - Museum } \\
\text { \& Art Gallery of the } \\
\text { Northern Territory }\end{array}$ & $\begin{array}{l}\text { Western Australian } \\
\text { Museum }\end{array}$ & 50 \\
\hline Mollusca: Polyplacophora & $\begin{array}{l}\text { Karen Gowlett-Holmes - } \\
\text { CSIRO }\end{array}$ & $\begin{array}{l}\text { Western Australian } \\
\text { Museum }\end{array}$ & 10 \\
\hline Mollusca: Cephalopoda & Mark Norman & Museum Victoria & 71 \\
\hline Echinodermata: Asteroidea & Loisette Marsh & $\begin{array}{l}\text { Western Australian } \\
\text { Museum }\end{array}$ & 187 \\
\hline Echinodermata: Crinoidea & Tim O’Hara & Museum Victoria & 53 \\
\hline Echinodermata: Echinoidea & Ashley Miskelly & Australian Museum & 103 \\
\hline Echinodermata: Holothuroidea & Mark O'Loughlin & Museum Victoria & 108 \\
\hline Echinodermata: Ophiuroidea & Tim O’Hara & Museum Victoria & 343 \\
\hline Crustacea: Decapoda & Gary Poore & Museum Victoria & 1109 \\
\hline Tunicata: Ascidicea & Patricia Mather & $\begin{array}{l}\text { Queensland } \\
\text { Museum }\end{array}$ & 80 \\
\hline
\end{tabular}


2B) Specimen lodgement details for the remaining non-target taxa with names of taxonomists who have an interest in working up the samples.

TAXONOMIC GROUP

TAXONOMIST
AND TAXONOMIST
AFFILIATION

TAXONOMIST

AFFILIATION

\section{SAMPLE LODGEMENT LOCATION}

NUMBER OF

SPECIMEN

LOTS RECORDED

IN FIELD DATA

Cnidaria: Medusozoa \&

Australian Museum

4

Ctenophora

Cnidaria: Hydrozoa

Museum Victoria

97

Cnidaria: Antipatharia

Karen Miller - Institute

Australian Museum

13

of Marine and Antarctic

Studies, University of

Tasmania

Tina Molodstova -

P.P. Shirshov Institute of

Oceanology, Russia

Cnidaria: Actinaria

Carden Wallace - Museum

Western Australian

108

Tropical Queensland

Museum

Cnidaria: Scleractinia

Marcelo Kitahara - Museum

Tropical Queensland

Western Australian

Museum

Stephen Cairns - National

Museum of Natural History,

Smithsonian Institute, USA

Australian Museum

187

Annelida: Polychaeta

Australian Museum

57

Echiura

Brachiopoda

Phil Bock

Australian Museum

33

Bryozoa

Museum Victoria

105

Crustacea: Cumacea

Crustacea: Mysidacea

Australian Museum

9

Australian Museum

5

Crustacea: Ostacoda

Crustacea: Copepoda

Australian Museum

Australian Museum

Crustacea: Cirripedia

Di Jones

Western Australian

Crustacea: Amphipoda

Museum

Australian Museum

32

Australian Museum

72

Shane Ahyong

Australian Museum

30

Claudia Arango -

Australian Museum

19 
(a)

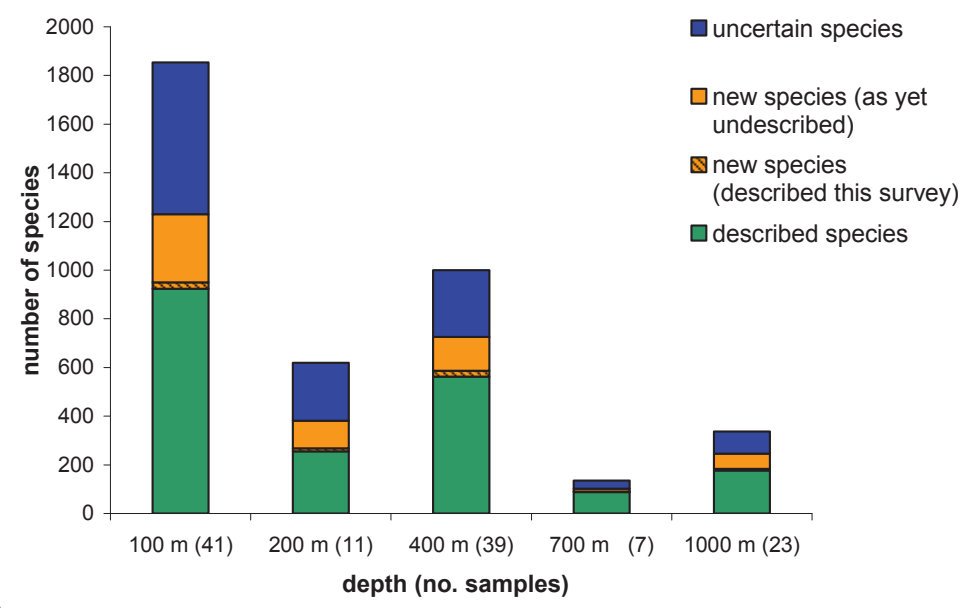

(b)

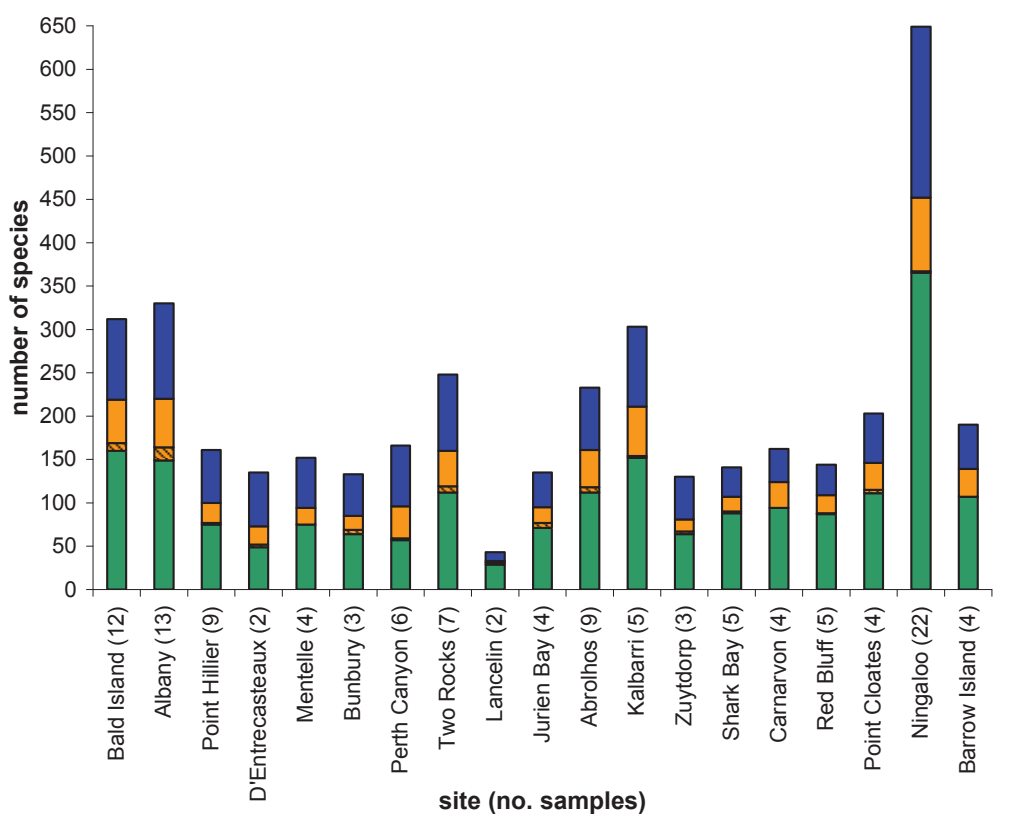

(c)

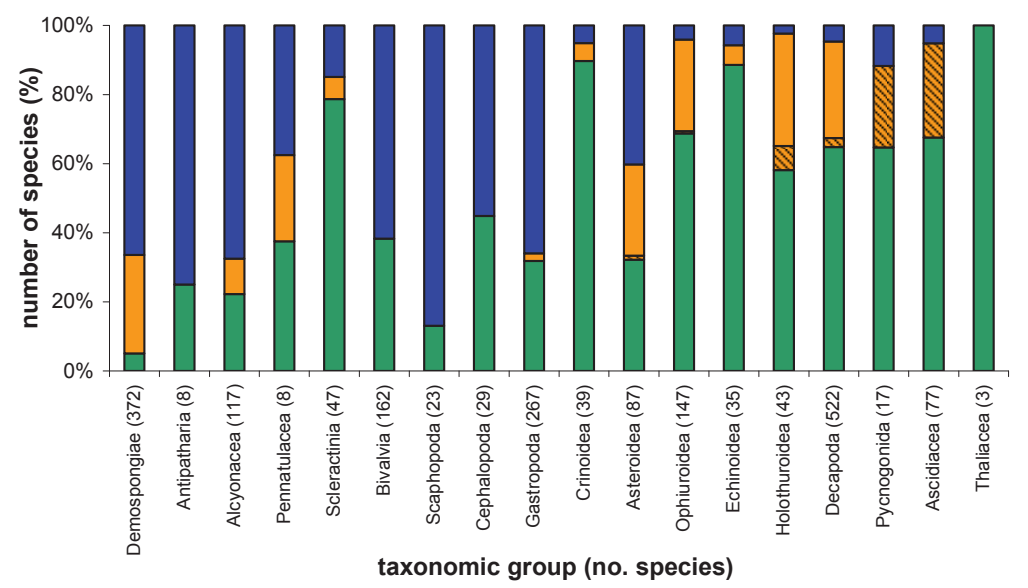

FIGURE 4 The numbers of described, 'unknown or uncertain' and new species of megabenthic invertebrates collected off the western continental margin of Australia: (a) by target depths, (b) by site and (c) by the major taxa identified (number of species shown in brackets after taxa name on x-axis). Note: species numbers are not standardised by sample size or swept area, and $1000 \mathrm{~m}$ also includes the two stations at $1200 \mathrm{~m}$ and $1500 \mathrm{~m}$. 


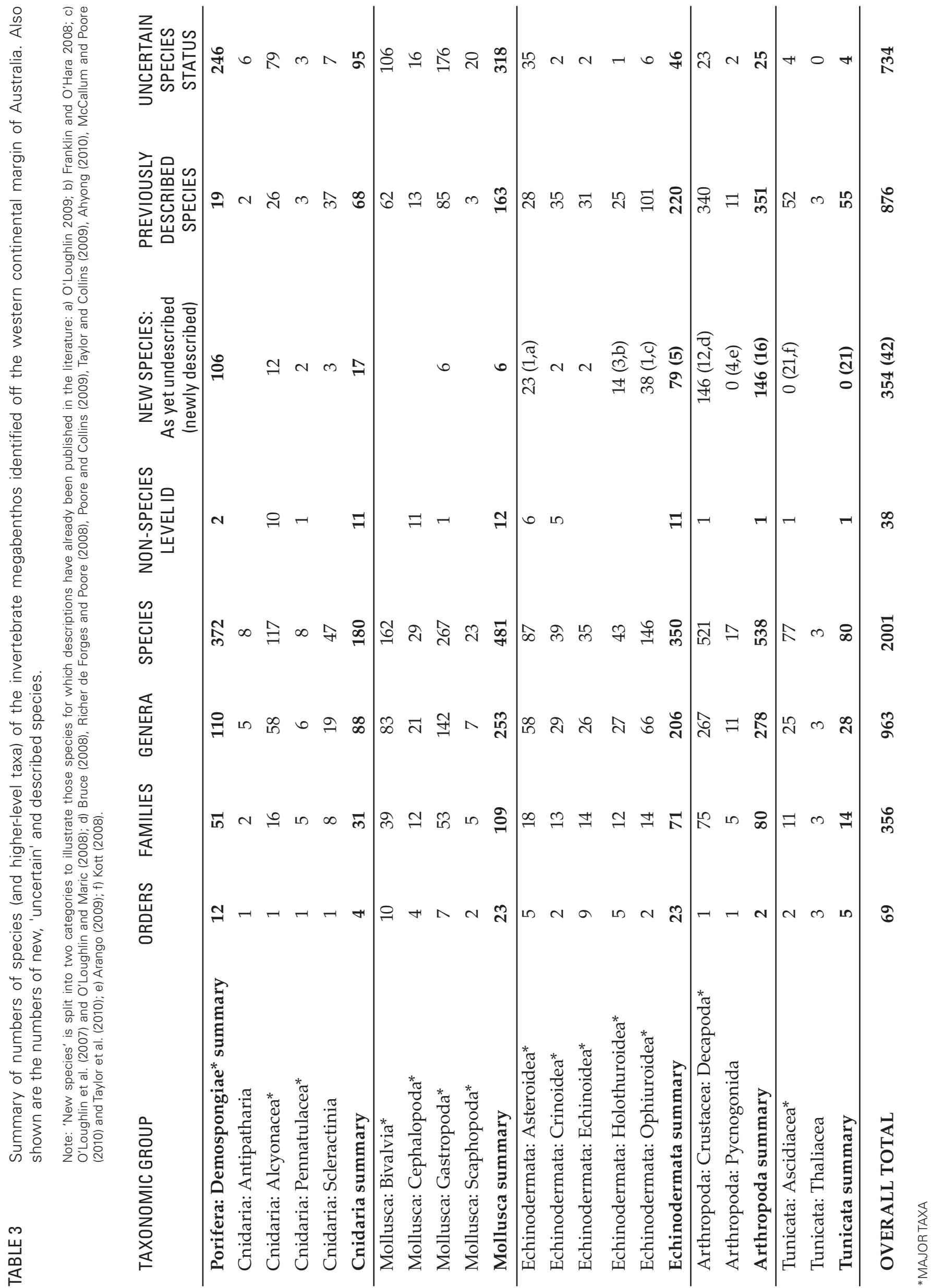




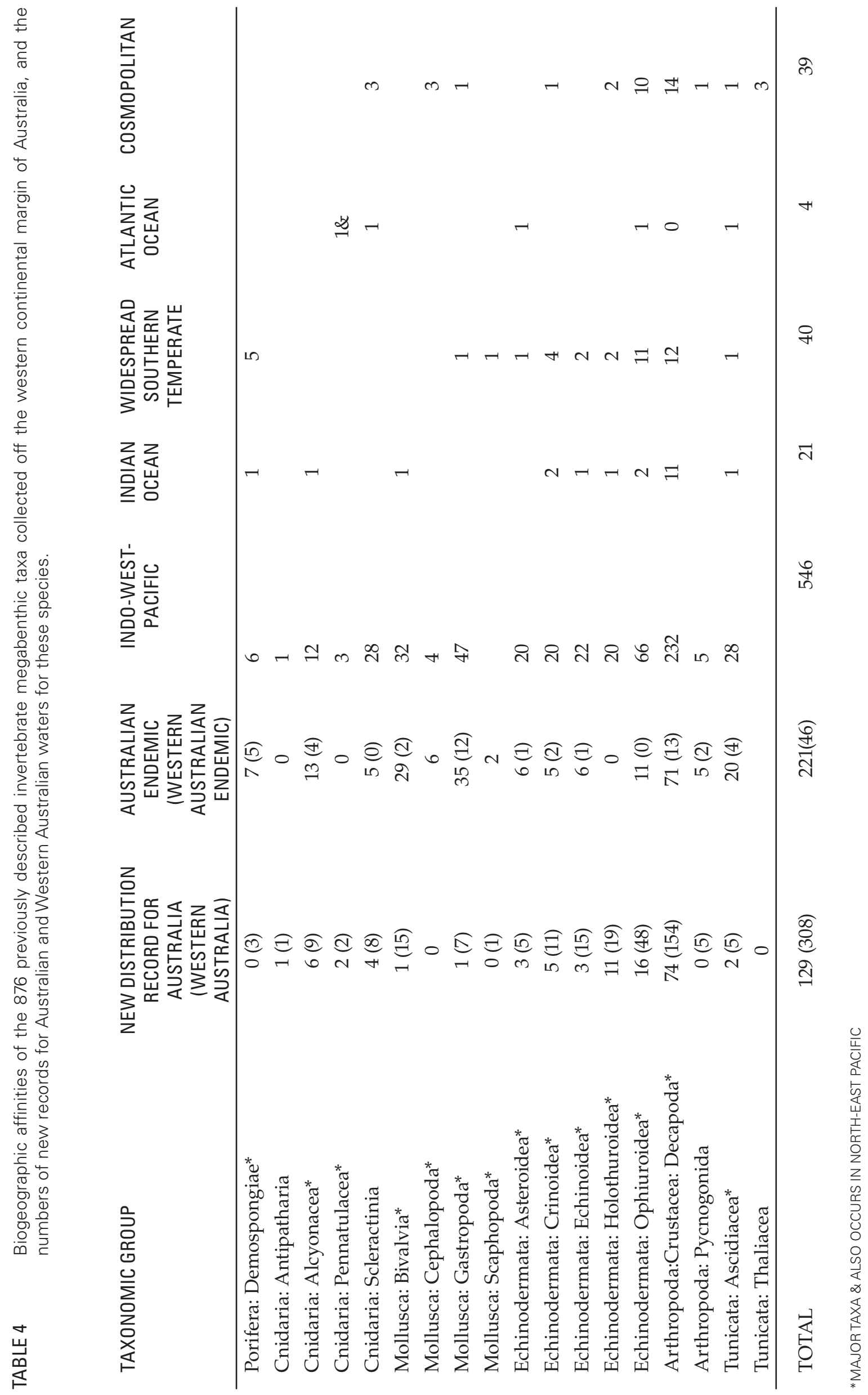


(a) $100 \mathrm{~m}$
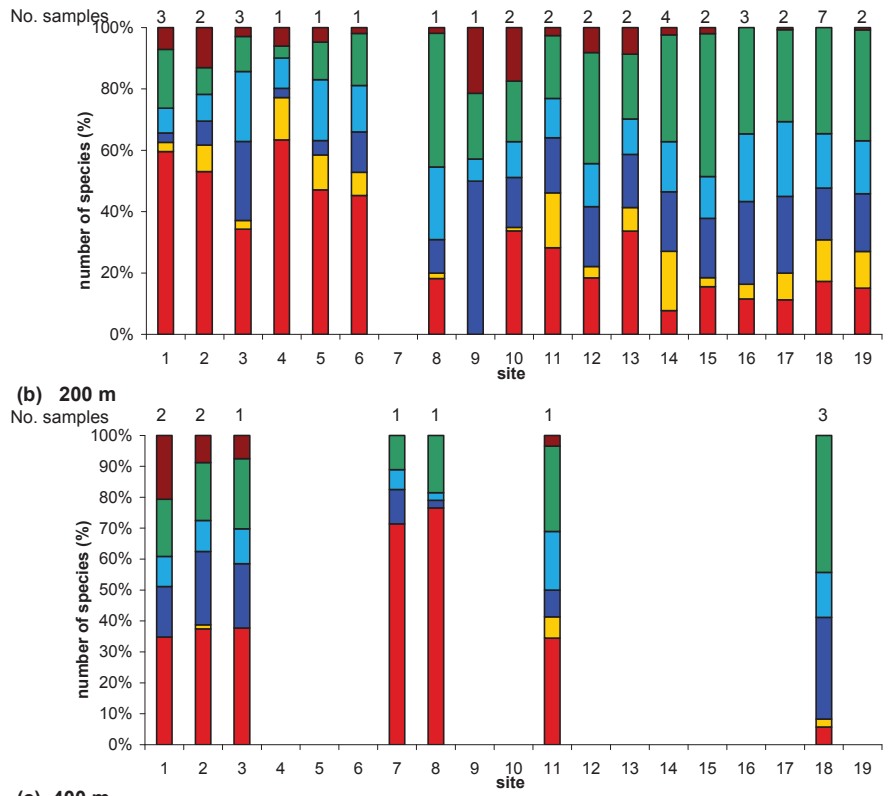

(c) $400 \mathrm{~m}$

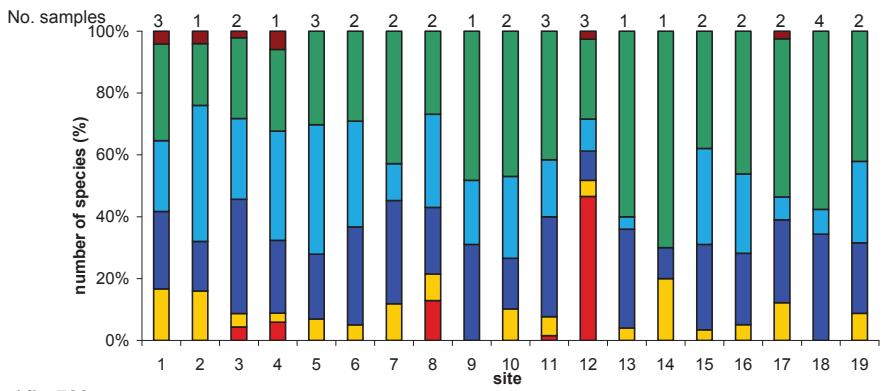

(d) $700 \mathrm{~m}$
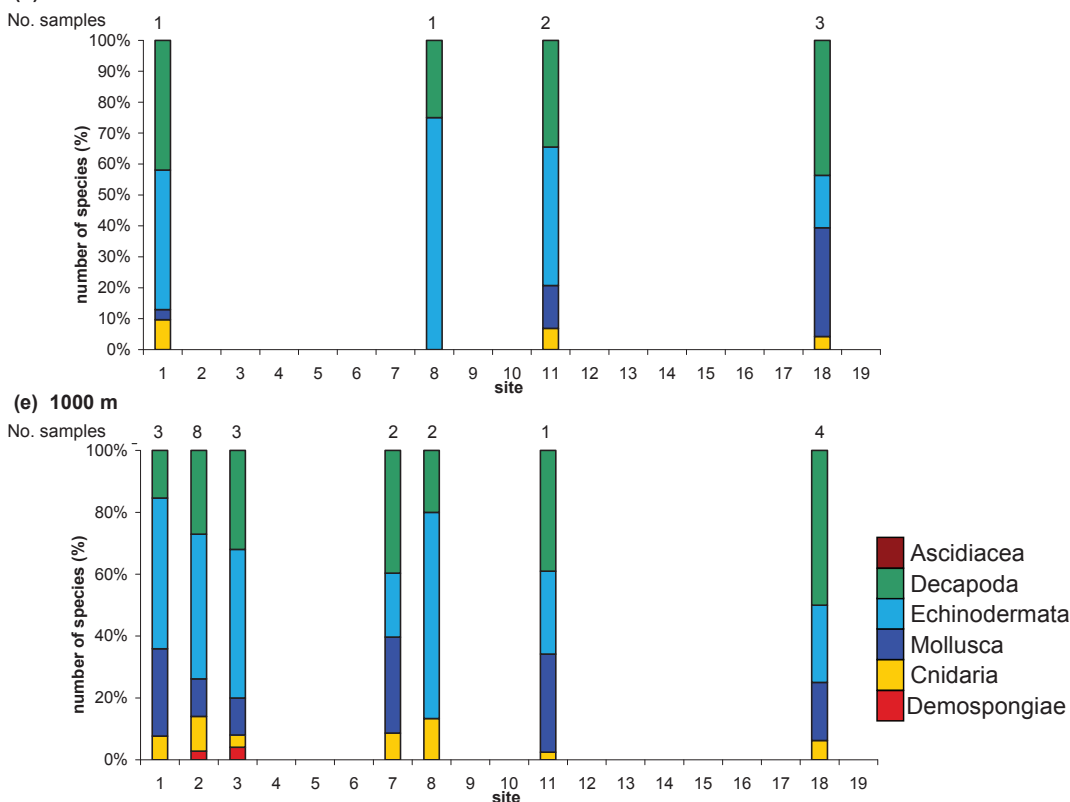

FIGURE 5 The proportions of the six major megabenthic invertebrate taxa off the western continental margin of Australia occurring at each site shown for five depth zones; (a) 100 m, (b) 200 m, (c) 400 m, (d) 700 m (e) $1000 \mathrm{~m}$; site names are shown in Figure 1 and Table 1. Number of stations sampled at each depth is shown at top of columns. Note: species numbers are not standardised by sample size or swept area, and $1000 \mathrm{~m}$ also includes the two stations at $1200 \mathrm{~m}$ and $1500 \mathrm{~m}$. 


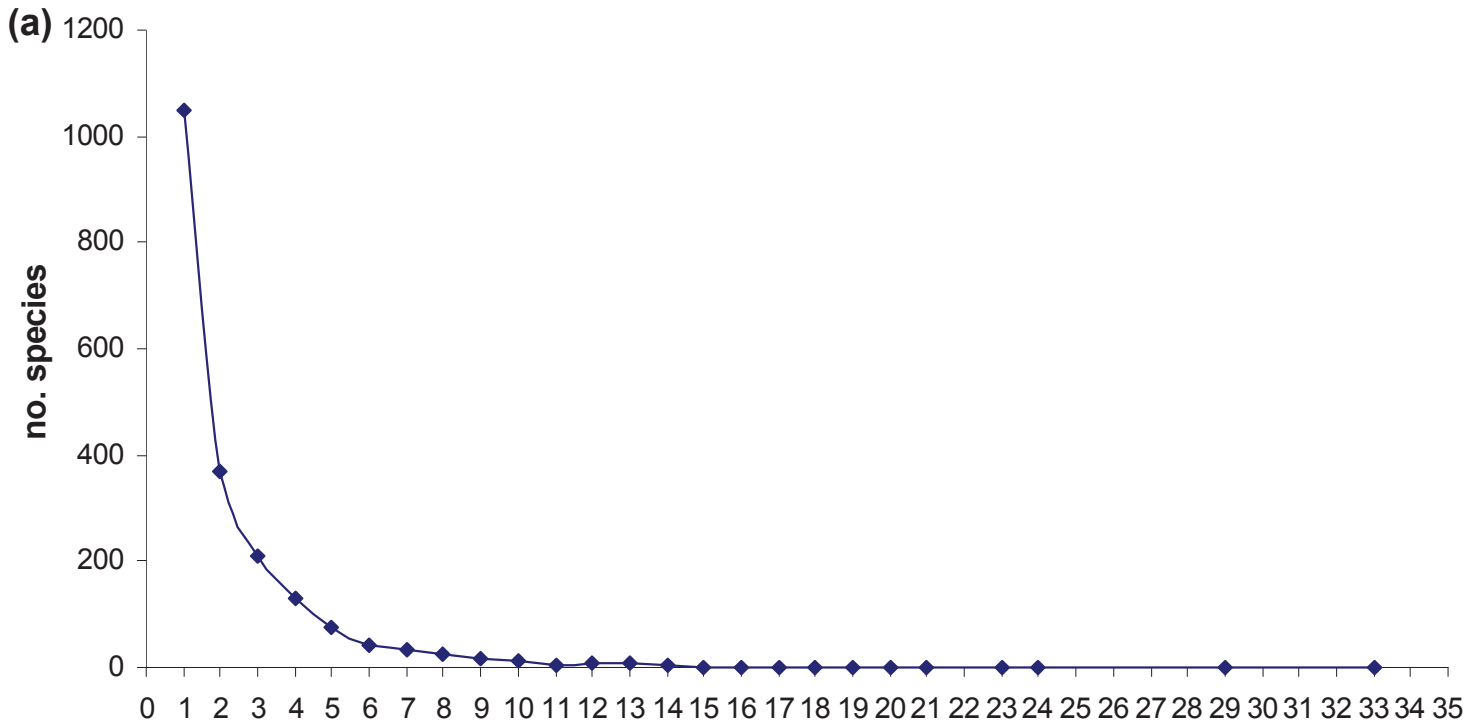

no. stations

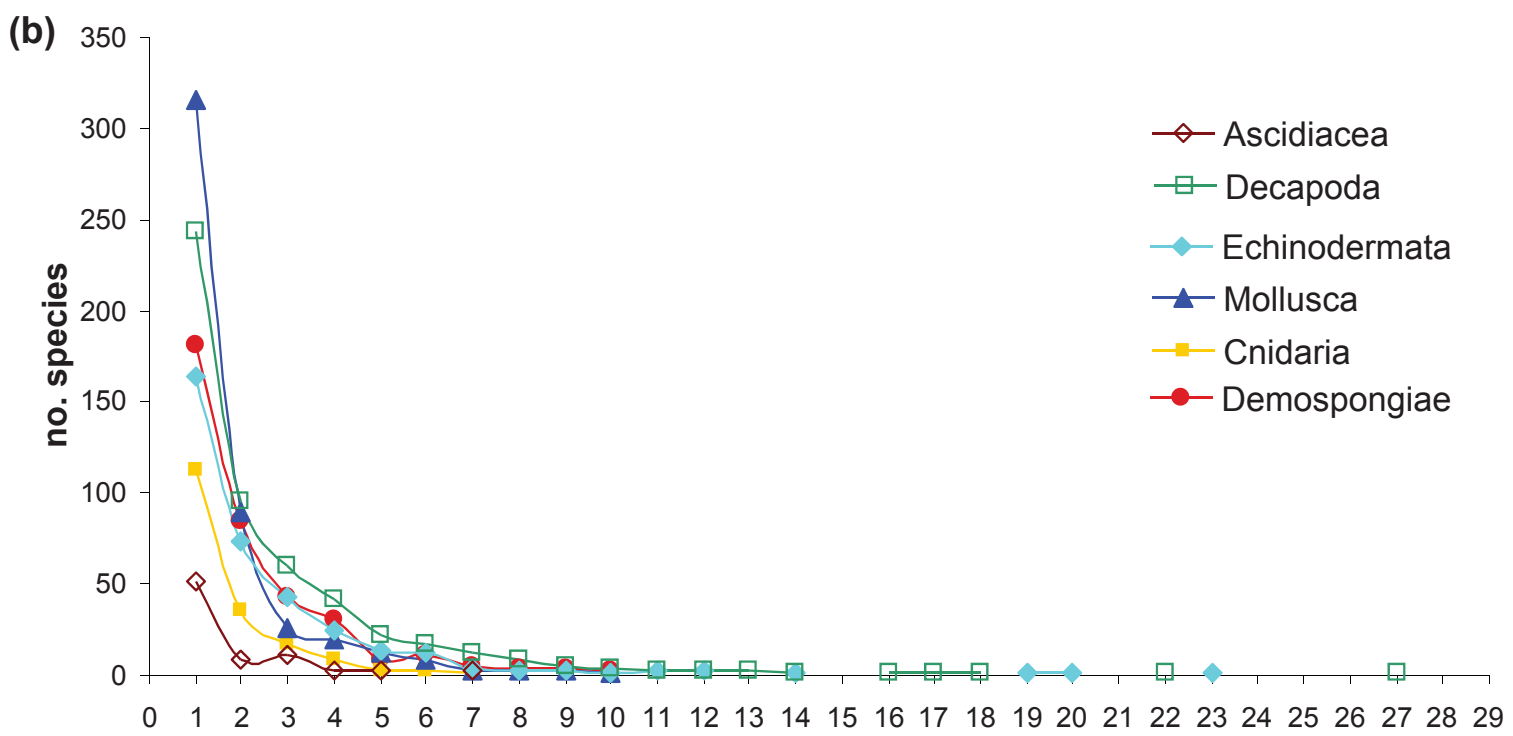

no. stations

FIGURE 6 The [ranked] occurrence of the megabenthic invertebrate taxa off the western continental margin of Australia by the number of stations each species was present: (a) summary of all species, (b) by the six major megabenthic invertebrate taxa.

$(44 \%)$ were identified as previously described species and $396(20 \%)$ were determined to be new species. The remaining 727 species $(36 \%)$ remain of uncertain identity. To date, 42 new species have been described from this collection. Some groups are better studied than others: $89 \%$ of the Echinoidea and $79 \%$ of the Scleractinia could be identified as described species, while only $5 \%$ of the demosponges and $22 \%$ of the Alcyonacea were recognised as described species (Table 3, Figure 4c).

Of the six major groups, the most speciose was the Decapoda with 521 species. The Mollusca with 481 species (excluding the Opisthobranchia and Polyplacophora) were the second most diverse group, followed by the Demospongiae (372), the Echinodermata (350), the Cnidaria (Octocorallia, Antipatharia and Scleractinia) (180) and the Ascidiacea (77). In addition, 17 species of Pycnogonida and 3 Thaliacea were recorded. Numerically the Echinodermata (6,829 specimens) and the Decapoda $(6,206)$ dominated the collection, followed by the Mollusca $(2,588)$ - excluding the 
Opisthobranchia and Polyplacophora, the Cnidaria (Octocorallia, Antipatharia and Scleractinia) $(1,916)$, the Demospongiae $(1,288)$ and the Ascidiacea $(458)$ (Table 3).

\section{FAUNAL DISTRIBUTIONS}

One-quarter (522) of all the species collected on the survey are new records for Australian waters. Of these, 129 records are the first record in Australian waters for a species previously described from elsewhere in the world; the remaining records are confirmed as new species. Over one-third (726) of all the species collected are new records for Western Australian waters, of these 308 are species known from elsewhere in the world (including the range extension of 175 Australian endemic species into Western Australia) and the remainder are confirmed as new species (Table 4).

\section{BIOGEOGRAPHY OF DESCRIBED SPECIES}

For the 876 species previously described in the literature (and hence with known distributions) collected on this survey, the largest proportion (61\%) had an Indo-West-Pacific biogeographic distribution, while only $4 \%$ were restricted to the Indian Ocean. Australian endemics accounted for $25 \%$ of species distributions, including $5 \%$ endemic to Western Australian waters. Southern Ocean or sub-Antarctic species represented 5\%, species previously recorded only from the Atlantic Ocean 1\%; and cosmopolitan species $4 \%$ of the biogeographic distributions. The biogeographic affinities of the identified megabenthos are shown in Table 4 along with the overall species totals for the taxonomic groups.

Depth appeared to strongly influence species diversity with many more species collected on the shallower outer shelf $(100 \mathrm{~m}$ depth) than on the upper slope $(400 \mathrm{~m})$ where sampling coverage and intensities were similar (41 compared with 39 sample collections) (Figure 4a). The proportions of the six major megabenthic invertebrate taxa caught at each site are shown for each of the five depth zones (Figure 5). The Demospongiae and the Ascidiacea were collected mostly from the shallow $100 \mathrm{~m}$ and $200 \mathrm{~m}$ stations and were considerably more diverse (with more species) in the temperate sites south of Two Rocks (Figure 5a,b). These shallower temperate sites appeared to contain abundant hard substrate that provided suitable habitat and access to favourable nutrient flow in the currents for these fixed sessile filter feeding taxa (also see Kott 2008; Williams et al. 2010). Corals were not diverse but were found to be locally abundant. No clear depth or site related patterns were discernible for the Mollusca, Decapoda or
Echinodermata at this high level of taxonomic resolution.

Overall, more species were collected where sampling effort was greatest - especially in the focus areas of Ningaloo, Albany and Bald Island, and lowest at sites where few collections were made (e.g. only two sample collections at Point D'Entrecasteaux and Lancelin) (Figure 4b). While unequal sample numbers made it difficult to compare site-level diversity across the entire data set, it is interesting to note that the total number of species collected was relatively consistent across all sites for which there were between two and six sampling stations with only Kalbarri (five stations) standing out as richer (Figure $4 b$ ).

The percentage of described species compared with the uncertain and new species per site was greater for sites north of Shark Bay; accounting for at least $55 \%$ of the species (Figure $4 b$ ).

For sites south of Shark Bay the opposite was found, driven by the large number of uncertain and new demosponge species recorded from the southernmost temperate sites at $100 \mathrm{~m}$ depth (Figures 4c, 5a,b).

The percentage of described species at each site ranged from $34-63 \%$, lowest at Perth Canyon and highest at Lancelin. The percentage of new species per site was fairly steady between $11-24 \%$, being lowest at Lancelin and highest at Abrolhos. Only two stations were sampled at the Lancelin site at $100 \mathrm{~m}$ and $400 \mathrm{~m}$ depths due to the narrowness of the shelf and lack of variation in the terrain (steep gradient, compressed habitat, soft substrate - no sponges were collected from these sites). The percentage of uncertain species per site ranged from $19-31 \%$, being lowest at Carnarvon and adjacent tropical sites and highest at Point D’Entrecasteaux.

The collection is dominated by a high percentage $(>50 \%)$ of singletons (occurring at one site only) (Figure 6a) and this pattern is consistent between the six major taxa (Figure 6b). For the six major taxa sampled, 1,075 of the 2,001 species were recorded at only one of the 172 stations sampled. The most frequently recorded species of the entire survey, the hermit crab Paguristes aciculus, occurred at 27 stations from Bald Island to Ningaloo in depths from $100-550 \mathrm{~m}$.

\section{DESCRIPTIVE SUMMARY AND OBSERVATIONS ON IDENTIFIED TAXA}

The following summaries are ordered taxonomically; please use the numerical sequence of the $C A A B$ numbers to facilitate comparison with the checklist of species in Appendix 1 and the species images in Appendix 2. The 
summaries include: a) a numerical summary of each taxa at relevant levels of taxonomic hierarchy, i.e. species, genera and families including numbers of new species; b) a breakdown of the biogeographical distributions of each taxa for the previously described species to illustrate worldwide affinities; and c) any other relevant points and interesting features of that taxa. For the larger groups, analyses by several different taxonomists and an overall summary at the higher level taxa are included, e.g. molluscs.

\section{PORIFERA: DEMOSPONGIAE (CAAB NUMBERS 1000\#\#\# \& 99100\#\#\#)}

\section{A) NUMERICAL SUMMARY}

The Class Demospongiae dominated the Porifera collected numerically with 1,312 specimens; 372 species were identified from 110 genera, 51 families and 12 orders, two additional OTUs were only identified to a higher taxonomic level. A further 21 and 30 specimens not identified to species level were from the sponge Classes Hexactinellida and Calcarea, respectively. A conservative estimate of the number of Demospongiae species new to science resulting from this study is 106 species (28\%), 246 species remain uncertain $(67 \%)$ and only 19 species (5\%) were determined to be described species (Table 3). Due to the large volume of the sponge collection, very small individuals and/or fragments $<5 \mathrm{~g}$ in wet weight were not identified due to time constraints, but these specimens have been kept for future taxonomic study. Of the 372 Demospongiae species recorded, 185 occurred at one station only, while the two most common Sarcotragus sp. WAM Ng1 (99100345) and Luffariella sp. WAM SS1 (99100343) (Order Dictyoceratida) occurred at 10 sampling stations. Agelas sp. WAM SS1 (99100448) (Order Agelasida) and Geodia sp. NG1 (99100423) (Order Astrophorida) occurred at nine sampling stations (Appendices 1, 2).

\section{B) BIOGEOGRAPHICAL DISTRIBUTIONS OF PREVIOUSLY DESCRIBED SPECIES}

Since $67 \%$ of the demosponges are uncertain species and $28 \%$ are new species, the following biogeographical summary applies to the 5\% (19 species) identified as previously described species (Table 4). Seven (37\%) are Australian endemics of which five (26\%) are Western Australian endemics. Of the remainder, six (32\%) have an Indo-West Pacific affinity, one $(5 \%)$ an Indian Ocean affinity and five $(26 \%)$ a Southern Ocean or sub-Antarctic origin. At least 106 are new species records for Australia and 109 are new records for Western Australia, this includes range extensions of three Australian endemic species (Table 4).
It was beyond the scope of this study to research the historical literature and to undertake major redescriptions of the Demospongiae species, some of which may have been found in this study. However, digitised species identification sheets (mudmaps) were compiled for each of the 372 species (see example: Fromont and Gomez 2007; Axinella sp. SS1 in Appendix 3. These mudmaps of Australian sponge OTUs are a standardised format for capturing the characteristics of each designated sponge OTU. They are used by taxonomic experts throughout Australia and thus allow an overall assessment of the biogeography of the sponge fauna, even though many of the species still need to be formally described and named. The synthesised OTUs will form the basis for new species descriptions.

The order Poecilosclerida is known to be the most speciose of the all the Demospongiae (Hooper and van Soest 2002). The Poecilosclerida had the highest numbers of species $(25 \%)$ followed by the Dictyoceratida (fibre sponges), Haplosclerida, Astrophorida and Halichondrida (18\%, 17\%, $13 \%$ and $11 \%$, respectively). Two comparable Australian deepwater projects, one in the Great Australian Bight (GAB) and the other in the Tasmanian seamounts also found Poecilosclerida dominated (30\% and $37 \%$, respectively) but the order of dominance of the other orders differed. In the GAB the Halichondrida and Haplosclerida, Dictyoceratida, Hadromerida and Astrophorida represented $19 \%, 19 \%, 14 \%, 11 \%$ and $4 \%$ of species, respectively (Sorokin et al. 2007), and in the southern Tasmanian seamounts, Dictyoceratida, Hadromerida, Haplosclerida, Halichondrida and Astrophorida represented 15\%,14\%,12\%,10\% and $9 \%$ of species, respectively (Schlacher-Hoenliger 2007).

This western continental margin study differs from the GAB and Tasmanian studies in recording sponges from the orders Agelasida, and the family Plakinidae. In addition, no species of the Order Verongida were found in the GAB and only one species was reported in the Tasmanian seamounts compared to eight species in the present study. This study included tropical locations and surveyed a larger area and a much greater latitudinal gradient compared with both the GAB and Tasmanian studies.

This is the first collection of sponges from depths greater than $100 \mathrm{~m}$ off the south-west coast of Australia. Recent shallow water collections in the region (Fromont et al. 2006; Fromont and Vanderklift 2009) have indicated a speciose shallow water fauna, and Hooper and Levi (1994) have suggested pockets of apparent endemism occur along the western continental margin of Australia. 


\section{CNIDARIA}

Only the subclass Octocorallia (Alcyonacea and Pennatulacea) was targeted for identification. However, specimens from the other coral taxa (Antipatharia and Scleractinia) were also identified and are discussed here.

\subsection{CNIDARIA: ALCYONACEA AND PENNATULACEA (CAAB NUMBERS 1117\#\#\#\#, 99110\#\#\# AND 112\#\#\#\#, 99110\#\#, RESPECTIVELY)}

\section{A) NUMERICAL SUMMARY}

Over 440 specimens within the Alcyonacea (soft corals, sea fans and sea whips) were identified from this survey. These comprised 117 species, 58 genera and 16 families. Of these, 26 species could be identified as described species, 12 recognised as new species and four as new genera (but as yet undescribed) and 79 species remain uncertain (Table 3). A further 10 OTUs were only identified at a higher taxonomic level and are not included in the following summary. The alcyonacean material was diverse, although the biomass was low for all but a few of the endemic species.

In addition, 29 specimens of the Order Pennatulacea (sea pens) were collected, including eight species, six genera and five families. Of these three could be identified as described species, two recognised as new and three remain uncertain (Table 3). A single OTU was too damaged to be identified to species level.

As in many other faunal groups, identifications are hindered by poor and inaccurate literature in a field where most genera need total revision using modern techniques. Given that there are virtually no comparative records of Octocorallia from the regions sampled, much of the material can be considered as new records. Of the 117 Alcyonacea and 9 Pennatulacea species recorded, 80 and 6 species, respectively, occurred at one station only. The most common Alcyonacea species, Mopsea encrinula (11188009) occurred at six stations at 100 $\mathrm{m}$ depths from Bald Island to Shark Bay. The most common Pennatulacea occurred at three stations, Umbellula huxleyi (11216003) was found at 1,000 m from Two Rocks to Abrolhos and Pteroeides sp. F (99110351) at $100 \mathrm{~m}$ at Ningaloo (Appendices 1, 2).

\section{B) BIOGEOGRAPHICAL DISTRIBUTIONS OF PREVIOUSLY DESCRIBED SPECIES}

This material included some surprising specimens; the biogeographic relationships of some of the species show unusual affinities and the data includes many new records for Australian waters. The data includes specimens previously known from both temperate and tropical regions and shows links to southern and northern Atlantic regions. It also provides more evidence for links with the Indonesian and northern Pacific (Japanese) fauna as well as containing some deep-water cosmopolitan species. Since $68 \%$ of the Alcyonacea are uncertain species and $10 \%$ are new species, the following biogeographical summary applies to the $22 \%$ ( 28 species) identified as previously described species (Table 4). Thirteen $(50 \%)$ are Australian endemics of which four $(15 \%)$ are Western Australian endemics. Of the remainder, 12 $(46 \%)$ have an Indo-West Pacific affinity $(46 \%)$ and a single species $(4 \%)$ has an Indian Ocean only origin.

Since $38 \%$ of the Pennatulacea are uncertain species and $25 \%$ are new species, the following biogeographical summary applies to the $38 \%$ (three species) identified as previously described species (Table 4). These species all have an Indo-West Pacific affinity with Umbellula huxleyi, additionally recorded from the north-east Atlantic (as well as the north-west Pacific).

For the alcyonaceans, 25 are new records for Australia (six are described species), 27 are new records for Western Australia, three of which are range extensions of Australian endemics. Oparinisis parkeri and O. viking were previously recorded from South Australia and Pseudoplumarella filicoides (1197015) was previously recorded in New South Wales only. For the pennatulaceans, two are new records for both Western Australia and Australia: Scytalium sarsii (11224001) and Umbellula huxleyi (11216003) (Appendices 1, 2).

\subsection{CNIDARIA: ANTIPATHARIA (CAAB NUMBERS 1115\#\#\#\# AND 99110\#\#\#)}

\section{A) NUMERICAL SUMMARY}

Only 12 specimens of Antipatharia were examined from this survey; these comprised eight species, five genera and two families, but other material remains to be examined (Table 3). Two described species were identified and six species remain uncertain and no new species were determined. Of the Antipatharia collected, six species occurred at one station only, while the most common, Stichopathes sp. A (99110615), occurred at three stations (Appendices 1, 2).

\section{B) BIOGEOGRAPHICAL DISTRIBUTIONS OF PREVIOUSLY DESCRIBED SPECIES}

Due to the low number of species examined the biogeographical summary of the antipatharian data reveals one species is an Australian endemic but not a Western Australian endemic (Table 4). The other described species has an Indo-West Pacific affinity. Myriopathes myriophylla (11158002), 
known from adjacent Indonesia is recorded for the first time in Australia from northern Ningaloo (Appendices 1, 2).

Preliminary determinations of the antipatharians from this cruise identified several species that are probably new records for Australian waters; however comparative material is required from the British Museum of Natural History for final identifications. Cupressopathes albies has a West Pacific distribution and the specimen Cupressopathes cf. albies examined from Point Cloates is possibly a new species. Stichopathes sp. A (recorded from Shark Bay and northern Ningaloo) may be Stichopathes cf. ceylonensis from the Indian Ocean.

All the black corals examined were collected from $100 \mathrm{~m}$ depth except for the endemic species, Parantipathes helicostricha (11161002), which was collected at 1,000 m off Albany (Appendices 1, 2). This species is previously known from deep water from the Great Australian Bight and southern Tasmania as well as New Zealand.

\subsection{CNIDARIA: AZOOXANTHELLATE SCLERACTINIA (CAAB NUMBERS 113\#\#\#\# AND 99110\#\#\#)}

\section{A) NUMERICAL SUMMARY}

Prior to this survey, 116 species of azooxanthellate Scleractinia were known from western and southern Australian (Rehberg 1892; Folkeson 1919; Hoffmeister 1933; Veron 1986; Cairns and Parker 1992; Cairns 1995; Cairns and Zibrowius 1997; Cairns 1998; Cairns 2004), representing 16.5\% all known species. Of these, 55 species are restricted to waters deeper than $200 \mathrm{~m}$, and an additional six occur both shallower and deeper than the $200 \mathrm{~m}$ isobath. The vast majority were recorded from the tropical region (north of Houtman Abrolhos), including nine endemic species (Cairns 1998). Another 18 species are restricted to waters south of Houtman Abrolhos, and only eight are reported from both regions (Cairns 2004).

This survey collected the majority of species previously recorded from deeper Western Australian waters. The azooxanthellate Scleractinia collected included over 1,400 specimens from 47 species, 19 genera and eight families. Of these, 37 species could be identified as described species, three recognised as new species and seven species remain uncertain (Table 3). Of the 47 Scleractinia species recorded, 20 occurred at one station only, while the most common, Stephanocyathus (Acinocyathus) spiniger (11314019) occurred at seven stations at $350-500 \mathrm{~m}$ depth from Bald Island to Shark Bay (Appendices 1, 2).

\section{B) BIOGEOGRAPHICAL DISTRIBUTIONS OF PREVIOUSLY DESCRIBED SPECIES}

Only $6 \%$ of the Scleractinia are uncertain species and $6 \%$ are new species, so the following biogeographical summary applies to the 79\% (37 species) identified as previously described species (Table 4). Five species (14\%) are Australian endemics but none are Western Australian endemics. The vast majority, 28 species $(76 \%)$ have an IndoWest Pacific affinity, three ( $8 \%$ ) are considered cosmopolitan and a single species (3\%) is known from the Atlantic Ocean only. Eight species are new records for Australian waters and 14 new for Western Australia, while two are range extensions of Australian endemics (Table 4). Dasmosmilia lymani, Phyllangia cf. papuensis, Trochocyathus cooperi, Notocyathus conicus and Truncatoflabellum candeanum are examples of species reported for the first time from Australian waters.

Biogeographically, the Anthemiphylliidae (Anthemiphyllia dentata and A. n. sp.) were collected in south-western Australian waters. All Caryophyllia species, except C. planilamellata (new record to Western Australia) and C. diomedeae, were collected in the tropical region of the western margin, with the deepest record for the genus being represented by C. scobinosa (new record to Western Australia) from $983-1,010 \mathrm{~m}$ depth. C. unicristata, another tropical species, was the most abundant species (see Kitahara et al. 2010). The distribution of Deltocyathus magnificus is extended to southern Western Australia and D. rotulus is reported for the first time in Western Australia.

Within the Dendrophylliidae, the most common genus was Balanophyllia with six species, including two new records to Western Australian waters: $B$. cf. dentata and B. desmophyllioides. The description of one new species of Balanophyllia from this survey is being prepared by Kitahara and colleagues at Museum of Tropical Queensland (pers. comm.).

Historically, the colonial corals Solenosmilia variabilis and to a lesser degree Madrepora oculata appear to be the most important deep-sea reef builders in Western Australia (Kitahara, pers. comm.). Solenosmilia variabilis appears to be an important substrate for the solitary species Caryophyllia diomedeae (Kitahara et al. 2010). Although no live Solenosmilia was collected during this survey, live coral was viewed on video footage taken on the survey SS200507. The live Solenosmilia observed was very patchy and most of the reef formations consisted of long dead, possibly subfossil, coral matrix.

Many images of the deep-sea scleractinian species from this survey included in Appendix 2 are the first ever taken of freshly collected specimens (examples include Anthemiphyllia n. sp. A (99110618); A. dentata (11304001); Fungiacyathus fragilis (11301001); Letepsammia formosissima (10299002); Caryophyllia planilamellata (11314042); 
Deltocyathus suluensis (11314021); Stephanocyathus spiniger (11314019); Flabellum tuthilli (11328010) and Truncatoflabellum macroeschara (11328015).

\section{MOLLUSCA}

Over 767 hard-shelled Mollusca species were identified. Of these, 425 species were live-taken and 342 represented only by dead-taken shells. Species from 109 families from the classes Gastropoda, Bivalvia, Scaphopoda and Polyplacophora have been identified to date.

Many specimens from this survey cannot be identified to species level at present. In some cases, specimens are recognised as belonging, or thought to belong, to undescribed species. In other cases, the lack of comparative material does not allow a definite identification to be made - this is not surprising as the molluscan fauna of Australia's western continental margin has been rarely accessed. In addition, many families of molluscs, particularly bivalves and especially those of the Indian Ocean are in urgent need of revision (some most-recently revised in the second half of the 19th century). Some groups, particularly those of interest to the shell-collecting fraternity, are reasonably well known and have enabled recognition of new and undescribed species previously not recorded in the Western Australian or Australian fauna, and records indicating an extension of the known latitudinal or longitudinal range of a species.

Dead-taken, as well as live-taken, Mollusca specimens were deliberately collected during the second half of the SS102005 survey, examined and lodged at the Western Australian Museum but their identifications are not included in the numerical summaries of this paper. However an example is given for the bivalves to illustrate the usefulness of such collections. The distinguishing of recentlydead from long-dead (or even sub-fossil) material can assist in forming a much more detailed record of a species distribution. During this survey, many species were either rarely or never collected as live specimens, even though their known geographic range was within the survey area. When the few instances in which dead-taken specimens have been included in the data set, they have reinforced or even extended the known geographic and depth distributional ranges.

\subsection{MOLLUSCA: BIVALVIA (CAAB NUMBERS 232\#\#\#\# AND 99230\#\#)}

\section{A) NUMERICAL SUMMARY}

Over 700 specimens of the Bivalvia, identified as belonging to at least 162 species from 83 genera, 39 families and 10 orders, were collected. Of these, only 62 species could be identified as described species and 101 remain uncertain, reflecting the lack of knowledge on this deep-sea fauna (Table 3). Of the 162 Bivalvia recorded, 100 species occurred at one station only, while the most common species, Venericardia rosulenta (23325016), occurred at 10 stations from Bald Island to Ningaloo in 100-200 m depths (Appendices 1, 2).

\section{B) BIOGEOGRAPHICAL DISTRIBUTIONS OF PREVIOUSLY DESCRIBED SPECIES}

No new species were identified and $68 \%$ of the bivalves were uncertain species and, so the following biogeographical summary applies to the $32 \%$ (62 species) identified as previously described species (Table 4). Twenty-nine species $(47 \%)$ are Australian endemics but only two (3\%) are Western Australian endemics. Of the non-native species, $32(52 \%)$ have an Indo-West Pacific affinity, and a single species $(3 \%)$ is known from the Indian Ocean only.

One previously described species, Amygdalum watsoni (23220073), is a new record for Australian waters and 15 are new for Western Australia, two of which are range extensions of Australian endemics (Table 4). Of interest were the dead-taken shells of Foramelina ?exempla, collected at just one station off Point Cloates in $400 \mathrm{~m}$ depth. Foramelina exempla is the only living species of an otherwise extinct family and was previously known only off south-eastern Australia. The Western Australian specimens were all dead-taken, but not fossil shells, and show some differences to the south-eastern species. Further study is required to determine whether they may represent a new species.

\subsection{MOLLUSCA: CEPHALOPODA (CAAB NUMBERS 236\#\#\#\# AND 99230\#\#\#)}

\section{A) NUMERICAL SUMMARY}

More than 120 specimens of the Cephalopoda were identified as belonging to 29 species from 21 genera, 12 families and four orders. Of these, only 13 could be identified as described species and 16 remain uncertain species (Table 3). Not all the specimens could be identified due to considerable damage to the soft tissues with 11 OTUs identified as higher level taxa, and no new species were discovered. The majority of specimens were collected by beam trawl over soft sediments with 42 lots collected at $400 \mathrm{~m}$, six at $200 \mathrm{~m}$ and 12 at $100 \mathrm{~m}$ depths, with only nine lots collected at $700 \mathrm{~m}$ or deeper. Not all cephalopods collected were benthic species, with some pelagic species inadvertently caught in the dredges in transit to and from the benthos; these species are thus under- 
represented in the areas surveyed but are included for information. Of the 40 Cephalopoda species recorded, 23 occurred at one station only, while the most common occurring at five stations each were indeterminate Sepia sp. (23607901) and Heteroteuthis spp. (23609902) (Appendices 1, 2).

\section{B) BIOGEOGRAPHICAL DISTRIBUTIONS OF PREVIOUSLY DESCRIBED SPECIES}

Since $55 \%$ of the Cephalopoda are uncertain species, the following biogeographical summary applies to the $45 \%$ (13 species) identified as previously described species (Table 4). Six species $(46 \%)$ are Australian endemics but none are Western Australian endemic species. Of the remainder $4(31 \%)$ have an Indo-West Pacific affinity and $3(23 \%)$ are cosmopolitan species. No new species were recorded for Australia. Ten Octopodidae species were collected, of which only one, Eledone palari, was previously described. One unidentifiable species of the meso- to bathy- pelagic Bolitaenidae was recorded from northern Ningaloo at a depth of $1,200 \mathrm{~m}$. One unnamed species of Benthoctopus from the deepwater subfamily Bathypolypodinae was recorded from northern Ningaloo waters in the relatively shallow depth of $400 \mathrm{~m}$. Included in the seven species of Sepiidae was the northern Australian endemic species, Sepia opipara (23607006). This species was found at Bald Island in the south-west, considerably extending its known distribution south of Fremantle. With the exception of one unidentifiable Sepiadarium species from deepwater near Bald Island in $1,000 \mathrm{~m}$ depth, the three remaining species of benthic Sepiadariidae were collected from $100 \mathrm{~m}$ or $400 \mathrm{~m}$ depths in both tropical and temperate waters. Sepioloidea lineolata (23608001), a southern Australian temperate species, was collected from Zuytdorp in $100 \mathrm{~m}$, just within its known geographical range, although it is generally found in shallower water to depths of only $20 \mathrm{~m}$ (Norman and Reid 2000). At least nine species of Sepiolidae were collected. Euprymna tasmanica (23609001), known from southern Australia, was collected at two northern sites - Point Cloates and northern Ningaloo - in $400 \mathrm{~m}$ and $100 \mathrm{~m}$, respectively (it is known to at least $80 \mathrm{~m}$; Norman and Reid 2000). Many specimens of the pelagic or benthopelagic genus Heteroteuthis from depths of $400-1,000 \mathrm{~m}$ in the south-west were unfortunately unidentifiable. Of the seven squid species collected, only four are described species. Enoploteuthidae undergo extensive daily vertical migrations and unidentified specimens of this family were collected at $400 \mathrm{~m}$. One unidentifiable species of Chiroteuthis, from the gelatinous, slow moving, deep-sea family Chiroteuthidae, was collected in 1,000 $\mathrm{m}$ off the Houtman Abrolhos.

\subsection{MOLLUSCA: GASTROPODA (CAAB NUMBERS 24\#\#\#\#\# AND 99240\#\#\#)}

\section{A) NUMERICAL SUMMARY}

More than 1,600 specimens of Gastropoda were identified as belonging to 267 species from 142 genera, 53 families and seven orders. Currently 85 described species have been identified, six new species (as yet undescribed) were distinguished and 176 remain uncertain species, reflecting the lack of knowledge on this deep-sea fauna (Table 3). Of the 267 Gastropoda species recorded, 187 occurred at one station only, while the most common, Comitas cf. galatheae (99240266), occurred at nine stations from Point Hillier to Barrow Island (Appendices 1, 2).

\section{B) BIOGEOGRAPHICAL DISTRIBUTIONS OF PREVIOUSLY DESCRIBED SPECIES}

Since $66 \%$ of the Gastropoda are uncertain species and $2 \%$ are new species, the following biogeographical summary applies to the 32\% (85 species) identified as previously described species (Table 4).

Thirty-five (41\%) are Australian endemics and of these $12(14 \%)$ are Western Australian endemics. The majority of the species, 47 (55\%) have an IndoWest Pacific affinity with the remainder of Southern Ocean or sub-Antarctic (1\%) origin or cosmopolitan $(1 \%)$.

At least one species is a new record for Australian waters and seven species are new records for Western Australia. Particularly interesting records for Australia include: Manaria kuroharai (24202215 - no image), previously known only from the Sea of Japan, and Akibumia orientalis (24173001 - no image), collected from 'soft' substrate off Houtman Abrolhos in $400 \mathrm{~m}$ depth. The only previous Australian record of $A$. orientalis is a single specimen taken in $>1,000 \mathrm{~m}$ depth off Sydney in New South Wales.

Not all the Opisthobranchia have been identified; to date 25 species have been recognised of which only one, the temperate Australian endemic Tylodina corticalis (24391001), has been identified as a previously known species.

\subsection{MOLLUSCA: SCAPHOPODA (CAAB NUMBERS 235\#\#\#\# AND 99230\#\#\#)}

\section{A) NUMERICAL SUMMARY}

The 130 specimens of the Scaphopoda were identified as belonging to 23 species from seven genera, five families and two orders (Table 3). Only three have been recognised as described species, the other 20 are uncertain species, reflecting the 
lack of knowledge on the deep-water species of this group of molluscs. Of the 23 species of Scaphopoda recorded, 14 occurred at one station only, while the most common, Fissidentalium cf. shirleyae (99230208), occurred at six stations from Lancelin to Ningaloo (Appendices 1, 2).

\section{B) BIOGEOGRAPHICAL DISTRIBUTIONS OF PREVIOUSLY DESCRIBED SPECIES}

Of the three $(13 \%)$ described species identified, one is a widespread southern temperate species and the other two are endemic Australian species (Table 4). One of these species, Dentalium potteri, was previously thought to be endemic to waters off eastern Australia and is a new record for Western Australia.

\subsection{OTHER MOLLUSCA (NOT EXAMINED)}

Of the other Mollusca, at least six species of the Polyplacophora remain unidentified and one lot of the Aplacophora remains unexamined. A small amount of additional live-taken material remains unidentified but is lodged at the WAM awaiting further work.

\section{ECHINODERMATA}

\subsection{ECHINODERMATA: ASTEROIDEA (CAAB NUMBERS 251\#\#\#\# AND 99250\#\#\#)}

\section{A) NUMERICAL SUMMARY}

The lack of knowledge of the taxonomy of the deep-water fauna of the western continental margin of Australian is reflected in the Asteroidea identifications. Of the (at least) 500 specimens from 87 species collected in 58 genera, 18 families and five orders, only 28 could be identified as described species (Table 3). An additional six OTUs could only be identified to higher taxa. Twenty-four new species are recorded while 35 remain uncertain. Some are tentatively identified or identified to genus only and probably include many more undescribed species. A new species in the Asterinidae, Aquilonastra shirleyae O'Loughlin, 2009, has already been described from $100 \mathrm{~m}$ at Point Cloates and Red Bluff (see Appendix 2: image 25140802). Many of the other new species are likely to remain undescribed for some time to come. Of the 87 Asteroidea species recorded, 54 occurred at one station only, while the most common species, occurring at six stations were Sclerasterias sp. 1 (99250364), Diplopteraster sp. 1 (99250292) and Hoplaster sp. 1 (99250343) (Appendices 1, 2).

\section{B) BIOGEOGRAPHICAL DISTRIBUTIONS OF PREVIOUSLY DESCRIBED SPECIES}

Since $40 \%$ of the Asteroidea are uncertain species and $28 \%$ are new species, the following biogeographical summary applies to the $32 \%$ ( 28 species) Six (21\%) are Australian endemics, and only one $(7 \%)$ is a Western Australian endemic species. The vast majority 21 species $(71 \%)$ have an Indo-West Pacific affinity. One species (7\%) has a Southern Ocean or sub-Antarctic origin and another species has only previously been recorded from the Atlantic Ocean (Table 4). Three of the described species are new records for Australian while five are new records for Western Australia, two of which are range extensions of Australian endemics. Eleven genera and six families (Odontasteridae, Labidiasteridae, Radiasteridae, Solasteridae, Porcellanasteridae and Ctenodiscidae) are newly recorded from Western Australia (the latter two families have previously been found off north-western Australia but the records are unpublished).

The Radiasteridae, Pterasteridae and Solasteridae are recognised as predominantly temperate cold water families, but were also collected in the cooler deep water in tropical areas on this survey. Of the 28 identified species, all but three are shelf species: Sidonaster vaneyi is known from the Indian Ocean and the Philippines, Ctenodiscus orientalis (see Appendix 2: image 25114001) is known from the Philippines, Indonesia and Tasmania, and Cnemidaster wyvillii known from the Philippines to the Arafura Sea. The affinities of the shelf species are predominantly with the tropical fauna to the north. There are close affinities with the asteroids of the Philippines (Fisher 1919) and Indonesia at the generic level and also with New Zealand (Clark and McKnight 2000, 2001; McKnight 2006).

Asterodiscides macroplax (see Appendix 2: image 25128004) was collected in $100 \mathrm{~m}$ off Carnarvon; this species is endemic to north-west Western Australia. Specimens lodged at the WAM have been collected from 30-40 m depth at the Houtman Abrolhos by museum staff and from trawls at 60-80 $\mathrm{m}$ depth off the Montebello Islands in 1979 on RV Soela (Marsh et al. 1999). Heteronardoa diamantinae (see Appendix 2: image 25125023), collected on this survey from a singe station in $100 \mathrm{~m}$ by beam trawl off Point Cloates, was described from a specimen from the 1964 HMAS Diamantina survey collected by beam trawl in $128 \mathrm{~m}$ off Point Cloates (Rowe 1976), suggesting a highly localised distribution of this species.

\subsection{ECHINODERMATA: CRINOIDEA (CAAB NUMBERS}




\section{A) NUMERICAL SUMMARY}

Over 250 specimens of the Crinoidea from 39 species from 29 genera, 13 families and two orders were collected, and an additional 6 OTUs were only identified to higher level taxa. Thirty-five species could be identified as described species, two recognised as new (but as yet undescribed) and two remain unknown or uncertain (Table 3). Of the 39 Crinoidea species recorded, 30 occurred at one station only, while the two most common species occurred at six stations; Phanogenia brevicirra (25030043) from Kalbarri to Point Cloates and Comatula pectinata (25030030) from Jurien to Barrow (Appendices 1, 2).

\section{B) BIOGEOGRAPHICAL DISTRIBUTIONS OF PREVIOUSLY DESCRIBED SPECIES}

As only $5 \%$ of the Crinoidea are uncertain species and $5 \%$ are new species, the following biogeographical summary applies to the majority of species, the $90 \%$ (35 species) identified as previously described species (Table 4). Five species (14\%) are Australian endemics and two of these (6\%) are Western Australian endemics. The remainder (61\%) have an Indo-West Pacific affinity or Indian Ocean only $(6 \%)$, Southern Ocean or sub-Antarctic $(11 \%)$ affinity or are cosmopolitan (3\%) (Table 4).

At least five are new species records for Australia, 11 are new records for Western Australia, and several others could only be identified to genus or family and may represent new species. The vast majority of specimens were comatulids (unstalked crinoids) with only one stalked crinoid (Bathycrinidae) collected. Crinoid identification was problematic, as many characters of the arm and cirri, traditionally used to separate species, are related to size of the animal and the ecological conditions prevailing at the collection site. Tissues from species collected by the SS200510 survey are being analysed by Eléaume and Hemery (Muséum National d'Histoire Naturelle de Paris; pers. comm.) using molecular methods to further understand their relationships. One species, provisionally identified as Stiremetra breviradia, was bioluminescent. Two species collected during this survey, Neometra conaminis and N. gorgonia, were originally described from this region by A.H. Clark (1914) from one of the few deep-water trawling collections on the FIS Endeavour off the mid-west coast of Western Australia (Fremantle to Geraldton in 146-219 m depths) in 1912.

\subsection{ECHINODERMATA: ECHINOIDEA (CAAB NUMBERS}

\section{A) NUMERICAL SUMMARY}

Australia and the Indo-Pacific Ocean have a diverse number of well studied Echinoidea species that occur in a wide range of habitats where they are often a conspicuous component of the marine fauna, making them easily identifiable (Miskelly 2002). This is reflected in the results of this survey. More than 400 specimens of Echinoidea identified as only 35 species from 26 genera, 14 families and nine orders were collected. During the survey 31 described species were identified, only two new species were distinguished and two remain uncertain (Table 3). Of the 35 Echinoidea species recorded, 14 occurred at one station only, while the most common, Prionocidaris baculosa (25202017) occurred at eight stations from Kalbarri to Ningaloo (Appendices 1, 2).

\section{B) BIOGEOGRAPHICAL DISTRIBUTIONS OF PREVIOUSLY DESCRIBED SPECIES}

As only $6 \%$ of the Echinoidea are uncertain species and $6 \%$ are new species, the following biogeographical summary applies to the majority of species, the $89 \%$ (31 species) identified as previously described species (Table 4).

The data reveals 6 (19\%) are Australian endemics, and only one of these species $(3 \%)$ is a Western Australian endemic. The remainder of the species have an Indo-West Pacific (71\%), Southern Ocean or sub-Antarctic $(6 \%)$ or Indian Ocean (3\%) affinity.

Three are new species records for Australia, while 15 are new records for Western Australia. The high number of new records for Western Australia (50\% of the echinoid species recorded from the survey) reflects the lack of knowledge of the deep-sea fauna of the western continental margin of Australia. Of these new records for Western Australia, five are extensions to the known distributions of Australian endemic species.

Over half of the urchin species were found only in the $100 \mathrm{~m}$ depth samples (with a few also recorded at the $200 \mathrm{~m}$ sites). Several Cidaridae species were only collected at $400 \mathrm{~m}$ depth from Mentelle and Bunbury (Rhopalocidaris gracilis and $R$. hirsutispina - no images) and at the Houtman Abrolhos Islands (Stereocidaris sceptriferoides) (25202023). The most widespread species was Caenopedina mirabilis (25220003), which was found at nine sites from Point Hillier to Ningaloo but all at $400 \mathrm{~m}$ depth except for the northernmost site where it occurred at $100 \mathrm{~m}$ (Appendices 1, 2).

The deep water southern temperate species, Dermechinus horridus and Gracilechinus multidentatus 
(25246002), were only recorded from the 1,000 m sites on the south coast from Point Hillier, Albany and Bald Island. While the deep water species, Phormosoma rigidum (25206002), was recorded at $700 \mathrm{~m}$ and $1,000 \mathrm{~m}$ on the south coast, it was also found at $700 \mathrm{~m}$ at the Houtman Abrolhos Islands and southern Ningaloo reflecting its Indo-Pacific distribution. Araeosoma owstoni (25205002) was the only deep water tropical echinoid species found at $700 \mathrm{~m}$ at southern and northern Ningaloo stations and but it was also found at $1,000 \mathrm{~m}$ in Perth canyon (Appendices 1, 2).

\subsection{ECHINODERMATA: HOLOTHUROIDEA (CAAB NUMBERS 254\#\#\#\# \& 99250\#\#\#)}

\section{A) NUMERICAL SUMMARY}

Our knowledge of the deep water Holothuroidea in Australian waters is poor and is reflected by the large number of new species found in this survey. A progressive systematic revision of known material incorporating molecular phylogenetic data is underway and more new species will certainly emerge.

More than 1,300 specimens of Holothuroidea were collected on this survey representing 43 species and 27 genera in 12 families and 5 orders. During the survey 25 described species were identified, 17 new species were distinguished and one remains uncertain (Table 3). Two new species of the family Holothuriidae have been described from this survey, extending from subtropical to warm temperate locations. This is noteworthy since the vast majority of the 157 species in the family Holothuriidae are recorded from shallow tropical waters predominantly on coral reefs $<50 \mathrm{~m}$ depth. The discovery of Holothuria (Panningothuria) austrinabassa O'Loughlin, 2007 (in O'Loughlin et al. 2007) represents the southern-most record for the family Holothuriidae in the world (25416062). Morphological and genetic analyses of the other new species, Holothuria (Halodeima) nigralutea O'Loughlin, 2007 (in O'Loughlin et al. 2007) found it to be a very recent offshoot of the wide-ranging Indo-West Pacific species H. (Halodeima) edulis. The etymology of this new species refers to the distinctive black and yellow colouring of the live individuals (25416063). Of the 43 Holothuroidea species recorded, 25 occurred at one station only, while the most common, Pseudostichopus hyalegerus (25418012) occurred at 20 stations from Bald Island to Abrolhos (Appendices 1, 2).

\section{B) BIOGEOGRAPHICAL DISTRIBUTIONS OF PREVIOUSLY DESCRIBED SPECIES}

As only $2 \%$ of the Echinoidea are uncertain species and $40 \%$ are new species, the following biogeographical summary applies to the 58\% (25 species) identified as previously described species (Table 4). The Holothuroidea data reveals none are Australian endemics. Twenty ( $80 \%)$ of the species have an Indo-West Pacific affinity, with the remainder with a widespread Southern Ocean or sub-Antarctic $(8 \%)$ or cosmopolitan distribution $(8 \%)$. A single species was previously recorded from the Indian Ocean only (4\%) affinity (Table 4).

At least 11 species are new records for Australia, 19 are new records for Western Australia of which two species are range extensions of Australian endemics. The recent revision of the genus Psolium (O'Loughlin and Maric 2008) described six new Australian species, including Psolium mccallumae collected during this survey, and re-assigned Psolus parmatus to Psolidium (25404011). The range of the latter species, described from the Siboga Expedition and previously known only from the Banda Sea in Indonesia, now extends south to Ningaloo in northwestern Australian waters. Psolium mccallumae is known only from the material collected at $100 \mathrm{~m}$ depth off Point Cloates ( $\mathrm{O}^{\prime}$ Loughlin and Maric 2008).

\subsection{ECHINODERMATA: OPHIUROIDEA (CAAB NUMBERS 2516\#\#\#\# AND 99250\#\#\#)}

\section{A) NUMERICAL SUMMARY}

Over 4,300 specimens of the Ophiuroidea were collected by this survey including 146 species from 66 genera, 14 families and two orders. Of these 101 described species were identified and at least 39 are undescribed, and probably new to science, while six remain unknown or uncertain (Table 3). One new species, Ophiomyxa crinita Franklin and O'Hara, 2008, has been described from this material, to date (see Appendix 2: image 25166006). Of the 146 Ophiuroidea species recorded, 45 occurred at one station only, while the most common, Ophiura ooplax (25176039) occurred at 23 stations from Bald Island to Ningaloo. Ophiomusium relictum occurred at 19 stations in a more restricted temperate distribution from Bald Island to Jurien (Appendices $1,2)$.

\section{B) BIOGEOGRAPHICAL DISTRIBUTIONS OF PREVIOUSLY DESCRIBED SPECIES}

As only $4 \%$ of the Ophiuroidea are uncertain species and $27 \%$ are new species, the following biogeographical summary applies to the 69\% (101 species) identified as previously described species (Table 4). The Ophiuroidea data reveals only $11 \%$ are Australian endemics, and none are Western Australian endemics. The remainder have fairly 
widespread distributions with 66 species $(65 \%)$ with an Indo-West Pacific affinity, (11\%) a Southern Ocean or sub-Antarctic Indian Ocean (10\%) and 10\% are cosmopolitan. A single species has previously only been recorded from the Atlantic Ocean (1\%) and two species from only the Indian Ocean (2\%). Biogeographically, the western continental margin was found to be an overlap zone of tropical and temperate faunas with a few species being endemic to the area.

At least 16 are new species records for Australia, while 48 are new records for Western Australia, of which Amphiura dolia (25191025) collected at Kalbarri and Ningaloo, is a considerable range extension of an east coast Australian endemic previously found from Tweed Heads, NSW to Bass Strait. The collection extended the southernmost records of tropical species - e.g. Amphilimna granulifera, A. transacta (25185063) and Sinophiura multispina - and northernmost records of temperate species - e.g. Ophionereis schayeri (25179009), Ophiothrix caespitose (25192002). Ophiactis brevis, previously known only from the shallows at its type locality near Darwin at 5-10 $\mathrm{m}$ depth and the Dampier Archipelago in 13-20 m depth (Marsh and Morrison 2004) was collected from northern Ningaloo in $160 \mathrm{~m}$ depth (Appendices 1, 2).

Of particular interest was the fauna from the deep-water seamounts near Albany that supported a Solenosmilia variabilis coral cover. Many of the ophiuroid species found at this site were previously recorded from Solenosmilia reef on deep-water seamounts south of Tasmania and on the Chatham Rise, west of New Zealand (O'Hara et al. 2008b).

At least 30 species tested from the first half of the voyage (the temperate sites from Albany to Perth) were found to be bioluminescent (Mallefet and $\mathrm{O}^{\prime}$ Hara, unpublished data; for techniques see: Mallefet et al. 2008).

\section{CRUSTACEA: DECAPODA (CAAB NUMBERS 28\#\#\#\#\# \& 99280\#\#\#)}

\section{A) NUMERICAL SUMMARY}

These data have been previously reported (Poore et al. 2008) but subsequent taxonomic changes and new species names are reported here. Over 6,200 specimens of the Decapoda, identified as 521 species and 267 genera from 75 families were collected. Of these species, 340 were recognised as previously described species, 158 new species were determined and for 23 OTUs the identifications were uncertain (Table 3). Within the described species, Retropluma cf. quadrata, doubtfully identified as the West Pacific species, is the first Australian record of the family Retroplumidae (Poore et al. 2008).
Twelve new species have been described since Poore et al.'s (2008) report, using material collected during this survey, a process that is continuing. Of the Brachyura, new species include Oxypleurodon wilsoni Richer de Forges and Poore, 2008 and Rochinia annae Richer de Forges and Poore, 2008. Of the Caridea, Lebbeus clarehannah McCallum and Poore, 2010 is described from $400 \mathrm{~m}$ off Bald Island; the holotype of this distinctly coloured species is shown in Appendix 2: see image 28767804. Pseudoclimenes holthuisi Bruce, 2008 is described from $100 \mathrm{~m}$ off Jurien. Also recently described is Lissosabinea lynseyae Taylor and Collins, 2009 (28781020) from $400 \mathrm{~m}$ off Bunbury.

In a recent review of the Axiidae, Poore and Collins (2009) described the following four new species from single locations from this survey: the new genus and species, Australocaris pinjarup from $400 \mathrm{~m}$ off Bunbury, Acanthaxius gathaagudu from $400 \mathrm{~m}$ off Shark Bay and Acanthaxius ningaloo from $200 \mathrm{~m}$ off northern Ningaloo. Images of these holotypes are in Appendix 2: see 28801806, 28801803, and 28801804, respectively. One new species, Calastacus myalup Poore and Collins, 2009 is described from $400 \mathrm{~m}$ off Bunbury and is a new record for the genus in the Indian Ocean as well as Australia.

Of the Anomura, Munidopsis comarge Taylor, Ahyong and Andreakis, 2010 (28840170) has been described from $900 \mathrm{~m}$ off Albany. Specimens from this survey in southern Western Australia were included in Ahyong's (2010) review of the Lithodidae which resulted in the new species, Lithodes rachelae (28836014), collected from $850 \mathrm{~m}$ to $1,000 \mathrm{~m}$ between Albany and Perth Canyon and Paralomis poorei (28836010) recorded at $900 \mathrm{~m}$ off Albany (Appendices 1, 2). Neopilumnoplax nieli (28917007) is also newly recognised from recent deep water collections in south-east Australia and New Zealand (Ahyong 2008) and was collected from Albany to Two Rocks collected from 200$900 \mathrm{~m}$ depths.

Since Poore et al.'s (2008) report several species have been identified, redetermined or moved to other genera (Appendix 1). The following are replacement names: Engystenopus palmipes, Philoceras incisus, Calaxius acutirostris, Bouvieraxius keiensis, Paraxiopsis pumilus, Planaxius brevifrons, Ciliopagurus shebae, Munida gordoae, Paragoneplax serenei, Neogoneplax renoculis, Stereomastis auriculatus and Stereomastis suhmi. For example, Philocheras MoV5422 from Poore et al.'s (2008) has now been identified as P. incisus and is the first record of this species in Australian waters (Taylor 2010) (see Appendix 2: image 28781804).

Of the 521 Decapoda species recorded, 238 species occurred at only one station. The most frequently recorded species of the entire survey, the hermit 
crab Paguristes aciculus (28827077) occurred at 27 stations. This species was found in depths of $95 \mathrm{~m}$ to $554 \mathrm{~m}$ at sites from Bald Island to Ningaloo (Appendices 1, 2).

\section{B) BIOGEOGRAPHICAL DISTRIBUTIONS OF PREVIOUSLY DESCRIBED SPECIES}

As only $5 \%$ of the Decapoda are uncertain species and $30 \%$ are new species, the following biogeographical summary applies to the 65\% (340 species) identified as previously described species (Table 4).

The data reveals $21 \%$ (71 species) are Australian endemics, but only 4\% (13 species) are Western Australian endemics. The majority of the species have widespread distributions, 68\% (232 species) have an Indo-West Pacific affinity, 4\% widespread Southern Ocean or sub-Antarctic affinity and 4\% cosmopolitan in their distribution. The remaining $3 \%$ have previously only been recorded from the Indian Ocean. At least 74 are new species records for Australia, 154 are new records for Western Australia. Of these, 20 species represent range extensions of Australian endemics to the west. Poore et al. (2008) detailed and summarised all new Australian records, new records for Western Australia and new records for southern Western Australia.

Of the commercially fished species collected during the survey, Ibacus alticrenatus, the White-tail Bug (28821001) was the most common, occurring around $400 \mathrm{~m}$. The commercial crab Hypothalassia acerba was collected only twice around $200 \mathrm{~m}$ while the other main large benthic crustaceans of commercial interest; Panulirus cygnus, Jasus edwardsii, Pseudocarcinus gigas and Chaceon albus (formerly confused with $C$. bicolor see Davie et al. 2007) were not collected at all, this is most likely an artefact of the types of sampling gear (no baits) used and terrain targeted. Only one commercial species of prawn, Haliporoides sibogae (28714005) was collected and this was numerous at $400 \mathrm{~m}$. Several species of scampi (Nephropidae) were also collected at this depth (Appendices 1, 2).

\section{PYCNOGONIDA (CAAB NUMBERS 33\#\#\#\#\#\# AND 99330\#\#)}

\section{A) NUMERICAL SUMMARY}

Forty specimens of the Pycnogonida from 17 species in 11 genera and five families were collected. Of these 11 could be identified as described species, four recognised as new species and two remain uncertain (Table 3). The new species, Hedgpethia calva, Tanystylum zuytdorpi, Pseudopallene difficile and Paranymphon bifilarium were described by Arango (2009). Photographs of the holotypes for the first two species can be viewed in Appendix 2: 33014024 and 33012054. Of the 17 species of Pycnogonida recorded, 14 occurred at one station only, while the most common, Pallenopsis cidaribatus (33020010), occurred at four stations in tropical waters from Shark Bay to Ningaloo (Appendices 1, 2)

\section{B) BIOGEOGRAPHICAL DISTRIBUTIONS OF PREVIOUSLY DESCRIBED SPECIES}

As only $12 \%$ of the Pycnogonida are uncertain species and $24 \%$ are new species, the following biogeographical summary applies to the 64\% (11 species) identified as previously described species (Table 4).

The data reveals $45 \%$ (5 species) are Australian endemics and of these $12 \%$ ( 2 species) are Western Australian endemics. The remainder have an IndoWest Pacific affinity $45 \%$ or are cosmopolitan (6\%) in their distributions. Five species are new records for Western Australia. Three of these new species records for Western Australia are range extensions of Australian endemics.

Three species were collected only in the deep waters off the south coast of Western Australia: Cilunculus sp. 1 from 700 m off Albany, Colossendeis colossea from 1,000 m off Port Hillier, and Hedgpethia calva from 1,000 m off Bald Island. The other 14 species were collected at only $100 \mathrm{~m}$ depths at a range of temperate and tropical sites (Appendix 1).

\section{TUNICATA: ASCIDIACEA (CAAB NUMBERS 35\#\#\#\#\# AND 99350\#\#\#)}

Over 450 specimens of the Ascidiacea from 77 species, 25 genera, 11 families and two orders were collected (Table 3), from 34 of the shallower surveyed sites, with $21100 \mathrm{~m}$ sites, seven $200 \mathrm{~m}$ sites and six $400 \mathrm{~m}$ depth sites recording specimens. During the survey, 52 described species were identified, 21 new species were described and four remain uncertain (Table 3 ). The majority of the new species were collected from the southern continental margin of Western Australia and the new species have been described by Kott (2008).

Prior to this survey there were 50 species known from the shallower coastal waters of Western Australia and adjacent areas (Hartmeyer 1919; Hartmeyer and Michaelsen 1928; Michaelsen 1930; Kott 1985, 1990, 1992, 2001, 2005a, 2005b). Only 21 of these previously known species were collected at more than one site on this survey of the deep shelf and slope. Of the 77 Ascidiacea species recorded, 49 occurred at one station only, while the most common species, Sigillina australis (35021011) occurred at seven stations from Albany to Carnarvon (Appendices 1, 2). 
Solitary species were rare and the collection was dominated by colonial species of the families Didemnidae and Polyclinidae. Species with flexible stalks were not often collected suggesting some bias associated with the collection method.

\section{B) BIOGEOGRAPHICAL DISTRIBUTIONS OF PREVIOUSLY DESCRIBED SPECIES}

As only $5 \%$ of the Ascidacea are uncertain species and $27 \%$ are new species, the following biogeographical summary applies to the $68 \%$ (52 species) identified as previously described species (Table 4). The data reveals 38\% (20 species) are Australian endemics but only $8 \%$ (2 species) are Western Australian endemics. The majority of the species, 54\% (28 species) have an Indo-West Pacific affinity, while a single species recorded a Southern Ocean or sub-Antarctic (4\%) or Indian Ocean (4\%) or Atlantic Ocean (4\%) affinity or cosmopolitan distribution (4\%).

A total of two species are new records for Australia, five are new records for Western Australia including Synoicum sphinctorum, a range extension of an Australian endemic species. This previously unsampled diverse offshore ascidian fauna extends the known biogeographical limits of many species around the Australian coastline. Species known from the tropical north have their range extended to the south, either to the central west coast (Pseudodistoma australe, Synoicum macroglossum, Aplidium caelestis, A. crateriferum, A. solidum, Polysyncraton millepore, Didemnum jedanense and Trididemnum sibogae) or to the south-western corner (Polysyncraton pavimentum, Didemnum ossium and D. perplexum). The distributions of the South Australian species, Pseudodiazona longigona and Leptoclinides fungiformis, has been extended westward to the continental shelf off Albany in $100 \mathrm{~m}$ depth. Two new species records for southwest Western Australian include the tropical, Herdmania momus, known from the western Pacific and Red Sea (also new open sea-floor habitat record at depths to $400 \mathrm{~m}$ ) and Synoicum laboutei, formerly restricted to Malagasy in the western Indian Ocean. Further details on this ascidian fauna can be found in the comprehensive paper by Kott (2008) and details on a subsequent deepwater survey of the north-western continental margin in Kott (2009).

\section{DISCUSSION}

Taxonomic study of the first regional scale collection of deepwater megabenthic invertebrates from Australia's western continental margin (100 $\mathrm{m}$ to $1,500 \mathrm{~m}$ depths and between $21-35^{\circ} \mathrm{S}$ latitude) reveals a previously undiscovered high level of species richness and novelty in the fauna. In the six major invertebrate groups targeted for this study - Demospongiae: Porifera, Octocorallia: Cnidaria, Mollusca, Echinodermata, Decapoda: Crustacea and Ascidiacea: Tunicata - 19,000 specimens yielded 2,021 species of which only $44 \%$ are described. One-quarter of all species identified are new records for Australian waters and over one-third are new records for Western Australian waters. From this survey material 42 new species have already been described: O'Loughlin (2009); Asteroidea, one species; Franklin and O'Hara (2008): Ophiuroidea, one species; O'Loughlin et al. (2007) and $\mathrm{O}^{\prime}$ Loughlin and Maric (2008): Holothuroidea, three species; Bruce (2008), Richer de Forges and Poore (2008), Poore and Collins (2009), Taylor and Collins (2009), Ahyong (2010), McCallum and Poore (2010) and Taylor et al. (2010): Decapoda, 12 species; Arango (2009): Pycnogonida, 4 species; and Kott (2008): Ascidiacea, 21 species. The high numbers of new records clearly reflects the lack of previous deep-sea research in this region, but also highlights the paucity of taxonomic knowledge or the lack of clarity in the literature for some groups. More than $50 \%$ of the species of Octocorallia, Antipatharia, Bivalvia, Cephalopoda, Gastropoda, Scaphopoda and Demospongiae collected are uncertain identifications. Further taxonomic work would ideally include revisions of some of the less well studied deep-sea taxa within otherwise well-known groups such as the Bivalvia.

Demosponges dominated in terms of biomass, but only $5 \%$ of species collected were described species. This reflects the lack of knowledge and potentially higher rate of endemism of this sessile taxonomic group. In contrast, $89 \%$ of echinoids, a more motile group with widespread distributions, could be identified as described species.

Overall, distributional patterns of described invertebrate taxa show the western margin is an overlap zone of tropical and temperate faunas. Knowledge from relatively well-known taxa confirmed, firstly, that many south-eastern Australian species were also recorded from Western Australia for the first time, and secondly, that the geographic distributions of many tropical/ sub-tropical species extend to temperate latitudes. This can be illustrated by the relatively well known southern Australian Decapoda (Poore 2004), for which this survey extended the known distributions of 62 documented species, and added 76 species to the temperate fauna - a $9.4 \%$ increase (Poore et al. 2008). Patterns in other groups indicated northwards extensions to their known distributions - an example being the penetration of several predominantly cold water asteroid families into deep waters of tropical latitudes.

The distributions of the 876 described species showed the strongest biogeographical pattern of the deep continental shelf fauna was an affinity with 
the wider Indo-West-Pacific region (61\% of species). Relatively few taxa appeared to be endemic to either the Indian Ocean $(4 \%)$ or to Western Australia (5\%) but $25 \%$ of species are endemic to Australia. This may be an underestimate given the high number (727) of uncertain species that could not be identified as known or new species until further taxonomic comparisons are undertaken, and the newly determined 396 species which as yet have not been found elsewhere in the world. Confirmation of this apparent endemism in such a poorly known region requires further taxonomic examination. This is epitomised by the Demospongiae of which the vast majority $(95 \%)$ of the 372 species are uncertain or new. In one wellstudied and species rich taxon, the Ophiuroidea, the western margin was found to be an overlap zone of tropical and temperate faunas with few endemic species confined to Western Australian waters.

Apparent rarity is high but typical of surveys of this kind, that is with few samples spread over a wide geographic range (e.g. fishes Williams et al. 2001). More than half of the species were collected from only one location. However, this is due, in part, to undersampling of species that may be of limited distribution or at low densities or difficult to catch with the sampling gears used. In addition, while this survey targeted the megabenthos $>25 \mathrm{~mm}$, some smaller macrofaunal specimens and pelagic cephalopods were incidentally collected, identified and included in this checklist. These macrofaunal data are a valuable record of presence in the previously unstudied western continental margin of Australia but not an accurate representation of the population.

Large intervals (typically of about 60 nautical miles) separated the 19 sites along the $\sim 2,000 \mathrm{~km}$ of continental margin surveyed, and there was insufficient replication at depths within sites to distinguish abundance from sampling efficiency or the degree of patchiness (Williams et al. 2010). The low density of samples, together with an unequal sampling intensity across sites, means that patterns of abundance must also be interpreted with care. While the sampling design aimed to systematically target soft and hard substrates at all of the sites on the $100 \mathrm{~m}$ and $400 \mathrm{~m}$ depth horizon, swath mapping of relatively large areas of seabed (10s to $100 \mathrm{~s} \mathrm{~km}^{2}$ per site) confirmed that both substrate types were not present at every site. On the other hand, mapping did identify special features at some sites enabling them to be sampled more intensively. For example, 13 collection stations at Ningaloo targeted a series of hard substratum types associated with drowned coral reefs and with rocky substratum within canyons.
As a result of the collections taken on the SS200510 survey and the continuing taxonomic work, we now have a substantially increased knowledge of the megabenthic invertebrate fauna from the deep western continental margin of Australia. All the invertebrate megabenthos collected during this survey are lodged in museums around Australia (including the ethanolpreserved material suitable for genetic analysis) and is available for further study. Together with a high quality data set on fishes collected in 1991 (Williams et al. 2001), these benthic invertebrate biodiversity data will make a large contribution to the Regional Marine Planning for the South West Region and inform the design of a representative system of marine protected areas.

\section{ACKNOWLEDGEMENTS}

Project funding was provided by CSIRO Wealth from Oceans flagship and the Australian Government Department of Environment Water Heritage and the Arts, with the assistance from Australia's Marine National Facility. Several authors (AW, FA, GP, TOH) are supported through the Commonwealth Environment Research Facilities (CERF) program, an Australian Government initiative, in particular by the CERF Marine Biodiversity Hub. This paper is a contribution to the Census of Marine Life project Continental Margin Ecosystems (COMARGE).

Special thanks are due to the crew of the FRV Southern Surveyor, who assisted with the collection of data at sea, the scientific personnel on-board responsible for sorting invertebrate catches and those who assisted the taxonomic experts in providing authoritative determinations for the megabenthos and specimen curation at the museums: John Keesing, Julia Phillips - CSIRO; Penny Berents, Steve Keable (Australian Museum); Mark Salotti, Corey Whisson, Oliver Gomez (Western Australian Museum); Jerome Mallefet, Anna McCallum, David Staples, Anna Syme, Robin Wilson, Joanne Taylor, Kate Naughton (Museum Victoria); Merrick Ekins (Queensland Museum); Sue Horner (Museum and Art Gallery of the Northern Territory).

Our thanks also goes to other taxonomists for contributing identifications on the material and continuing to work on the samples: Mark O'Loughlin, Mark Norman and Phil Bock (Museum Victoria); Ashley Miskelly, Steve Keable (Australian Museum); Richard Willan (Museum and Art Gallery of the Northern Territory); Karen Miller (Institute of Marine and Antarctic Studies); Stephen Cairns (Smithsonian Institute - National Museum of Natural History, USA); Tina Moldstova (P.P. Shirshov Institute of Oceanology RAS, Russia); Claudia Arango, Sandy Bruce (Queensland 
Museum); Di Jones (Western Australian Museum); Carden Wallace (Museum of Tropical Queensland); Richer de Forges (Institut de Recherché pour le Developpment, New Caledonia); Jerome Mallefet, Catholic University of Louvain, Belgium; Marc Eléaume, Lenaïg Hemery (Museum National d'Histoire Naturelle, Paris).

The authors also wish to acknowledge the willing support provided by the museums of Australia who underpinned this and other similar projects, by providing staff to participate at sea, and by subsequently processing and curating the samples.

Among the many staff from CSIRO who assisted and contributed to this massive project, we would like to specifically thank the following: Mark Lewis, Rudy Kloser, Nic Bax, Bruce Barker, Gordon Keith, Tony Cracknell, Tanya Gorenc, Miroslaw Ryba, Pamela Brodie, Lea Crosswell, Caroline Sutton and Joel MacKeen.

\section{REFERENCES}

Agassiz, A. (1879). Preliminary report on the 'Challenger' echini. Proceedings of the American Academy of Arts and Sciences, 3rd series. 14: 190-212.

Ahyong, S.T. (2008). Deepwater crabs from seamounts and chemosynthetic habitats off Eastern New Zealand (Crustacea : Decapoda : Brachyura). Zootaxa 1708: $1-72$.

Allen, G.R. and Steene, R. (1994). Indo-Pacific coral reef field guide. Tropical Reef Research: Singapore.

Arango, C.P. (2009). New species and new records of sea spiders (Arthropoda: Pycnogonida) from deep waters in Western Australia. Zootaxa 1977: 1-20.

Balss, H. (1935). Brachyura of the Hamburg Museum expedition to south-western Australia, 1905. Journal of the Royal Society of Western Australia 21: 113-151.

Broch, H. (1910). Pennatulida. In: Michaelsen, W. and Hartmeyer. R. (eds), Die fauna Südwest-Australiens. Ergebnisse der Hamburger sudwest-australischen Forschungsreise 3(2): 109-122. Verlag von Gustav Fischer: Jena.

Broch, H. (1916). Alcyonaria. In: Results of Dr E. Mjöberg's Swedish scientific expeditions to Australia 1910-13. Kungliga Svenska Vetenskaps-Akademiens Handlingar 52(11): 1-48.

Bruce, A.J. (2008). Palaemonoid shrimps from the Australian North West Shelf. Zootaxa 1815: 1-24.

Bryce, C. (2009). Marine biological survey of Mermaid Reef (Rowley Shoals), Scott and Seringapatam Reef, Western Australia 2006. Records of the Western Australian Museum, Supplement 77: 1-255.

Buhl-Mortensen, L., Vanreusel, A., Gooday, A.J., Levin, L.A., Priede, I.G., Buhl-Mortensen, P., Gheerardyn, H., King, N.J. and Raes, M. (2010). Biological structures as a source of habitat heterogeneity and biodiversity on the deep ocean margins. Marine Ecology 31: 21-50.

Cairns, S.D. (2004). The azooxanthellate Scleractinia (Coelenterata: Anthozoa) of Australia. Records of the
Australian Museum 56: 259-329.

Cairns, S.D. (1998). Azooxanthellate Scleractinia (Cnidaria: Anthozoa) of Western Australia. Records of the Western Australian Museum 18: 361-417.

Cairns, S.D. (1995). The marine fauna of New Zealand: Scleractinia (Cnidaria Anthozoa). New Zealand Oceanographic Institute Memoir 103: 1-210.

Cairns, S.D. and Parker, S.A. (1992). Review of the Recent Scleractinia of South Australia, Victoria, and Tasmania. Records of the South Australian Museum, Monograph Series 3: 1-82.

Cairns, S.D. and Zibrowius, H. (1997). Cnidaria: Anthozoa: azooxanthellate Scleractinia from the Philippine and Indonesian regions. Mémoires $d u$ Muséum National D’Histoire Naturelle 172: 27-243.

Clark, A.H. (1911). Crinoidea. In: Michaelsen, W. and Hartmeyer, R. (eds), Die fauna Südwest-Australiens, Ergebnisse der Hamburger sudwest-australischen Forschungsreise 3(13): 435-467. Verlag von Gustav Fischer: Jena.

Clark, A.H. (1914). The crinoids collected by the 'Endeavour' between Fremantle and Geraldton (Western Australia). Records of the Western Australian Museum 1: 113-131.

Clark, H.L. (1916). Report of the sea-lilies, starfishes, brittle-stars and sea-urchins obtained by the F.I.S Endeavour on the coasts of Queensland, New South Wales, Tasmania, Victoria, South Australia and Western Australia. Endeavour Research 4(1): 1-123.

Clark, H.L. (1923). Some echinoderms from West Australia. Journal of the Linnaean Society (Zoology) 35:229-252.

Clark, H.L. (1938). Echinoderms from Australia. An account of the collections made in 1929 and 1932. Memoirs of the Museum of Comparative Zoology at Harvard College 55: 1-596.

Clark, H.E.S. and McKnight, D.G. (2000). The Marine Fauna of New Zealand: Echinodermata: Asteroidea (Sea Stars), Order Paxillosida and Order Notomyotida. NIWA Biodiversity Memoir 116: 1-196.

Clark, H.E.S. and McKnight, D.G. (2001). The Marine Fauna of New Zealand: Echinodermata: Asteroidea (Sea Stars), Order Valvatida. NIWA Biodiversity Memoir 117: $1-269$.

Dampier, W. (1703). A voyage to New Holland, \&c. in the year 1699. James and John Knapton: London.

Davie, P.J.F., Ng, P.K.L. and Dawson, E.W. (2007). A new species of deep-sea crab of the genus Chaceon Manning \& Holthuis, 1989 (Crustacea : Decapoda : Brachyura : Geryonidae) from Western Australia. Zootaxa 1505: 51-62.

Doderlein, L. (1914). Echinoidea. In: Michaelsen, W. and Hartmeyer, R. (eds), Die fauna Südwest-Australiens, Ergebnisse der Hamburger sudwest-australischen Forschungsreise 4: 443-492. Verlag von Gustav Fischer: Jena.

Domingues, C.M., Maltrud, M.E., Wijffels, S.E., Church, J.A. and Tomczak, M. (2007). Simulated Lagrangian pathways between the Leeuwin Current System and 
the upper-ocean circulation of the southeast Indian Ocean. Deep-Sea Research Part II-Topical Studies in Oceanography 54: 797-817.

Edgar, G.J. (1997). Australian marine life - the plants and animals of temperate waters. Reed Books: Victoria.

Erwe, W. (1913). Holothurioidea. In: Michaelsen, W. and R. Hartmeyer (eds), Die fauna Südwest-Australiens, Ergebnisse der Hamburger sudwest-australischen Forschungsreise 4(9): 349-402.

Escobar-Briones, E.G., Gaytan-Caballero, A. and Legendre, P. (2008). Epibenthic megacrustaceans from the continental margin, slope and abyssal plain of the south-western Gulf of Mexico: Factors responsible for variability in species composition and diversity. DeepSea Research Part II-Topical Studies in Oceanography 55: 2667-2678.

Fell, H.B. (1958). Deep sea echinoderms of New Zealand. Zoology Publications from Victoria University of Wellington 24: 1-40.

Feng, M., Slawinski, D., Beckley, L.E. and Keesing, J.K. (2010). Retention and dispersal of shelf waters influenced by interactions of ocean boundary current and coastal geography. Marine and Freshwater Research 61: 1259-1267.

Fisher, W.K. (1919). Starfishes of the Philippine Seas and adjacent waters. Bulletin of the United States National Museum 100(3): XI + 712, 156 pls.

Folkeson, F. (1919). Madreporaria In: Results of Dr E. Mjöberg's Swedish scientific expeditions to Australia 1910-13. Kungliga Svenska Vetenskaps-Akademiens Handlingar. 59(1): 1-23.

Forest, J. (1981). Compte rendu et remarques générales (texte bilingue) / Report and general comments (bilingual text). Résultats des campagnes MUSORSTOM 1 Mémoires ORSTOM 91.

Franklin, A.M. and O'Hara, T.D. (2008). A new species in the genus Ophiomyxa from south-west Australian waters (Echinodermata: Ophiuroidea: Ophiomyxidae). Memoirs of Museum Victoria 65: 57-61.

Fromont, J. and Gomez, O. (2007). Mudmap for Axinella sp. SS1 from SW Southern Surveyor Sponges, Western Australia, Western Australian Museum: Perth.

Fromont, J., Vanderklift, M.A. and Kendrick, G.A. (2006). Marine Sponges of the Dampier Archipelago, Western Australia: patterns of species distributions, abundance and diversity. Biodiversity and Conservation 15: 37313750 .

Fromont, J. and Vanderklift, M.A. (2009). Porifera (sponges) of Mermaid Reef (Rowley Shoals), Scott and Seringapatam Reefs, Western Australia. Records of the Western Australian Museum, Supplement 77: 89-104.

Gage, J.D., Roberts, J.M., Hartley, J.R. and Humphery, J.D. (2005). Potential impacts of deep-sea trawling on the benthic ecosystem along the northern European continental margin: a review. Benthic Habitats and the Effects of Fishing 41: 503-517.

George, R.W. (1962). Description of Panulirus cygnus sp. nov., the commercial crayfish (or spiny lobster) of Western Australia. Journal of the Royal Society of Western Australia 45: 100-110.
George, R.W. (1966). Hypothalassia armata (De Haan) in Western Australia. Crustaceana 10: 223-224.

Gowlett-Holmes, K. (2008). A field guide to the marine invertebrates of South Australia. Notomares: Sandy Bay, Australia.

Gray, J.S., Poore, G.C.B., Ugland, K.I., Wilson, R.S., Olsgard, F. and Johannessen, O. (1997). Coastal and deep-sea benthic diversities compared. Marine Ecology-Progress Series 159: 97-103.

Griffith, J.K. and Fromont, J. (1998). A catalogue of recent Cnidaria type specimens in the Western Australian Museum of Natural Science, Perth. Records of the Western Australian Museum 19: 223-239.

Hartmeyer, R. (1919). Ascidien. In: Results of Dr E. Mjöberg's Swedish scientific expeditions to Australia 1910-13. Kungliga Svenska Vetenskaps-Akademiens Handlingar 60(4): 1-150.

Hartmeyer, R. and Michaelsen, W. (1928). Ascidiae Diktyobranchiae und Ptychobranchiae. In: Michaelsen, W. and Hartmeyer, R. (eds), Die Fauna Südwest-Australiens. Ergebnisse der Hamburger sudwestaustralischen Forschungsreise 5: 251-460. Verlag von Gustav Fischer: Jena.

Heap A.D., Harris P.T., Hinde A. and Woods M. (2005). Benthic marine bioregionalisation of Australia's Exclusive Economic Zone. Report to the National Oceans Office on the Development of a National Benthic Marine Bioregionalisation in support of Regional Marine Planning. The Departments of the Environment and Heritage (National Oceans Office): Hobart, Australia.

Hentschel, E. (1909). Tetraxonida. 1. In: Michaelsen, W. and Hartmeyer, R. (eds), Die Fauna SüdwestAustraliens. Ergebnisse der Hamburger sudwestaustralischen Forschungsreise 2(21): 347-402.

Hentschel, E. (1911). Tetraxonida. 2. In: Michaelsen, W. and Hartmeyer, R. (eds), Die Fauna SüdwestAustraliens. Ergebnisse der Hamburger sudwestaustralischen Forschungsreise 3(10): 277-393.

Hoffmeister, J.E. (1933). Report on deep sea corals obtained by the F.I.S. 'Endeavour', on the coasts of New South Wales, Victoria, South Australia, and Tasmania. Biological Results of the Fishing Experiments carried by the F.I.S. 'Endeavour', 1909-1914 6(1): 1-16.

Hooper, J.N.A. and Levi, C. (1994). Biogeography of IndoWest Pacific sponges: Microcionidae, Raspailiidae, Axinellidae (pp. 191-212). In: van Soest, R.W.M., van Kempen, Th.M.G. and Braekman, J-C. (eds), Sponges in time and space. Balkema: Rotterdam.

Hooper J.N.A. and van Soest R.W.M. (2002). Systema Porifera: a guide to the classification of sponges. Kluwer Academic/Plenum Press: New York.

Howell, K.L., Billett, D.S.M. and Tyler, P.A. (2002). Depth-related distribution and abundance of seastars (Echinodermata: Asteroidea) in the Porcupine Seabight and Porcupine abyssal plain, NE Atlantic. Deep-Sea Research Part I-Oceanographic Research Papers 49: 1901-1920.

Jones D.S. (2004). Aquatic fauna of the waters of the Dampier Archipelago, Western Australia. Report of the Woodside Energy Ltd/Western Australia Museum 
partnership to explore the marine biodiversity of the Dampier Archipelago, 1998-2001. Records of the Western Australian Museum Supplement 66: 1-401.

Jones, D.S. and Morgan, G.J. (2002). A field guide to crustaceans of Australian waters, second edition. Reed New Holland: Sydney.

Kitahara, M.V., Cairns, S.D. and Miller, D.J. (2010). Monophyletic origin of Caryophyllia (Scleractinia, Caryophyllidae), with descriptions of six new species. Systematics and Biodiversity 8: 157-168.

Kloser, R.J., Williams, A. and Butler, A. (2007). Exploratory surveys of seabed biotopes in Australia's deep ocean using remote sensing - needs and realities (93-110). In Todd, B.J. and Greene, H.G. (eds), Mapping the seafloor for habitat characterization: Geological Association of Canada, Special Paper. The Geological Association of Canada: St Johns, Newfoundland.

Koehler, R. (1907). Ophiuroidea. In: Michaelsen, W. and Hartmeyer, R. (eds), Die fauna Südwest-Australiens. Ergebnisse der Hamburger sudwest-australischen Forschungsreise 1(4): 241-254. Verlag von Gustav Fischer: Jena.

Koslow, J.A., Gowlett-Holmes, K., Lowry, J.K., O'Hara, T., Poore, G.C.B. and Williams, A. (2001). Seamount benthic macrofauna off southern Tasmania: community structure and impacts of trawling. Marine Ecology Progress Series 213: 111-125.

Kott, P. (1985). The Australian Ascidiacea Part 1. Phlebobranchia and Stolidobranchia. Memoirs of the Queensland Museum 23: 1-440.

Kott, P. (1990). The Australian Ascidiacea Part 2. Aplousobranchia. (1). Memoirs of the Queensland Museum 29: 1-266.

Kott, P. (1992). The Australian Ascidiacea. Part 3: Aplousobranchia (2). Memoirs of the Queensland Museum 32: 375-620.

Kott, P. (2001). The Australian Ascidiacea Part 4, Aplousobranchia (3), Didemnidae. Memoirs of the Queensland Museum 47: 1-407.

Kott, P. (2005a). Catalogue of Tunicata in Australian Waters. Catalogue of Tunicata in Australian waters.: i-iv, 1-301.

Kott, P. (2005b). New and little-known species of Didemnidae (Ascidiacea, Tunicata) from Australia (Part 2). Journal of Natural History 39: 2479-2479.

Kott, P. (2008). Ascidiacea (Tunicata) from deep waters of the continental shelf of Western Australia. Journal of Natural History 42: 1103-1217.

Kott, P. (2009). Taxonomic revision of Ascidiacea (Tunicata) from the upper continental slope off North-Western Australia. Journal of Natural History 43: 1947-1986.

Kükenthal, W. (1910). Alcyonaria 1. In: Michaelsen, W. and Hartmeyer, R. (eds), Die fauna Südwest -Australiens, Ergebnisse der Hamburger sudwestaustralischen Forschungsreise. 3(1): 1-108. Verlag von Gustav Fischer: Jena.

Lamarck, J.B.P. De Monet, Comte De (1813-1814). Sur les polypiers empâtés. Suite du mémoire intitulé: Sur les Polypiers empâtés. Suite des éponges. Annales $d u$ Muséum national d'histoire naturelle, Paris 20(6): 294312 (published 1813), 370-386, 432-458 (published 1814).

Last P.R., Lyne V.D., Yearsley G.K., Gledhill D.C., Gomon M.F., Rees A.J.J. and White W. (2005). Validation of national demersal fish datasets for the regionalisation of the Australian continental slope and outer shelf (>40 m depth). Department of the Environment and Heritage and CSIRO Marine Research. The National Oceans Office: Hobart.

Lewis, M. (1999). CSIRO-SEBS (Seamount, Epibenthic Sampler), a new epibenthic sled for sampling seamounts and other rough terrain. Deep-Sea Research Part I-Oceanographic Research Papers 46: 1101-1107.

Lewis, M. (2009). Sherman the epibenthic sled for rough terrain (CSIRO Marine and Atmospheric Research paper 029). CSIRO Marine and Atmospheric Research: Canberra.

Lewis, M. (2010). The CSIRO $4 m$ Beam Trawl (CSIRO Marine and Atmospheric Research Paper 033) Hobart, Tas. CSIRO Marine and Atmospheric Research. 17p.

Macpherson, E. (2002). Large-scale species-richness gradients in the Atlantic Ocean. Proceedings of the Royal Society of London Series B-Biological Sciences 269: 1715-1720.

Macpherson, E. (2003). Species range size distributions for some marine taxa in the Atlantic Ocean: effect of latitude and depth. Biological Journal of the Linnaean Society 80: 437-455.

Mallefet, J., Quevy, F. and O’Hara, T. (2008). Distribution of luminescence in Ophiuroidea (Echinodermata). Luminescence 23: 84.

Marsh, L.M. (1976). Western Australian Asteroidea since H. L. Clark. Thalassia Jugoslavica 12(1): 213-225.

Marsh, L.M., Fromont, J. and Salotti, M. (1999). A catalogue of Recent echinoderm type specimens in the Western Australian Museum, Perth. Records of the Western Australian Museum 19: 391-411.

Marsh, L.M. and Morrison, S.M. (2004). Echinoderms of the Dampier Archipelago, Western Australia. Records of the Western Australian Museum, Supplement 66: 293-342.

McCallum, A.W. and Poore, G.C.B. (2010). Two crested and colourful species of Lebbeus (Crustacea: Caridea: Hippolytidae) from the continental margin of Western Australia. Zootaxa 2372: 126-137.

McKnight, D.G. (2006). The marine fauna of New Zealand: Echinodermata: Asteroidea (sea stars). 3. Orders Velatida, Spinulosida, Forcipulatida, Brisingida with addenda to Paxillosida, Valvatida. NIWA Biodiversity Memoir 120: 1-187.

Melville-Smith, R., Gould, R. and Bellchambers, L. (2006). The Crystal Crab fishery in Western Australia: First steps in the development of a sustainable deepwater crab fishery. FAO Fisheries Proceedings 3: 117-129.

Melville-Smith, R., Norton, S.M.G. and Thomson, A.W. (2007). Biological and fisheries data for managing deep sea crabs in Western Australia. Final FRDC Report Project 2001/055. Fisheries Western Australia 
Fisheries Research Report 165: 1-95.

Michaelsen, W. (1930). Ascidae Krikobranchiae. In: Michaelsen, W. and Hartmeyer, R. (eds), Die fauna Südwest-Australiens, Ergebnisse der Hamburger sudwestaustralischen Forschungsreise 1905. 5(7): 463-558. Verlag von Gustav Fischer: Jena.

Miskelly, A. (2002). Sea urchins of Australia and the IndoPacific. Capricornia Publications: Australia.

Morgan, G.J. and Jones, D.S. (1991). Checklist of marine decapod Crustacea of southern Western Australia (pp. 483-497). In: Wells, F.E., Walker, D.I., Kirkman, H. and Lethbridge, R. (eds), Proceedings of the Third International Marine Biological Workshop: the marine flora and fauna of Albany, Western Australia. Volume 2. Western Australian Museum: Perth.

Narayanaswamy, B.E., Bett, B.J. and Hughes, D.J. (2010). Deep-water macrofaunal diversity in the FaroeShetland region (NE Atlantic): a margin subject to an unusual thermal regime. Marine Ecology 31: 237-246.

National Marine Facility survey report (Williams, A., Kloser, R., Bax, N. and Poore, G.C.B.) (2005a). Voyage summary SS07/2005. Mapping benthic ecosystems on the deep continental shelf and slope in Australia's 'South West Region' to understand evolution and biogeography and support implementation of the SW Regional Marine Plan and Commonwealth Marine Protected Areas. Phase 1. (21 July-17 August 2005). 2005 RV Southern Surveyor program. 27pp. Marine National Facility. Australia. Available at: http://www.marine.csiro.au/ nationalfacility/voyagedocs/2005/summary_ss072005.pdf

National Marine Facility survey report (Williams, A., Kloser, R., Bax, N. and Poore, G.C.B.) (2005b). Voyage summary SS10/2005. Mapping benthic ecosystems on the deep continental shelf and slope in Australia's 'South West Region' to understand evolution and biogeography and support implementation of the SW Regional Marine Plan and Commonwealth Marine Protected Areas. Phase 2. (18 Nov-14 Dec 2005). 2005 RV Southern Surveyor program. 19p. Marine National Facility, Australia. Available at: http://www.marine.csiro.au/nationalfacility/ voyagedocs/2005/Summary_SS10-2005.pdf

National Marine Facility survey report (Williams, A., Kloser, R. and Bax, N.) (2007). Voyage summary SS02/2007. Survey and monitoring for SE MPAS including the Tasman Seamounts Marine Reserve. RV Southern Surveyor program. Marine National Facility, Australia. Available at: http://www.marine.csiro.au/ nationalfacility / voyagedocs / 2007/MNF-SS02-07_ sum.pdf

Norman, M. and Reid, A. (2000). A guide to the squid, cuttlefish and octopuses of Australasia. CSIRO Publishing: Victoria.

O'Hara, T.D. (2007). Seamounts: centres of endemism or species richness for ophiuroids? Global Ecology and Biogeography 16: 720-732.

O'Hara, T. (2008a). Bioregionalisation of Australian waters using brittle stars (Echinodermata: Ophiuroidea), a major group of marine benthic invertebrates. Department of the Environment, Water, Heritage and the Arts: Canberra.
O'Hara, T. (2008b). Bioregionalisation of the waters around Lord Howe and Norfolk Islands using brittle stars (Echinodermata: Ophiuroidea). Department of the Environment, Water, Heritage and the Arts: Canberra.

O'Hara, T.D. and Poore, G.C.B. (2000). Patterns of distribution of southern Australian marine echinoderms and decapods. Journal of Biogeography 27: 1321-1335.

O'Hara, T., Rowden, A.A. and Williams, A. (2008). Coldwater coral habitats on seamounts: do they have a specialist fauna? Diversity and Distribution 16: 925-934.

O'Loughlin, P.M. (2009). New asterinid species from Africa and Australia (Echinodermata: Asteroidea: Asterinidae). Memoirs of Museum Victoria 66: 203-213.

O'Loughlin, P.M. and Maric, D. (2008). Australian species of Psolidium Ludwig (Echinodermata: Holothuroidea: Psolidae). Memoirs of Museum Victoria 65: 1-21.

O'Loughlin, P.M., Paulay, G., VandenSpiegel, D. and Samyn, Y. (2007). New Holothuria species from Australia (Echinodermata: Holothuroidea: Holothuriidae), with comments on the origin of deep and cool holothuriids. Memoirs of Museum Victoria 64: $35-52$.

Péron, F. and de Freycinet, L. (1824). Voyage de découvertes aux Terres Australes, fait par ordre du gouvernement, sur les corvettes le Géographe, le Naturaliste et la goëlette le Casuarina, pendant les années 1800, 1801, 1802, 1803 et 1804, 4 volumes and atlas, Paris.

Poore G.C.B. (2004) Marine decapod Crustacea of southern Australia: a guide to identification. CSIRO Publishing: Melbourne.

Poore, G.C.B. (2007). Thalassinidean shrimps (Crustacea: Decapoda) from north-western Australia, including five new species. Records of the Western Australian Museum Supplement 73: 161-179.

Poore, G.C.B. and Collins, D.J. (2009). Australian Axiidae (Crustacea: Decapoda: Axiidea). Memoirs of Museum Victoria 66: 221-287.

Poore G.C.B., Just J. and Cohen B.F. (1994). Composition and diversity of Crustacea Isopoda of the southeastern Australian continental-slope. Deep-Sea Research Part I-Oceanographic Research Papers 41: 677-693.

Poore, G.C.B., McCallum, A.W. and Taylor, J. (2008). Decapod Crustacea continental margin of southwestern and central Western Australia: preliminary identifications of 524 species from FRV Southern Surveyor voyage SS10-2005. Museum Victoria Science Reports 11: 1-106.

Rehberg, H. (1892). Neue und wenig bekannte Korallen. Abhandlungen aus dem Gebiete der Naturwissenschaften. 12(1): 1-50.

Richer de Forges, B. and Poore, G.C.B. (2008). Deep-sea majoid crabs of the genera Oxypleurodon and Rochinia (Crustacea: Decapoda: Brachyura: Epialtidae) mostly from the continental margin of Western Australia. Memoirs of Museum Victoria 65: 63-70.

Roberts, C.M., McClean, C.J., Veron, J.E.N., Hawkins, J.P., Allen, G.R., McAllister, D.E., Mittermeier, C.G., Schueler, F.W., Spalding, M., Wells, F., Vynne, C. 
and Werner T.B. (2002) Marine biodiversity hotspots and conservation priorities for tropical reefs. Science 295(5558): 1280-1284.

Rowe, F.W.E. (1976). The occurrence of the genus Heteronardoa (Asteroidea: Ophidiasteridae) in the Indian Ocean, with the description of a new species. Records of the Western Australian Museum 4: 85-100.

Rowe, G.T. and Kennicutt, M.C. (2008). Introduction to the Deep Gulf of Mexico Benthos Program. Deep-Sea Research Part II - Topical Studies in Oceanography 55: 2536-2540.

Ruhl, H.A. (2007). Abundance and size distribution dynamics of abyssal epibenthic megafauna in the north-east Pacific. Ecology 88: 1250-1262.

Schlacher, T.A., Schlacher-Hoenlinger, M.A., Williams, A., Althaus, F., Hooper, J.N.A. and Kloser, R. (2007). Richness and distribution of sponge megabenthos in continental margin canyons off south-eastern Australia. Marine Ecology-Progress Series 340: 73-88.

Shortis, M.R., Seager, J.W., Williams, A., Barker, B.A. and Sherlock, M. (2008). Using stereo-video for deep water benthic habitat surveys. Marine Technology Society Journal 42: 28-37.

Smith, K.D., Hall, N.G. and Potter, I.C. (2004). Relative abundances and size compositions of champagne crabs, Hypothalassia acerba, on two coasts and in different water depths and seasons. Marine and Freshwater Research 55: 653-661.

Sorokin, S., Fromont, J. and Currie, D. (2007). Demosponge biodiversity in the Great Australian Bight's benthic protection zone. Transactions of the Royal Society of South Australia 131: 192-204.

Taylor, J. (2010). The sand shrimp genus Philocheras (Caridea: Crangonidae) from the continental margin of Western Australia including the description of new species and a key to Australian species. Zootaxa 2373: 157-168.

Taylor, J. and Collins, D.J. (2009). New shrimp records of the genus Lissosabinea (Caridea: Crangonidae) from Australia including descriptions of three new species and a key to the world species. Memoirs of Museum Victoria 66: 175-187.

Thiele, J. (1930). Gastropoda und Bivalvia. In: Michaelsen, W. and Hartmeyer, R. (eds), Die fauna SüdwestAustraliens. Ergebnisse der Hamburger sudwestaustralischen Forschungsreise 5: 561. Verlag von Gustav Fischer: Jena.

Thouzeau, G., Robert, G. and Ugarte, R. (1991). Faunal assemblages of benthic megainvertebrates inhabiting sea scallop grounds from Eastern Georges Bank, in relation to environmental-factors. Marine EcologyProgress Series 74: 61-82.

Topsent, E. (1930). Eponges de Lamarck conservées au Muséum de Paris Annales du Muséum national d'histoire naturelle, Paris 6(5): 1-56.

Topsent, E. (1932). Eponges de Lamarck conservées au Muséum de Paris, Deuxième partie. Annales du Muséum national d'histoire naturelle, Paris 6(8): 61-124.

Topsent, E. (1933). Eponges de Lamarck conservées au Muséum de Paris. Fin, Annales du Muséum national d'histoire naturelle, Paris 6(10): 1-60.
Veron, J.E.N. (1986). Corals of Australia and Indo-Pacific. Angus and Robertson: Sydney.

Verco, J.C. and Cotton, B.C. (1935). Combing the Southern Seas / by Sir Joseph Verco; Edited by B.C. Cotton. The Mail Newspapers Ltd.: Adelaide.

Wadley, V. and Evans, D. (1992) Crustaceans from the deepwater trawl fisheries of Western Australia. Western Australian Museum: Perth.

Waite, A.M., Thompson, P.A., Pesant, S., Feng, M., Beckley, L.E., Domingues, C.M., Gaughan, D., Hanson, C.E., Holl, C.M., Koslow, T., Meuleners, M., Montoya, J.P., Moore, T., Muhling, B.A., Paterson, H., Rennie, S., Strzelecki, J. and Twomey, L. (2007). The Leeuwin Current and its eddies: an introductory overview. Deep-Sea Research Part Ii-Topical Studies in Oceanography 54: 789-796.

Walker, D.I. and Wells, F.E. (1999). The seagrass flora and fauna of Rottnest Island, Western Australia. Proceedings of the Ninth International Marine Biological Workshop, January 1996, Rottnest Island, Western Australia. Western Australian Museum: Perth.

Ward, T.J. and Rainer, S.F. (1988). Decapod Crustaceans of the North-West Shelf, a tropical continental-shelf of north-western Australia. Australian Journal of Marine and Freshwater Research 39: 751-765.

Ward, T.M., Sorokin, S.J., Currie, D.R., Rogers, P.J. and McLeay, L.J. (2006). Epifaunal assemblages of the eastern Great Australian Bight: effectiveness of a benthic protection zone in representing regional biodiversity. Continental Shelf Research 26: 25-40.

Wells, F.E. (1997). The flora and fauna of the Houtman Abrolhos Islands, Western Australia. Volumes $1 \mathcal{E} 2$. Proceedings of the Seventh International Marine Biological Workshop. Western Australian Museum/AMSA: Perth.

Wells, F.E. and Bryce, C.W. (1988). Seashells of Western Australia. Western Australian Museum: Perth.

Wells, F.E. and Bryce, C.W. (1993). Sea Slugs of Western Australia. Western Australian Museum: Perth.

Wells, F.E., Walker, D.I., Kirkman, H. and Lethbridge, R. (1990). The flora and fauna of Albany, Western Australia. Volume 1. Proceedings of the Third International Marine Biological Workshop. Western Australian Museum/ AMSA: Perth.

Wells, F.E., Walker, D.I., Kirkman, H. and Lethbridge, R. (1991). The flora and fauna of Albany, Western Australia. Volume 2. Proceedings of the Third International Marine Biological Workshop. Western Australian Museum/ AMSA: Perth.

Wells, F.E., Walker, D.I., Kirkman, H. and Lethbridge, R. (1993). The flora and fauna of Rottnest Island, Western Australia. Volumes 1E 2. Proceedings of the Fifth International Marine Biological Workshop. Western Australian Museum/AMSA: Perth.

Wells, F.E., Walker, D.I. and Jones, D.J. (2003). The marine flora and fauna of Dampier, Western Australia. Proceedings of the Twelfth International Marine Biological Workshop. Western Australian Museum: Perth.

Wells, F.E., Walker, D.I. and Kendrick, G.A. (2005). The marine flora and fauna of Esperance, Western Australia. Proceedings of the Twelfth International Marine Biological Workshop. Western Australian Museum: Perth. 
Weltner, W. (1910). Spongillidae. In: Michaelsen, W. and Hartmeyer, R. (eds), Die fauna Südwest-Australiens. Ergebnisse der Hamburger sudwest-australischen Forschungsreise 3(5): 135-144.

Whitelegge, T. (1900). Scientific results of the trawling expedition of H.M.C.S. 'Thetis' off the coast of New South Wales in February and March, 1898. Crustacea. Part I. Memoirs of the Australian Museum 4: 135-199, pls 132-135.

Wicksten, M.K. and Packard, J.M. (2005). A qualitative zoogeographic analysis of decapod crustaceans of the continental slopes and abyssal plain of the Gulf of Mexico. Deep-Sea Research Part I-Oceanographic Research Papers 52: 1745-1765.

Williams, A., Althaus, F., Dunstan, P.K., Poore, G.C.B., Bax, N.J., Kloser, R.J. and McEnnulty, F.R. (2010).
Scales of habitat heterogeneity and megabenthos biodiversity on an extensive Australian continental margin (100-1100 m depths). Marine Ecology (an Evolutionary Perspective) 31: 222-236.

Williams, A., Bax, N.J., Kloser, R.J., Althaus, F., Barker, B. and Keith, G. (2009). Australia's Deep-Water Reserve Network: Implications of false homogeneity for classifying abiotic surrogates of biodiversity. ICES Journal of Marine Science 66: 214-224.

Williams, A., Koslow, J.A. and Last, P.R. (2001). Diversity, density and community structure of the demersal fish fauna of the continental slope off Western Australia (20 to 35 degrees S). Marine Ecology-Progress Series 212: 247-263.

MANUSCRIPT RECEIVED JUNE 2010; ACCEPTED MARCH 2011. 

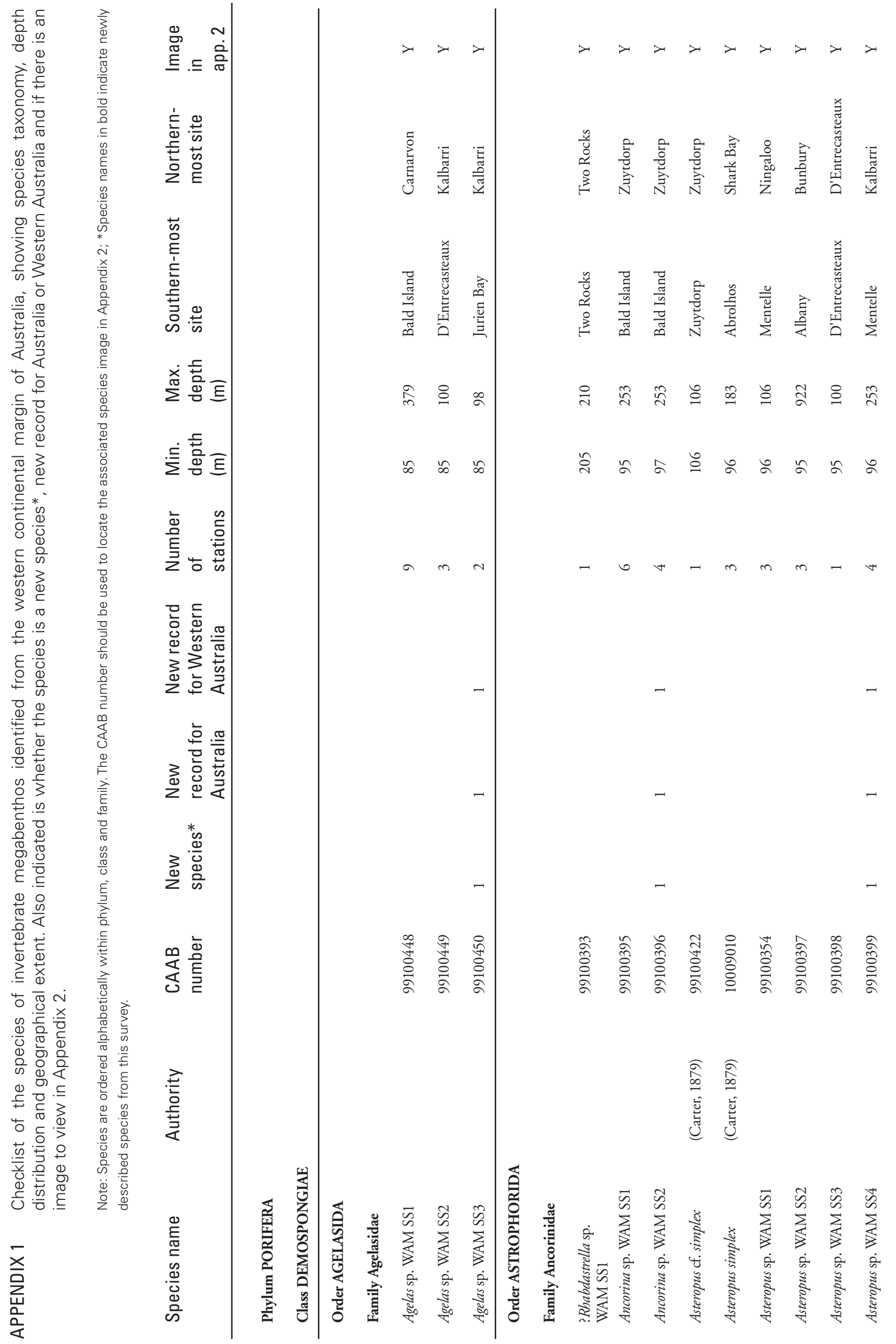


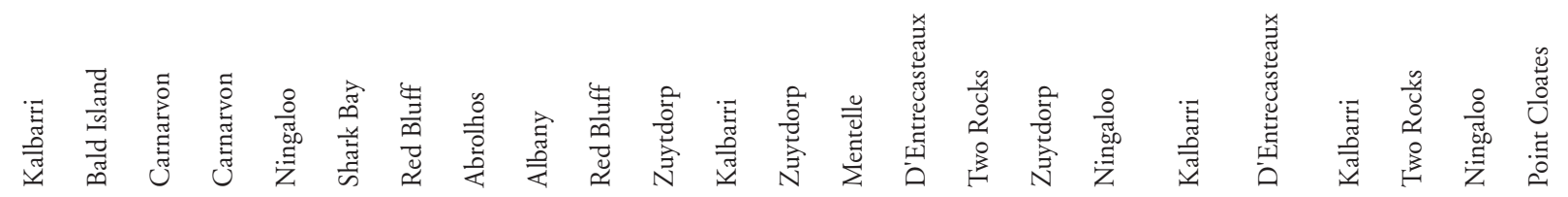

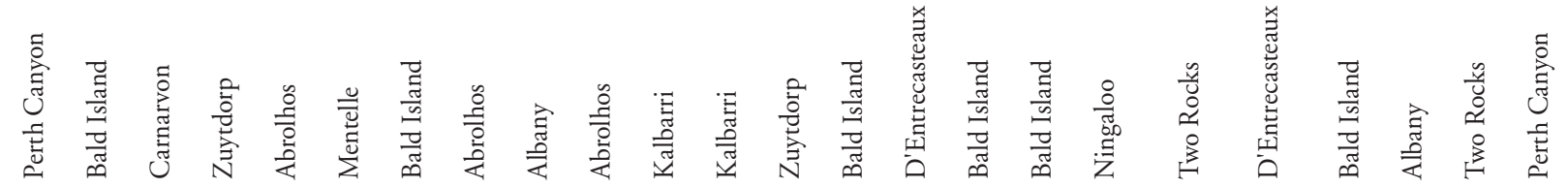

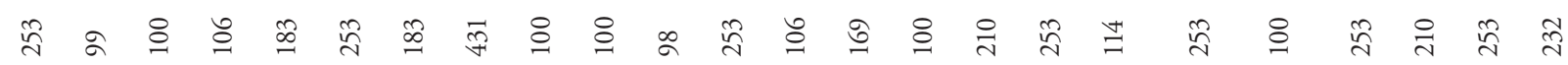

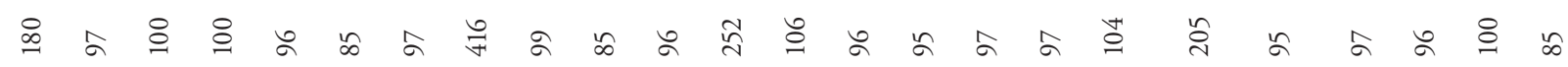

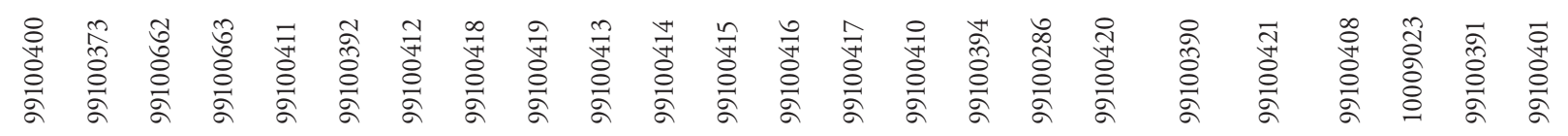

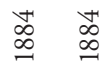

$$
\begin{aligned}
& \text { 莗 }
\end{aligned}
$$

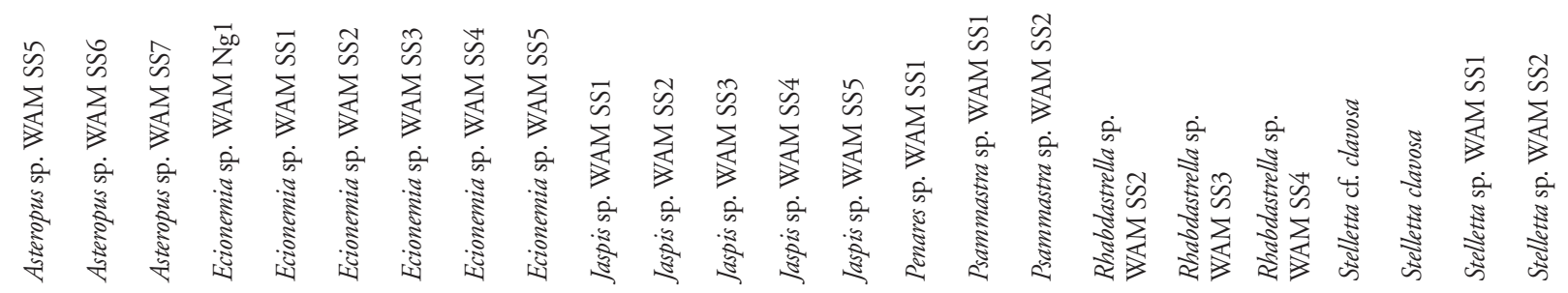




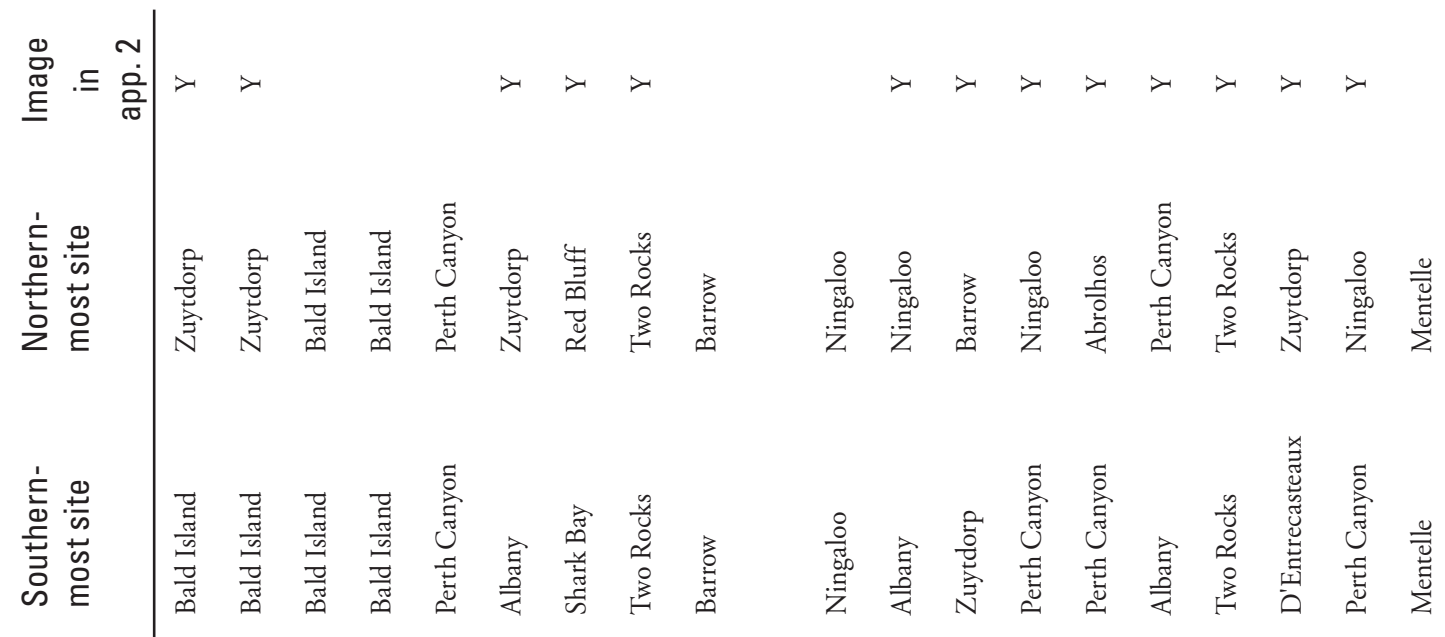

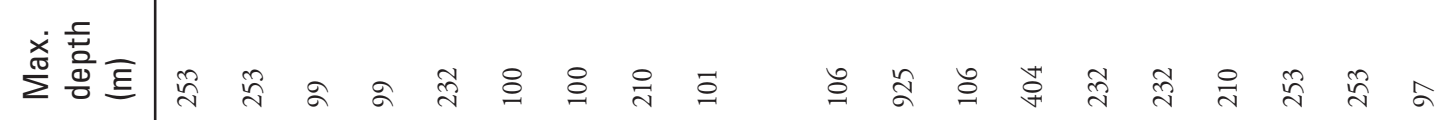

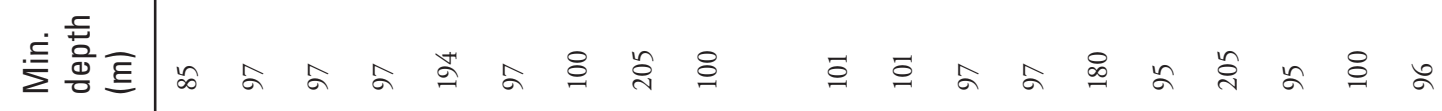

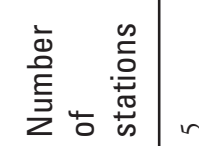

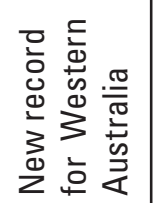

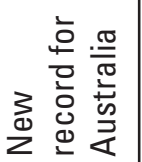

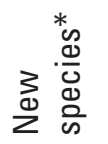

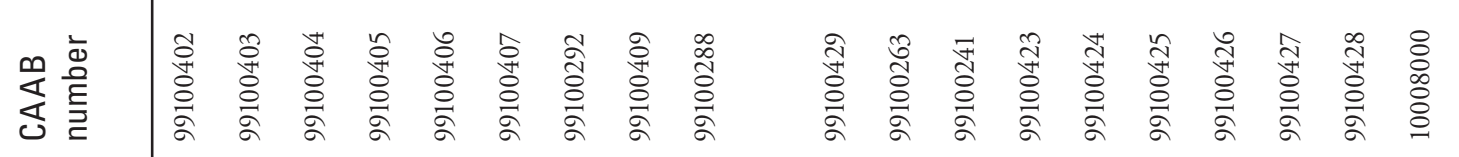

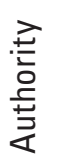

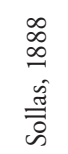

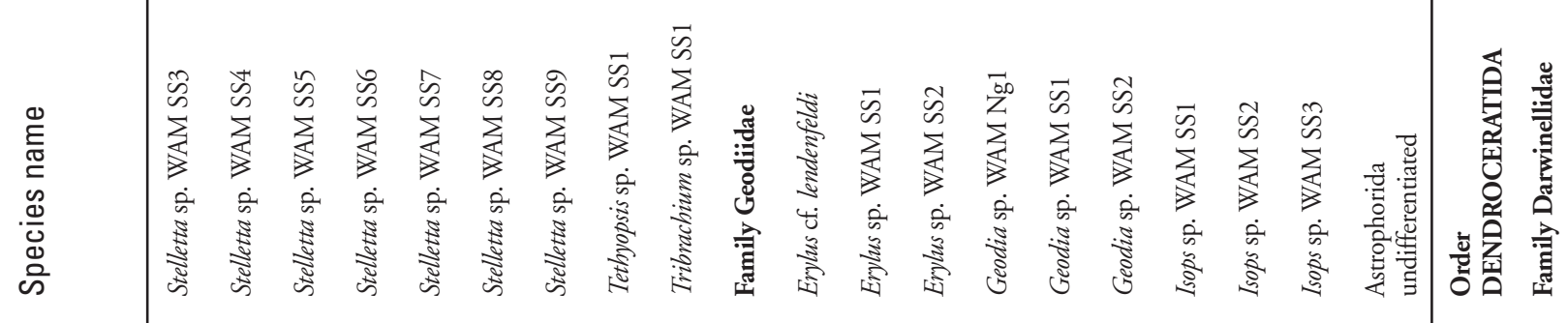




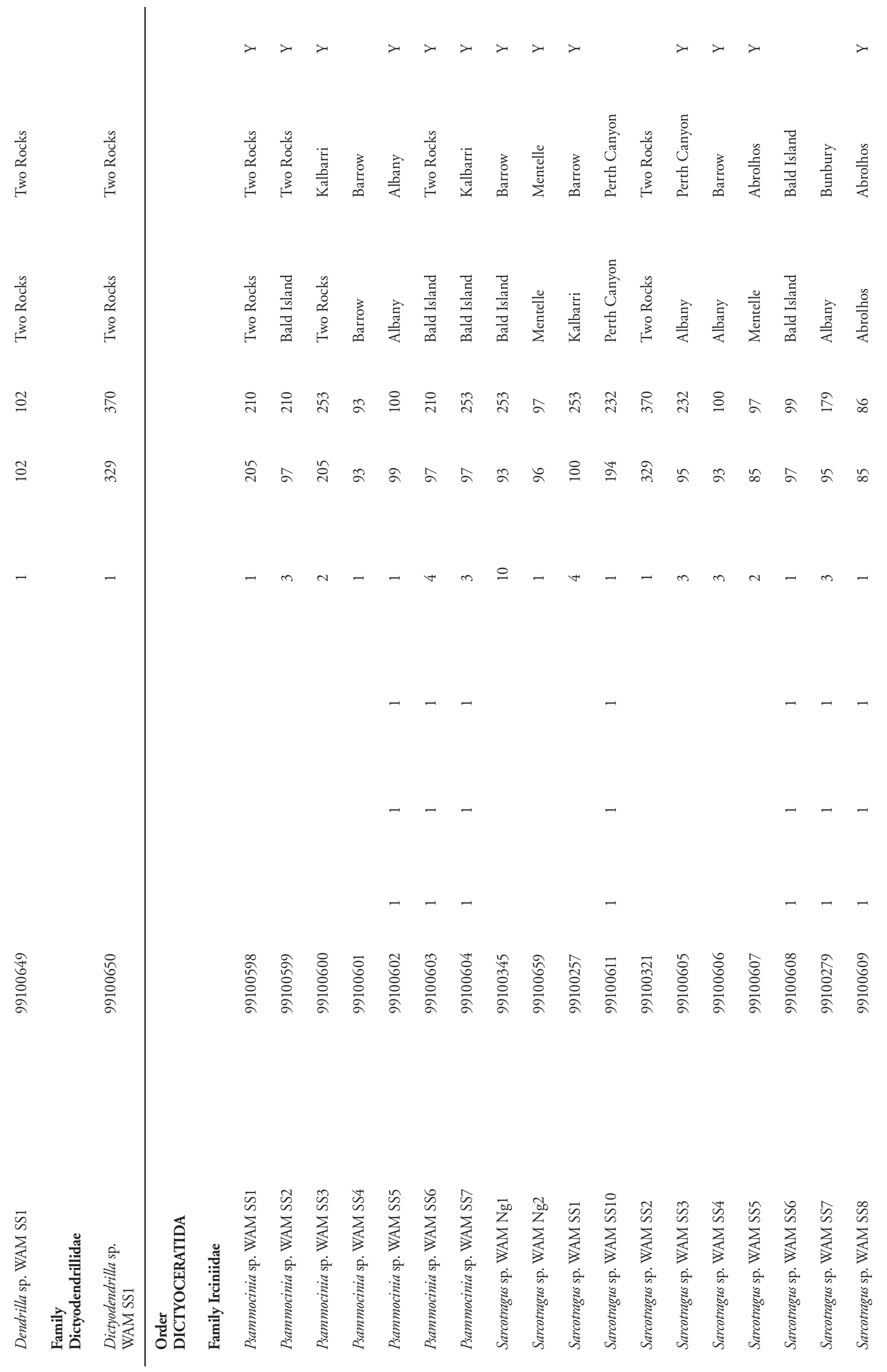




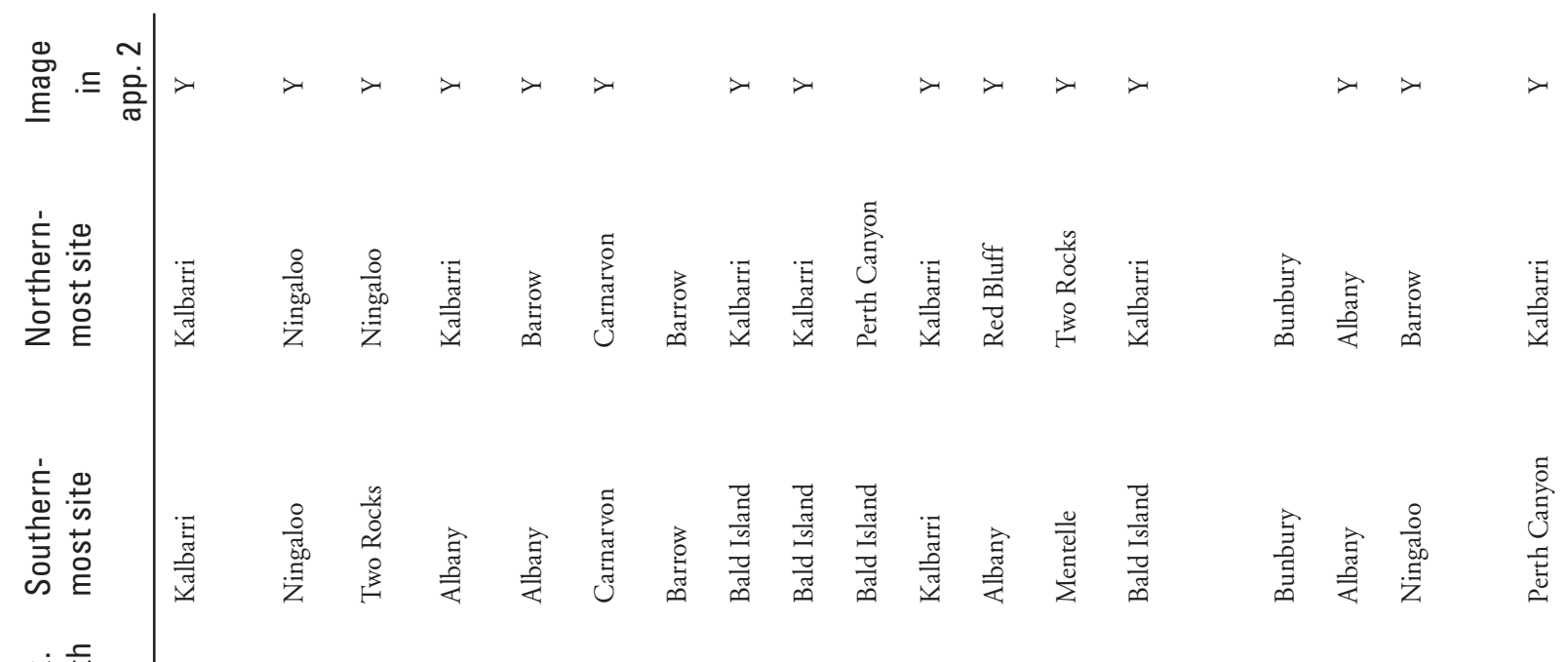

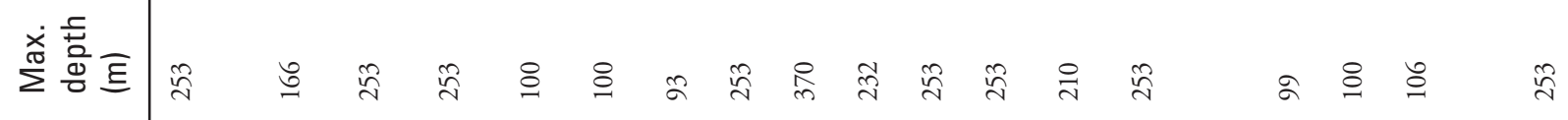

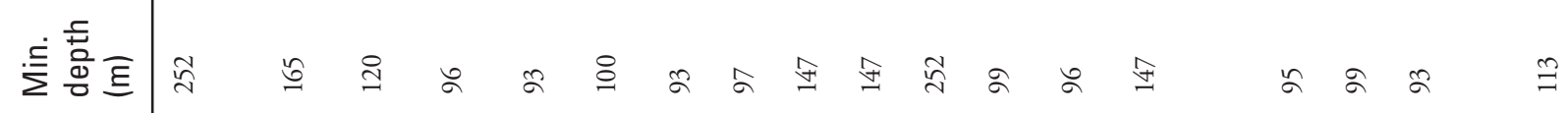

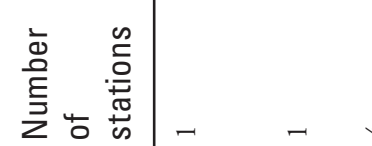

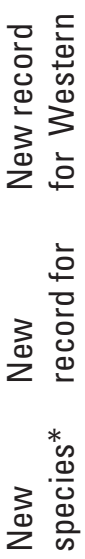

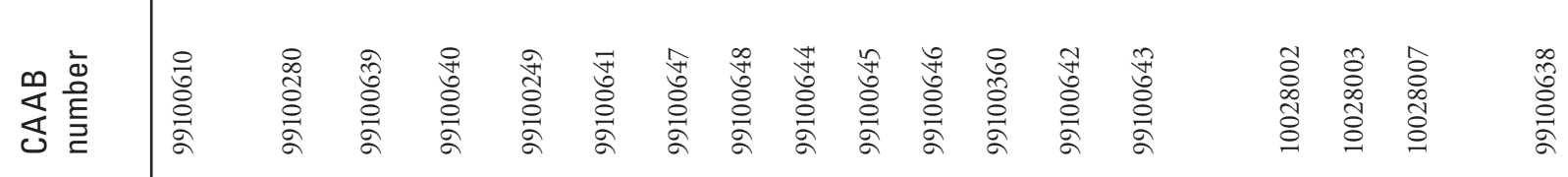
른

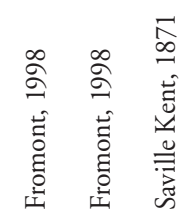

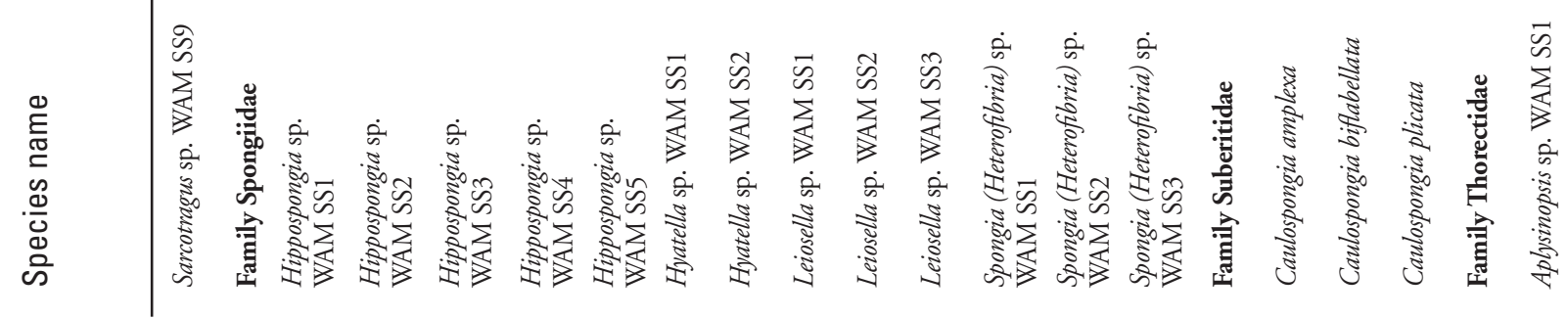




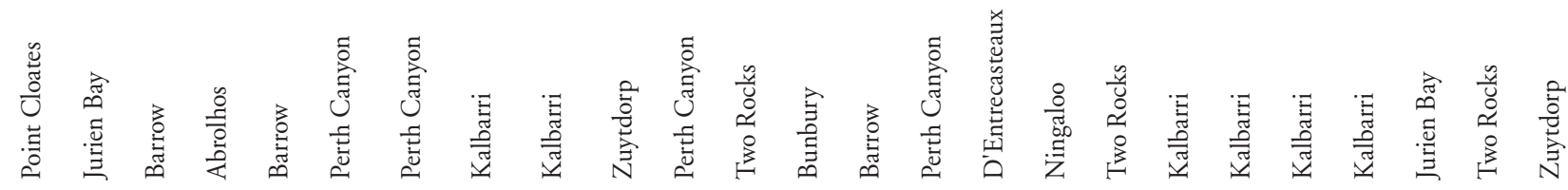

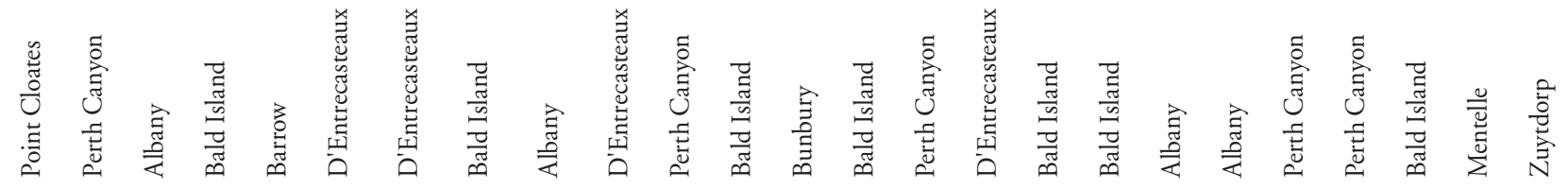

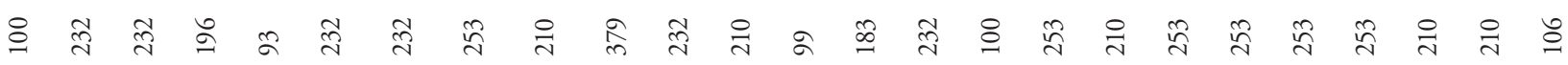

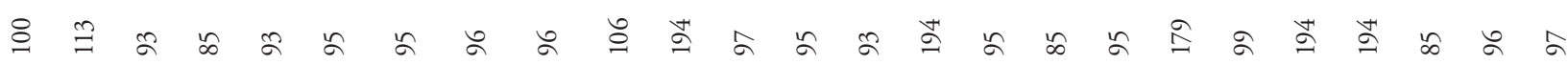

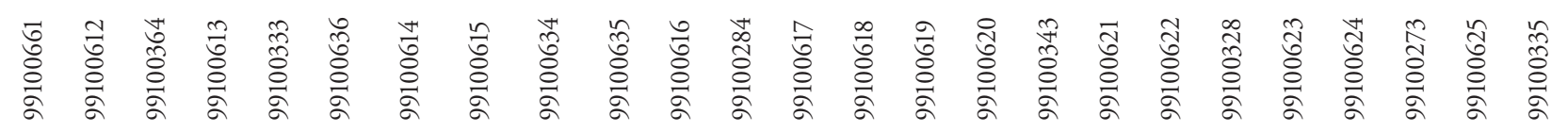

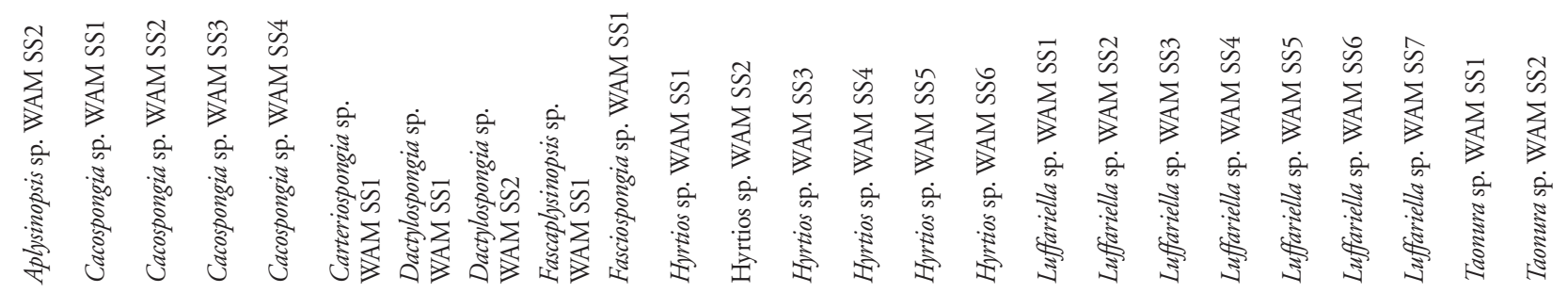




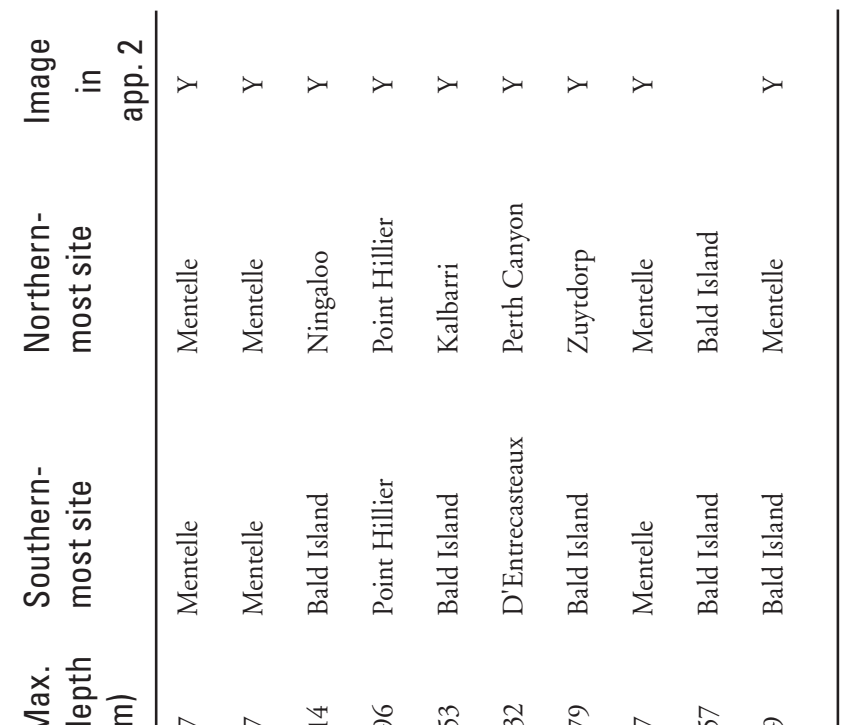

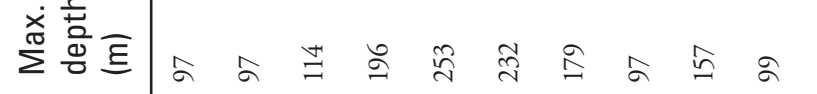

妾葶主

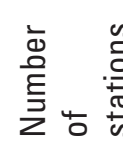

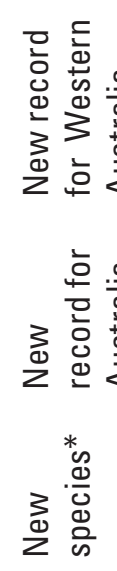

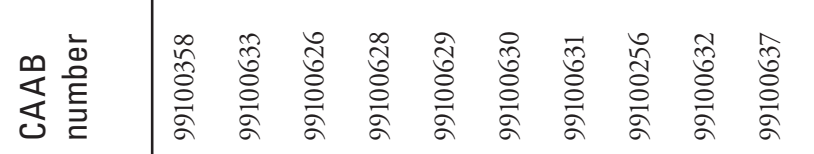

辛

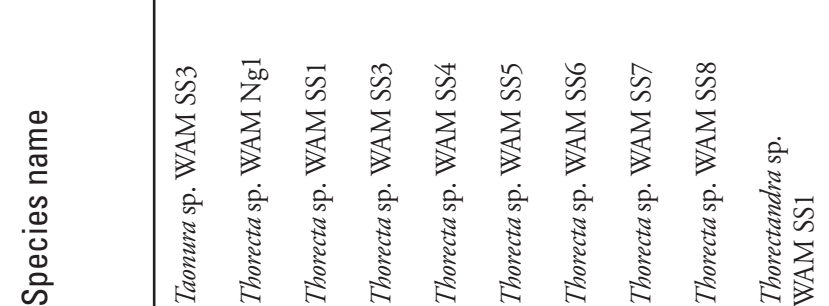

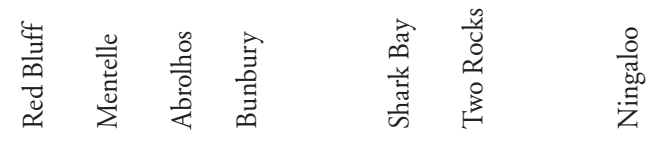

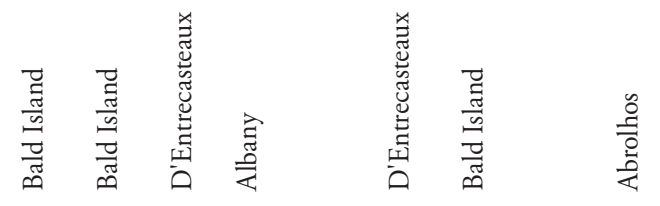

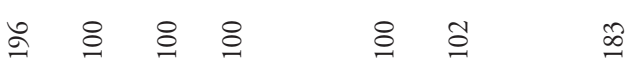

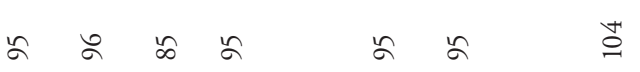

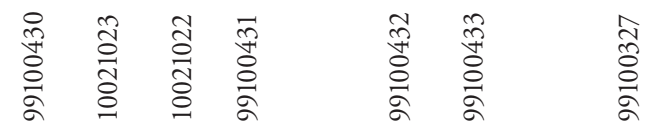

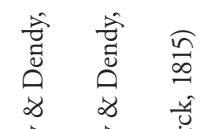

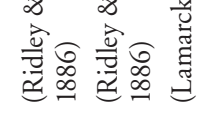

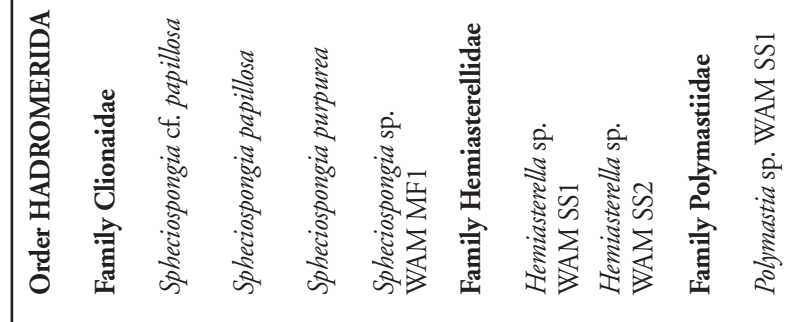




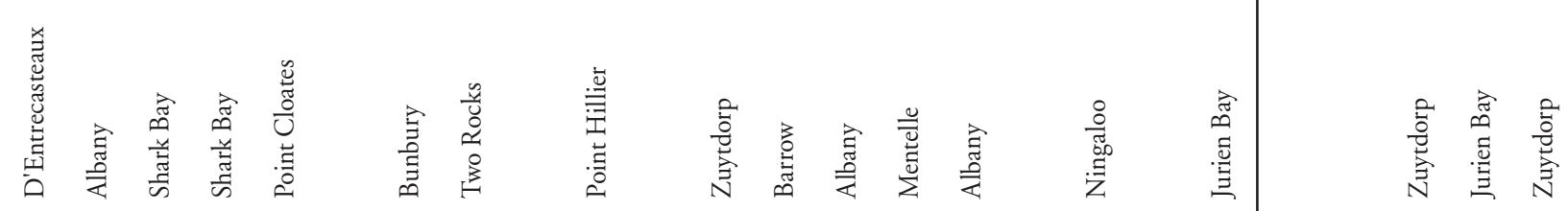

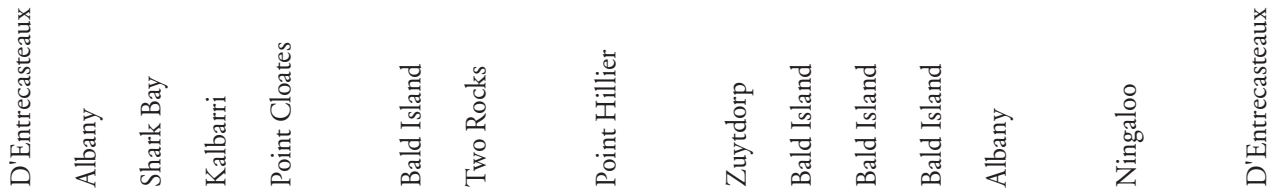

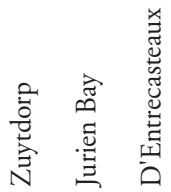

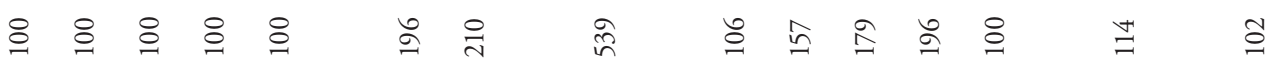

$\approx \approx \approx$

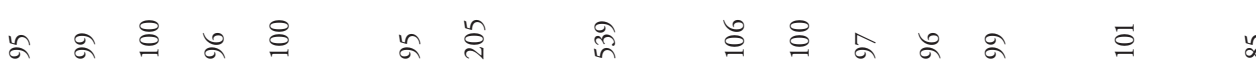

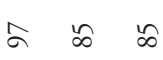

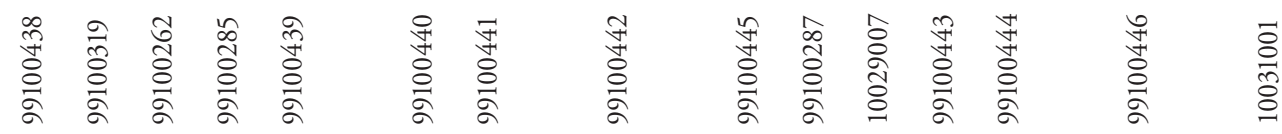

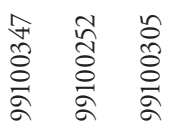

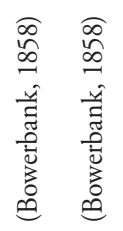

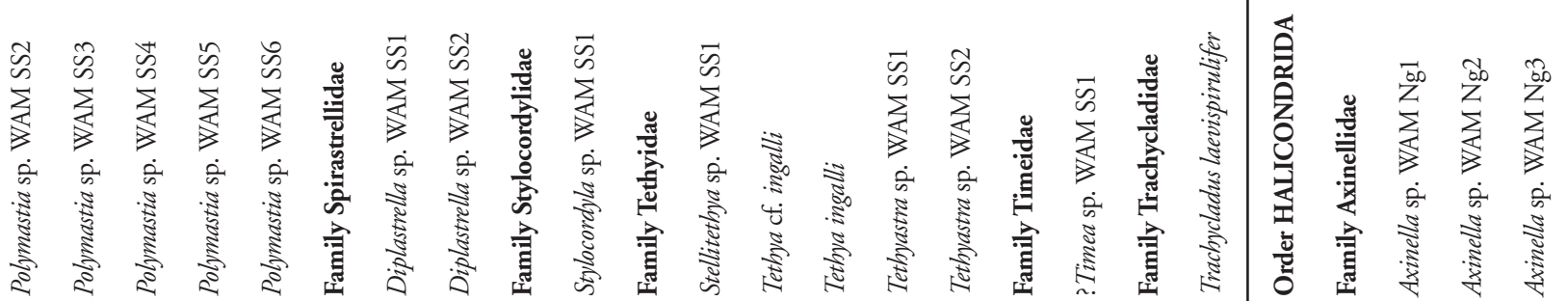




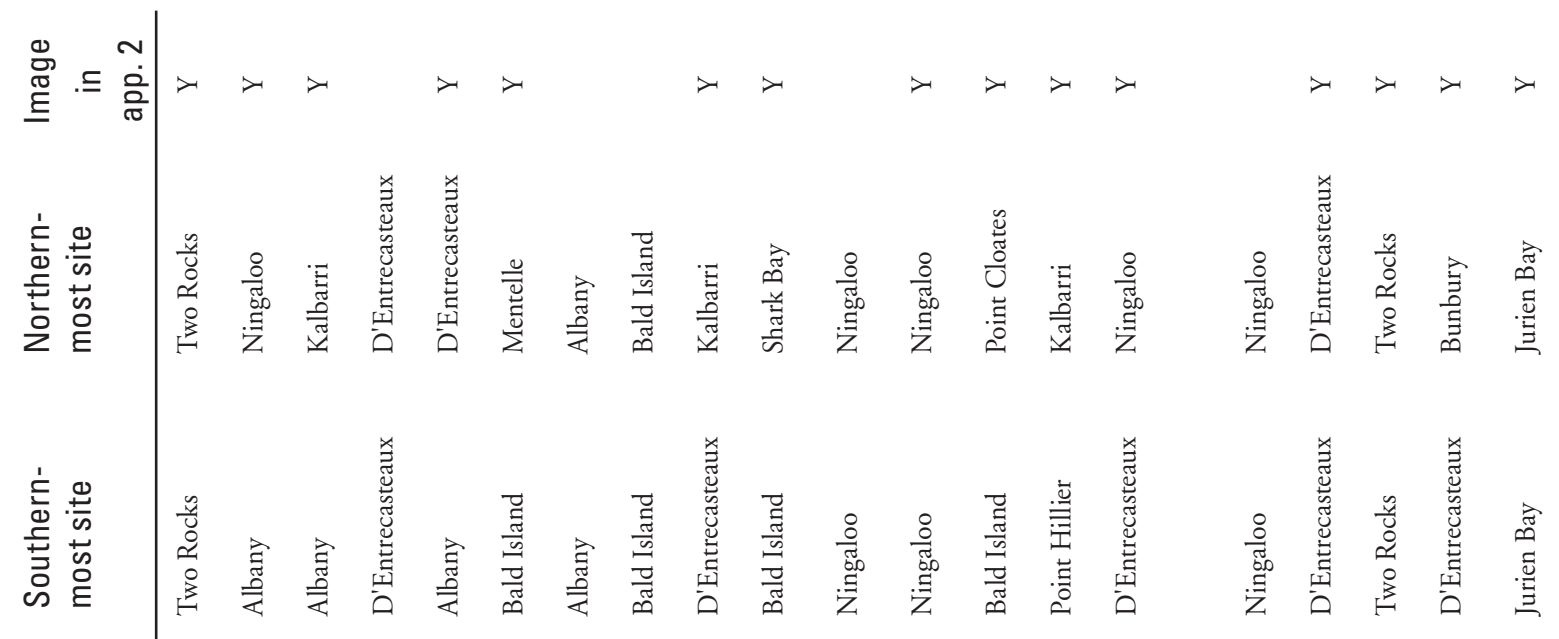

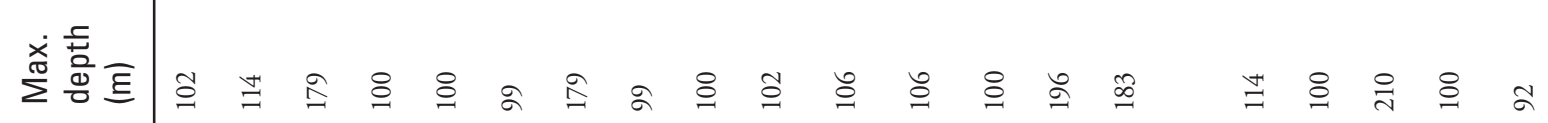

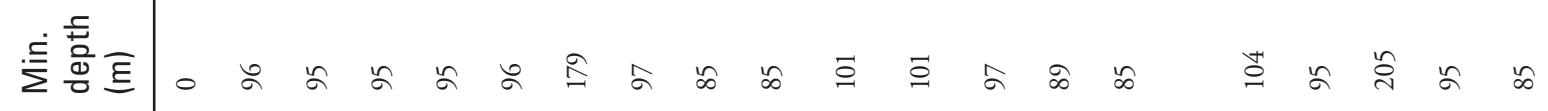

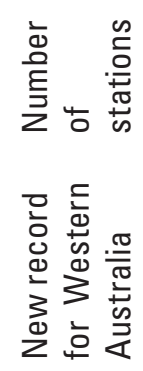

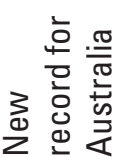

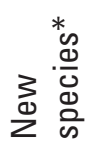

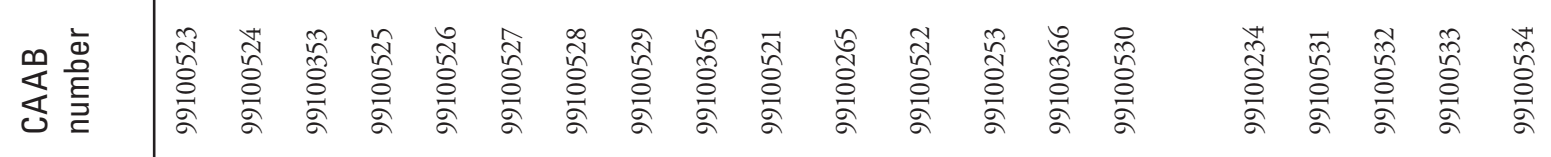

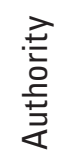

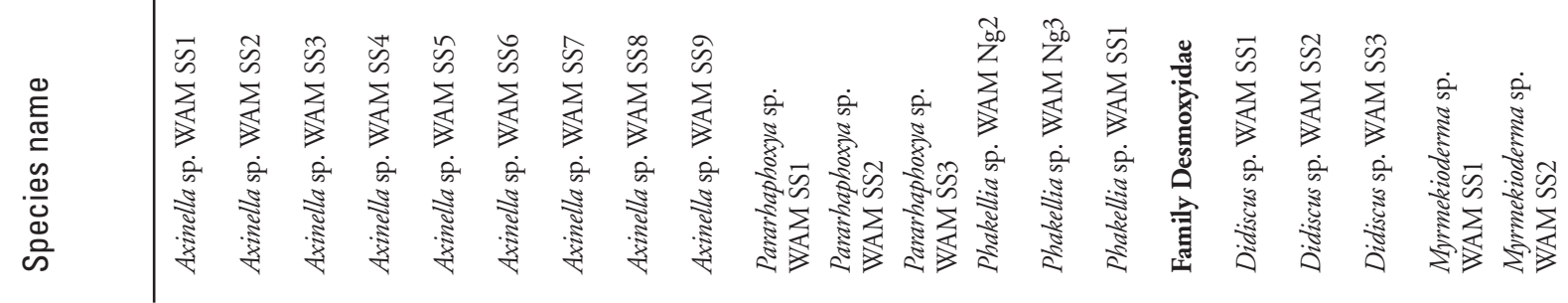




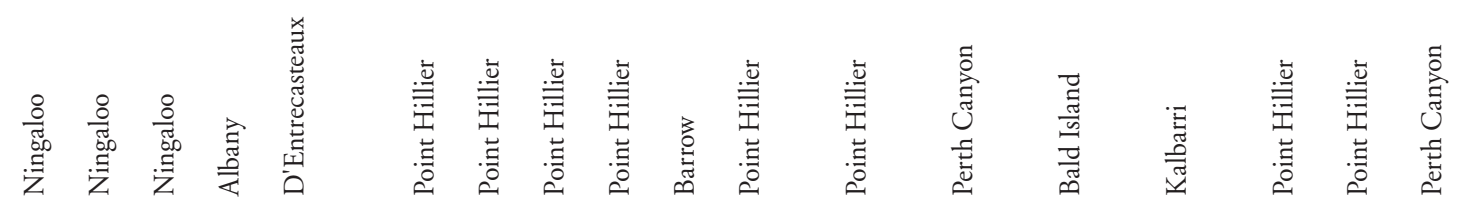

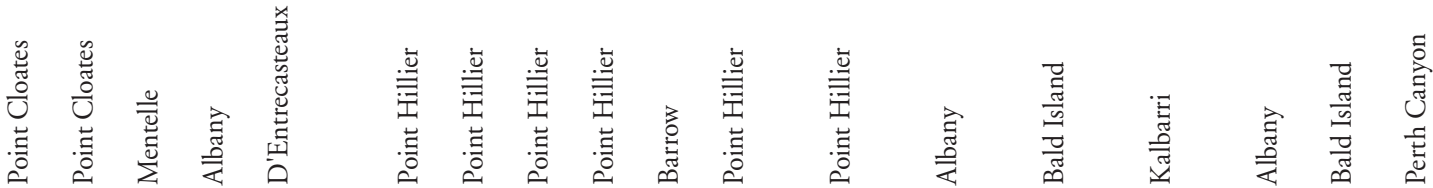

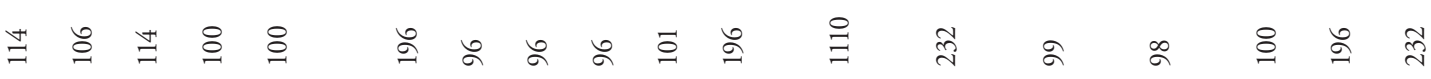

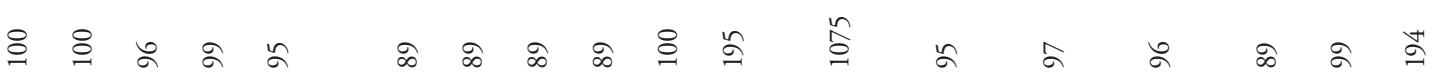

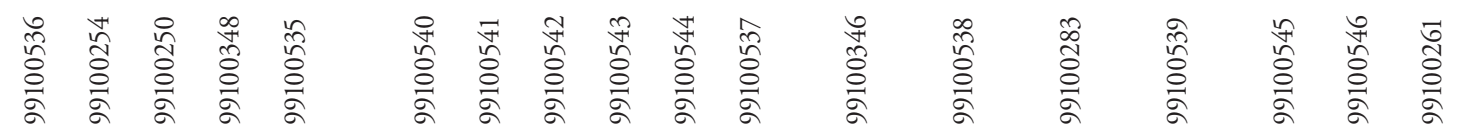

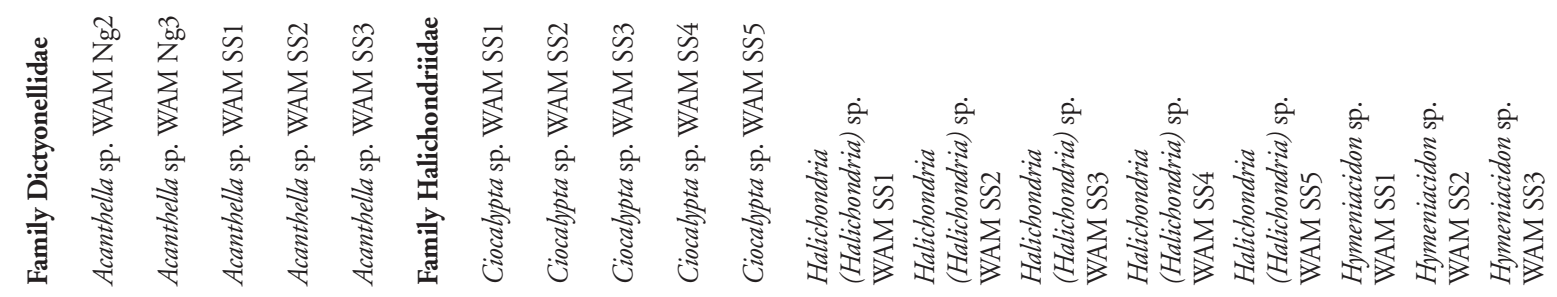




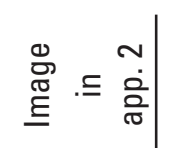

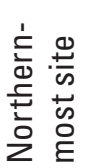

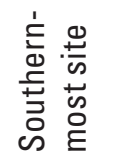

离营可

这营馬

离

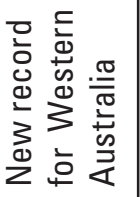

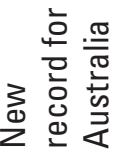

zon

这离

言

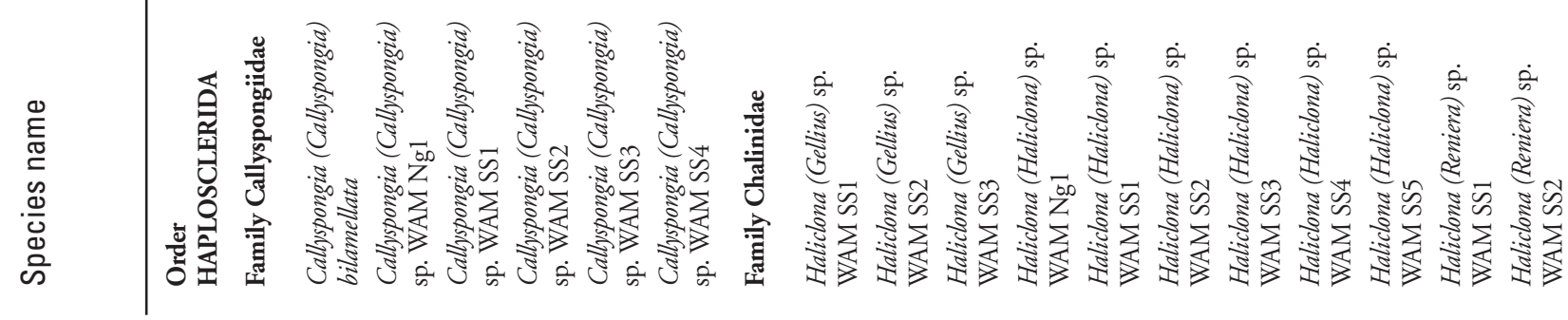




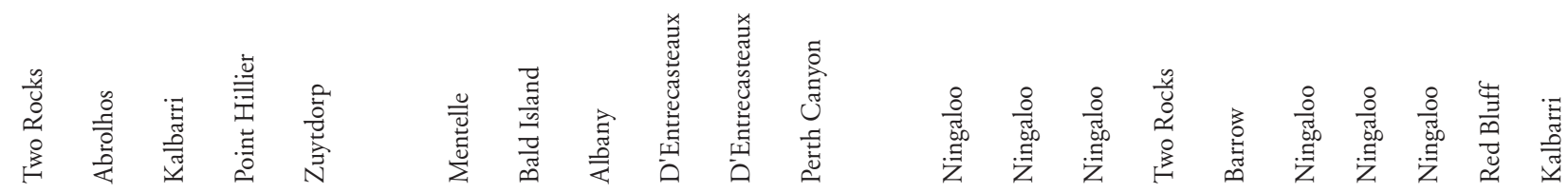

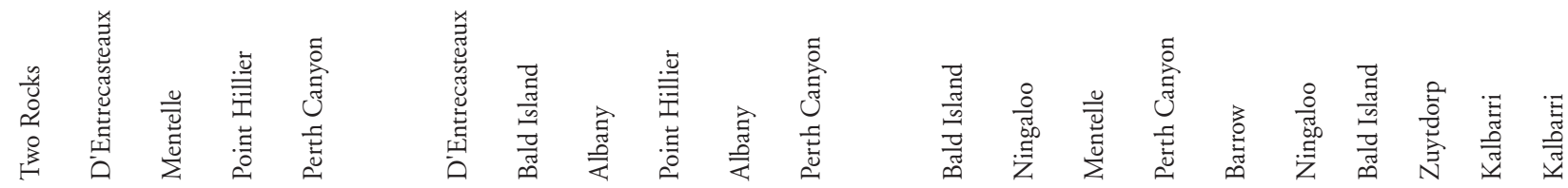

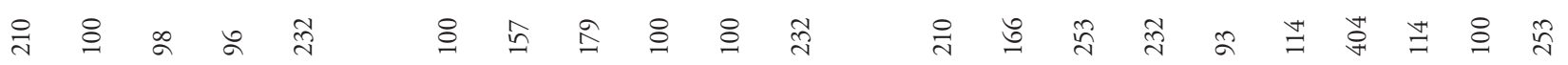
藏

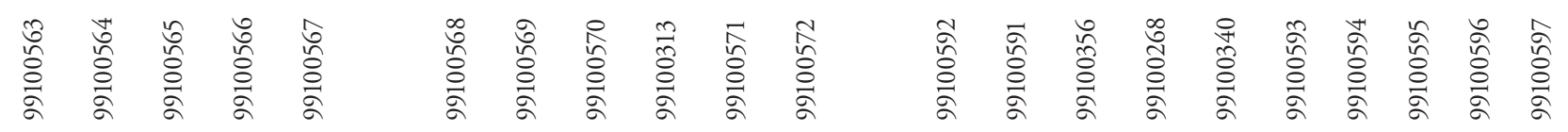

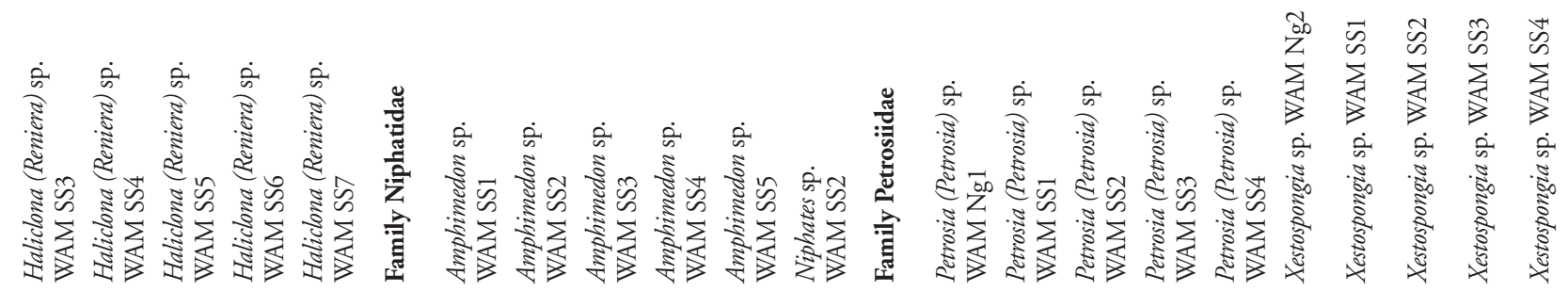




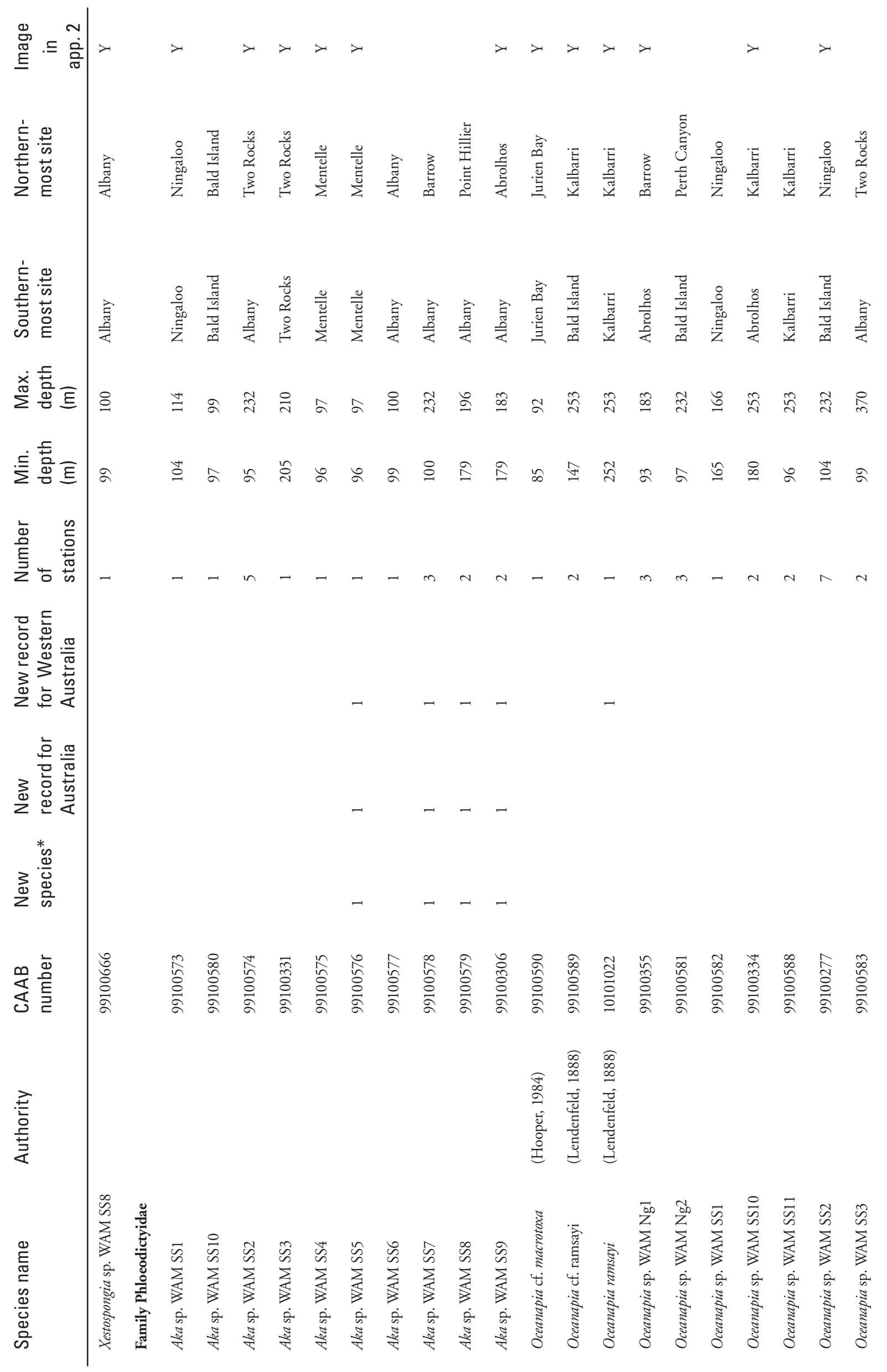




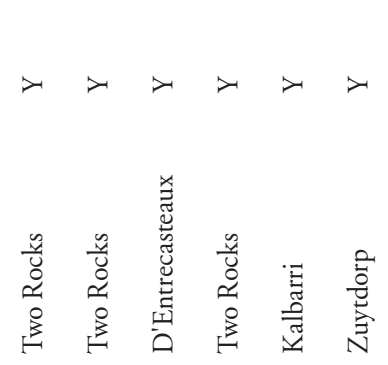

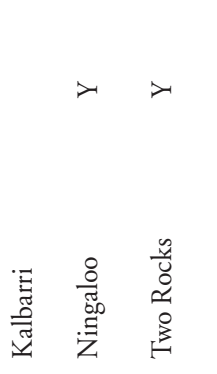

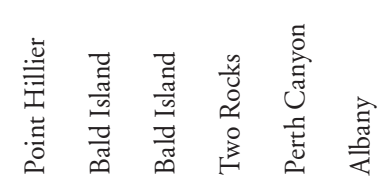

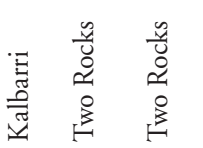

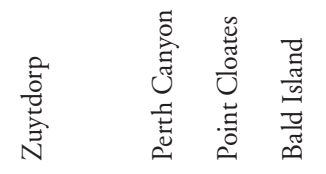

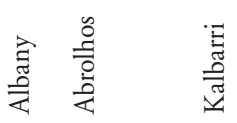

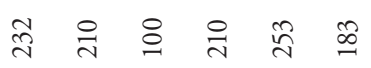

$$
\begin{aligned}
& \text { ิํㄱㅇำ }
\end{aligned}
$$

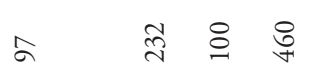

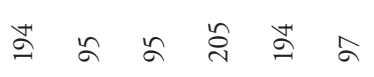

$$
\begin{aligned}
& \text { สิ ฉ } \\
& \text { ล } \stackrel{+}{2} \Xi
\end{aligned}
$$

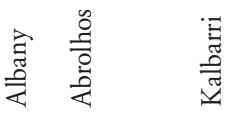

$$
\begin{aligned}
& \cong \cong \cong \\
& \curvearrowright \stackrel{\infty}{\because}
\end{aligned}
$$

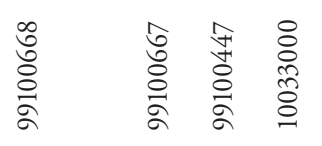

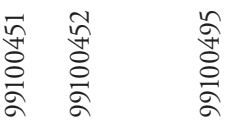

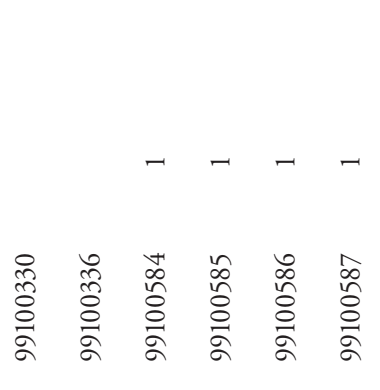

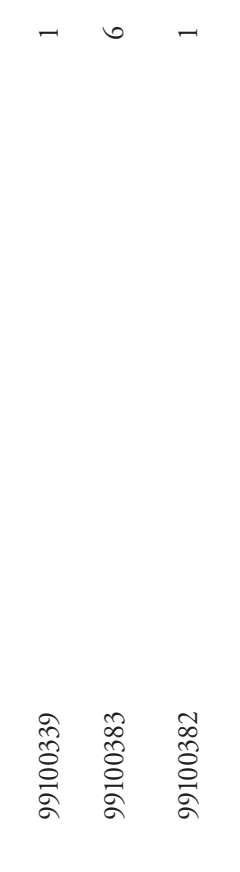

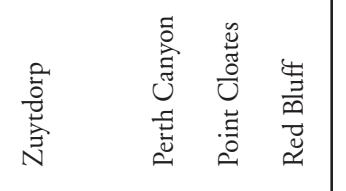

$$
\begin{aligned}
& \text { 를 } \\
& m 0 n-n \\
& -+n \\
& -\quad-
\end{aligned}
$$

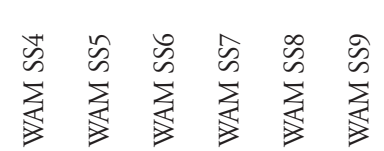

$$
\begin{aligned}
& \text { मे के के के के के }
\end{aligned}
$$

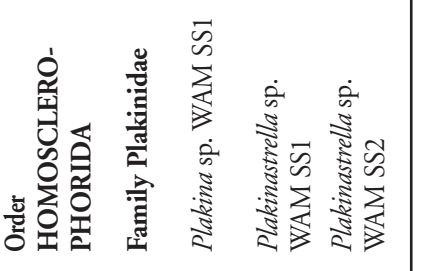

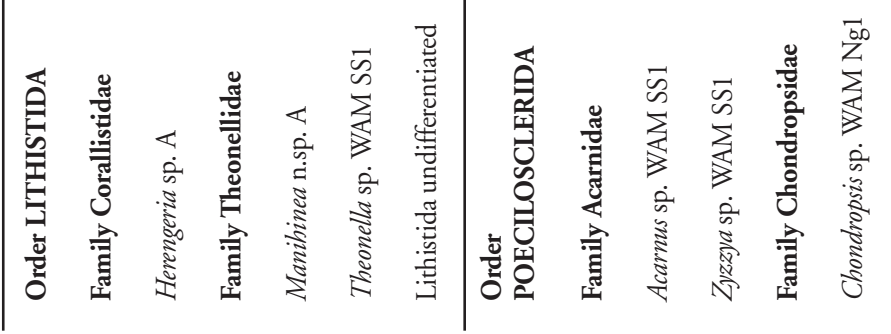




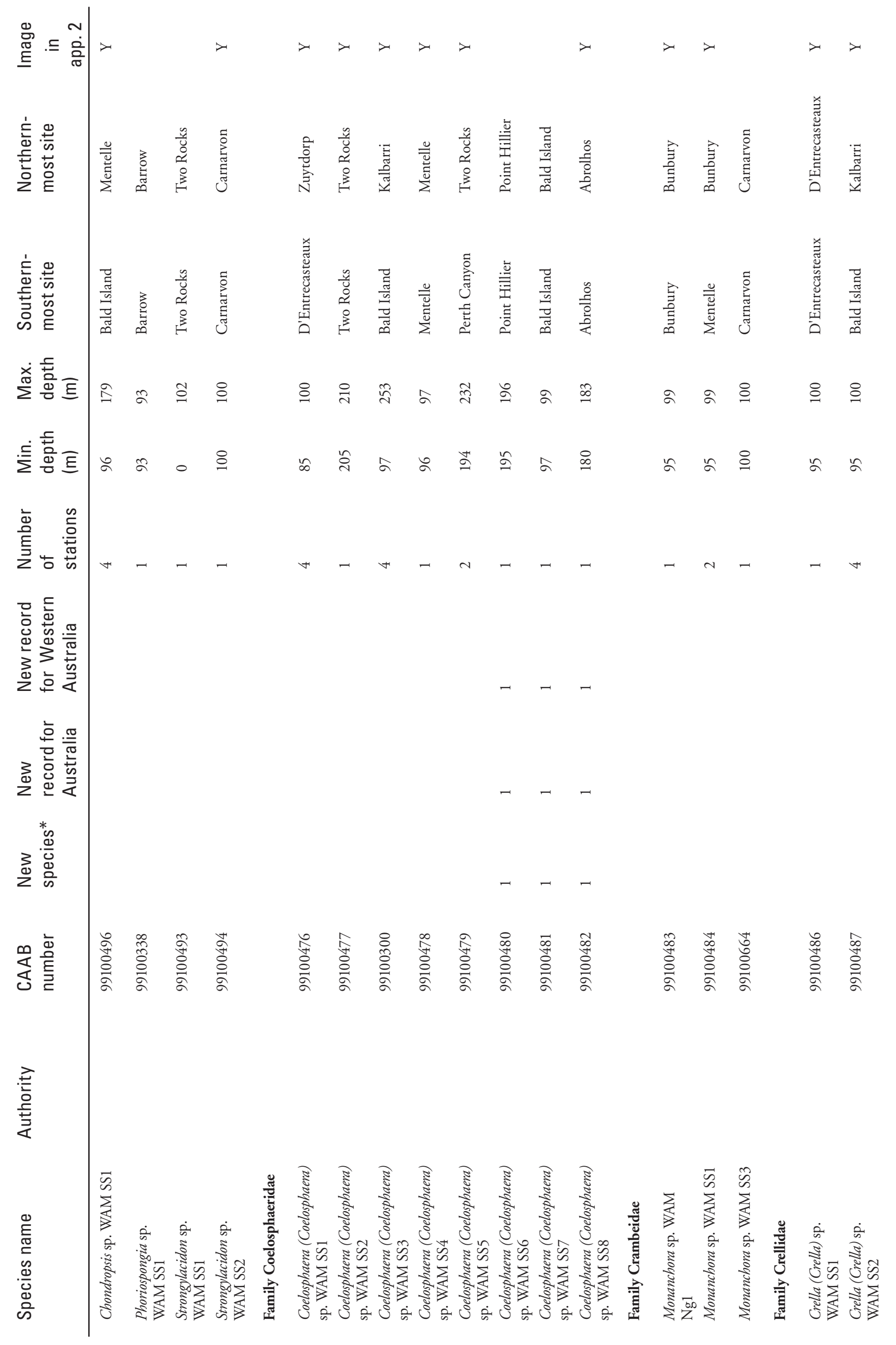




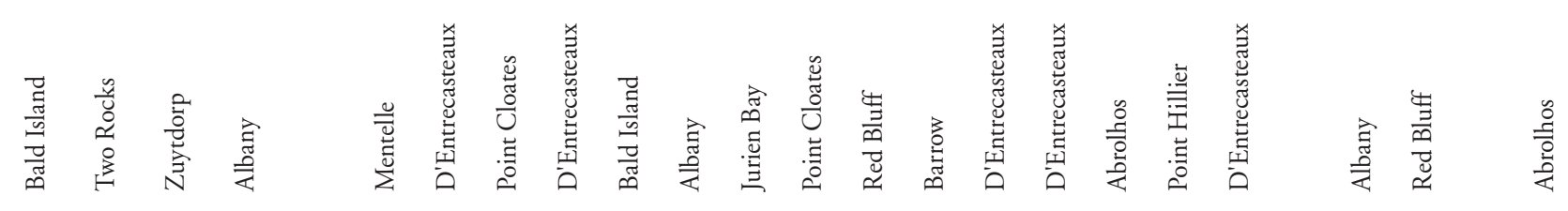

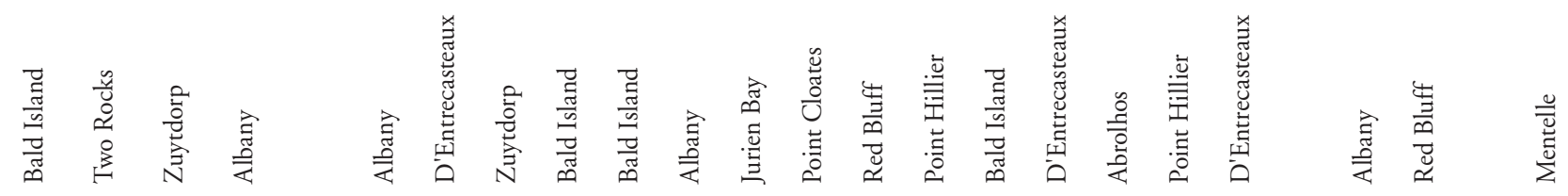

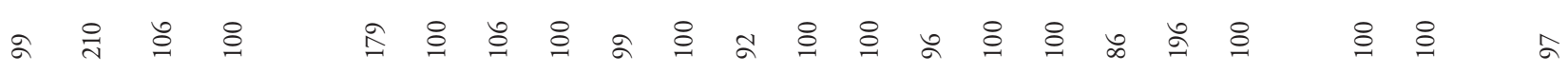

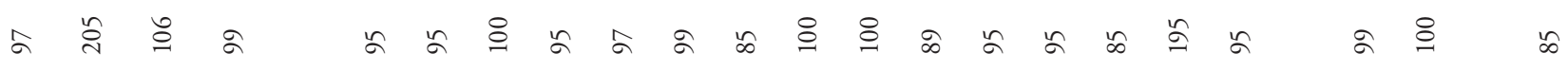

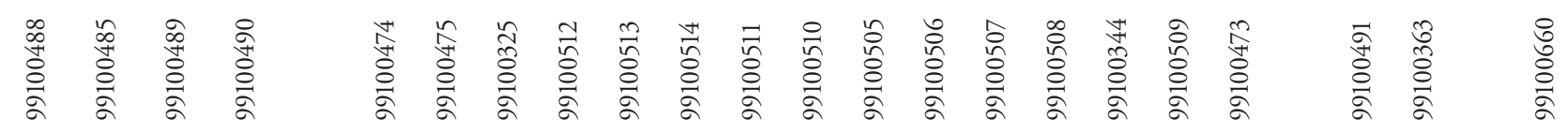

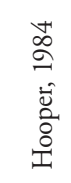

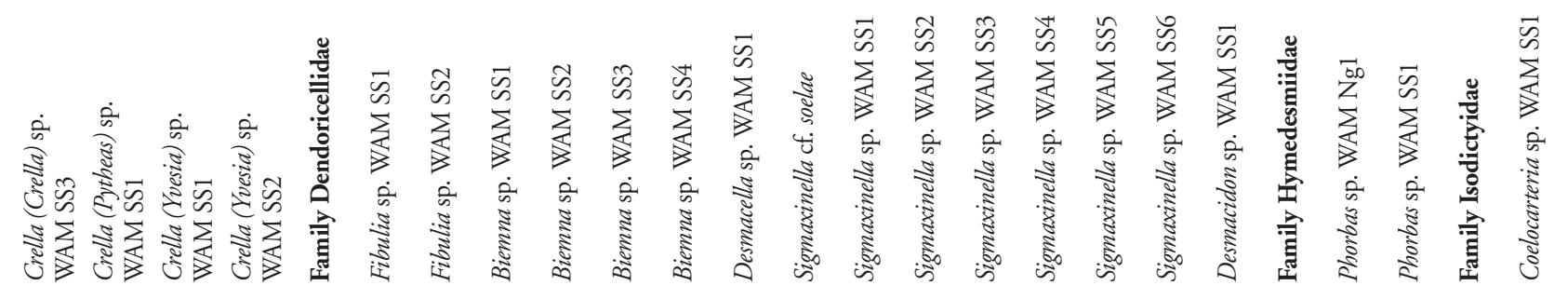




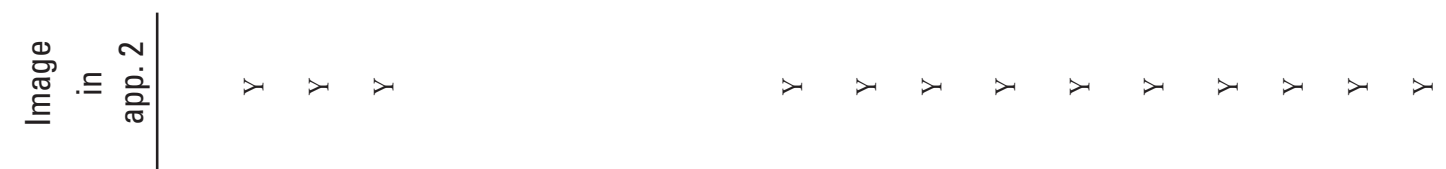

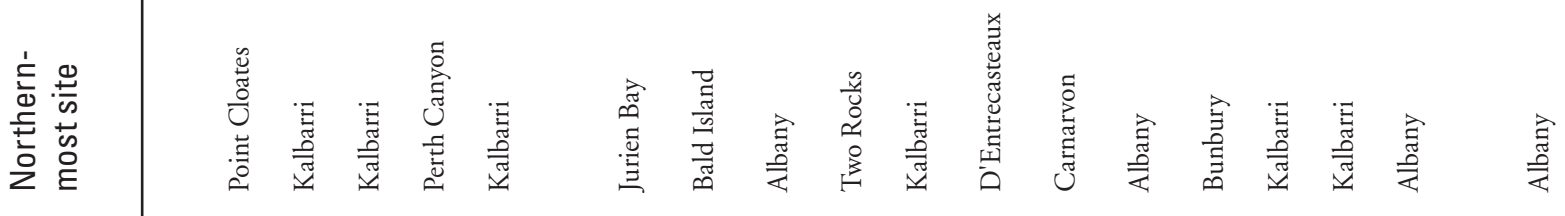

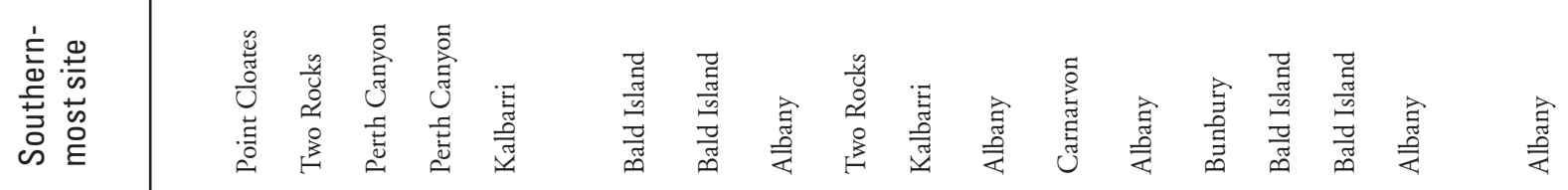

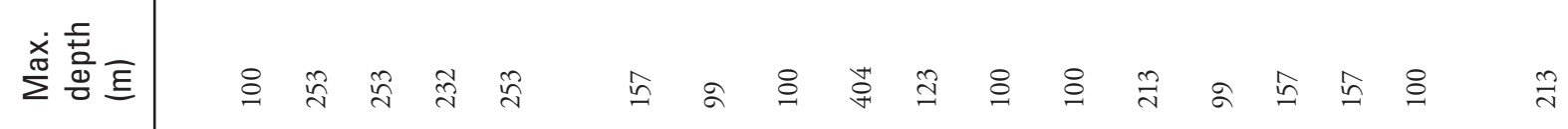

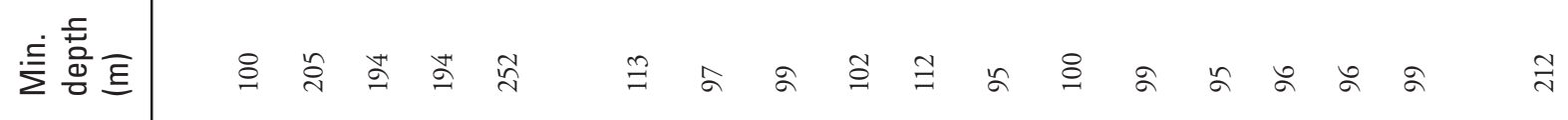

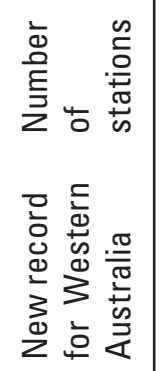

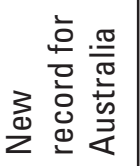

$\sum \frac{*}{\infty}$

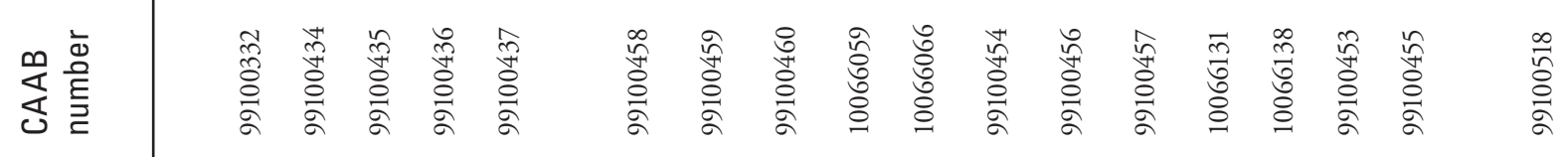

吾

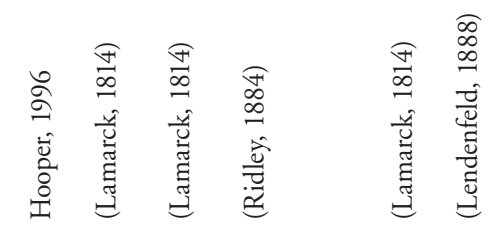

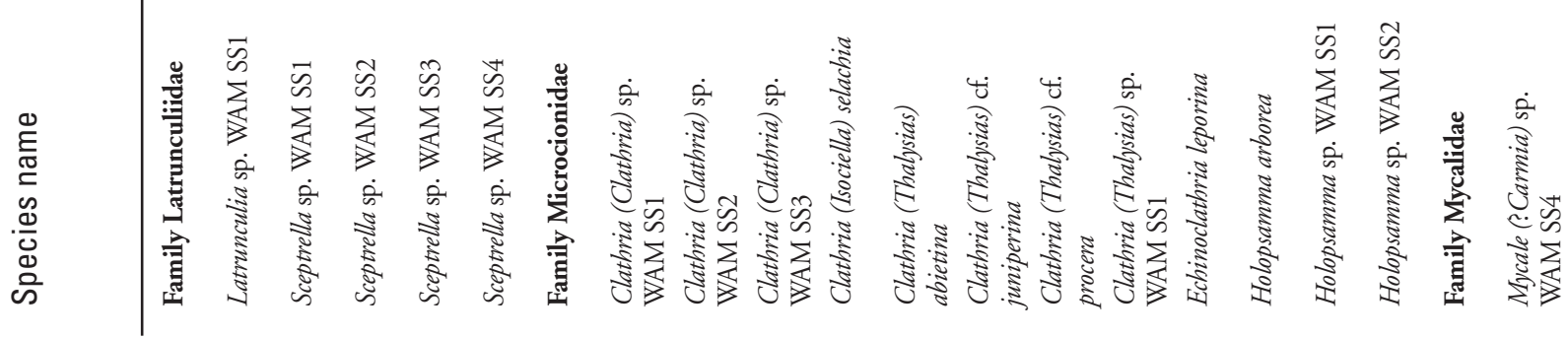




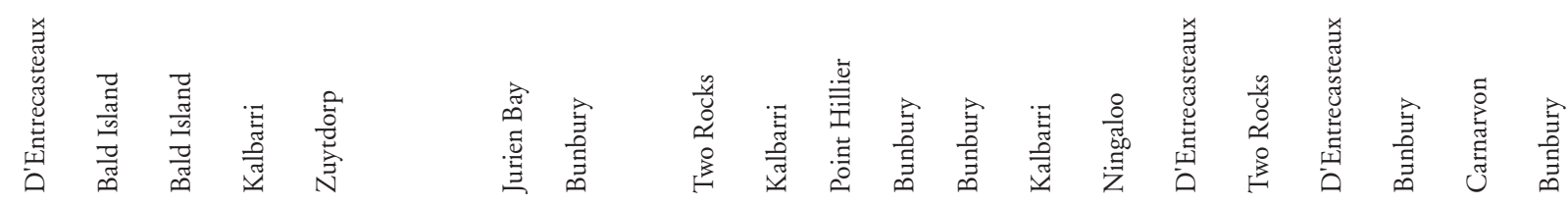

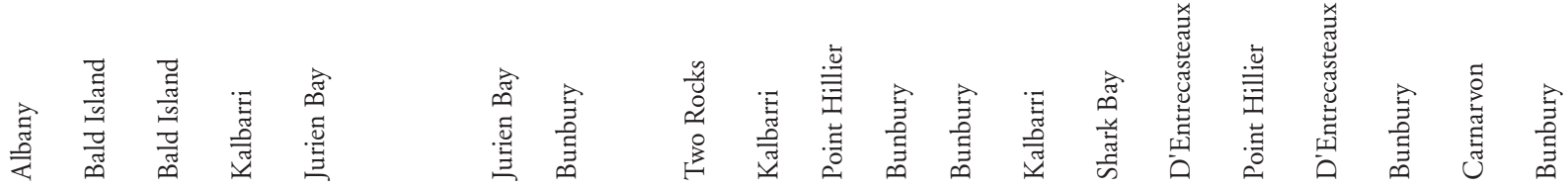

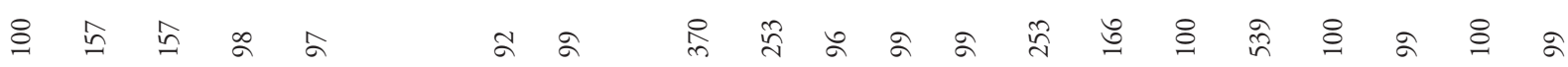

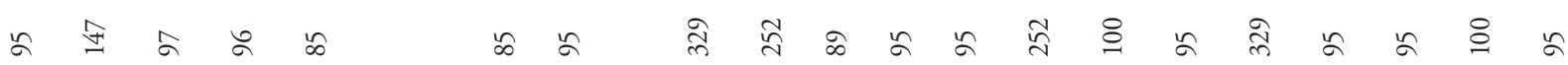

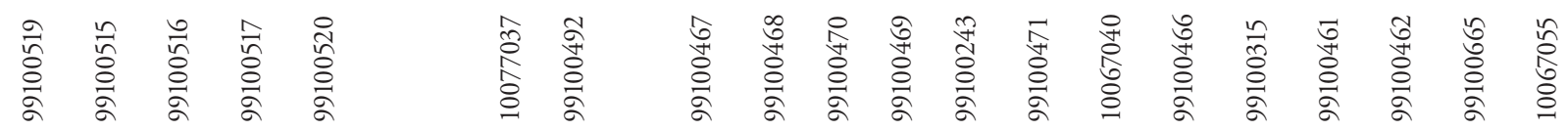

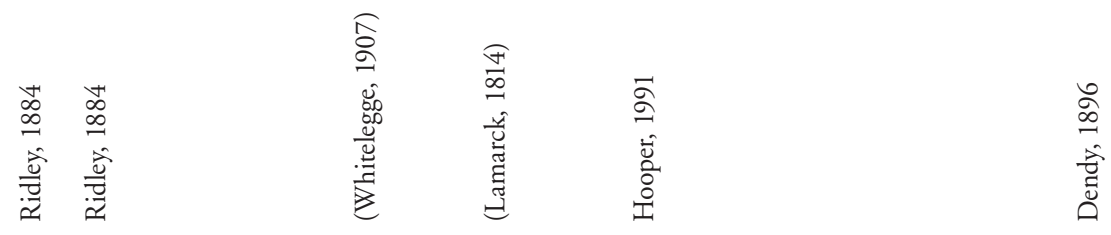

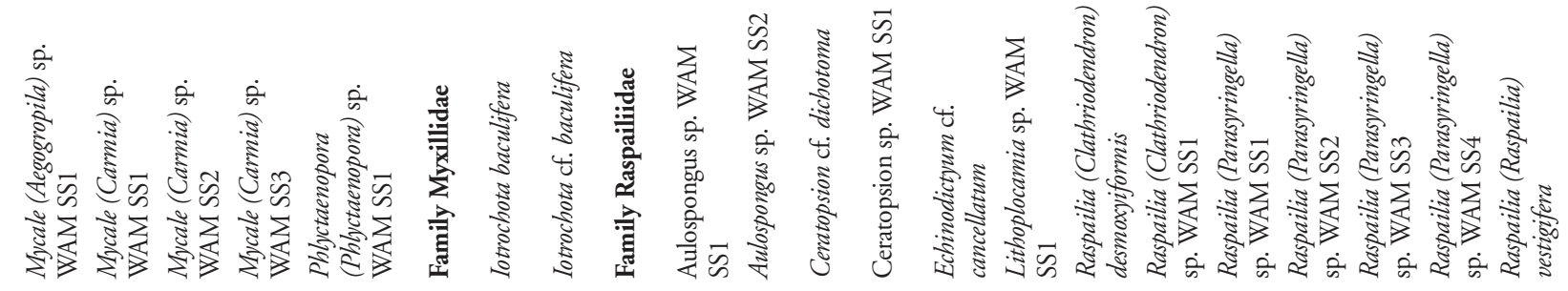




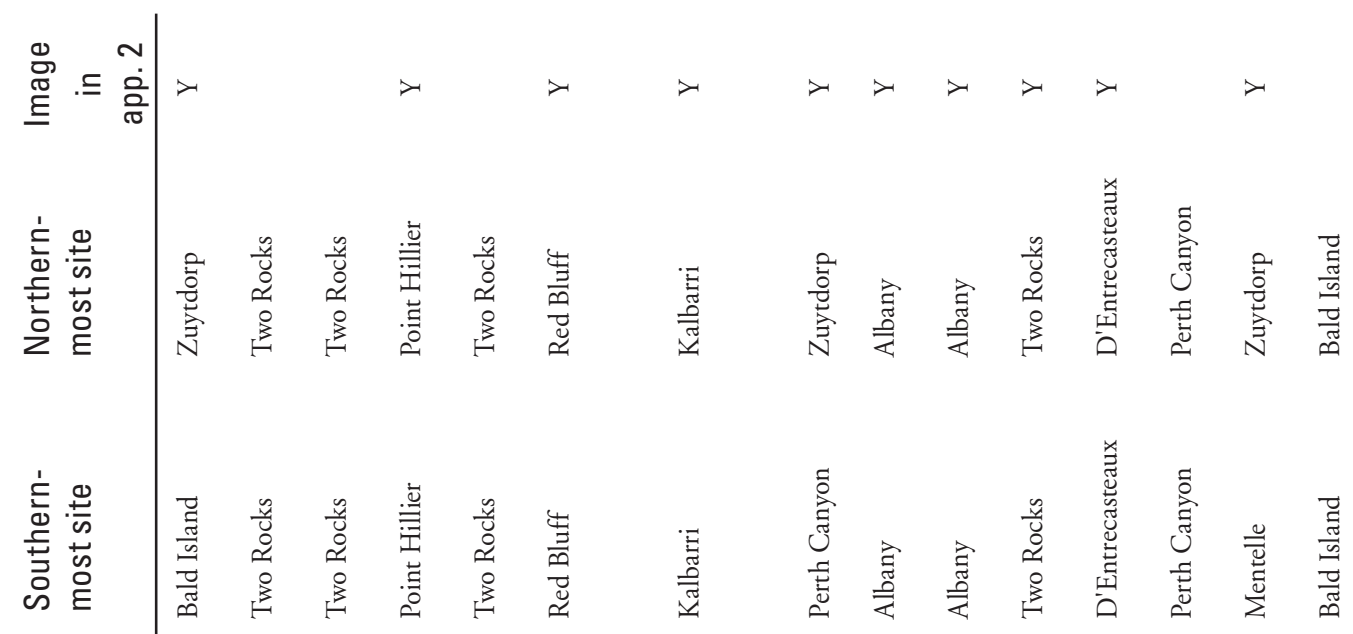

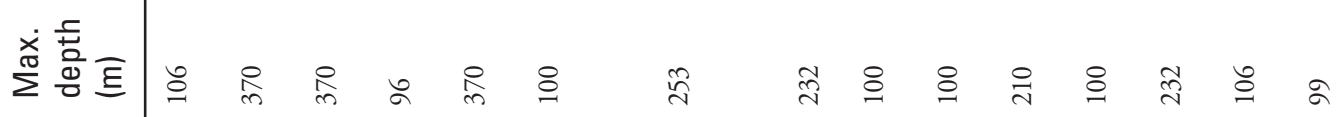

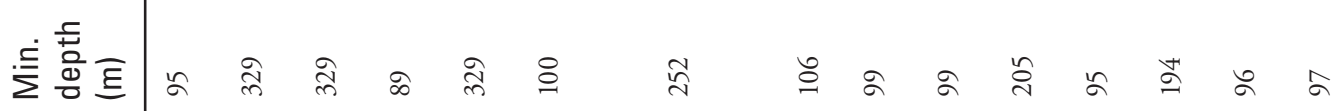

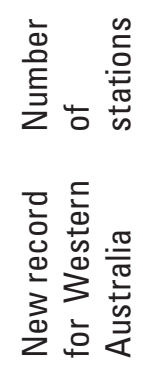

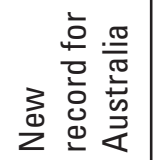
$\frac{2}{2}$

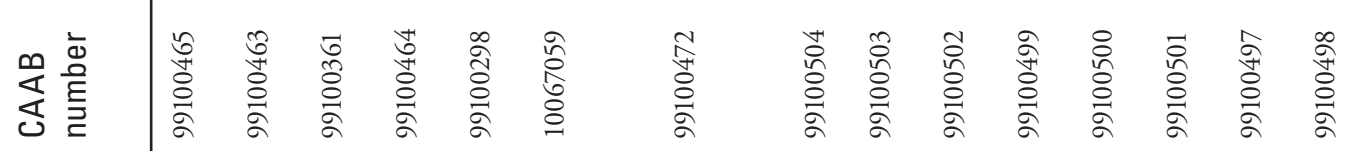

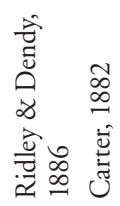

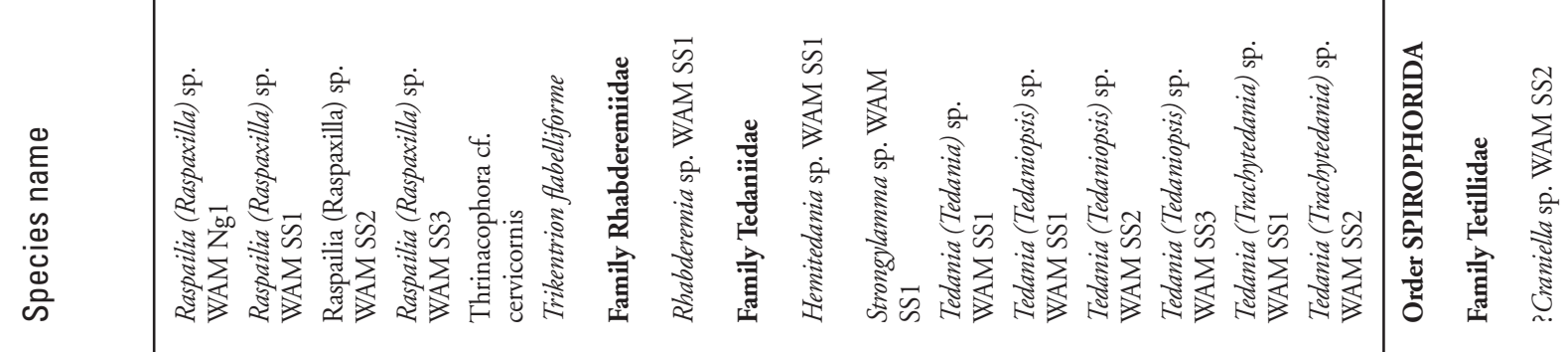




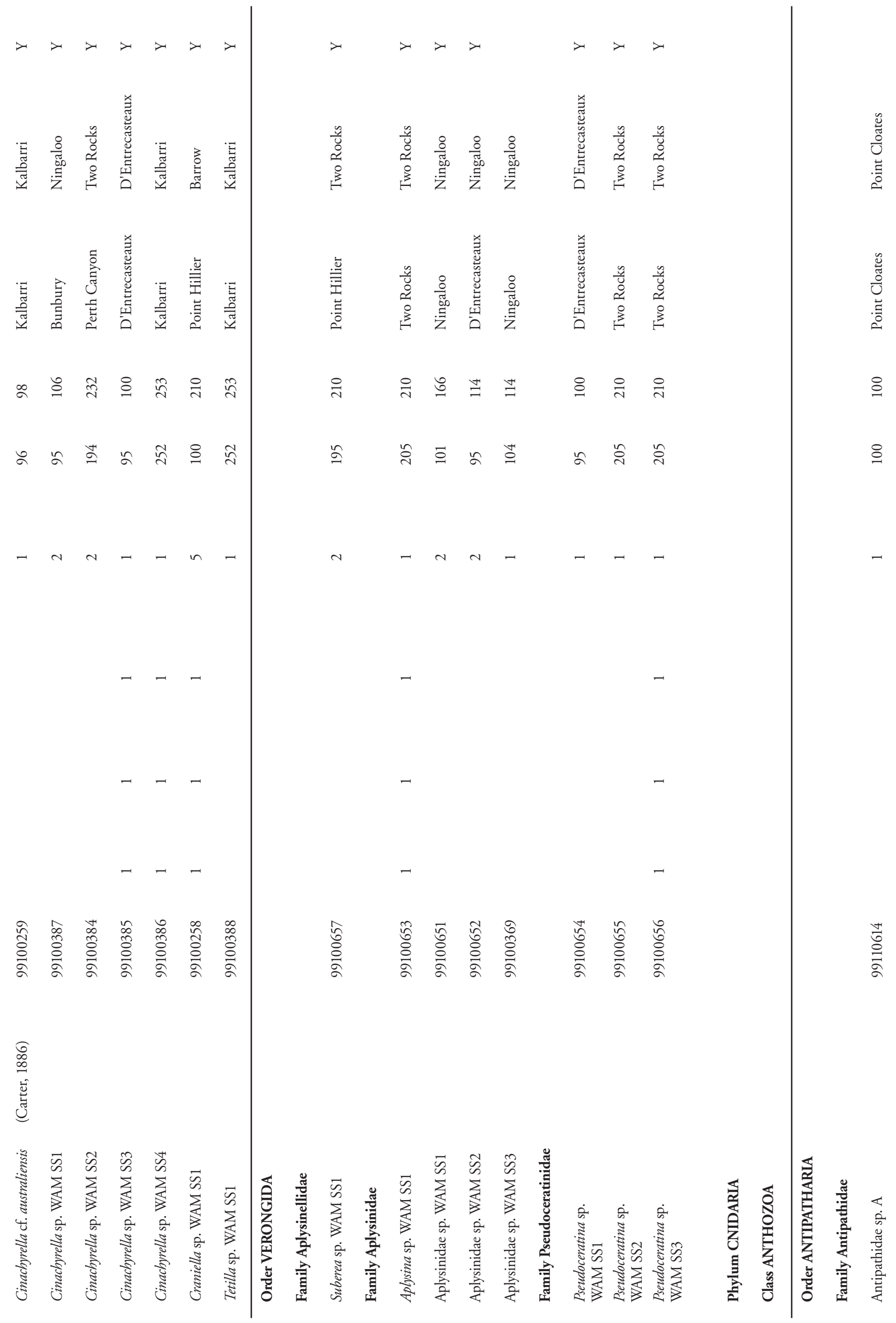



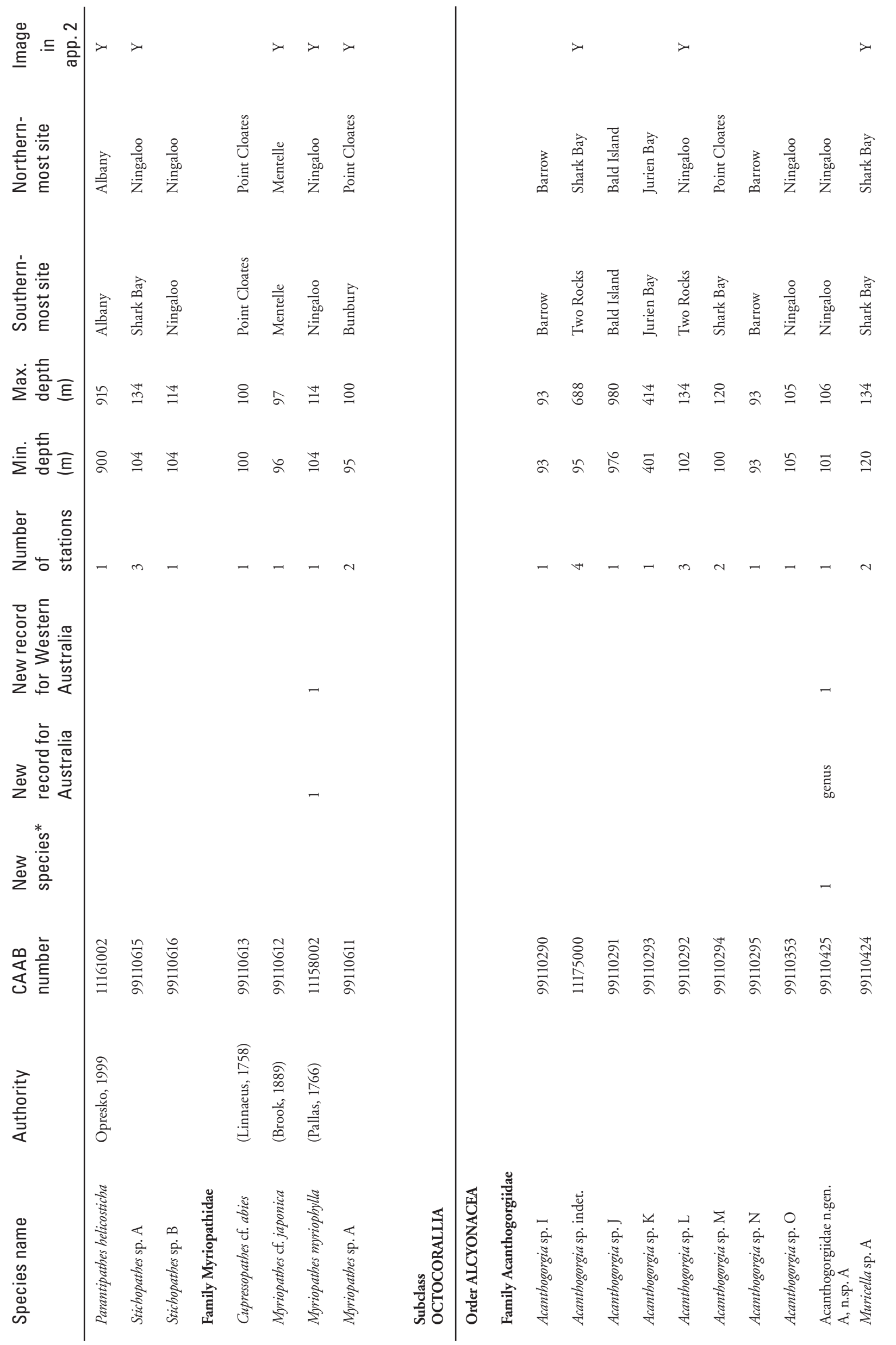


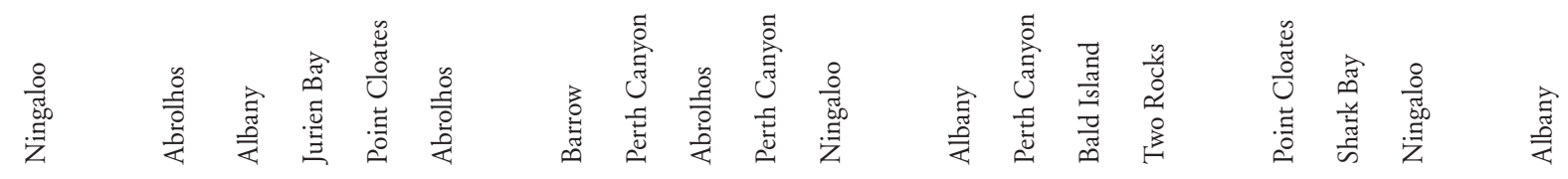

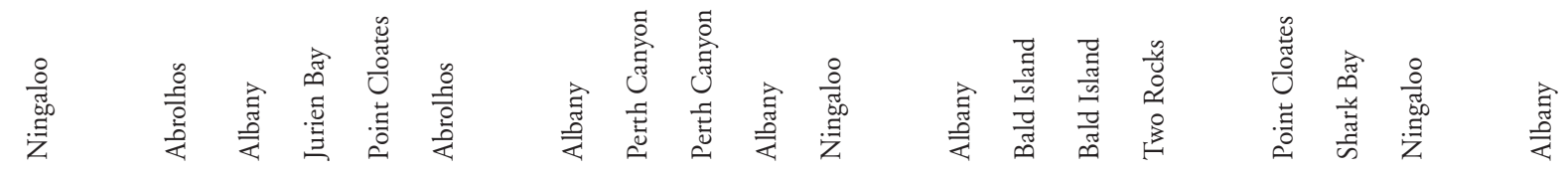

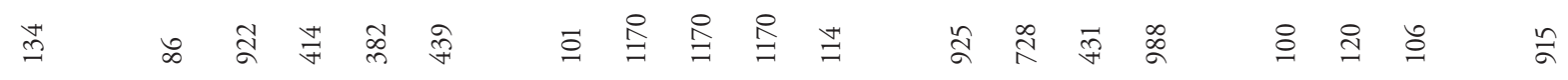

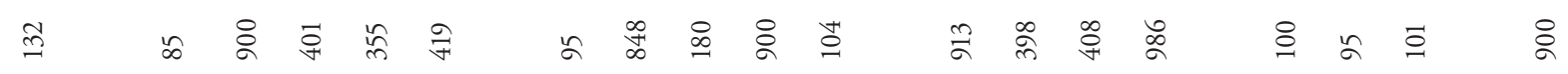

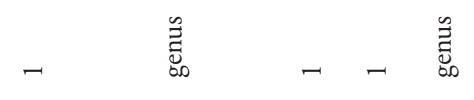

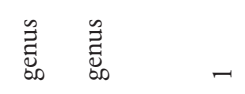

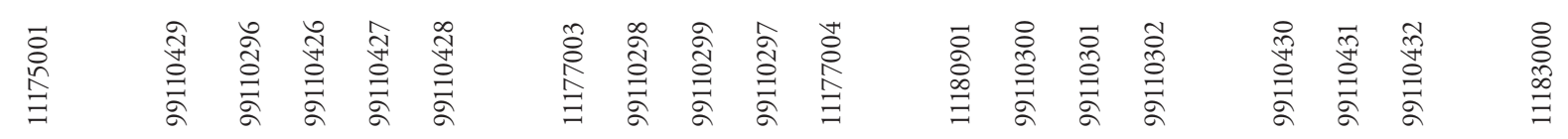

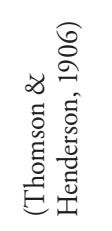<smiles>CC1CC1C1CC1</smiles>

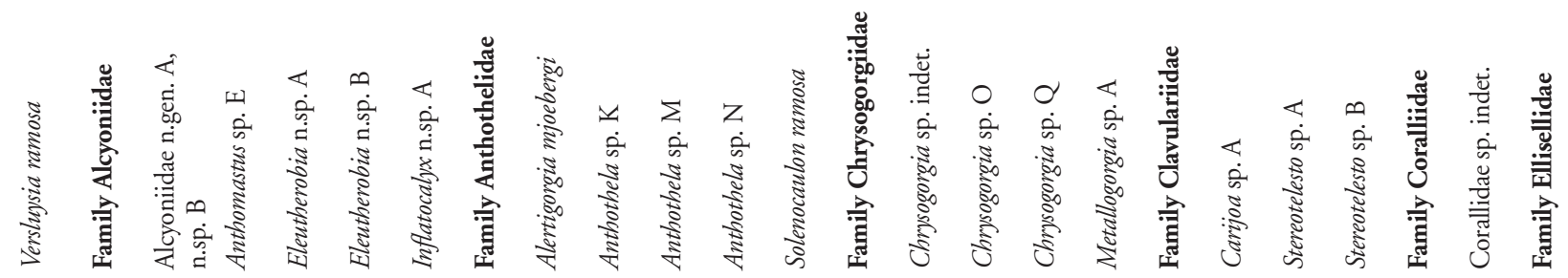




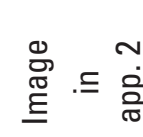

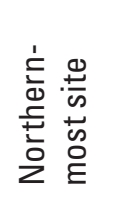

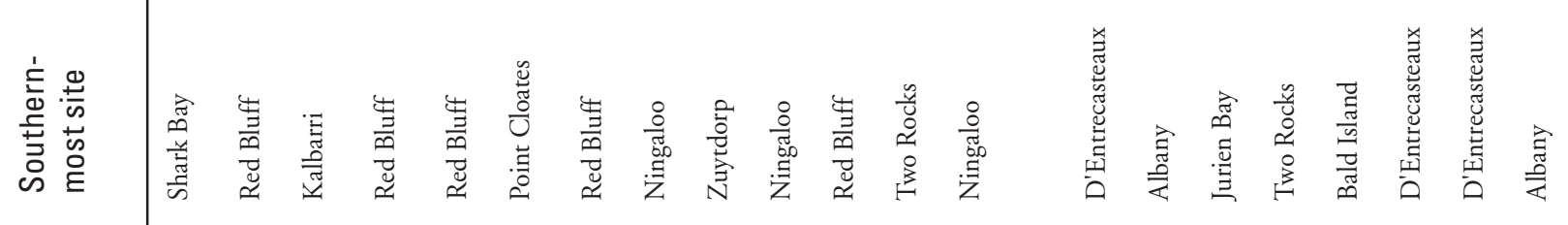

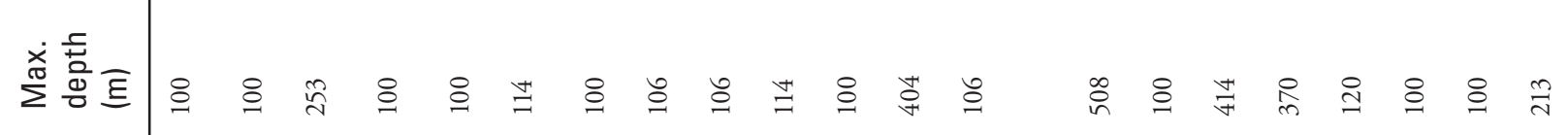

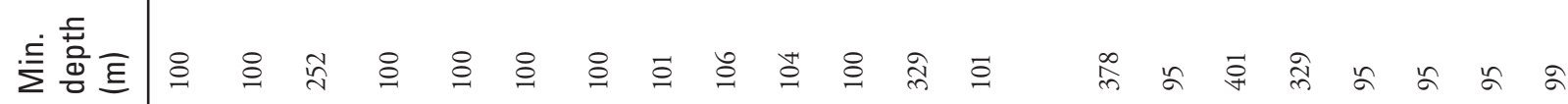

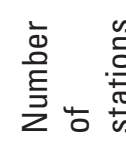

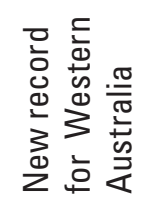

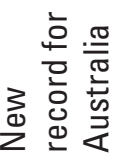

$\sum \frac{*}{\infty}$

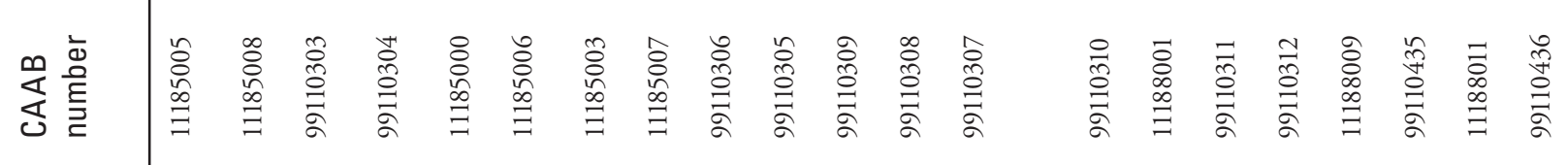

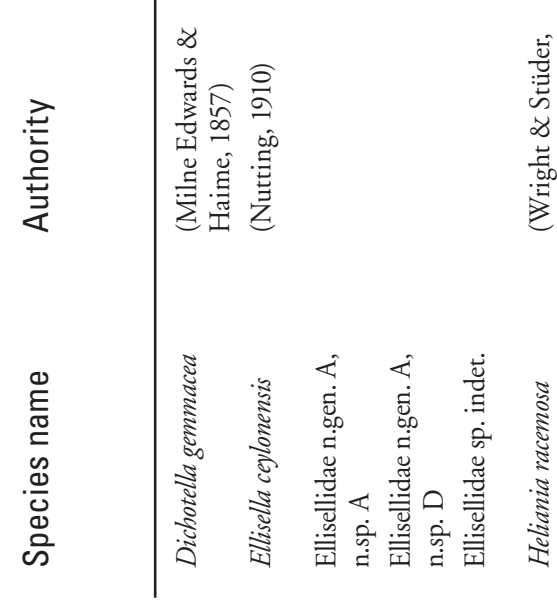

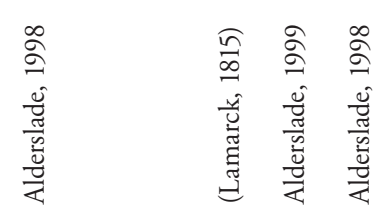




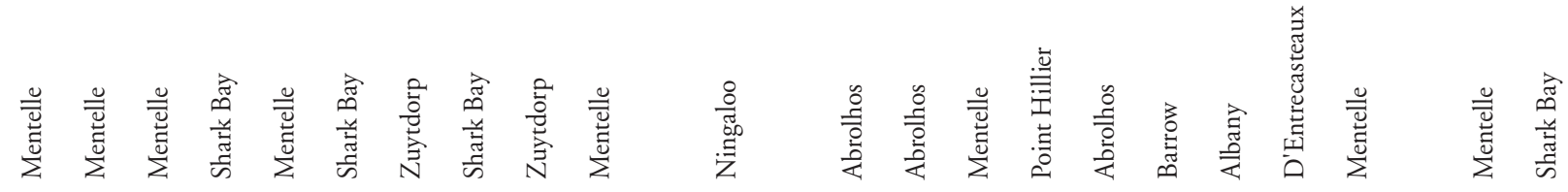

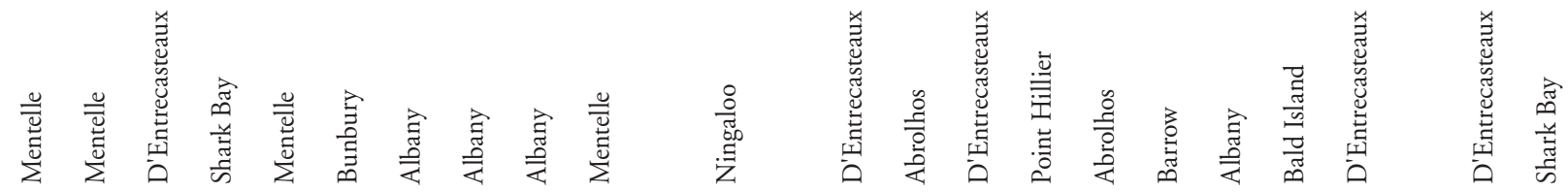
ล

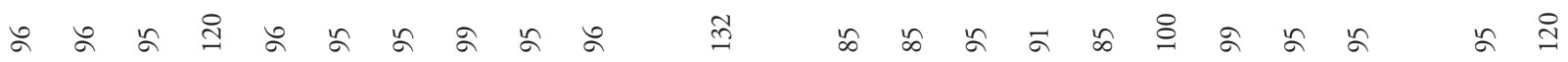

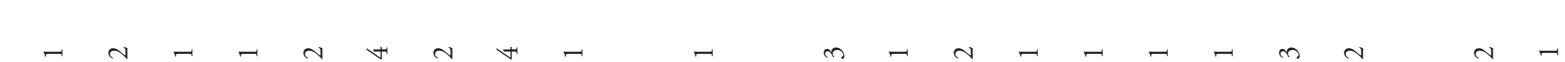

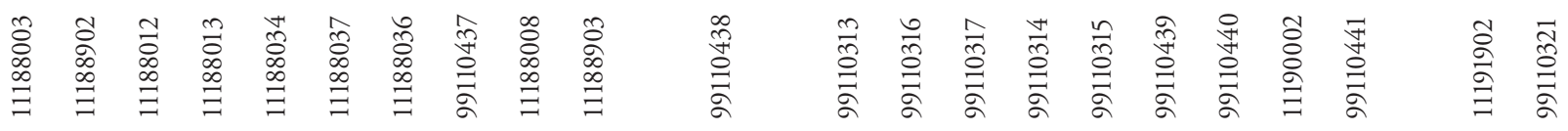

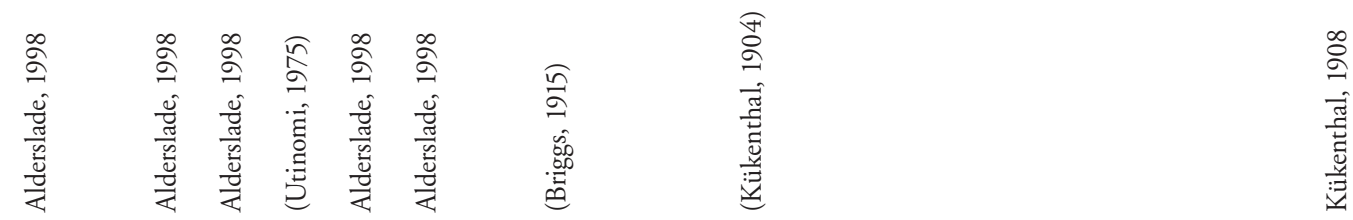

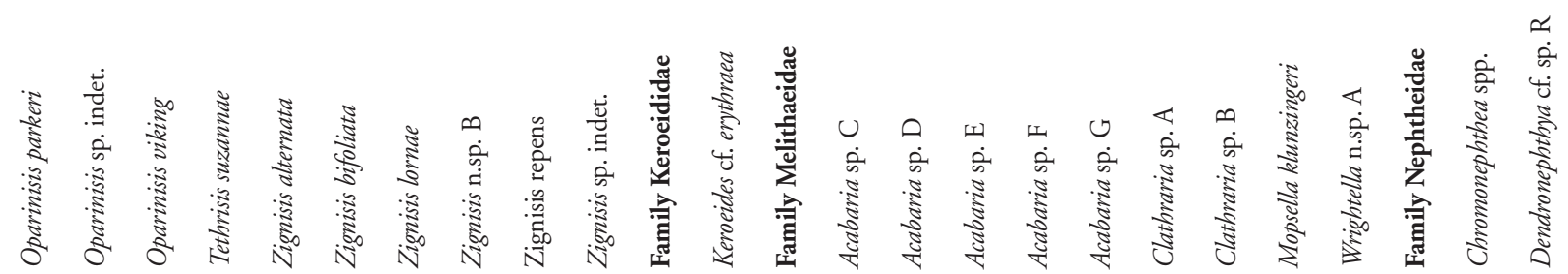




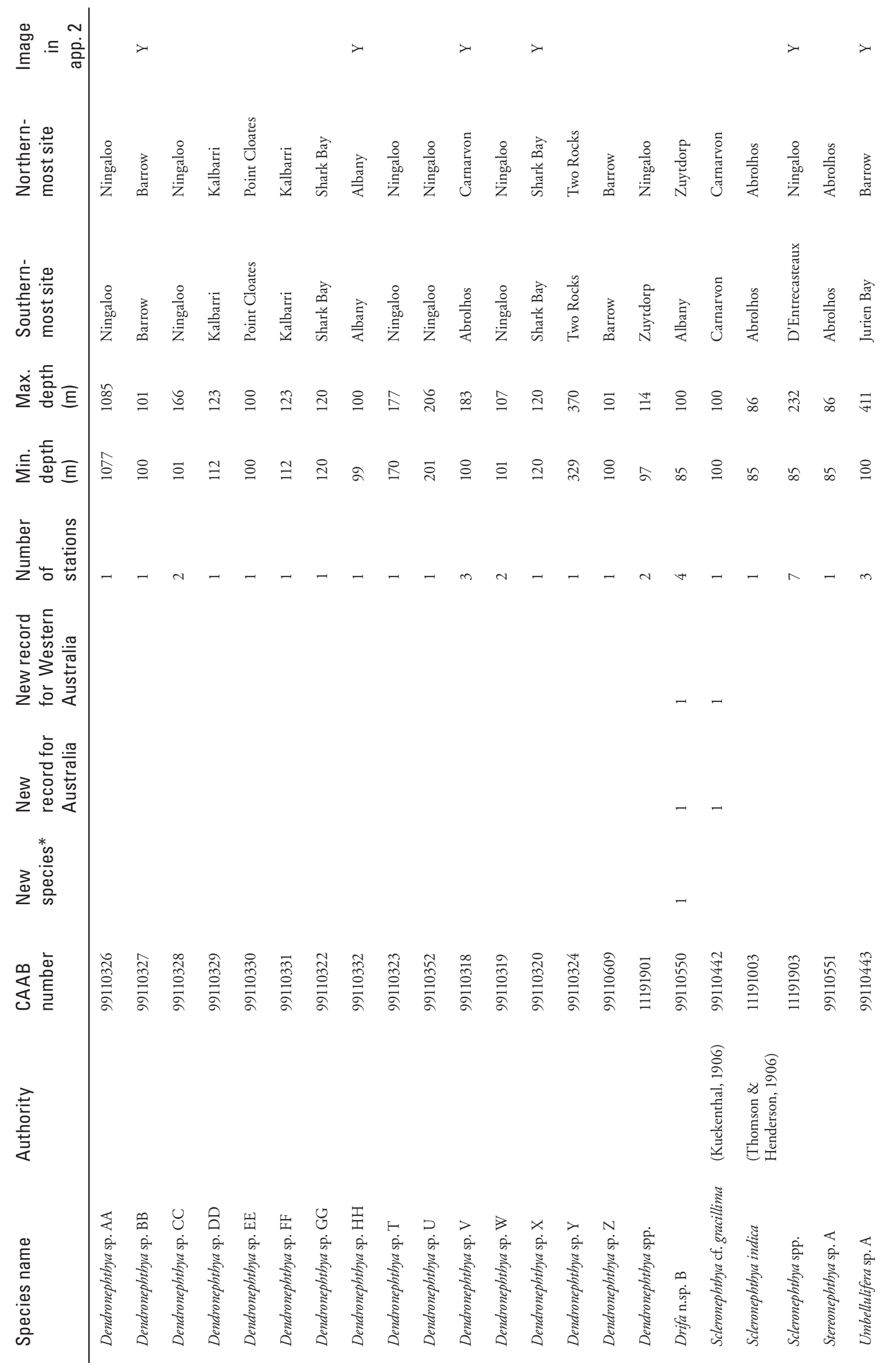




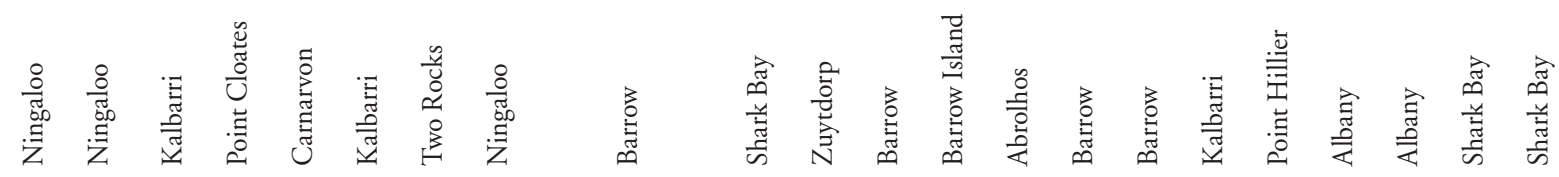

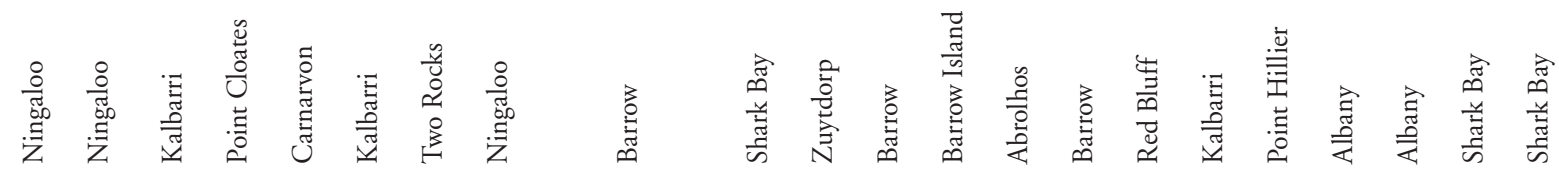

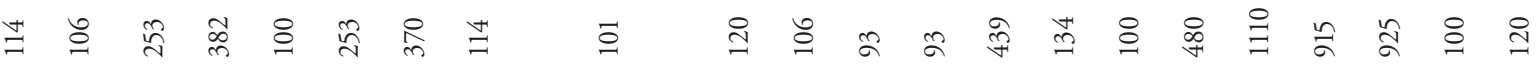

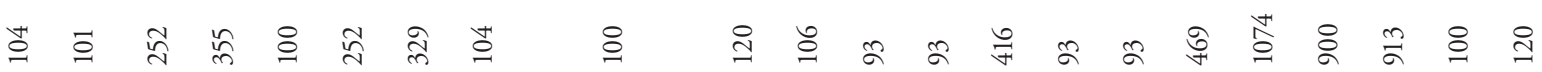

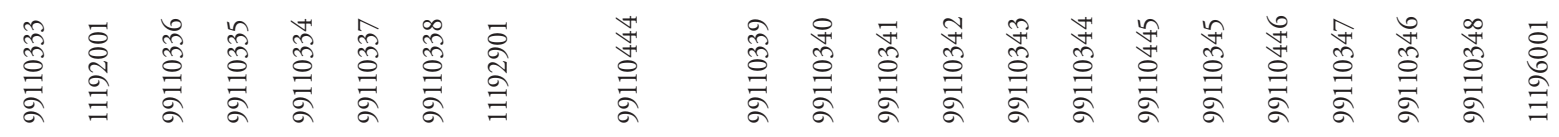

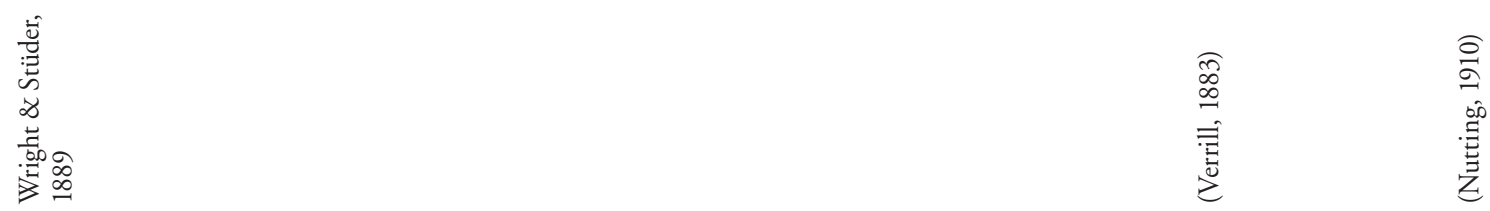

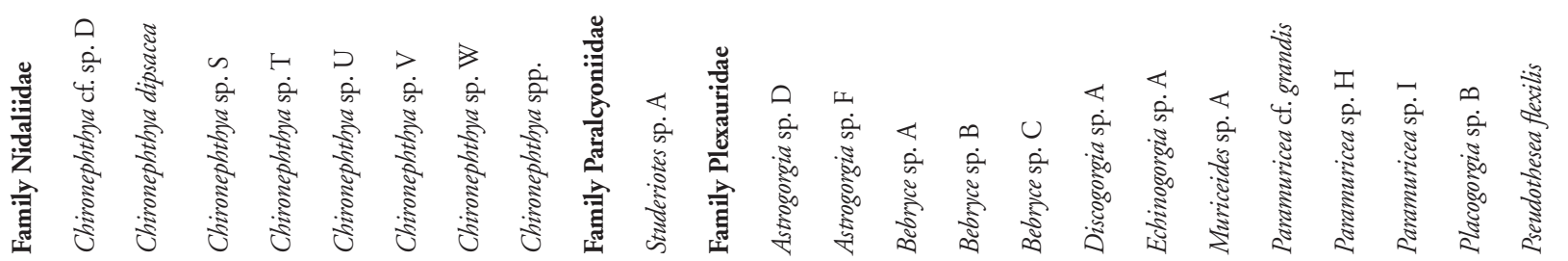




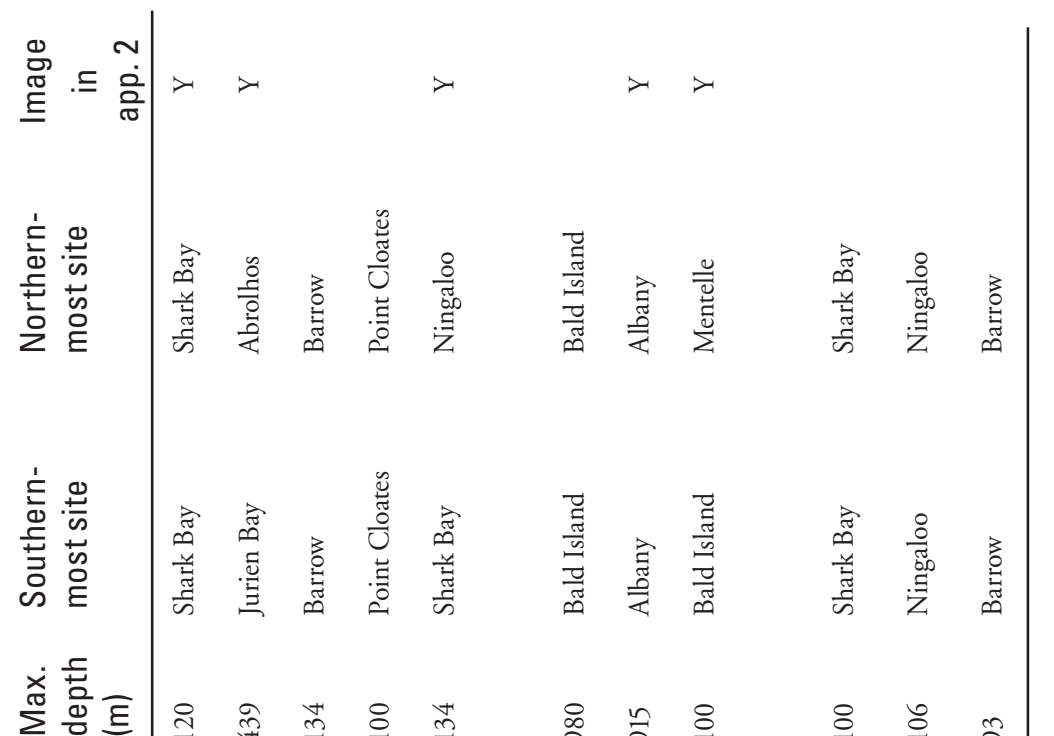

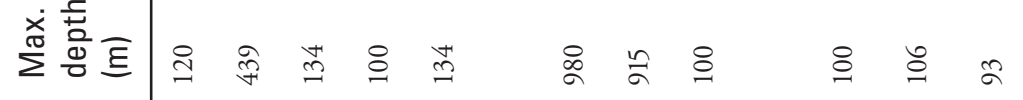

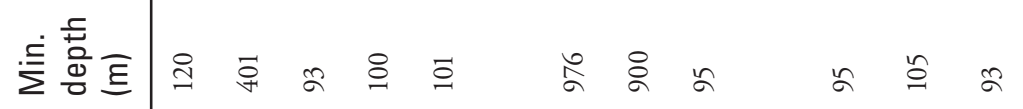

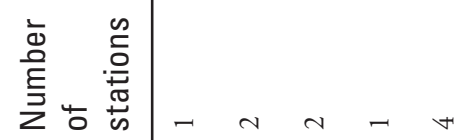

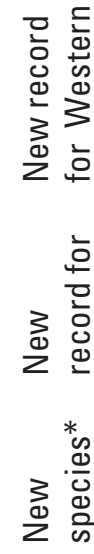

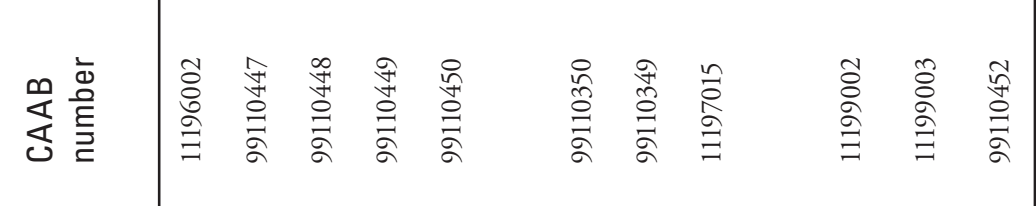

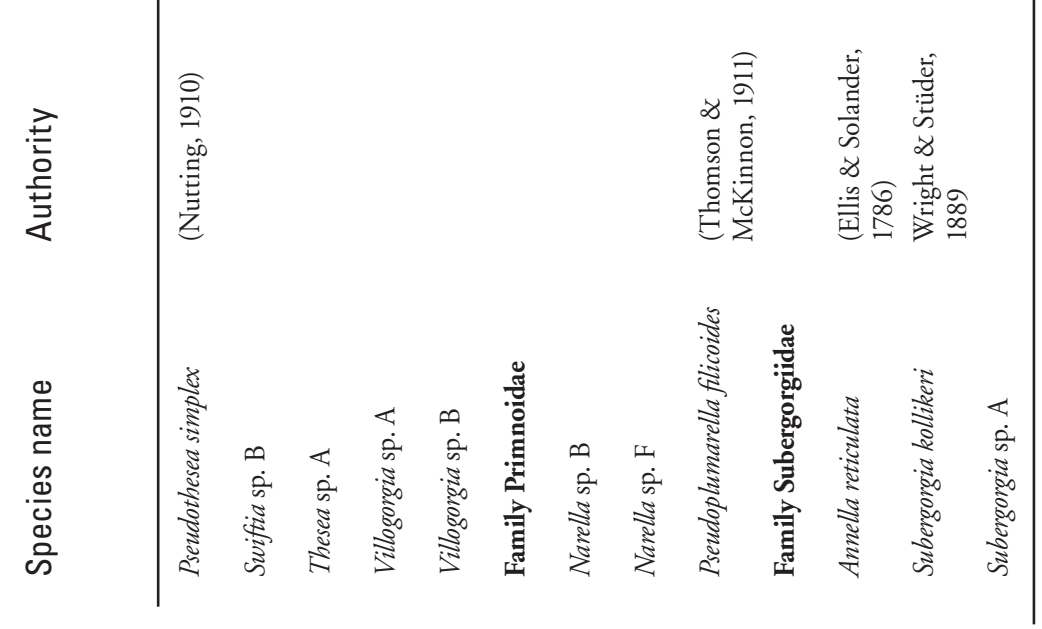

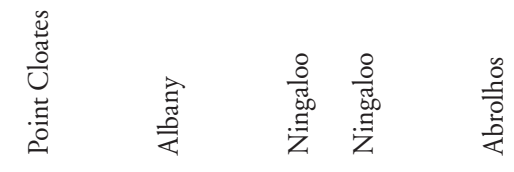

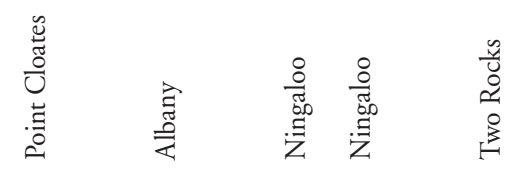

ஓे के ‡ڤ

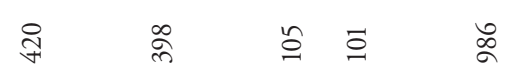

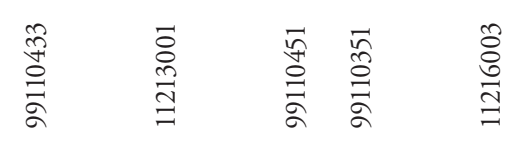

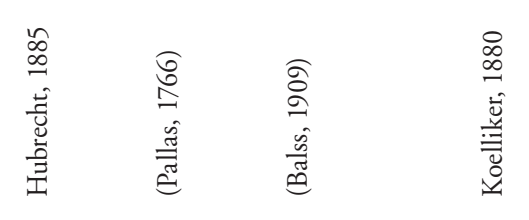

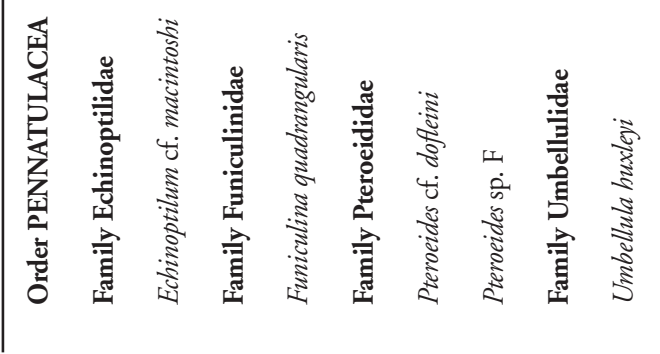




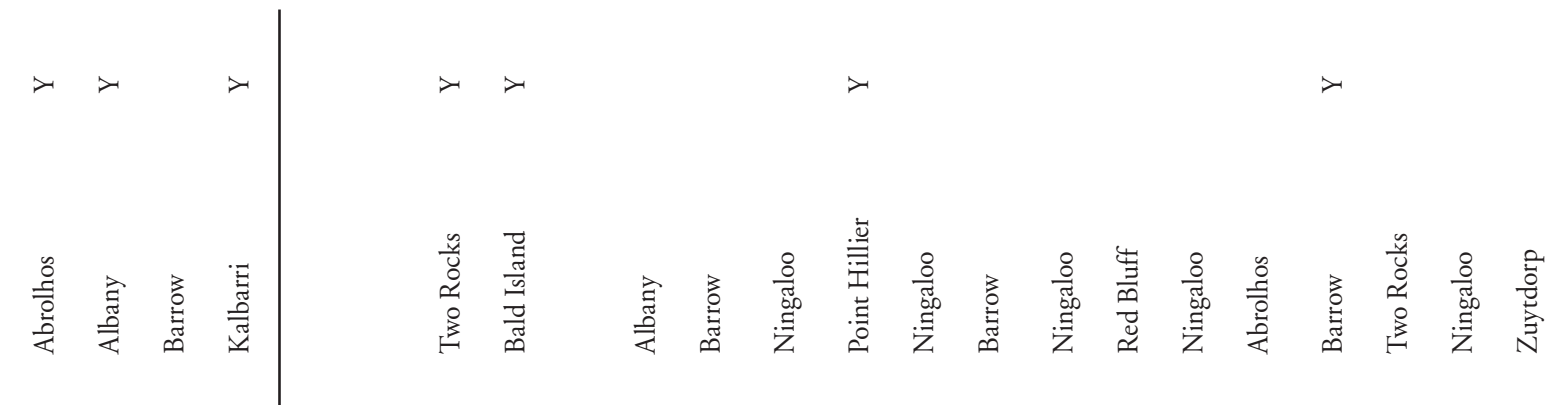

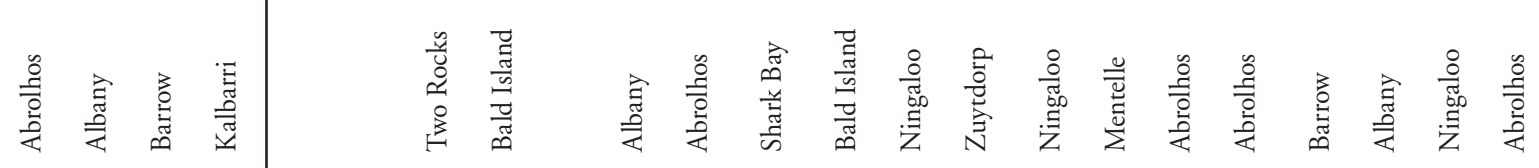

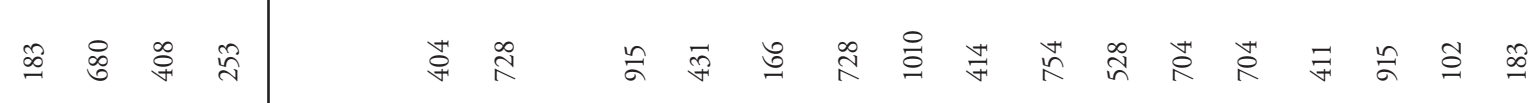

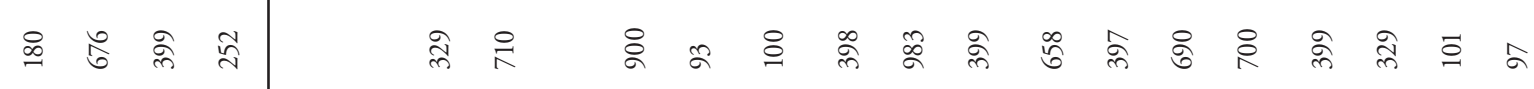

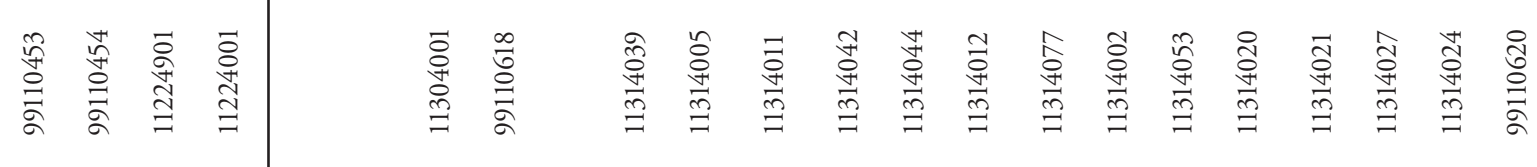

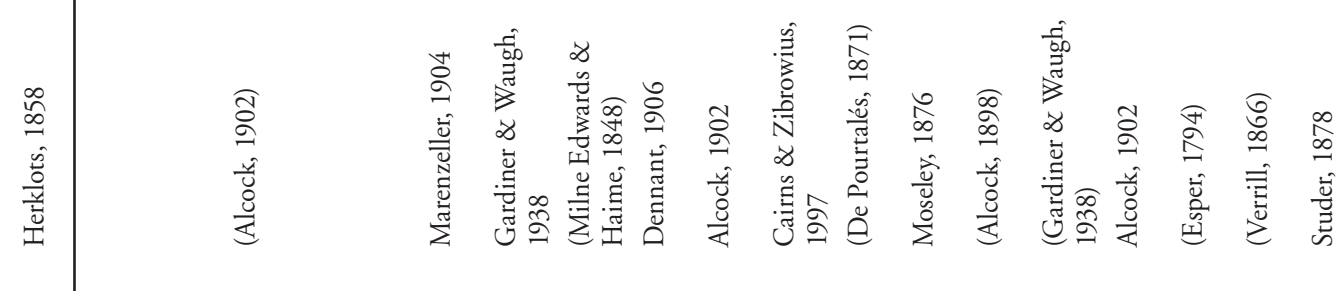

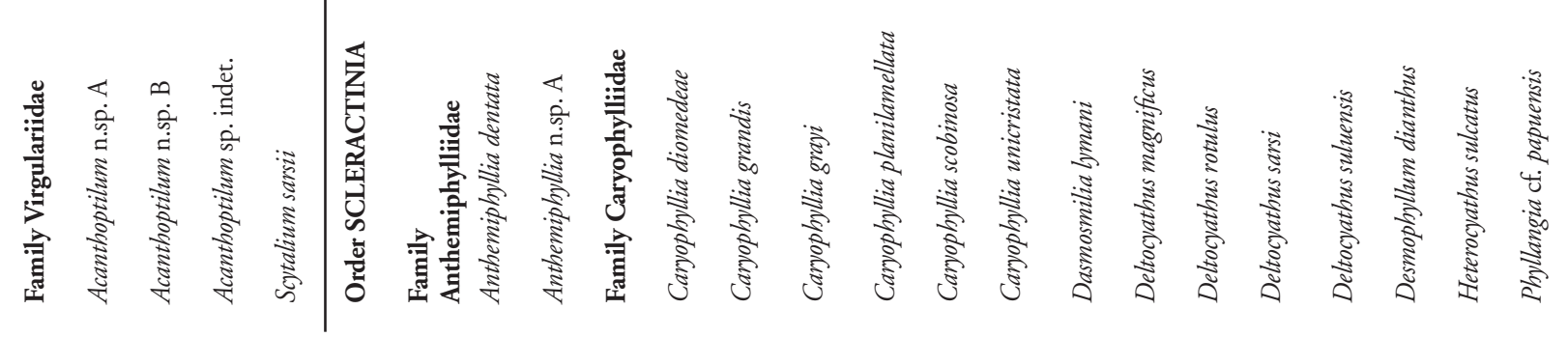




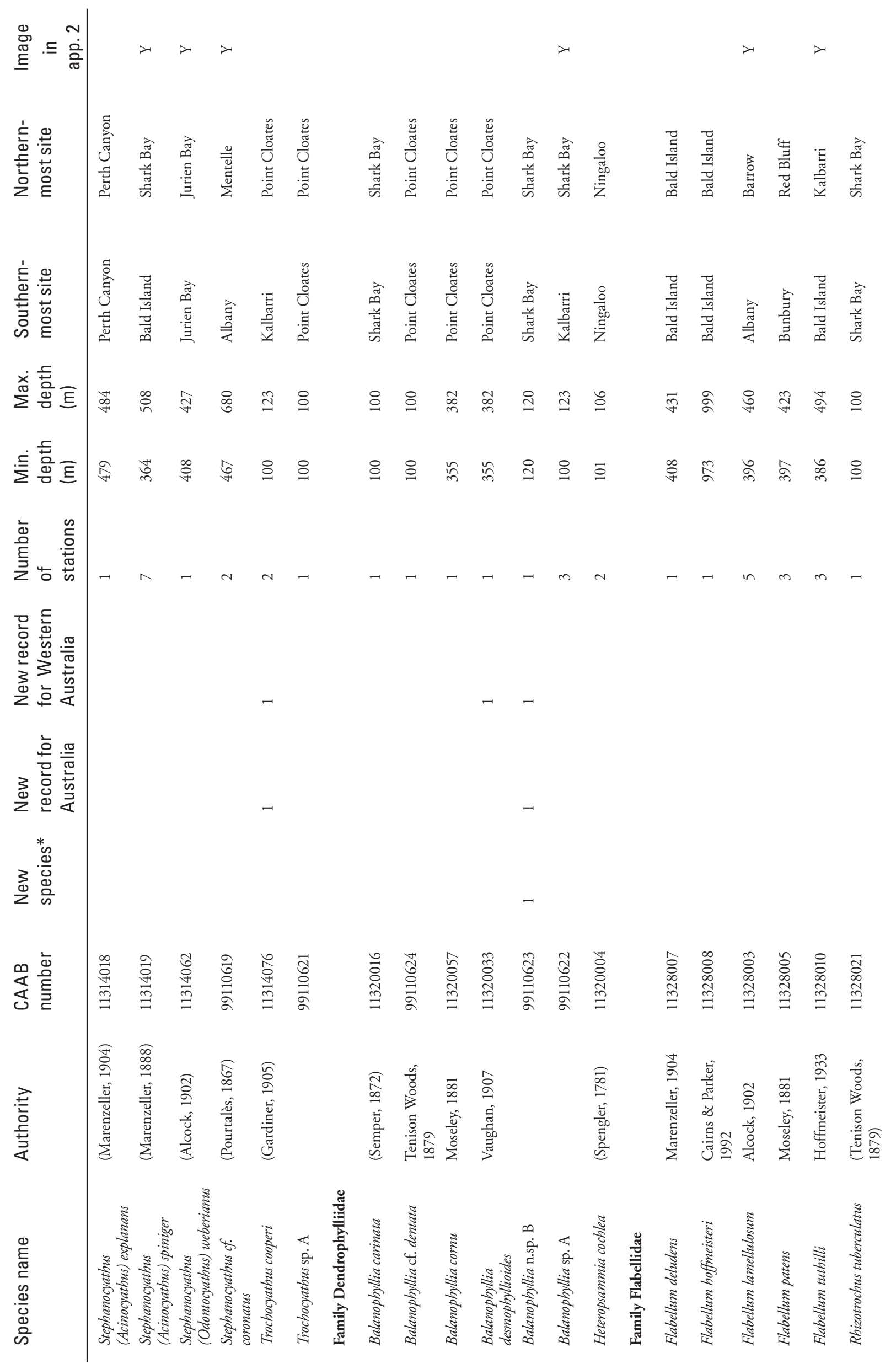




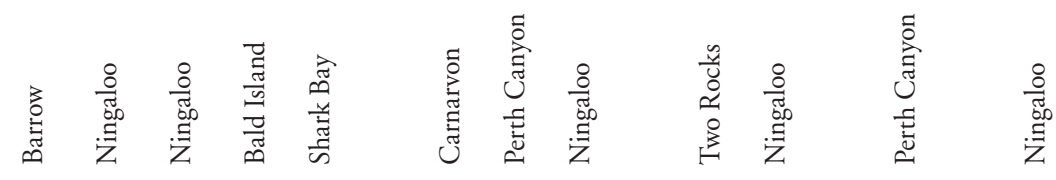

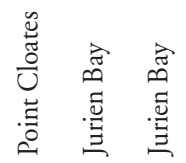

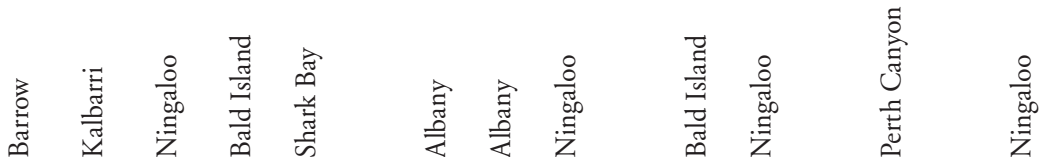

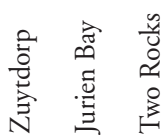

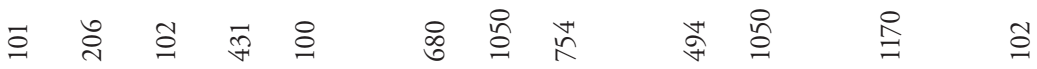

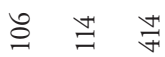

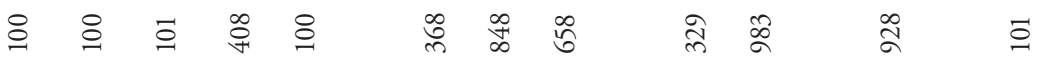

$\approx \stackrel{2}{=}$

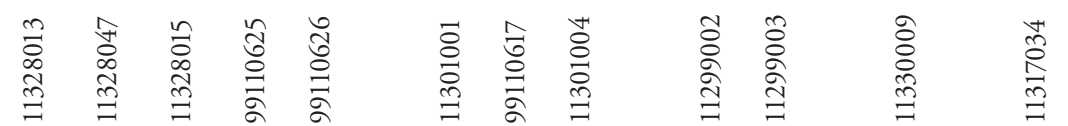

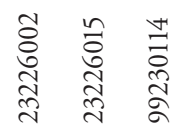

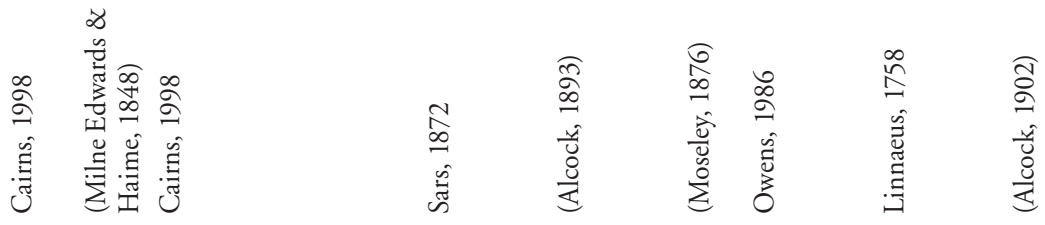

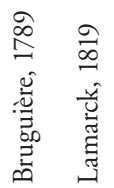

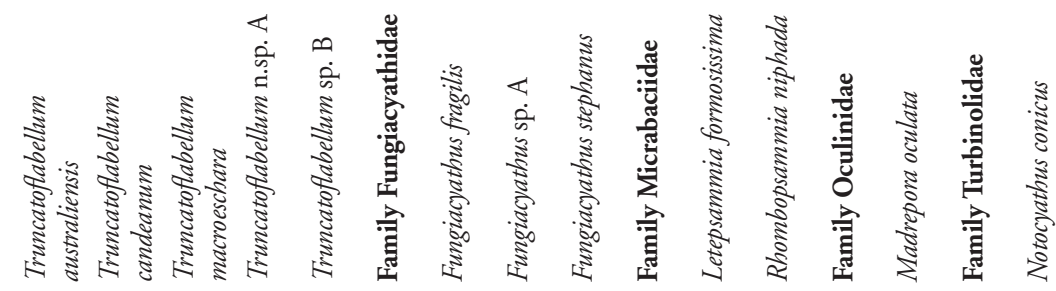

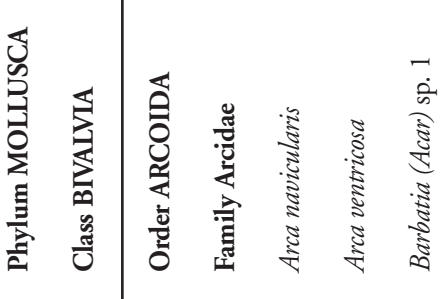




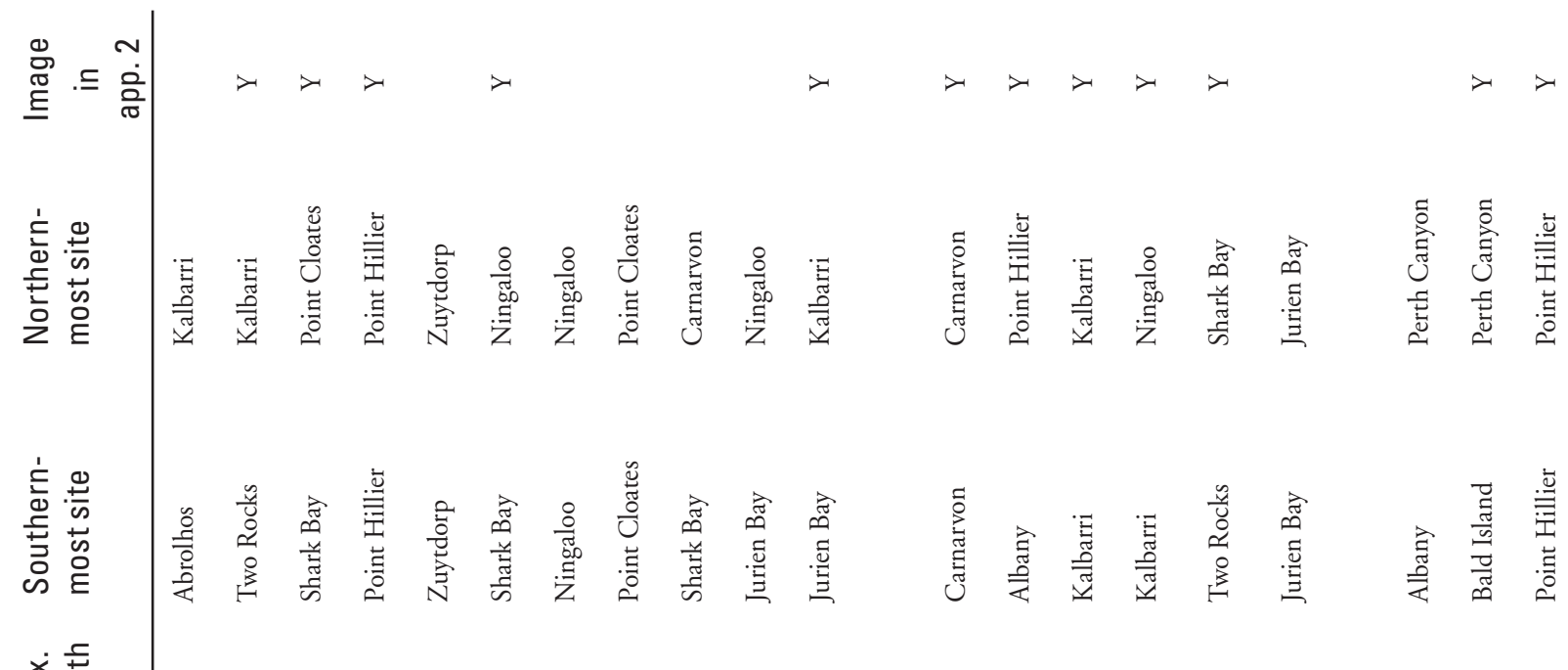

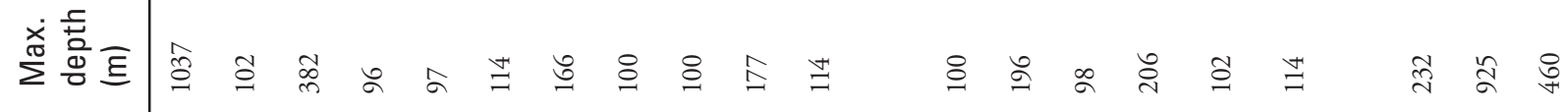

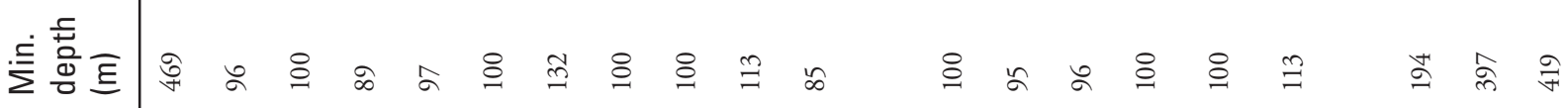

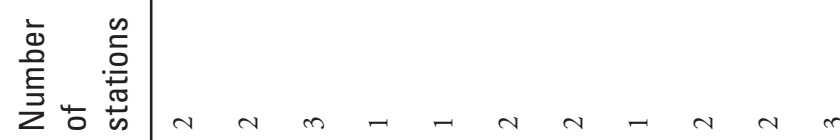

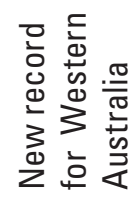

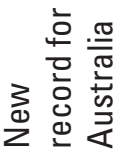
zis

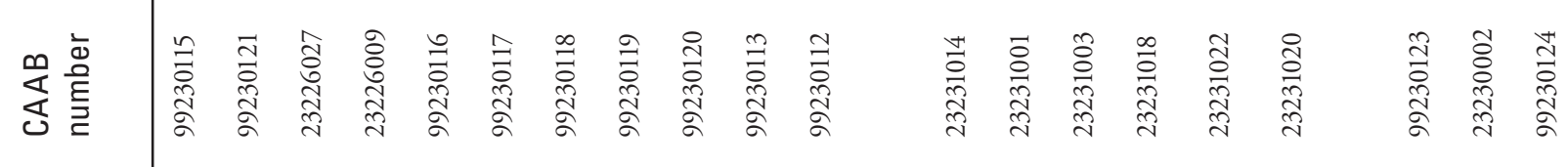

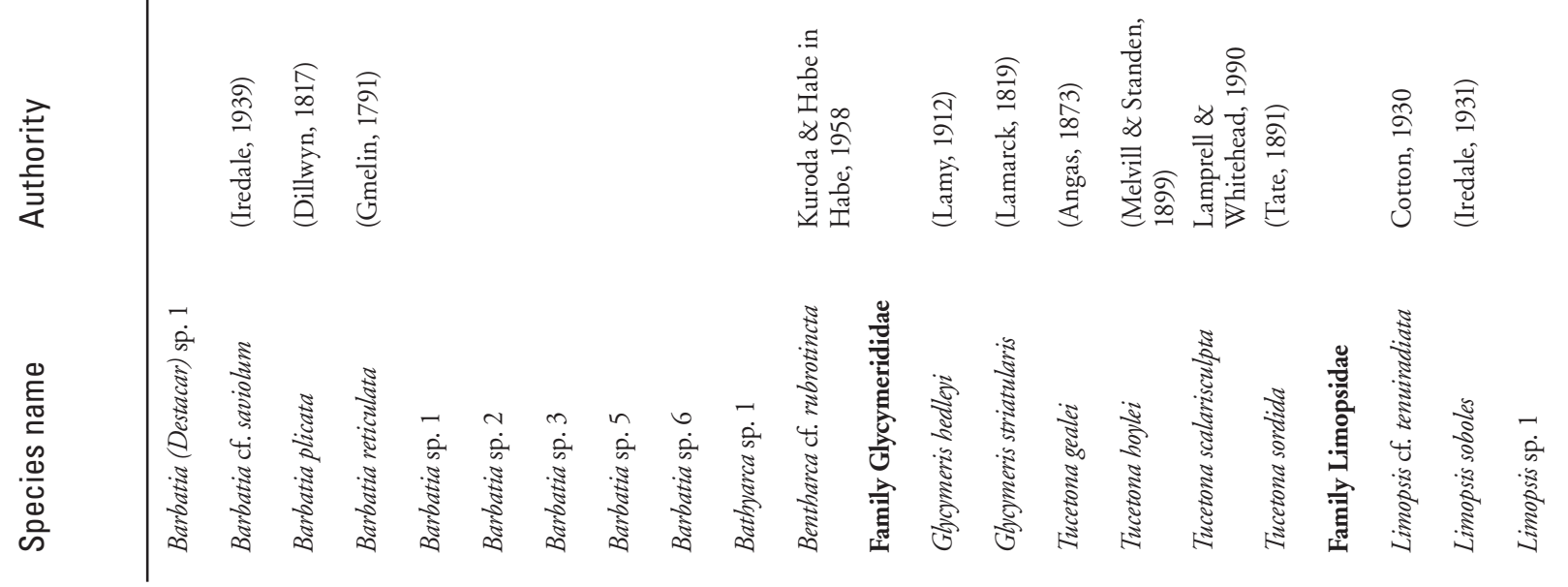




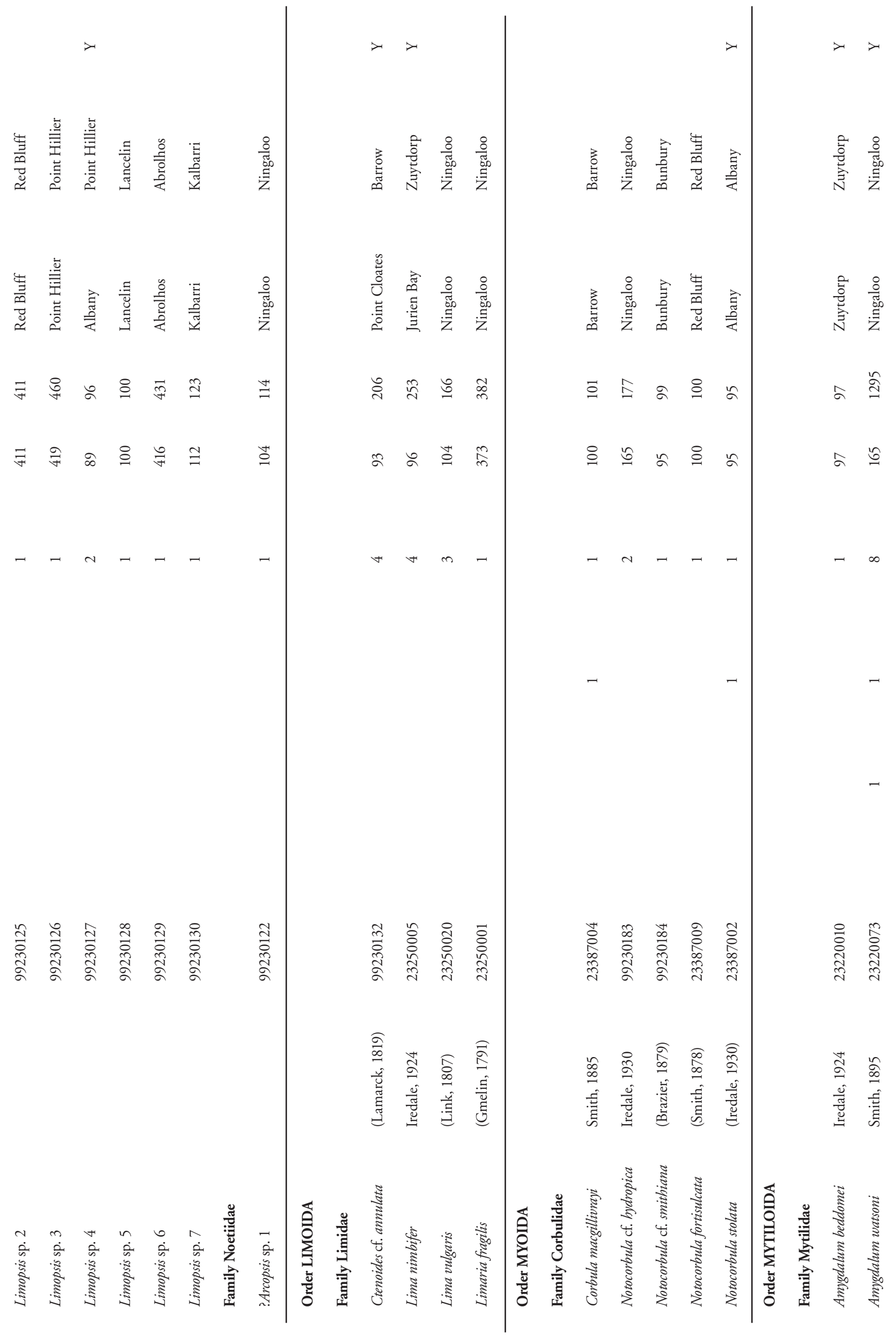




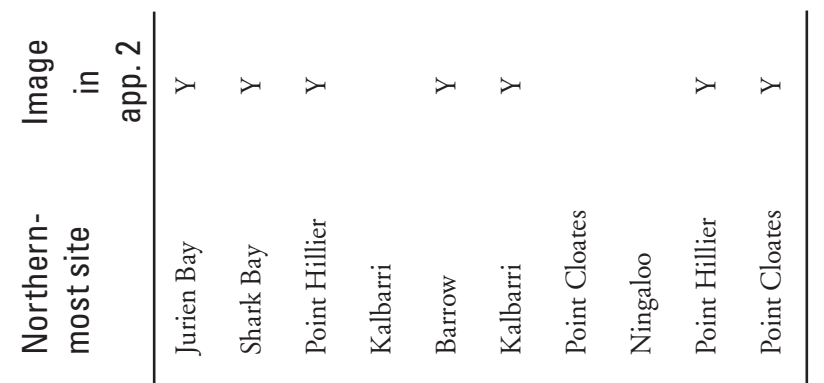

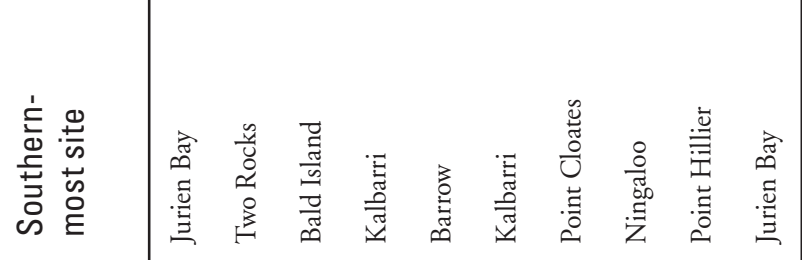
㐫营至 這蓄主

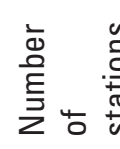

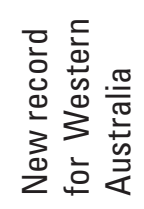

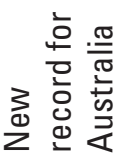

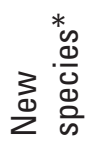

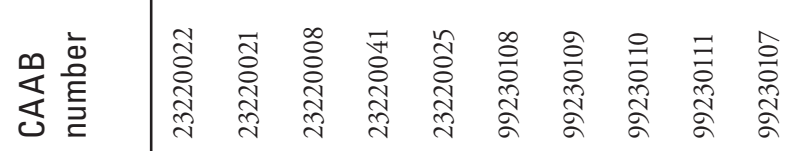
त

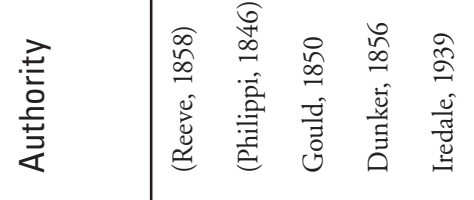

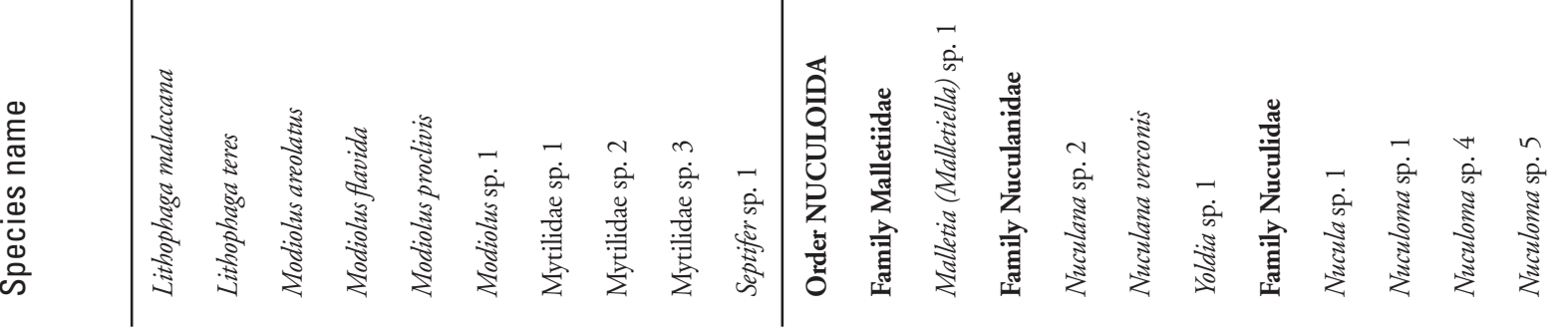




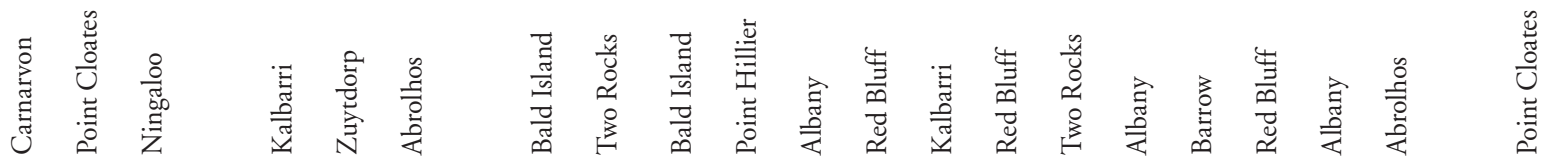

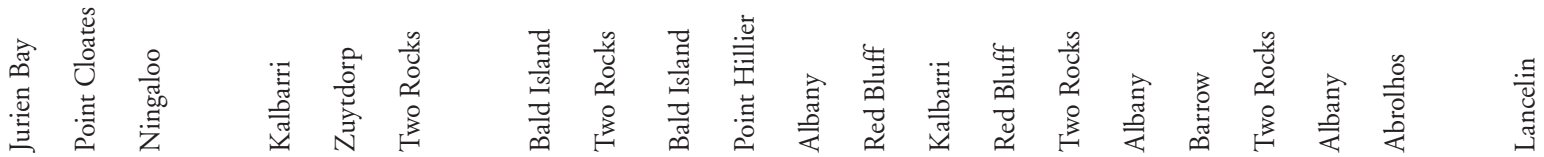

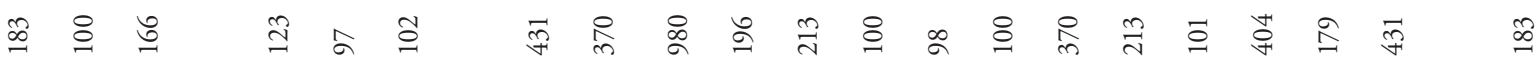

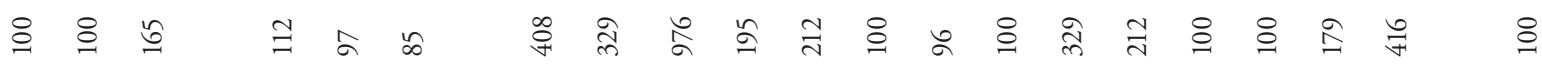

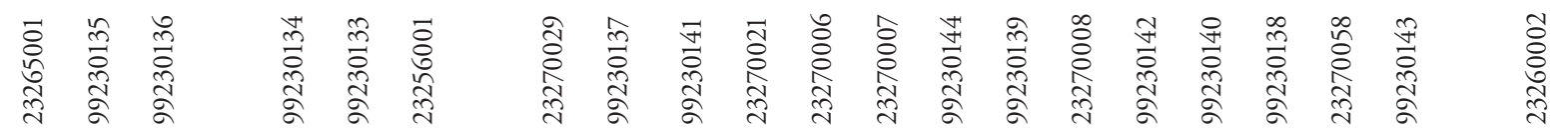

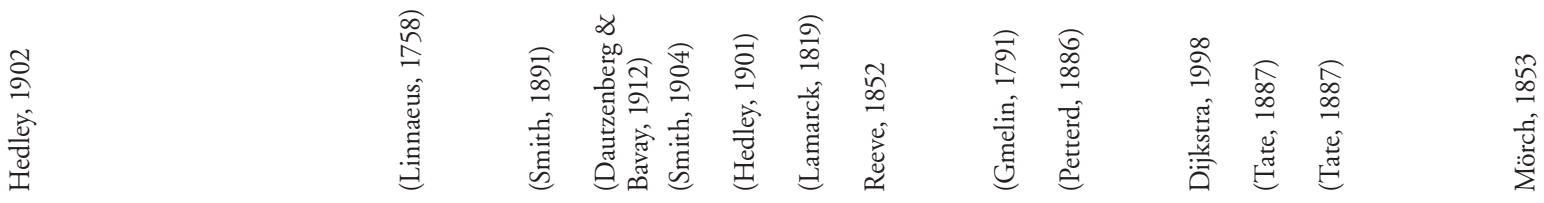

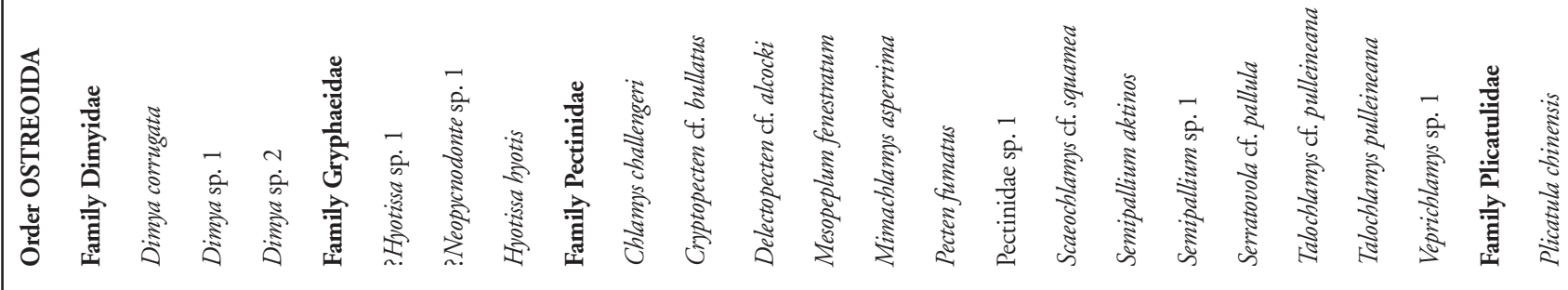




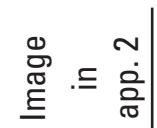

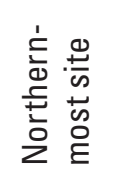

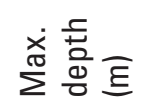

它营至

离

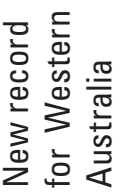

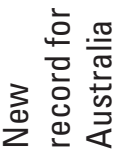

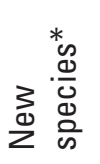

辛

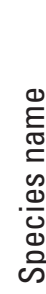

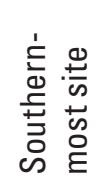

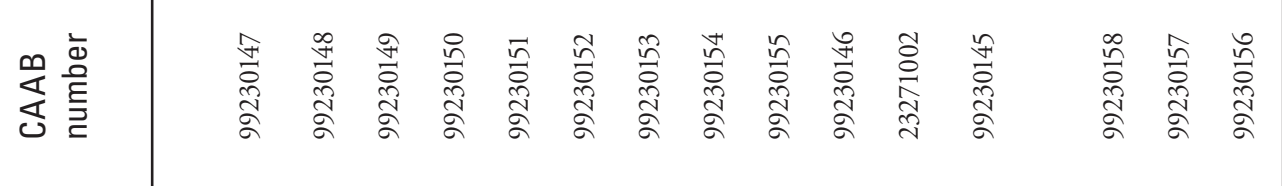

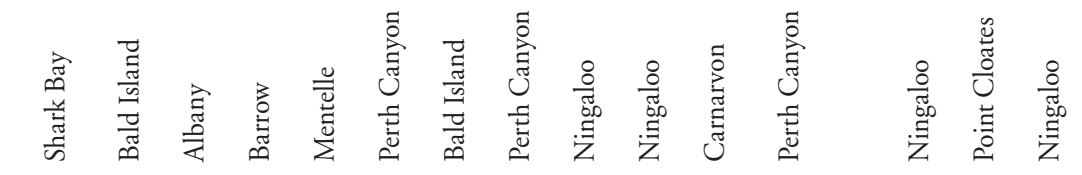

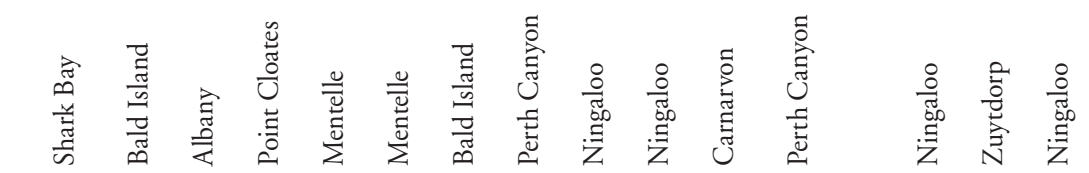

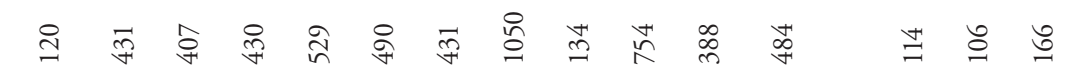

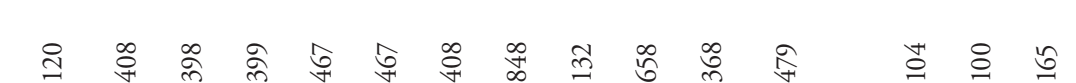

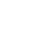

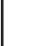

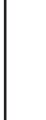
(1)

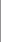

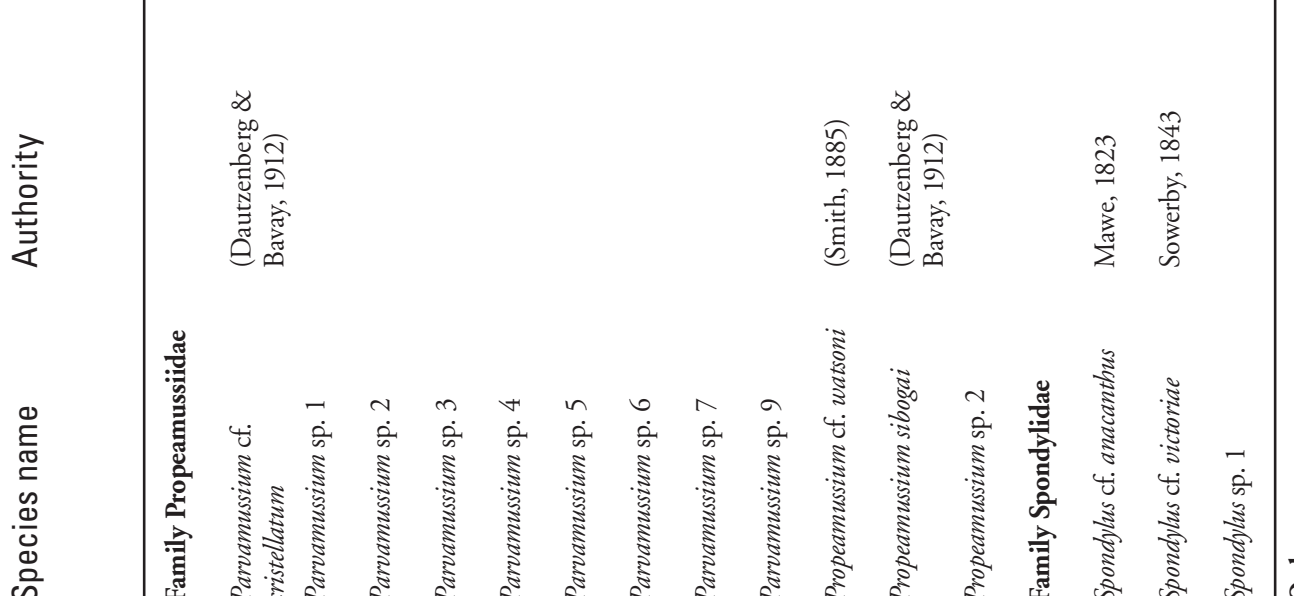

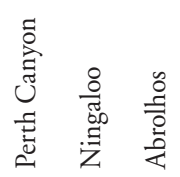

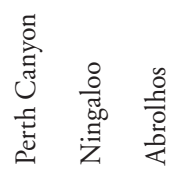

号苑

$\underset{\infty}{\infty}$

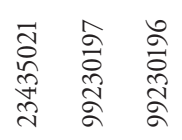

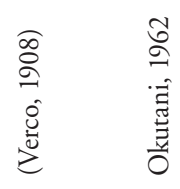

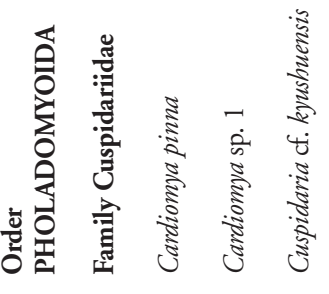




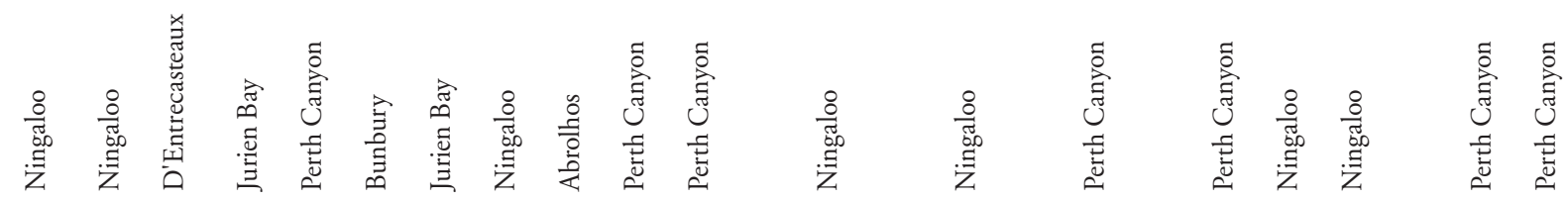

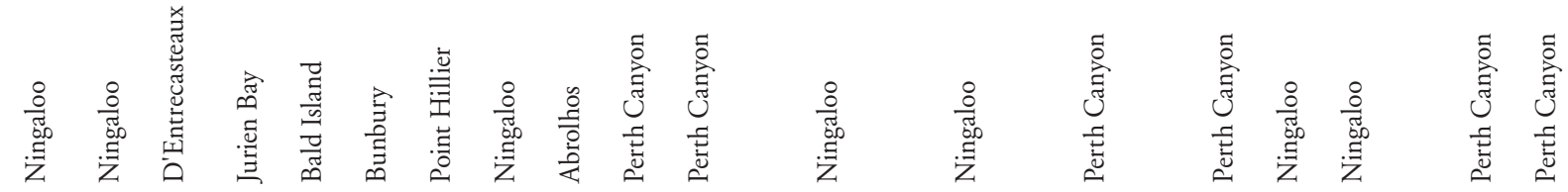

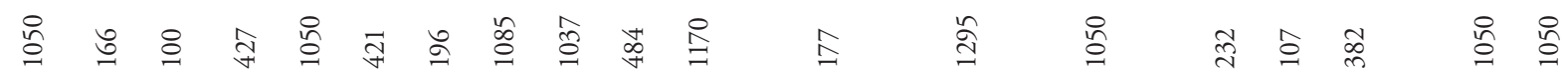

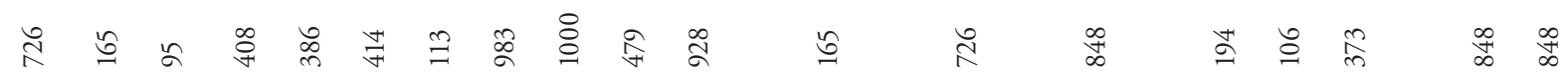

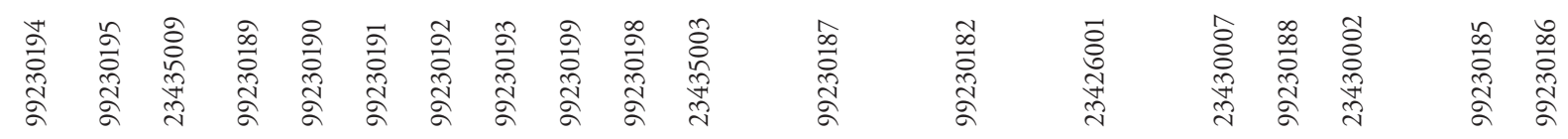

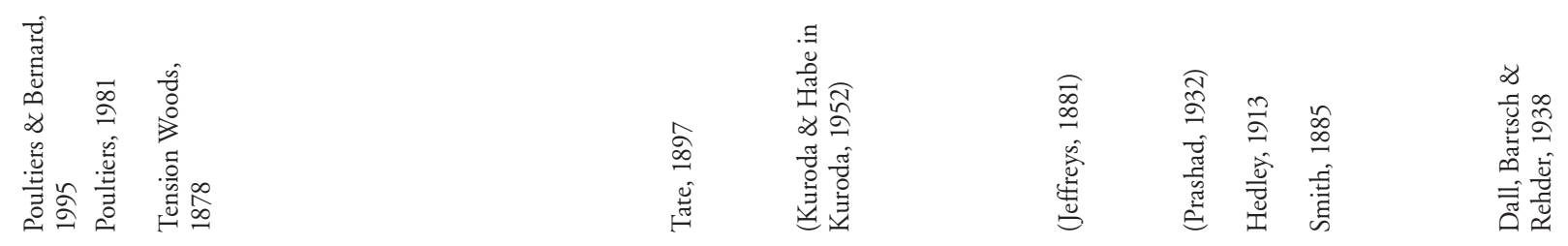

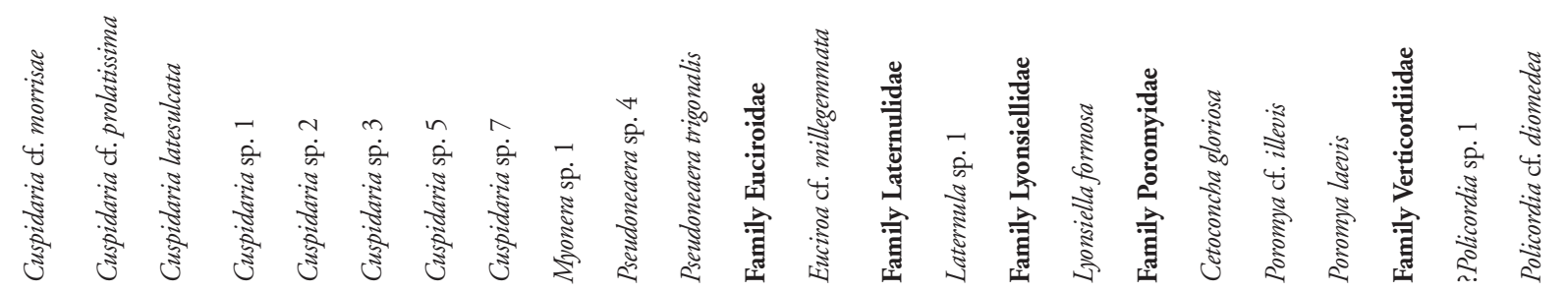




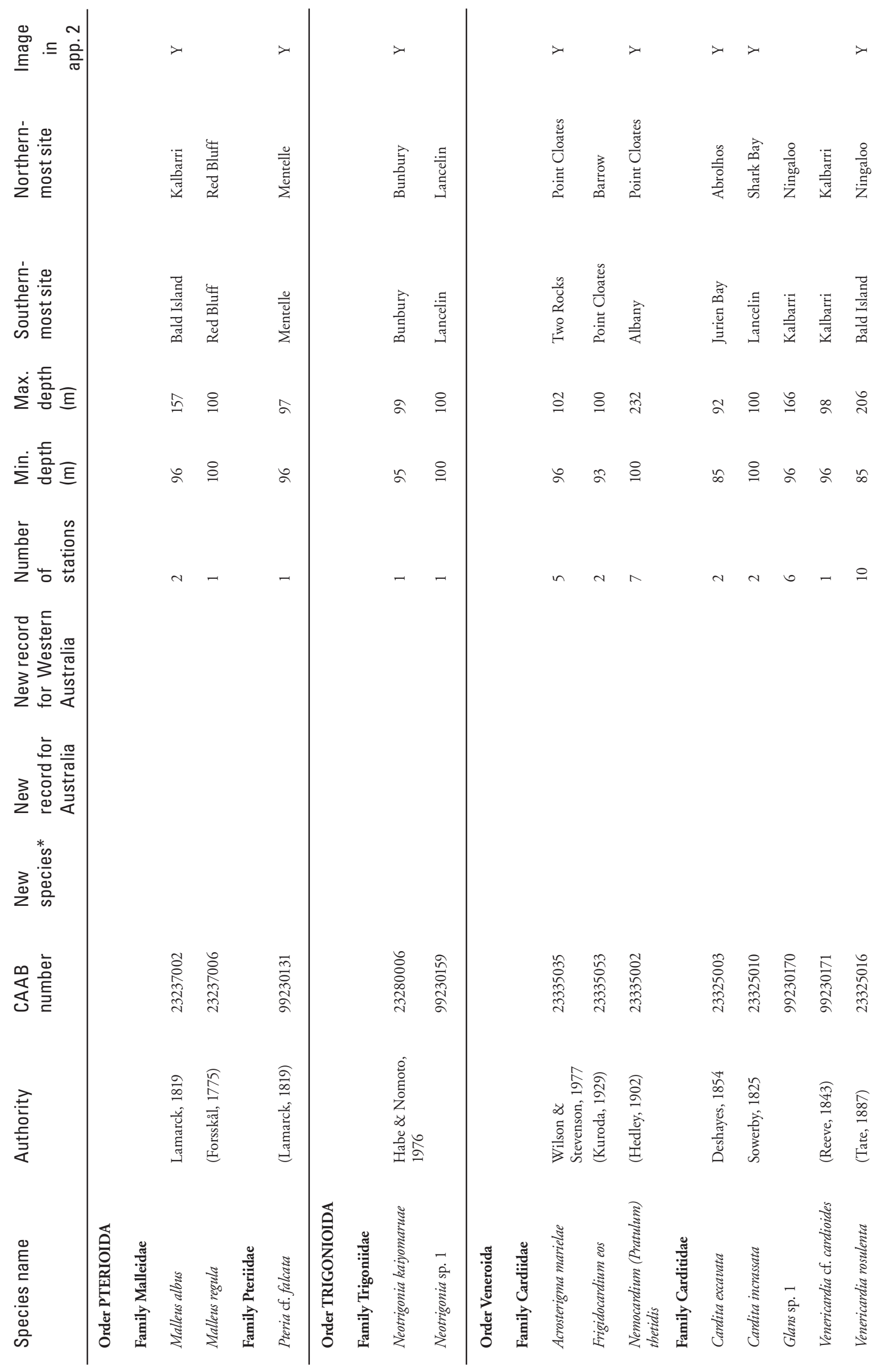




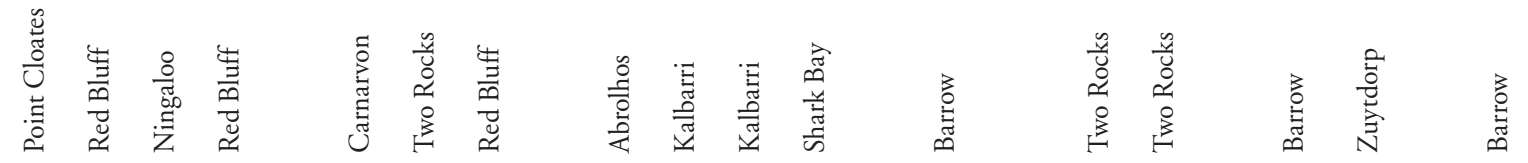

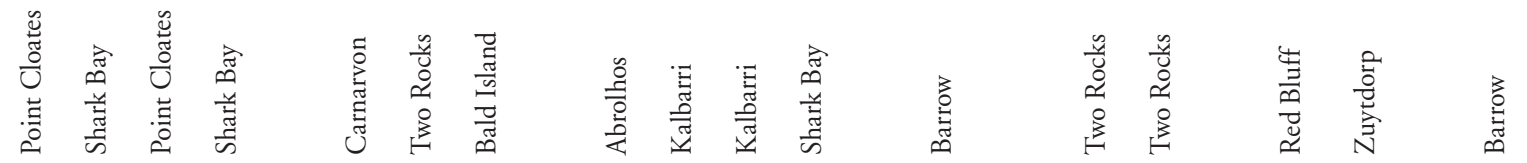

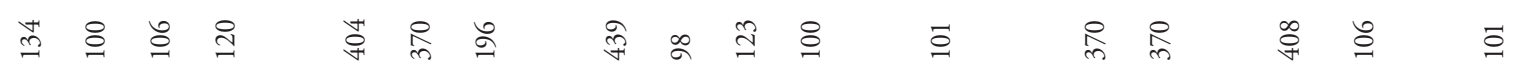

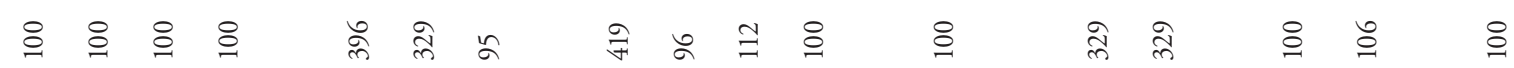

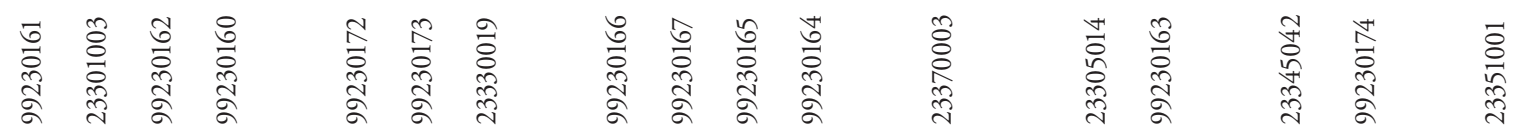

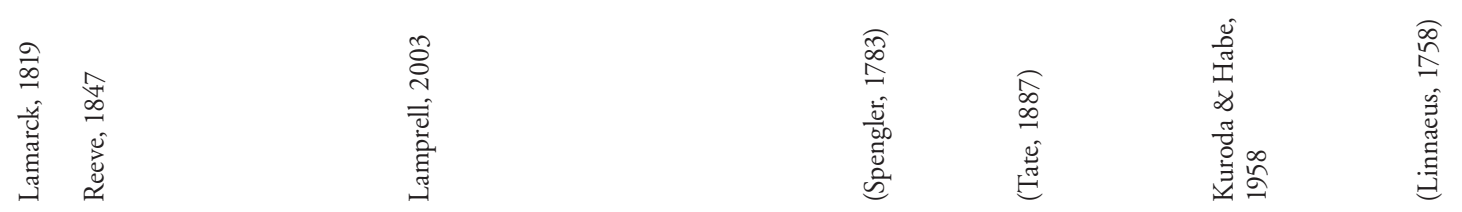

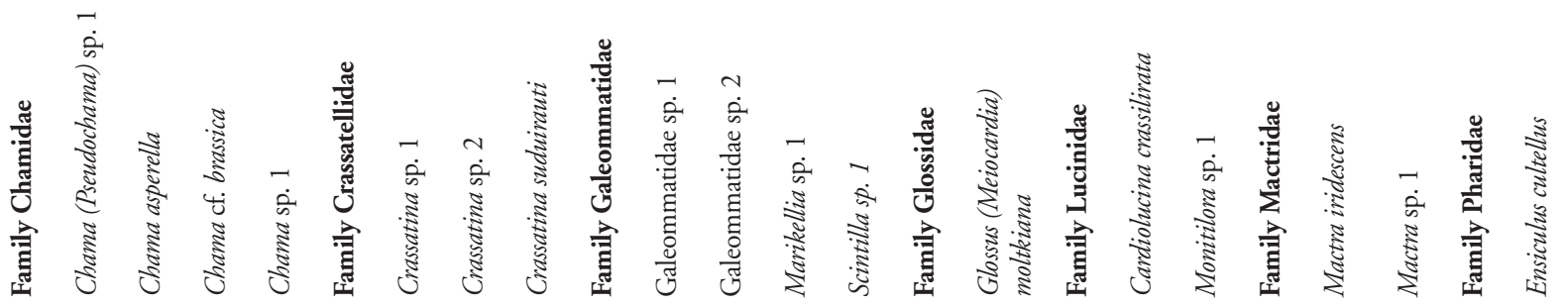




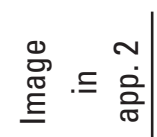

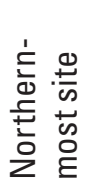

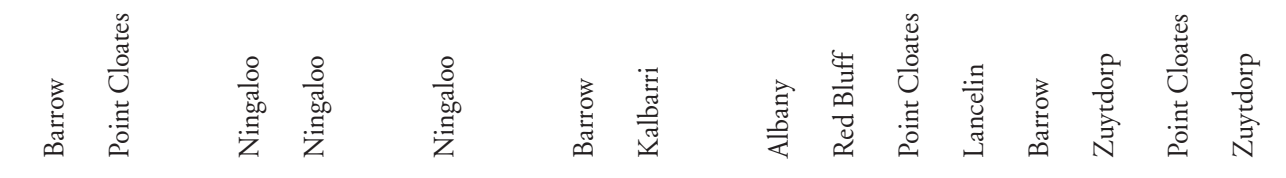

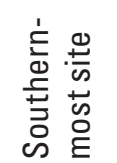

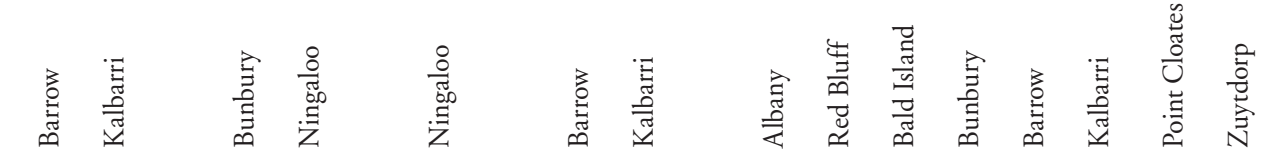

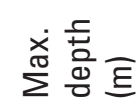

ఏ

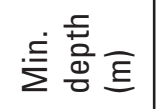

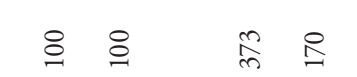

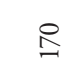

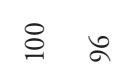

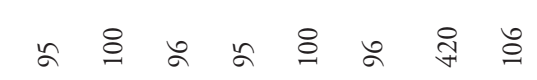

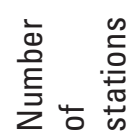

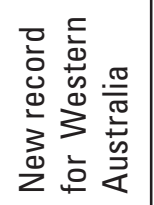

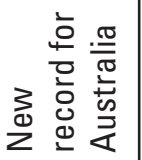

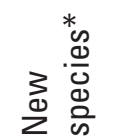

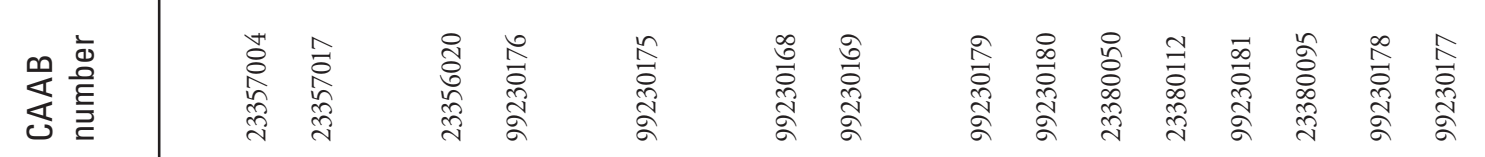

言

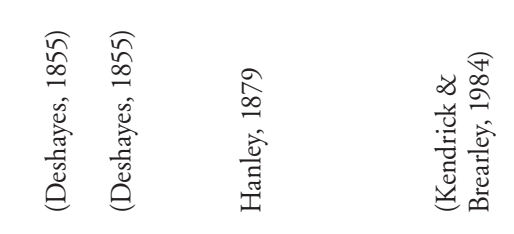

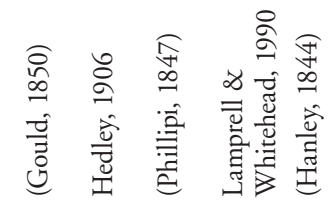

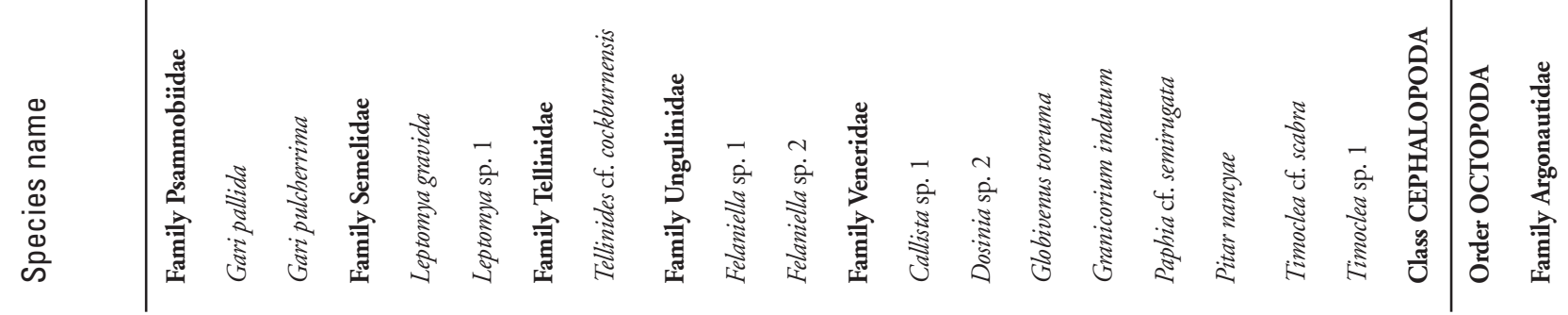




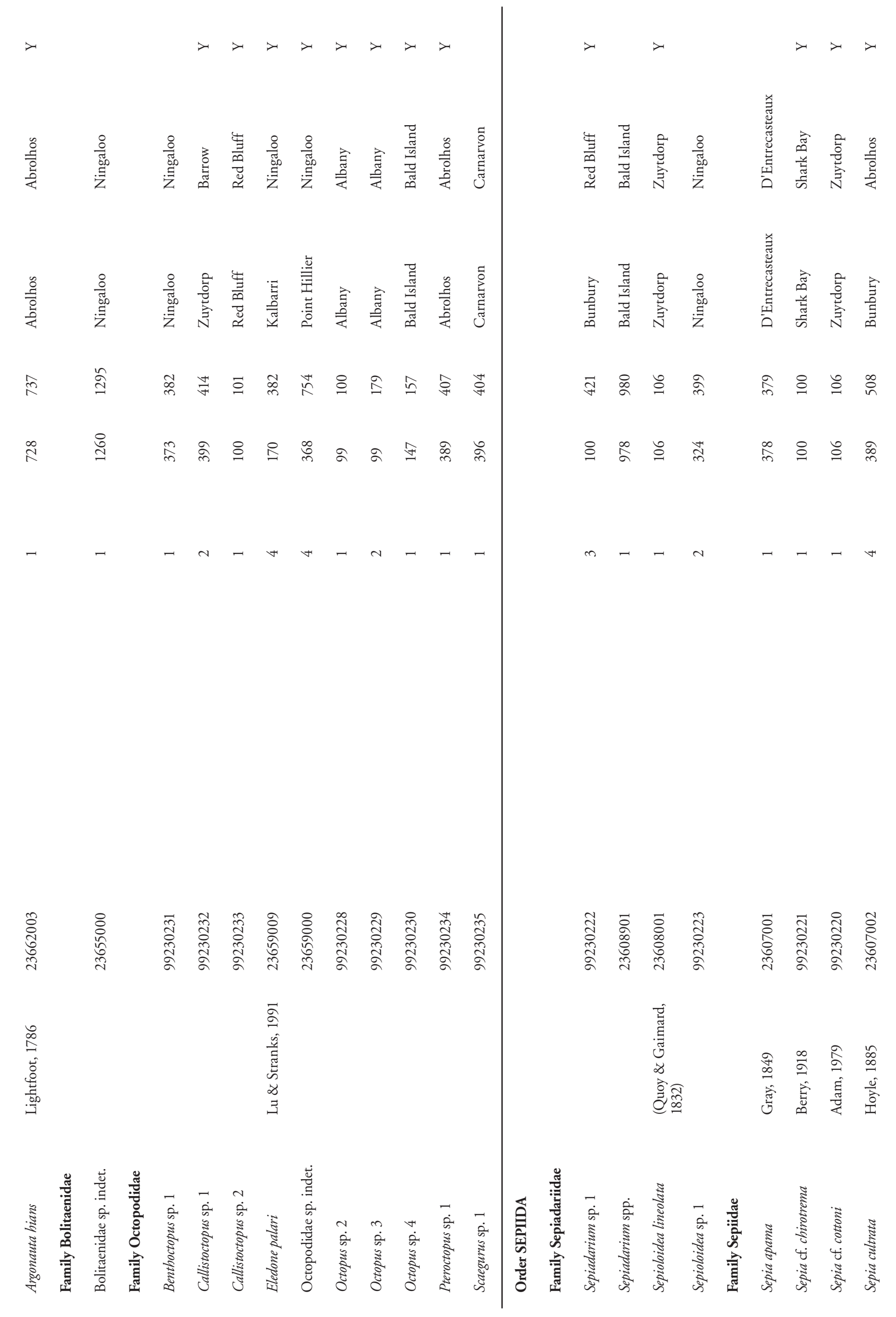




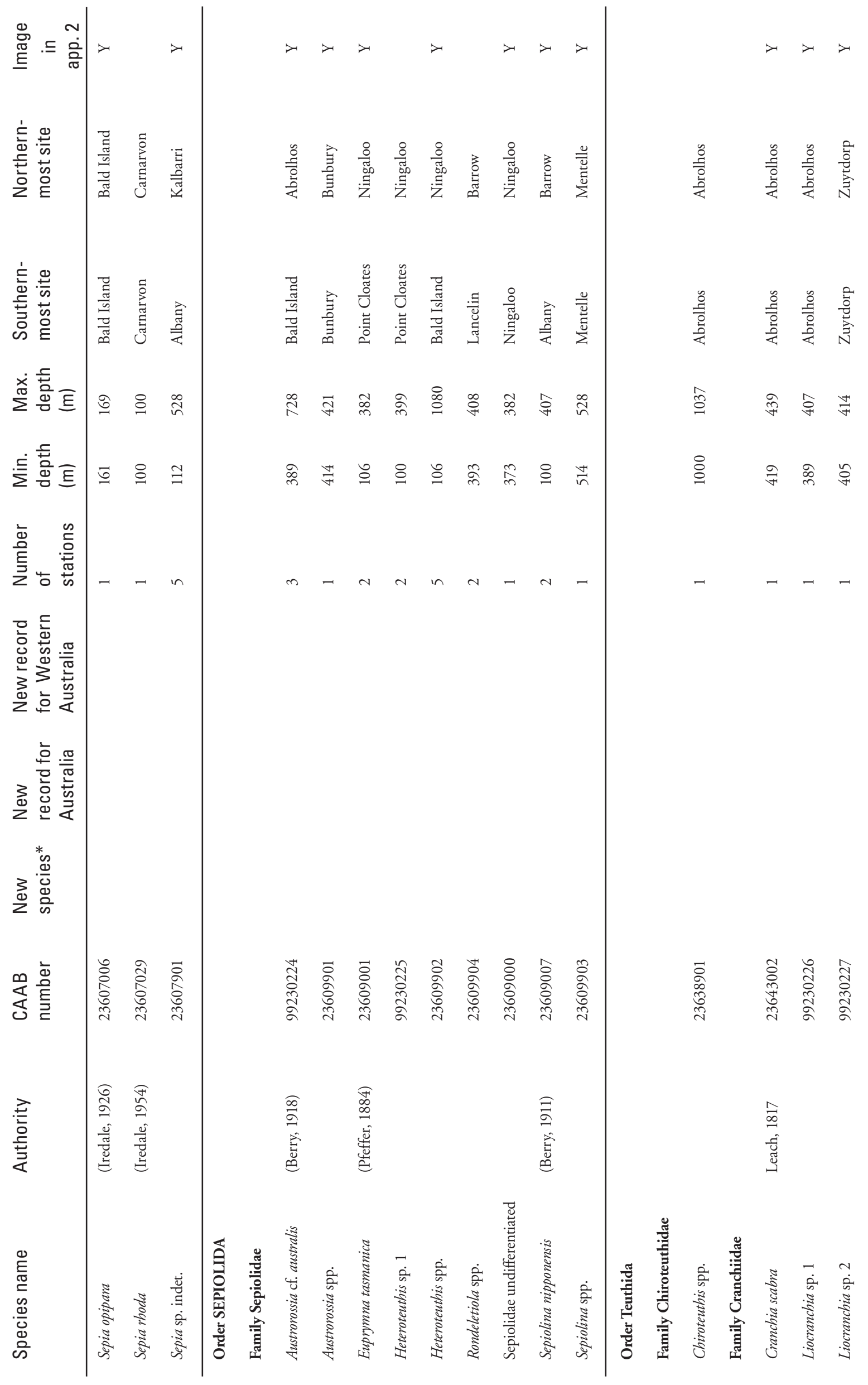




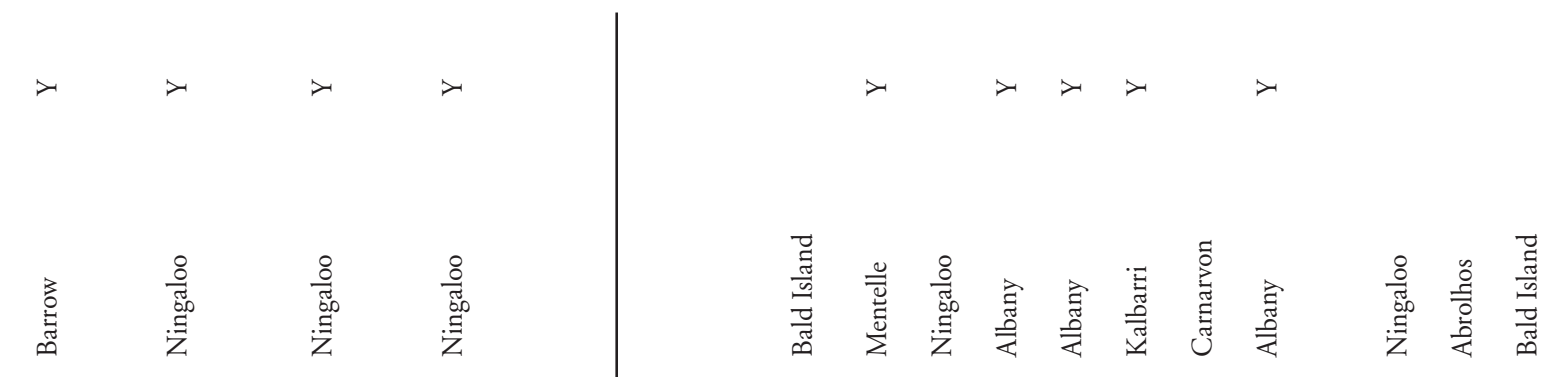

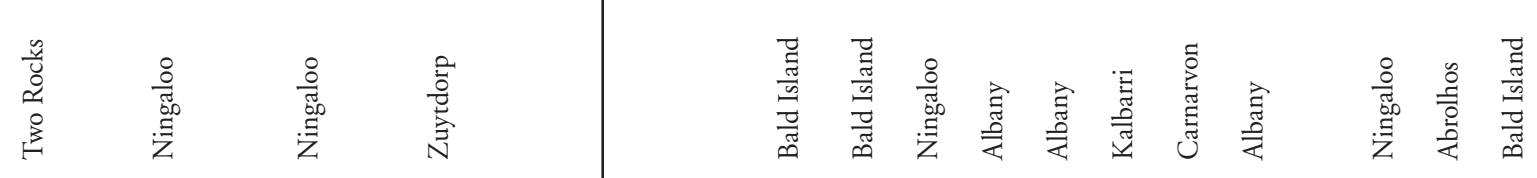

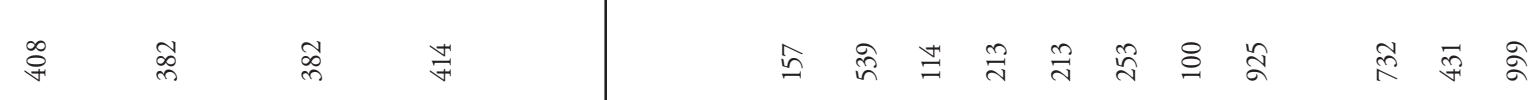

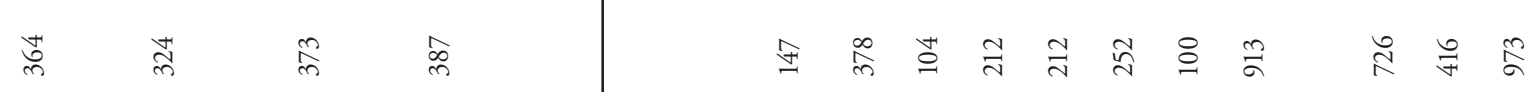

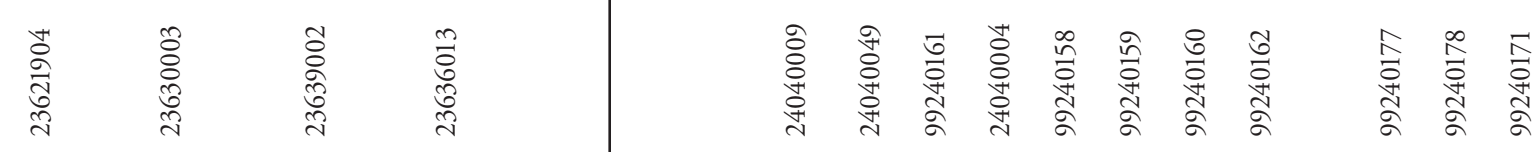

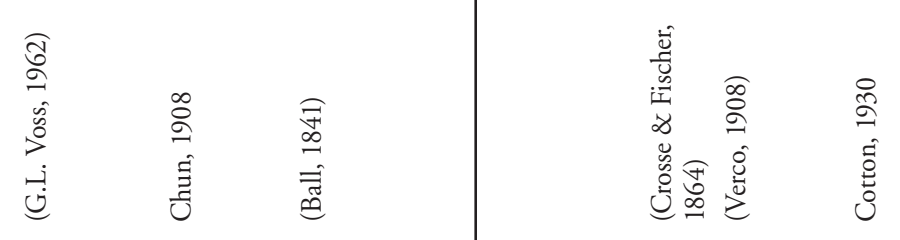

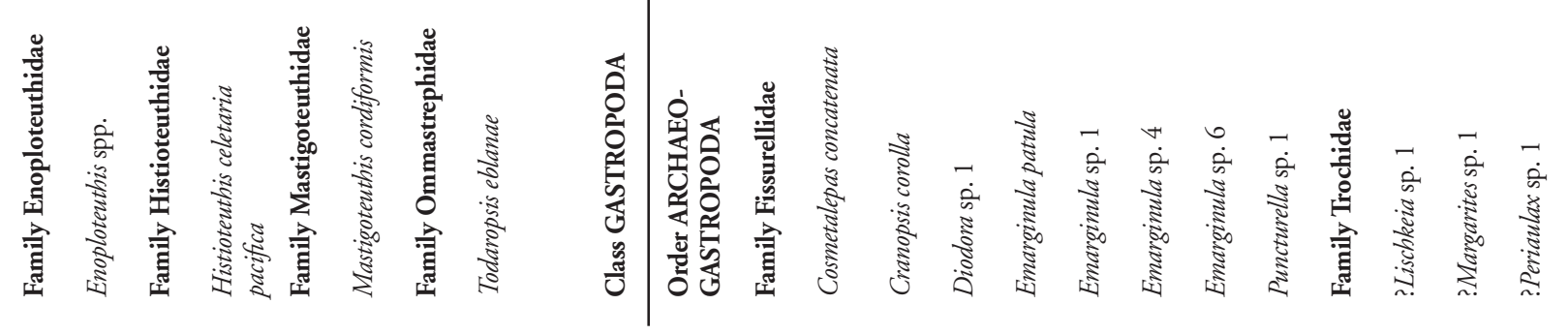




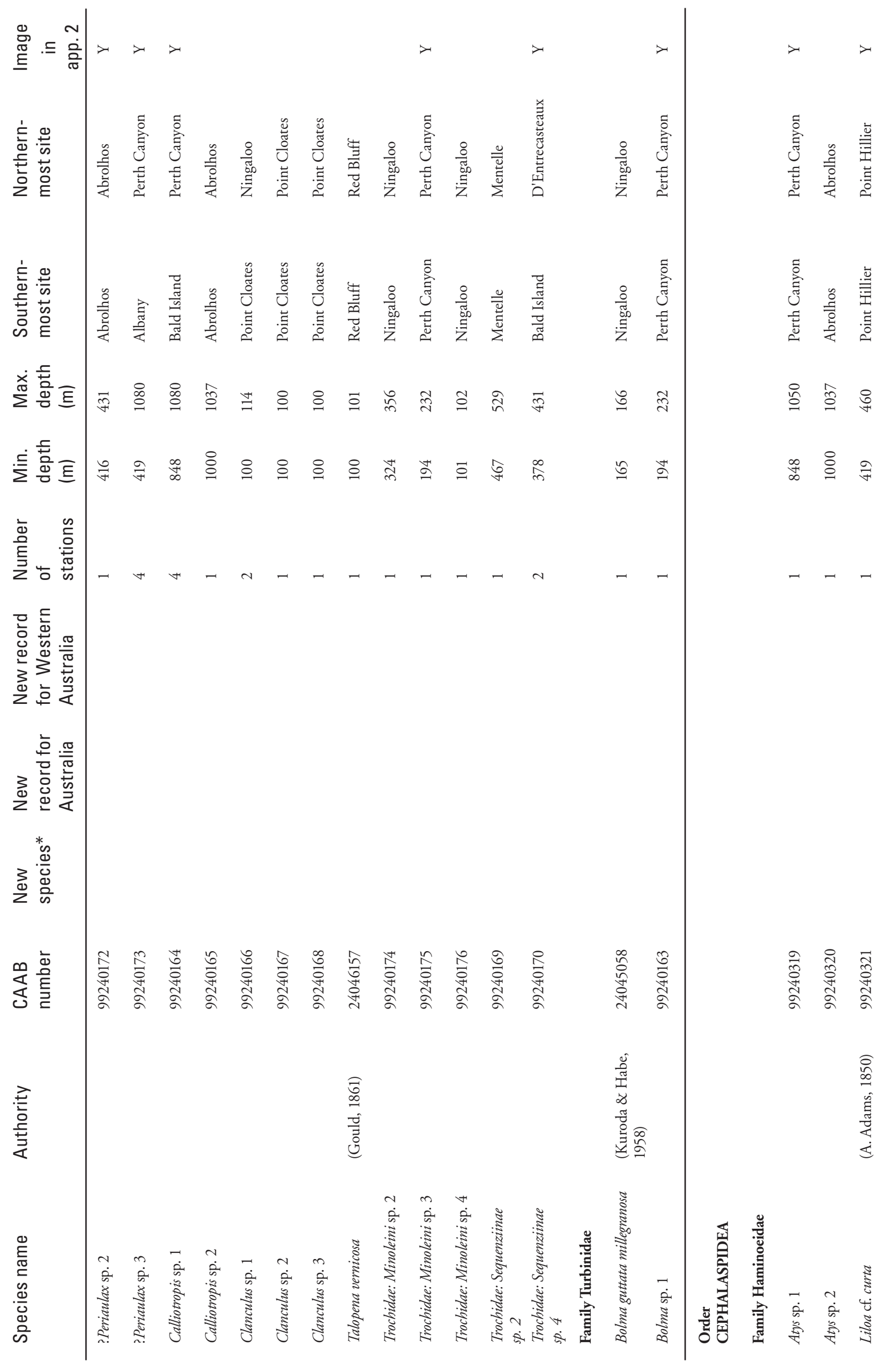




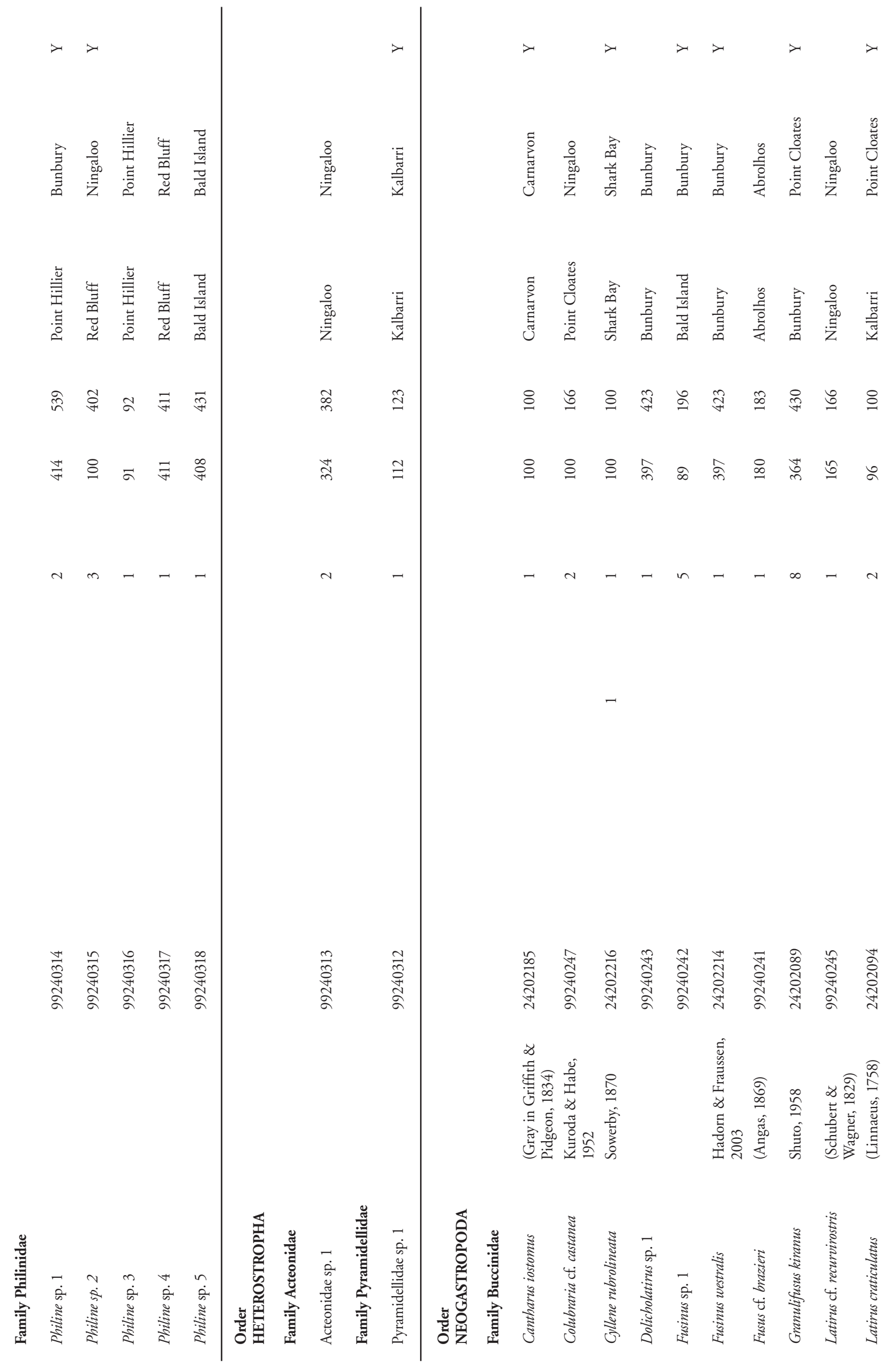




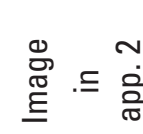

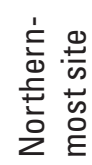

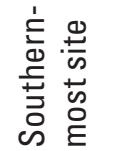

离营馬

紊

这券宐

离

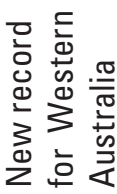

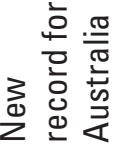

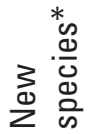

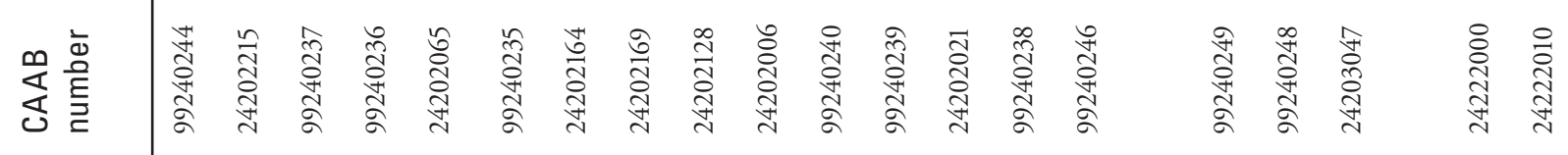

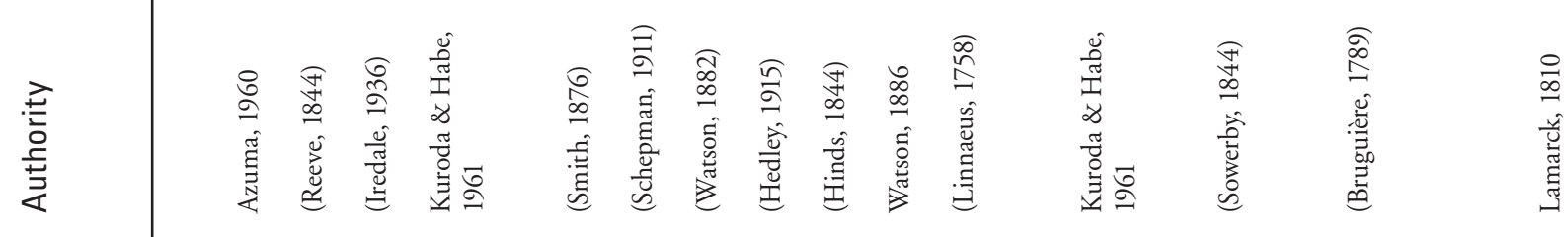

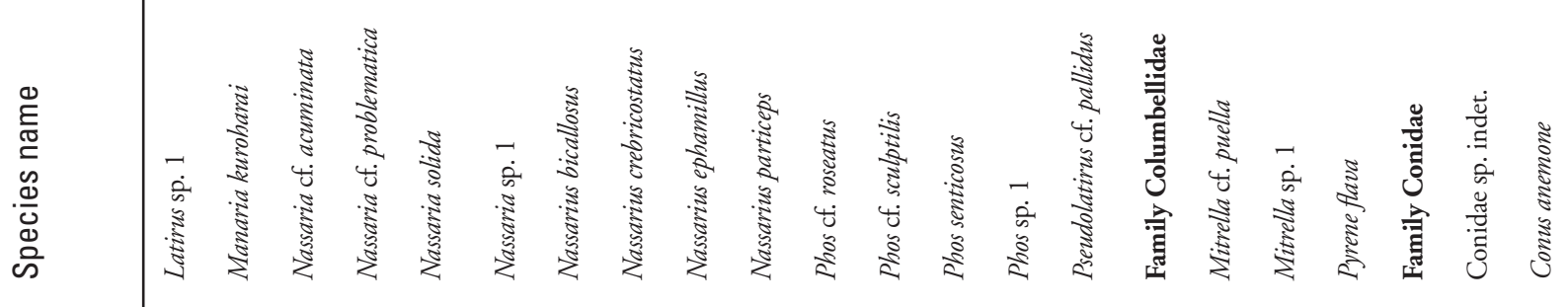




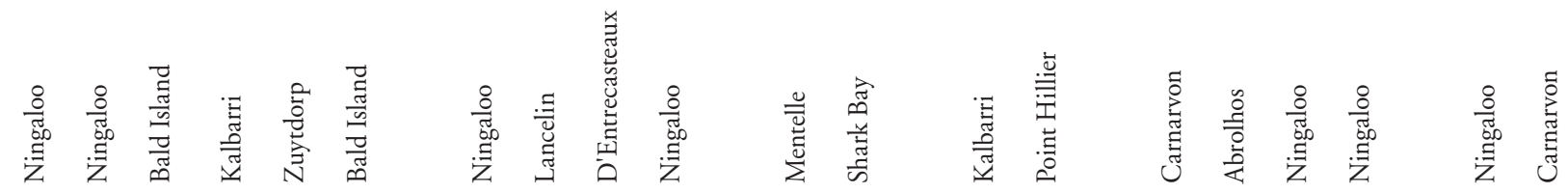

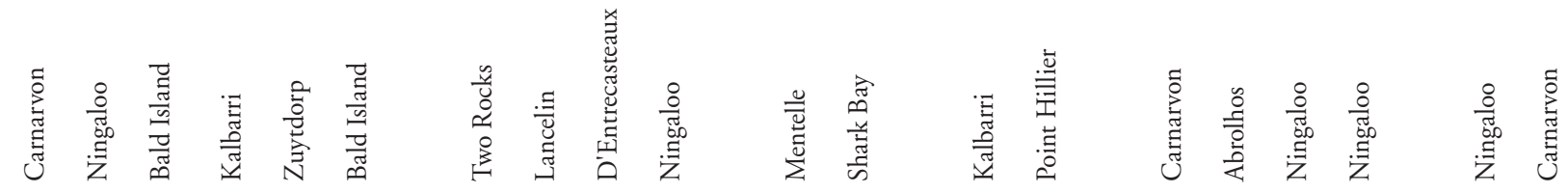

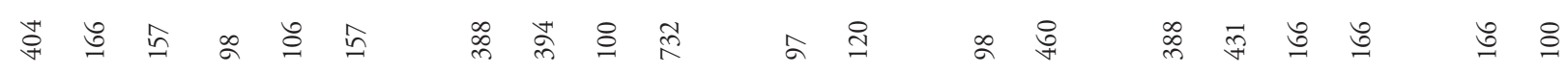

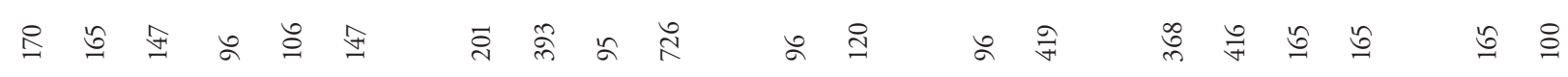

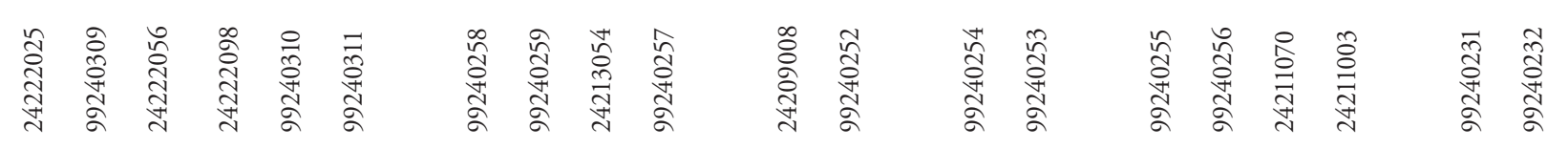

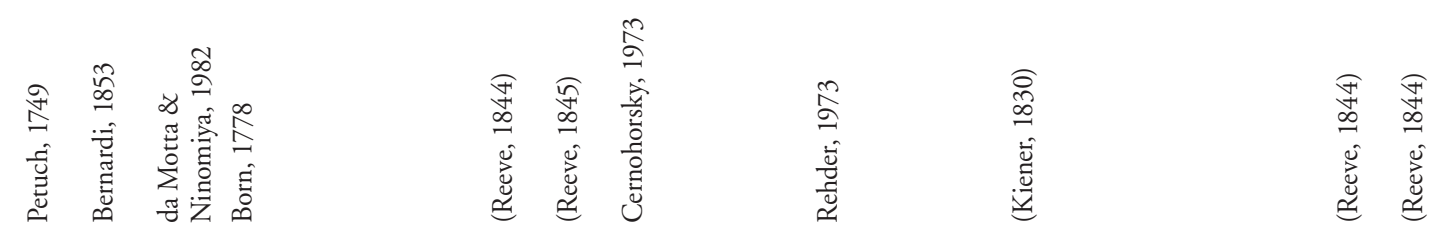

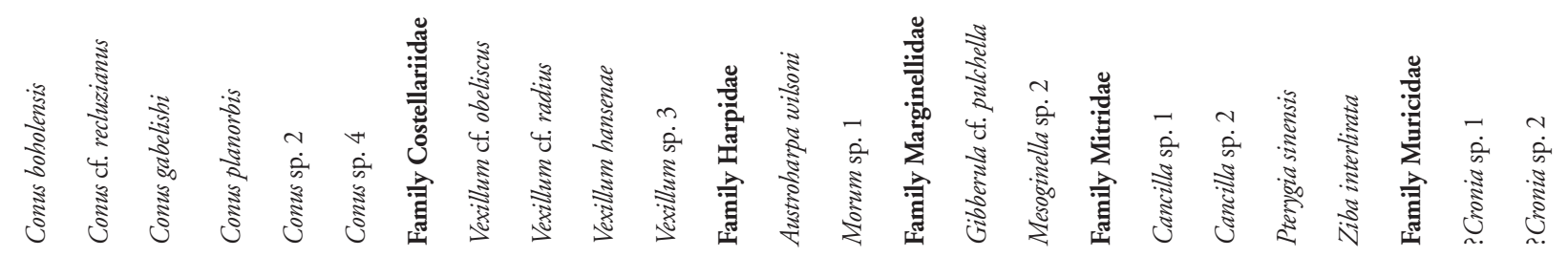




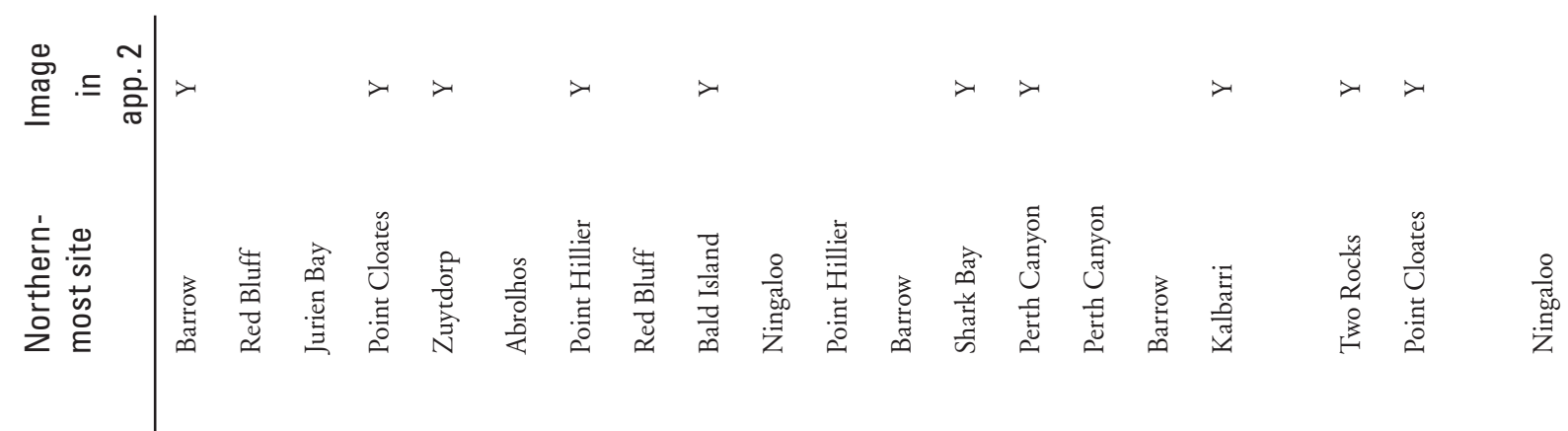

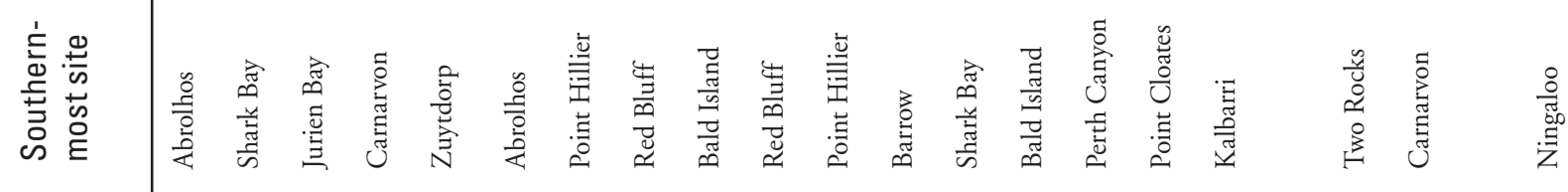

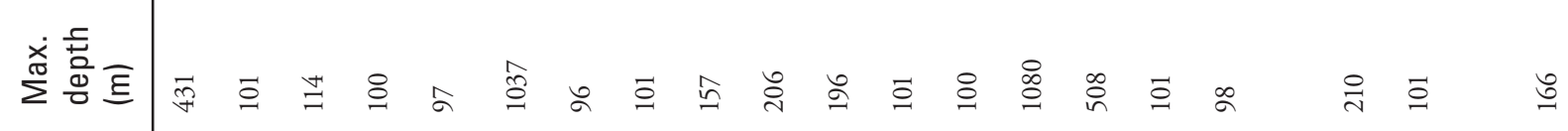

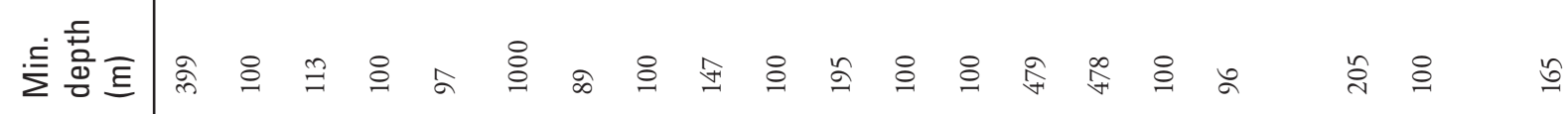

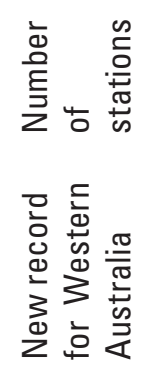

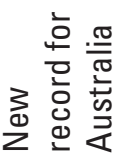

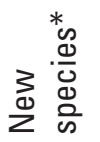

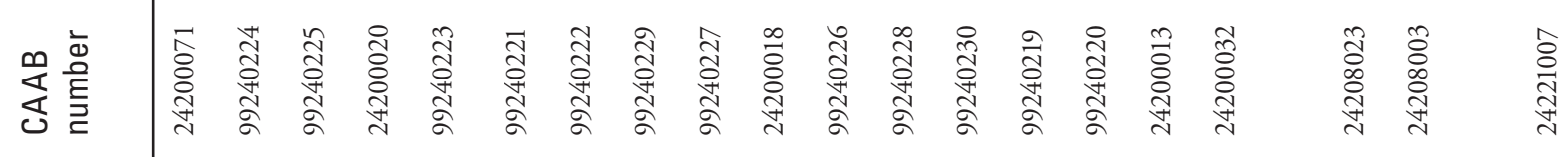

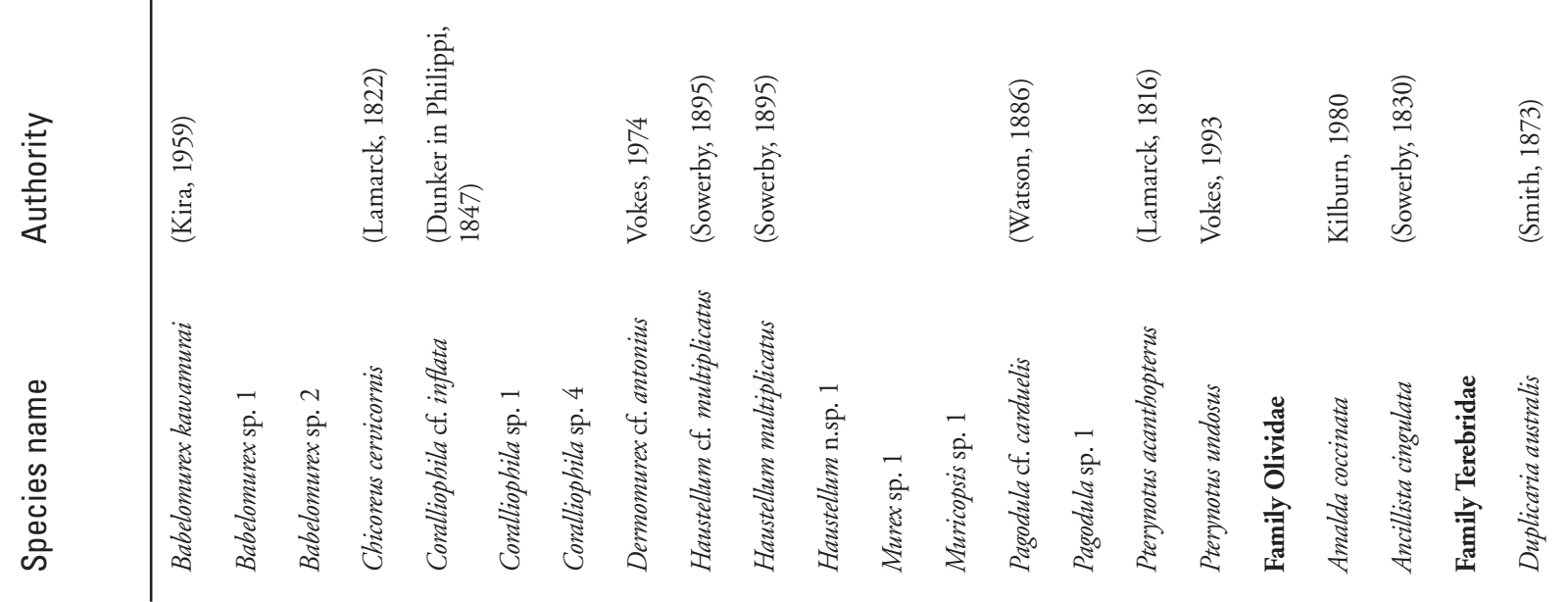




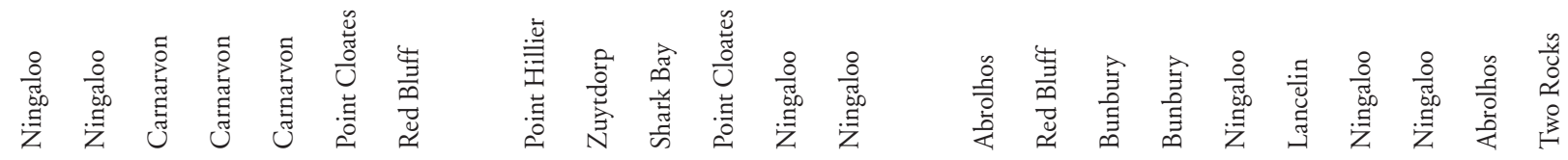

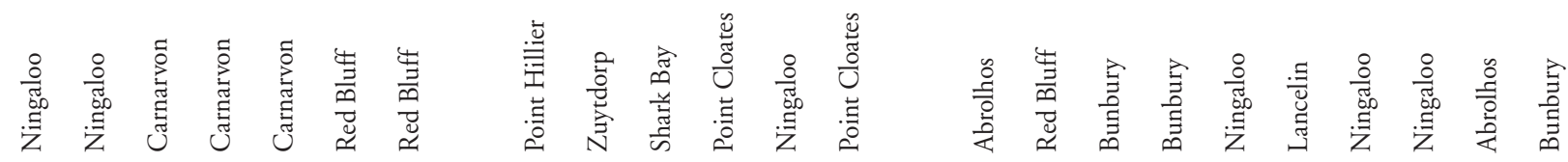
ఫั่

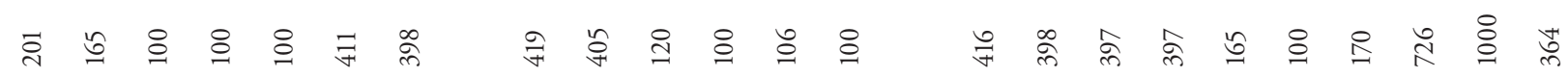

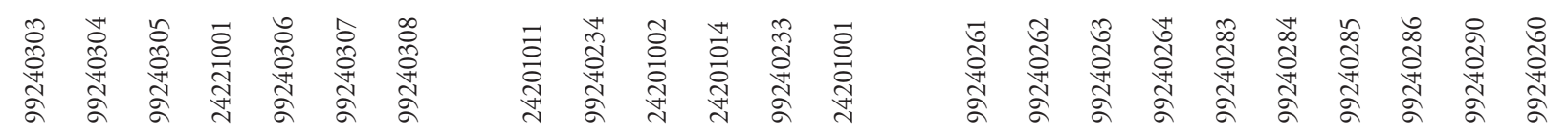
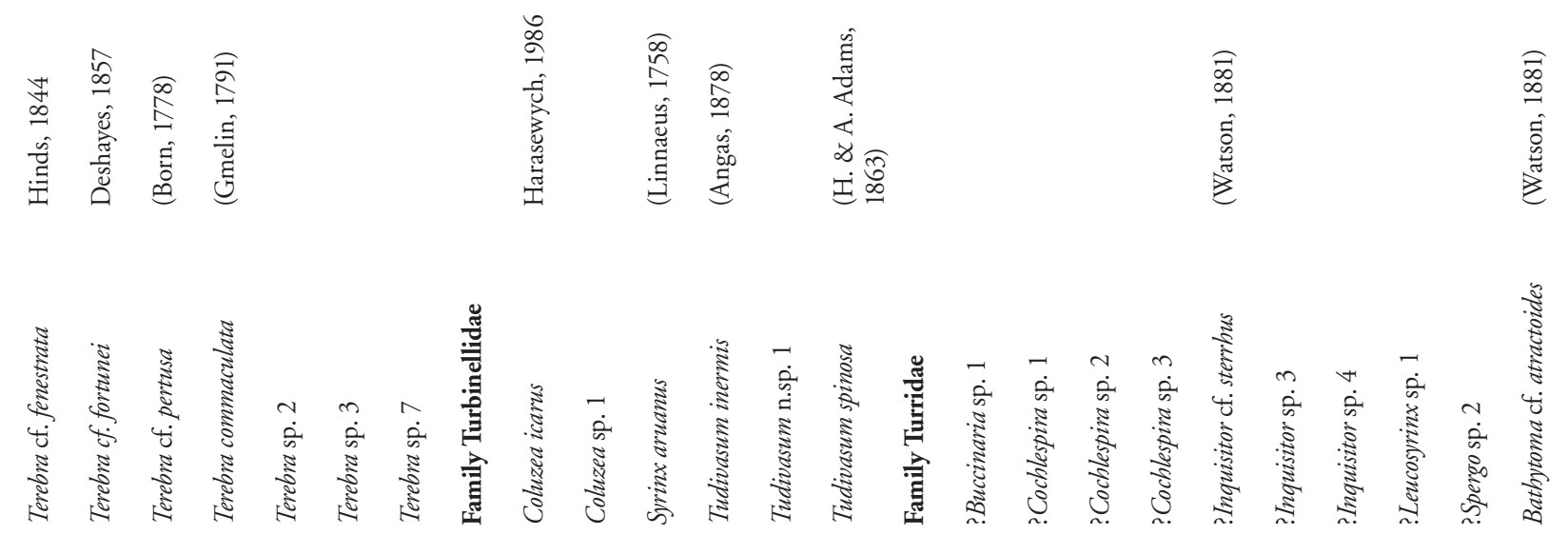


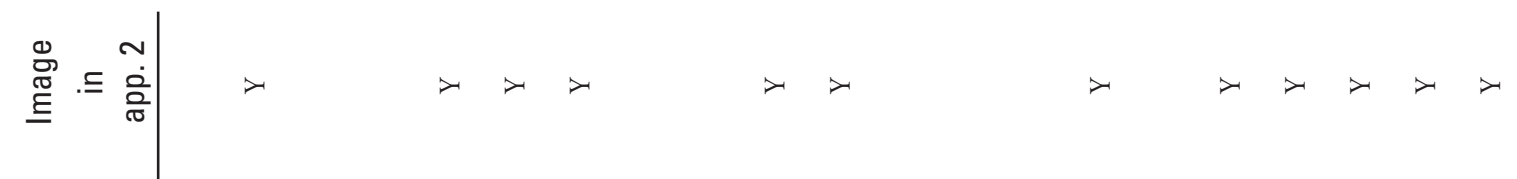

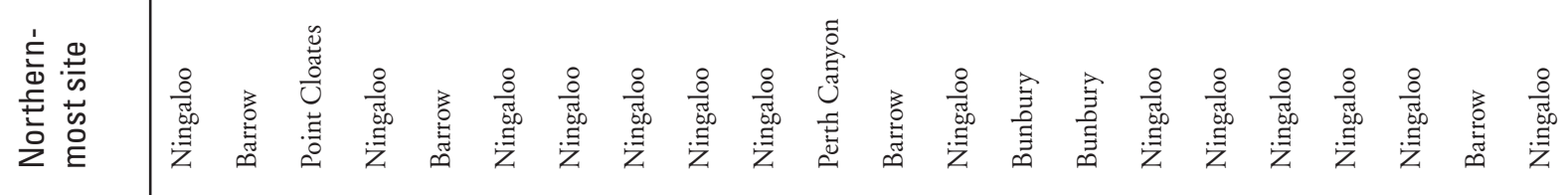

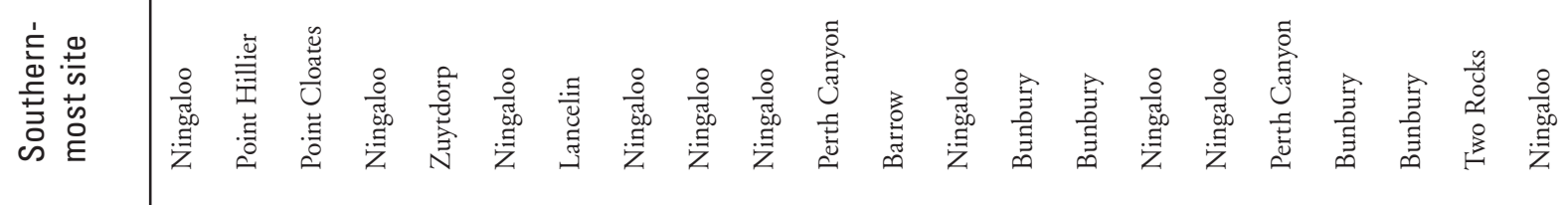

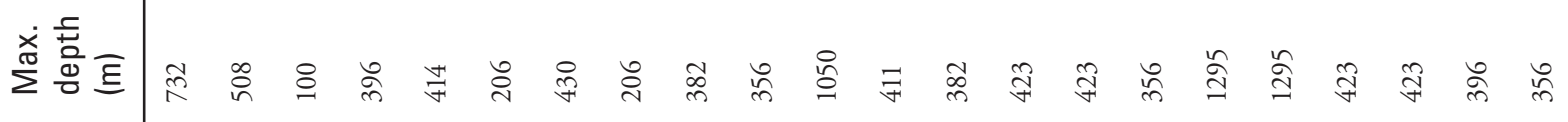

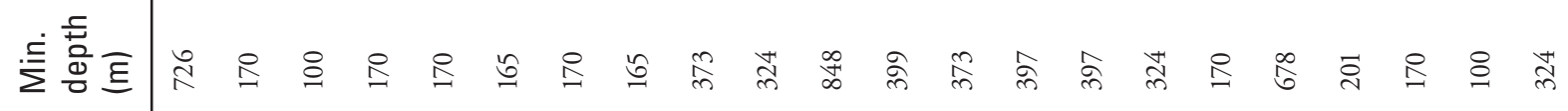

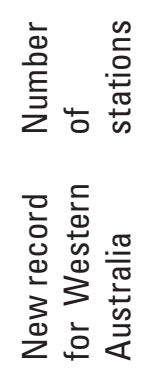

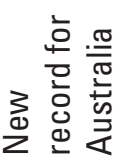

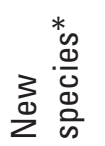

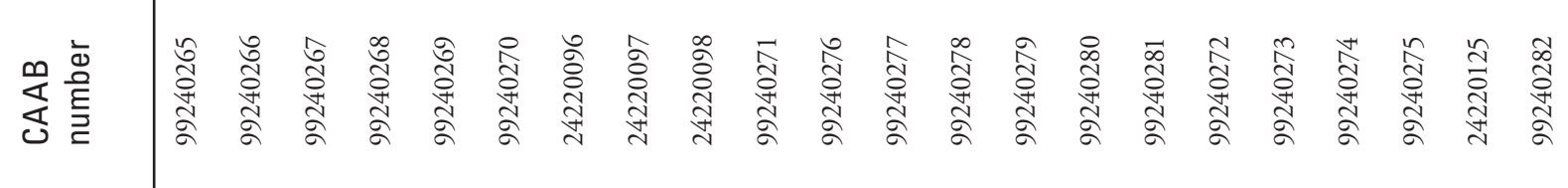

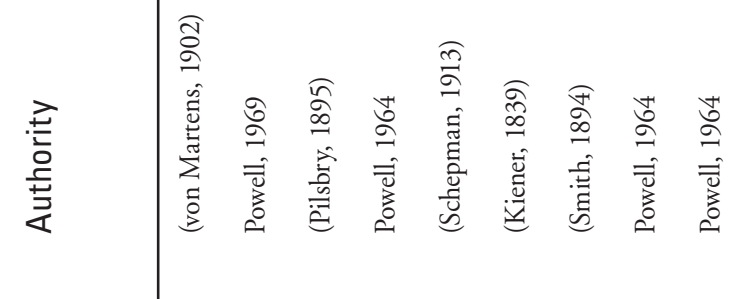

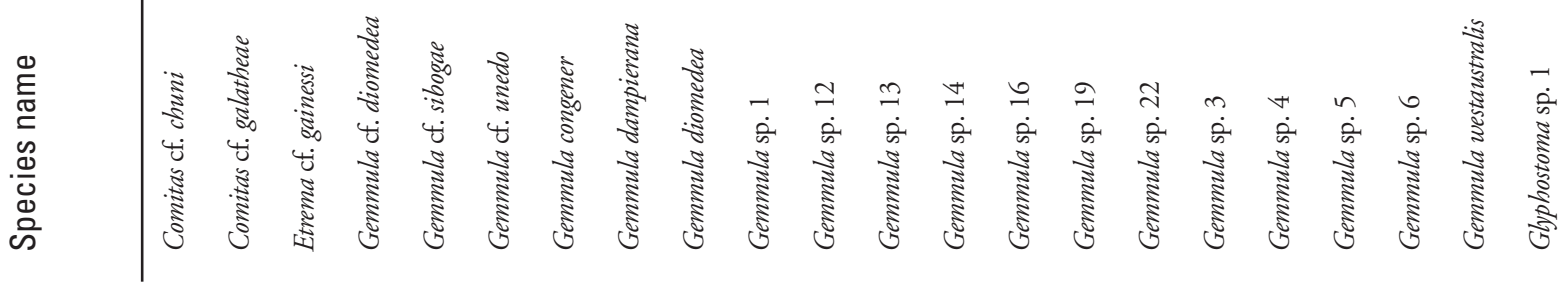




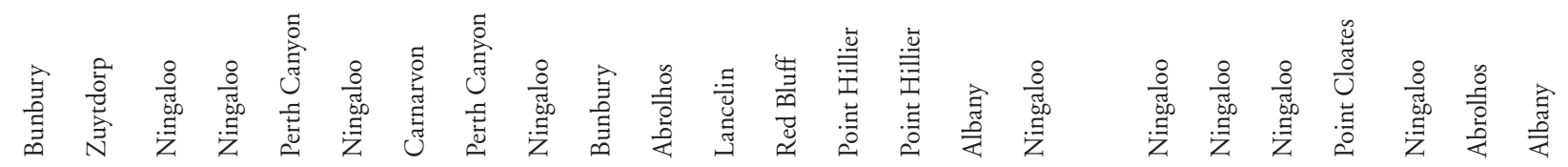

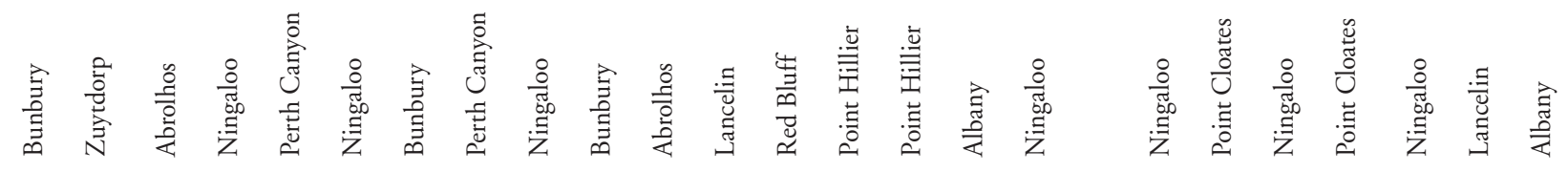

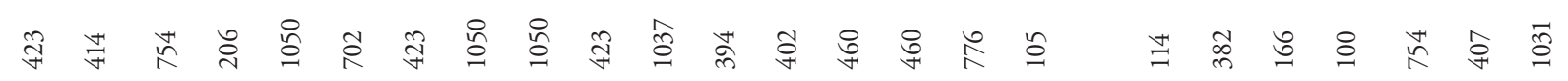
aे 尓

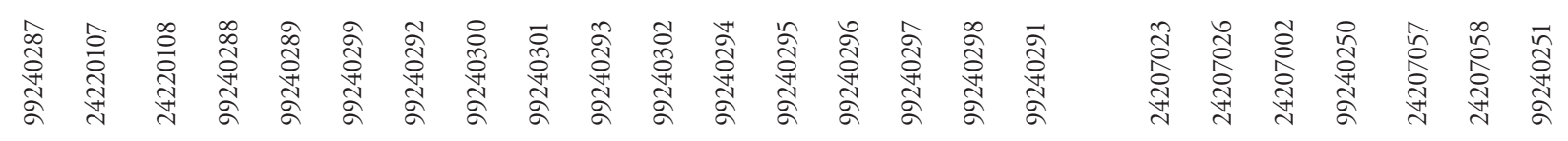

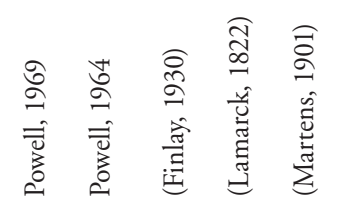

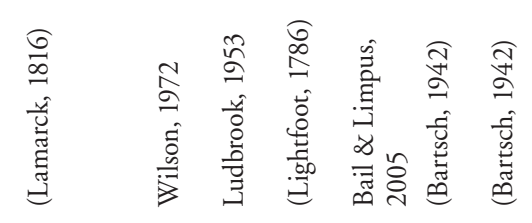

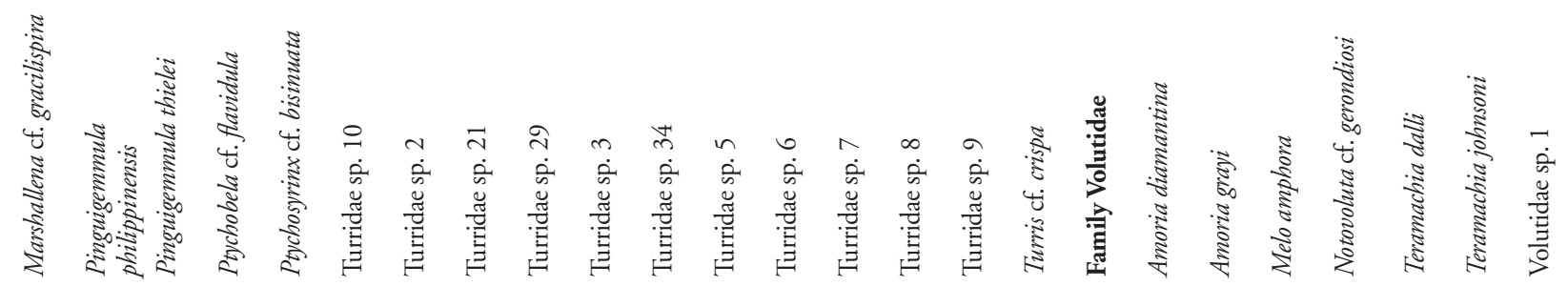




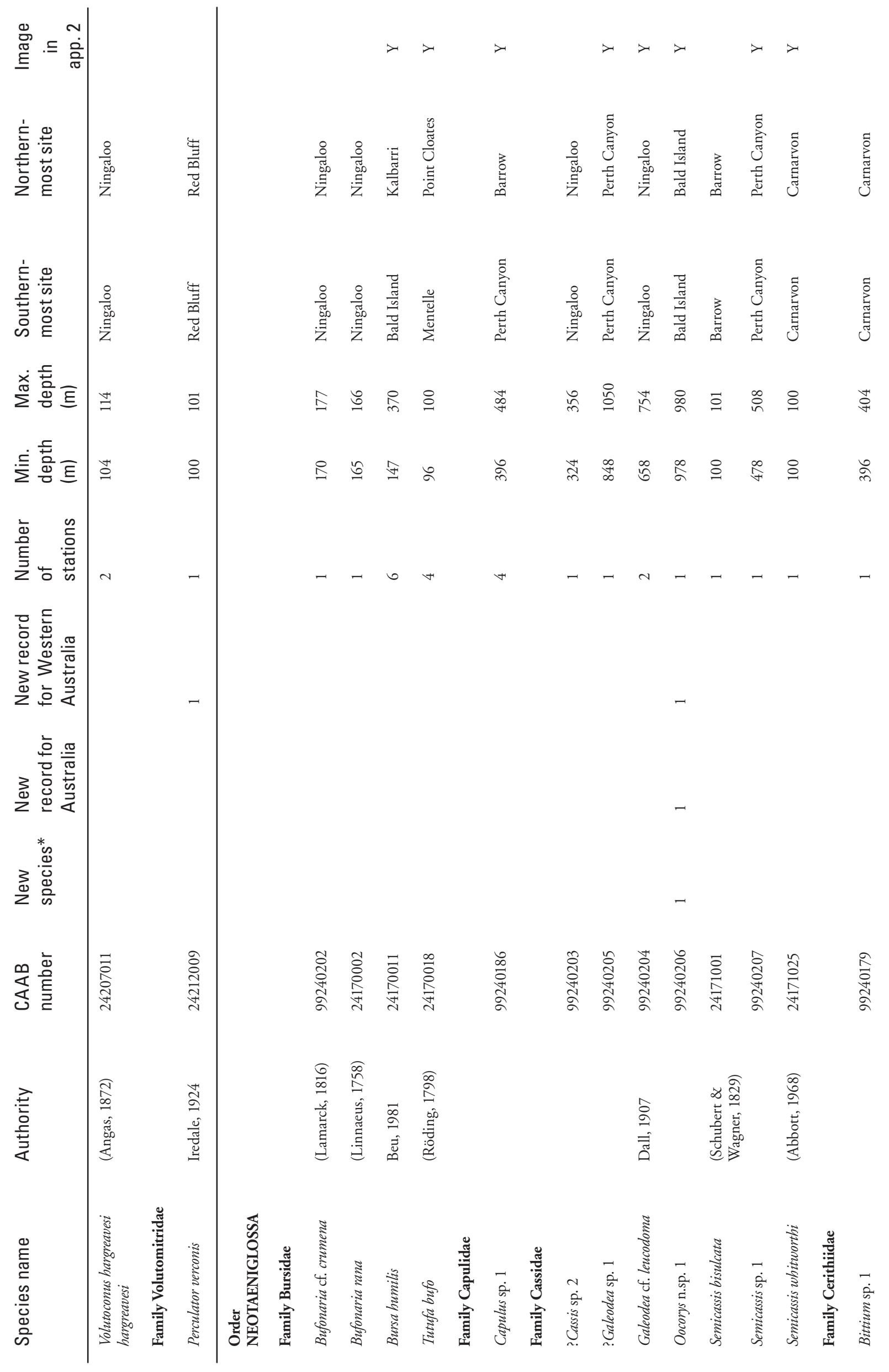




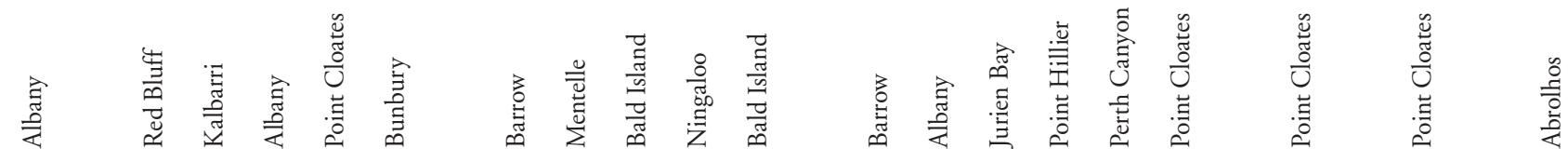

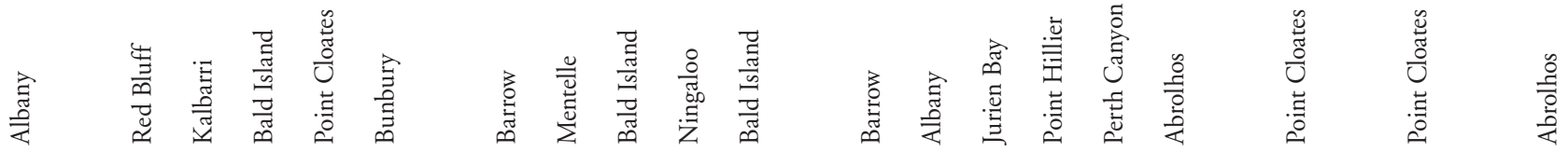

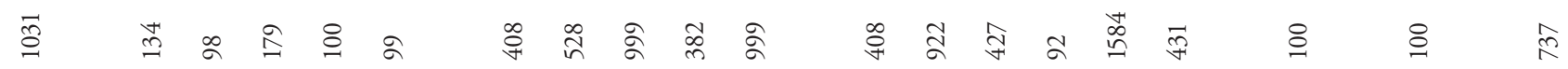

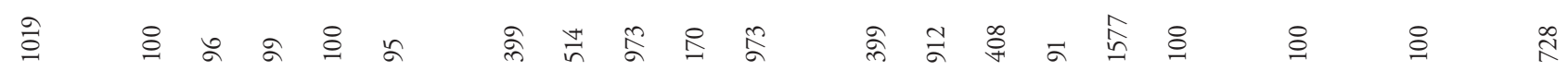

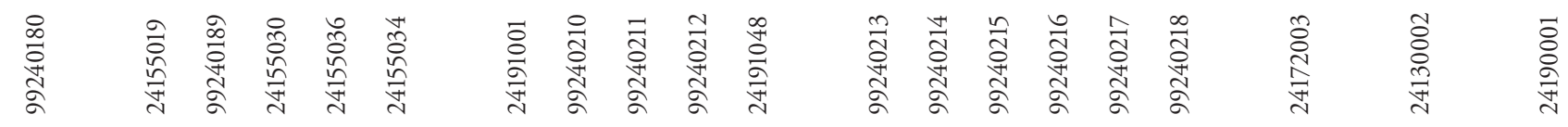

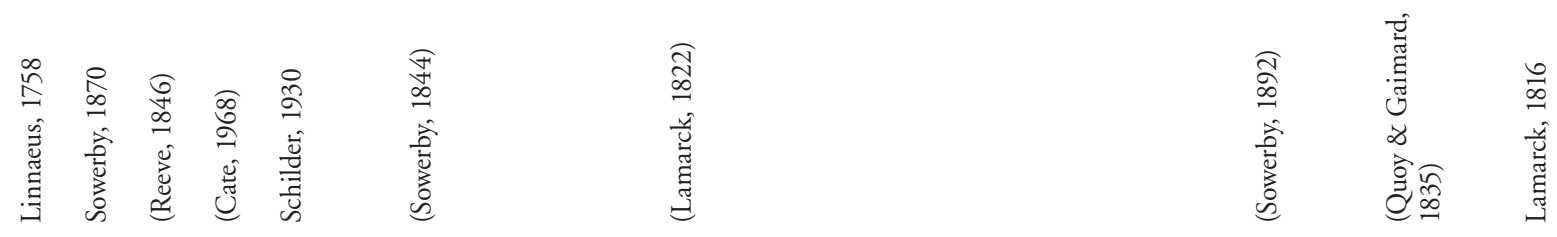

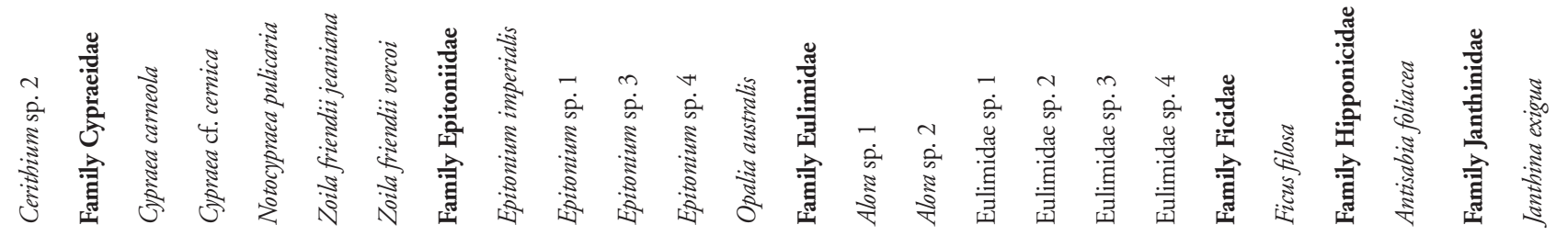




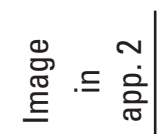

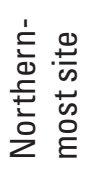

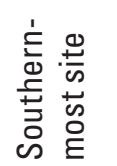

产产

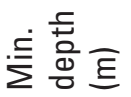

히를

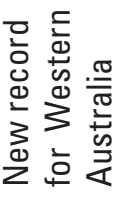

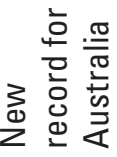

竞尊

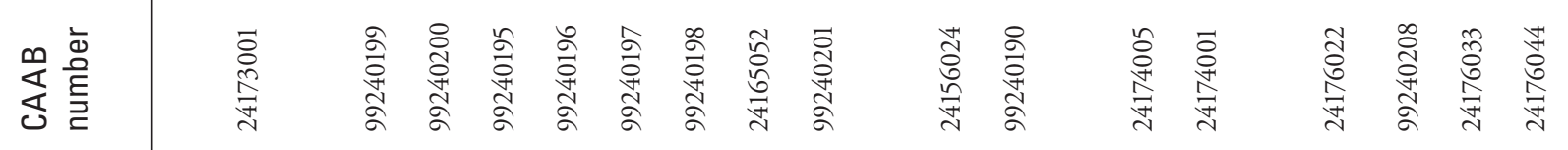

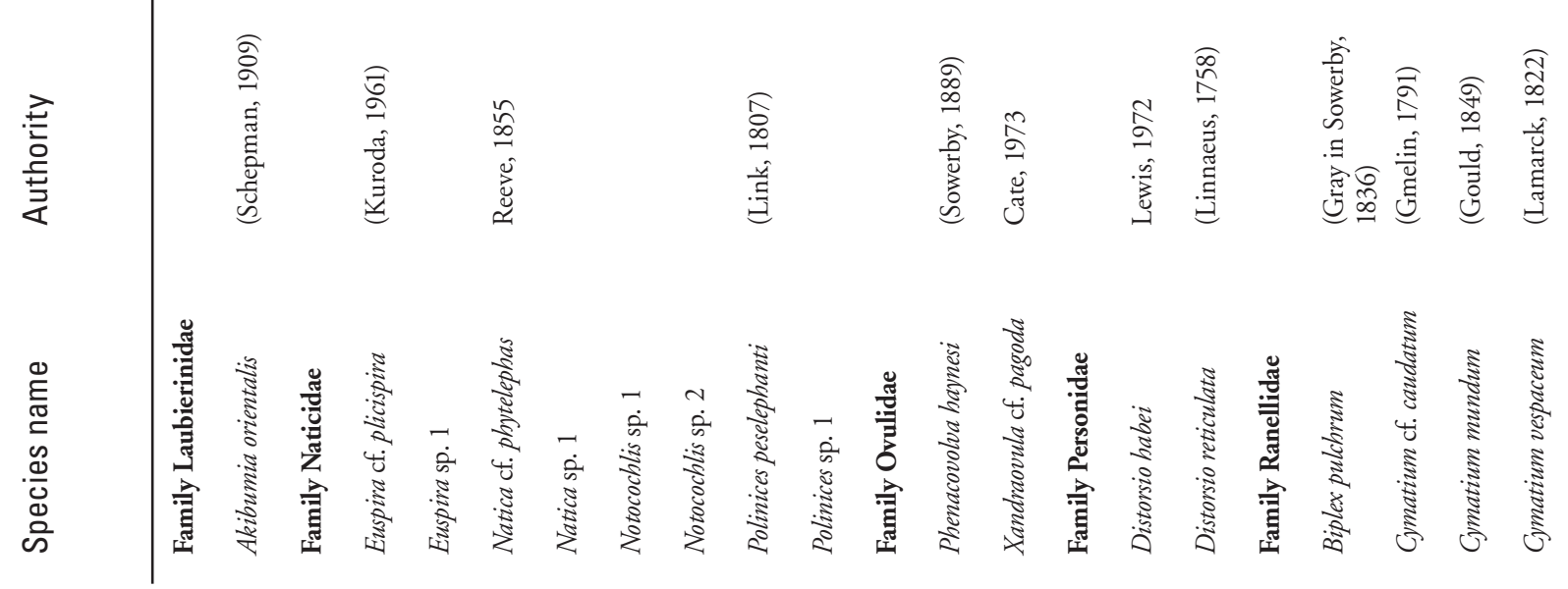




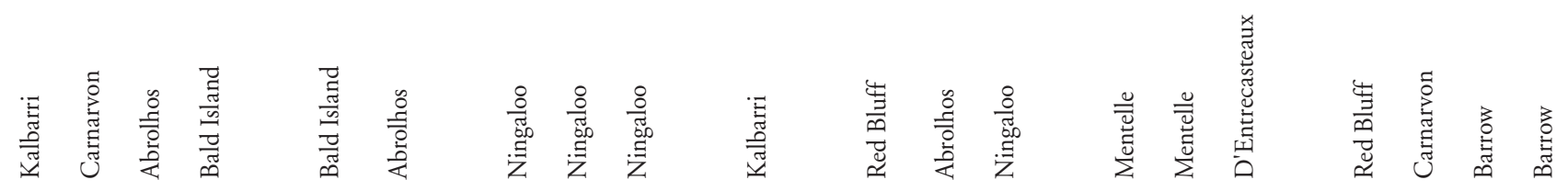

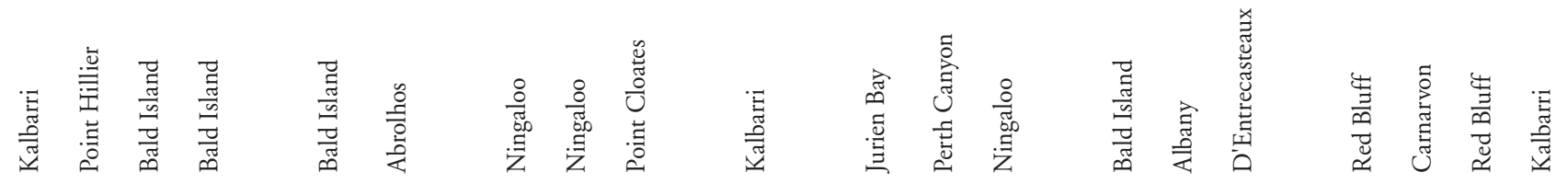

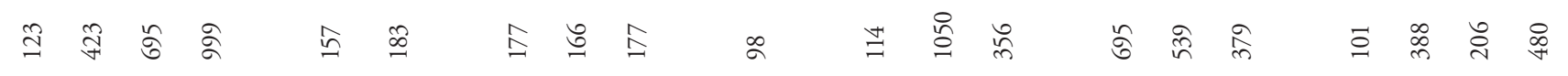

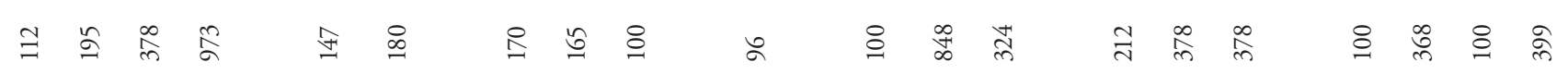

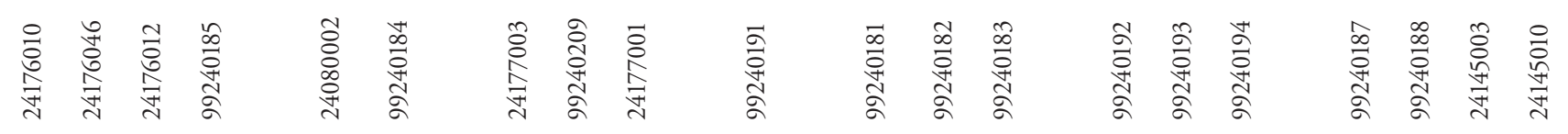

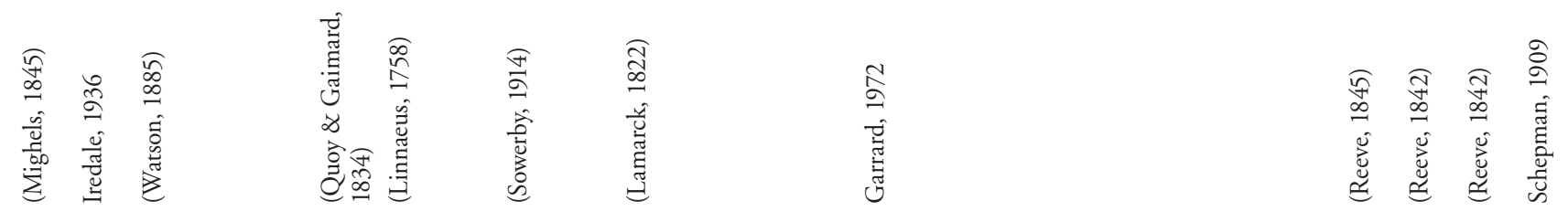

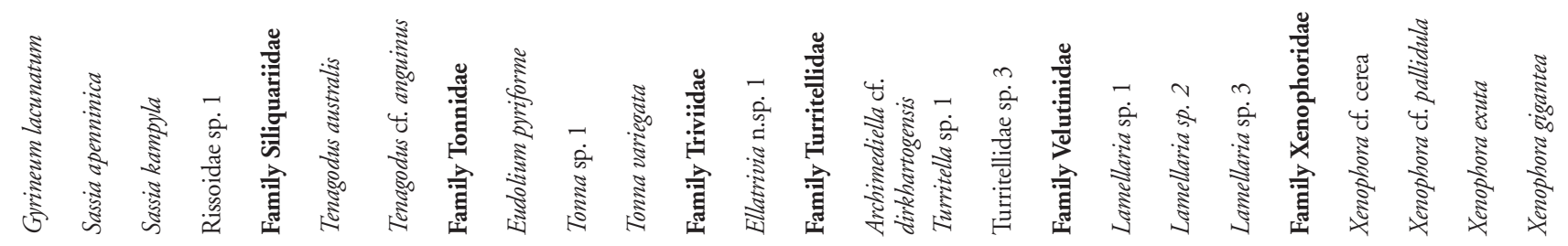




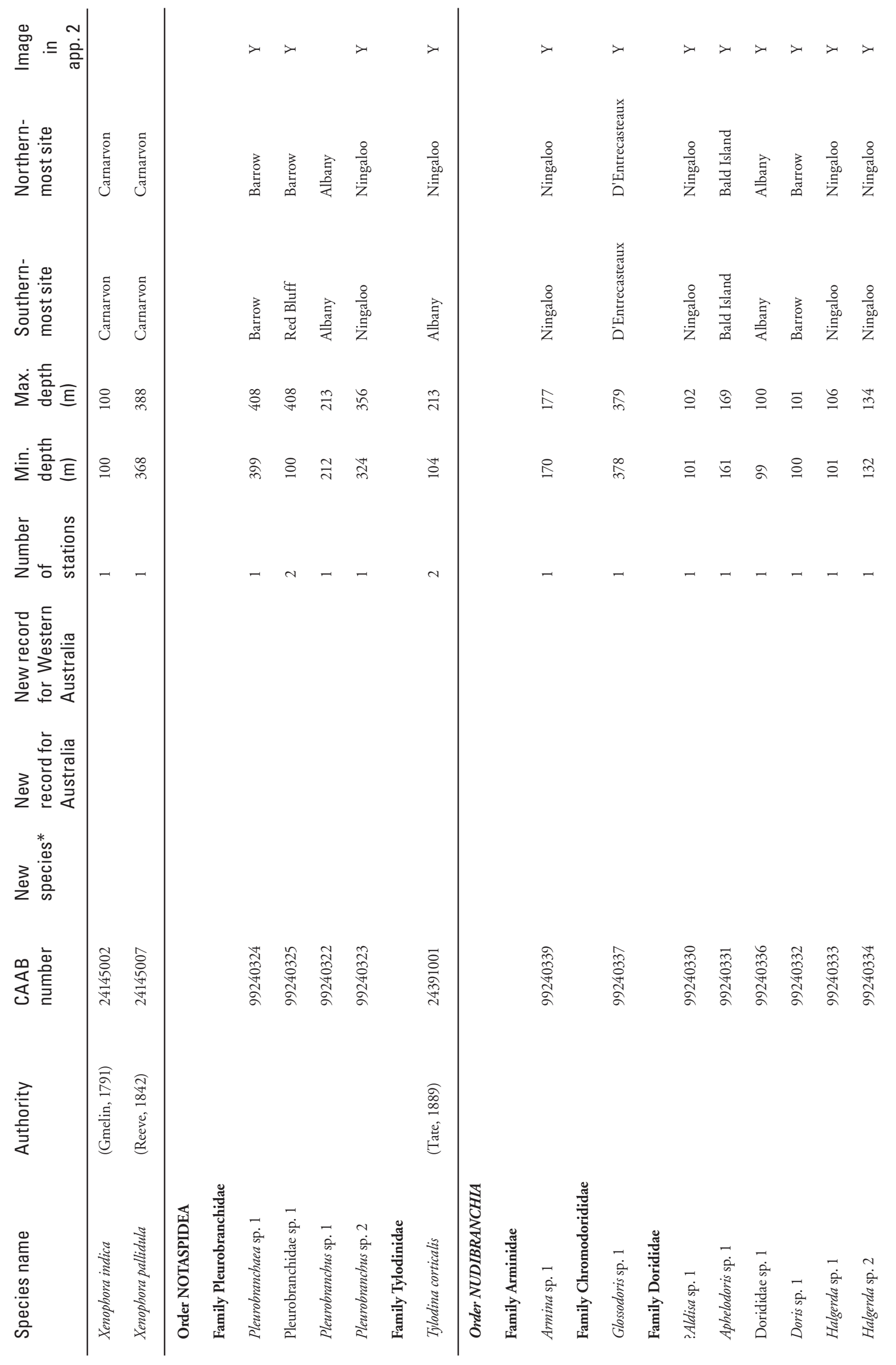




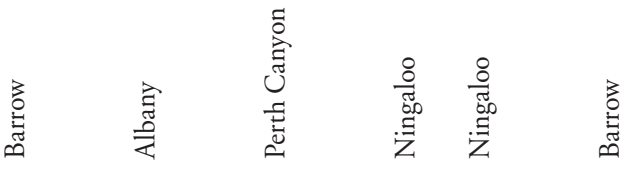

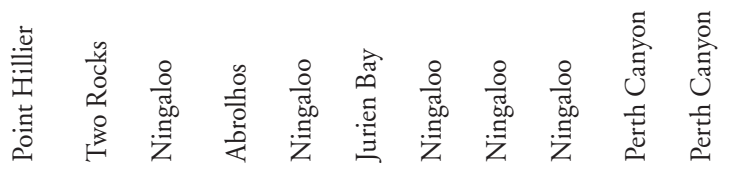

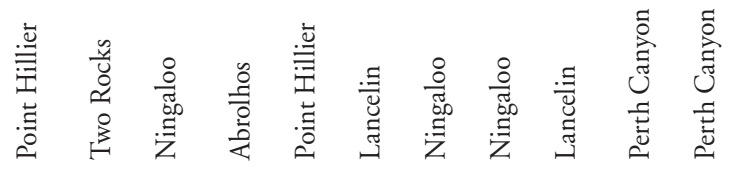

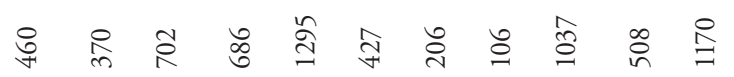

₹ ते ฉ్ర

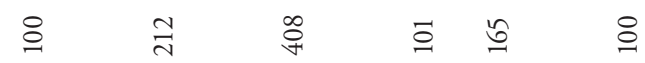

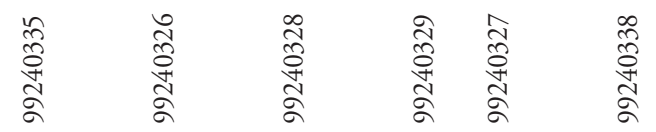

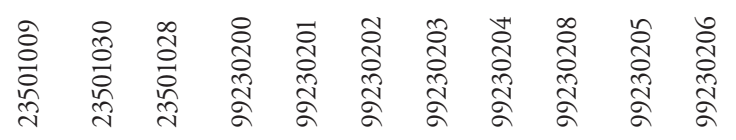

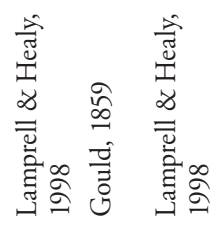

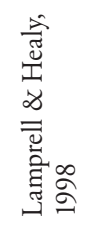

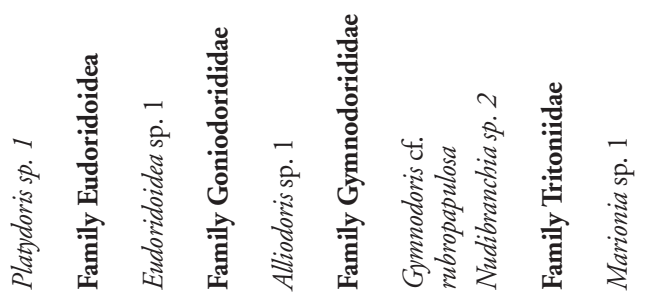

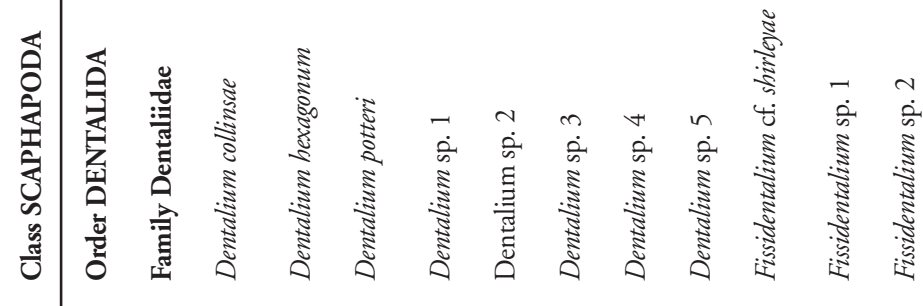




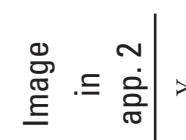

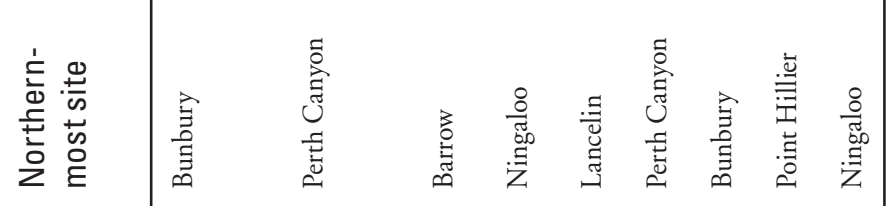

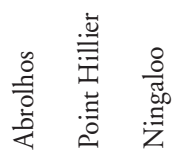

衰

产劳

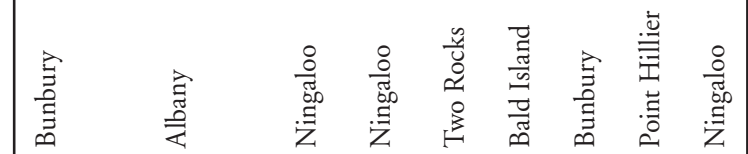

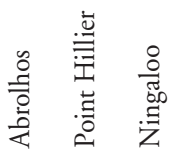

衰

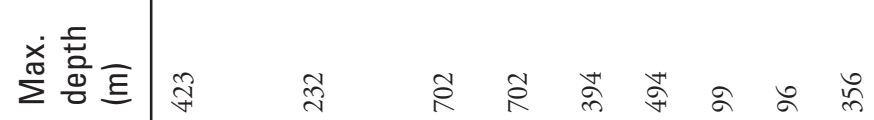

\&

$\approx$

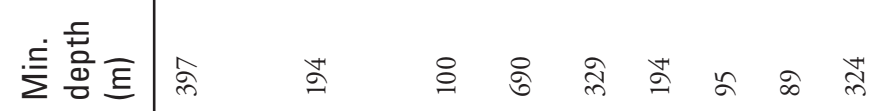

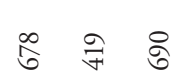

ঃ

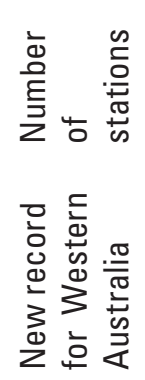

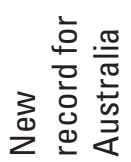

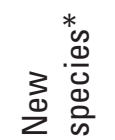

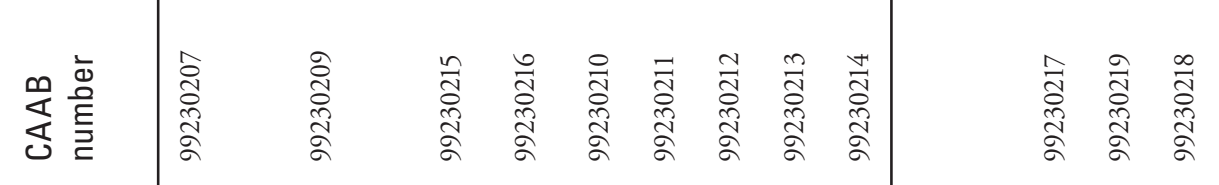

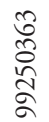

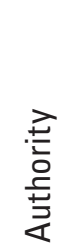

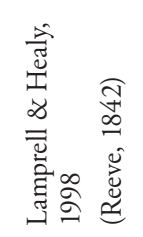

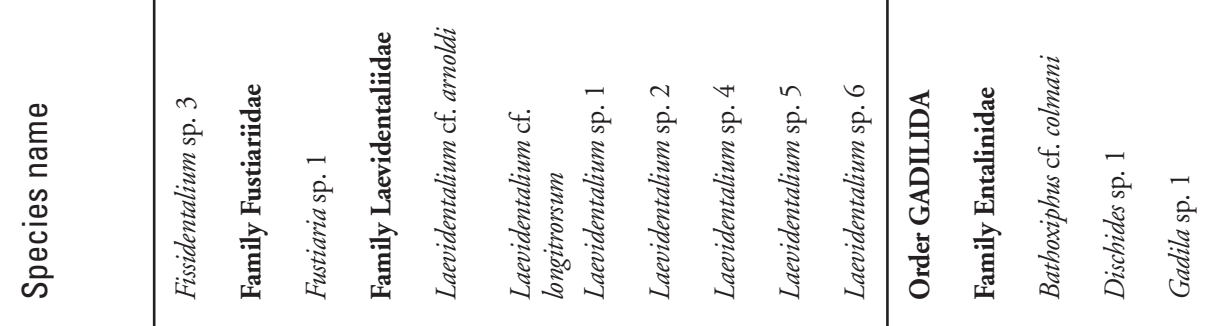

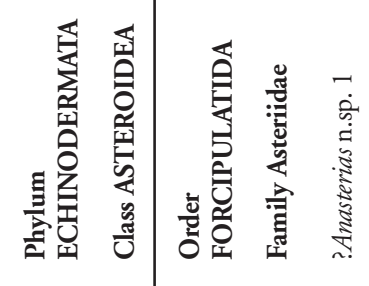




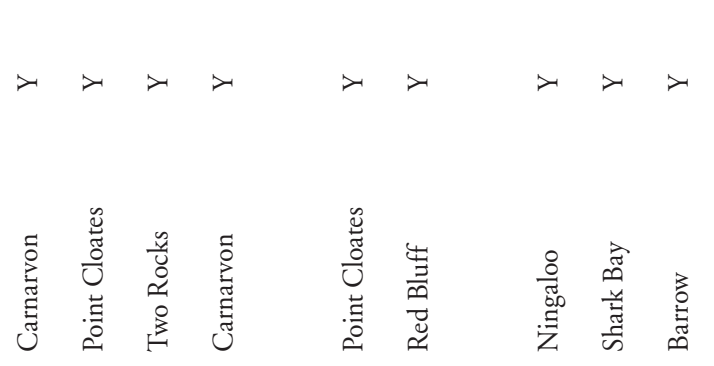

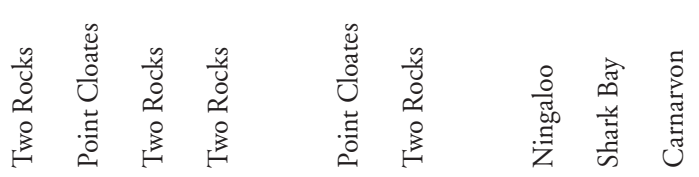

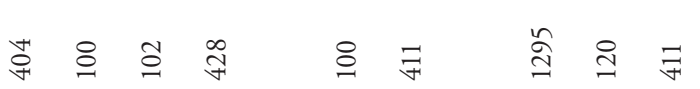

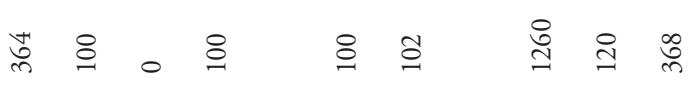

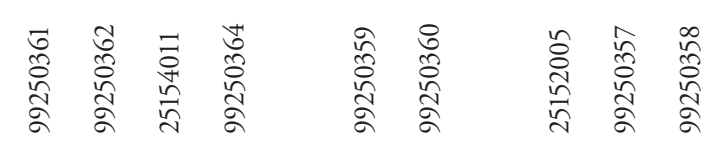

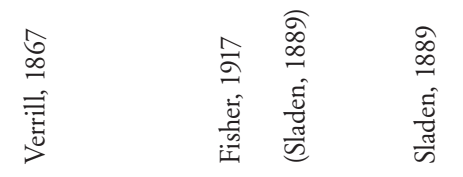

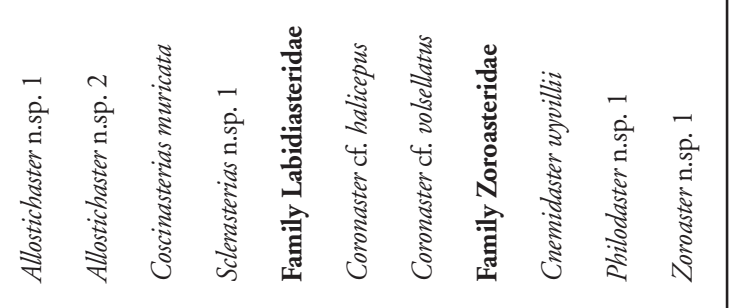

$$
\begin{aligned}
& \text { 范 }
\end{aligned}
$$

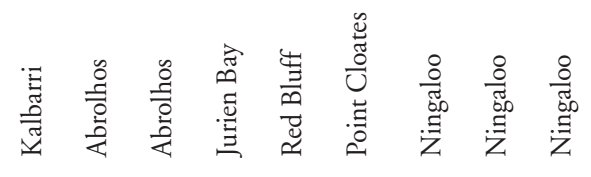

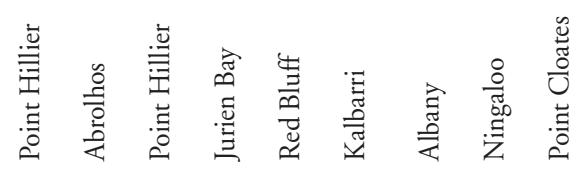

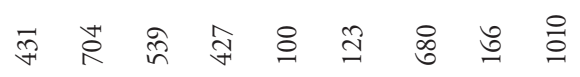

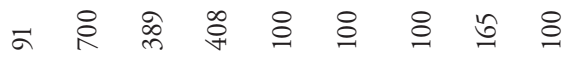

$$
\begin{aligned}
& \text { ๙े } \\
& \text { ๙్రి }
\end{aligned}
$$

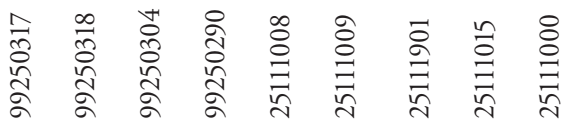

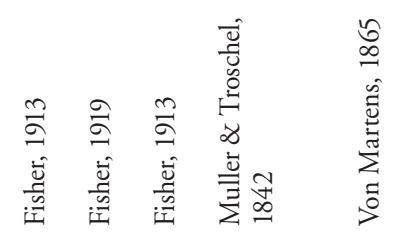

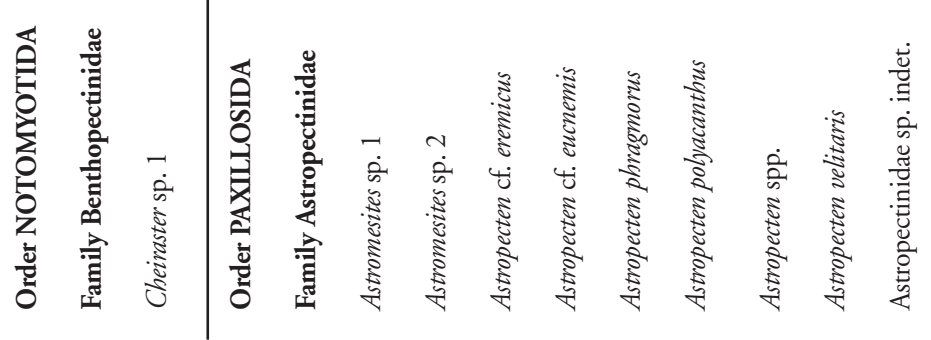




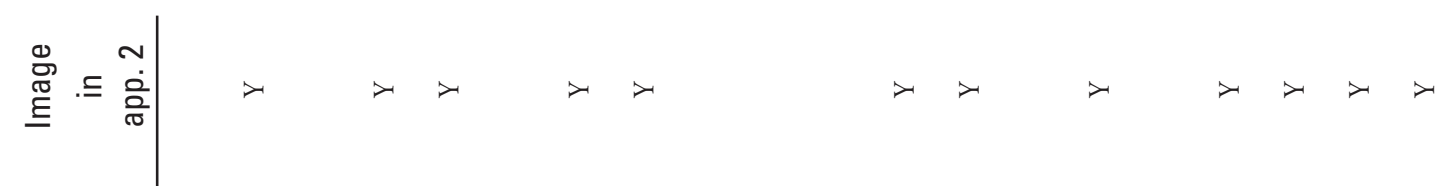

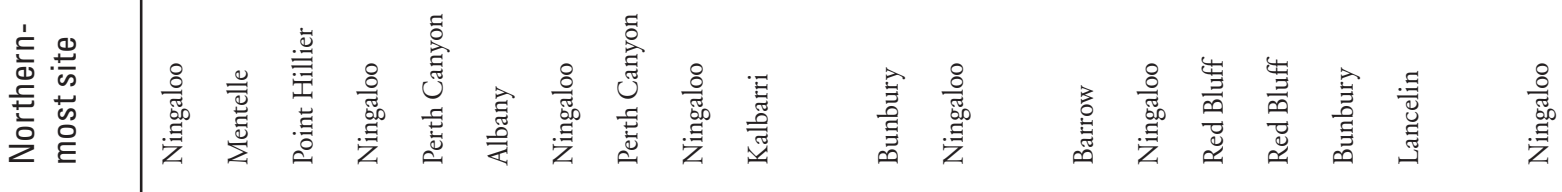

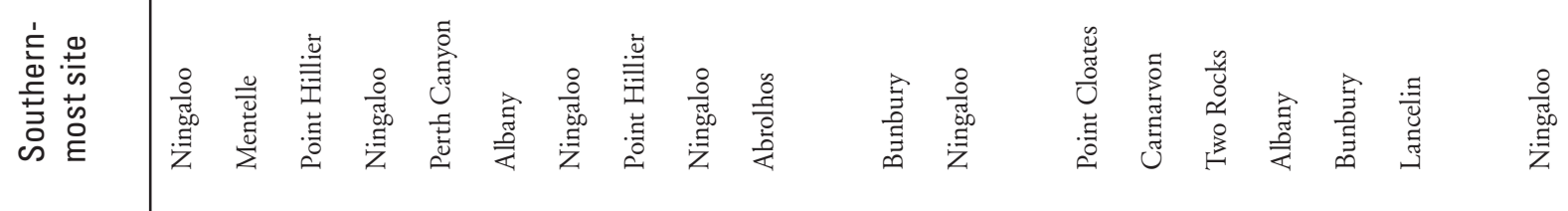

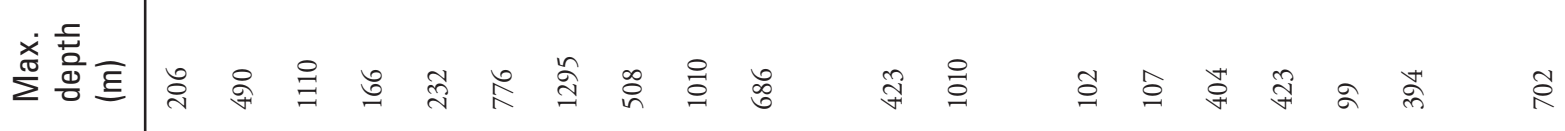

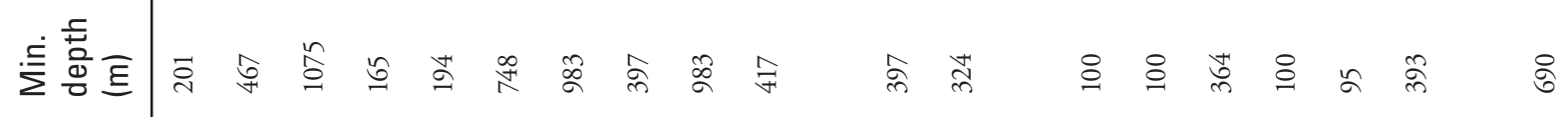

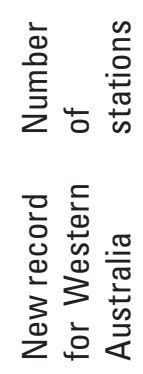

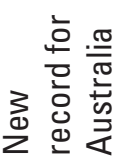

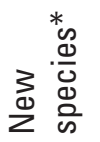

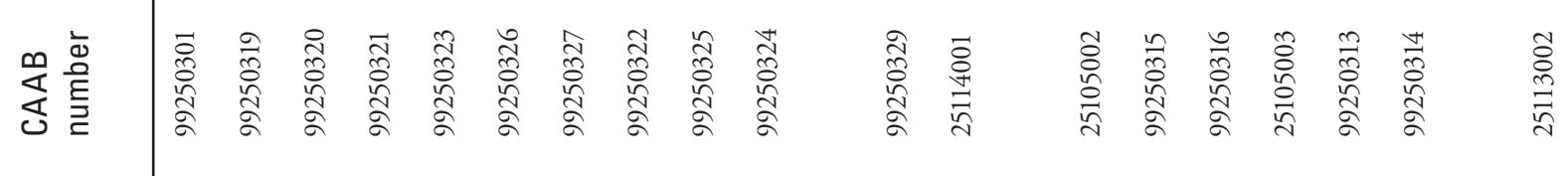

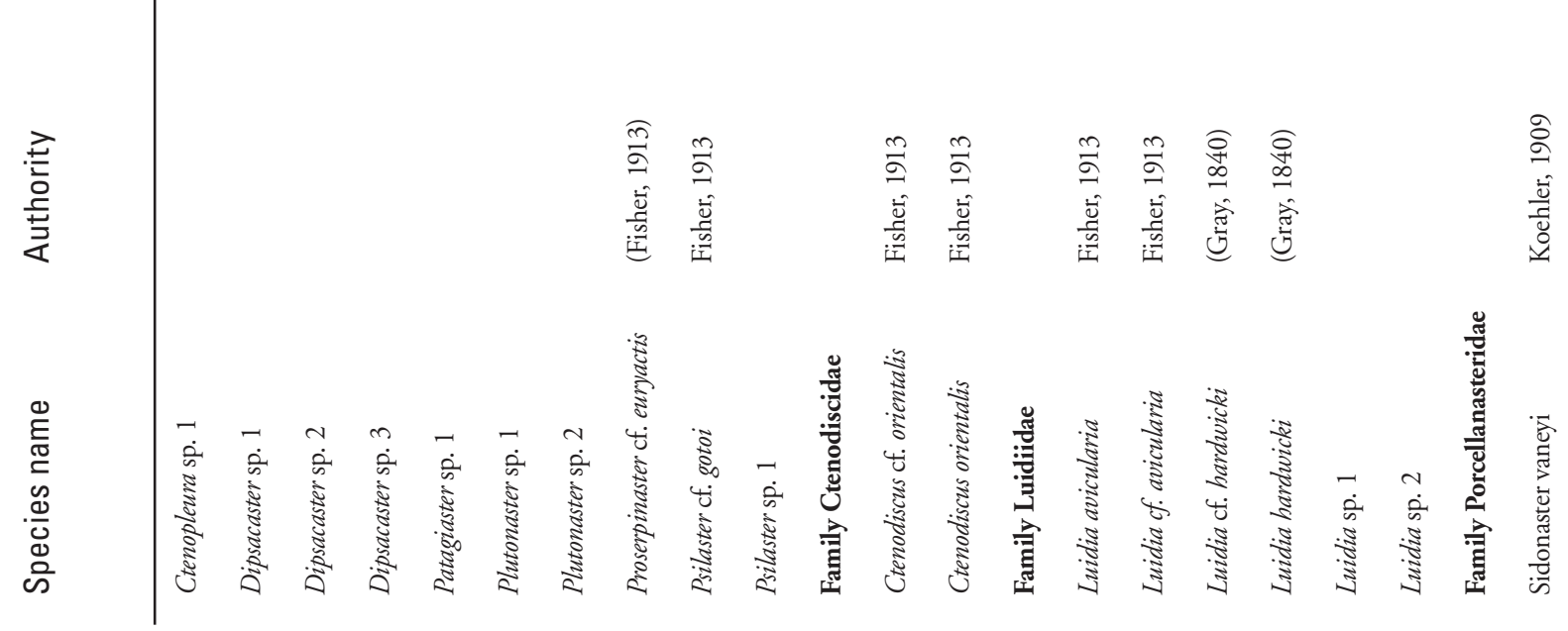




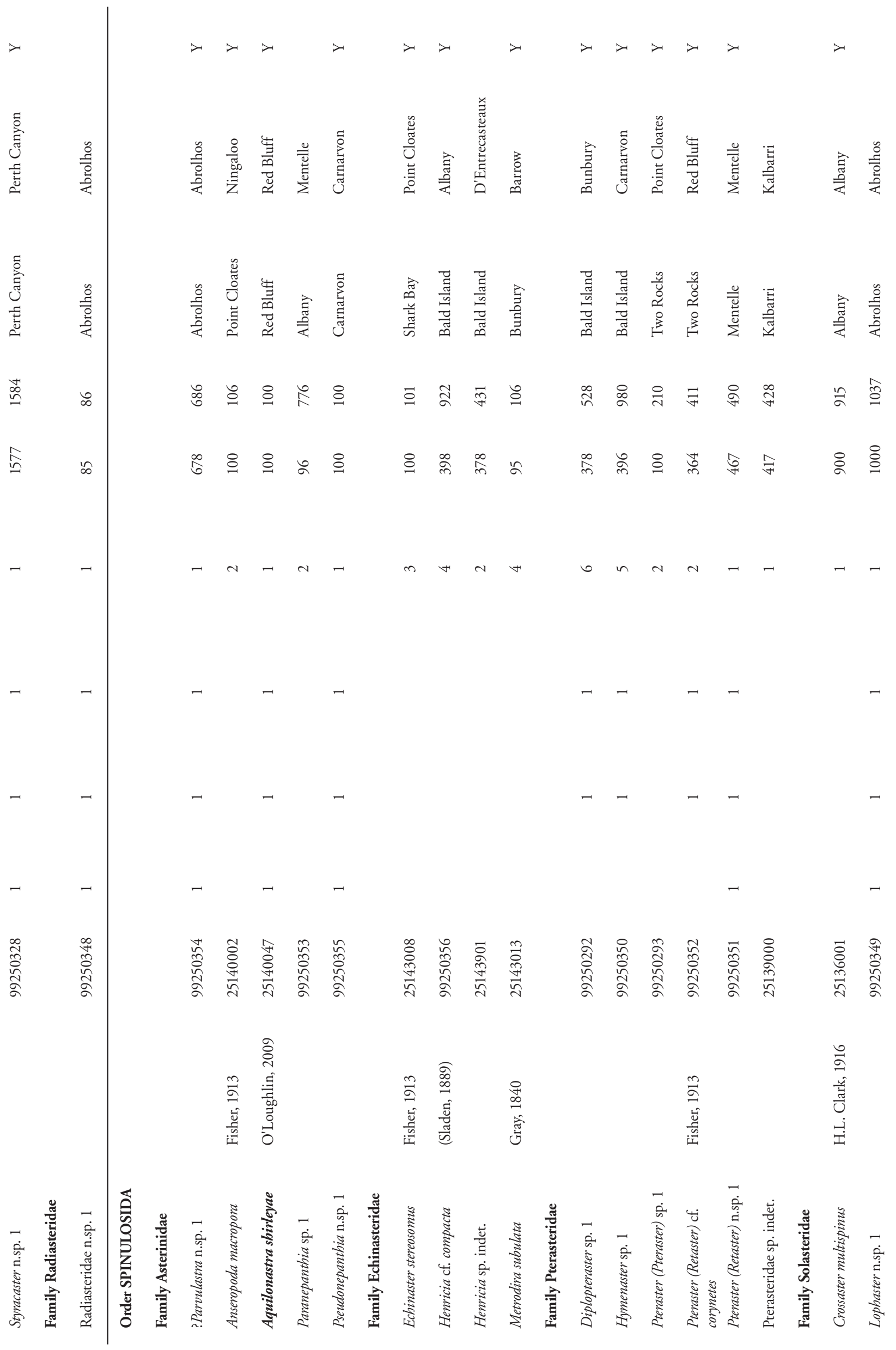




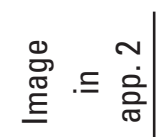

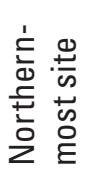

童:

离营馬

它营馬

离

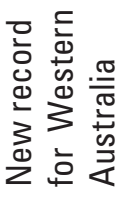

童亭高

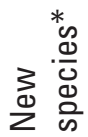

迡產

言

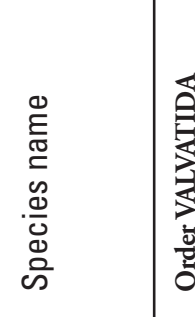

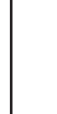

:

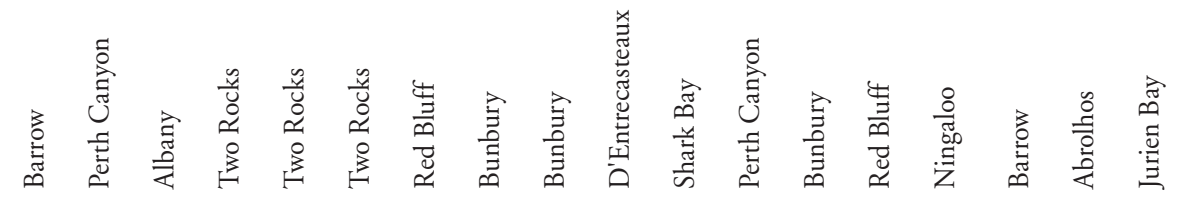

节

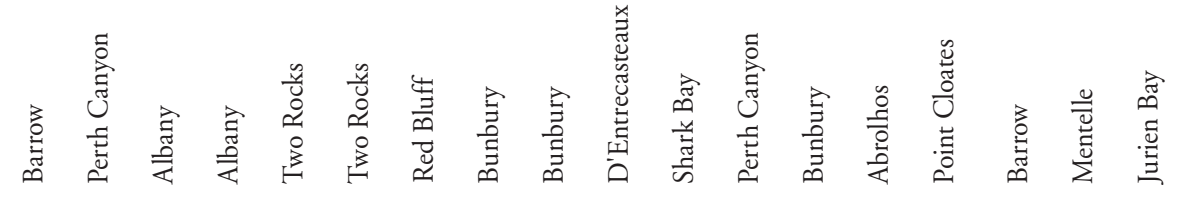

\&

承

مू

$\approx$

శ্

ธิ ฐ

ঞ

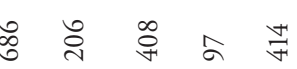

$@$

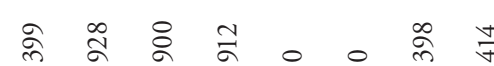

$+2$

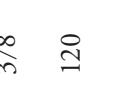

s

ळ

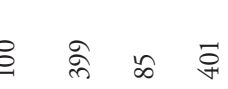

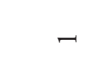

率

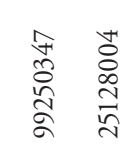

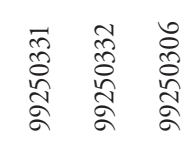

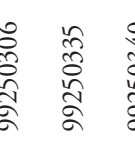

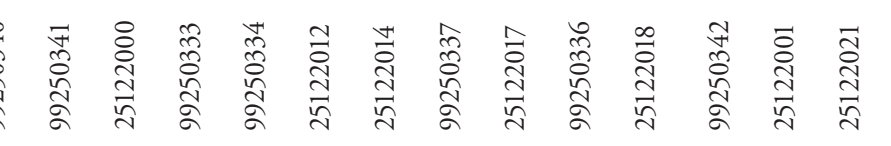

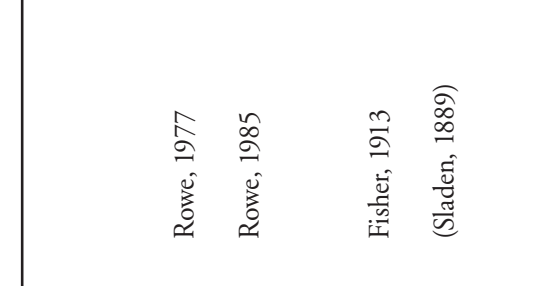

|

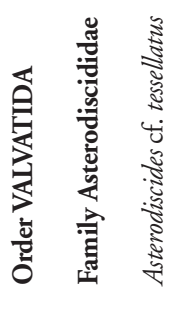

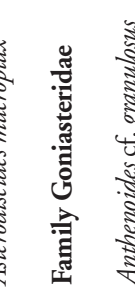

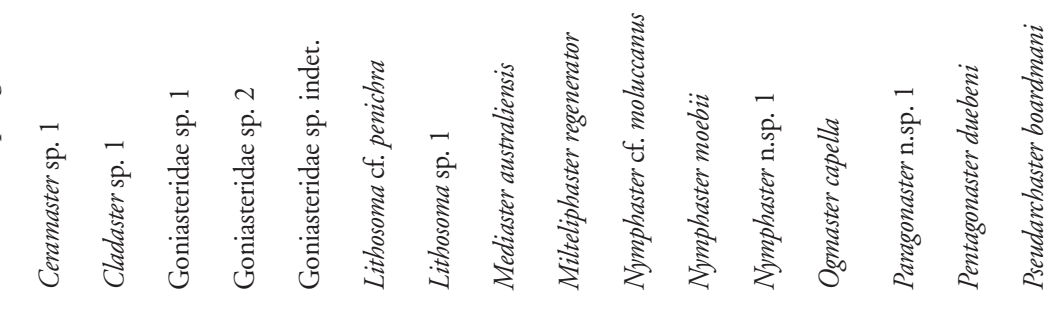




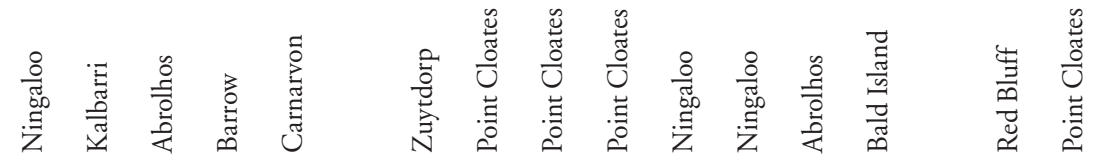

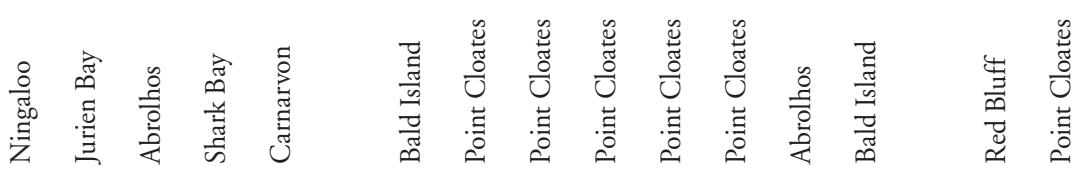

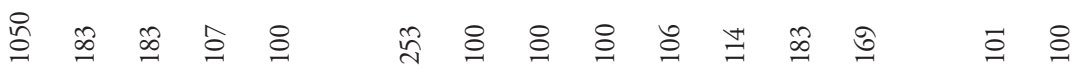

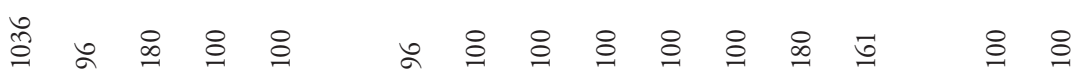

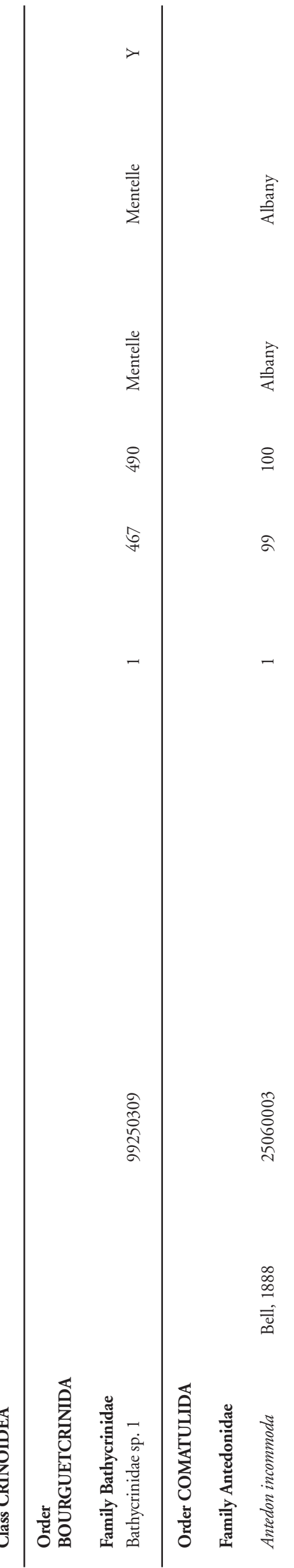

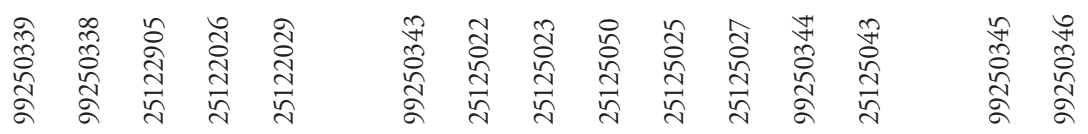

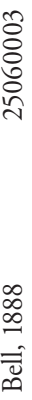

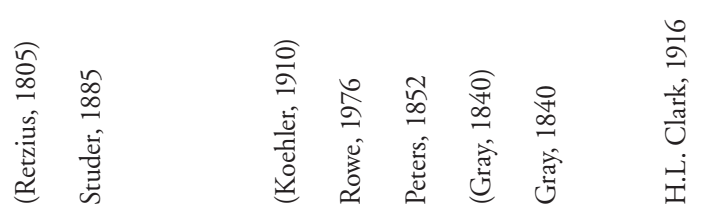

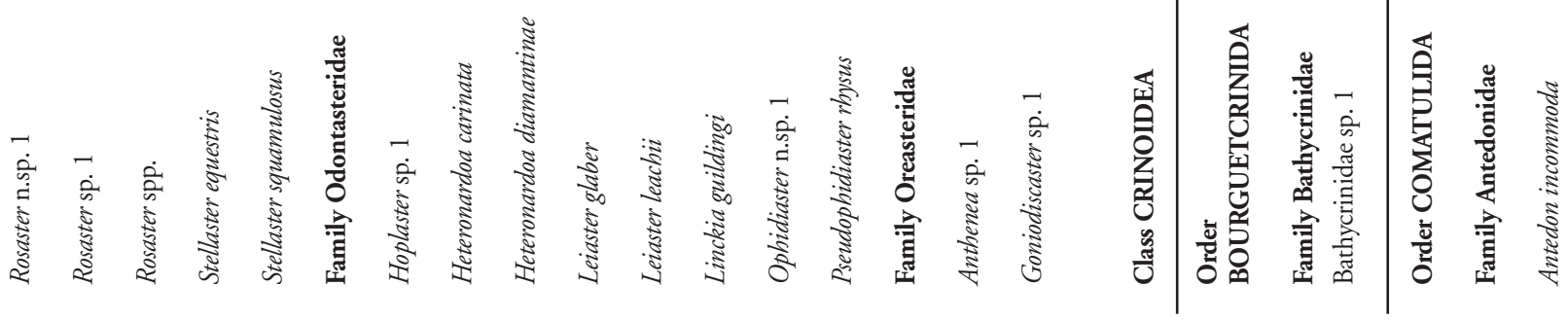




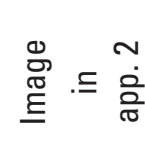

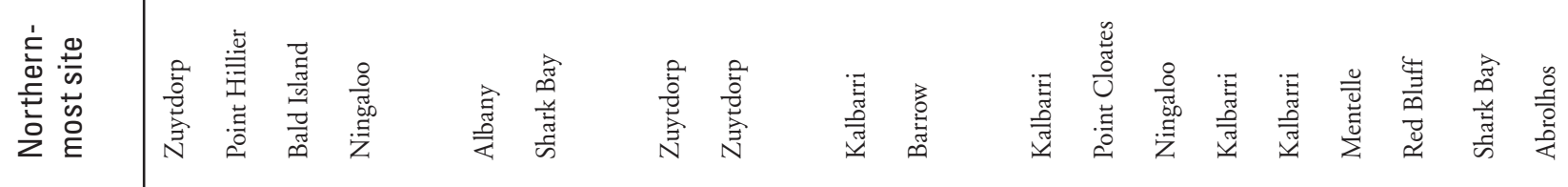

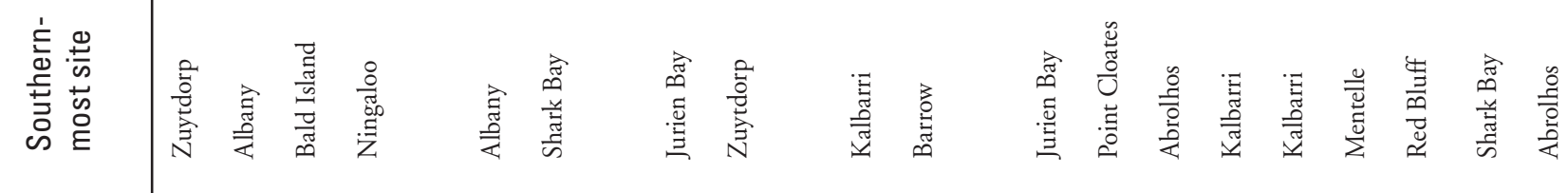

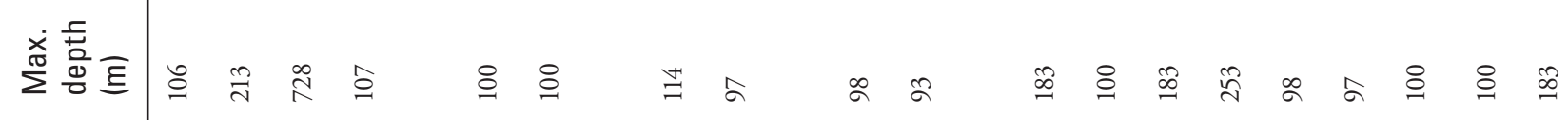
这营互

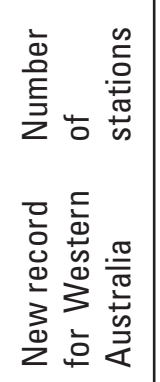

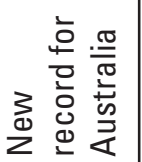

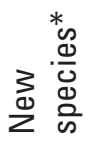

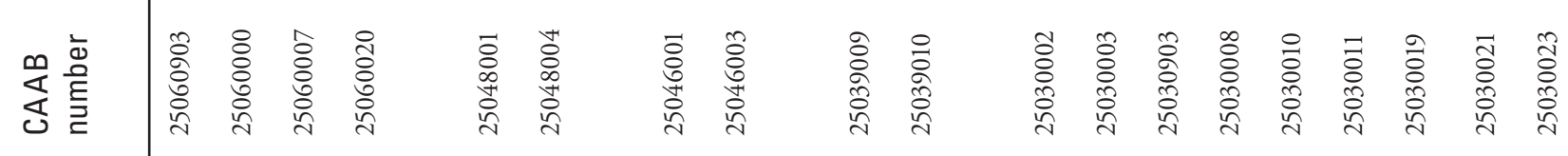

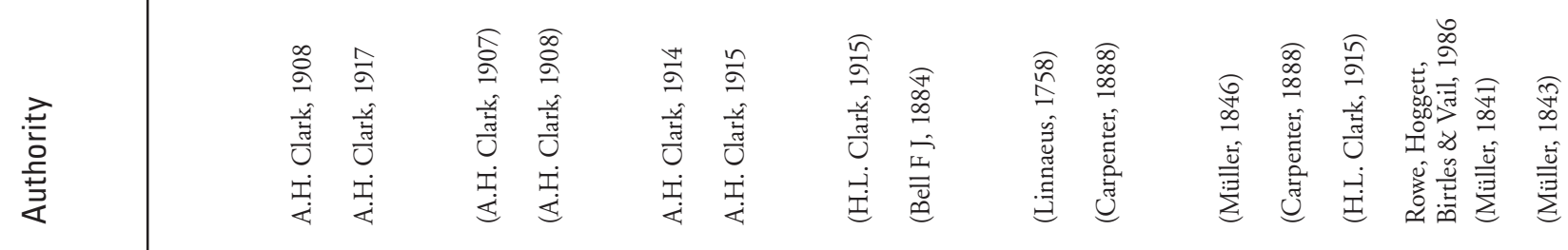

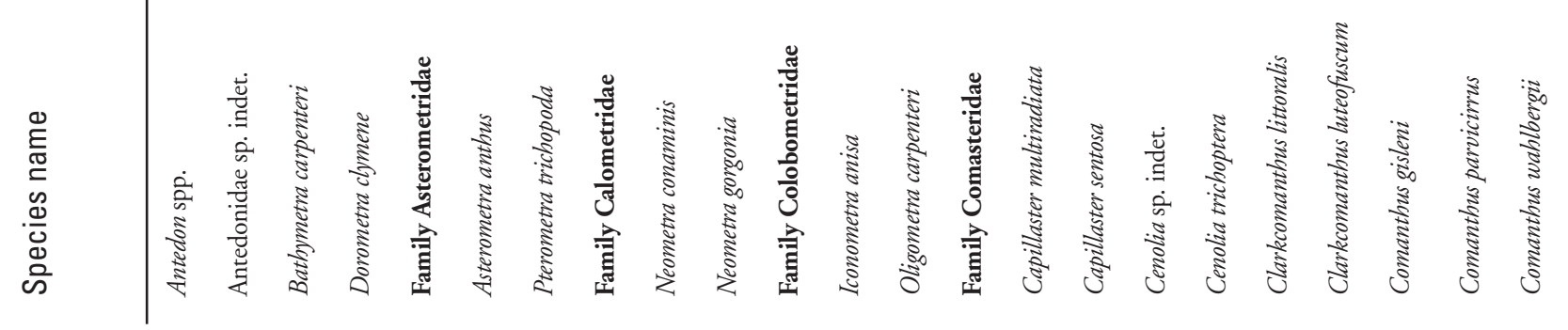




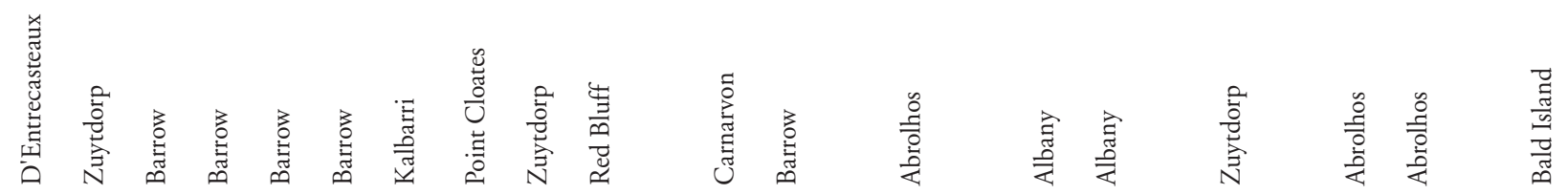

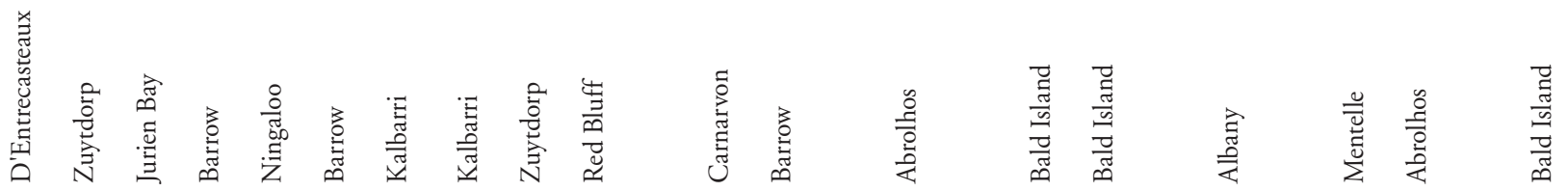
๙

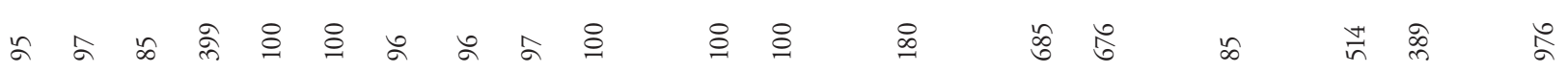

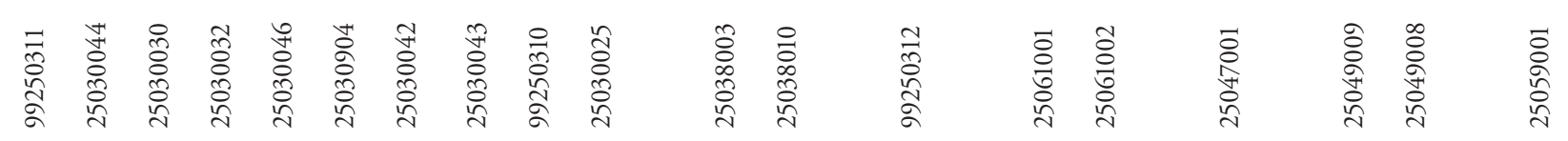

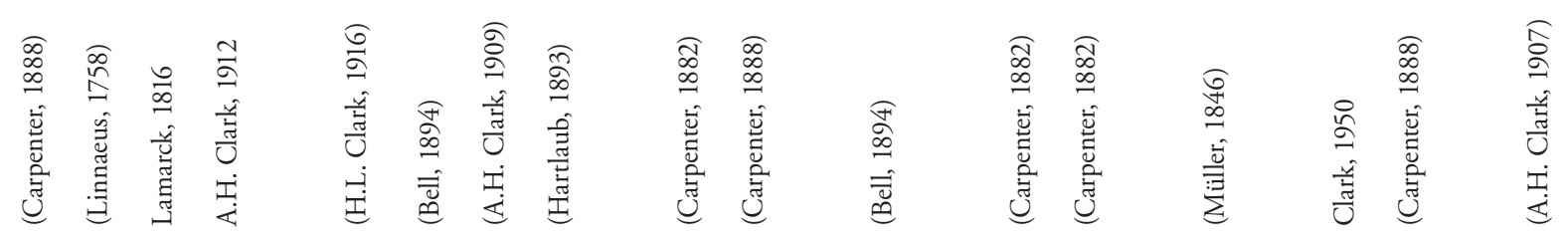

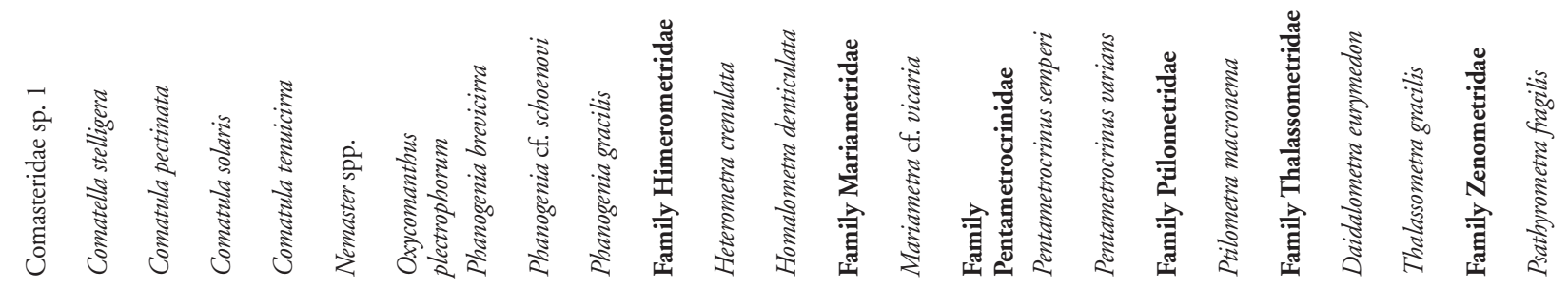




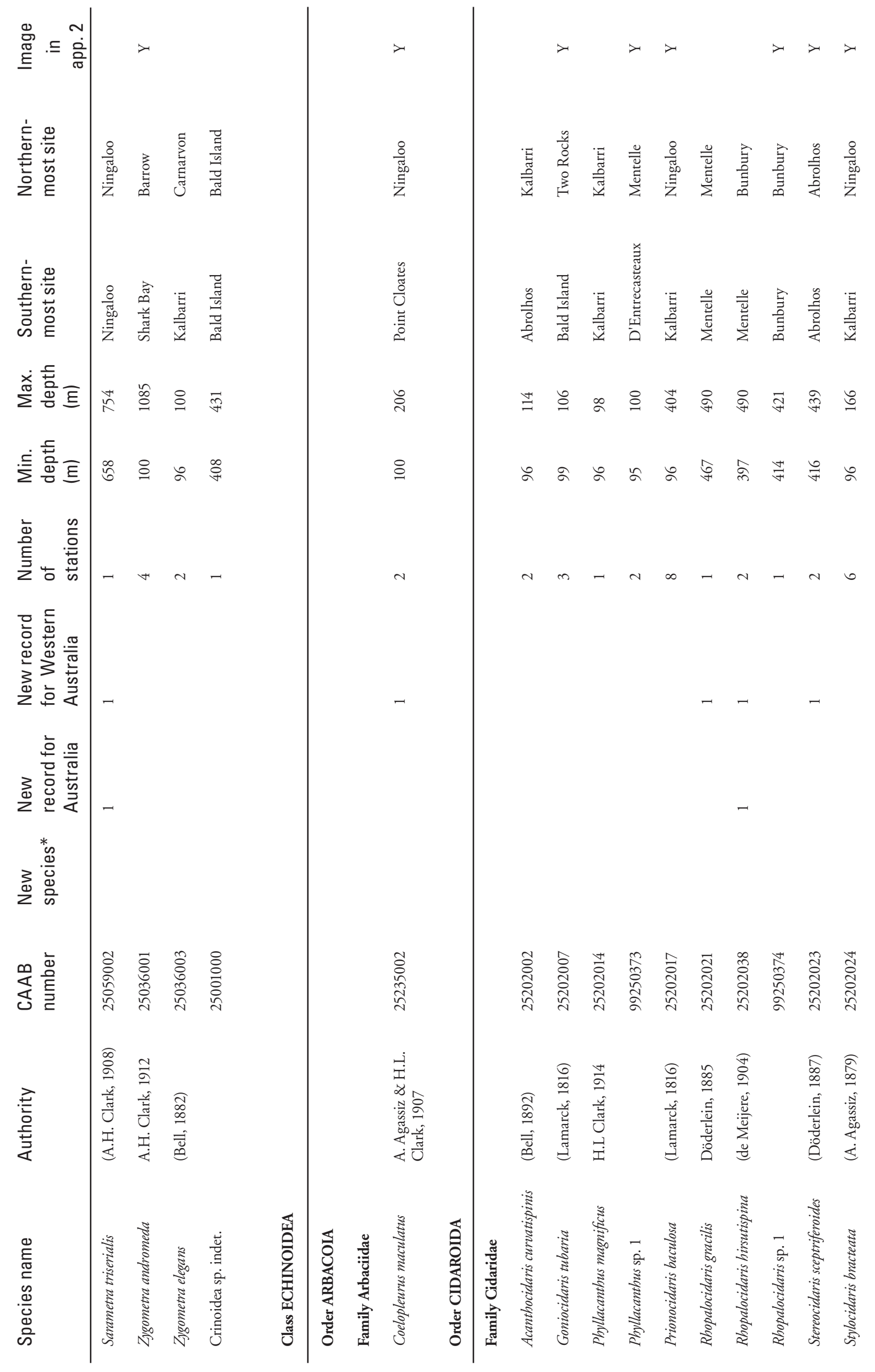




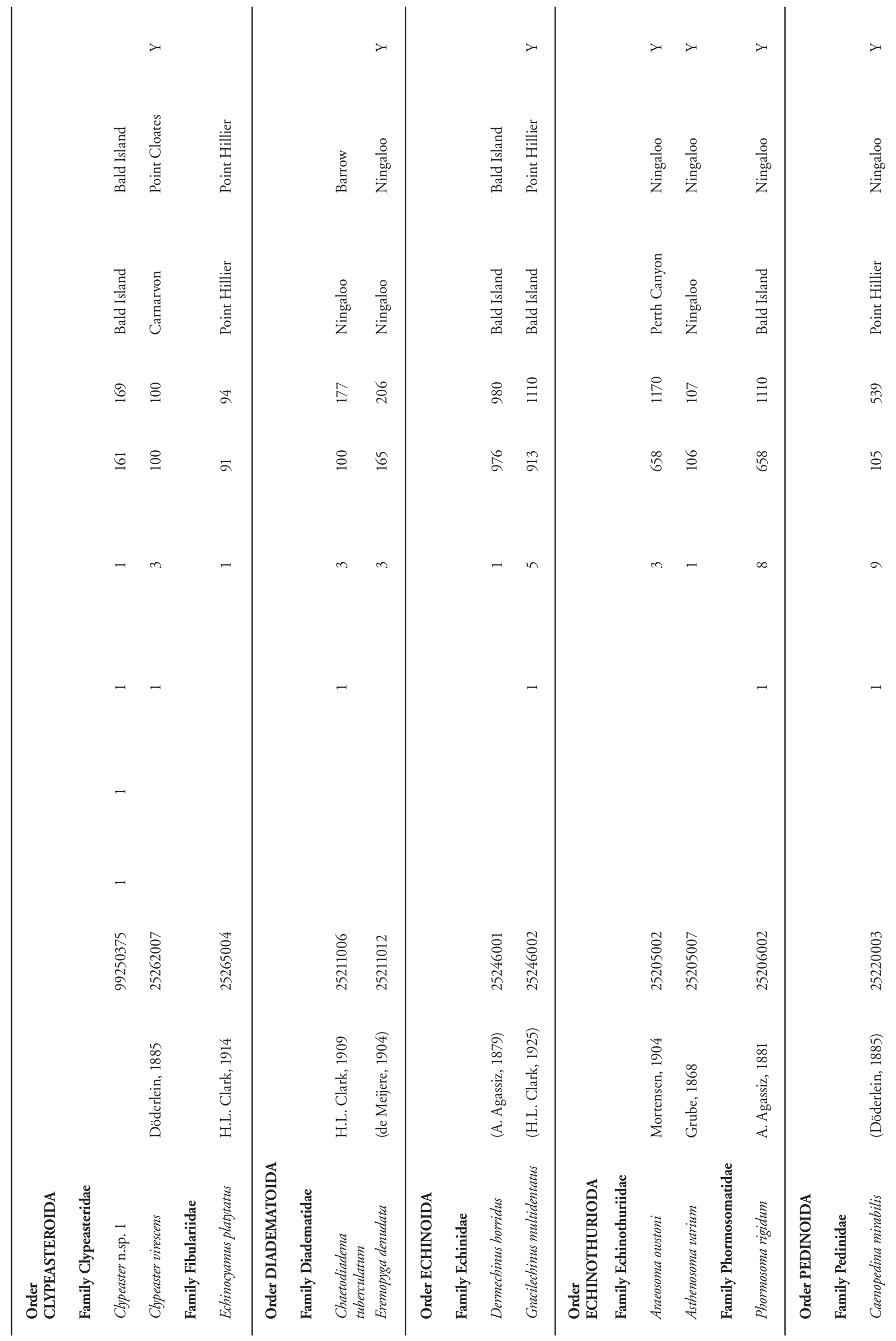




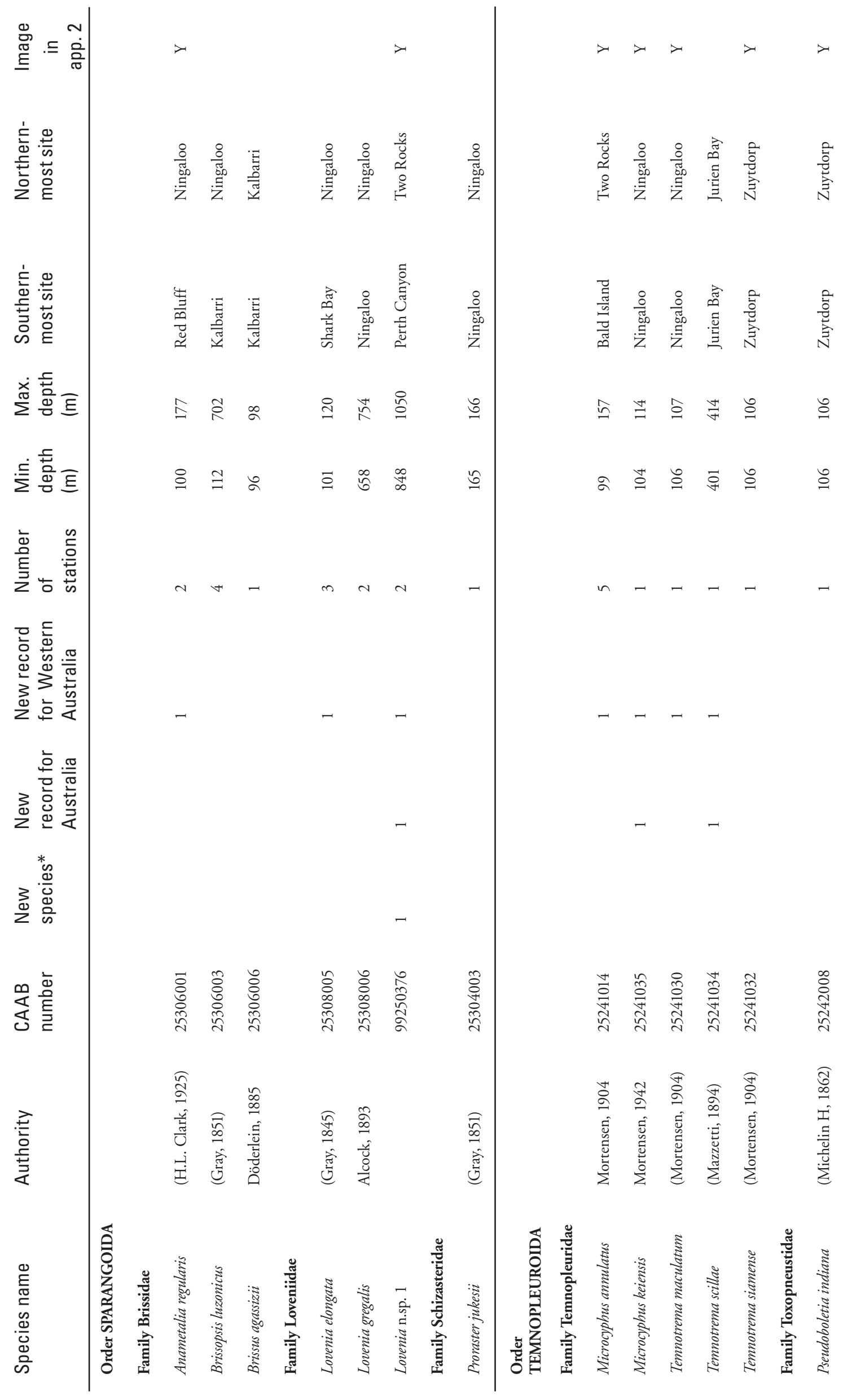




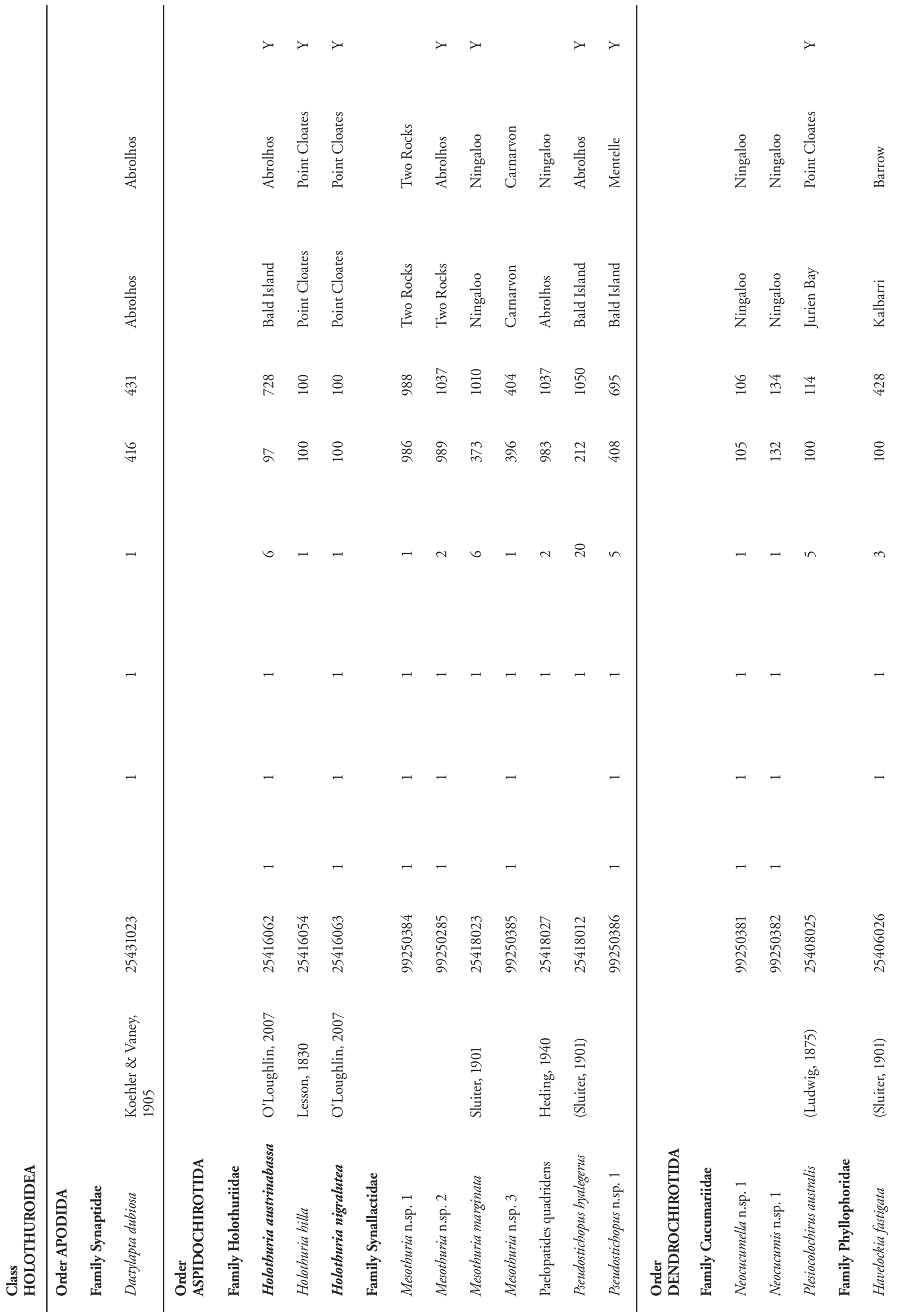




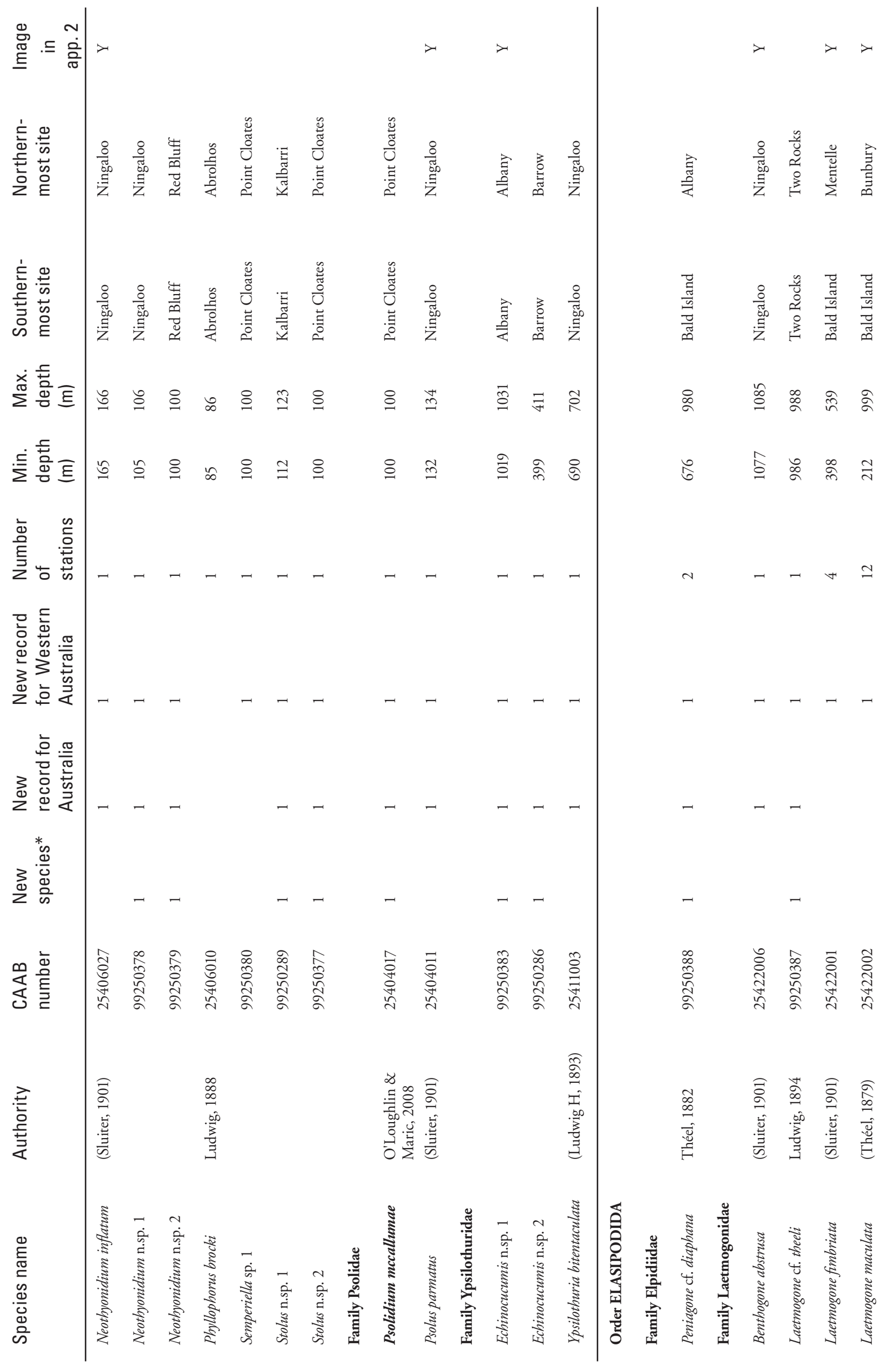




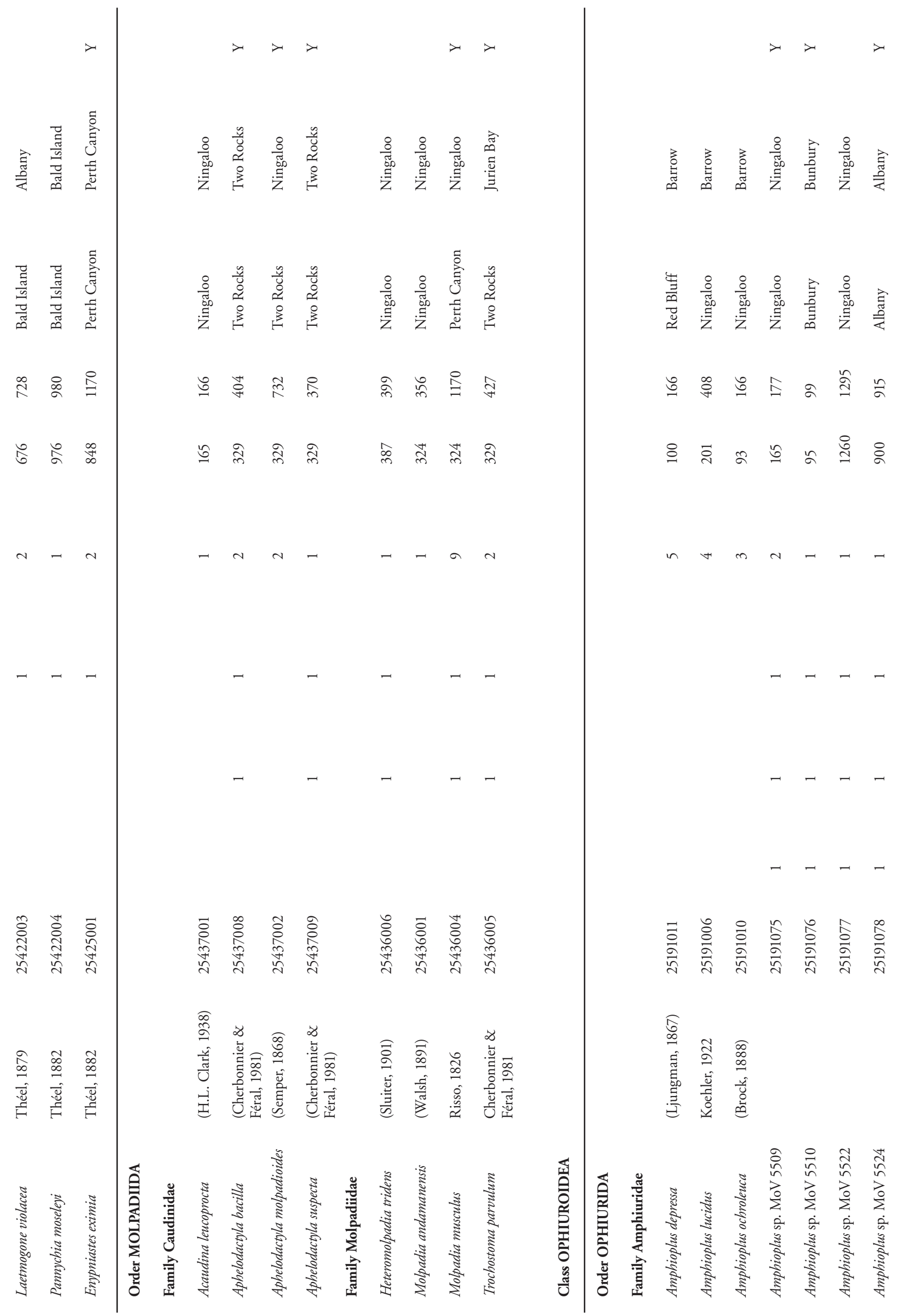




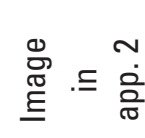

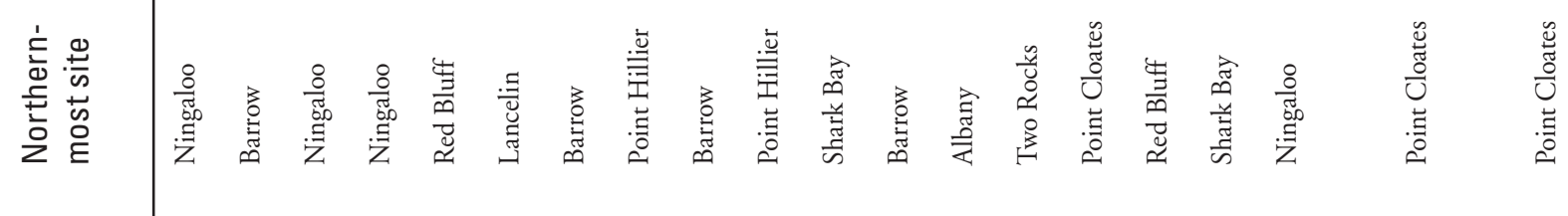

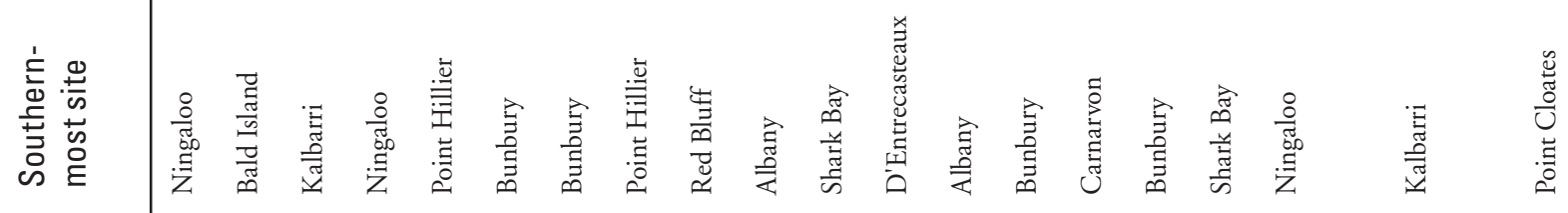

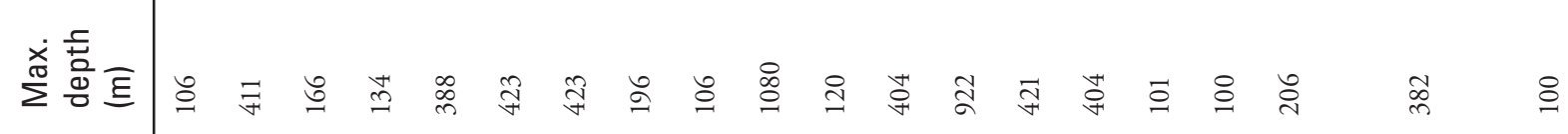

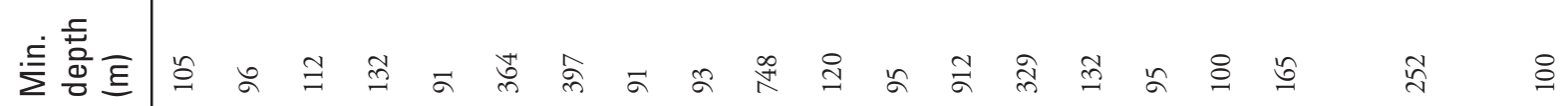

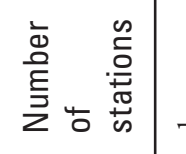

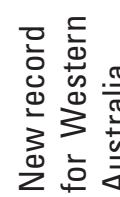

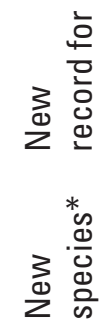

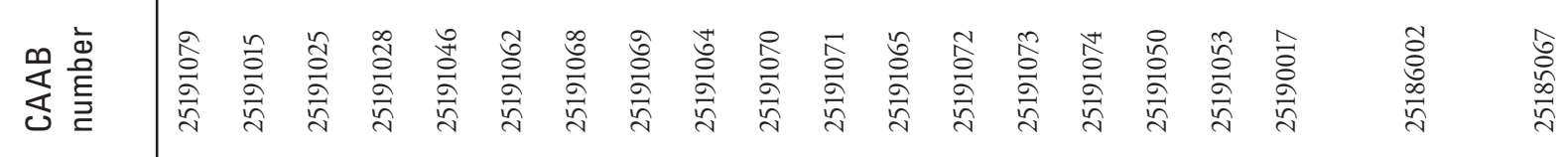

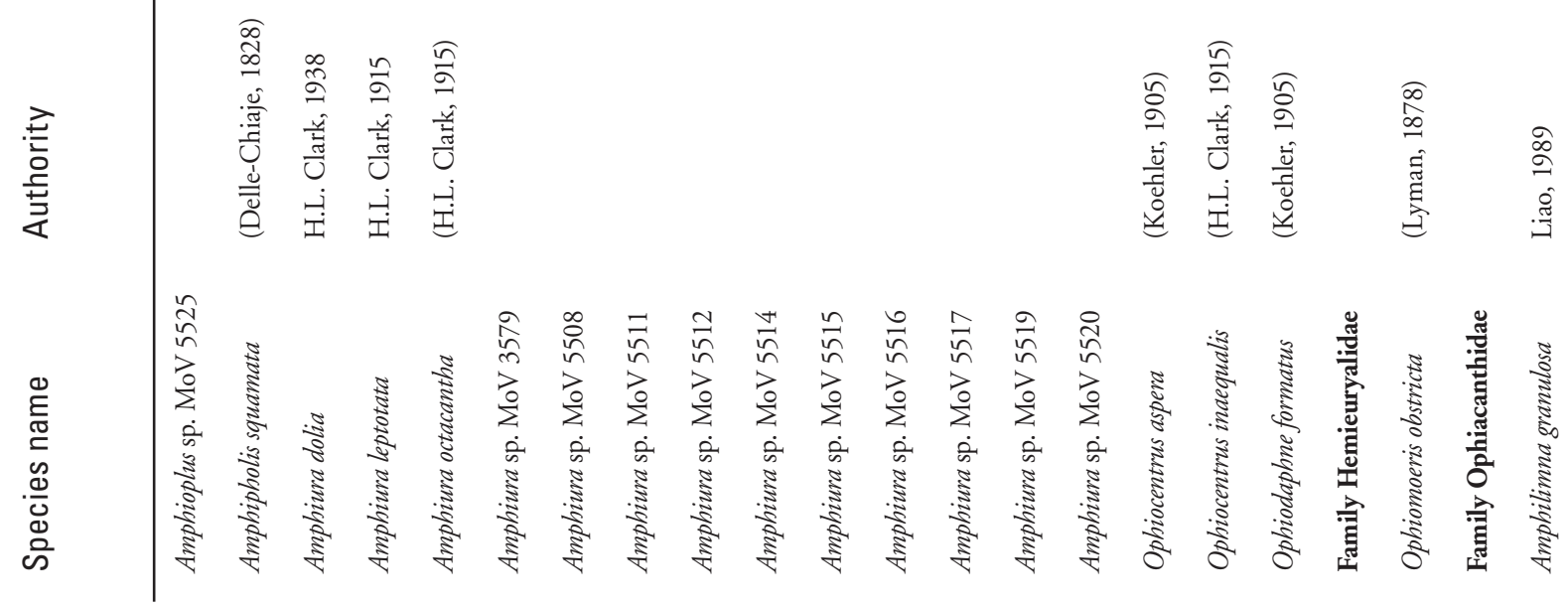




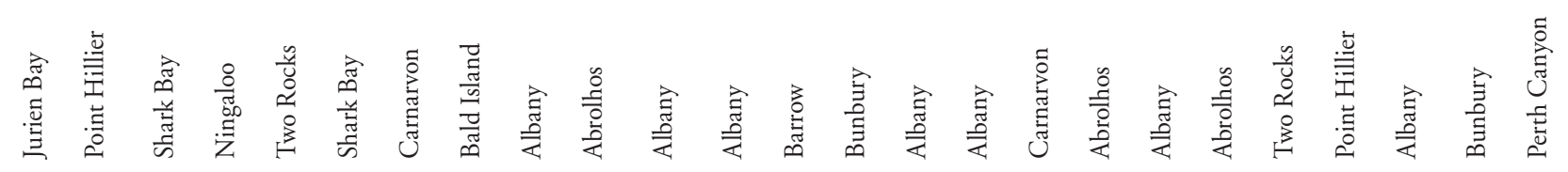

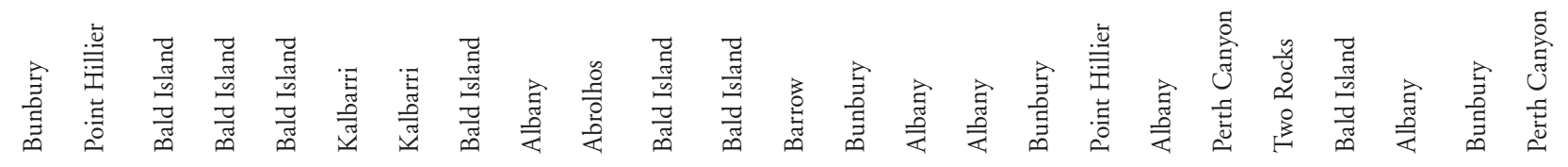

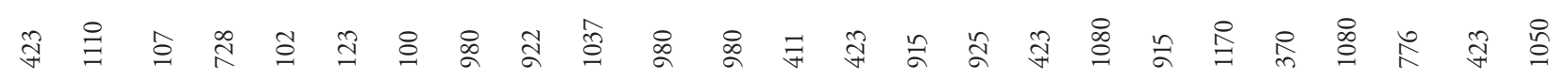

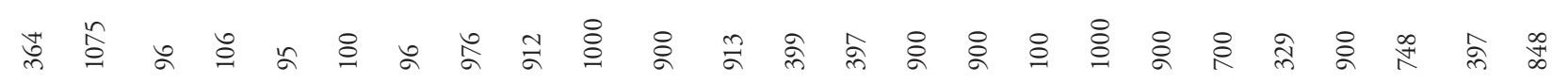

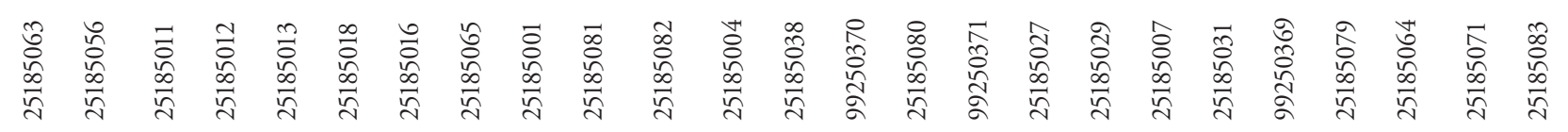

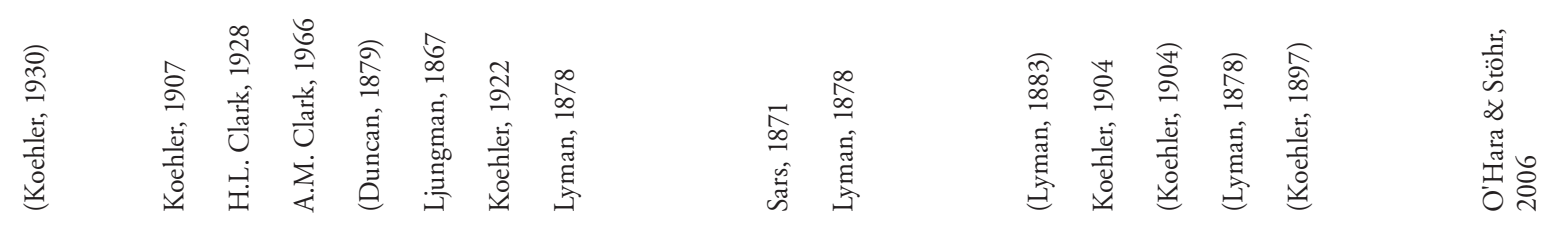

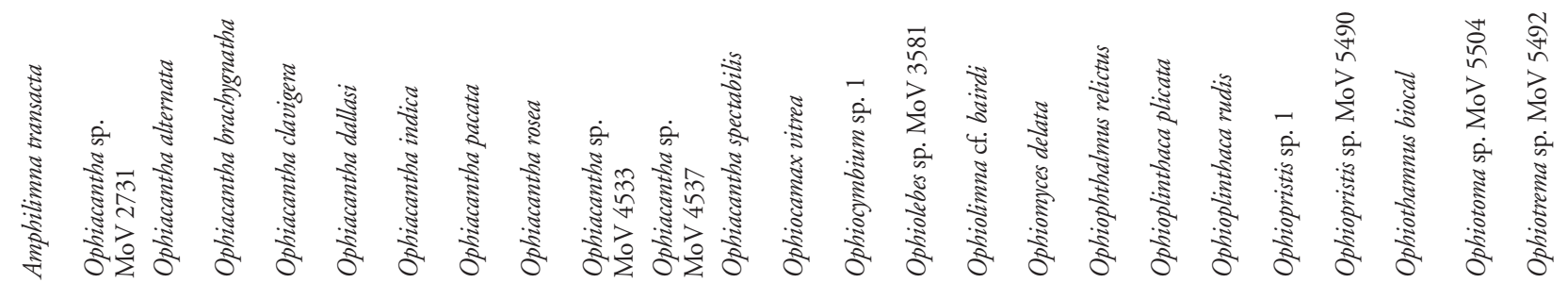




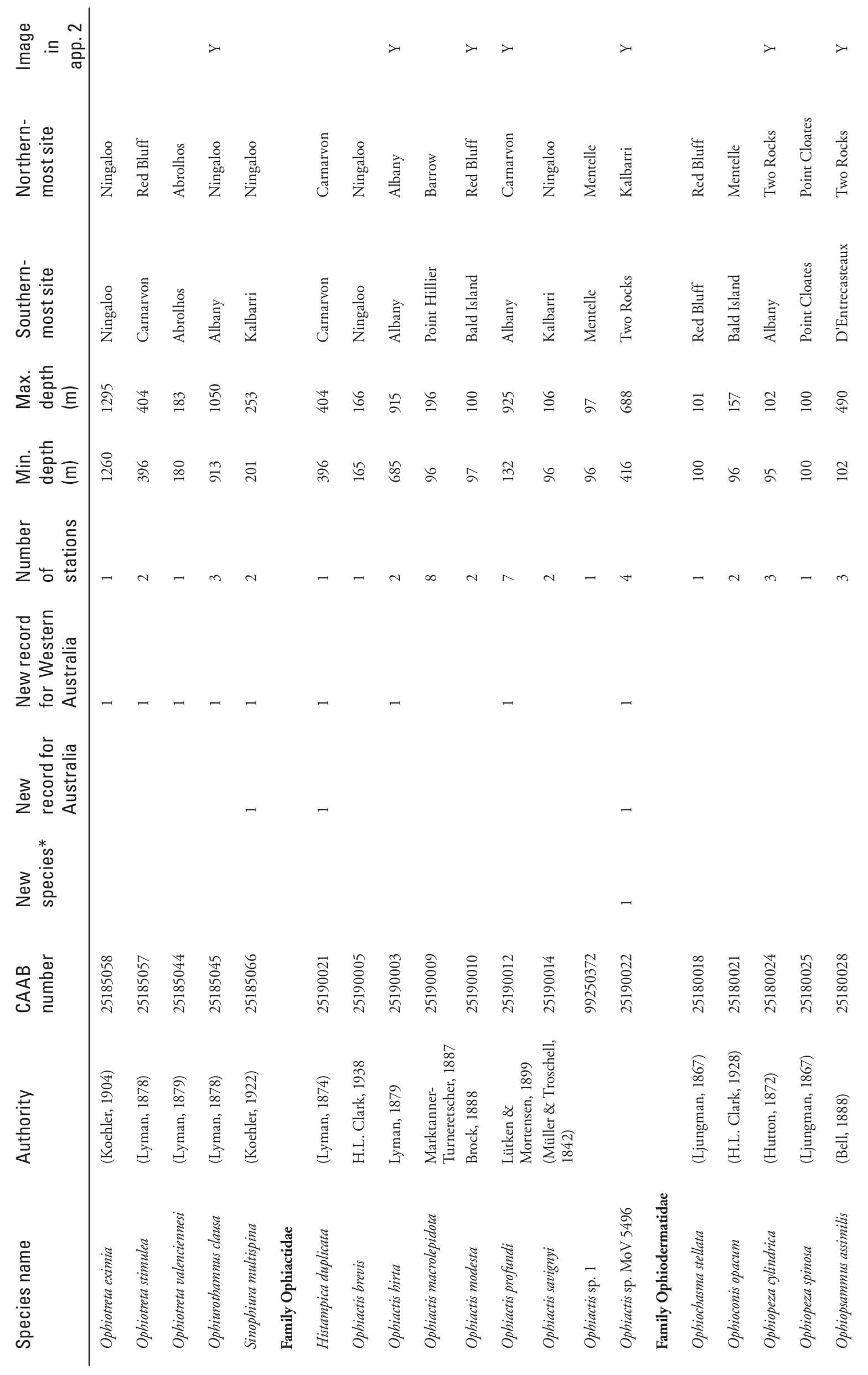




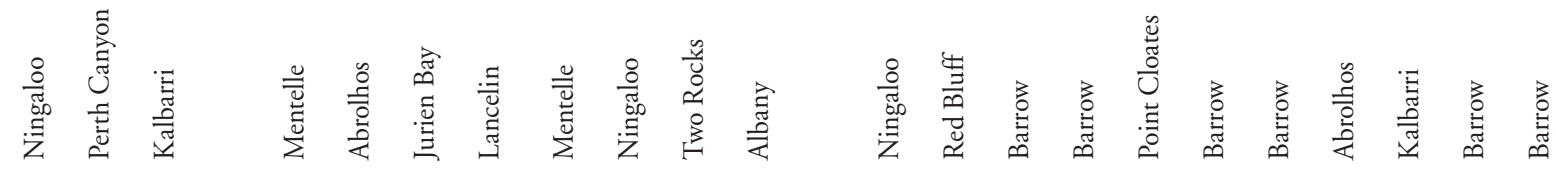

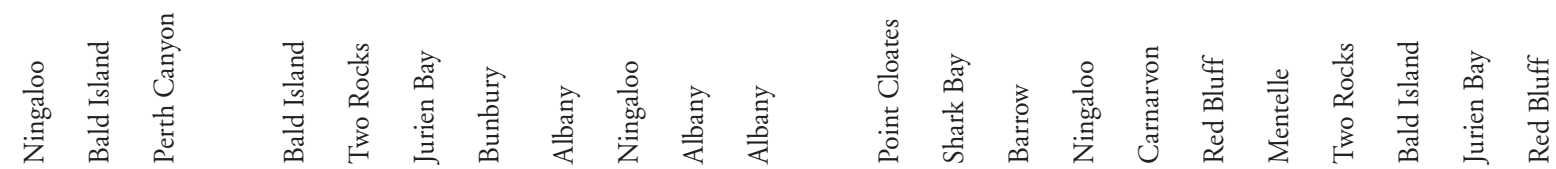

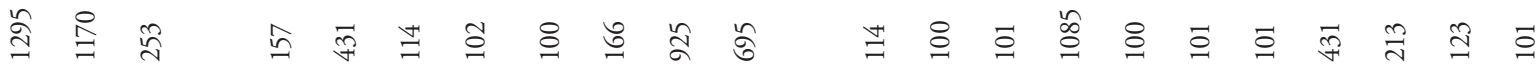

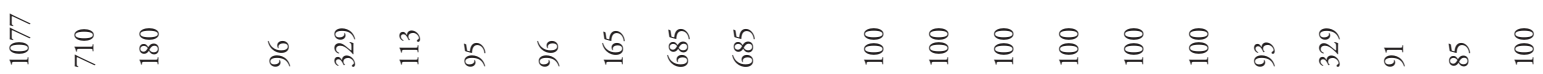

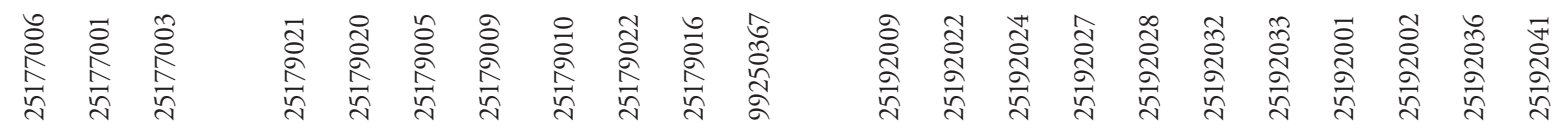

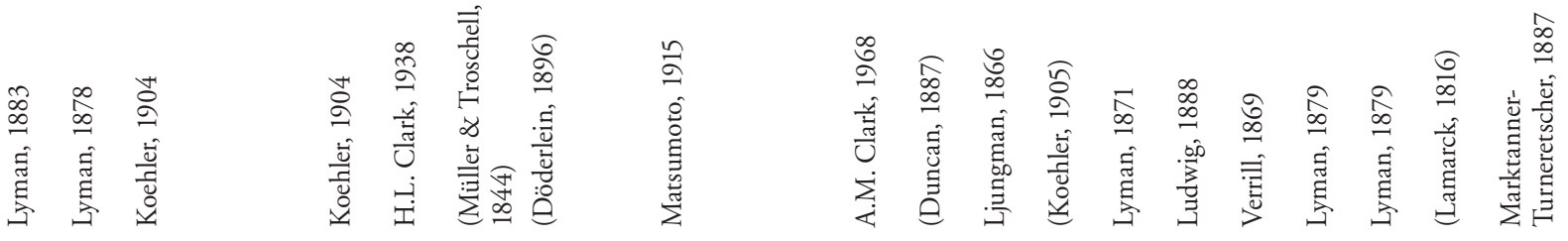

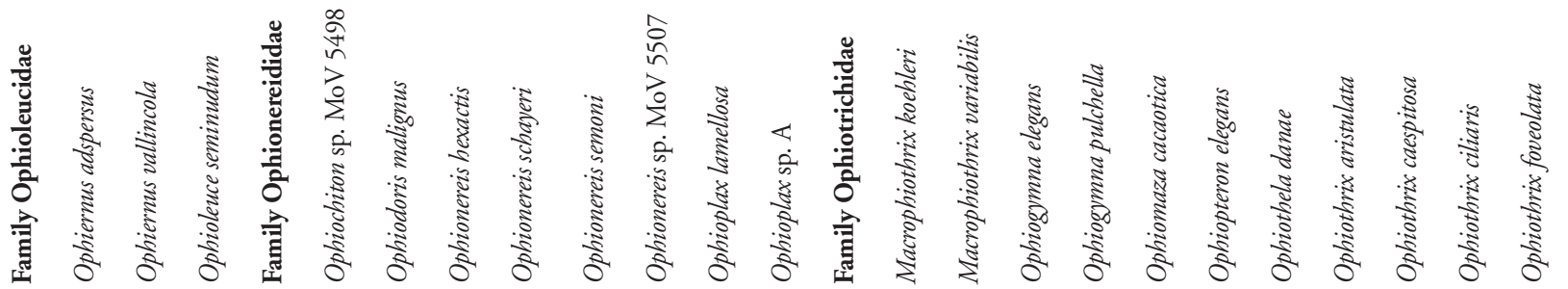




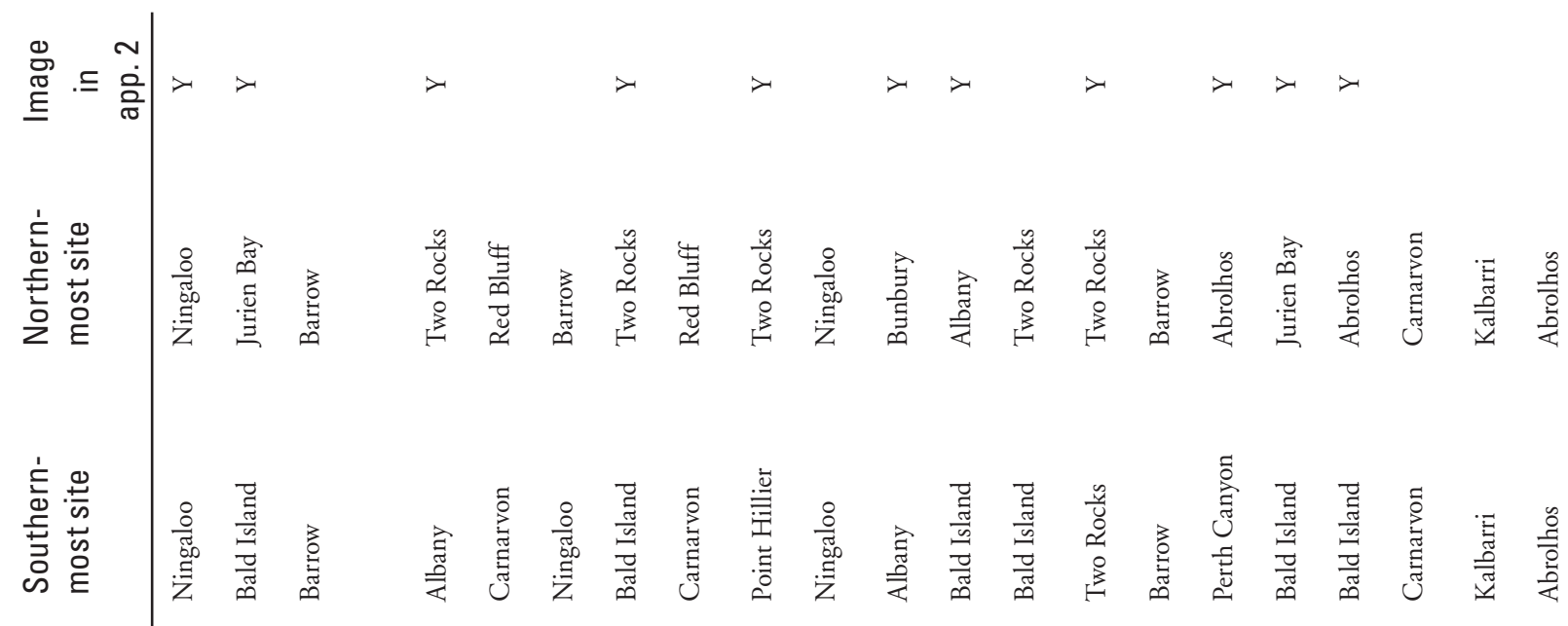
离蓄主

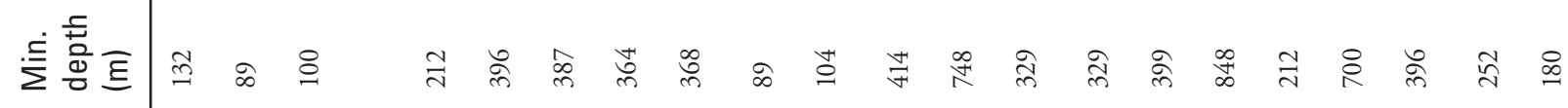

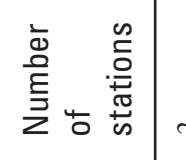

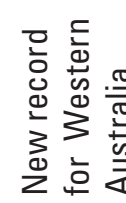

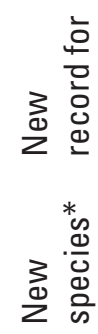

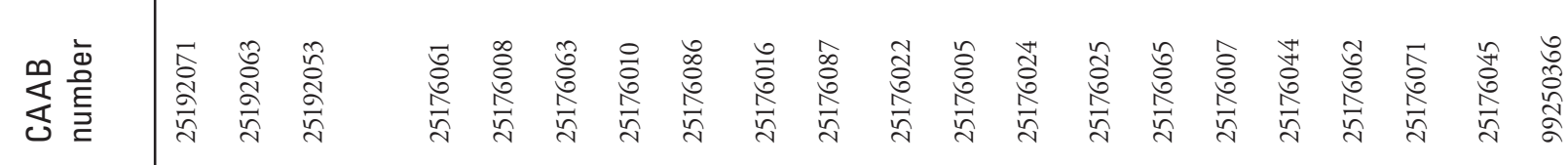

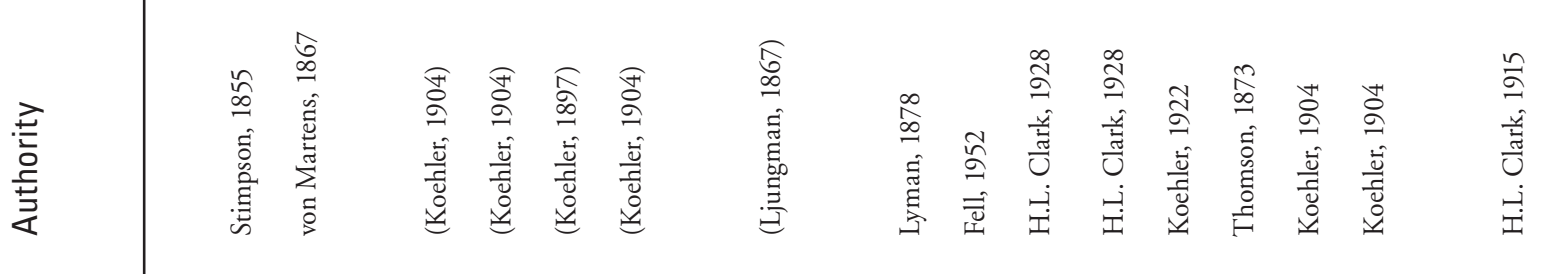

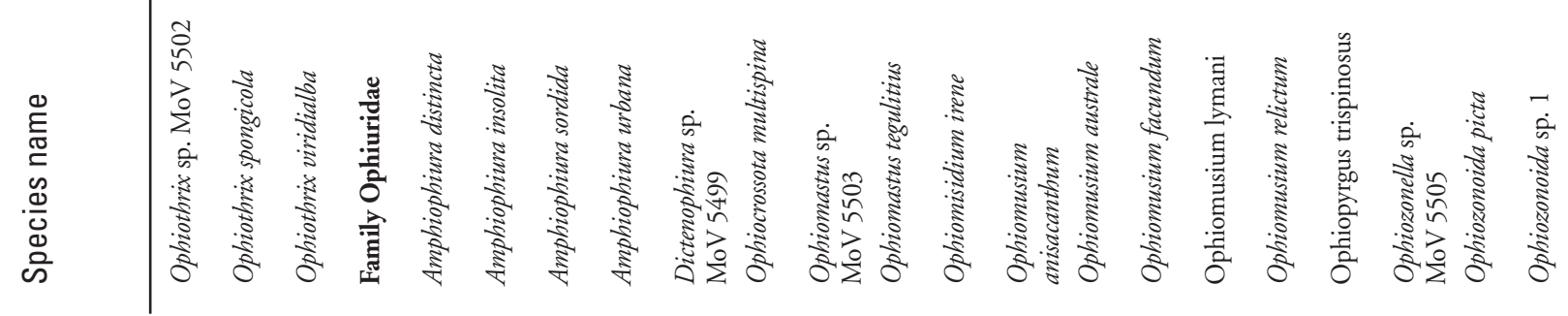




$$
\begin{aligned}
& \succ>>>\succ \succ
\end{aligned}
$$

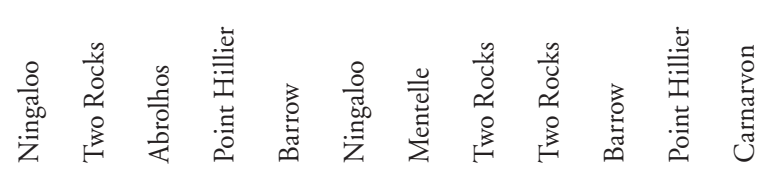

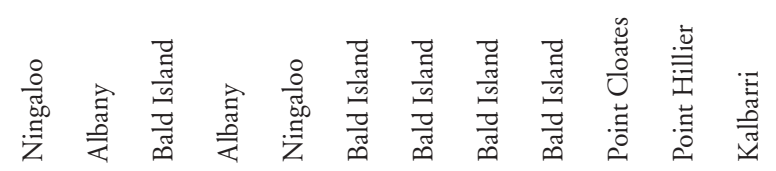

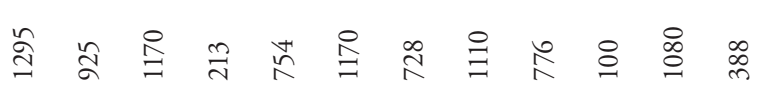

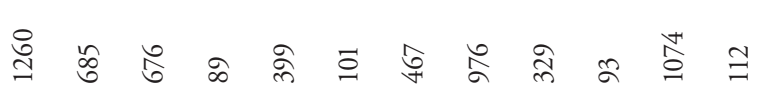

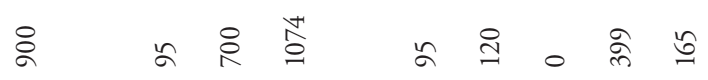

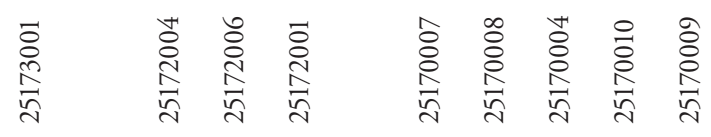

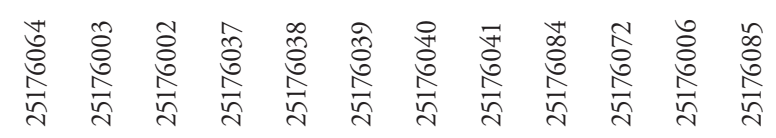

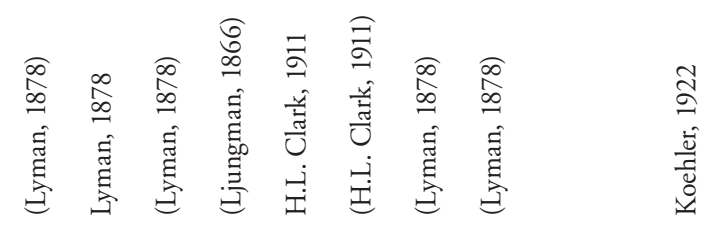

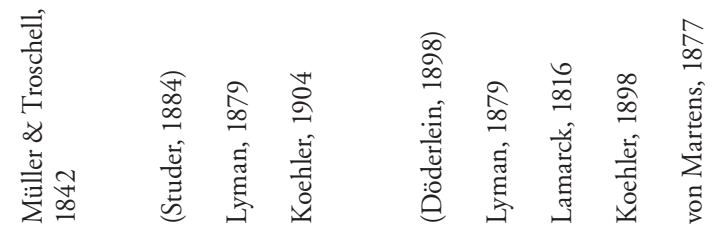

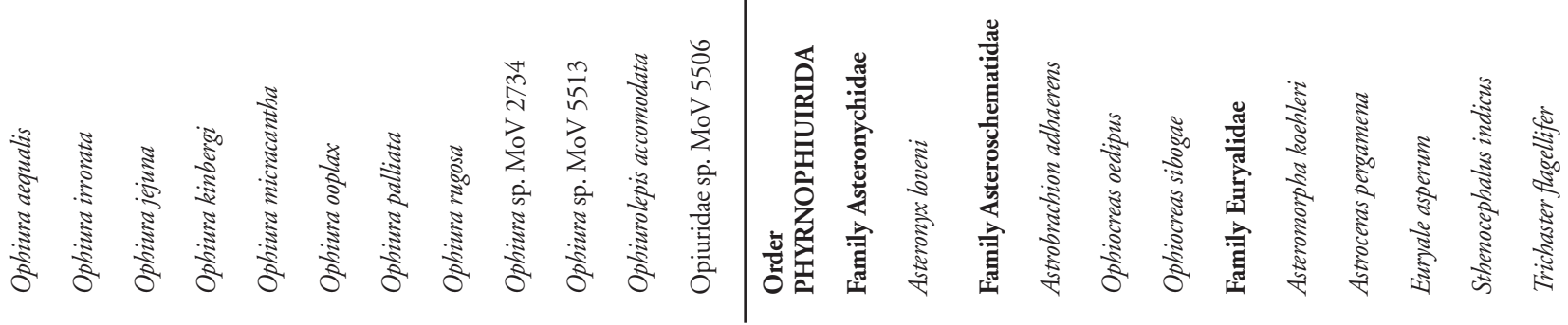




\begin{tabular}{|c|c|c|c|c|c|c|c|c|c|c|c|}
\hline 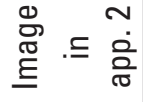 & $\lambda$ & $\lambda$ & $\lambda$ & $\lambda$ & $>$ & $\lambda$ & $>$ & $>$ & & $\succ$ & $\lambda$ \\
\hline 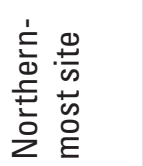 & 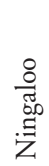 & $\begin{array}{l}\text { o } \\
\stackrel{0}{0} \\
\text { : }\end{array}$ & 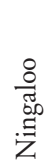 & 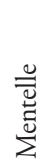 & 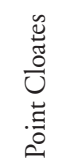 & 䓂 & 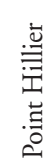 & 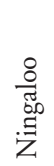 & 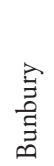 & 密 & $\begin{array}{l}\tilde{y} \\
0 \\
0 \\
0 \\
3\end{array}$ \\
\hline 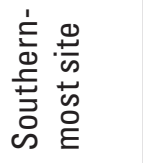 & 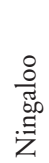 & 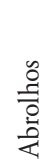 & 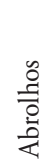 & 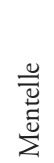 & 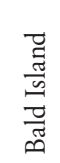 & 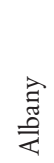 & 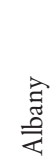 & $\begin{array}{l}\stackrel{8}{\circ} \\
\stackrel{5}{\pi} \\
\stackrel{5}{Z}\end{array}$ & 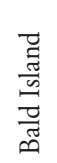 & 宓 & 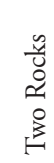 \\
\hline
\end{tabular}

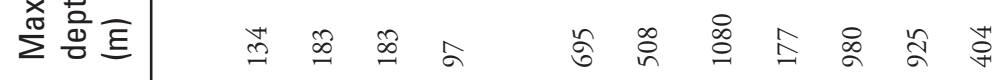

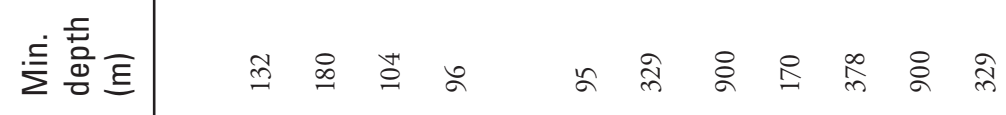

$\underset{\substack{0 \\: 0}}{\stackrel{0}{\circ}}$

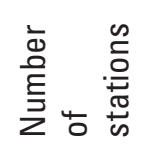

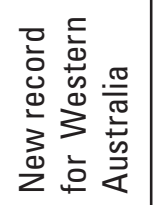

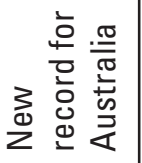

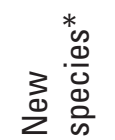

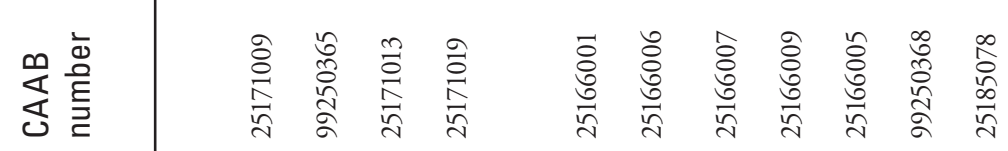

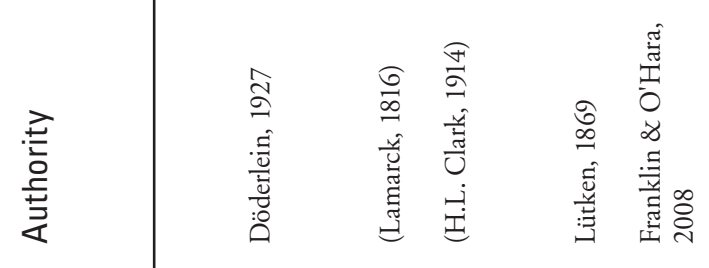

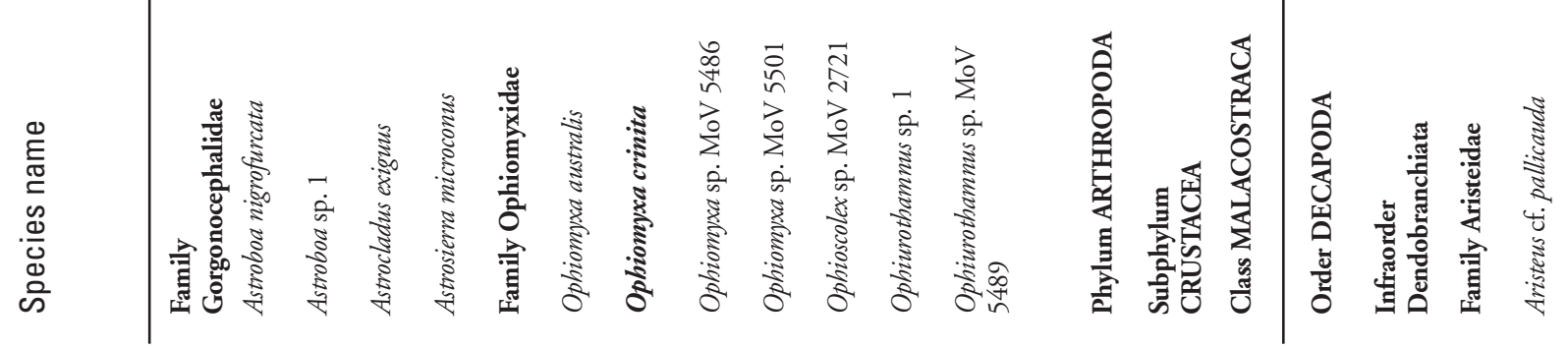




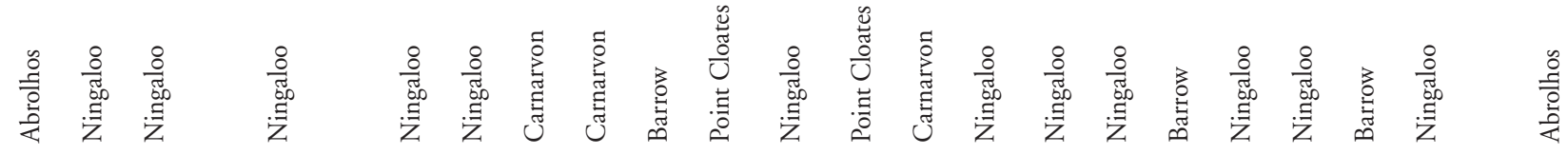

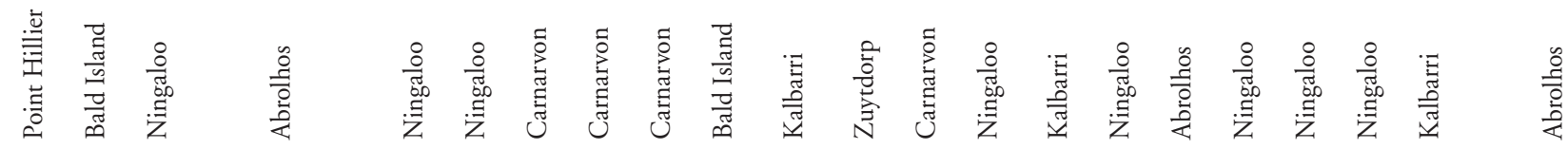

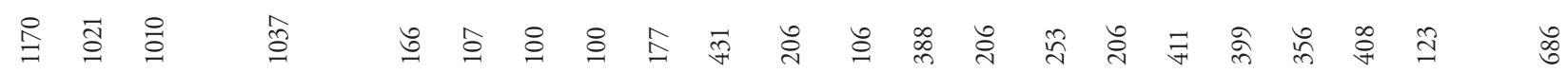

హ్

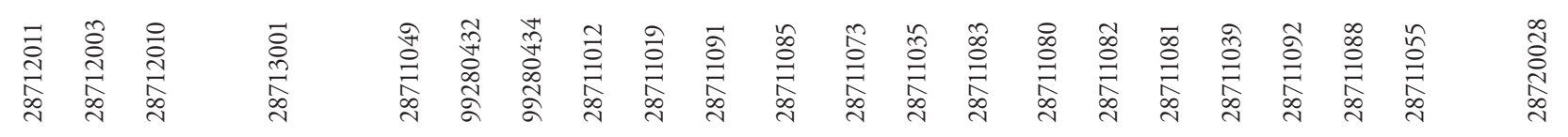

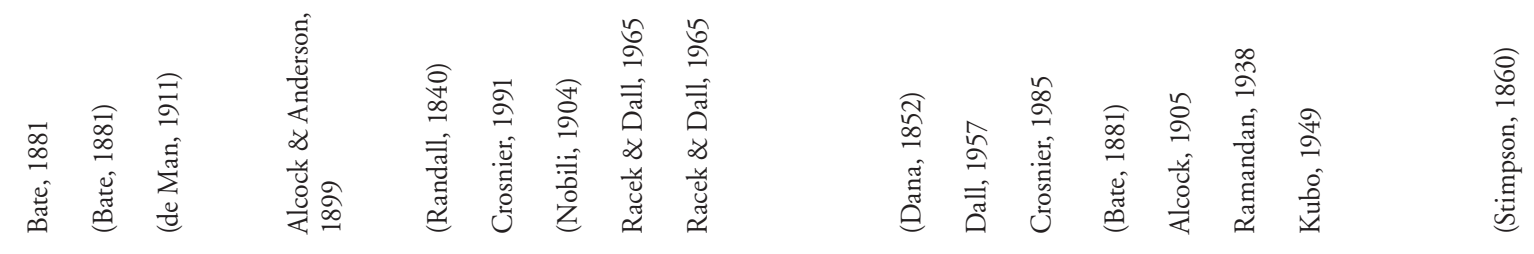

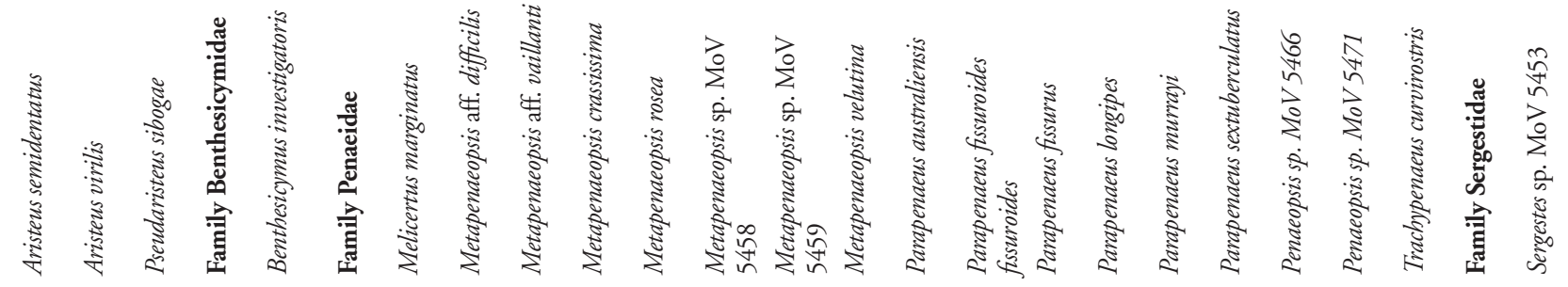




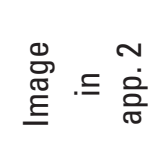

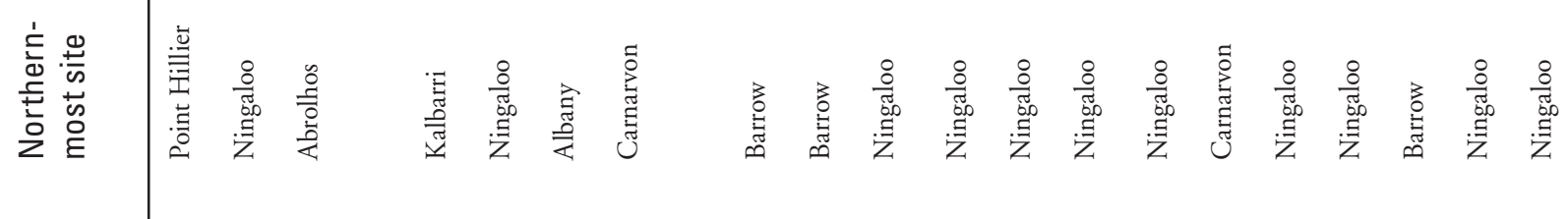

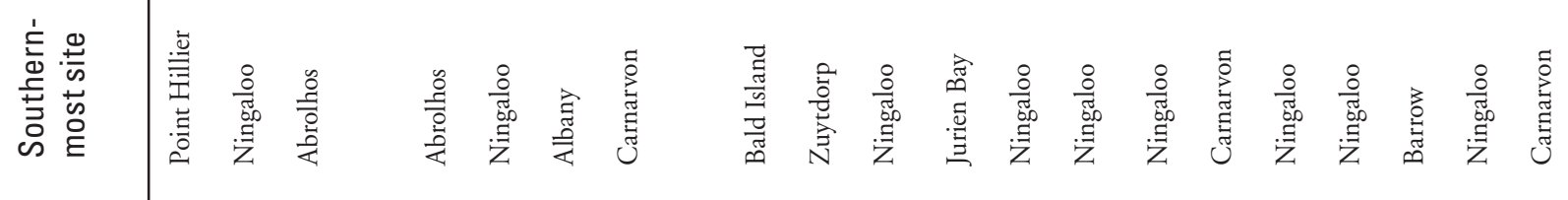

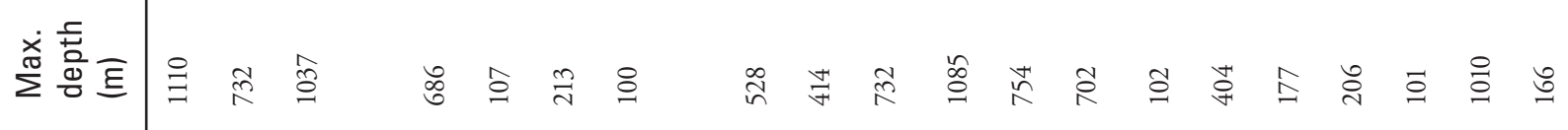

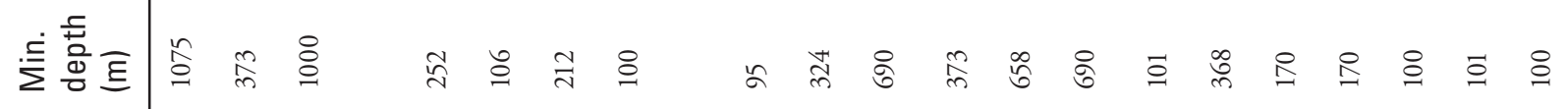

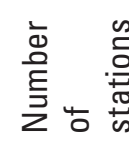

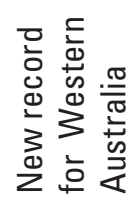

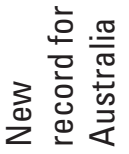

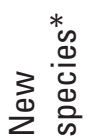

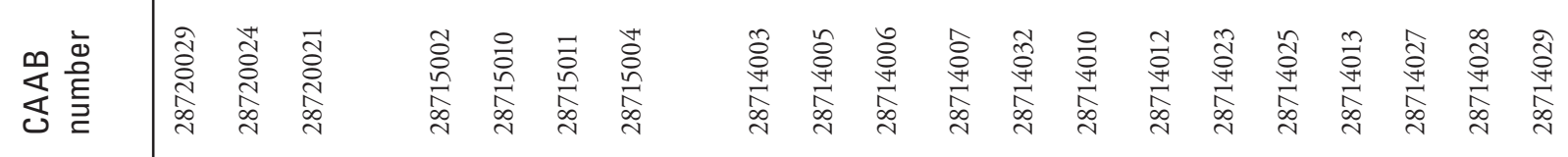

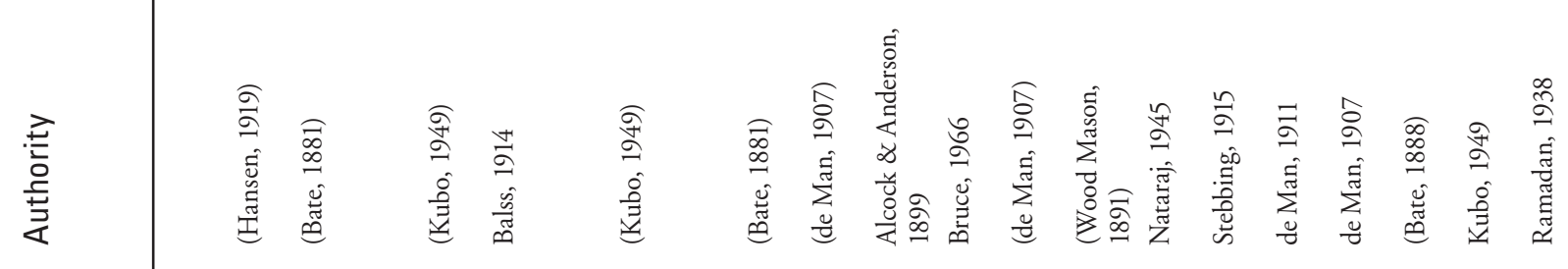

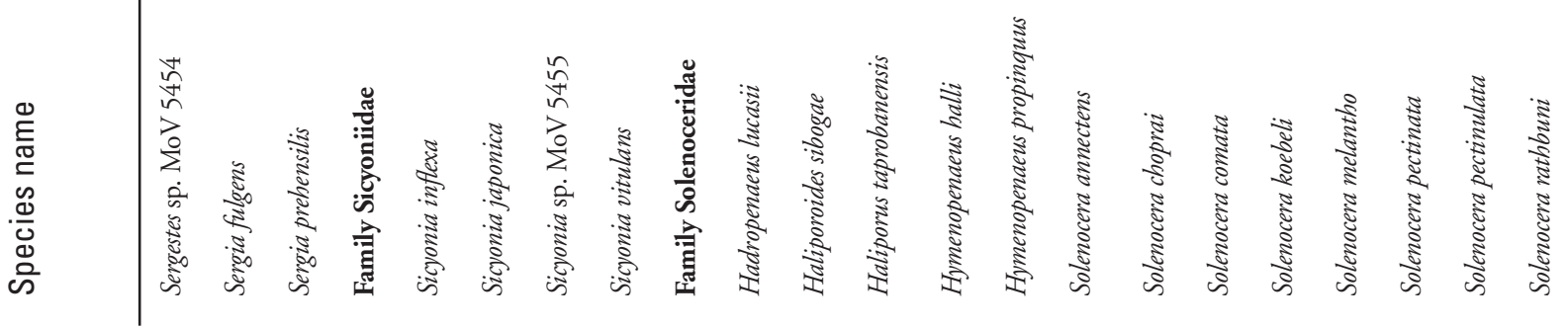




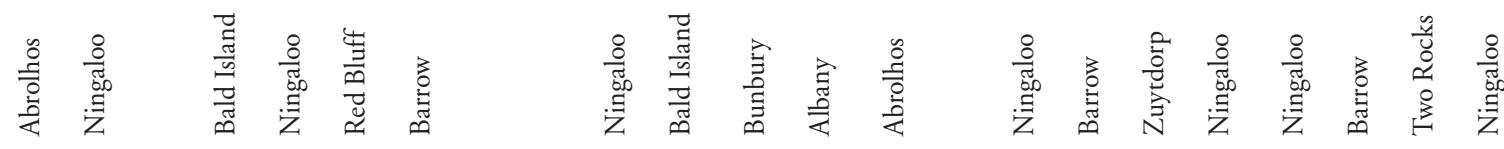

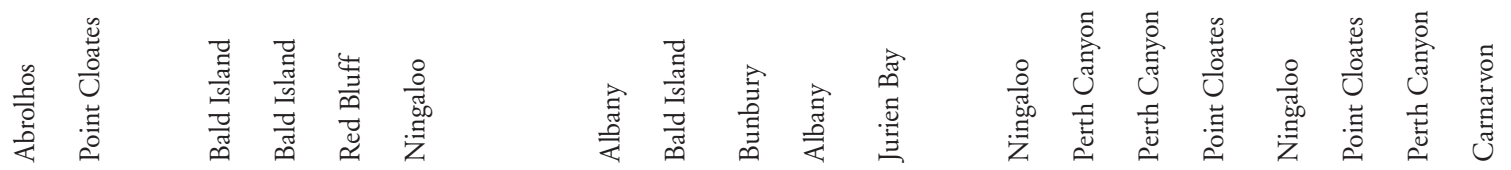

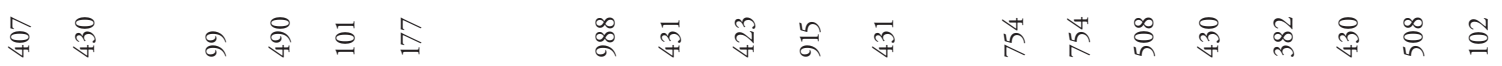

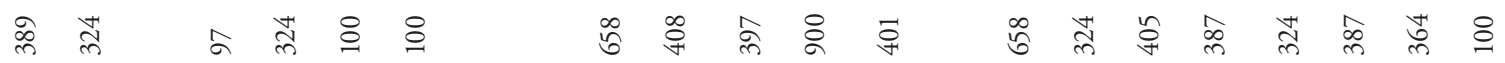

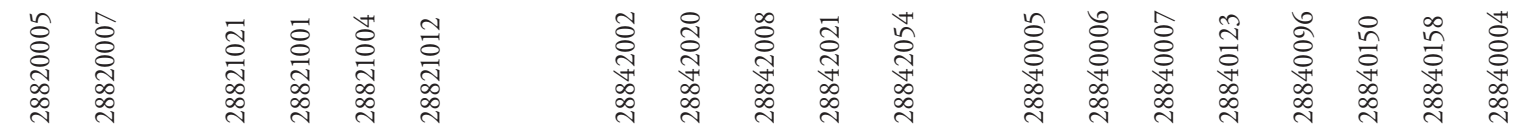

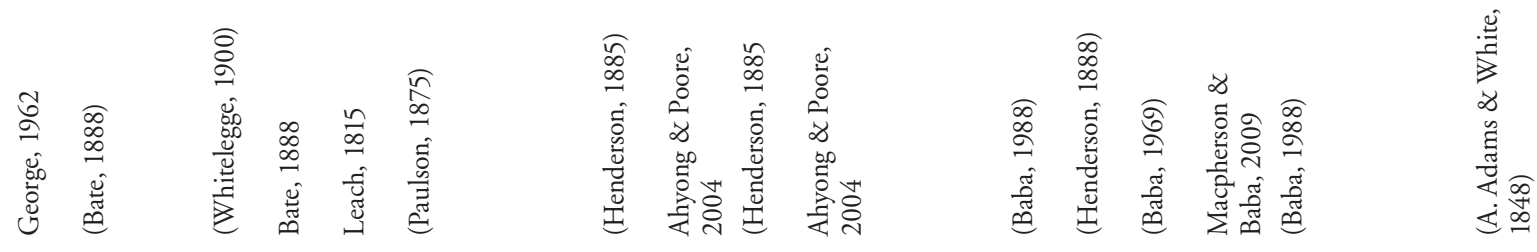

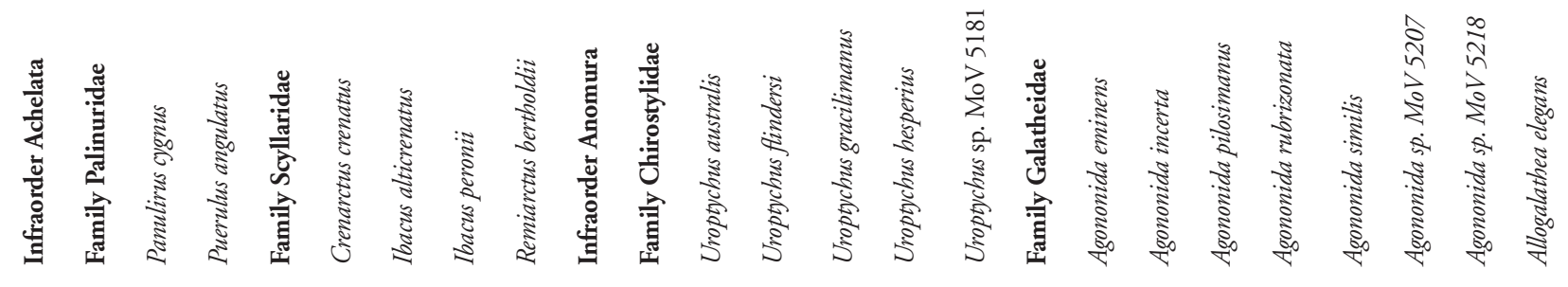




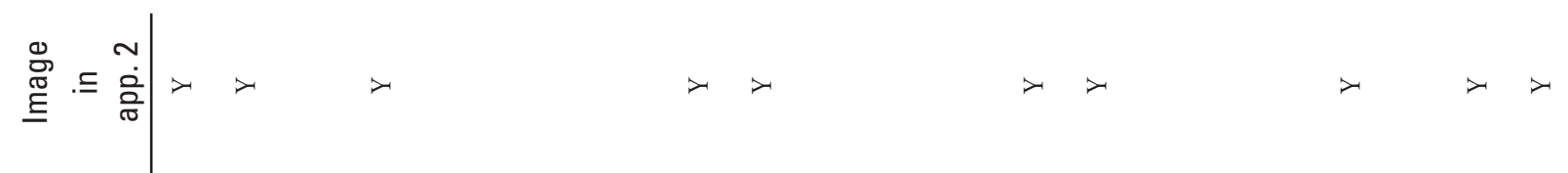

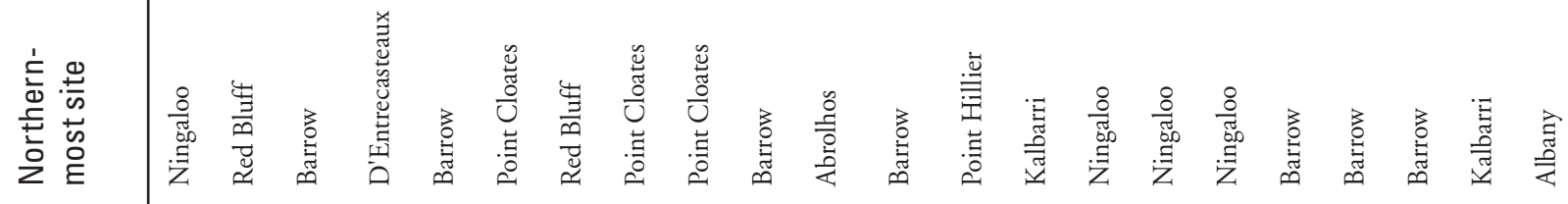

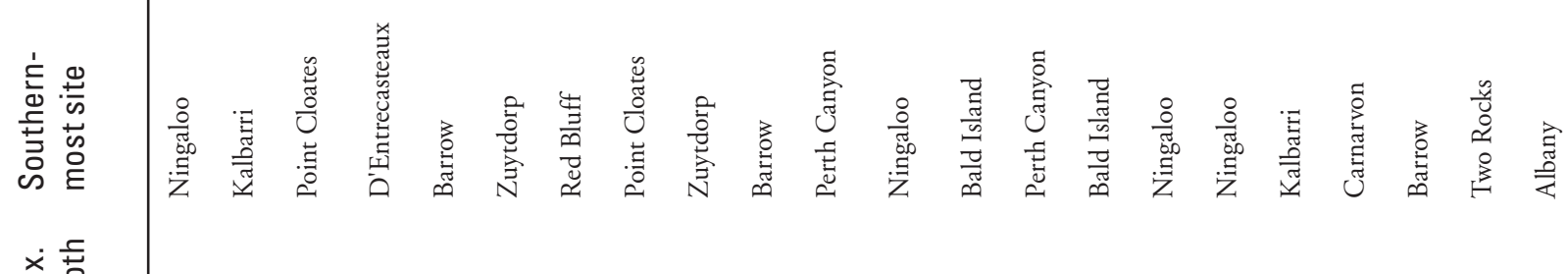

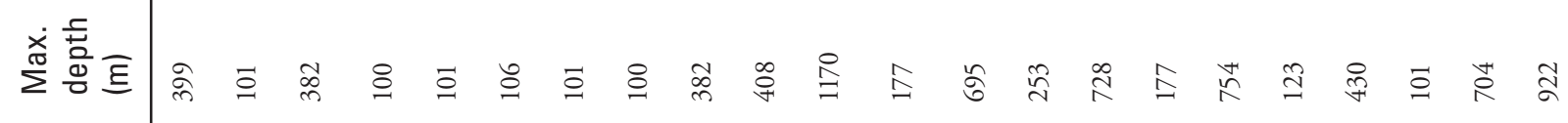

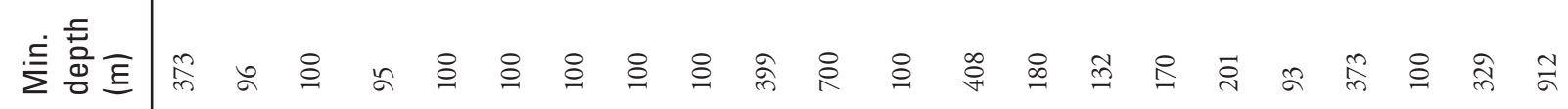

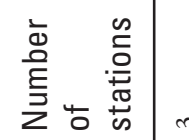

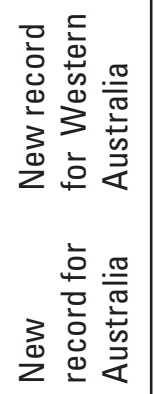

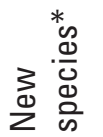

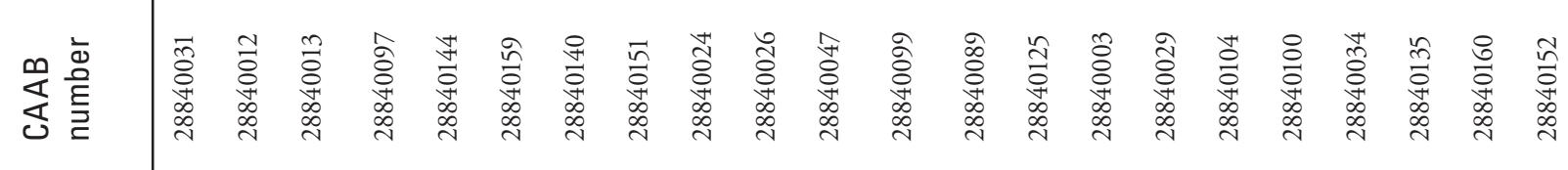

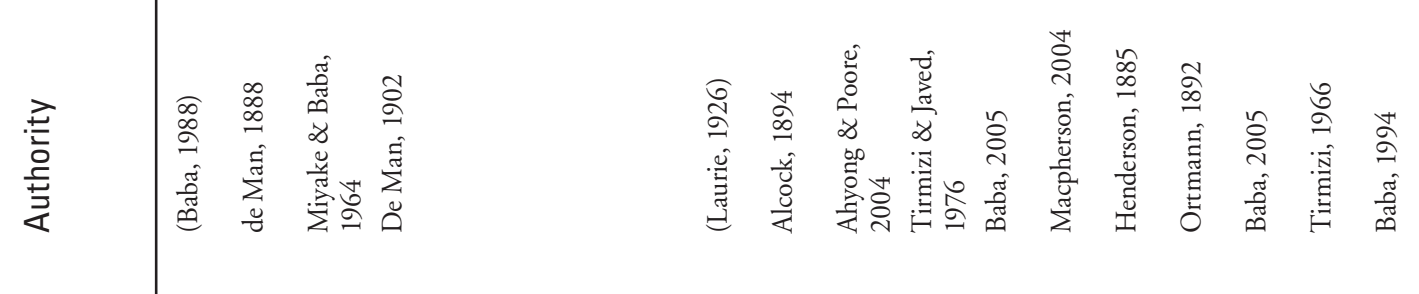

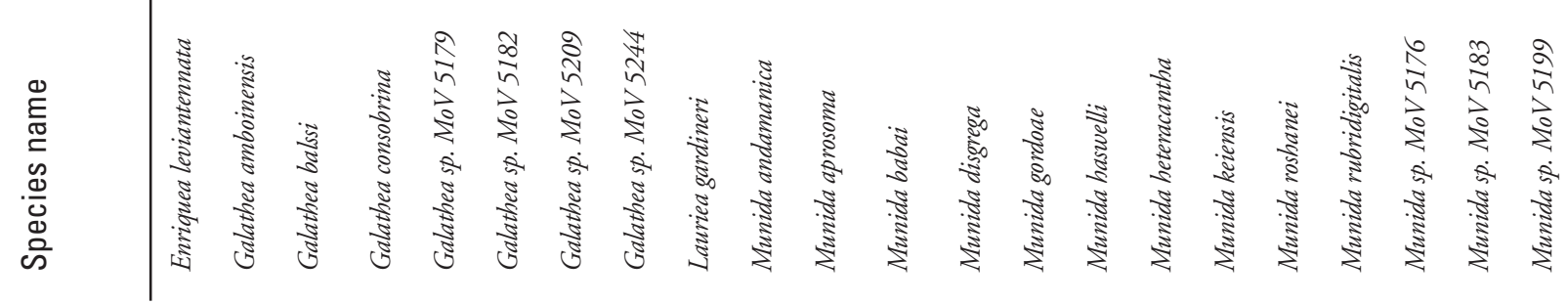




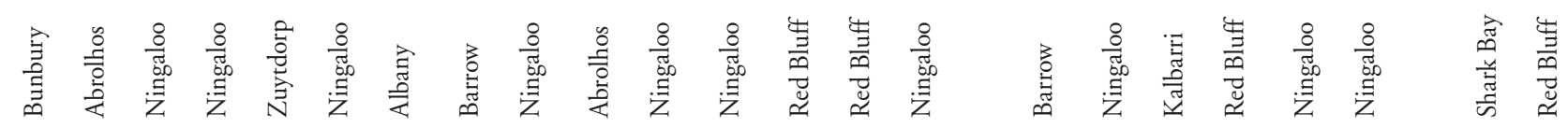

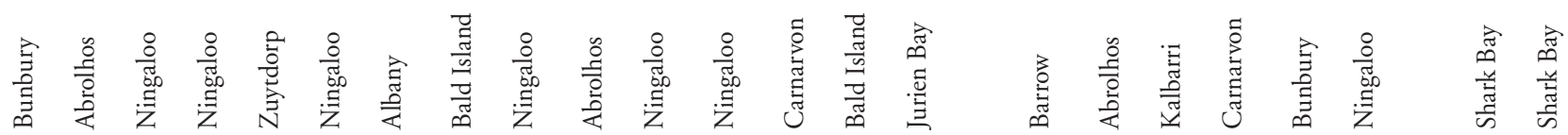

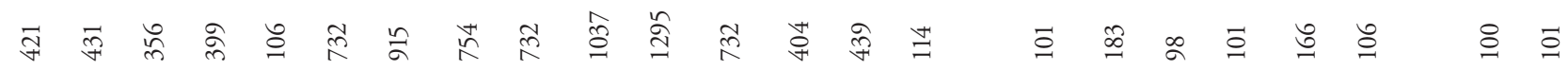
将

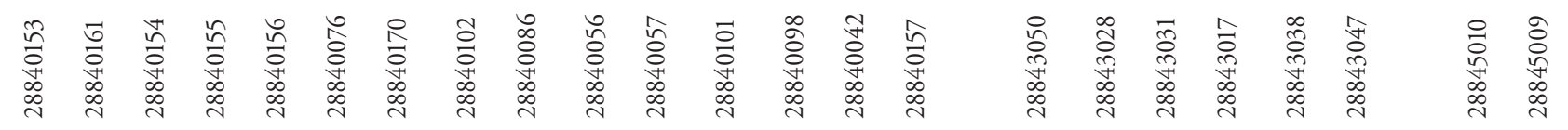

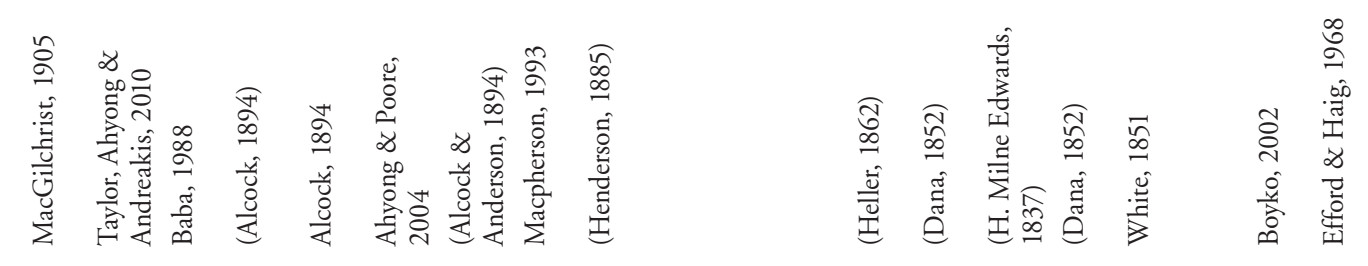

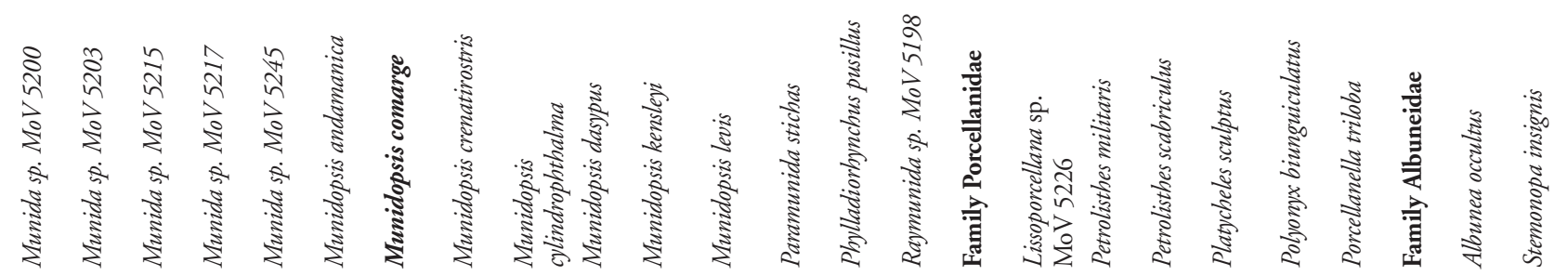




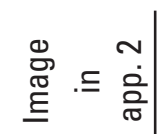

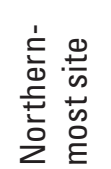

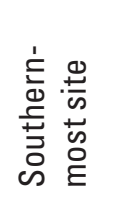

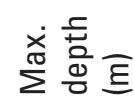

这营至

离兽惫

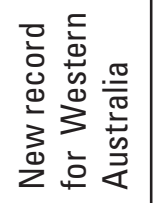

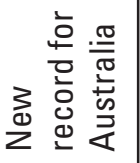

zon

这离

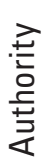

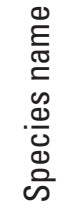

苍

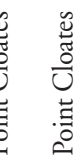

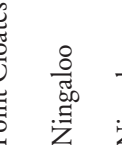

竞密

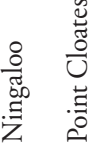

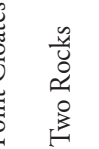

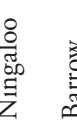

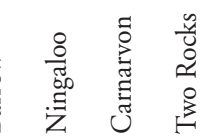

党 营

苞芯

莺

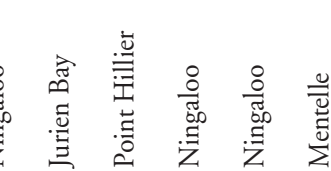

苞

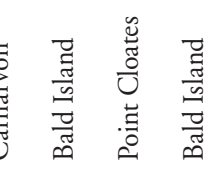

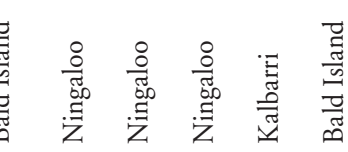

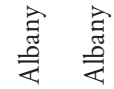

○ 응

ֻ

$\stackrel{0}{\ddots}$

in

$\stackrel{ }{\circ}$

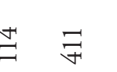

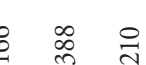

$\stackrel{\circ}{a}$

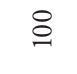

$\bar{\sigma}$

$\stackrel{n}{0}$

○

$\kappa$

흥

๘



$\stackrel{\infty}{\stackrel{\infty}{\circ}}$

$-$

更

$-$
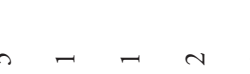

$-$

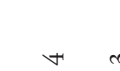

$-$ 


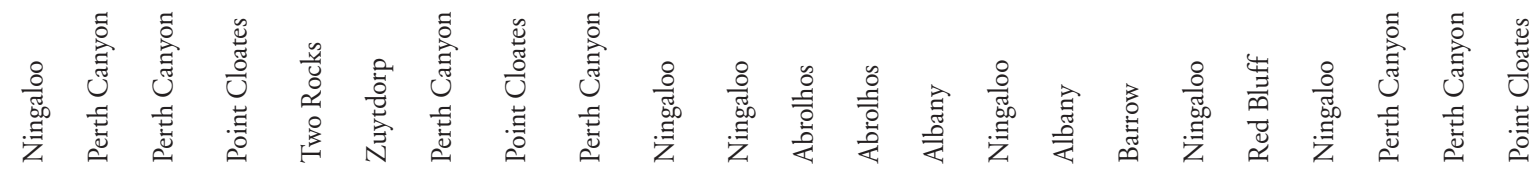

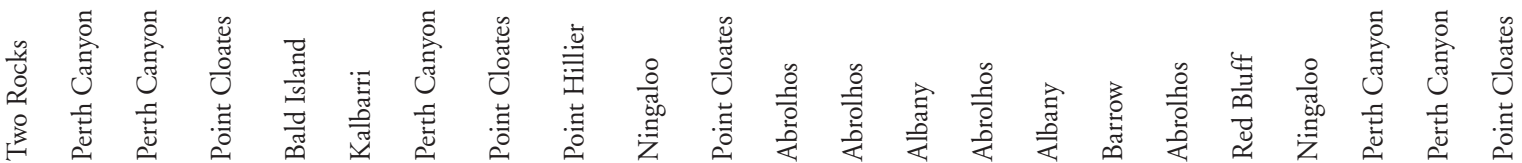

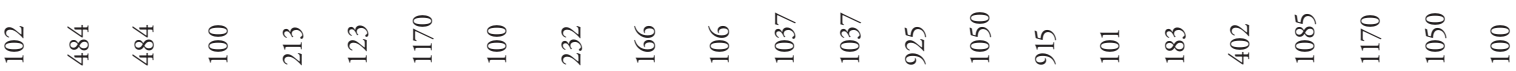

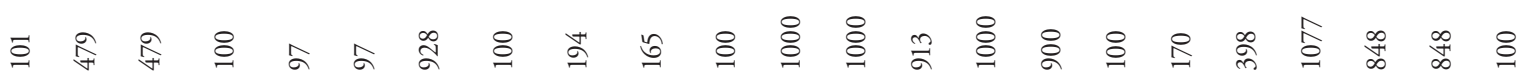

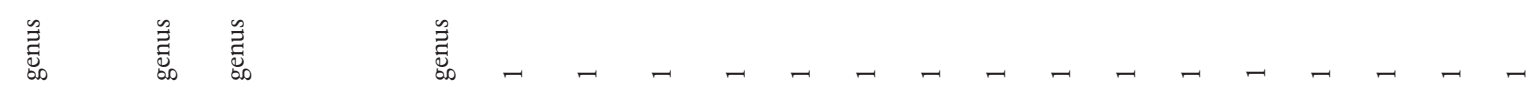

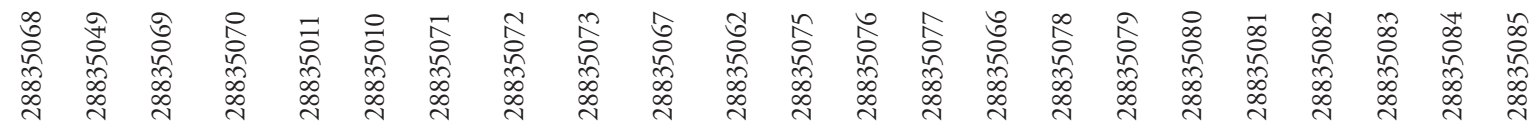

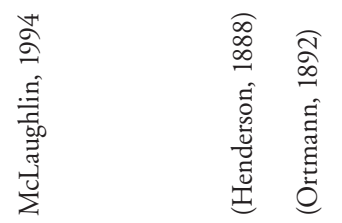

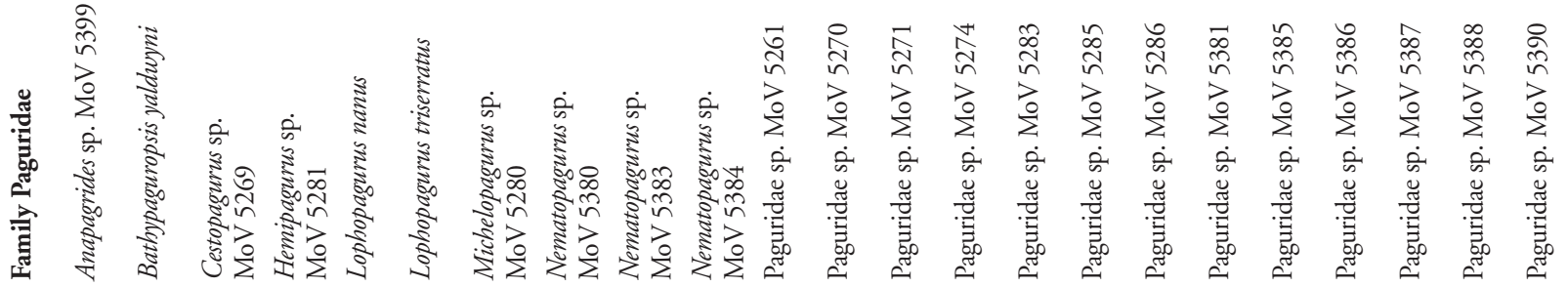




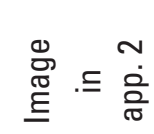

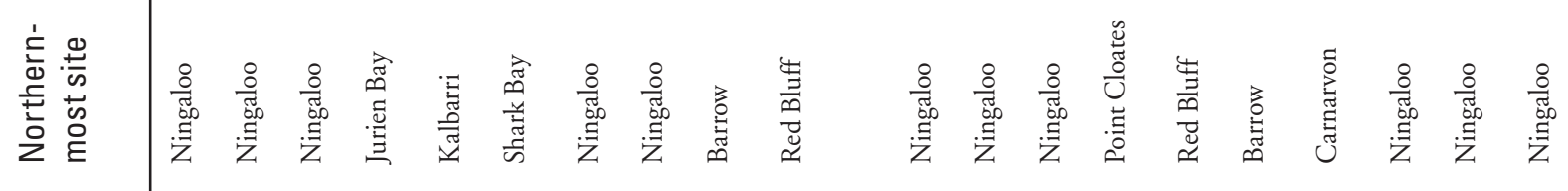

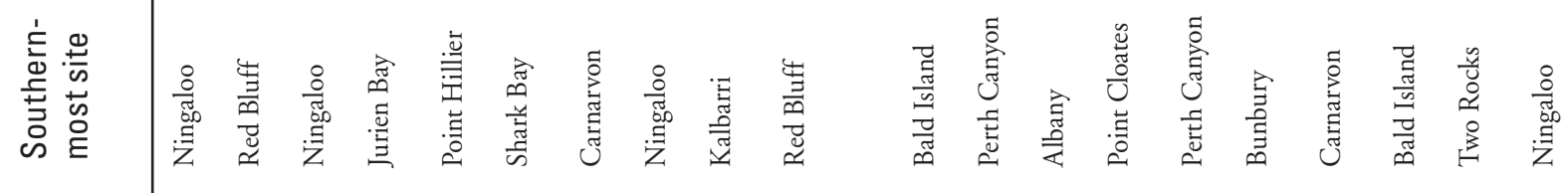

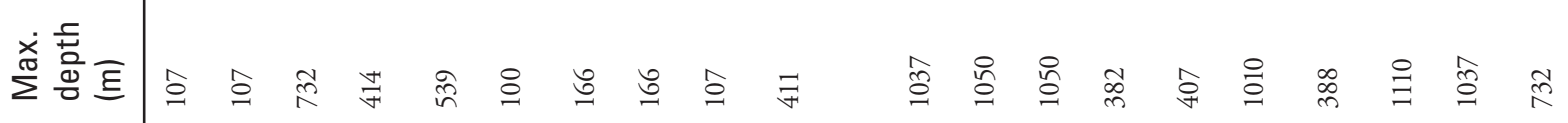

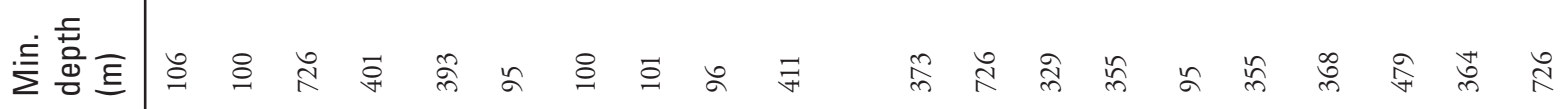

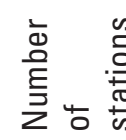

焉离

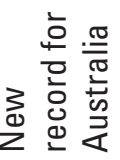

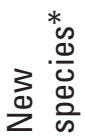

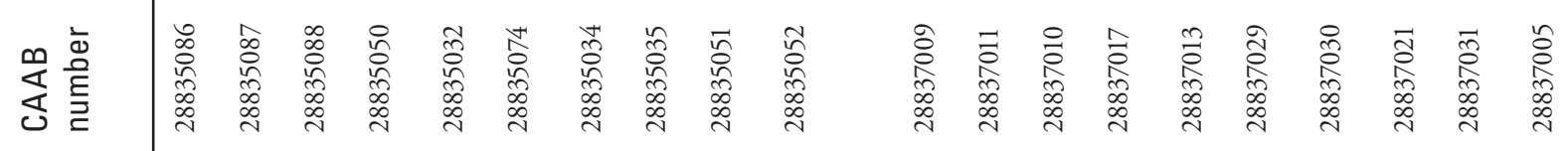

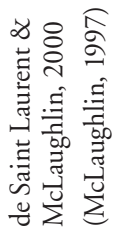

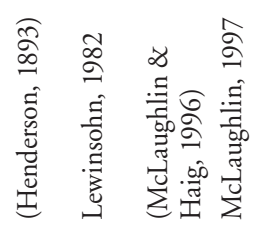

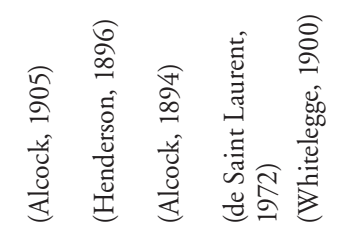

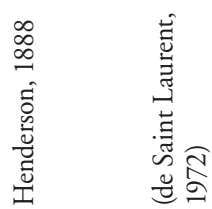

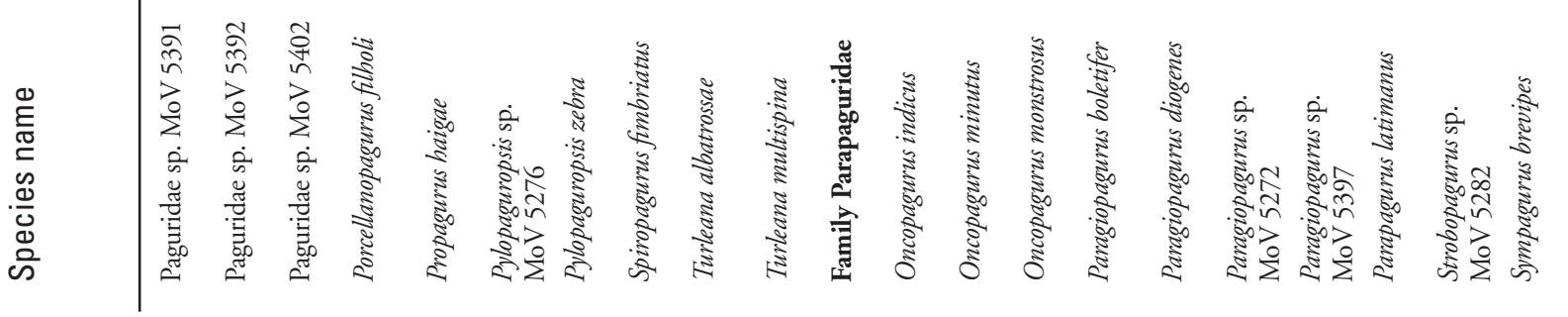




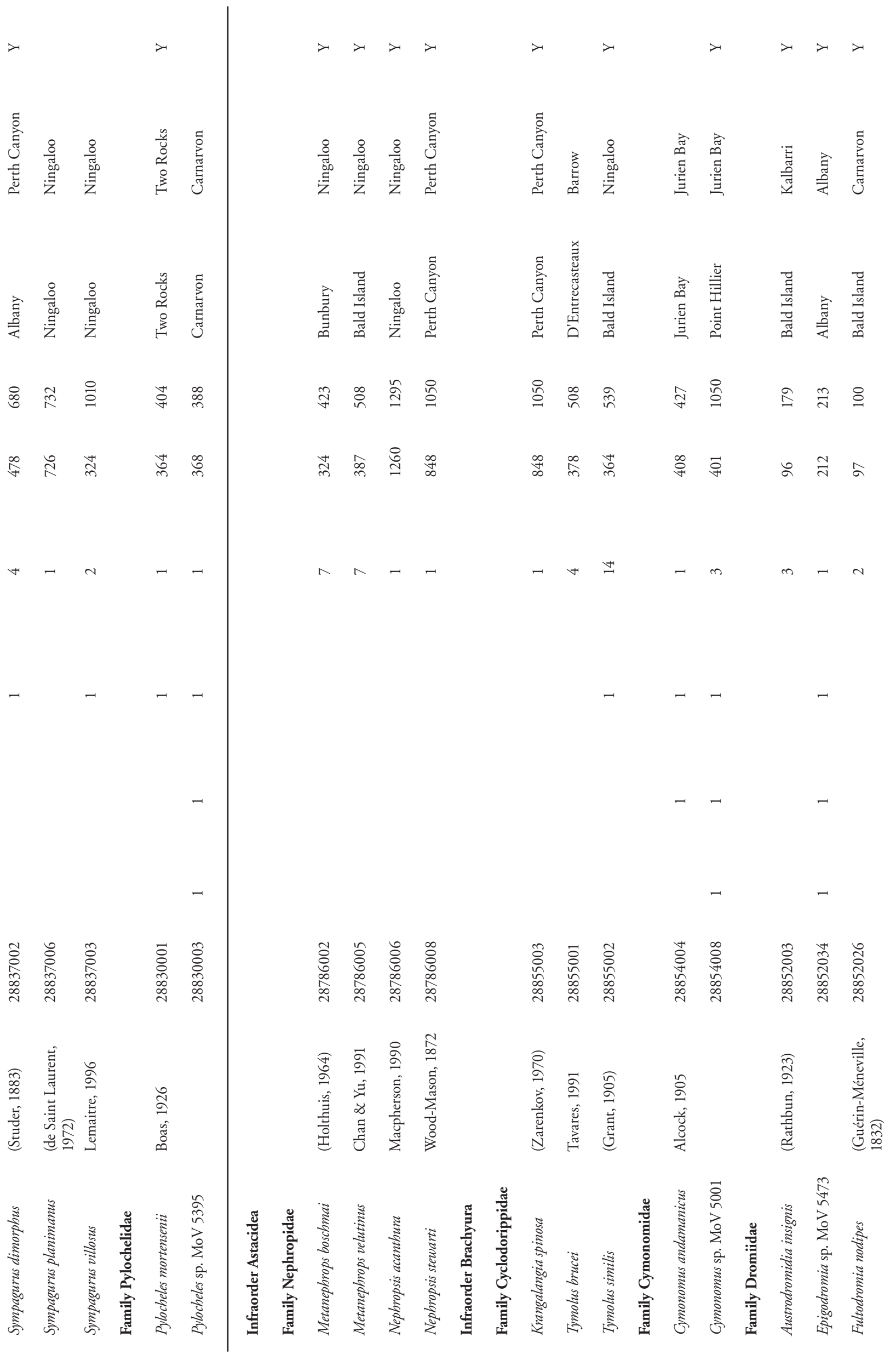




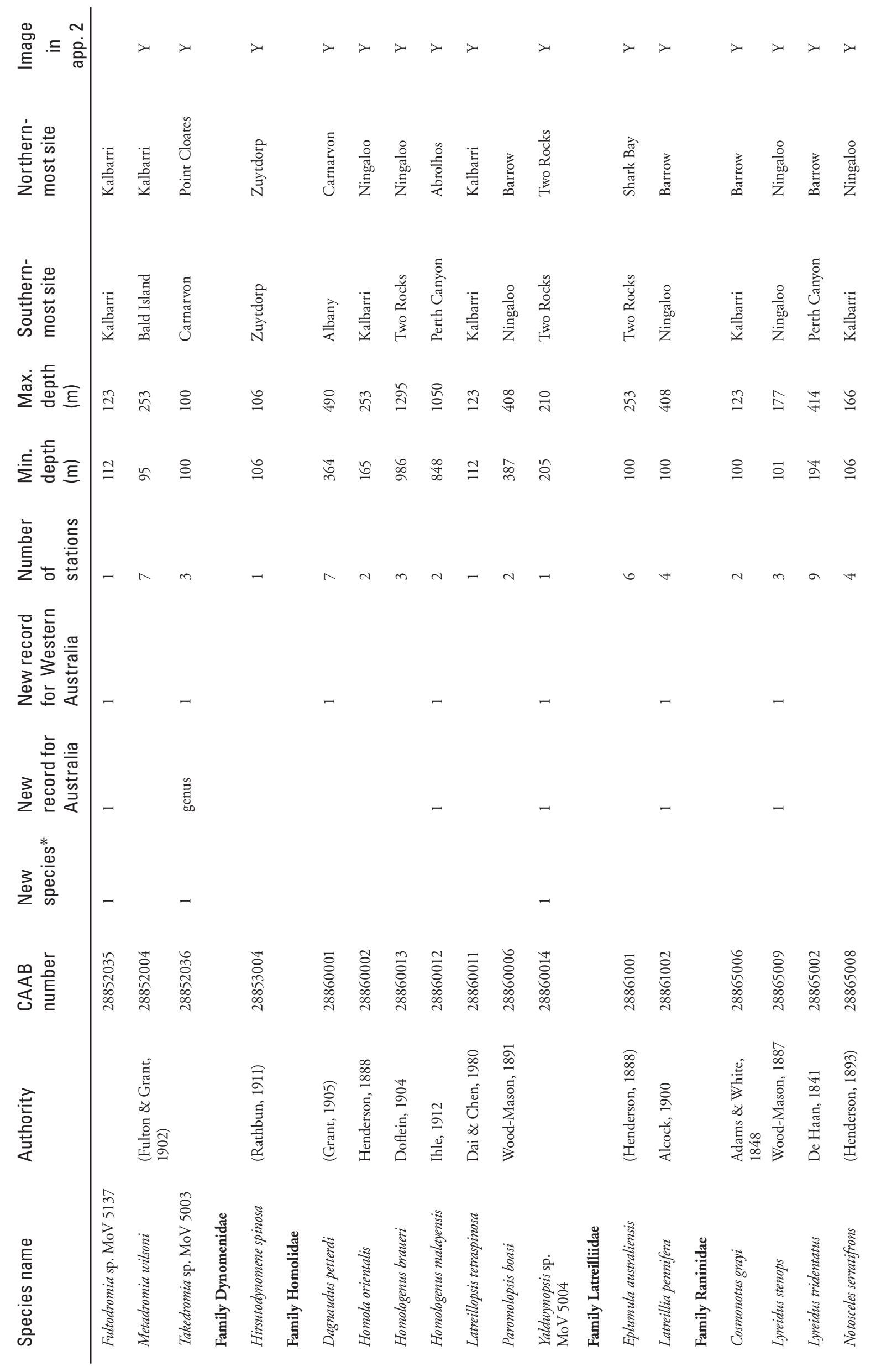




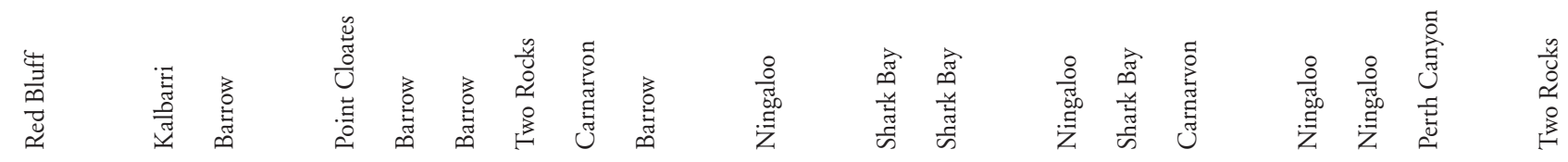

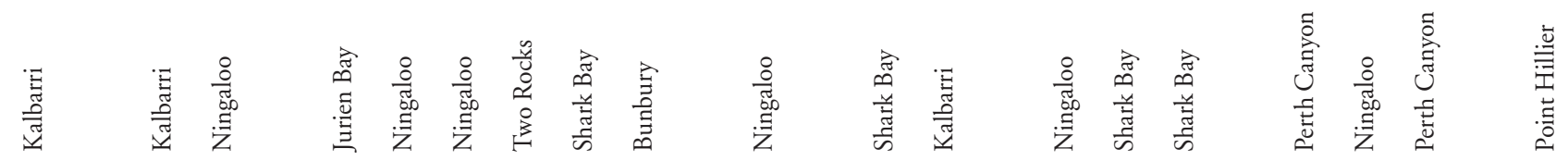
‡

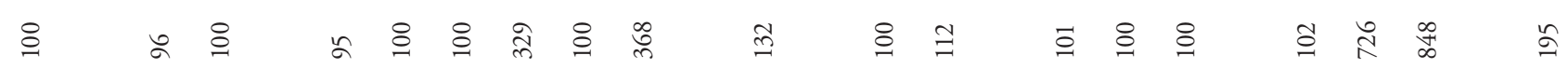

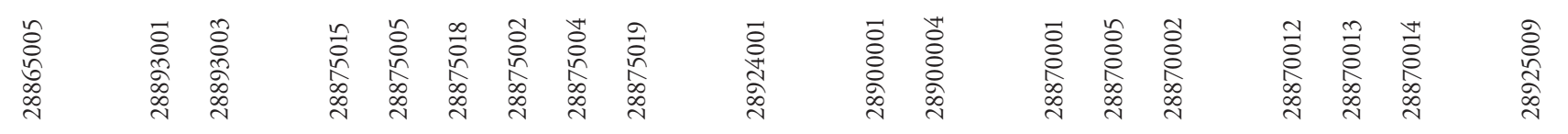

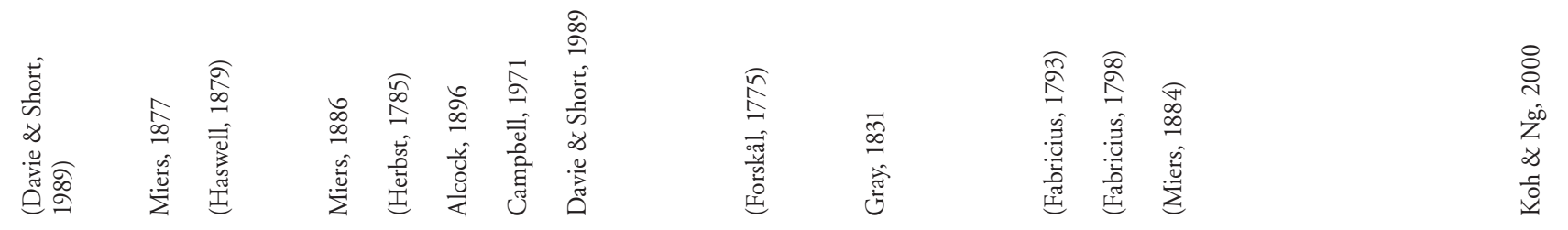

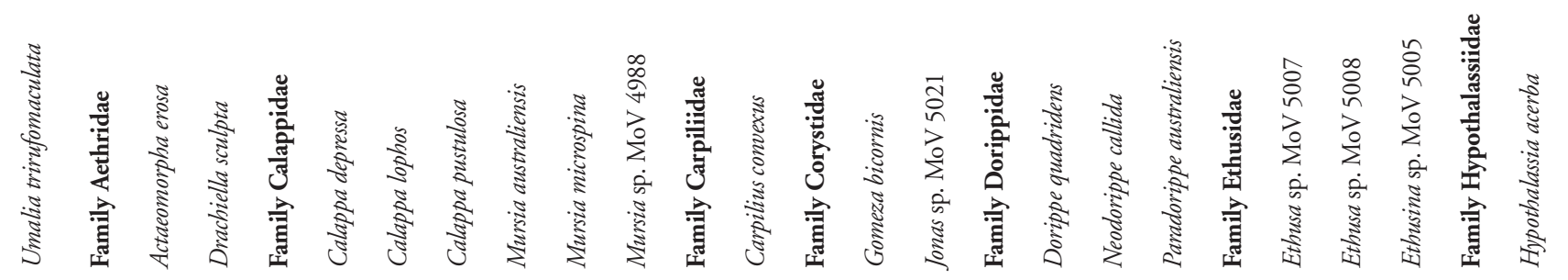




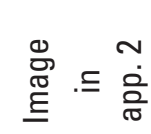

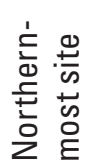

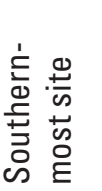

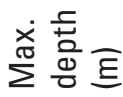

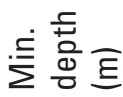

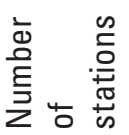

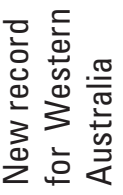

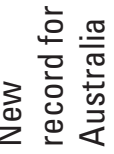

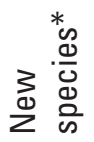
言

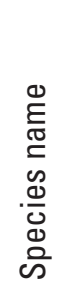

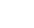

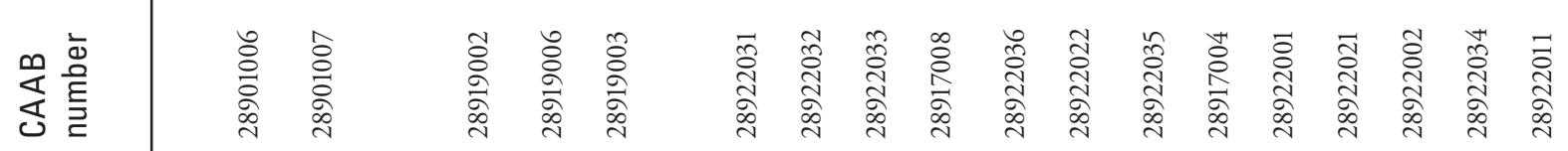

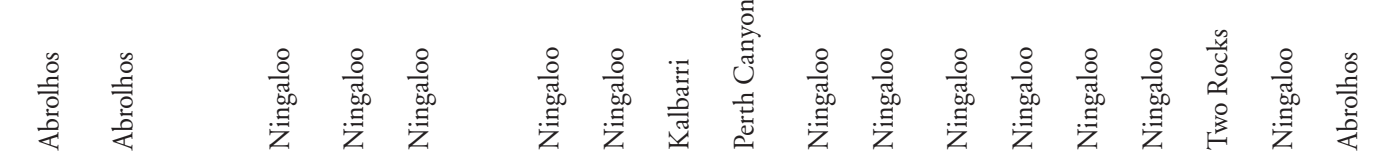

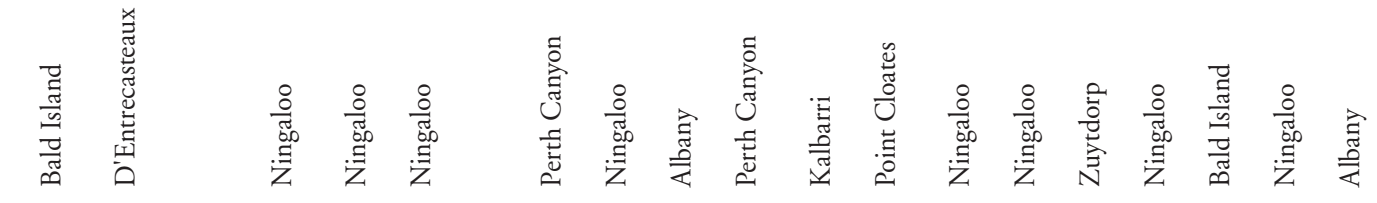

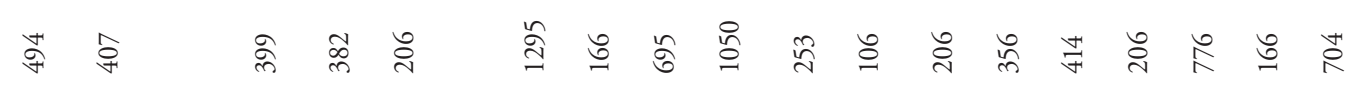

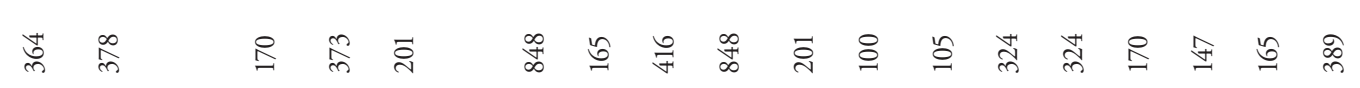

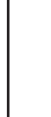

$\infty \quad$ r

$-$

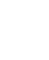<smiles>C1CCCCCC1</smiles>

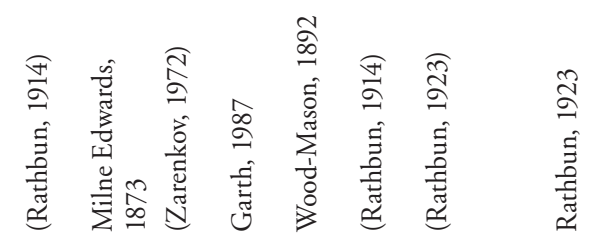

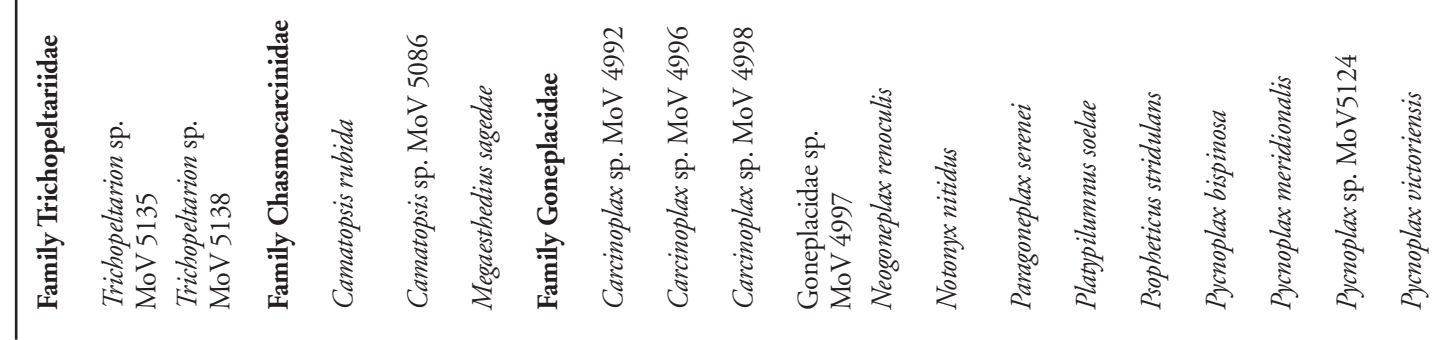




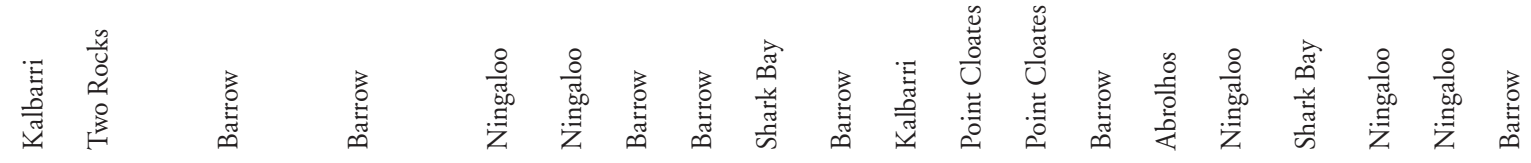

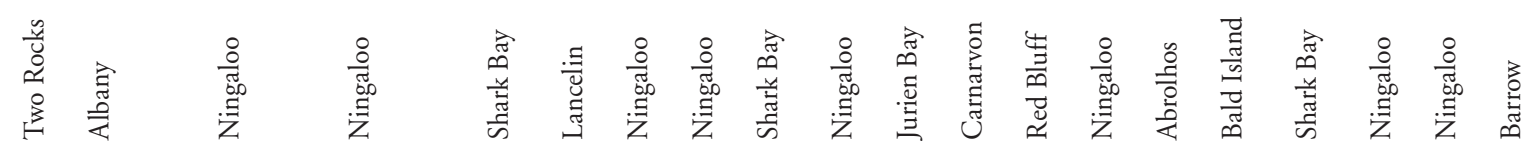
๙ู่

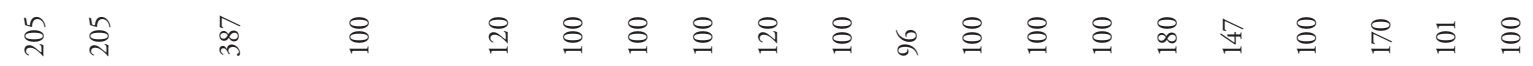

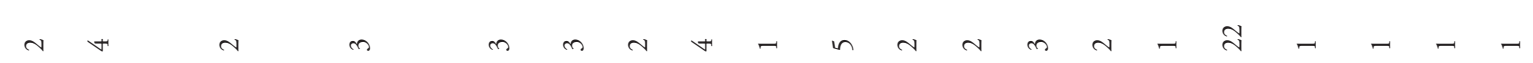

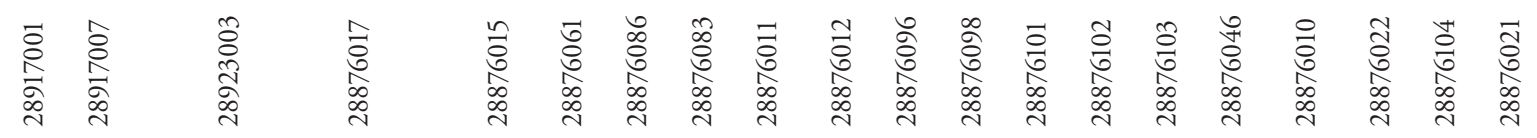

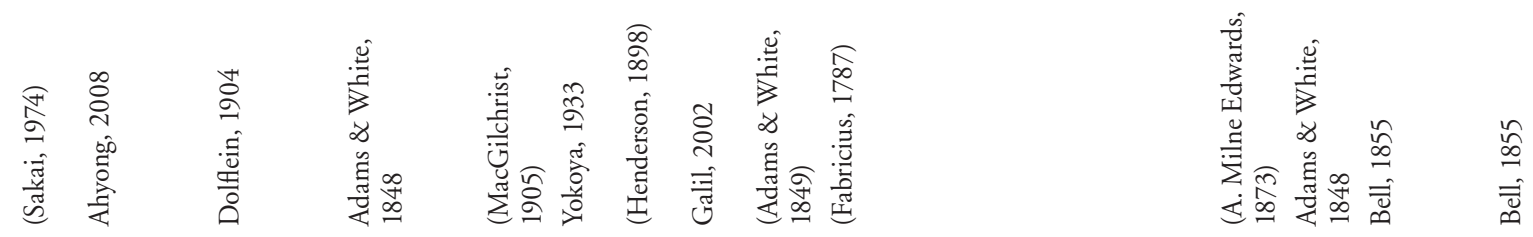

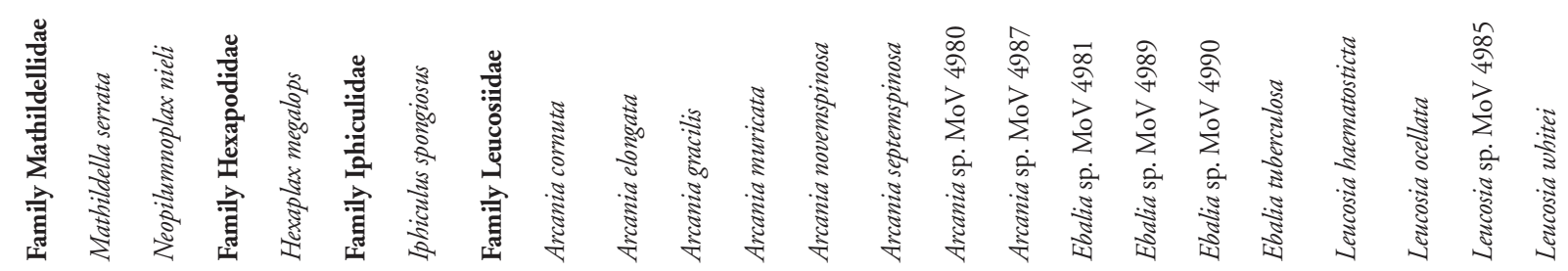




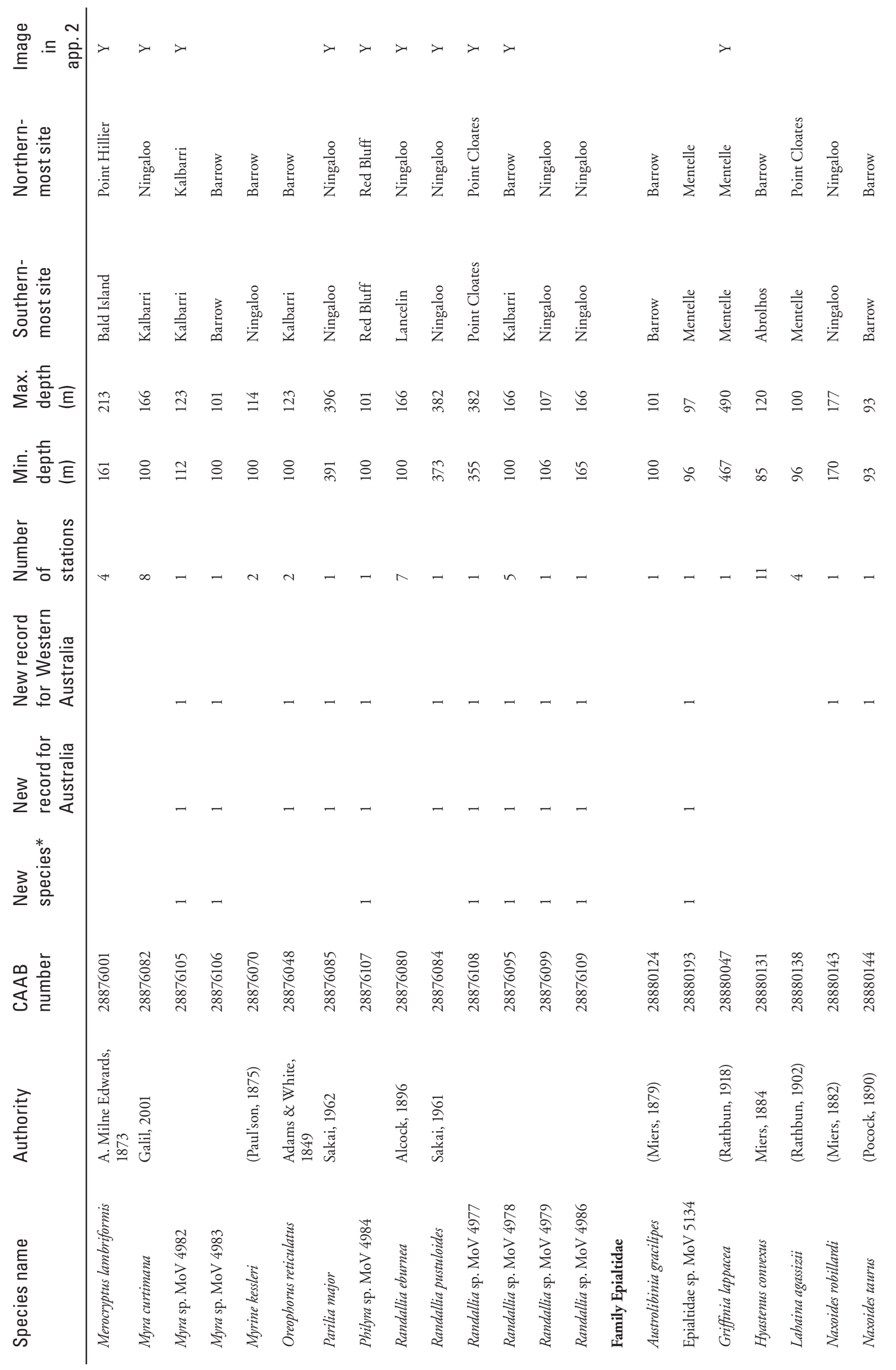




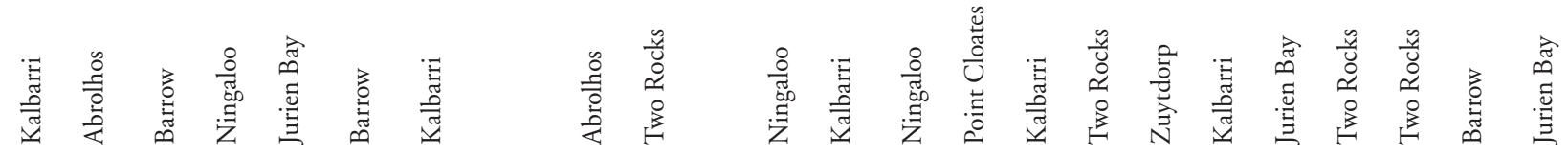

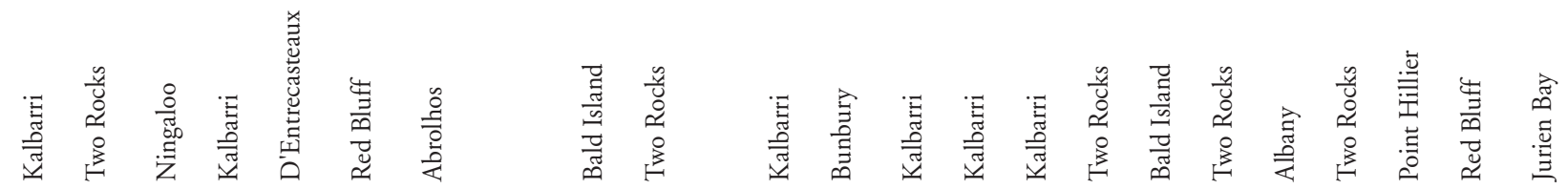

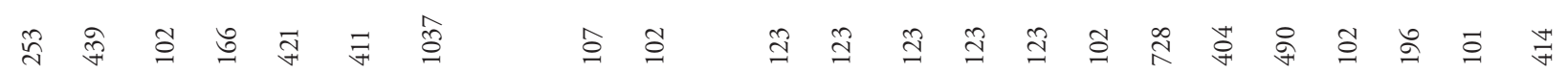

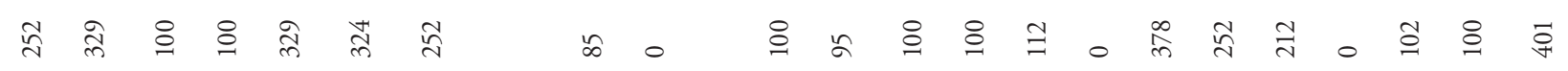

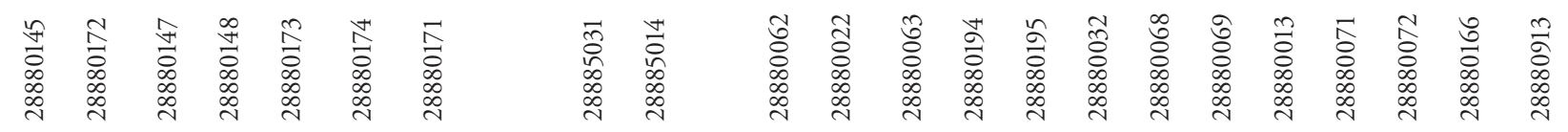

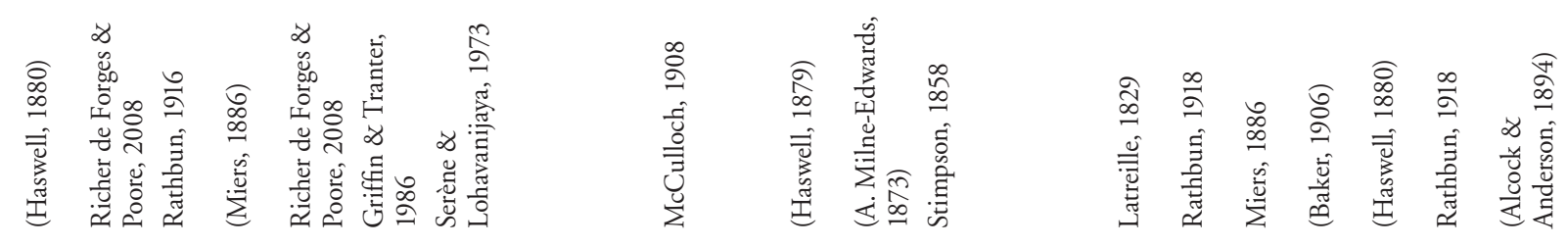

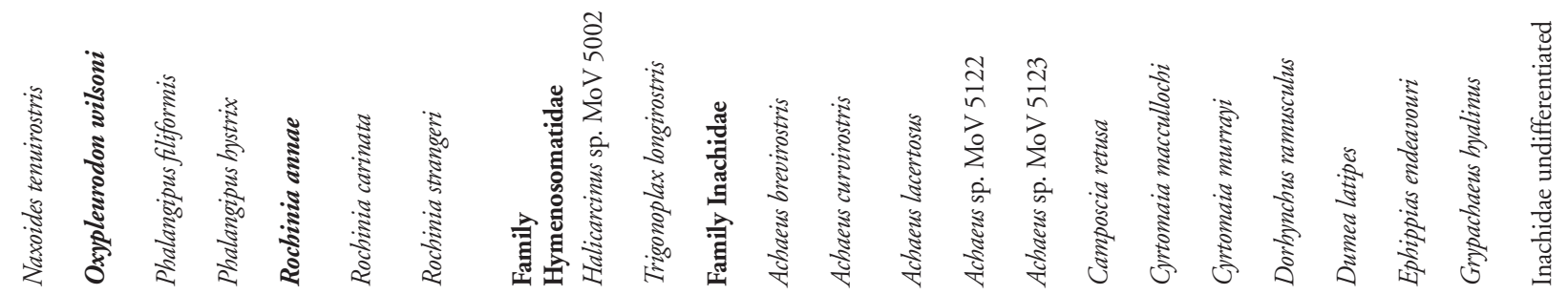




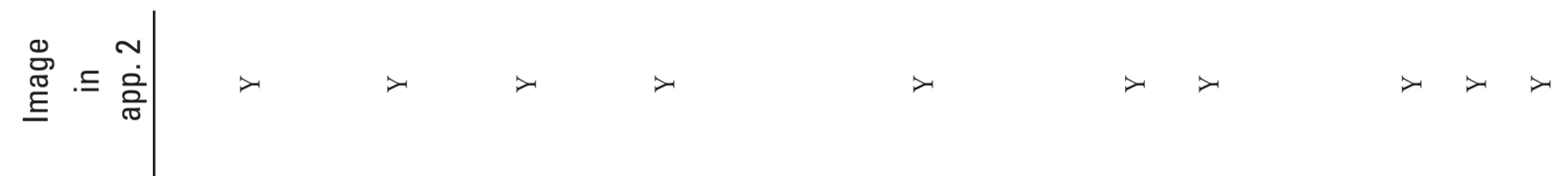

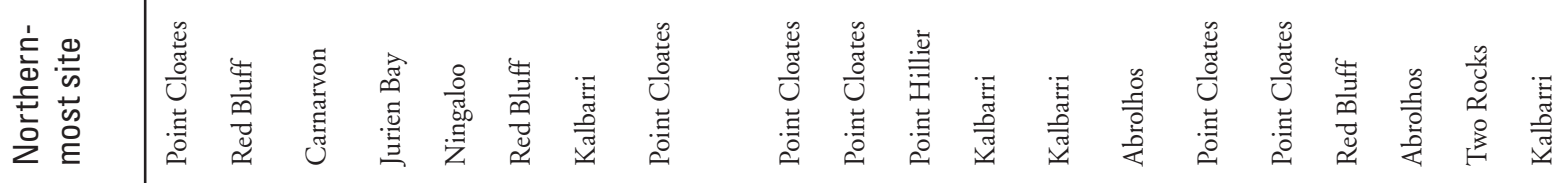

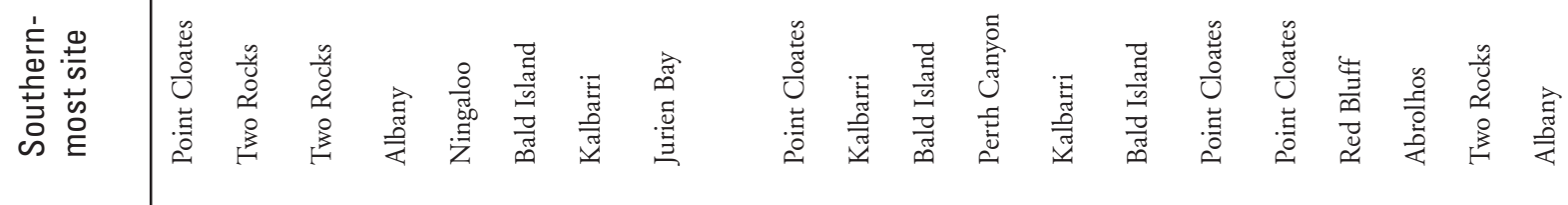

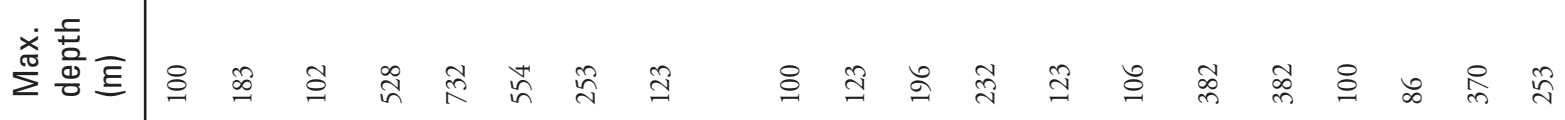

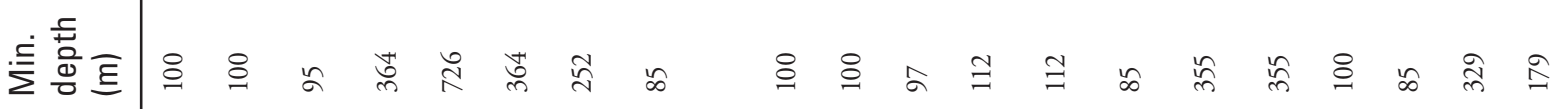

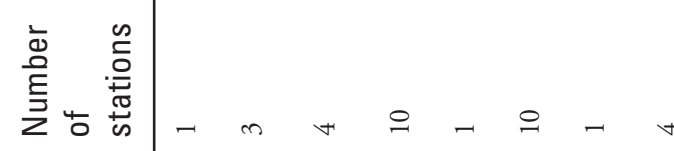

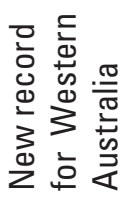

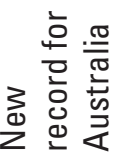

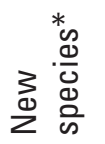

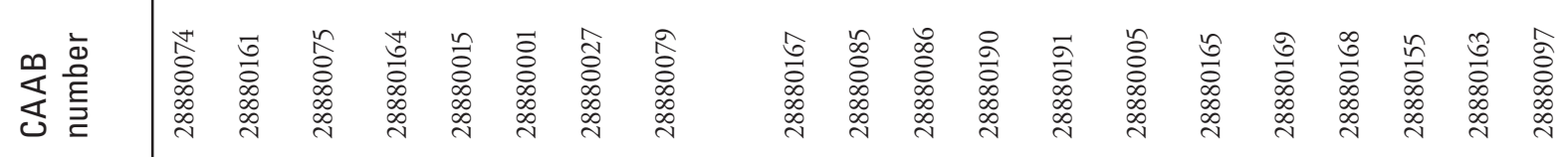

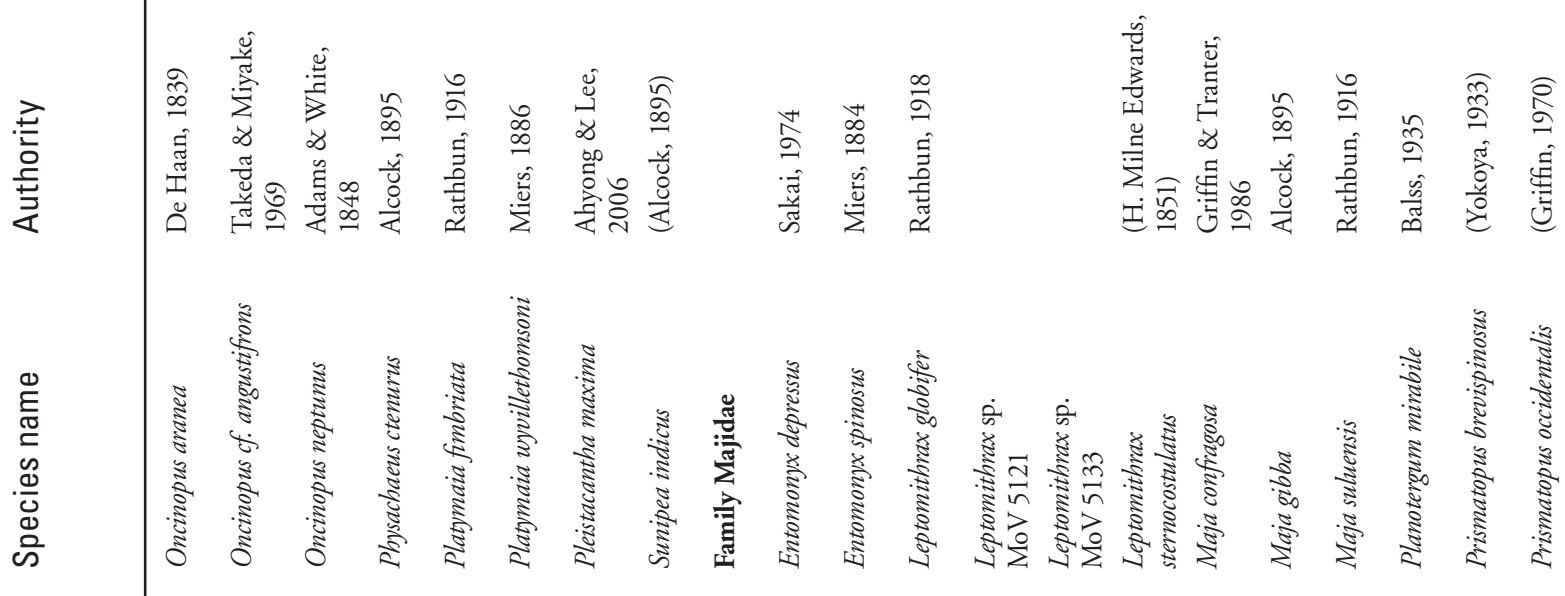




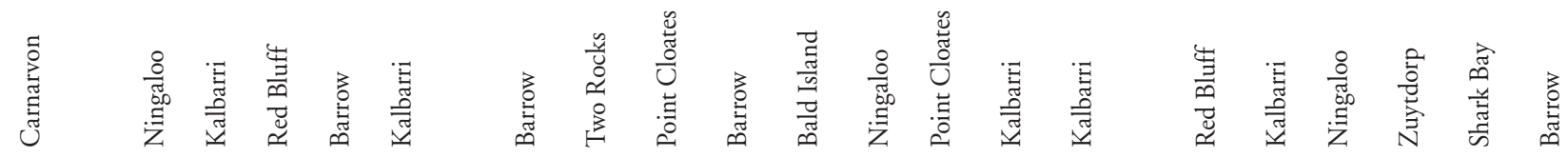

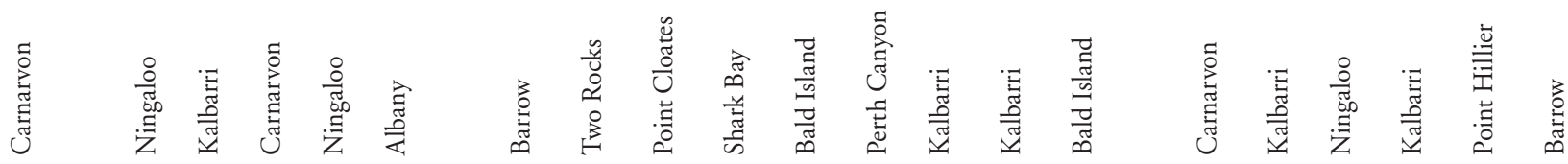

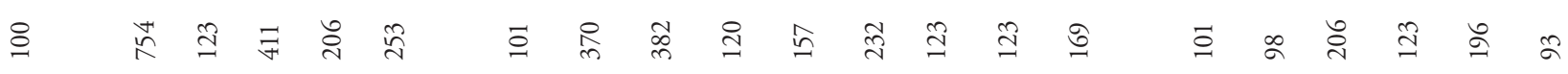

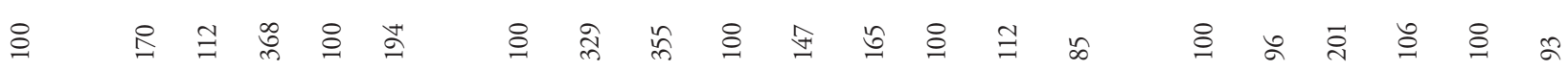

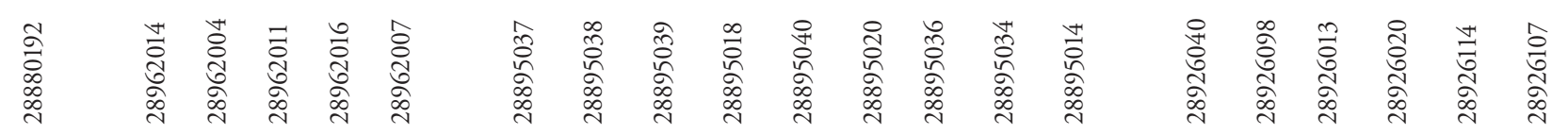

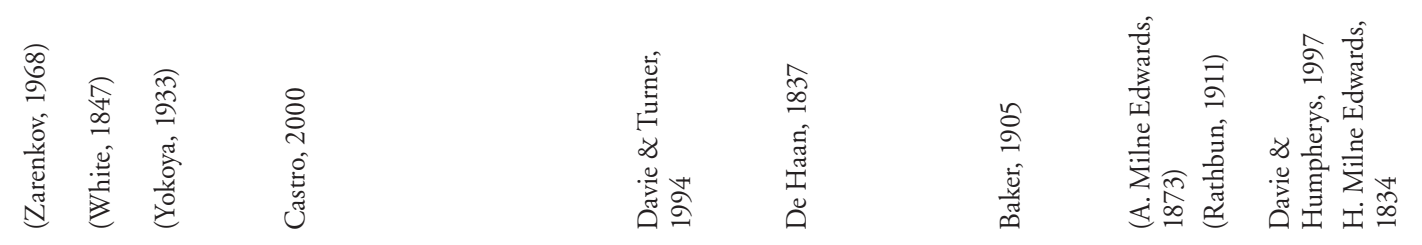

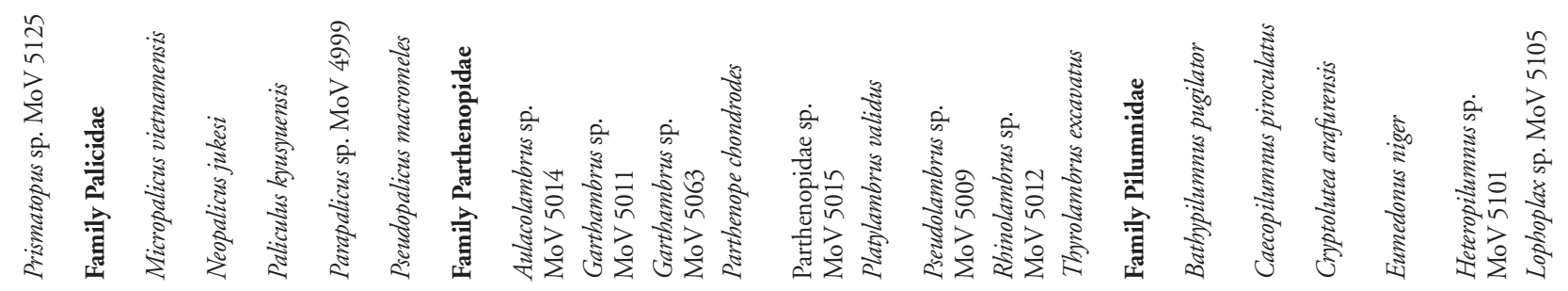




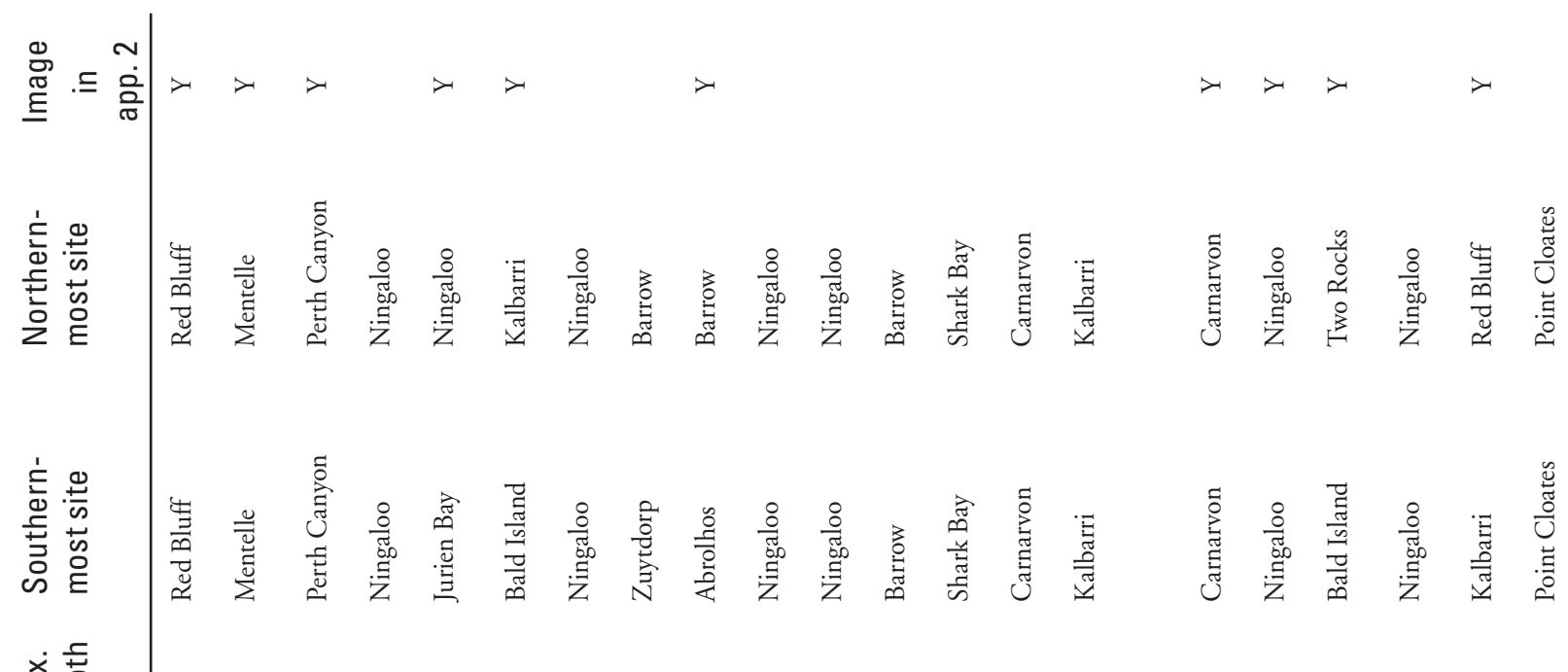

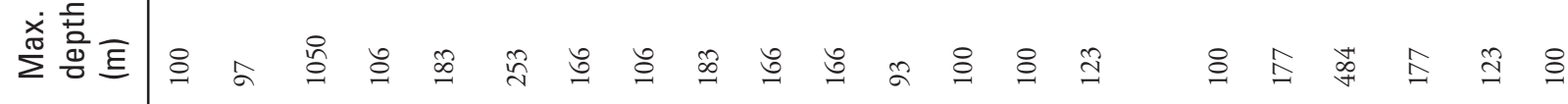

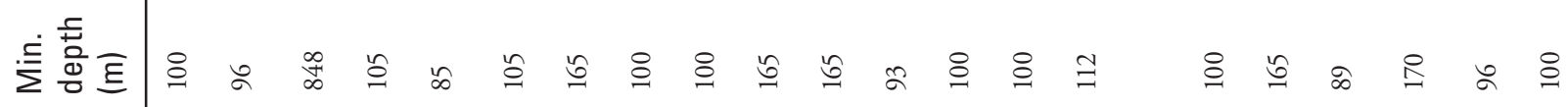

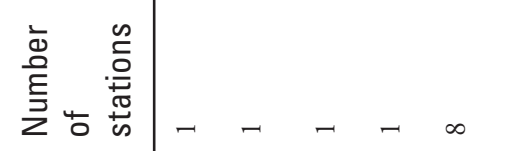

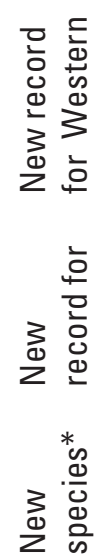

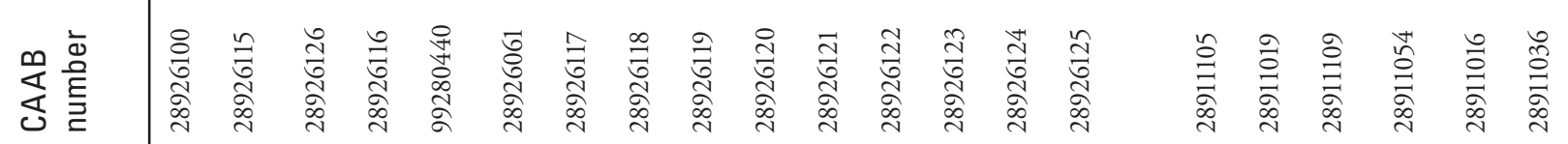

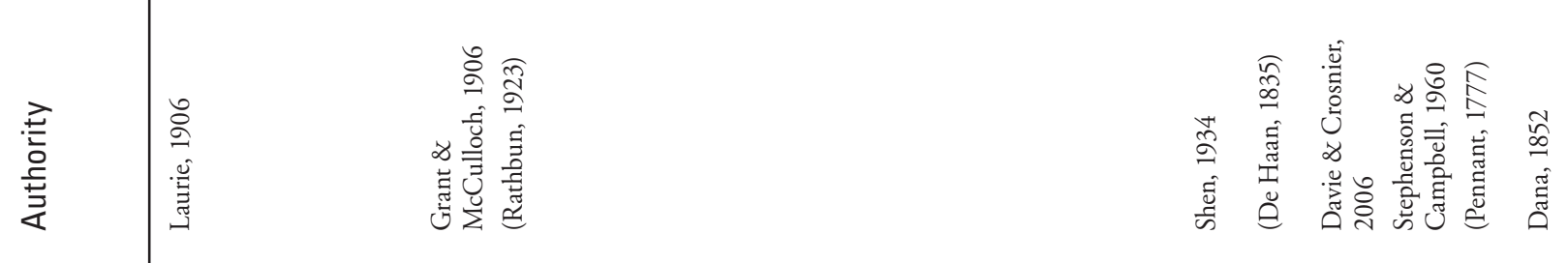

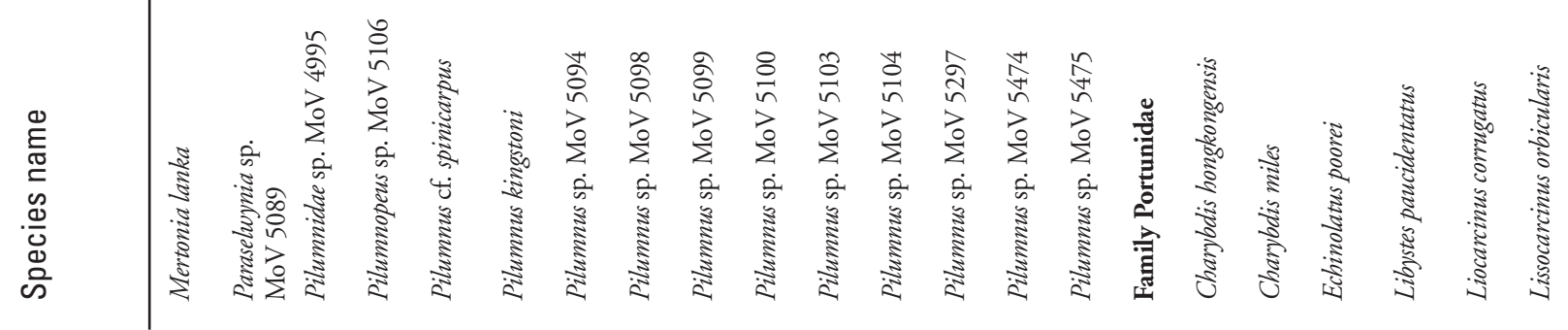




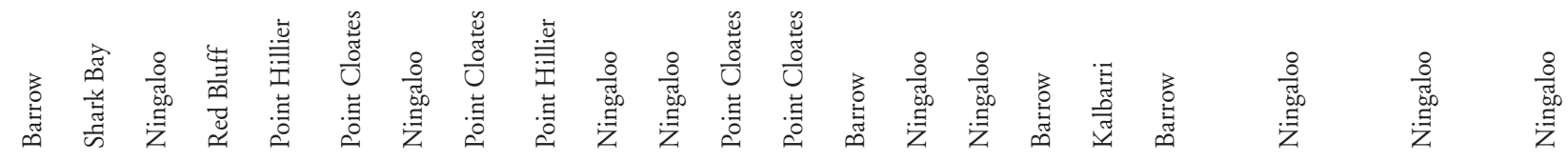

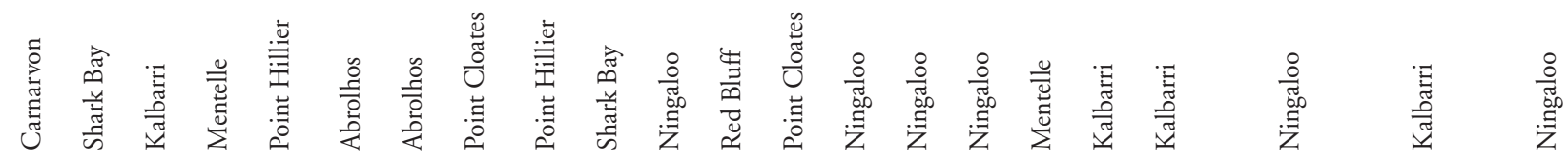

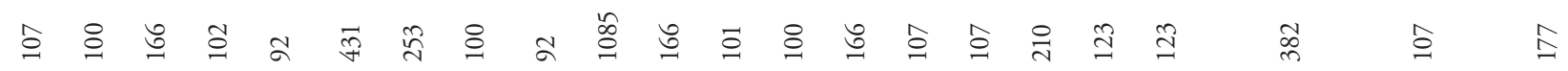

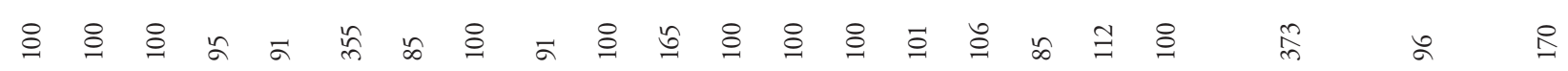

绻泀

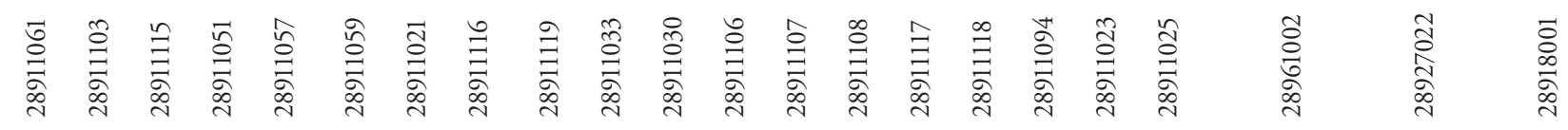

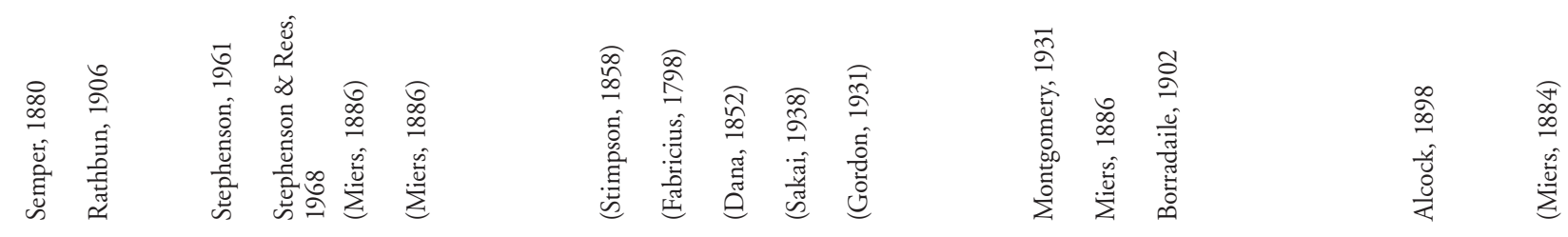

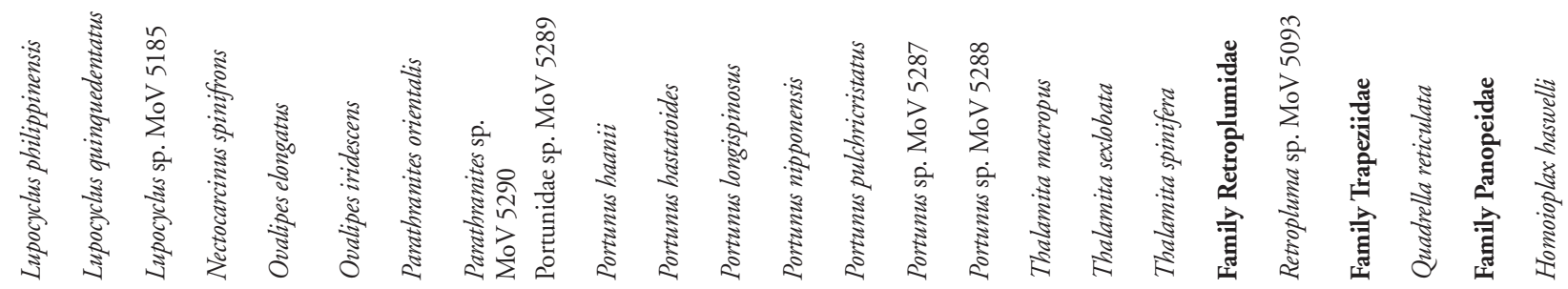




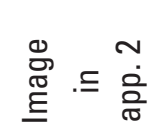

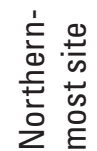

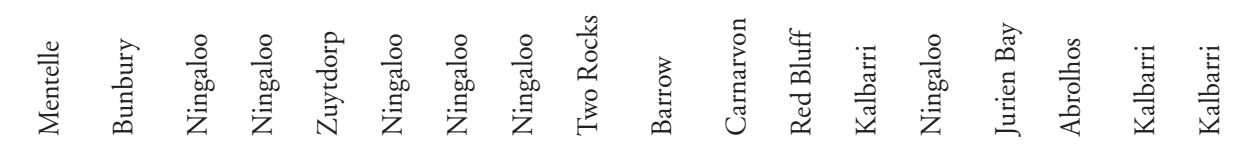

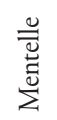

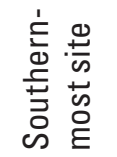

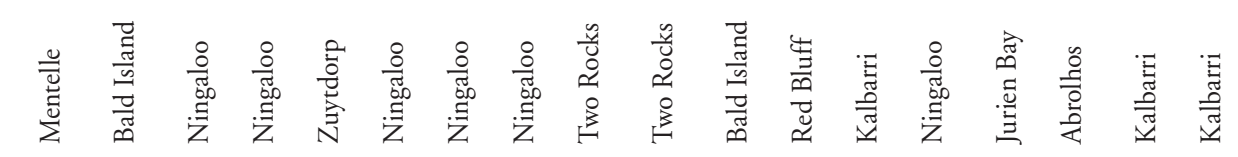

离营馬

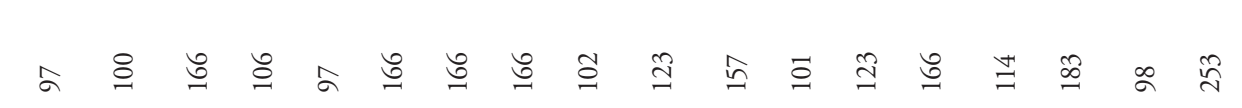

运营互

衰

离

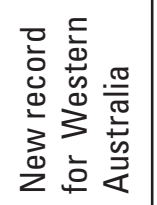

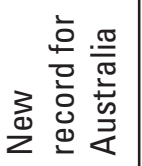

z)

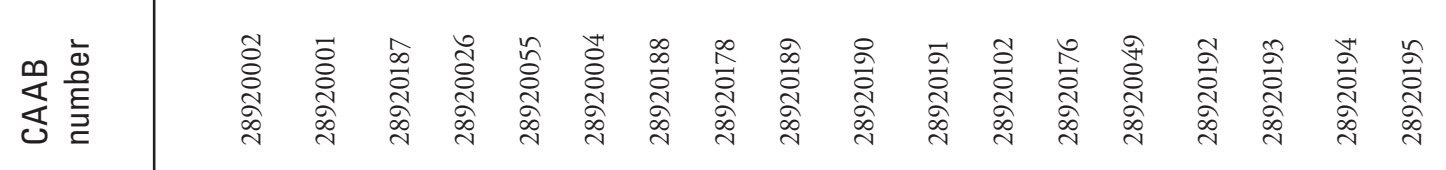

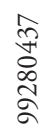

:

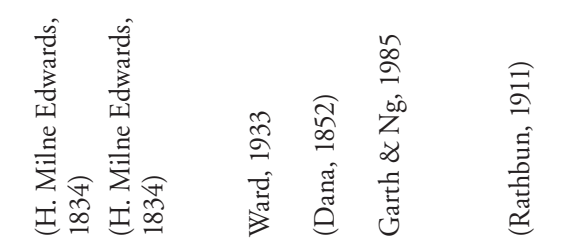

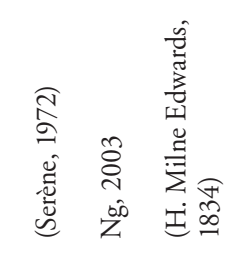

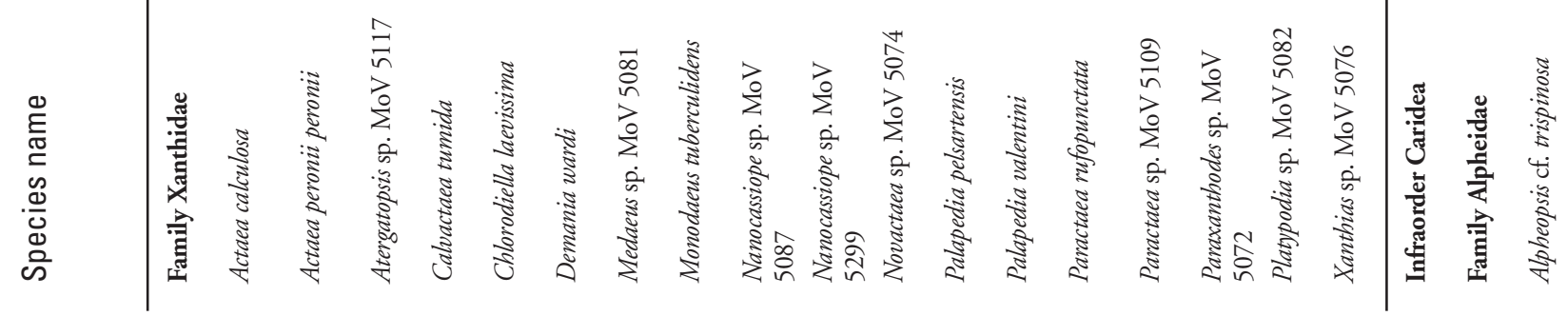




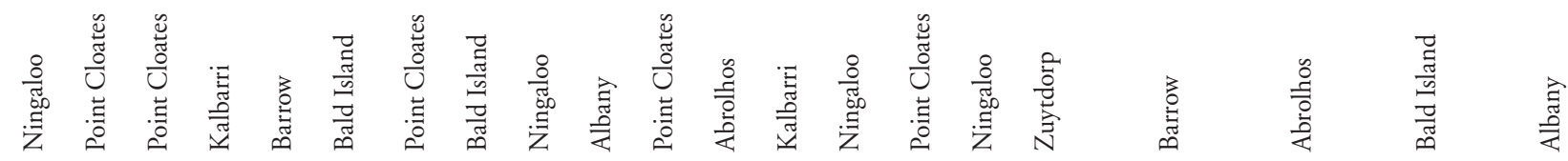

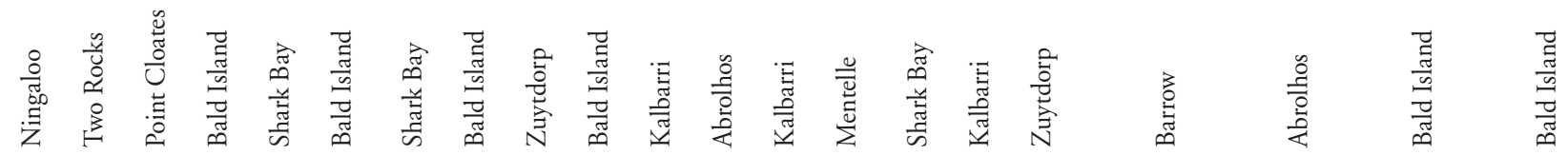

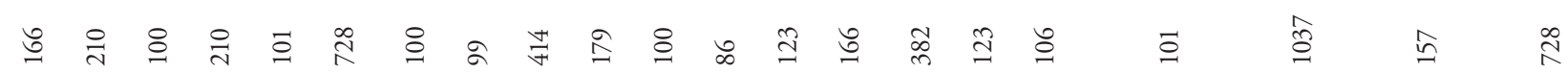

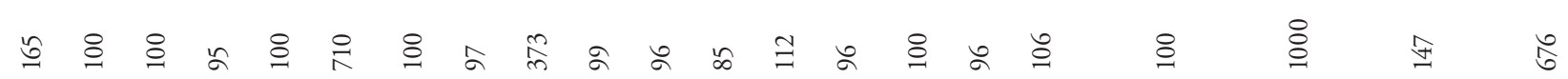

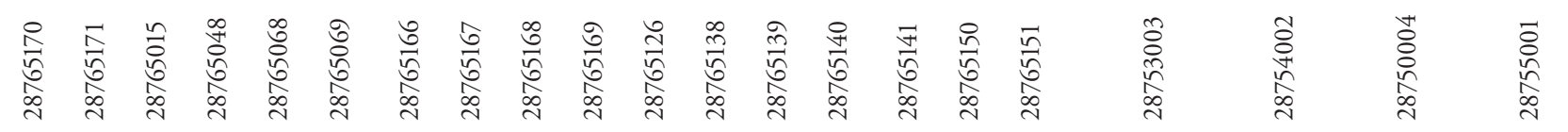

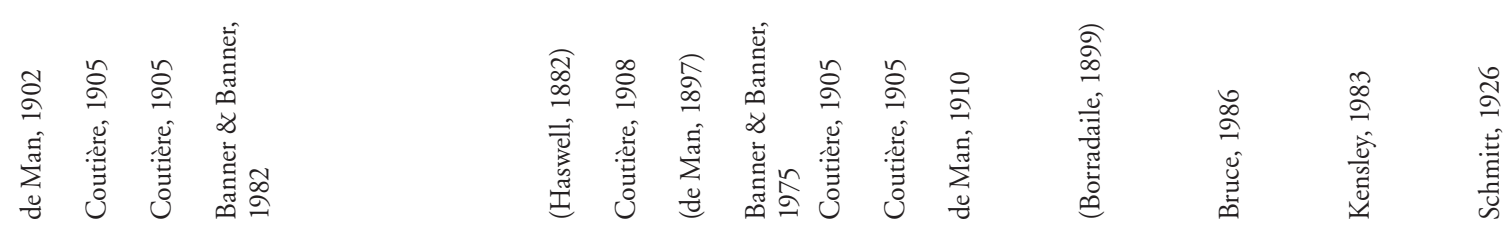

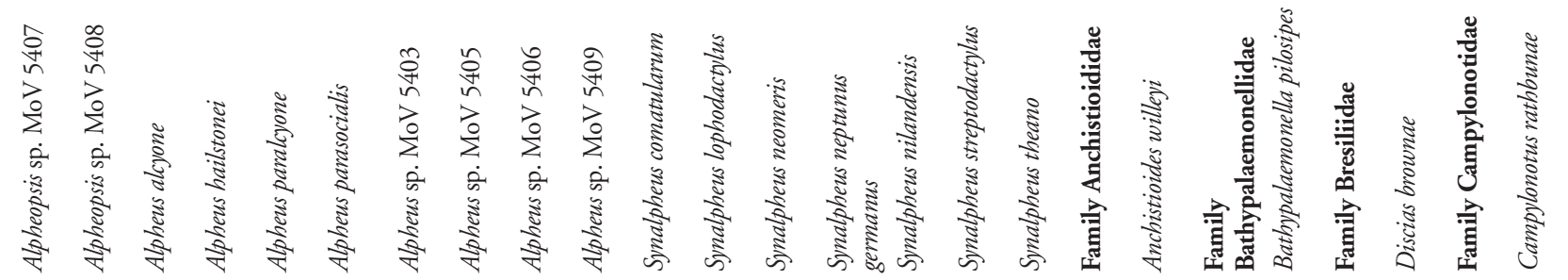




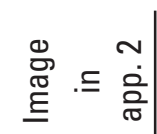

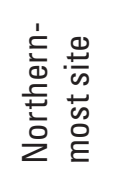

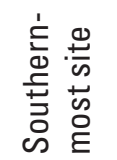

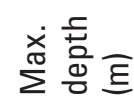

它营总

离产峷

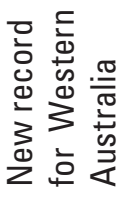

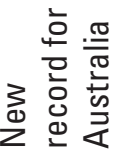

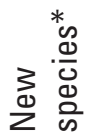

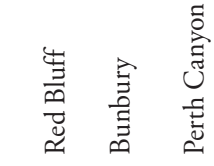

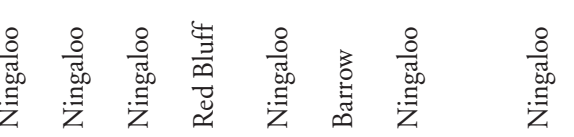

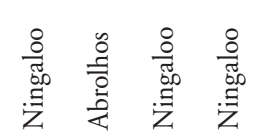

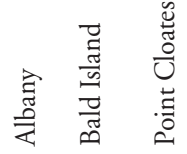

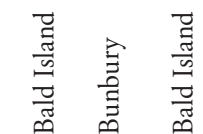

品

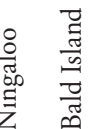

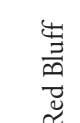

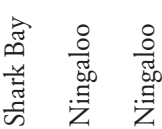

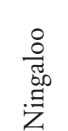

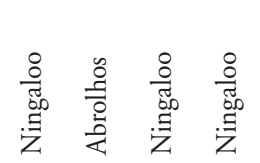

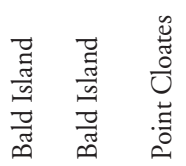

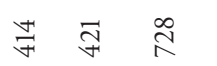

ळे

$\stackrel{\circ}{\stackrel{0}{0}=}$

త

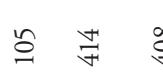

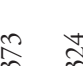

ริ

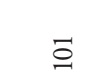

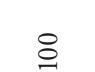
.

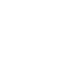

.

.

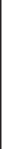

这离

辛

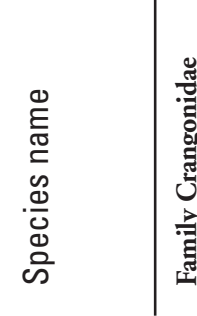

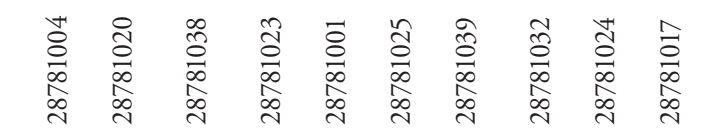

$\vec{\circ}$
$\stackrel{2}{n}$
$\infty$
d

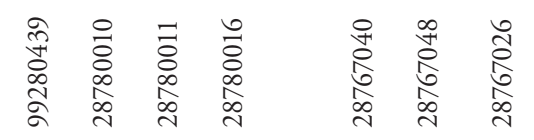

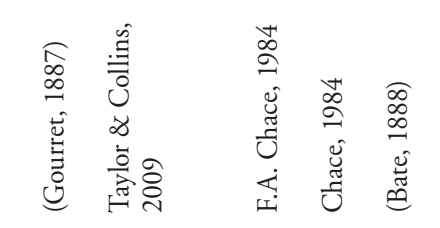

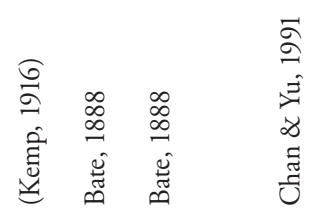

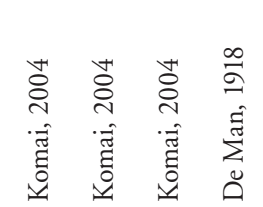

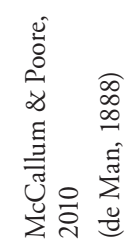

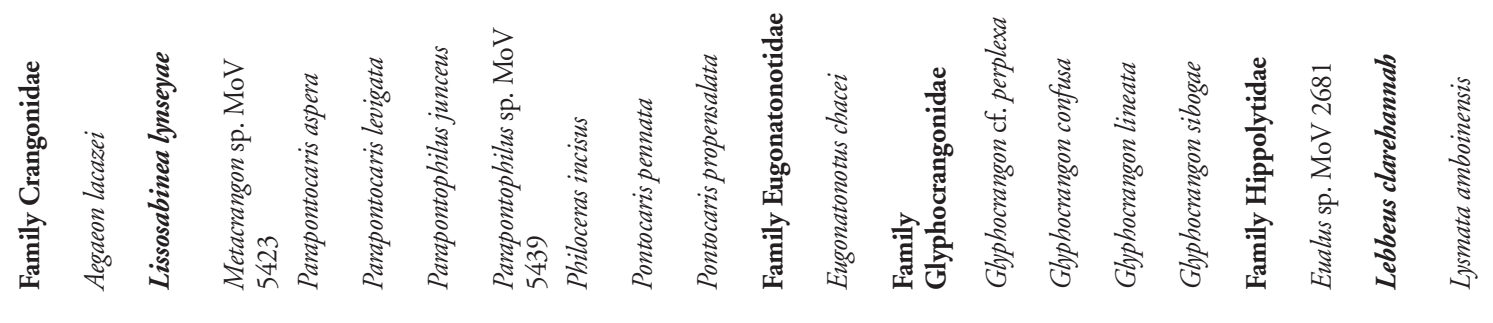




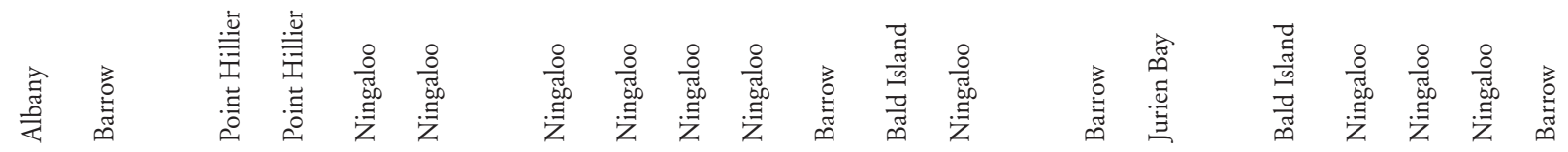

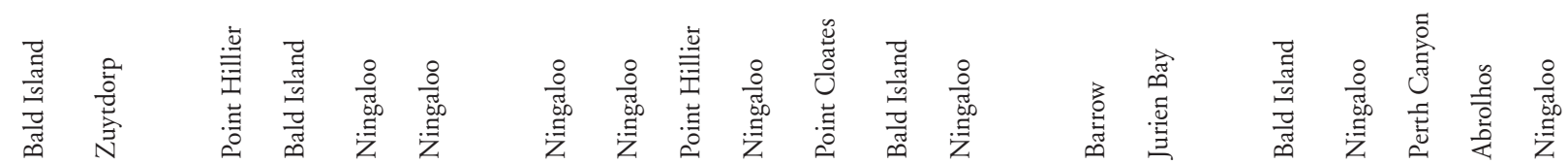

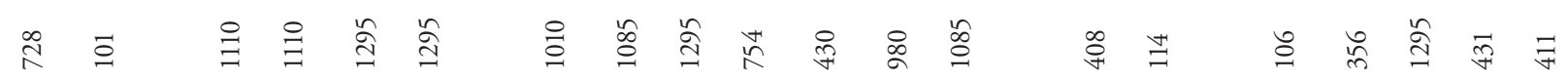
\&్ర

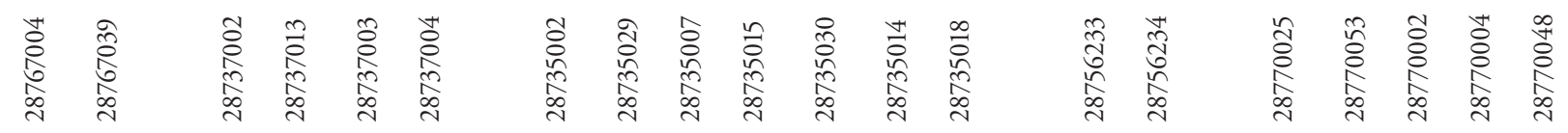

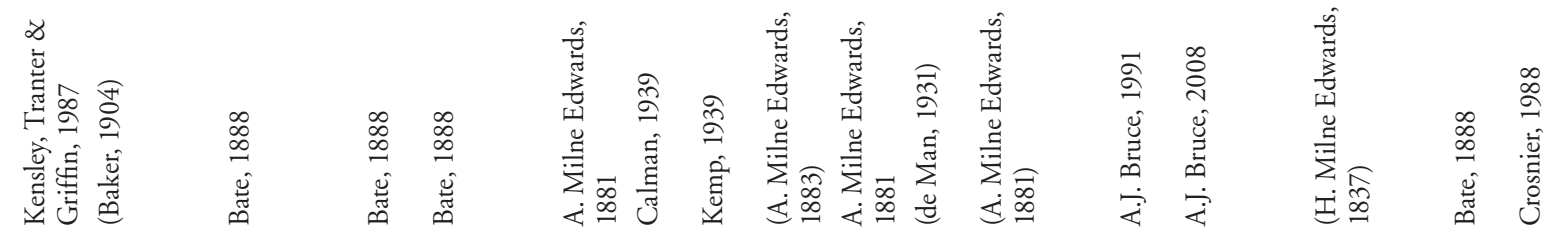

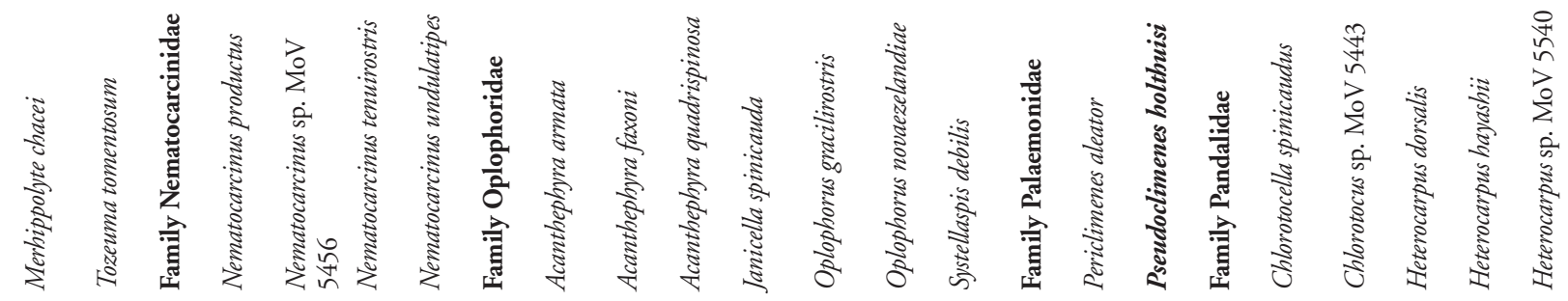




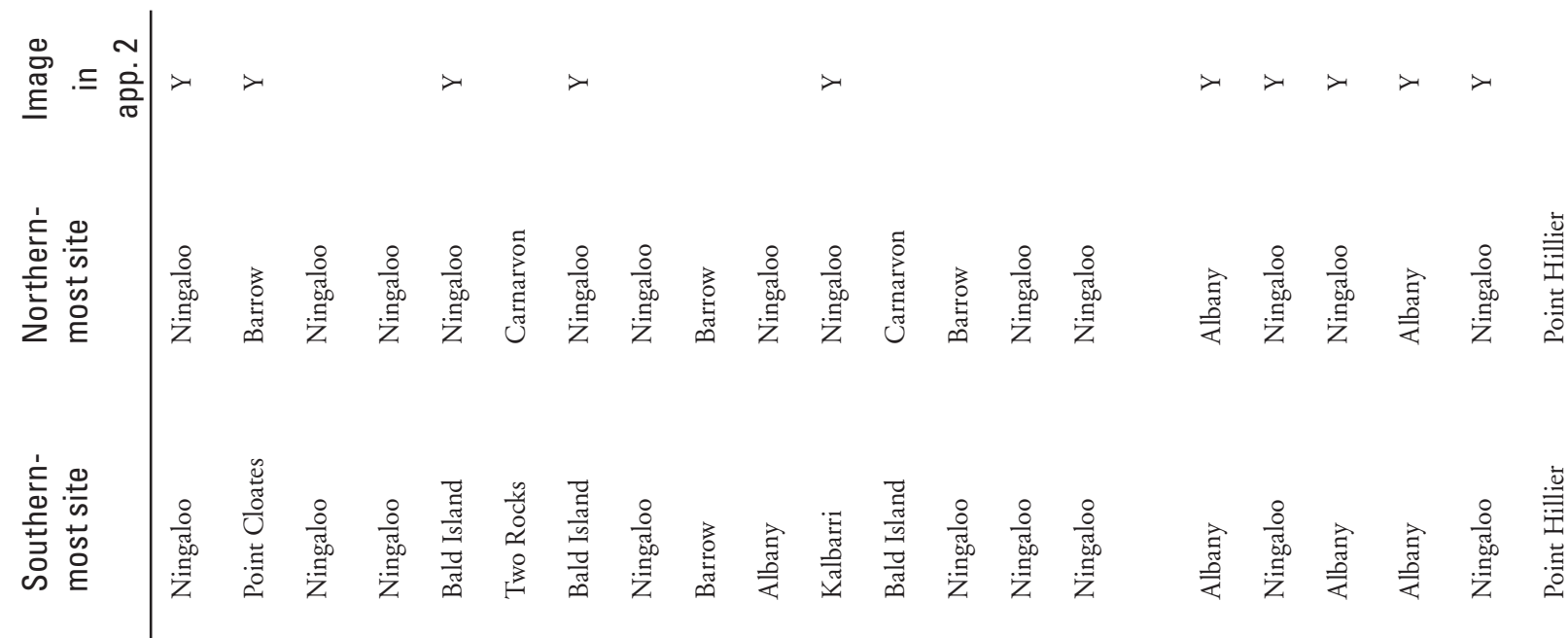

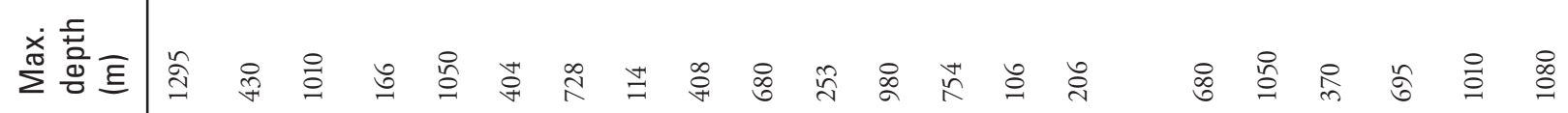

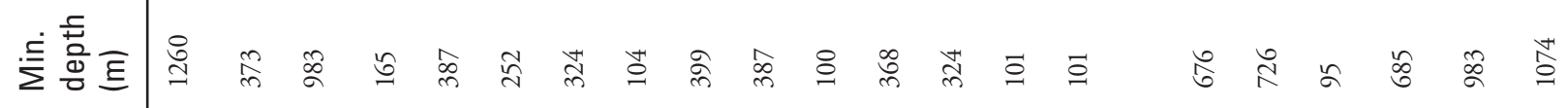

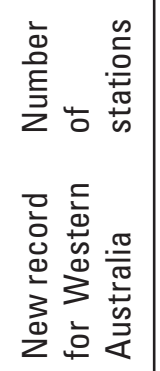

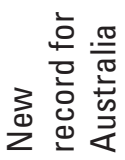

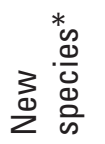

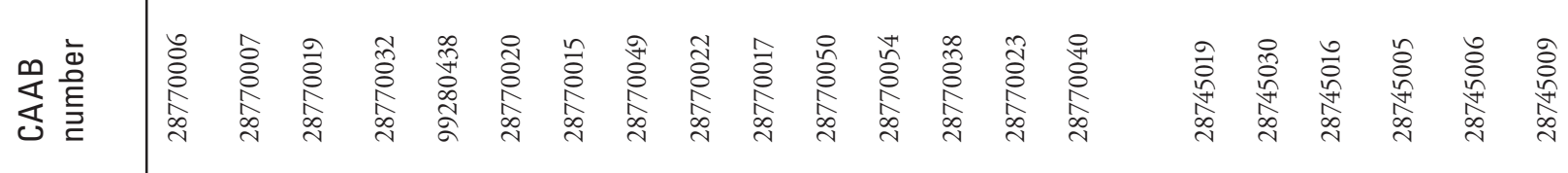

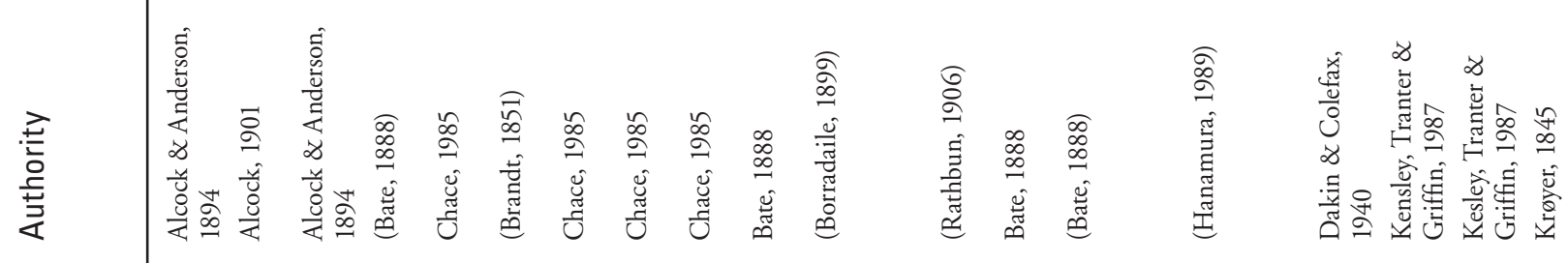

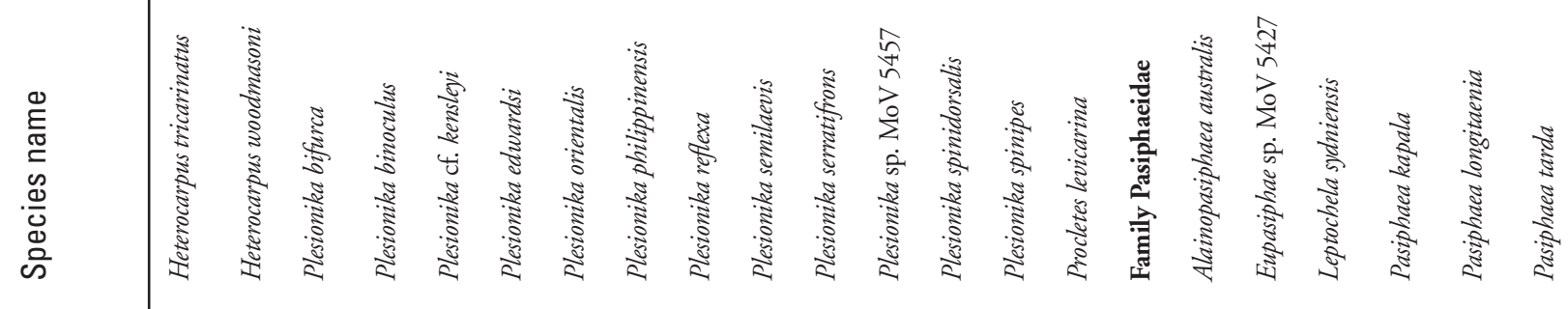




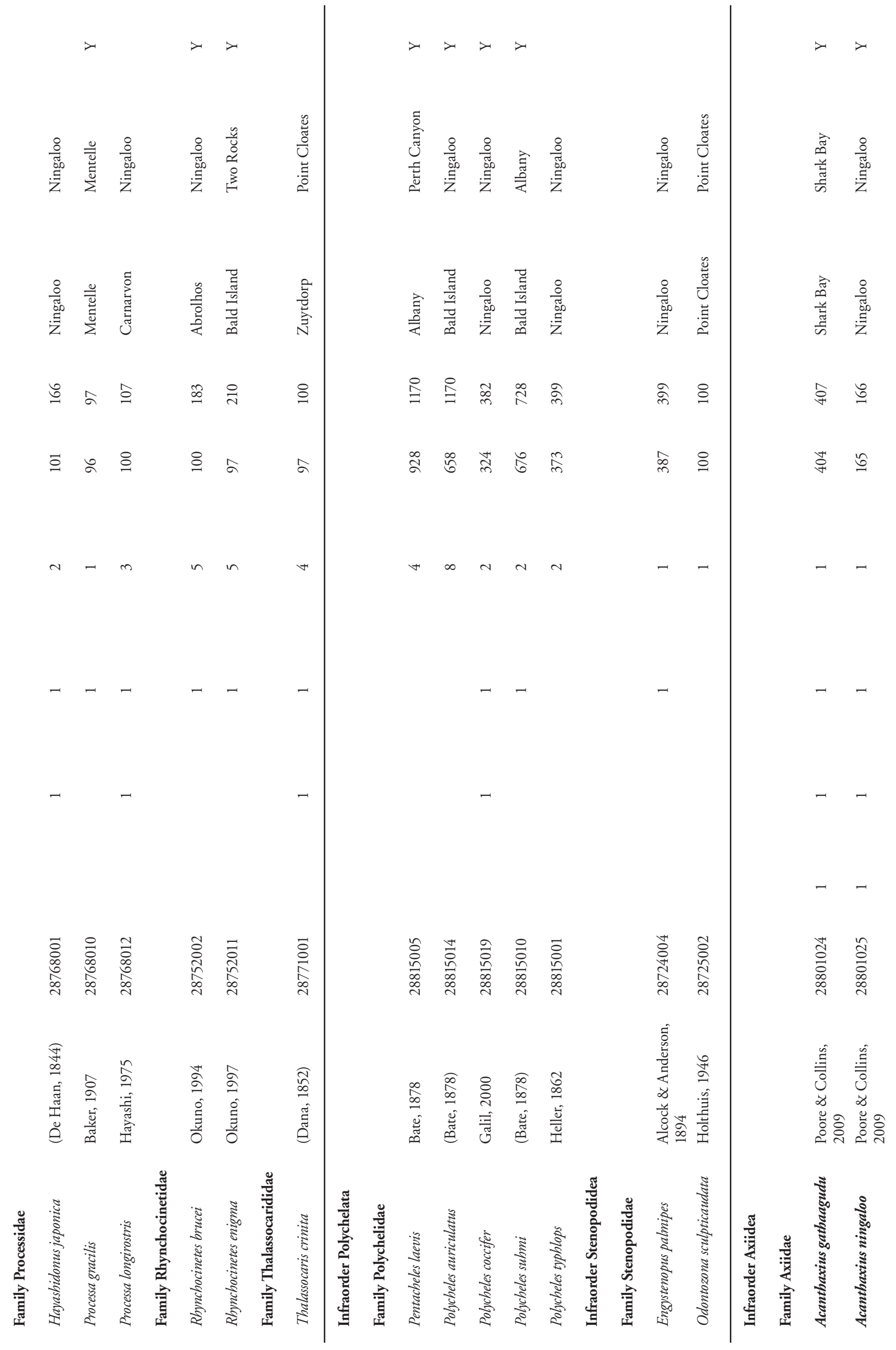




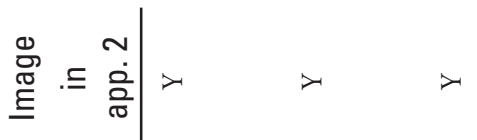

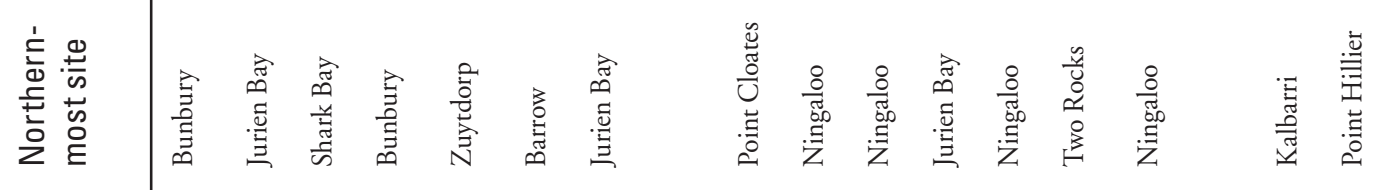

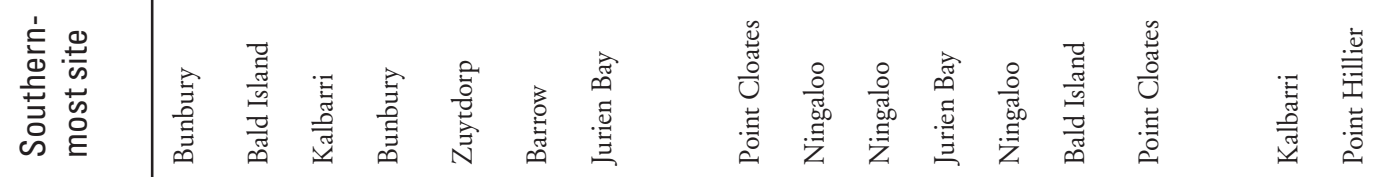

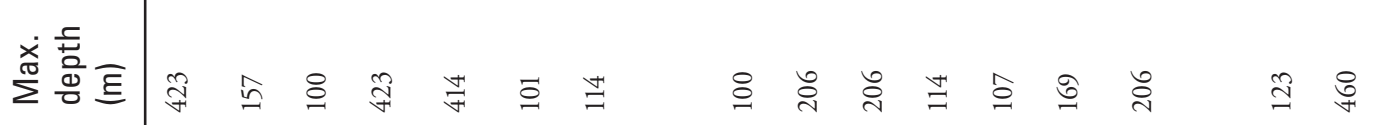

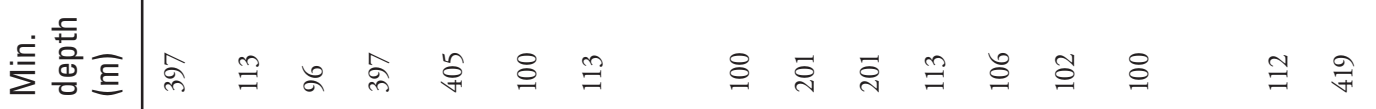

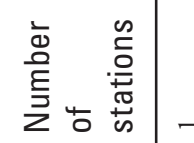

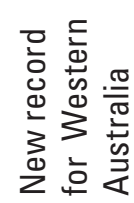

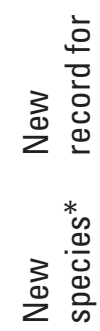

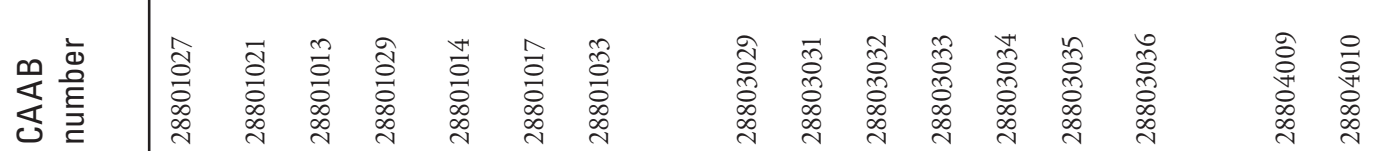

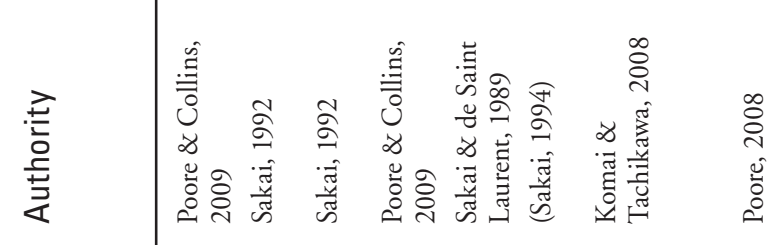

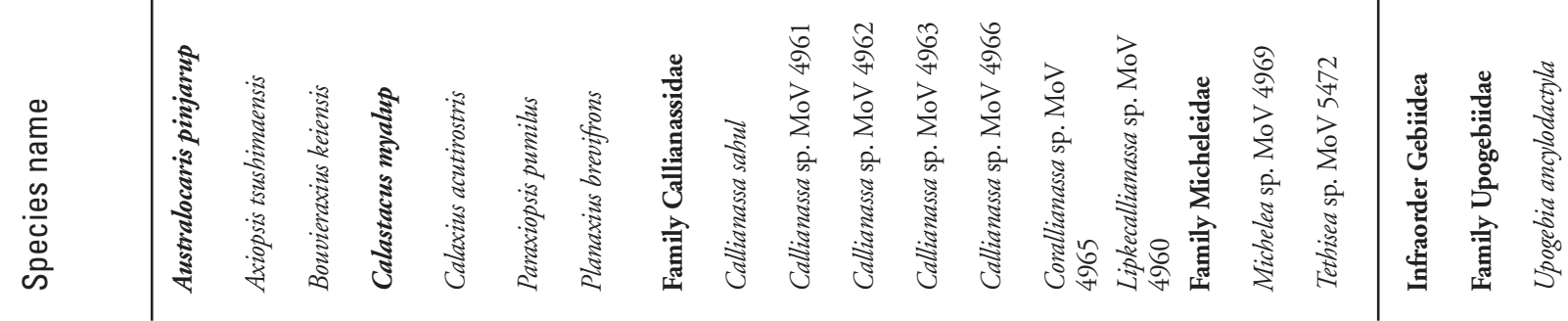




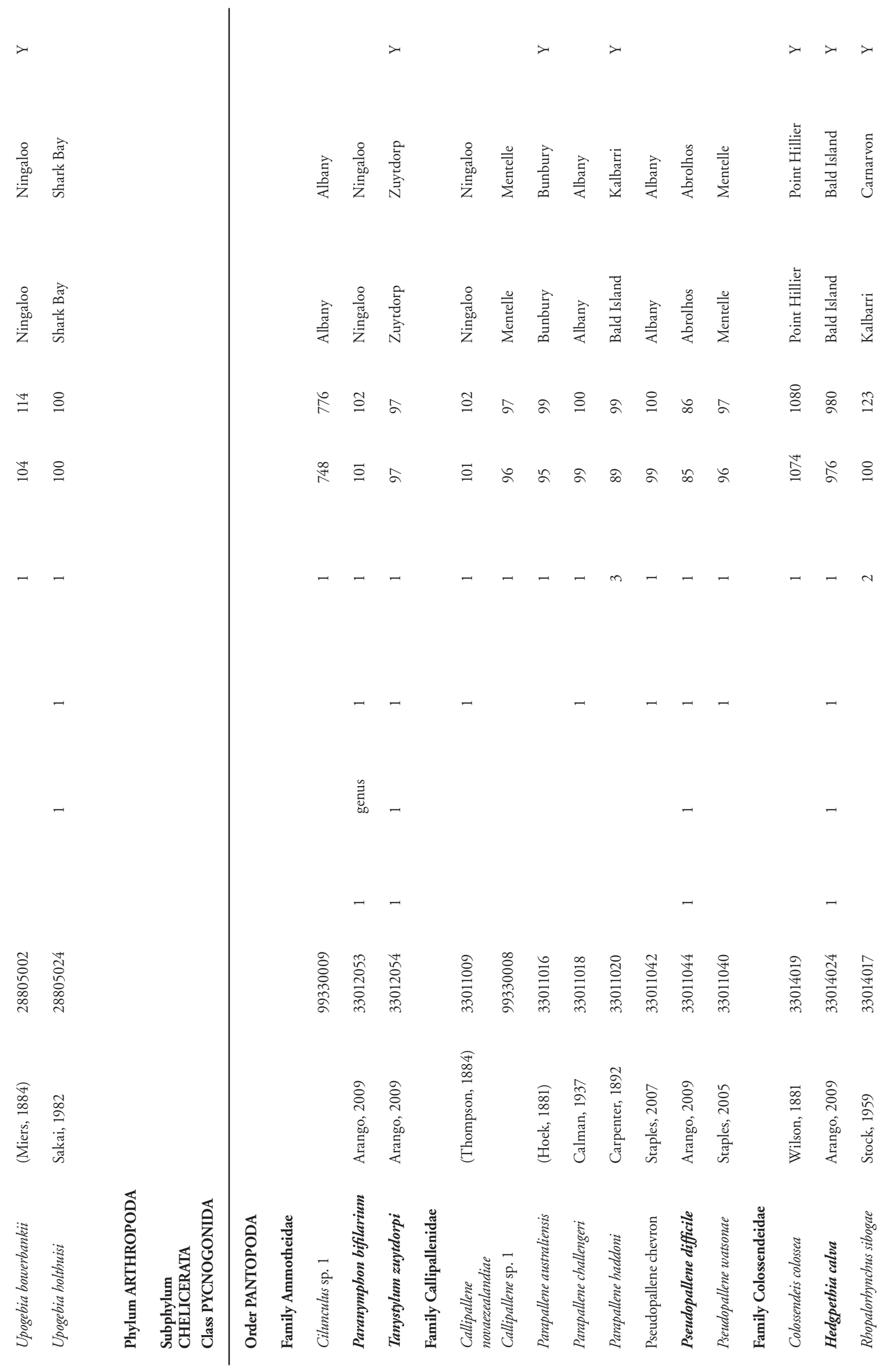




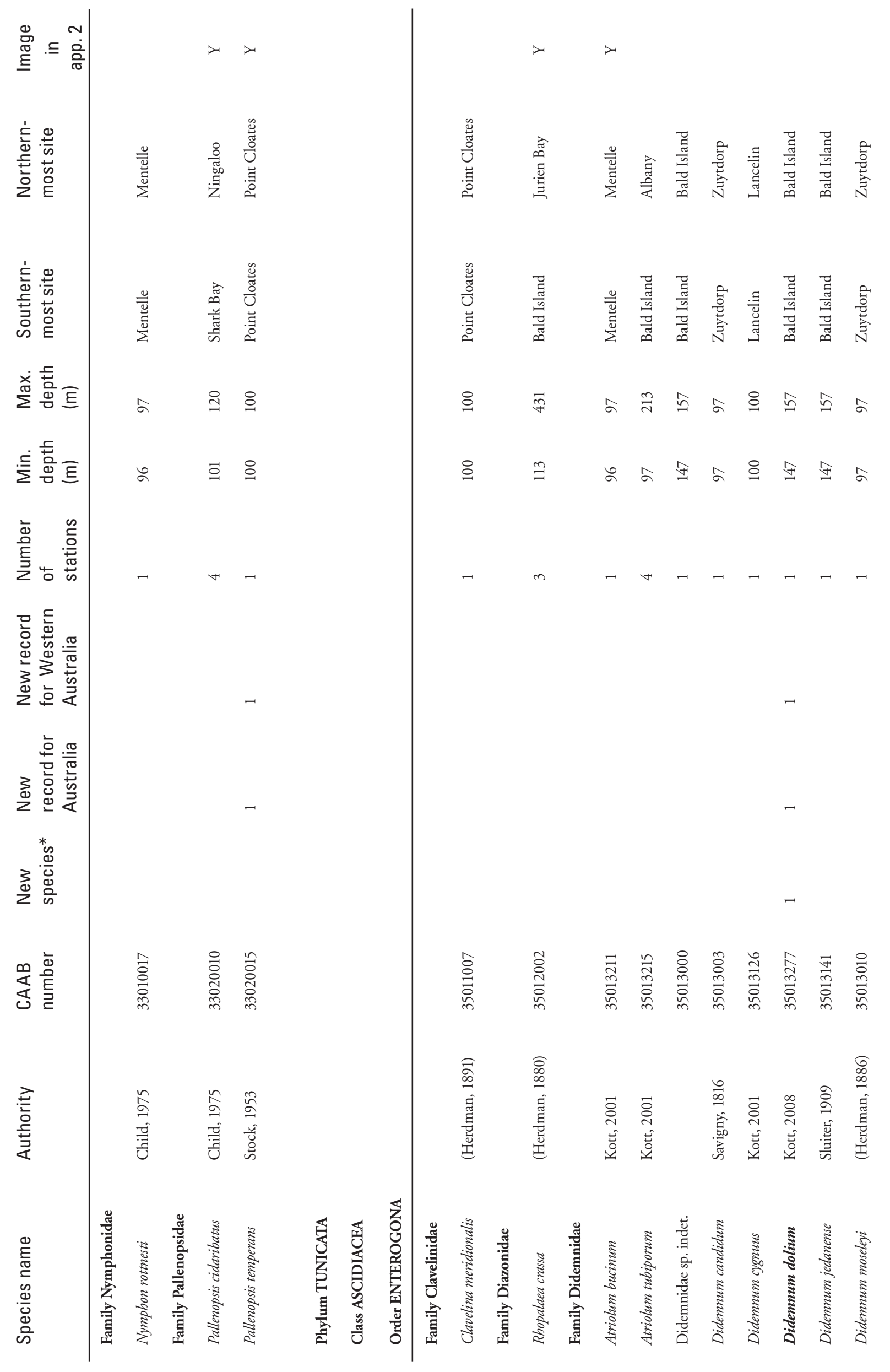




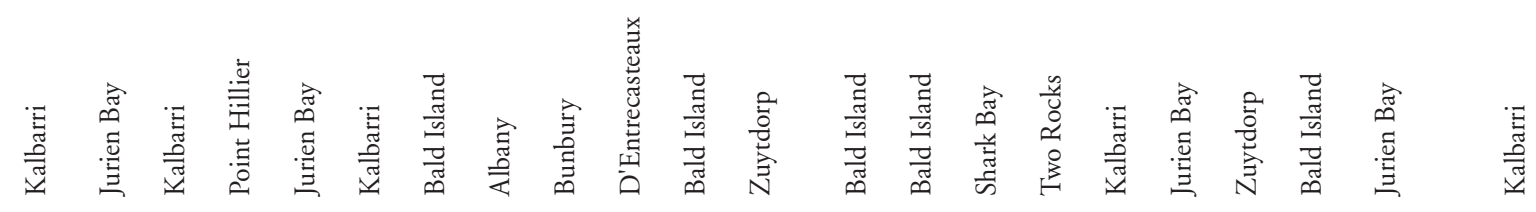

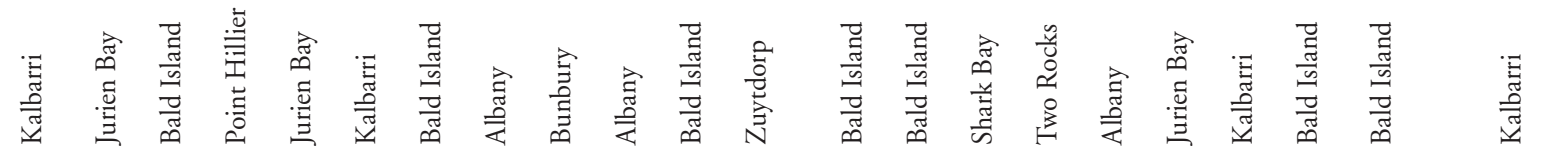

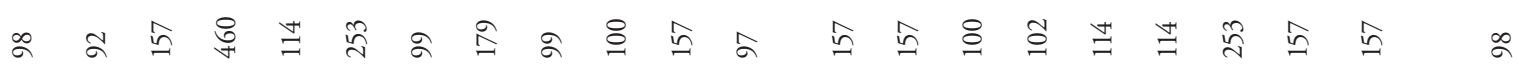

ฉ

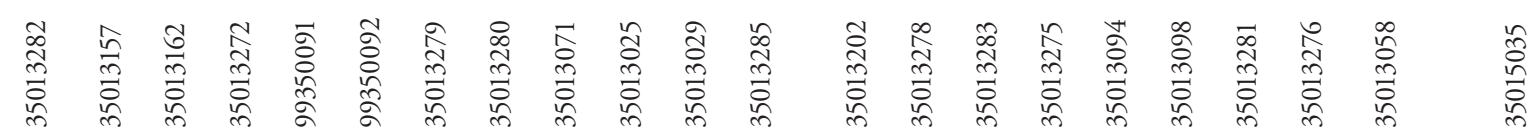

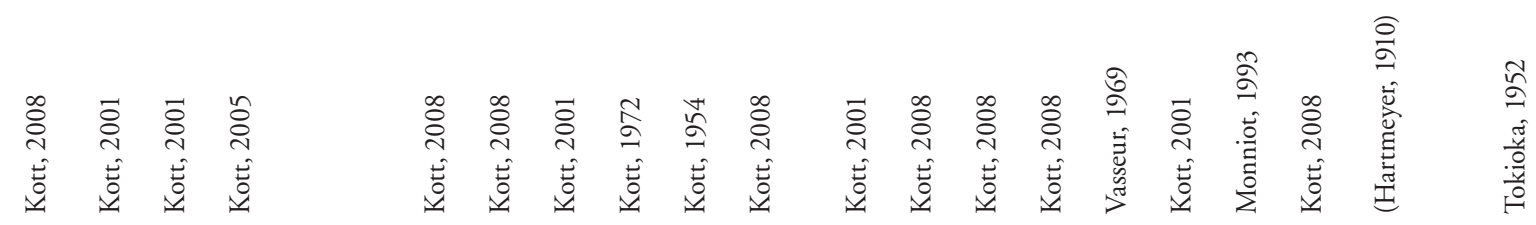

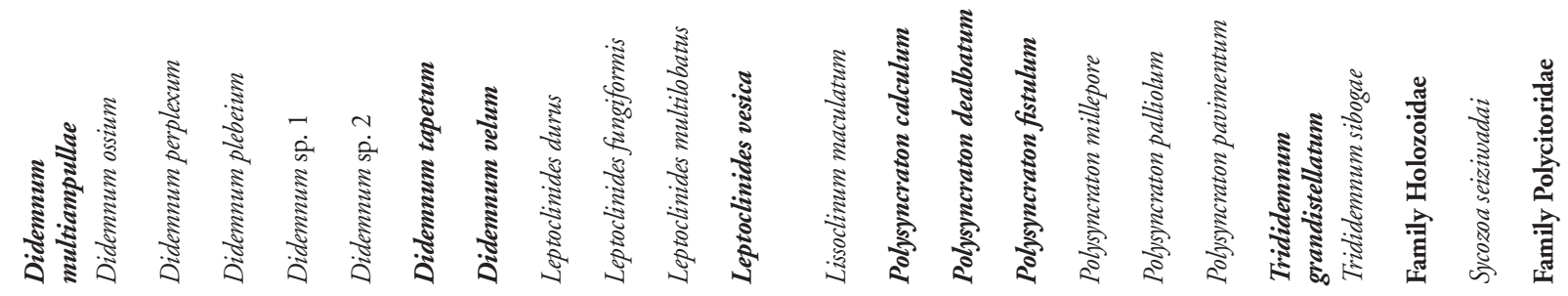




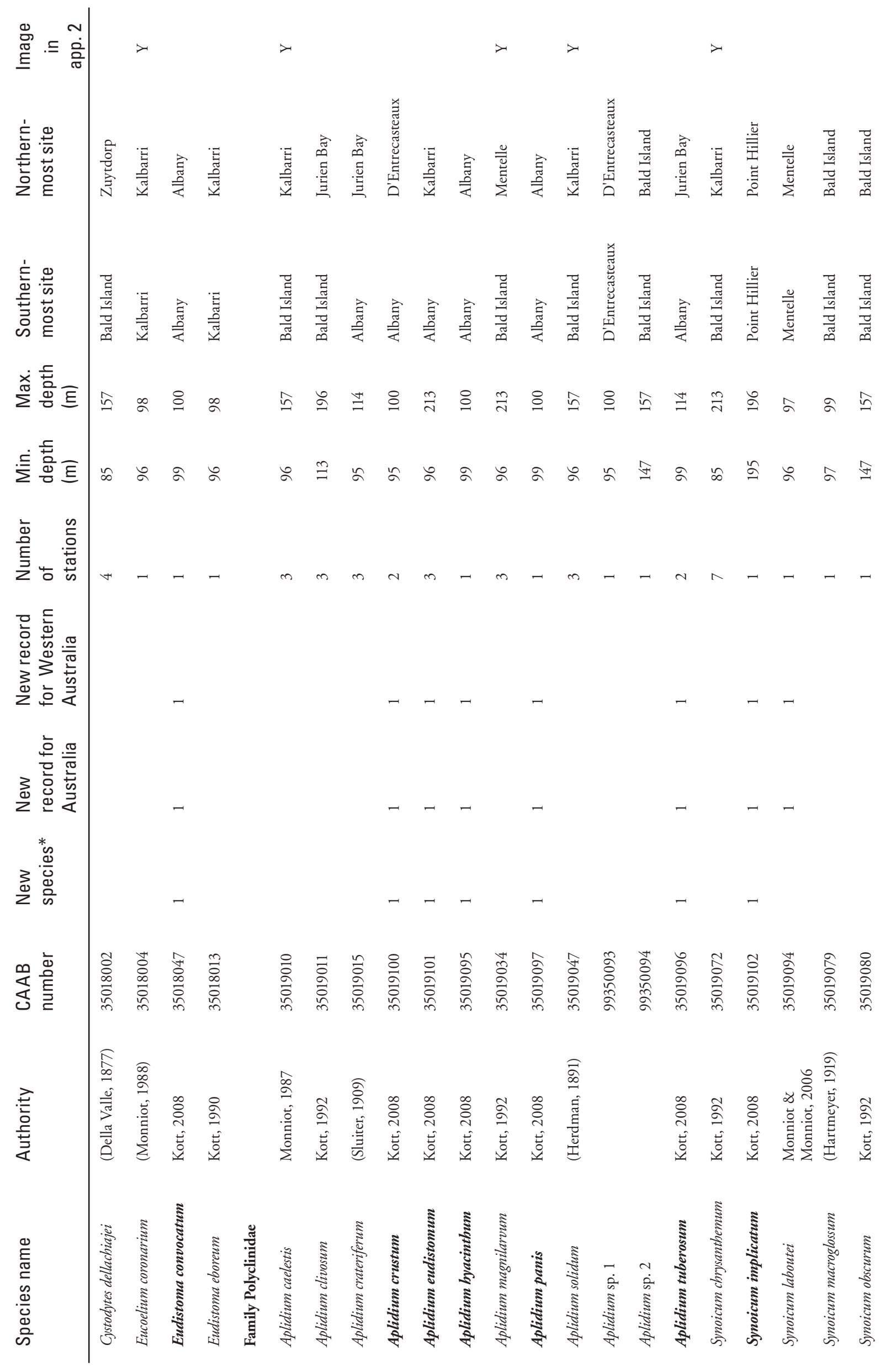




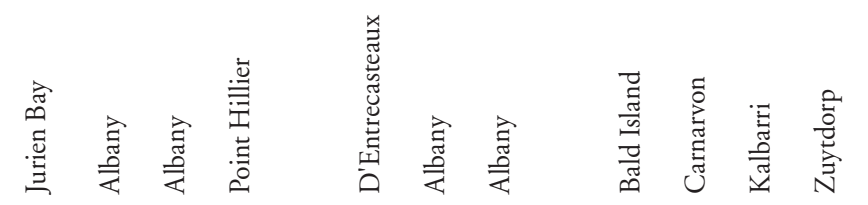

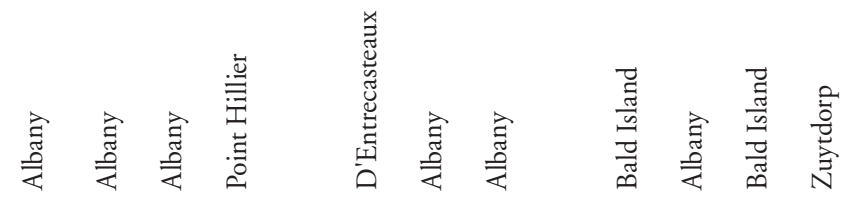

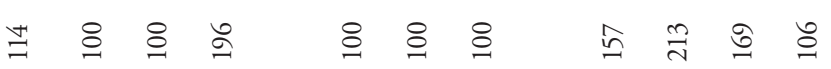

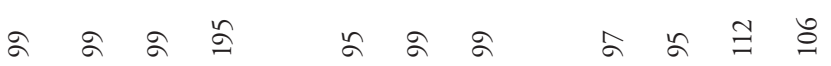

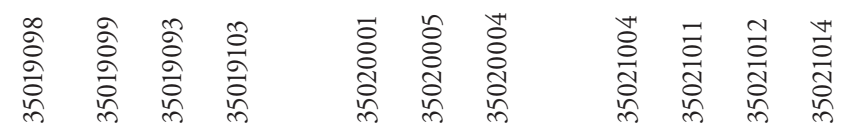

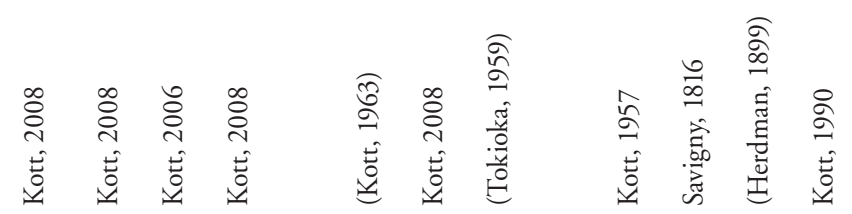

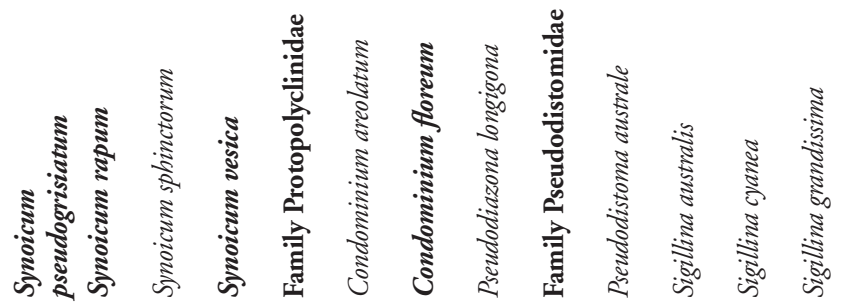

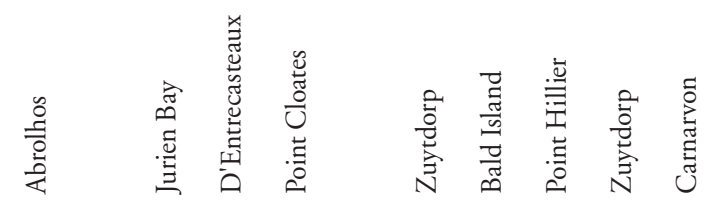

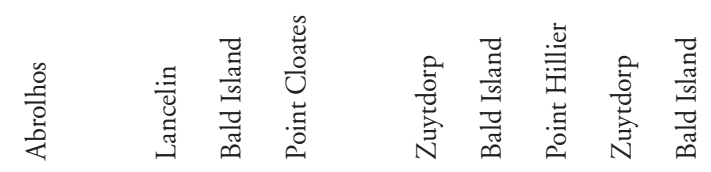

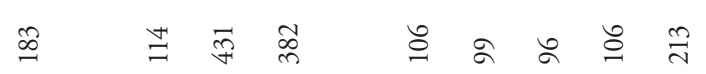

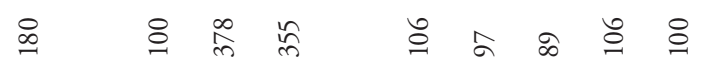

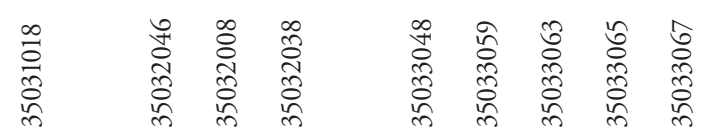

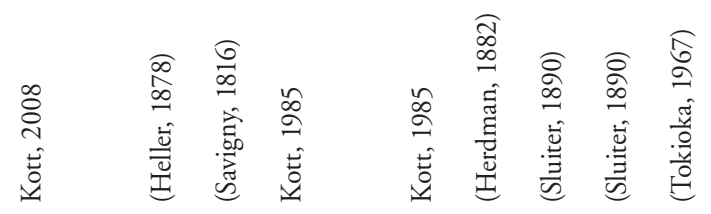

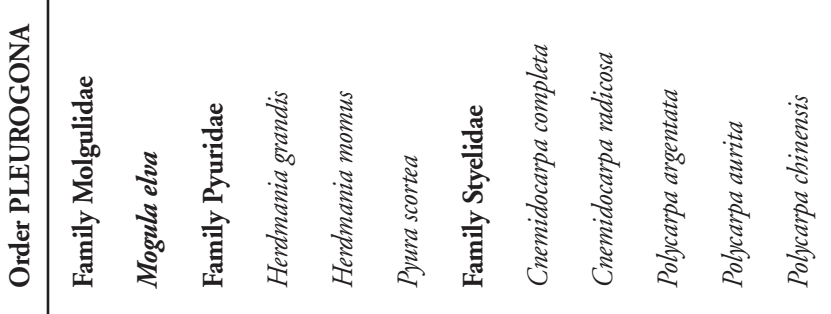




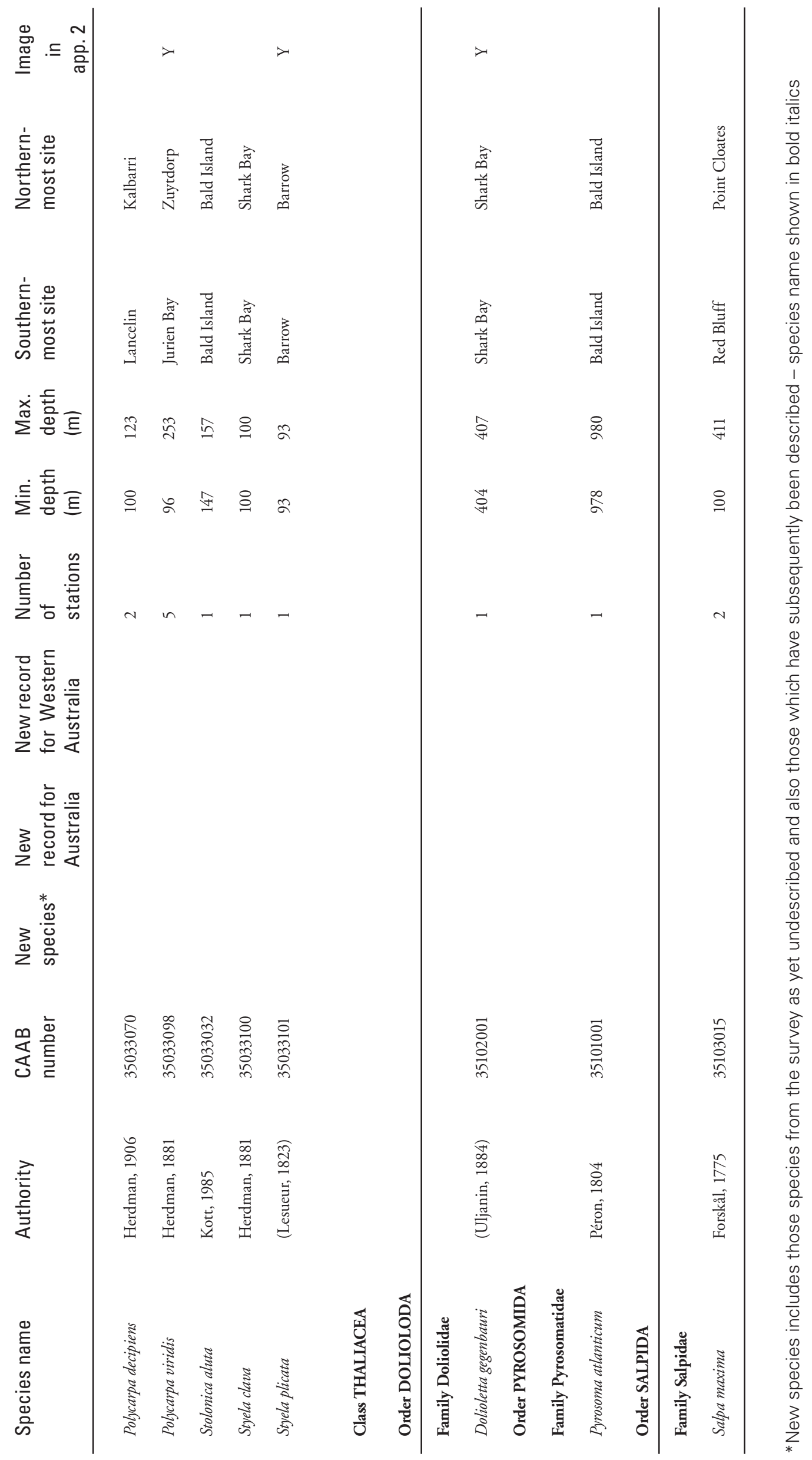


APPENDIX 2 Gallery of live colour images of over half the species of invertebrate megabenthos identified from the western continental margin of Australia.

Note: Image names show the CAAB number plus the species name - this can be matched to Appendix 1 for further information each the species. Note not all species are photographed due to the level of rough sorting permissible at sea.

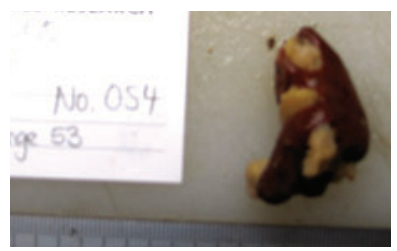

99100382-Plakinastrella-sp.WAMSS2

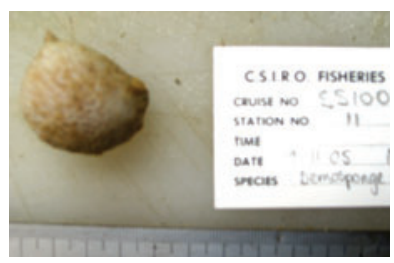

99100258-Craniella-sp.WAMSSI

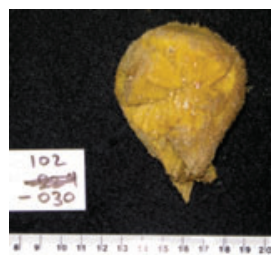

99100259-Cinachyrella-cf.-australiensis

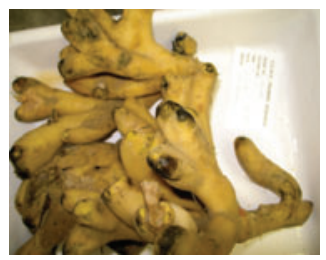

99100390-Rhabdastrella-sp.WAMSS3

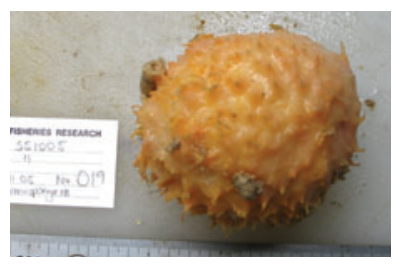

99100394-Psammastra-sp.WAMSSI

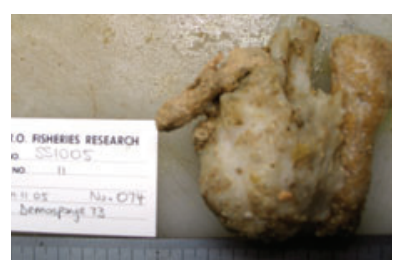

99100409-Tethyopsis-sp.WAMSS I

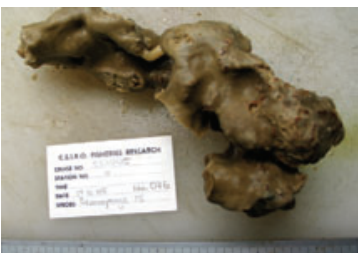

99100383-Plakinastrella-sp.WAMSS

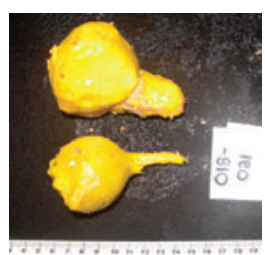

99100385-Cinachyrella-sp.WAMSS3

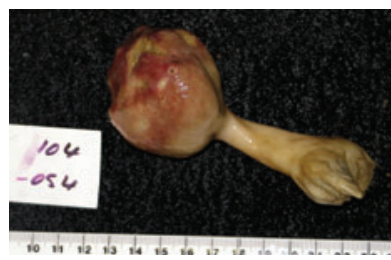

99100389-Craniella-sp.WAMSS2

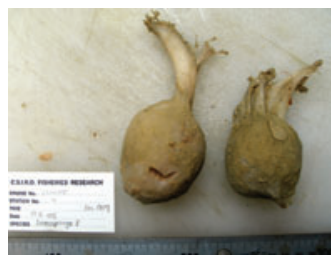

99100391-Stelletta-sp.WAMSS I

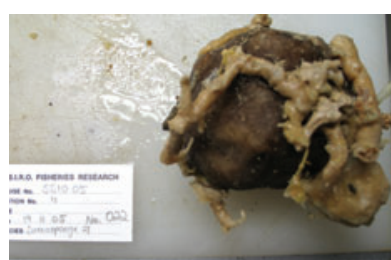

99100395-Ancorina-sp.WAMSS I

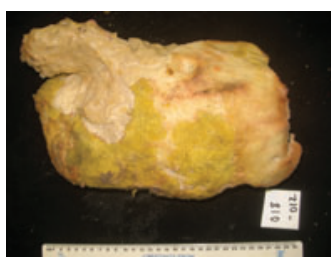

99100397-Asteropus-sp.WAMSS2

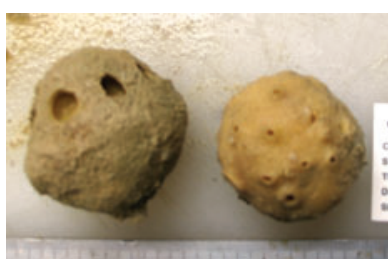

99100384-Cinachyrella-sp.WAMSS2

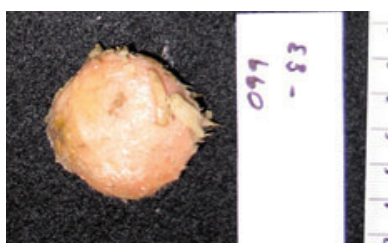

99100386-Cinachyrella-sp.WAMSS4

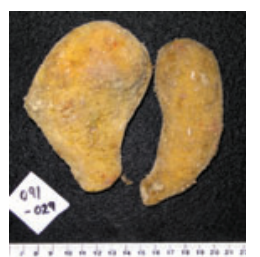

10009010-Asteropus-simplex

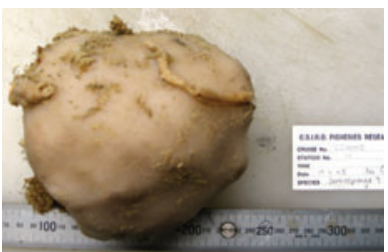

99100392-Ecionemia-sp.WAMSS2

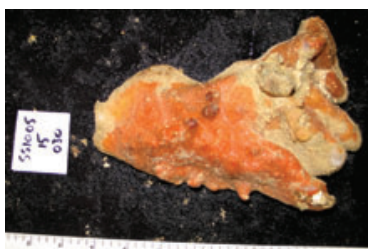

99100399-Asteropus-sp.WAMSS4

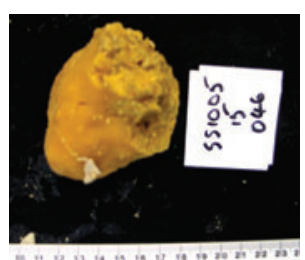

99100354-Asteropus-sp.WAMSS ।

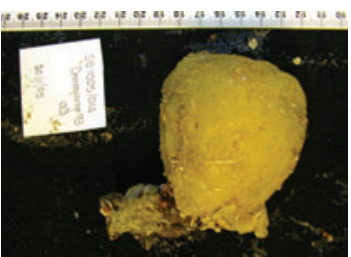

99100387-Cinachyrella-sp.WAMSS

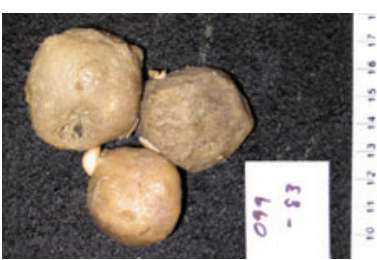

99100388-Tetilla-sp.WAMSSI

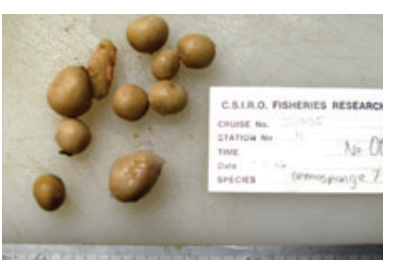

I0009023-Stelletta-clavosa

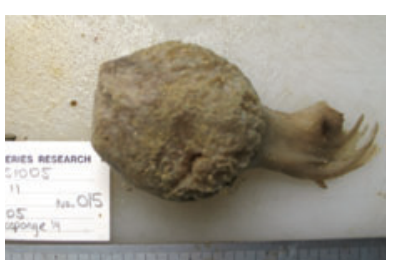

99100393-Rhabdastrella-sp.WAMSSI

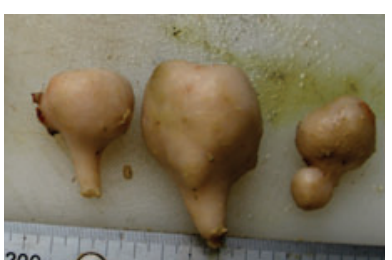

9910040 I-Stelletta-sp.WAMSS2

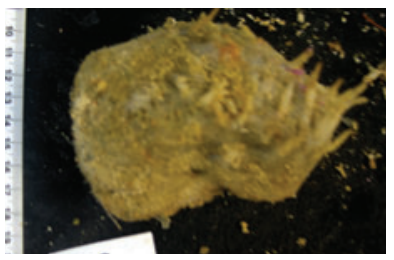

99100398-Asteropus-sp.WAMSS3 


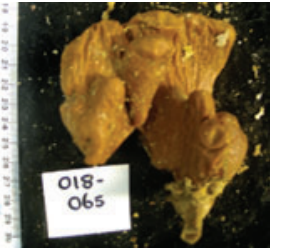

99100410-Penares-sp.WAMSSI

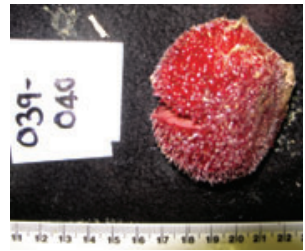

99100286-Psammastra-sp.WAMSS2

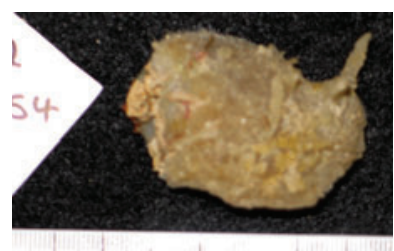

991004।3-Jaspis-sp.WAMSS I

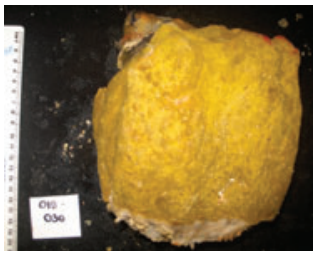

9910042I-Rhabdastrella-sp.WAMSS4

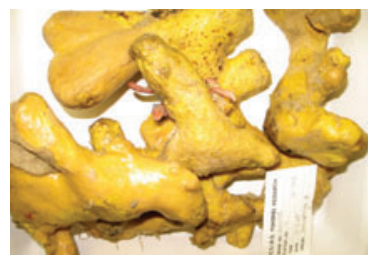

99100423-Geodia-sp.WAMNGI

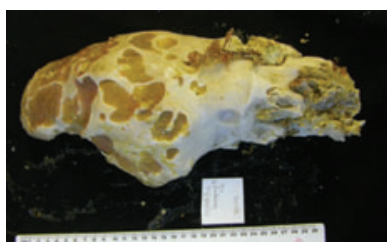

99100425-Geodia-sp.WAMSS2

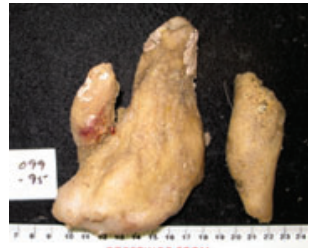

99100403-Stelletta-sp.WAMSS4

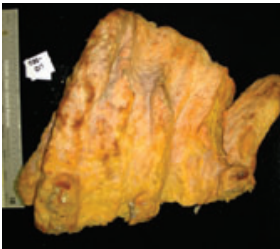

99100396-Ancorina-sp.WAMSS2

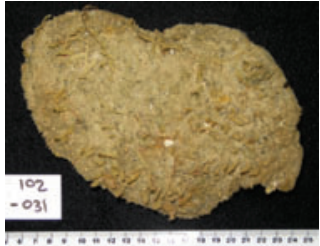

99|004|4-Jaspis-sp.WAMSS2

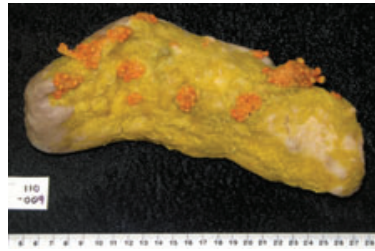

991004 I6-Jaspis-sp.WAMSS4

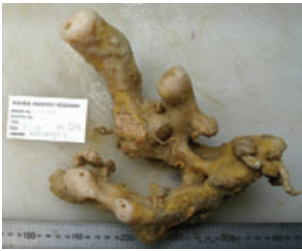

99100426-Isops-sp.WAMSSI

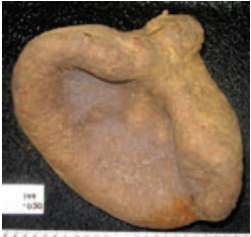

99100263-Erylus-sp.WAMSSI

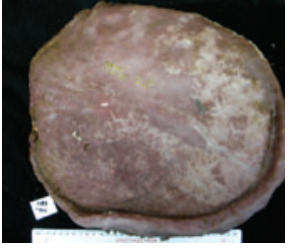

99100402-Stelletta-sp.WAMSS3

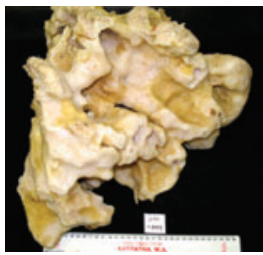

99100400-Asteropus-sp.WAMSS5

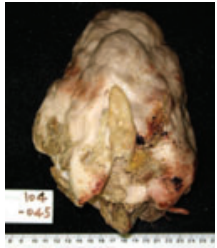

99100407-Stelletta-sp.WAMSS8

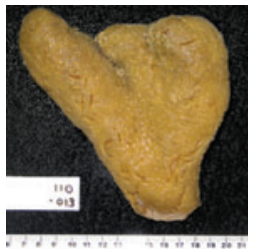

99100422-Asteropus-cf.-simplex

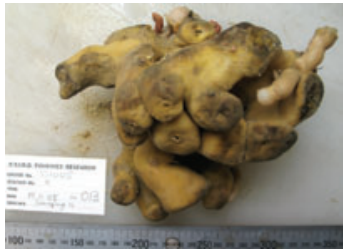

99100424-Geodia-sp.WAMSS I

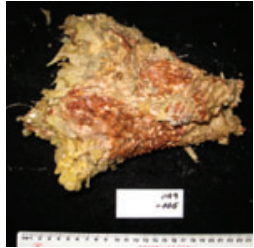

99100428-Isops-sp.WAMSS3

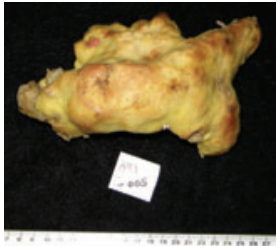

99100412-Ecionemia-sp.WAMSS3

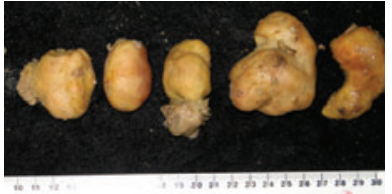

99|004 | I-Ecionemia-sp.WAMSSI

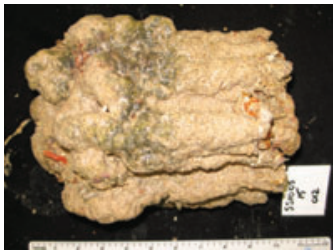

991004 I7-Jaspis-sp.WAMSS5

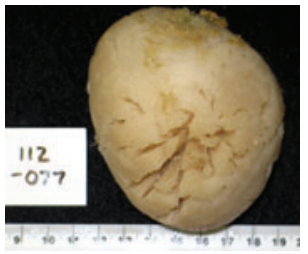

99100292-Stelletta-sp.WAMSS9

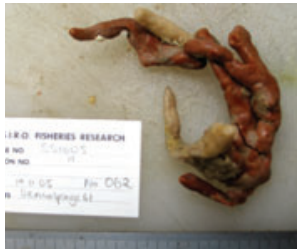

99100427-Isops-sp.WAMSS2

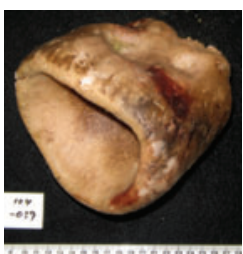

9910024|-Erylus-sp.WAMSS2 


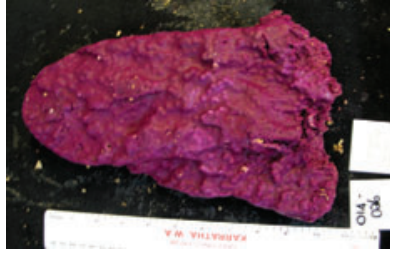

10021022-Spheciospongia-purpurea

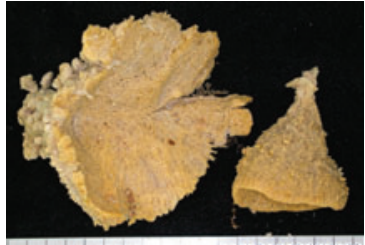

99100433-Hemiasterella-sp.WAMSS2

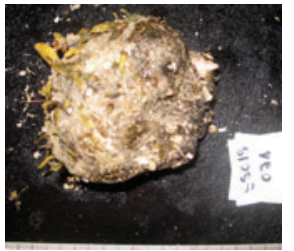

99100438-Polymastia-sp.WAMSS2

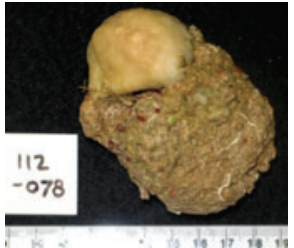

99100262-Polymastia-sp.WAMSS4

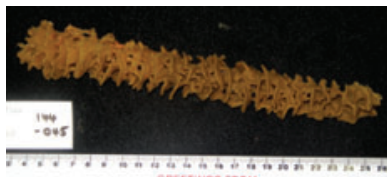

I0028007-Caulospongia-plicata

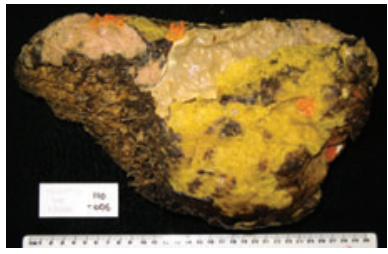

99100445-Stellitethya-sp.WAMSS I

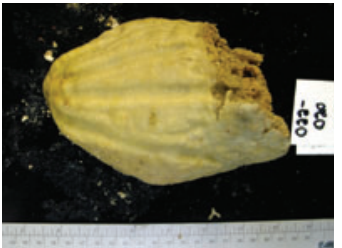

10021023-Spheciospongia-papillosa

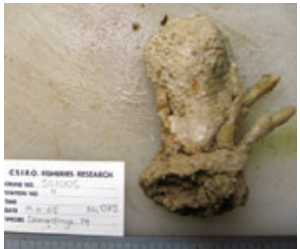

99100434-Sceptrella-sp.WAMSS I

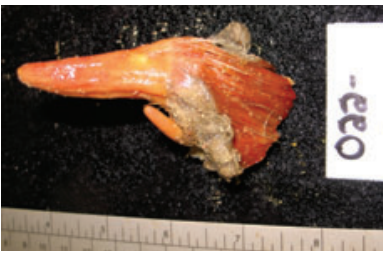

99|003 I9-Polymastia-sp.WAMSS3

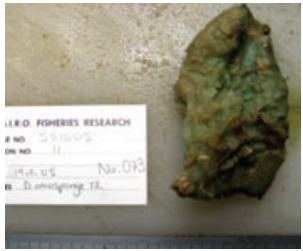

9910044I-Diplastrella-sp.WAMSS2

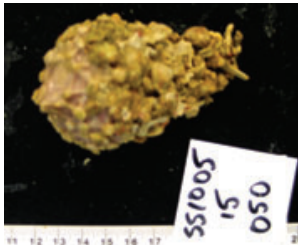

99100443-Tethyastra-sp.WAMSSI

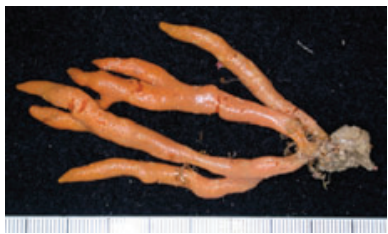

I003 |00 I-Trachycladus-laevispirulifer

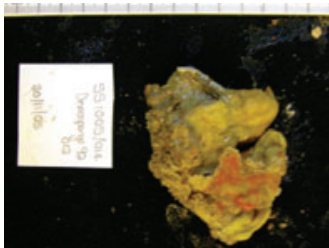

99100430-Spheciospongia-cf.-papillosa

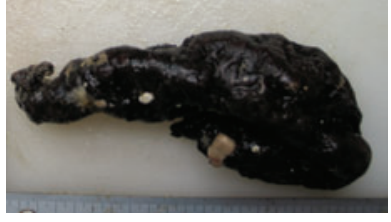

99100435-Sceptrella-sp.WAMSS2

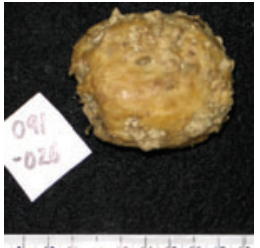

99100327-Polymastia-sp.WAMSSI

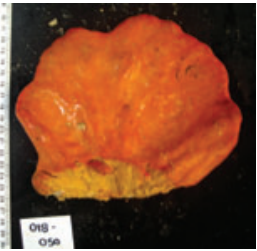

99100440-Diplastrella-sp.WAMSS I

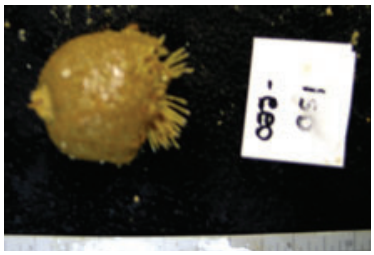

99100444-Tethyastra-sp.WAMSS2

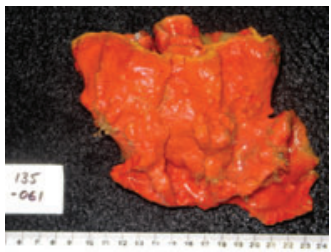

99100447-Theonella-sp.WAMSS

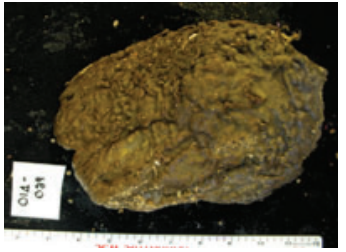

9910043I-Spheciospongia-sp.WAMMFI

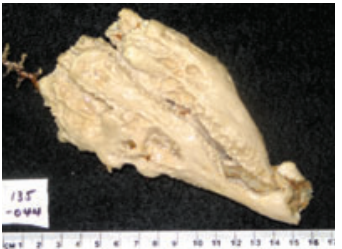

99100332-Latrunculia-sp.WAMSSI

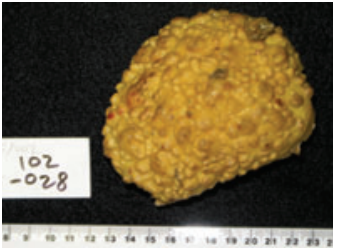

99100285-Polymastia-sp.WAMSS5

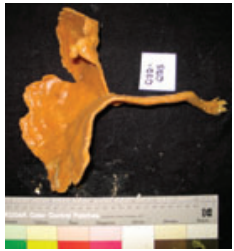

I0028003-Caulospongia-biflabellata

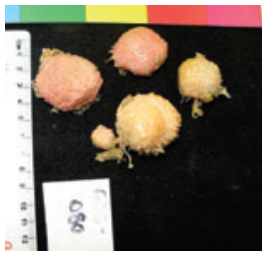

99100287-Tethya-cf.-ingalli

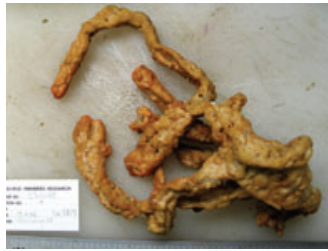

99100448-Agelas-sp.WAMSS I 


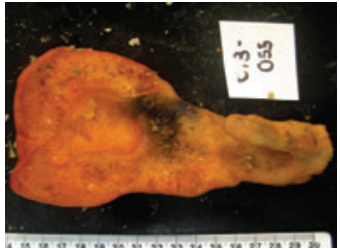

99100449-Agelas-sp.WAMSS2

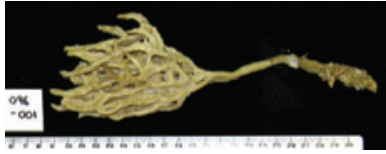

10066066-Clathria-(Thalysias)-abietina

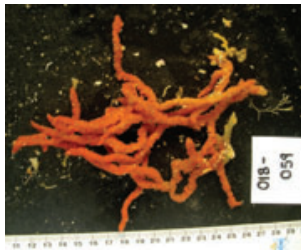

99100454-Clathria-(Thalysias) cf.-juniperina
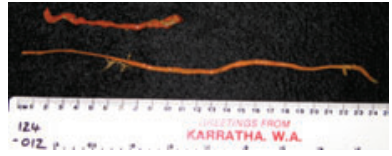

99100456-Clathria-(Thalysias) cf.-procera

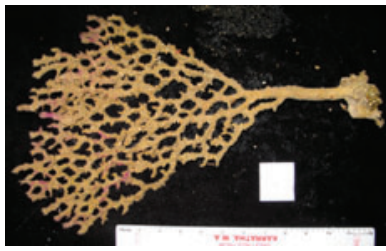

99100243-Echinodictyum-cf. cancellatum

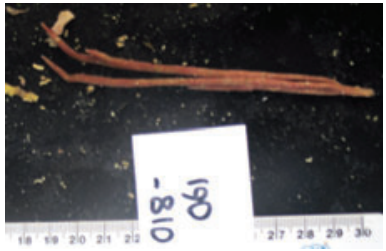

9910046I-Raspailia-(Parasyringella) sp.WAMSS2

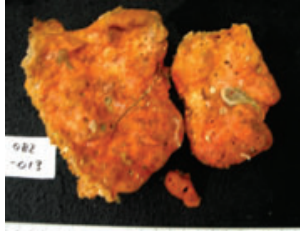

99100450-Agelas-sp.WAMSS3

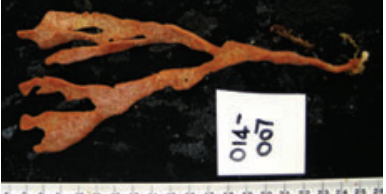

|0066 |3|-Echinoclathria-leporina

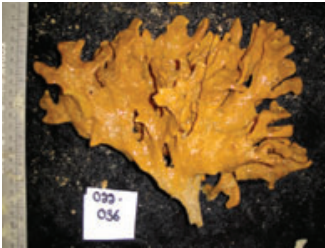

99100460-Clathria-(Clathria)-sp.WAMSS3

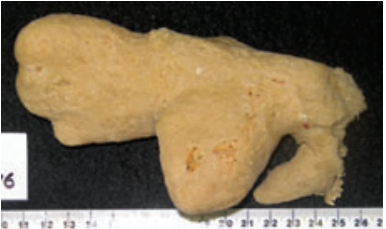

I0067040-Raspailia-(Clathriodendron) desmoxyiformis

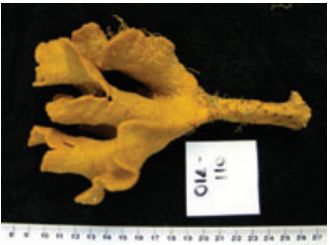

99100462-Raspailia-(Parasyringella) sp.WAMSS3

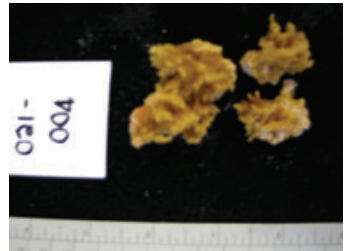

99100464-Raspailia-(Raspaxilla) sp.WAMSS3

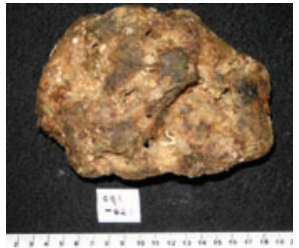

99100452-Zyzzya-sp.WAMSSI

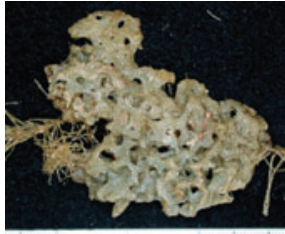

|0066|38-Holopsamma-arborea

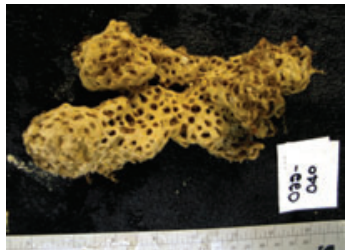

99100455-Holopsamma-sp.WAMSS2

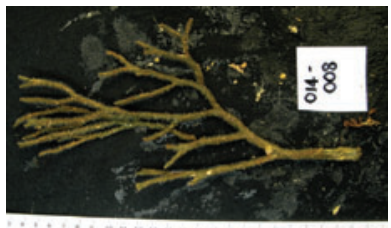

I0067055-Raspailia-(Raspailia)-vestigifera

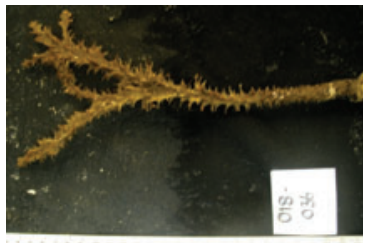

99100466-Raspailia-(Clathriodendron) sp.WAMSS I

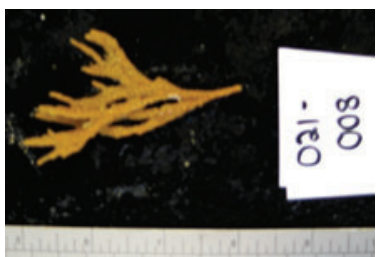

99100470-Ceratopsion-cf.-dichotoma

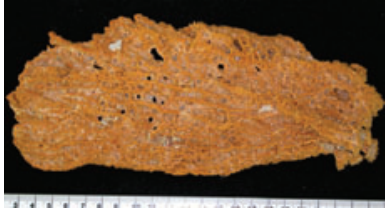

0066059-Clathria-selachia

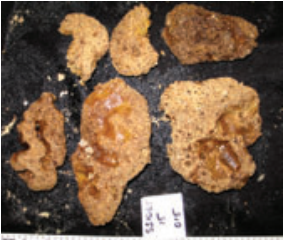

99100453-Holopsamma-sp.WAMSS I

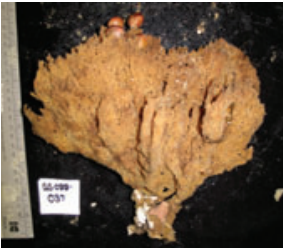

99100457-Clathria-(Thalysias)-sp.WAMSS

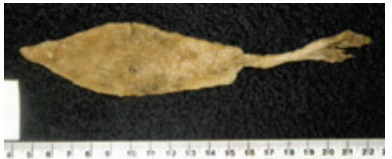

10067059-Trikentrion-flabelliforme

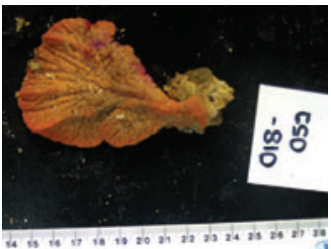

99100465-Raspailia-(Raspaxilla) sp.WAMNG I

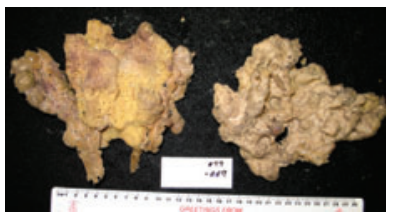

9910047I-Lithoplocamia-sp.WAMSS 


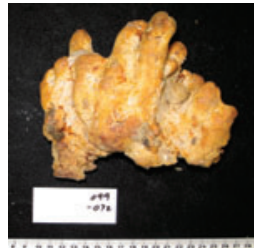

99100468-Aulospongus-sp.WAMSS2

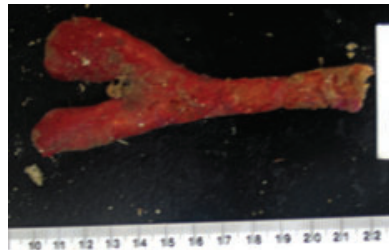

99100475-Fibulia-sp.WAMSS2

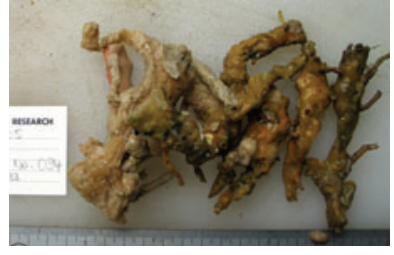

99100477-Coelosphaera-(Coelosphaera) sp.WAMSS2

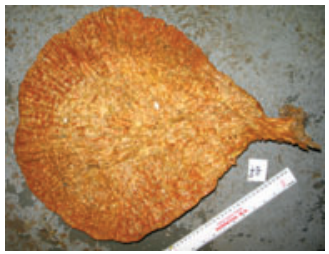

99100484-Monanchora-sp.WAMSSI

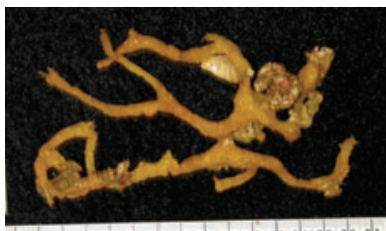

99100489-Crella-(Yvesia)-sp.WAMSSI

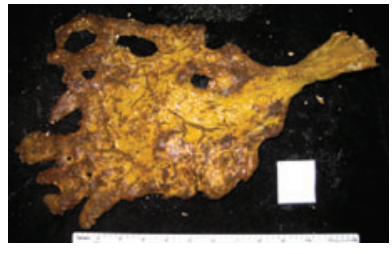

99100492-lotrochota-cf.-baculifera

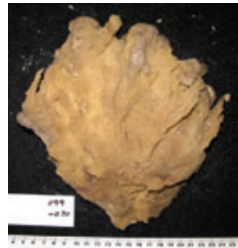

99100472-Rhabderemia-sp.WAMSS I

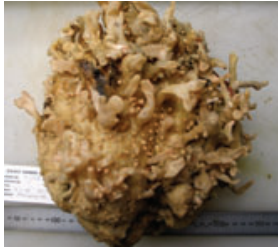

99100300-Coelosphaera-(Coelosphaera) sp.WAMSS3

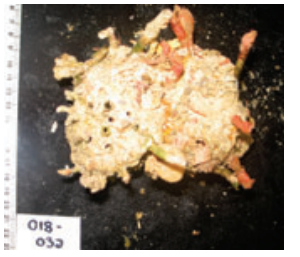

99100476-Coelosphaera-(Coelosphaera) sp.WAMSSI

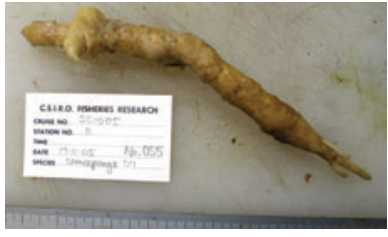

99100485-Crella-(Pytheas)-sp.WAMSS I

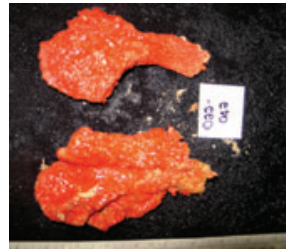

9910049|-Phorbas-sp.WAMNG I

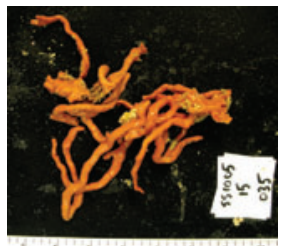

99100496-Chondropsis-sp.WAMSSI

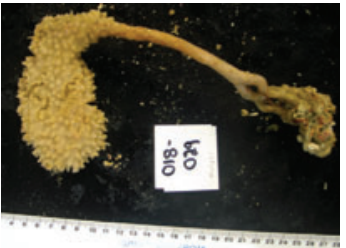

99100473-Desmacidon-sp.WAMSS I

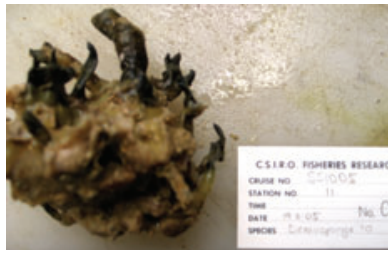

99100479-Coelosphaera-(Coelosphaera) sp.WAMSS5

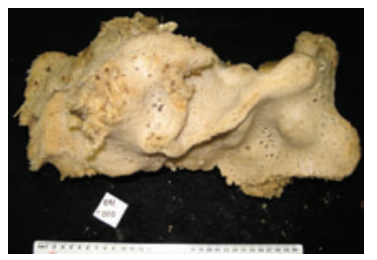

99100482-Coelosphaera-(Coelosphaera) sp.WAMSS8

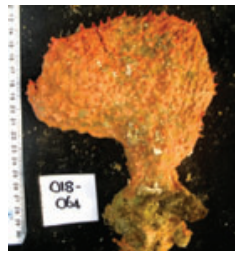

99100486-Crella-(Crella)-sp.WAMSSI

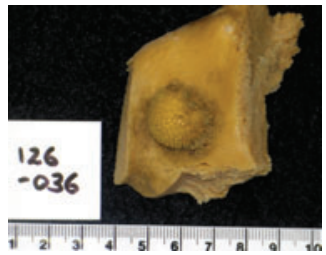

99100363-Phorbas-sp.WAMSSI

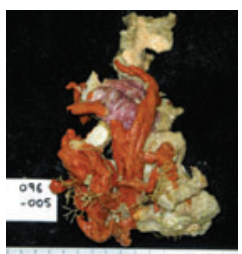

99100495-Chondropsis-sp.WAMNG I

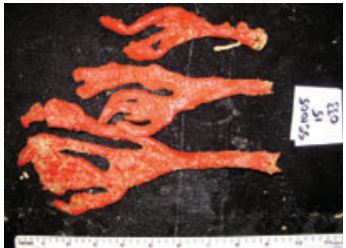

99100474-Fibulia-sp.WAMSSI

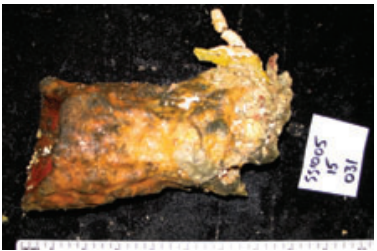

99100478-Coelosphaera-(Coelosphaera) sp.WAMSS4

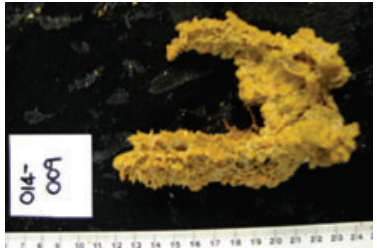

99100483-Monanchora-sp.WAMNG I

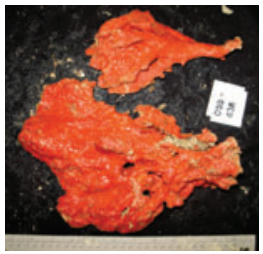

99100490-Crella-(Yvesia)-sp.WAMSS2

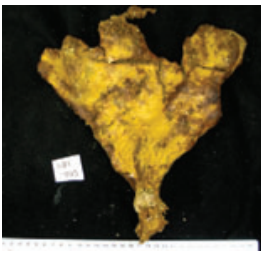

I0077037-lotrochota-baculifera

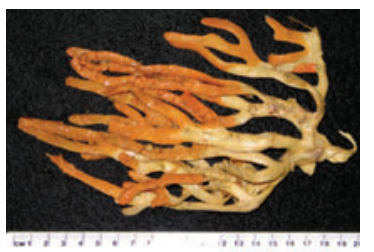

99100494-Strongylacidon-sp.WAMSS2 


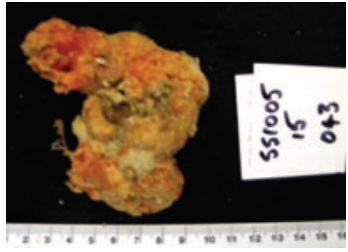

99100497-Tedania-(Trachytedania) sp.WAMSSI

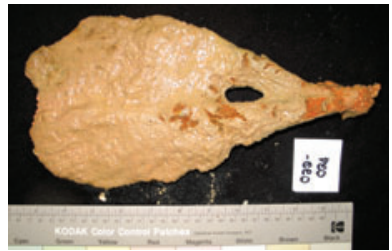

99100503-Strongylamma-sp.WAMSSI

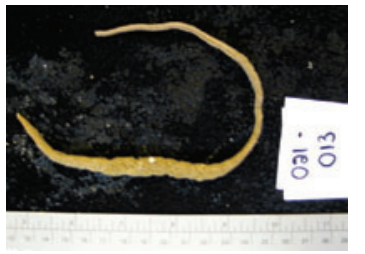

99100506-Sigmaxinella-sp.WAMSS2

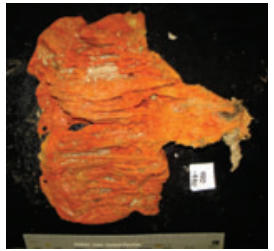

991005|4-Biemna-sp.WAMSS4

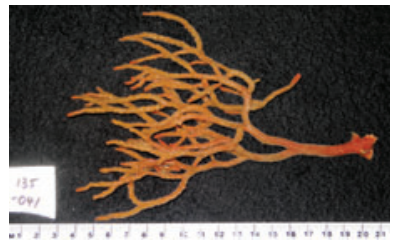

991005 I0-Sigmaxinella-cf.-soelae

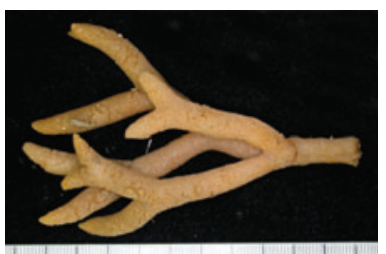

99100521-Pararhaphoxya-sp.WAMSSI

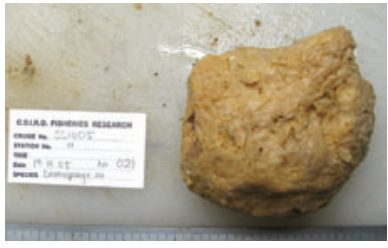

99100499-Tedania-(Tedaniopsis) sp.WAMSSI

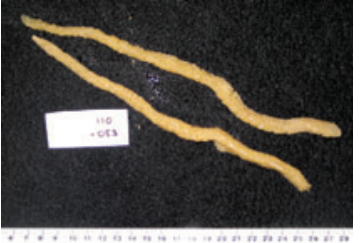

99100504-Hemitedania-sp.WAMSSI

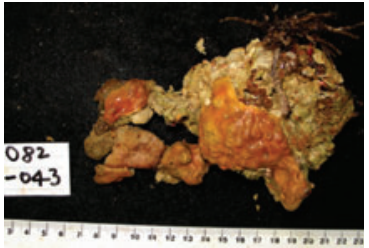

99|005 I I-Desmacella-sp.WAMSS I

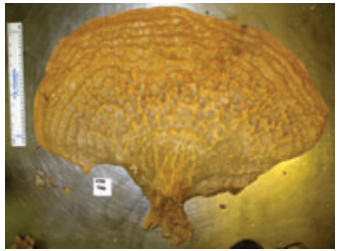

991005।3-Biemna-sp.WAMSS3

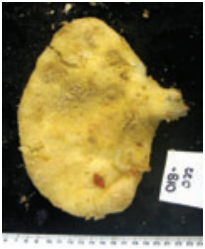

99|005 I9-Mycale-(Aegogropila) sp.WAMSSI

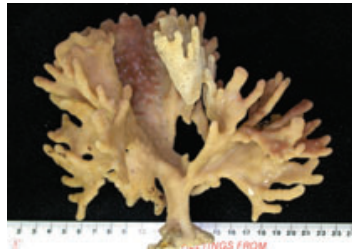

99100523-Axinella-sp.WAMSS।

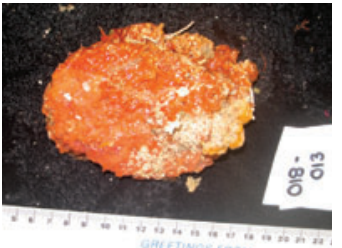

99100500-Tedania-(Tedaniopsis) sp.WAMSS2

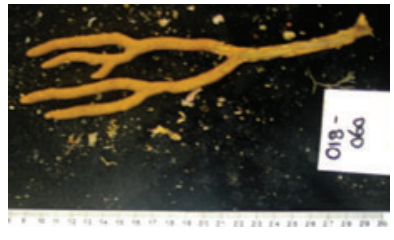

99100508-Sigmaxinella-sp.WAMSS4

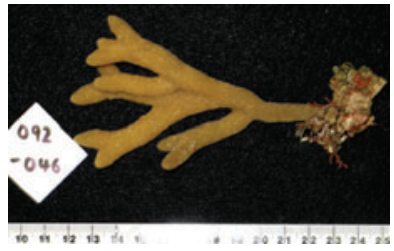

99100344-Sigmaxinella-sp.WAMSS5

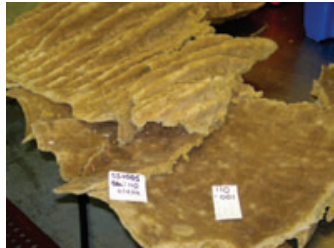

99100325-Biemna-sp.WAMSSI

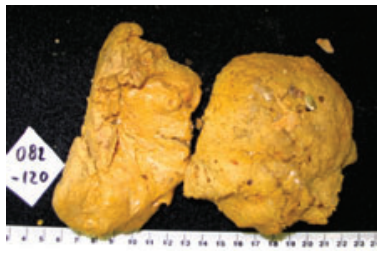

99100520-Phlyctaenopora-(Phlyctaenopora) sp.WAMSSI

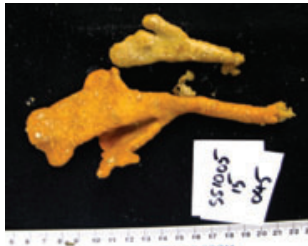

99100366-Phakellia-sp.WAMNG3

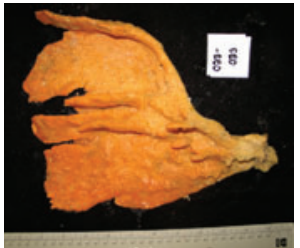

99100502-Tedania-(Tedania) sp.WAMSSI

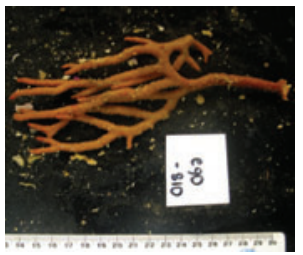

99100507-Sigmaxinella sp.WAMSS3

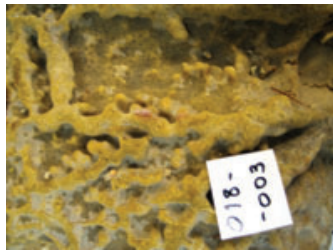

991005/2-Biemna-sp.WAMSS2

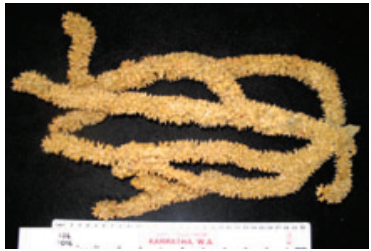

99100505-Sigmaxinella-sp.WAMSS |

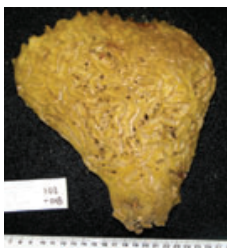

991005 I7-Mycale-(Carmia) sp.WAMSS3

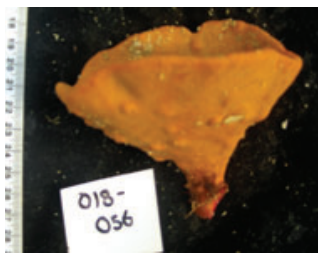

99100305-Axinella-sp.WAMNG3 


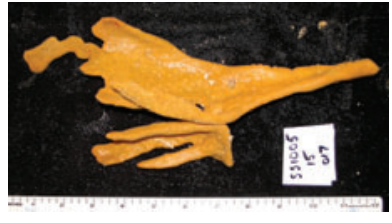

99100524-Axinella-sp.WAMSS2

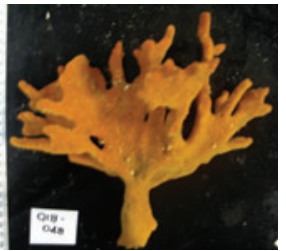

99100526-Axinella-sp.WAMSS5

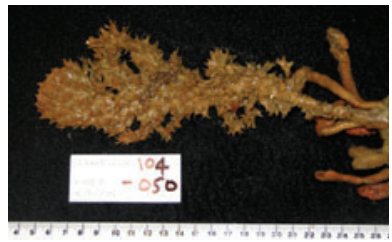

99100347-Axinella-sp.WAMNG I

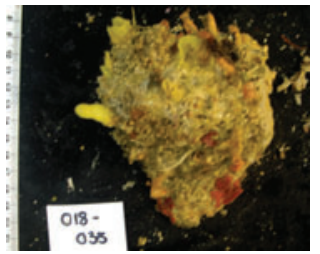

9910053I-Didiscus-sp.WAMSS2

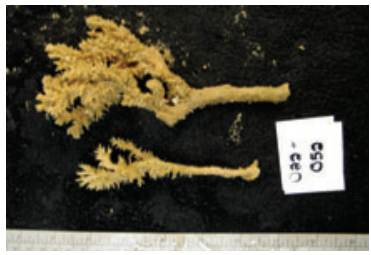

99100348-Acanthella-sp.WAMSS2

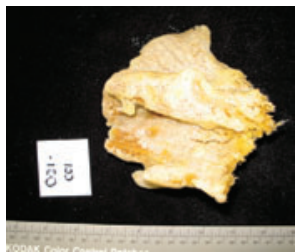

99100545-Hymeniacidon-sp.WAMSSI

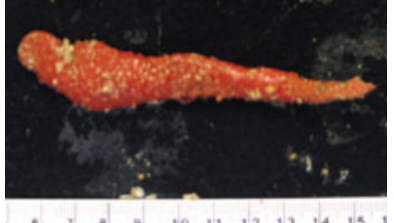

99100527-Axinella-sp.WAMSS6

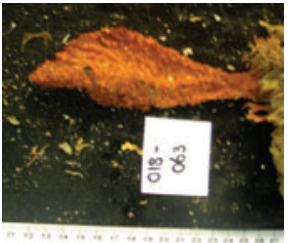

99100365-Axinella-sp.WAMSS9

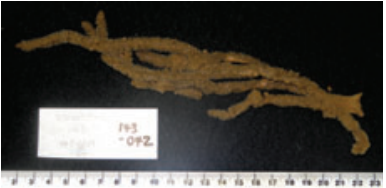

99100522-Pararhaphoxya-sp.WAMSS3

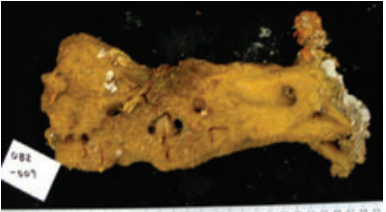

99100534-Myrmekioderma-sp.WAMSS2

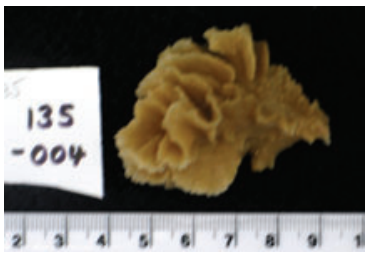

99100254-Acanthella-sp.WAMNG3

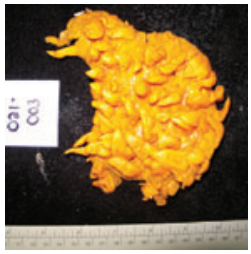

99100540-Ciocalypta-sp.WAMSSI

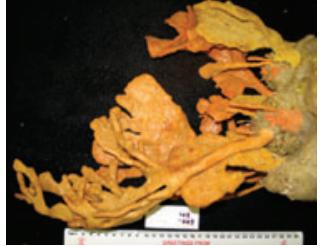

99100353-part-Axinella-sp.WAMSS3

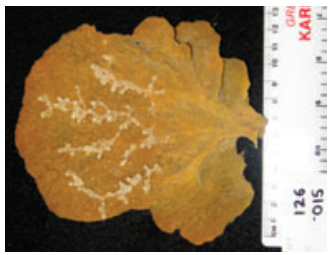

99100253-Phakellia-sp.WAMNG2

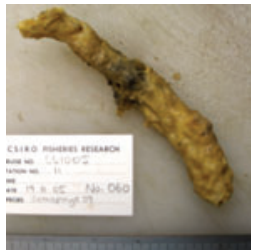

99100532-Didiscus-sp.WAMSS3

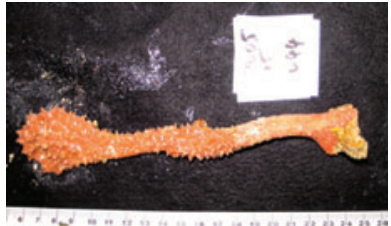

99100250-Acanthella-sp.WAMSSI

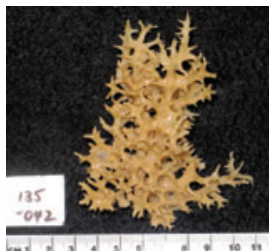

99100536-Acanthella-sp.WAMNG2

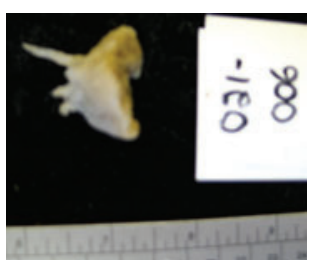

99100542-Ciocalypta-sp.WAMSS3

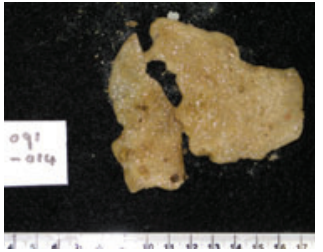

99100530-Phakellia-sp.WAMSS I

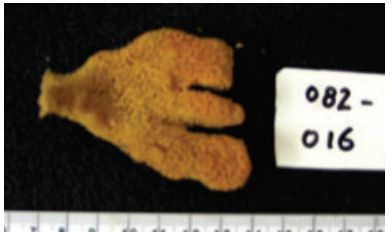

99100252-Axinella-sp.WAMNG2

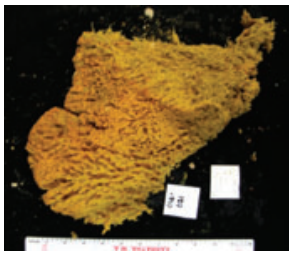

99100533-Myrmekioderma sp.WAMSSI

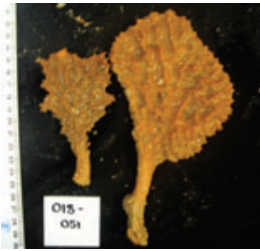

99100535-Acanthella-sp.WAMSS3

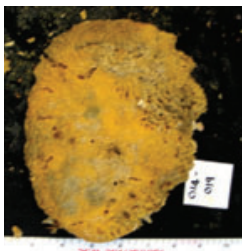

99100538 -Halichondria-(Halichondria) sp.WAMSS3

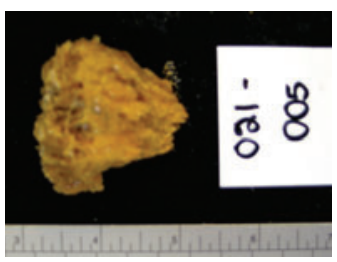

99100543-Ciocalypta-sp.WAMSS4 


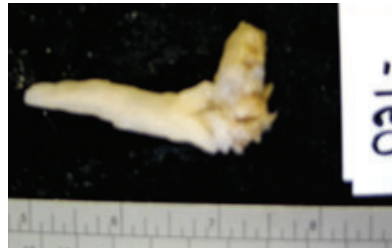

9910054I-Ciocalypta-sp.WAMSS2

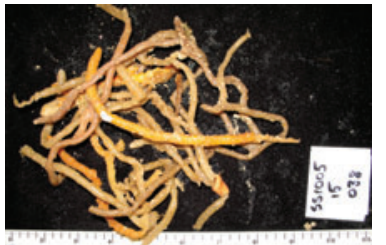

99100548-Callyspongia-(Callyspongia) sp.WAMSSI

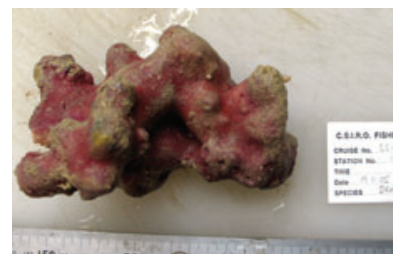

99100559-Haliclona-(Gellius)-sp.WAMSS2

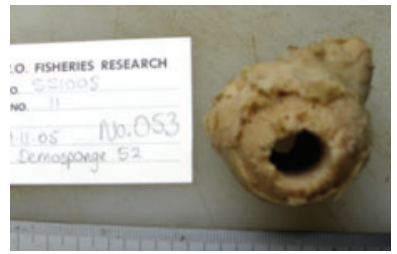

99100560-Haliclona-(Gellius) sp.WAMSS3

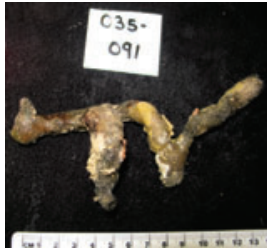

99100553-Haliclona-(Haliclona) sp.WAMSSI

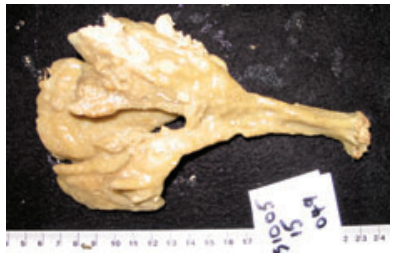

99100568-Amphimedon-sp.WAMSSI

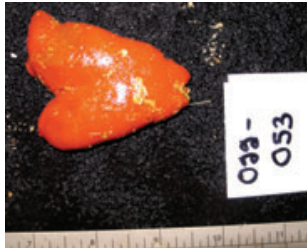

99100546-Hymeniacidon sp.WAMSS2

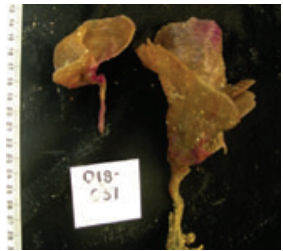

99100547-Callyspongia-(Callyspongia) sp.WAMNG I

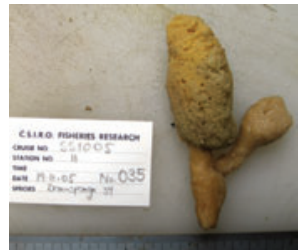

99100562-Haliclona-(Reniera) sp.WAMSS2

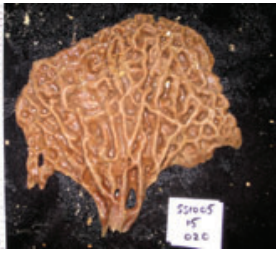

99100565-Haliclona-(Reniera) sp.WAMSS5

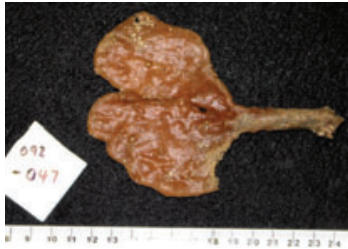

99100552-Haliclona-(Haliclona) sp.WAMNGI

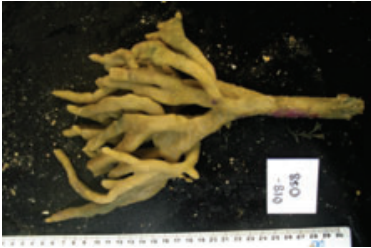

991003 I3-Amphimedon-sp.WAMSS4

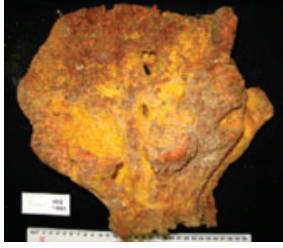

99100539-Halichondria-(Halichondria) sp.WAMSS5

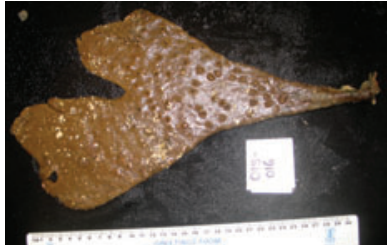

99100549-Callyspongia-(Callyspongia) sp.WAMSS2

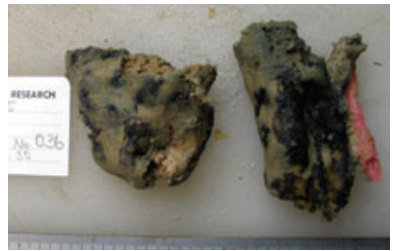

99|0056I-Haliclona-(Reniera) sp.WAMSSI

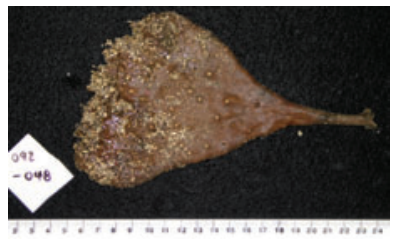

99100564-Haliclona-(Reniera) sp.WAMSS4

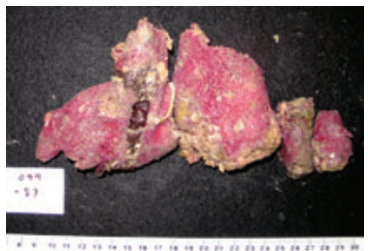

99I 00557-Haliclona-(Haliclona) sp.WAMSS5

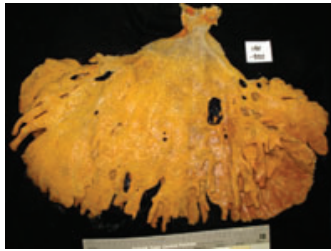

9910057I-Amphimedon-sp.WAMSS5

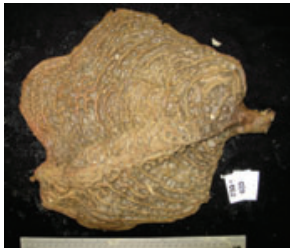

I00980 I0-Callyspongia-(Callyspongia) bilamellata

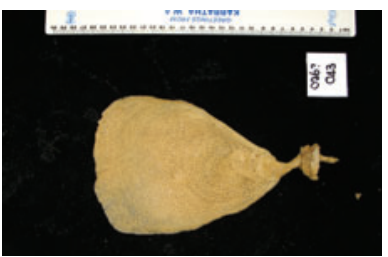

9910055 I-Callyspongia-(Callyspongia) sp.WAMSS4

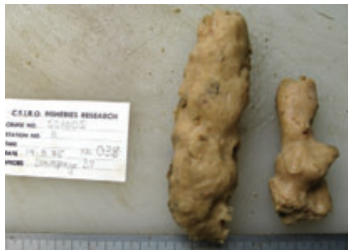

99100563-Haliclona-(Reniera) sp.WAMSS3

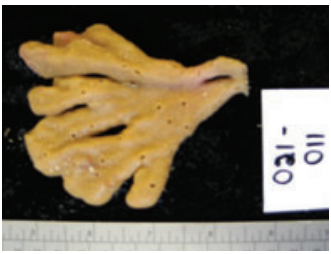

99100566-Haliclona-(Reniera) sp.WAMSS6

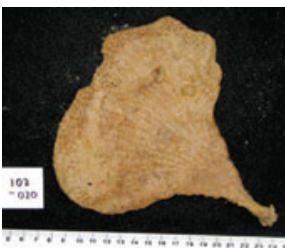

99100555-Haliclona-(Haliclona) sp.WAMSS3

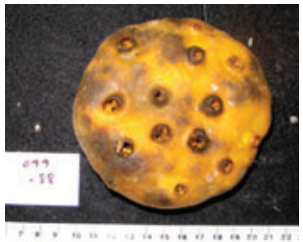

10101022-Oceanapia-ramsayi 


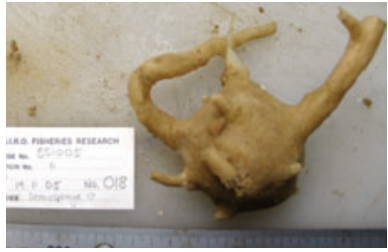

99100336-Oceanapia-sp.WAMSS5

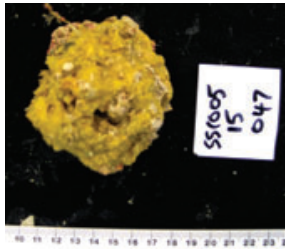

99100575-Aka-sp.WAMSS4

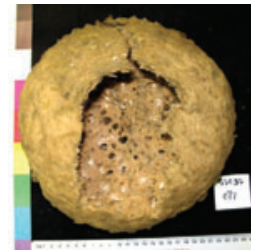

99100589-Oceanapia-cf.-ramsayi

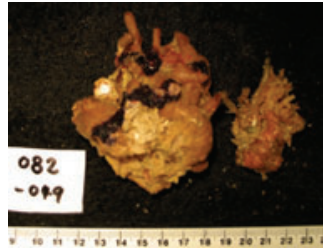

99100590-Oceanapia-cf. macrotoxa

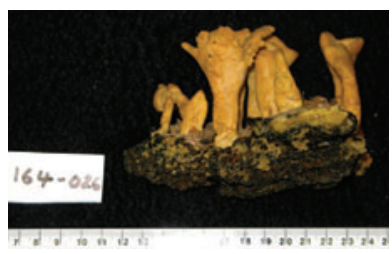

99100573-Aka-sp.WAMSS

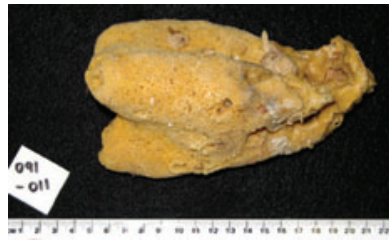

99100594-Xestospongia-sp.WAMSS

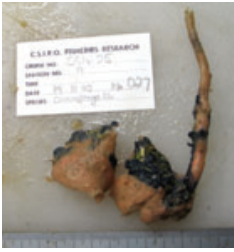

99100330-Oceanapia-sp.WAMSS4

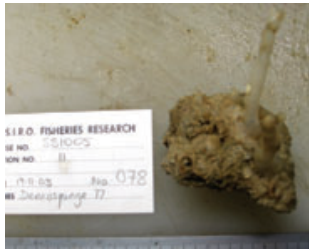

9910033I-Aka-sp.WAMSS3

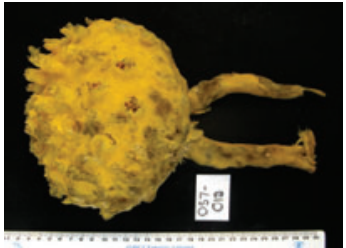

99100277-Oceanapia sp.WAMSS2

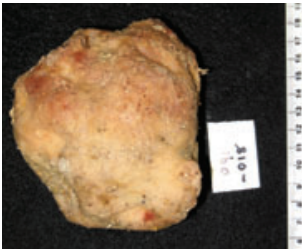

99100334-Oceanapia-sp.WAMSSI0

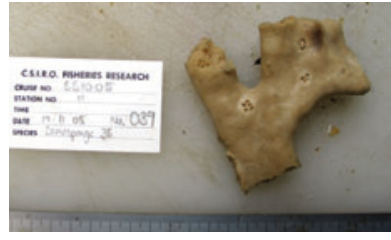

99100592-Petrosia-(Petrosia)-sp.WAMNG I

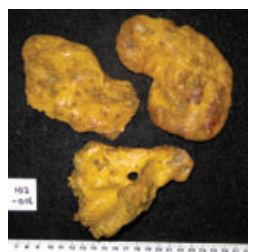

99100596-Xestospongia-sp.WAMSS3

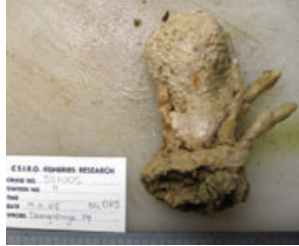

99100574-Aka-sp.WAMSS2

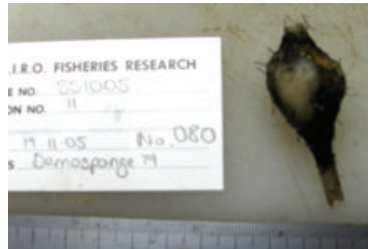

99100585-Oceanapia-sp.WAMSS7

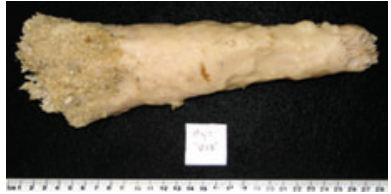

99100306-Aka-sp.WAMSS9

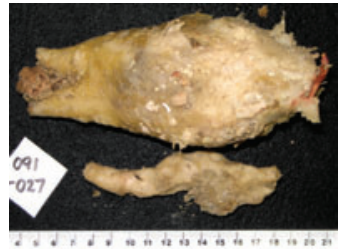

99100355-Oceanapia-sp.WAMNG I

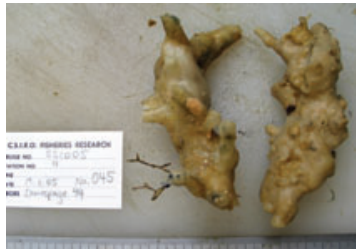

99100268-Petrosia-(Petrosia)-sp.WAMSS3

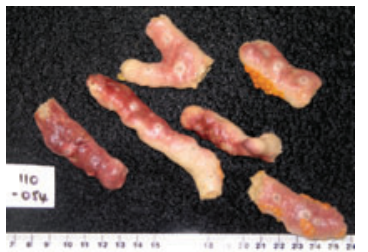

99100593-Xestospongia-sp.WAMNG2

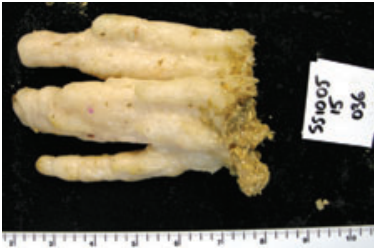

99100576-Aka-sp.WAMSS5

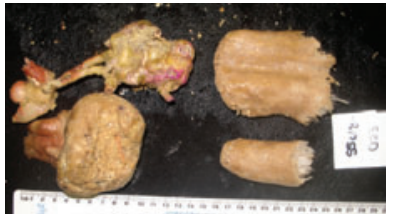

99100584-Oceanapia-sp.WAMSS6

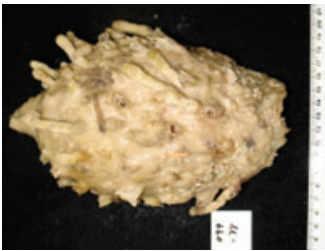

99100586-Oceanapia-sp.WAMSS8

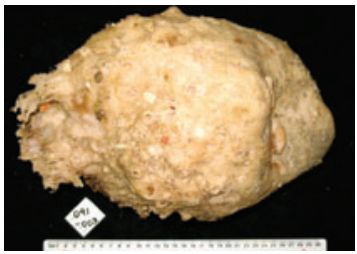

99100587-Oceanapia-sp.WAMSS9

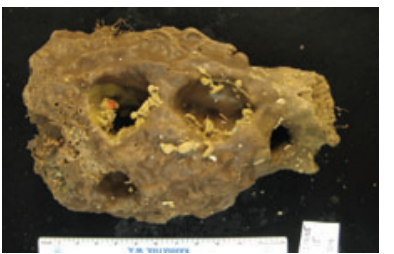

99100356-Petrosia-(Petrosia)-sp.WAMSS2

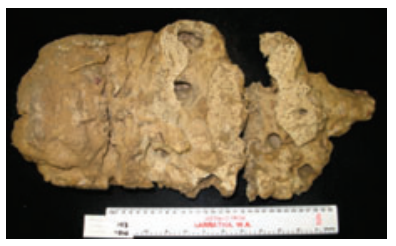

9910059|-Petrosia-(Petrosia)-sp.WAMSSI 


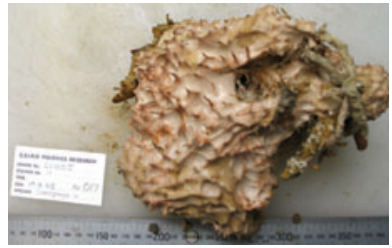

99100345-Sarcotragus-sp.WAMNG |

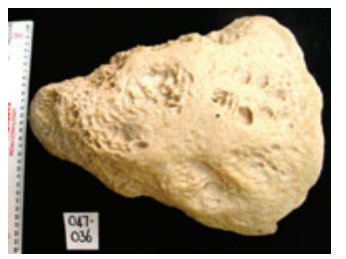

99100605-Sarcotragus-sp.WAMSS3

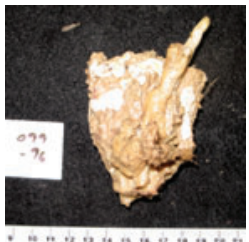

99100604-Psammocinia-sp.WAMSS7

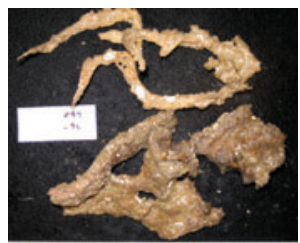

99100257-Sarcotragus-sp.WAMSSI

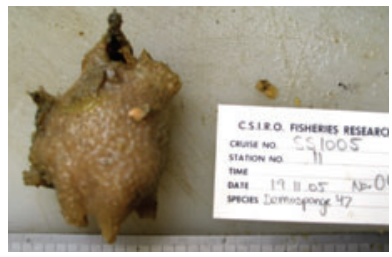

99100343-Luffariella-sp.WAMSS I

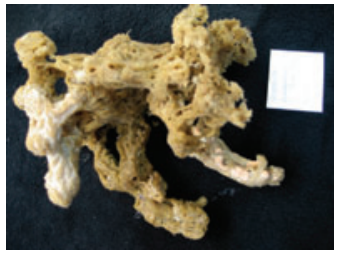

99100635-Fasciospongia-sp.WAMSS

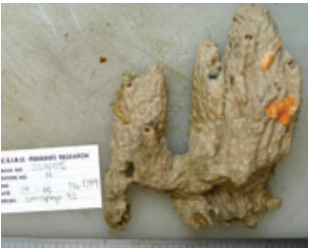

99100600-Psammocinia-sp.WAMSS3

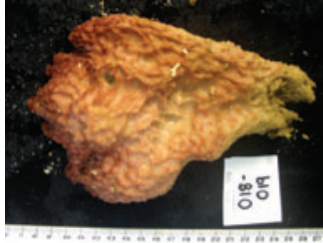

99100606-Sarcotragus-sp.WAMSS4

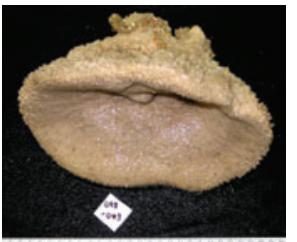

99100607-Sarcotragus-sp.WAMSS5

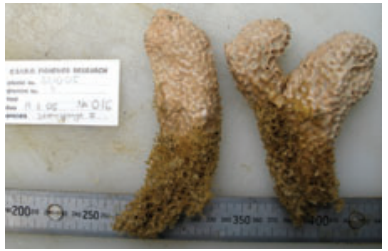

99100625-Taonura-sp.WAMSSI

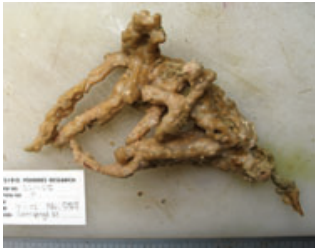

9910062I-Luffariella-sp.WAMSS2

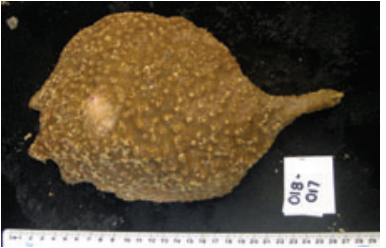

99|006 |4-Dactylospongia-sp.WAMSS I

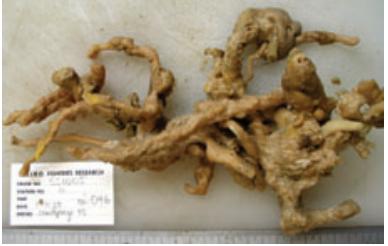

99100599-Psammocinia-sp.WAMSS2

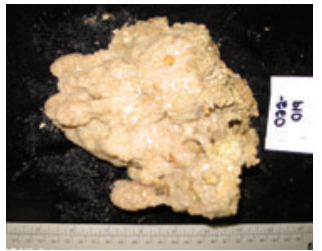

99100602-Psammocinia-sp.WAMSS5

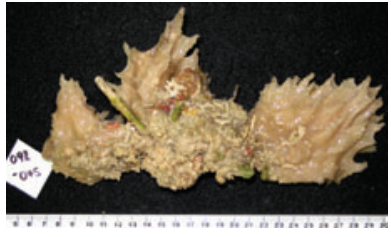

99100609-Sarcotragus-sp.WAMSS8

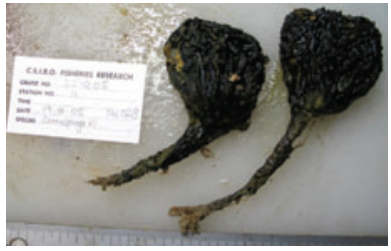

99100629-Thorecta-sp.WAMSS4

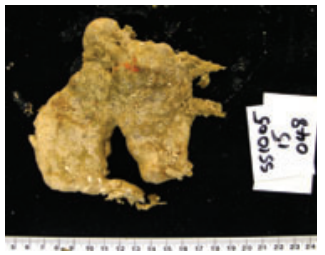

99100633-Thorecta-sp.WAMNG I

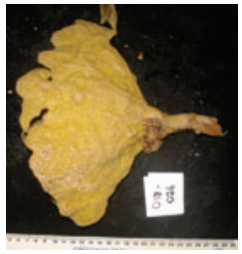

99100636-Carteriospongia-sp.WAMSS I

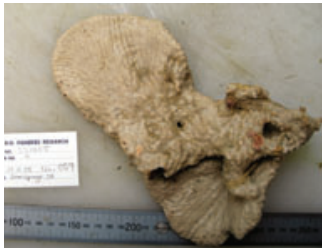

99100598-Psammocinia-sp.WAMSSI

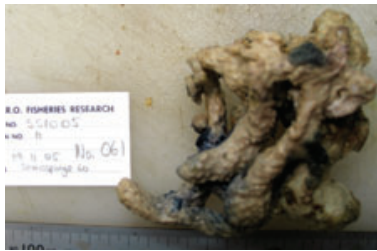

99100603-Psammocinia-sp.WAMSS6

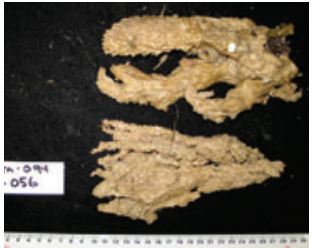

99100610-Sarcotragus-sp.WAMSS9

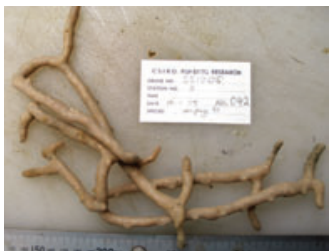

991006|5-Dactylospongia-sp.WAMSS2

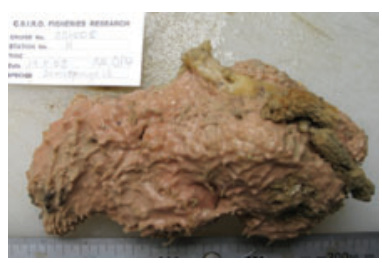

99100634-Fascaplysinopsis-sp.WAMSS I

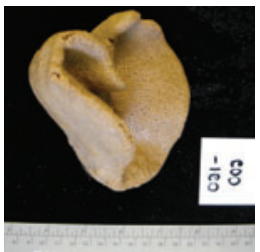

99100628-Thorecta-sp.WAMSS3 


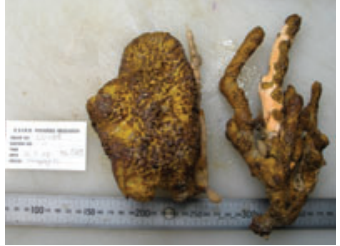

99100273-Luffariella-sp.WAMSS7

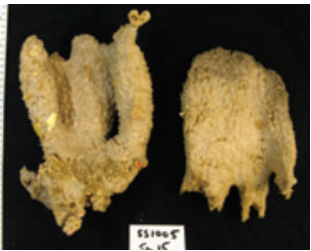

99100613-Cacospongia-sp.WAMSS3

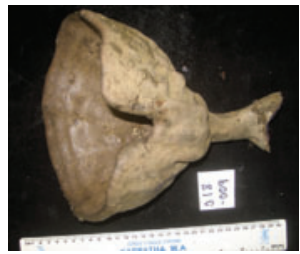

99100620-Hyrtios-sp.WAMSS6

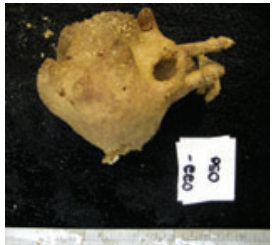

99100631-Thorecta-sp.WAMSS6

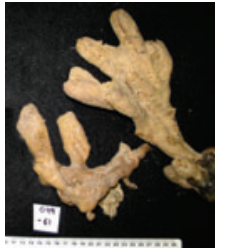

99100638-Aplysinopsis-sp.WAMSSI

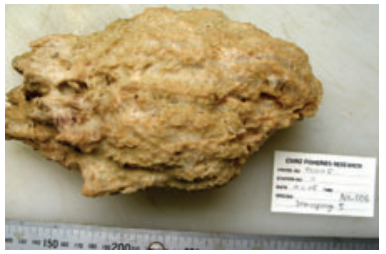

99100639-Hippospongia-sp.WAMSS2

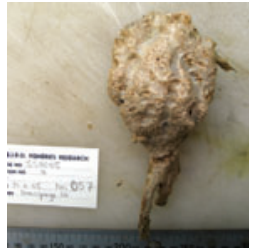

99100624-Luffariella-sp.WAMSS6

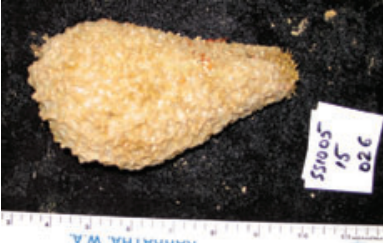

99100358-Taonura-sp.WAMSS3

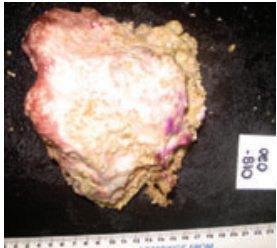

99100618-Hyrtios-sp.WAMSS4

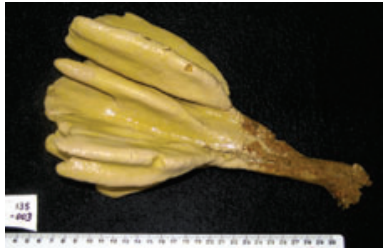

99100626-Thorecta-sp.WAMSS I

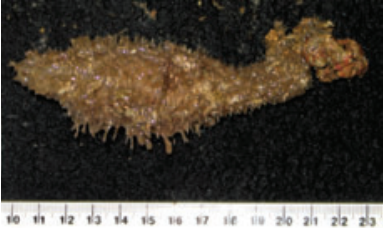

99100335-Taonura-sp.WAMSS2

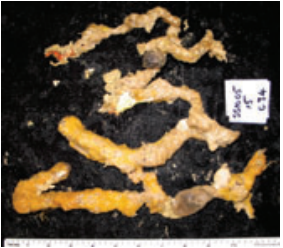

99100640-Hippospongia-sp.WAMSS3

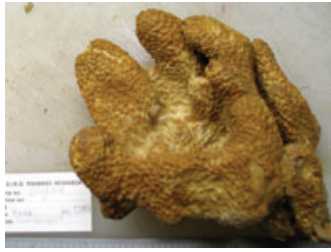

99100284-Hyrtios-sp.WAMSS2

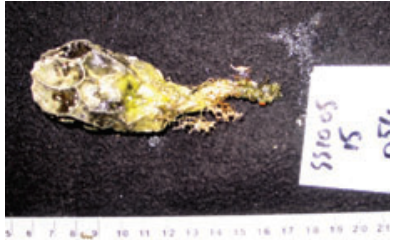

99100637-Thorectandra-sp.WAMSSI

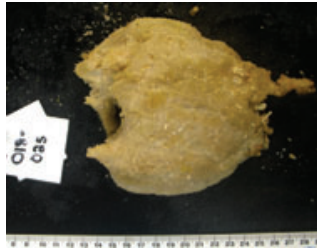

99100630-Thorecta-sp.WAMSS5

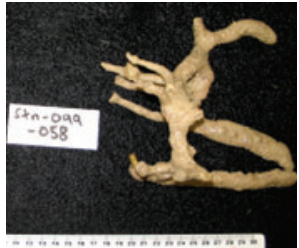

99100622-Luffariella-sp.WAMSS3

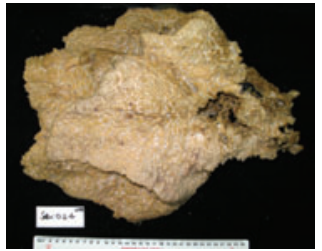

99100644-Leiosella-sp.WAMSS I

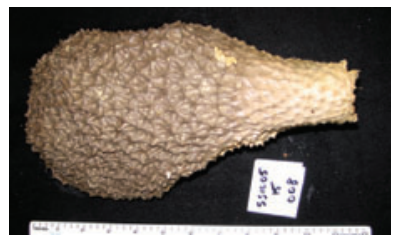

99100642-Spongia-(Heterofibria) sp.WAMSS2

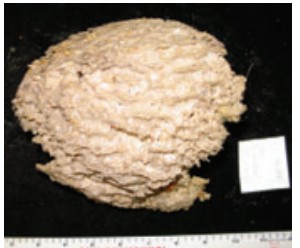

99100617-Hyrtios-sp.WAMSS3

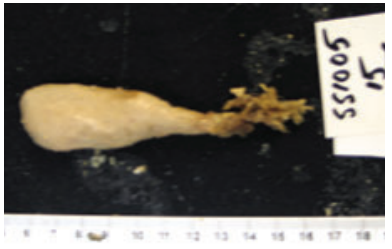

99100256-Thorecta-sp.WAMSS7

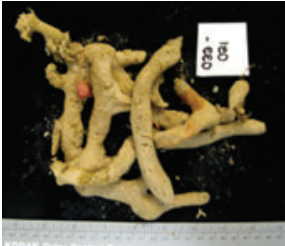

99100328-Luffariella-sp.WAMSS4

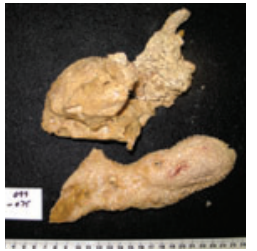

99100623-Luffariella-sp.WAMSS5

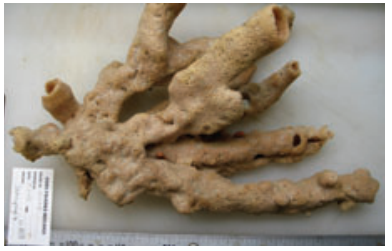

99100648-Hyatella-sp.WAMSS2

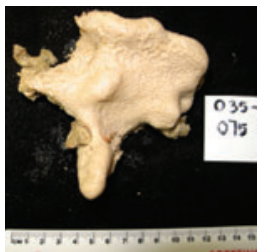

99100643-Spongia-(Heterofibria) sp.WAMSS3 


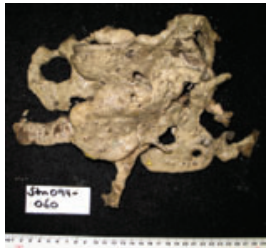

99100646-Leiosella-sp.WAMSS3

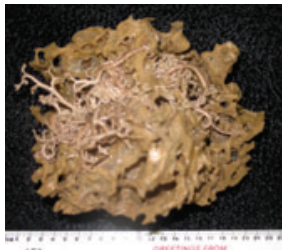

99100280-Hippospongia-sp.WAMSSI

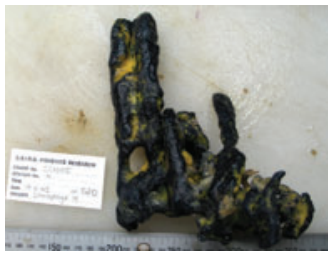

99100656-Pseudoceratina-sp.WAMSS3

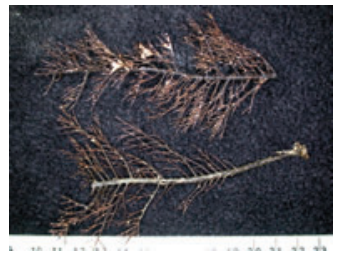

99| |06 | I-Myriopathes-sp.A

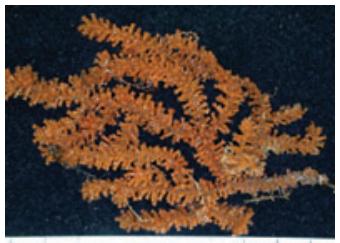

99| 10292-Acanthogorgia-sp.D

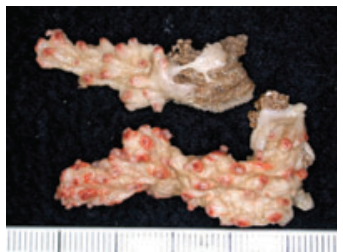

99110426-Eleutherobia-n.sp.A

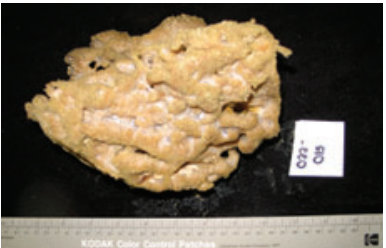

99100360-Spongia-(Heterofibria) sp.WAMSSI

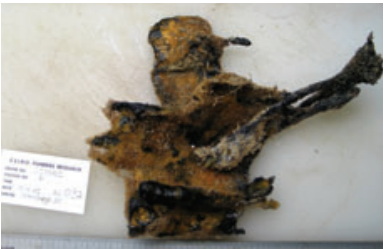

99100653-Aplysina-sp.WAMSS I

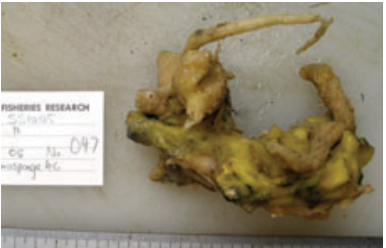

99100655-Pseudoceratina-sp.WAMSS2

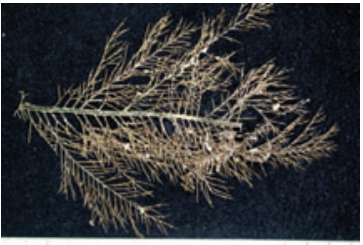

99| I06 I2-Myriopathes-cf.-japonica

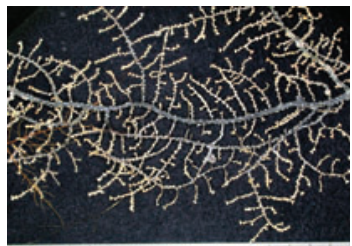

99| I0424-Muricella-sp.A

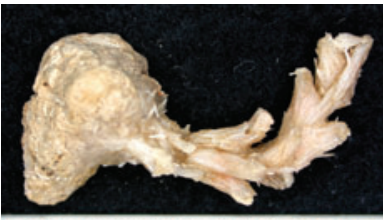

||1|||||||||||||||||||||||||ii||

99|10428-Inflatocalyx-n.sp.A

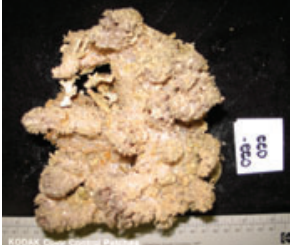

99100249-Hippospongia-sp.WAMSS4

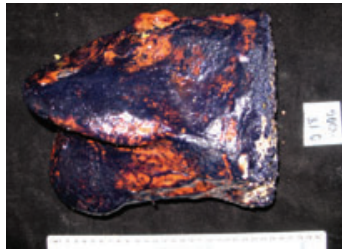

99100652-Aplysinidae-sp.WAMSS2

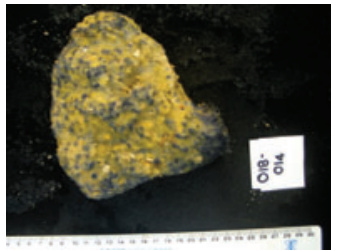

99100654-Pseudoceratina-sp.WAMSSI

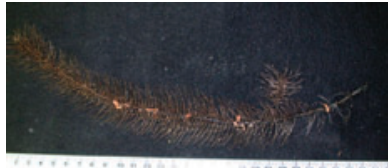

II |61002-Parantipathes-helicosticha

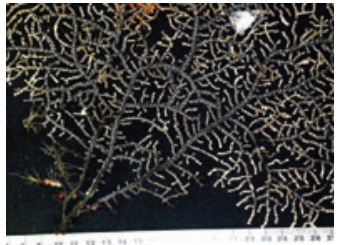

II I75000-Acanthogorgia-sp.-indet
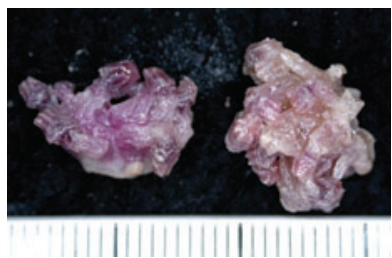

99I 10297-Anthothela-sp.N

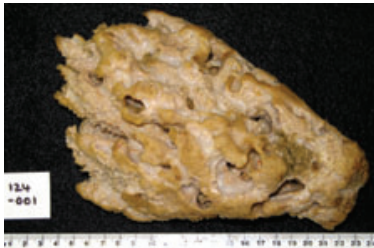

9910064I-Hippospongia-sp.WAMSS5

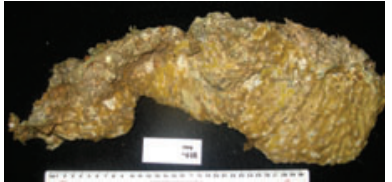

9910065I-Aplysinidae-sp.WAMSSI

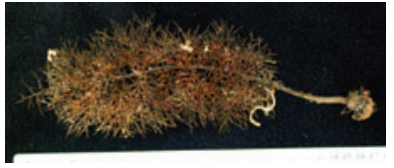

I I 58002-Myriopathes-myriophylla

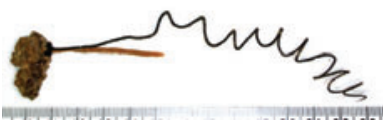

99| I06 |5-Stichopathes-sp.A

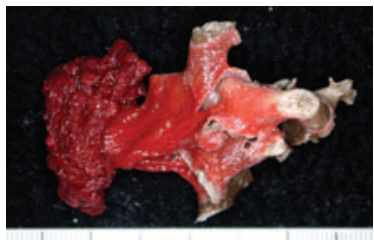

99| I0296-Anthomastus-sp.A

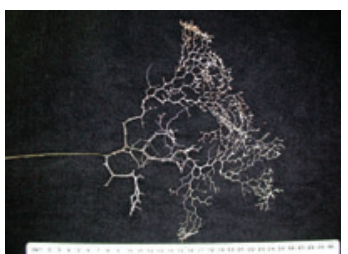

991 10302-Metallogorgia-sp.A 


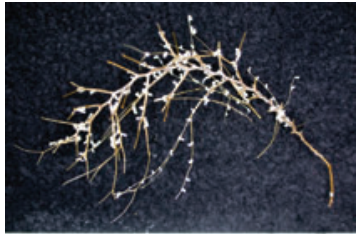

991 10300-Chrysogorgia-sp.O

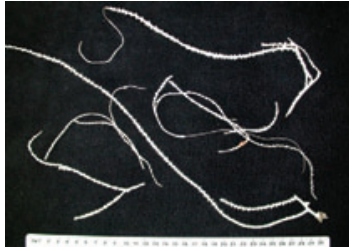

99| I0308-Viminella-sp.H

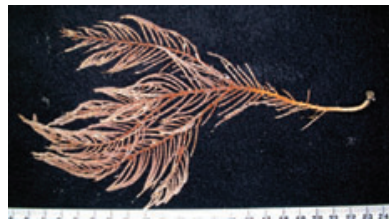

I I I88009-Mopsea-encrinula

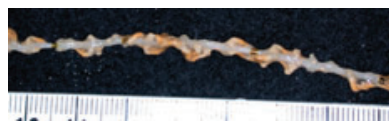

99| I03 | |-Lepidisis-sp.N

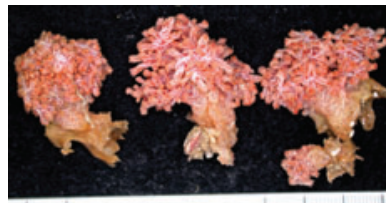

III91903-Scleronephthya-spp

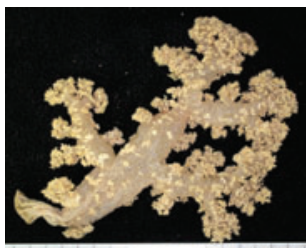

99|I0327-Dendronephthya-sp.H

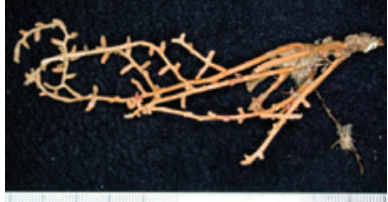

99| I043|-Stereotelesto-sp.A

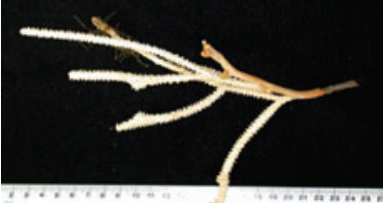

I I 85000-Ellisellidae-sp.-indet.

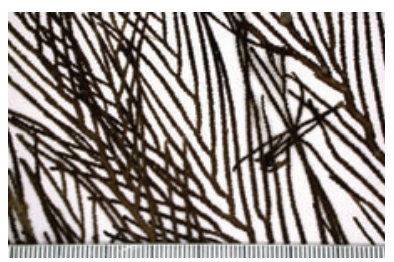

I | |880|3-Tethrisis-suzannae

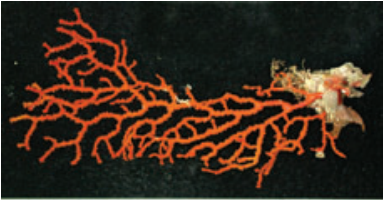

I I 90002-Mopsella-klunzingeri

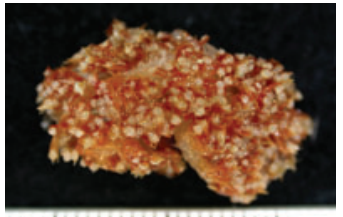

99| I03 I8-Dendronephthya-sp.V

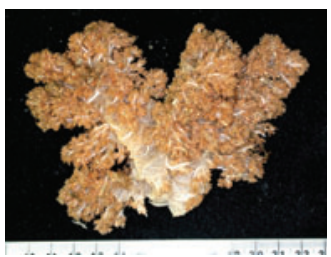

99| I0332-Dendronephthya-sp.HH

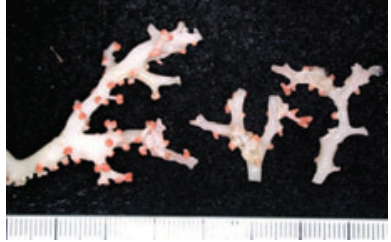

I | |83000-Corallidae-sp.-indet.

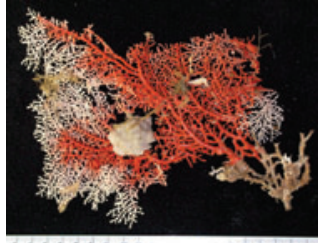

99|10305-Verrucella-sp.G

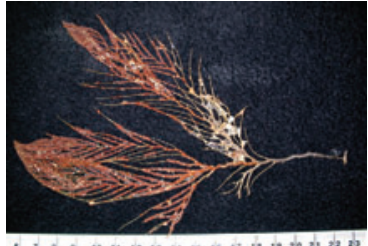

I | | 88037-Zignisis-bifoliata

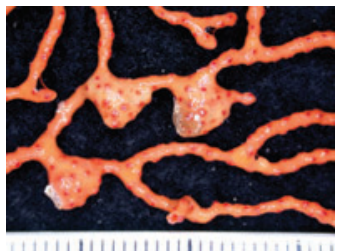

99| I 044 I-Wrightella-n.sp.A

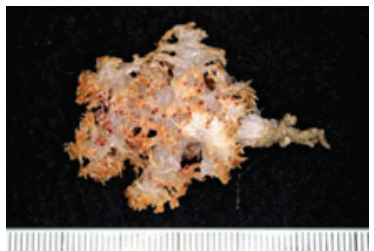

991 10320-Dendronephthya-sp.X

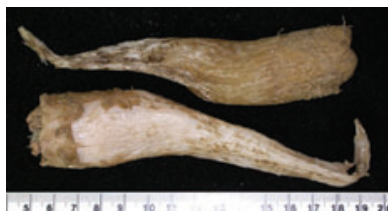

991 I0444-Studeriotes-sp.A

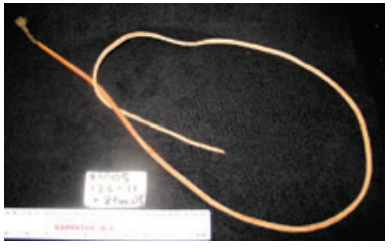

I I 85003-junceella-juncea

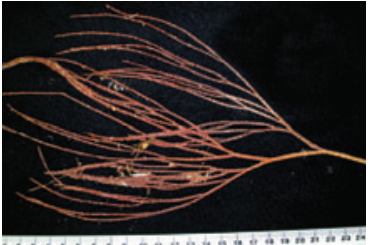

| | |8800 |-Annisis-sprightly

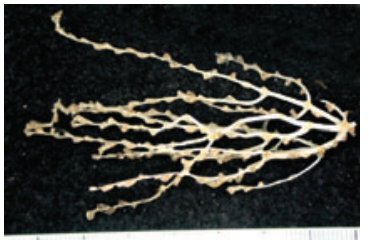

99|I03I0-Acanella-sp.C

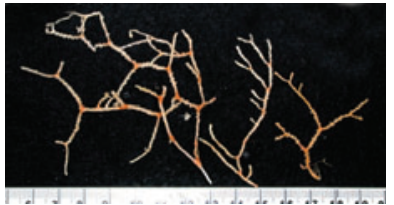

99|I03|7-Acabaria-sp.E

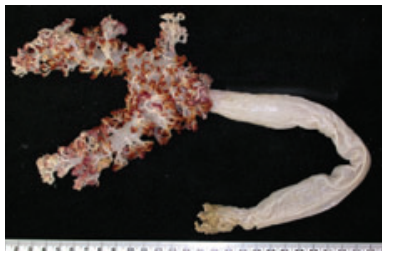

99I 10443-Umbellulifera-sp.A

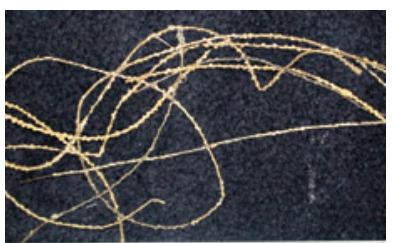

1 | 196002-Pseudothesea-simplex 


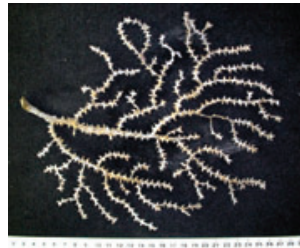

991 10446-Paramuricea-cf.-grandis

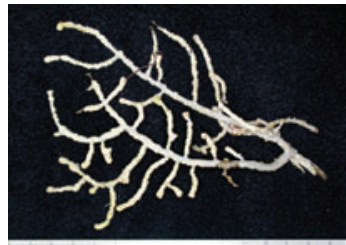

99| I0339-Astrogorgia-sp.D

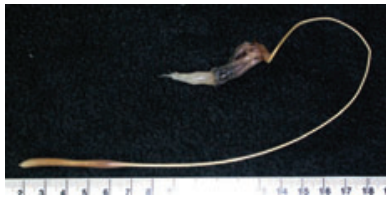

II216003-Umbellula-huxleyi

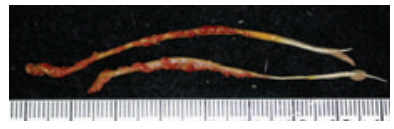

991 10453-Acanthoptilum-n.sp.A

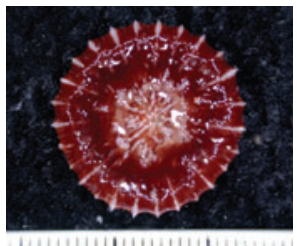

99| 10618-Anthemiphyllia-n.sp.A

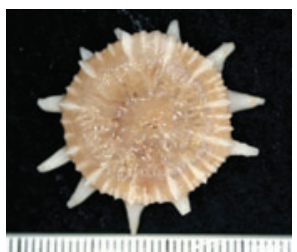

I|3|4062-Stephanocyathus-weberianus

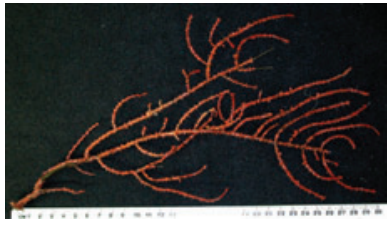

99| I0447-Swiftia-sp.B

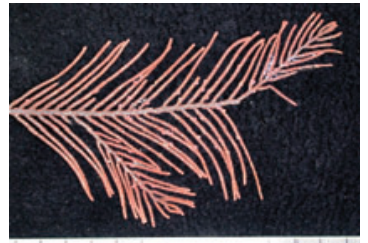

I I 970 I5-Pseudoplumarella-filicoides

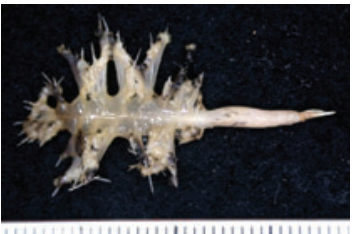

99| I035।-Pteroeides-sp.F

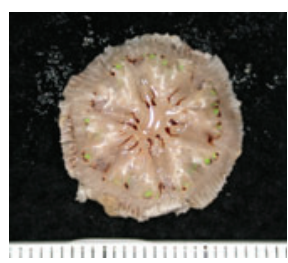

I I299002-Letepsammia-formosissima

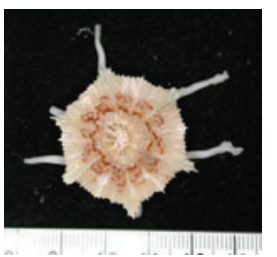

I|3|40 |9-Stephanocyathus-spiniger

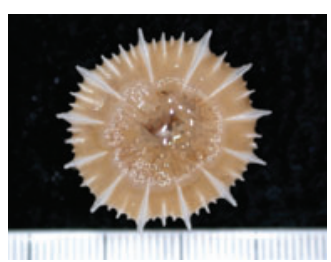

99|10619-Stephanocyathus-cf.-coronatus

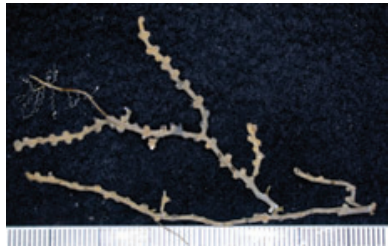

99110343-Bebryce-sp.C

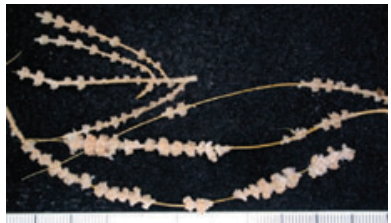

99| I0349-Narella-sp.F

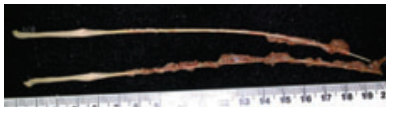

| | 22400 I-Scytalium-sarsii

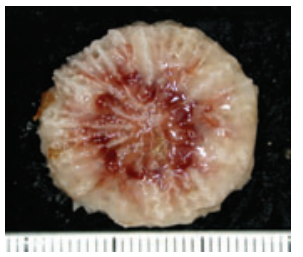

I I30 I00 I-Fungiacyathus-fragilis

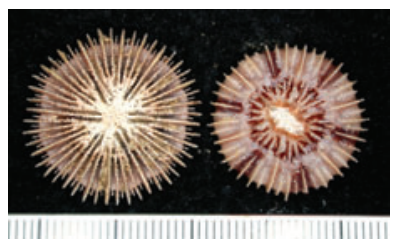

| |3|402 |-Deltocyathus-suluensis

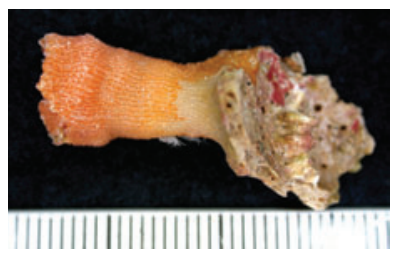

991 10622-Balanophyllia-sp.A

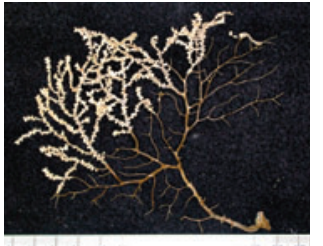

99I 1 0450-Villogorgia-sp.B

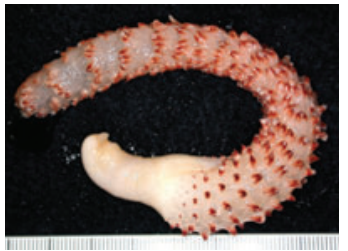

99I I0433-Echinoptilum-cf.-macintoshi

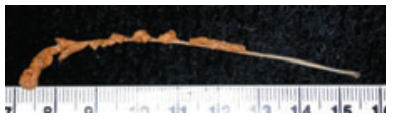

99| I0454-Acanthoptilum-n.sp.B

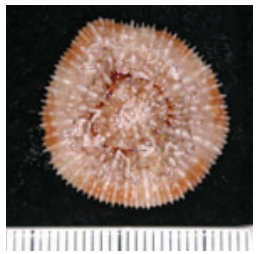

I I30400 I-Anthemiphyllia-dentata

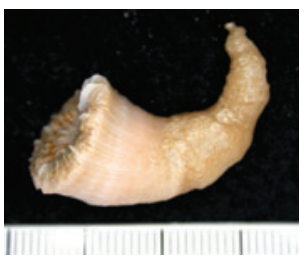

| | 3 |4042-Caryophyllia-planilamellata

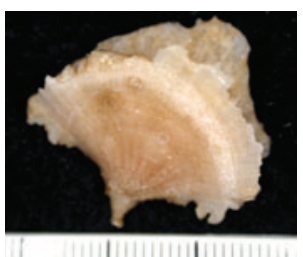

I 328003-Flabellum-lamellulosum 


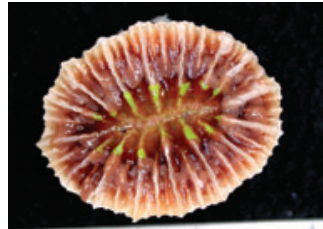

| 3280 |0-Flabellum-tuthilli

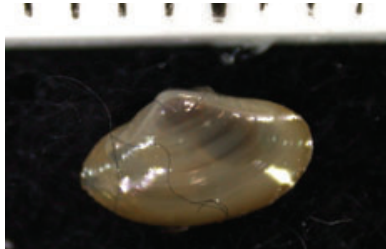

99230105-Yoldia-sp.I

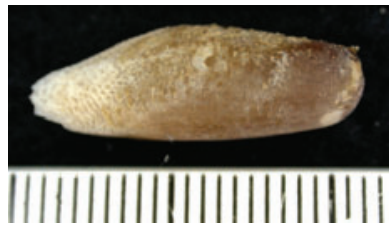

23220022-Lithophaga-malaccana

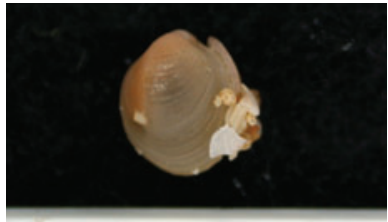

i i i 1 i i l a i i i i

99230 I I I-Mytilidae-sp.3

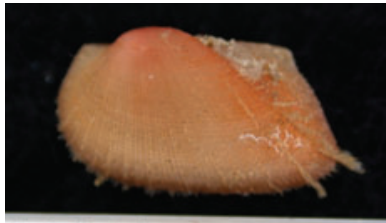

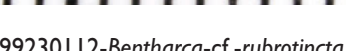

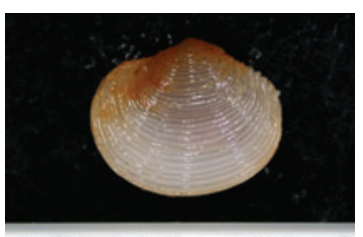

141111111119

99230124-Limopsis-sp.I

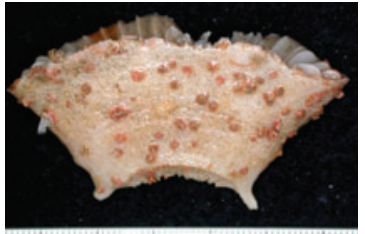

| |3280| 5-Truncatoflabellummacroeschara

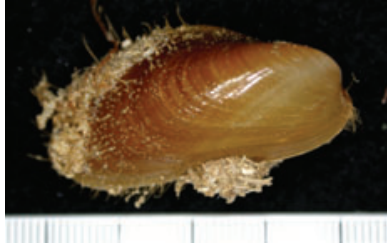

23220008-Modiolus-areolatus

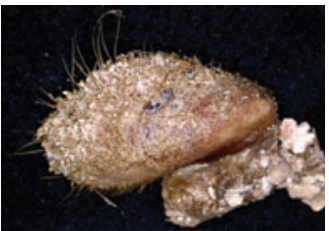

23220025-Modiolus-proclivis

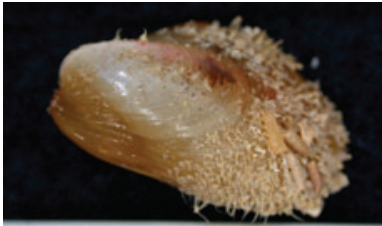

111111111111111111111

99230 108-Modiolus-sp. I

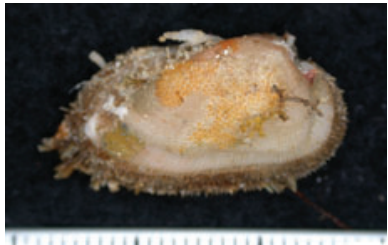

99230 I 2I-Barbatia-cf.-saviolum

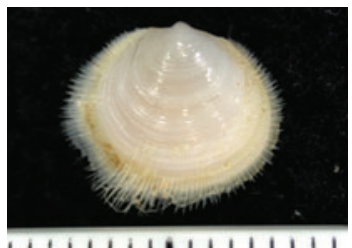

$99230127-L i m o p s i s-s p .4$

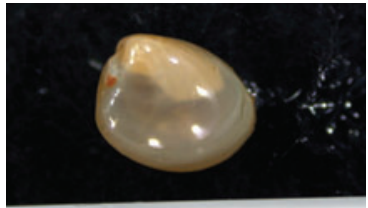

1 I I I I I I I I

99230100-Nucula-sp.I

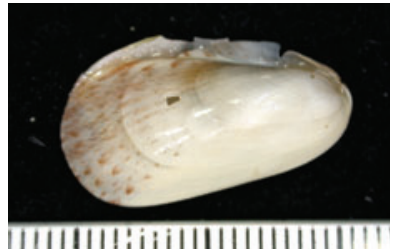

232200 I0-Amygdalum-beddomei

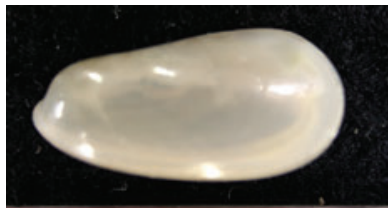

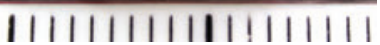

23220073-Amygdalum-watsoni

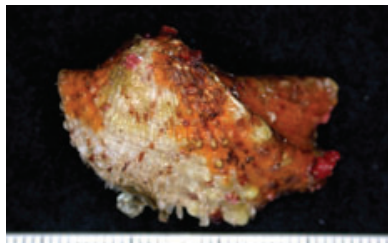

23226002-Arca-navicularis

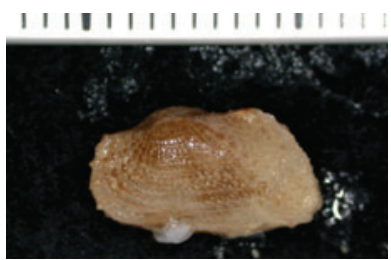

99230 I | 4-Barbatia-(Acar)-sp.|

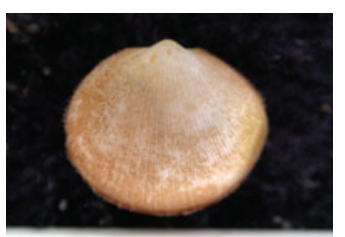

1 1 1 1 1 1 1 1 i 1 1 1 I

2323100 I-Glycymeris-striatularis

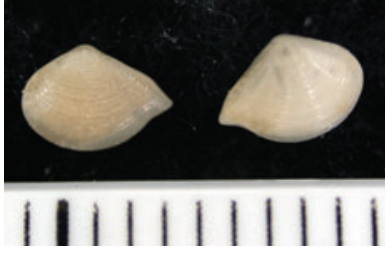

23207019-Nuculana-verconis

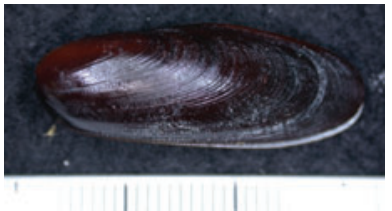

2322002I-Lithophaga-teres

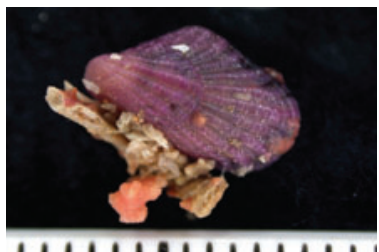

99230107-Septifer-sp.I

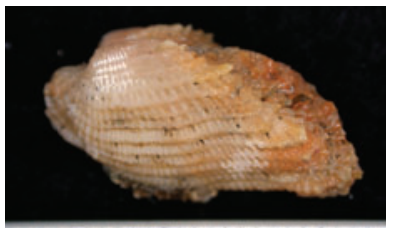

T111IIIIIIIIIIIIIIIII

23226027-Barbatia-plicato

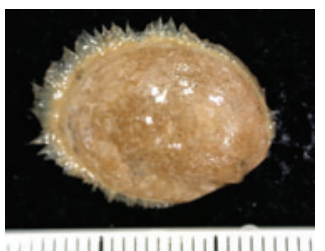

23230002-Limopsis-soboles

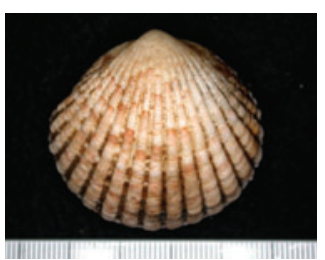

23231003-Tucetona-gealei 


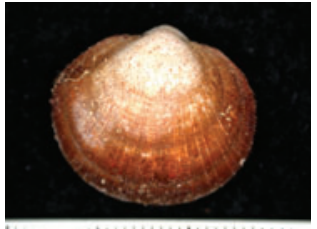

23231014-Glycymeris-hedleyi

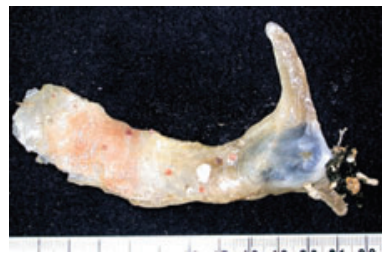

23237002-Malleus-albus

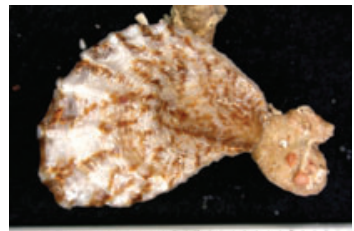

|11IIIIIIIIIIIIIIIIIIIIIIII

2326500I-Dimya-corrugata

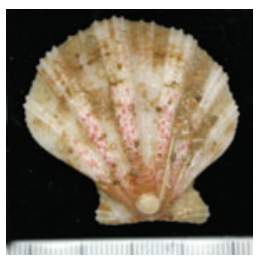

2327002I-Mesopeplum-fenestratum

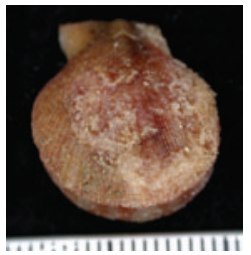

99230 I44-Pectinidae-sp.I

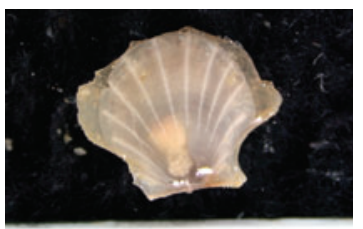

$11,1,1,1,111$

99230 I49-Parvamussium-sp.2

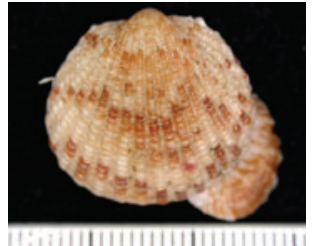

232310|8-Tucetona-hoylei

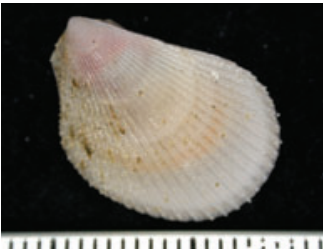

23250005-Lima-nimbifer

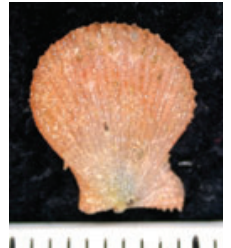

23270006-Mimachlamys-asperrima

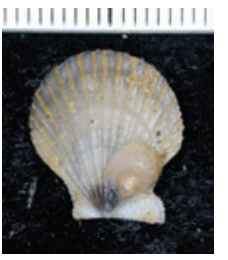

23270029-Chlamys-challengeri

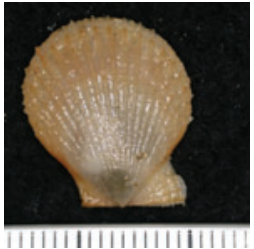

99230 I 38-Talochlamys-cf.-pulleinean

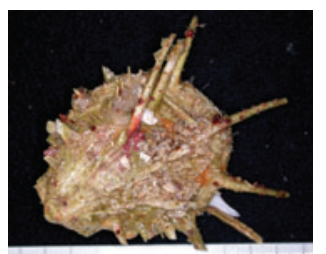

99230157-Spondylus-cf.-victoriae

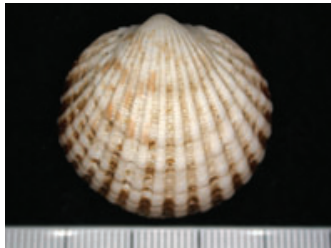

23231022-Tucetona-scalarisculpta

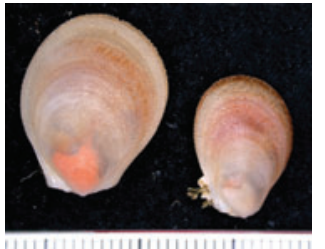

99230 I32-Ctenoides-cf.-annulata

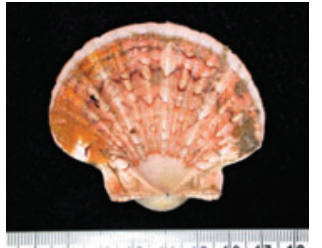

23270007-Pecten-fumatus

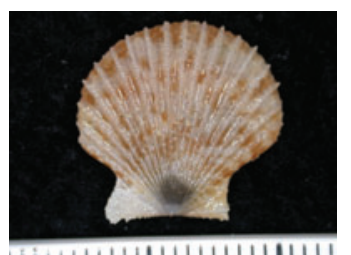

99230137-Cryptopecten-cf.-bullatus

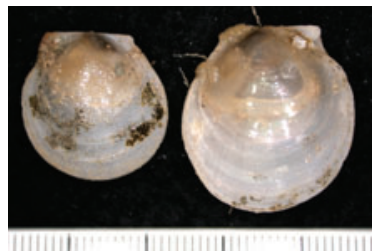

99230 I 4 I-Delectopecten-cf.-alcocki

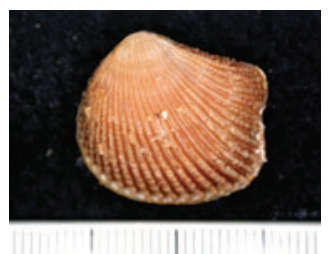

23280006-Neotrigonia-kaiyomaruae

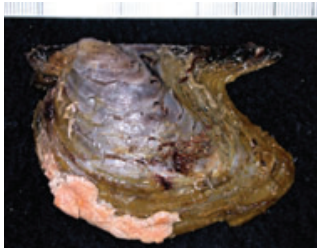

99230 I3I-Pteria-cf.-falcata

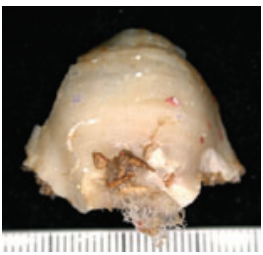

99230 I33-Neopycnodonte-sp. I

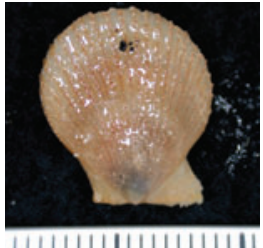

23270008-Semipallium-aktinos

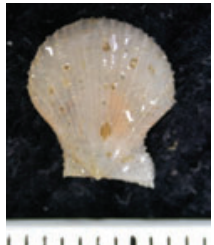

99230 I42-Semipallium-sp.I

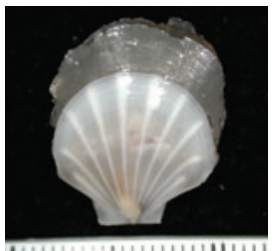

99230 I45-Propeamussium-sp.2

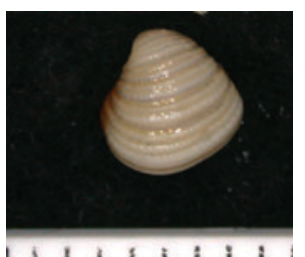

233050 |4-Cardiolucina-crassilirata 


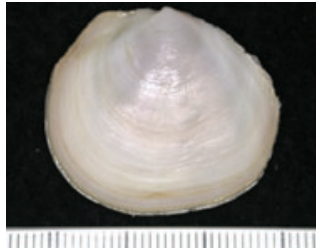

99230 I63-Monitilora-sp. I

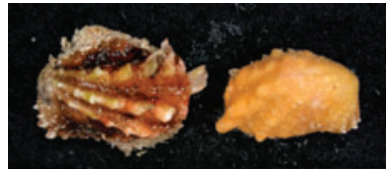

||||||||||||||||||||||||||'ili|

23325003-Cardita-excavata

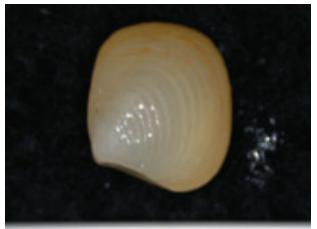

1111111111111111

99230 I73-Crassatina-sp.2

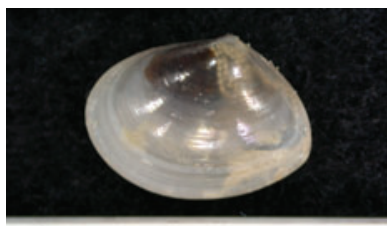

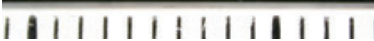
23356020-Leptomya-gravida

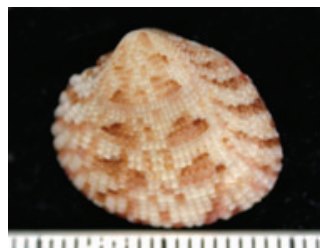

99230I77-Timoclea-sp.I

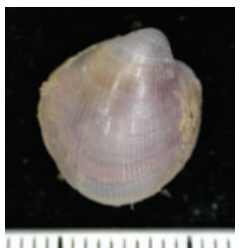

99230186-Policordia-cf.-diomedea

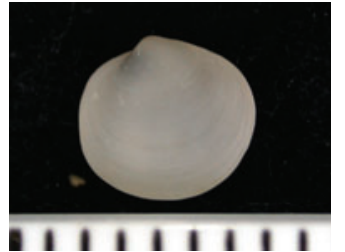

99230169-Felaniella-sp.2

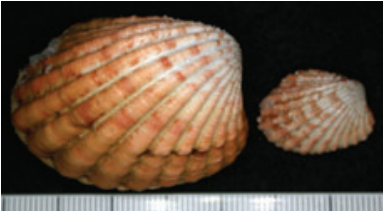

233250 I0-Cardita-incrassata

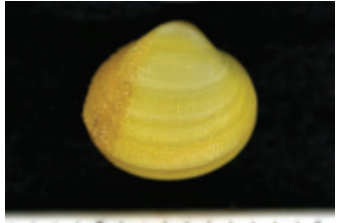

$|1|||||||||||||$

23335002-Nemocardium-thetidis

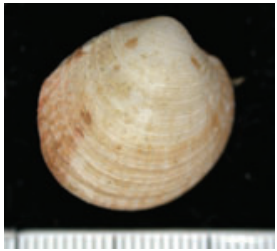

23380050-Globivenus-toreuma

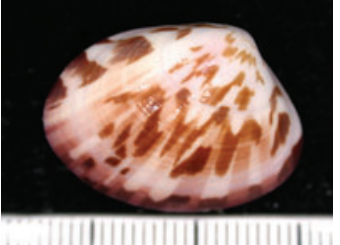

99230 I79-Callista-sp. I

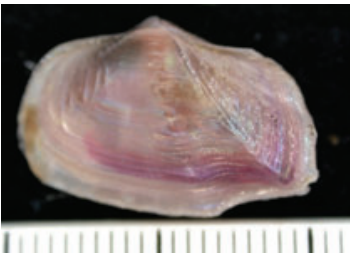

2342600 I-Lyonsiella-formosa

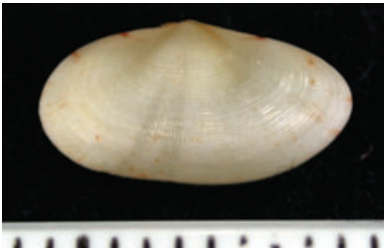

99230167-Galeommatidae-sp.2

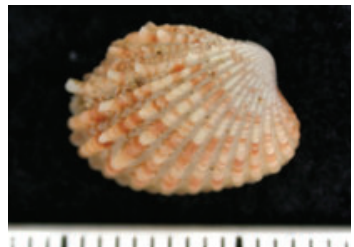

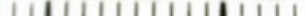

23325016-Venericardia-rosulento

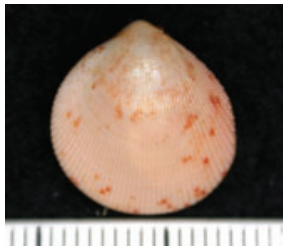

23335035-Acrosterigma-marielae

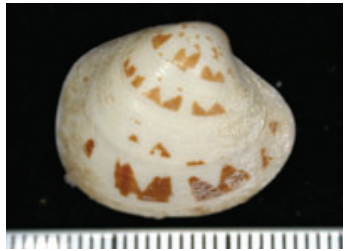

23380095-Pitar-nancyae

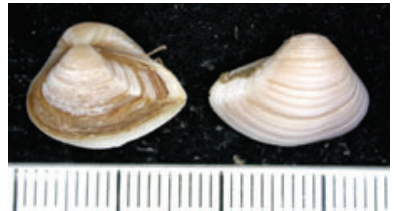

23387002-Notocorbula-stolata

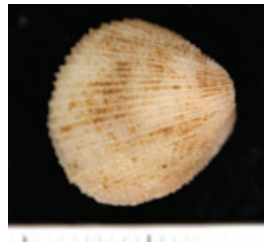

99230 I 87-Euciroa-cf.-millegemmata

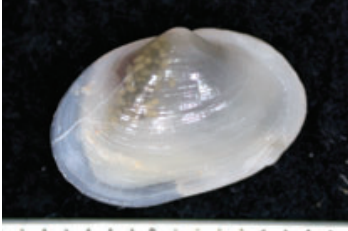

i i 1 1 1 1 i i i i 1 1 1

99230 I64-Scintilla-n.sp.I

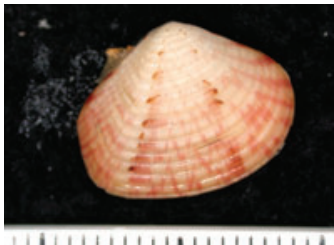

233300 I9-Crassatina-suduirauti

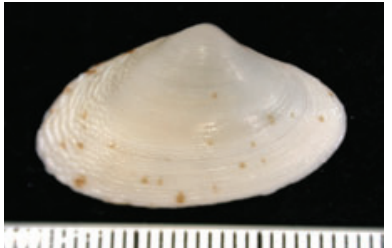

99230 I74-Mactra-sp.I

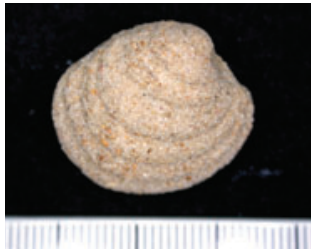

23380 I 12-Granicorium-indutum

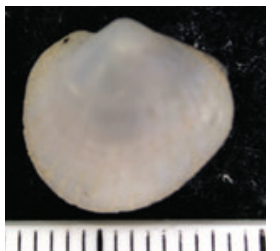

99230 I85-Policordia-sp. I

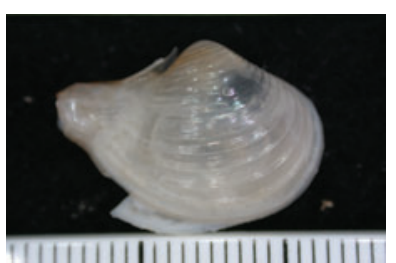

23435003-Pseudoneaera-trigonalis 


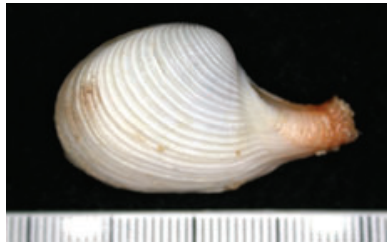

23435009-Cuspidaria-latesulcata

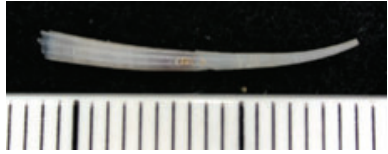

99230208-Fissidentalium-cf.-shirleyae

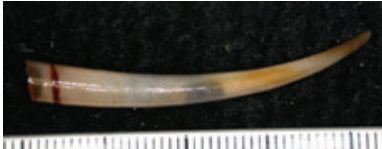

|||||||||||||||||||||||||||||||||||||||||||||

992302 I I-Laevidentalium-sp.2

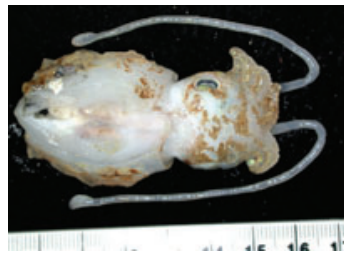

2360790 I-Sepia-sp-indet.

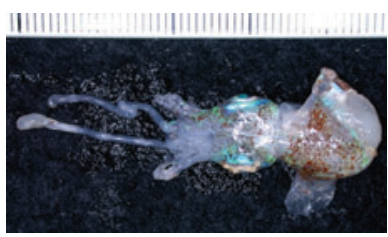

99230222-Sepiadarium-sp.l

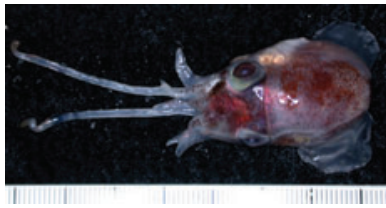

23609902-Heteroteuthis-spp.

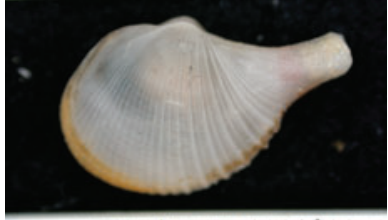

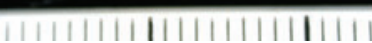

2343502I-Cardiomya-pinna

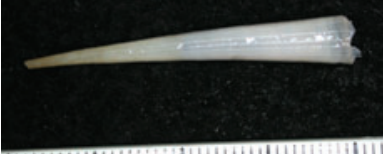

| ||||||||||||||||||||||||||||||||||||||||||||

99230207-Fissidentalium-sp.3

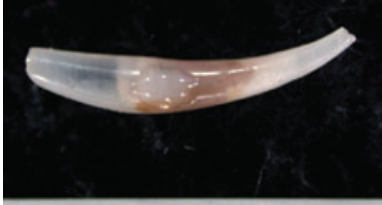

4 1 1 1 1 1 1 1 1 1

992302 I9-Dischides-sp. I

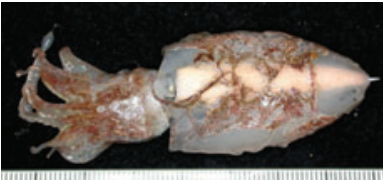

99230220-Sepia-cf-cottoni

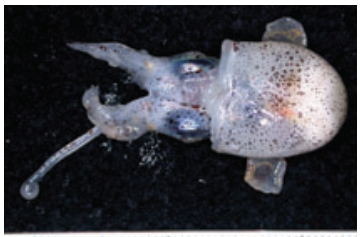

2360900 I-Euprymna-tasmanica

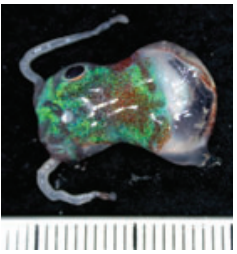

23609903-Sepiolina-spp.

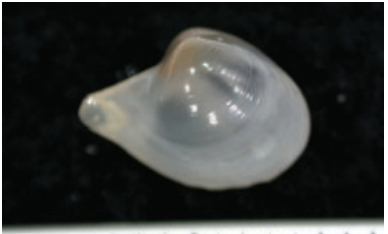

$9230198-P s e u d o n e a e r a-s p .4$

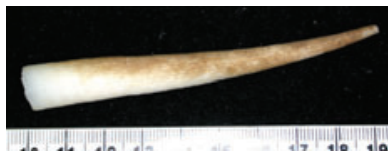

99230205-Fissidentalium-sp.I

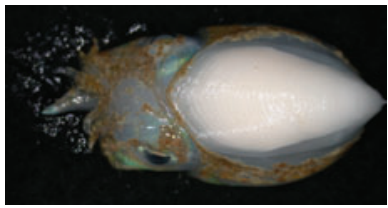

23607002-Sepia-cultrata

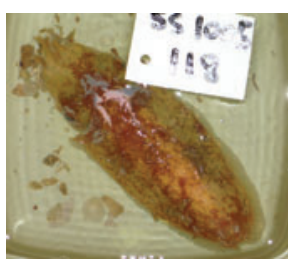

9923022I-Sepia-cf.-chirotrema

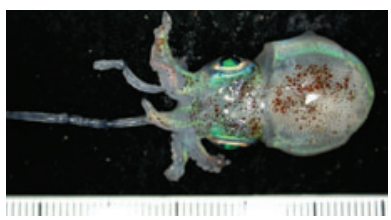

23609007-Sepiolina-nipponensis

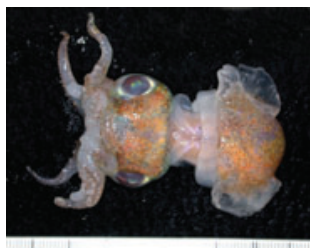

99230224-Austrorossia-cf.-australis

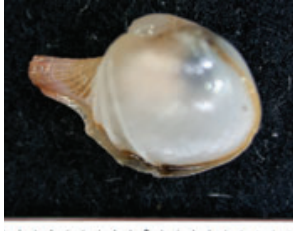

1111111111111411111 99230197-Cardiomya-sp. I

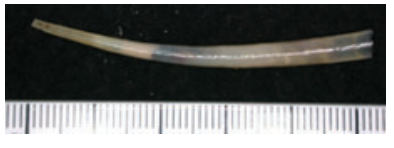

992302 I0-Laevidentalium-sp. I

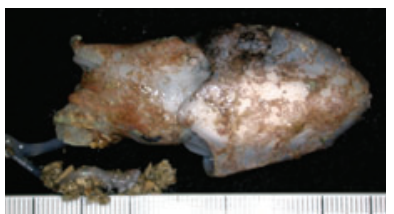

23607006-Sepia-opipara

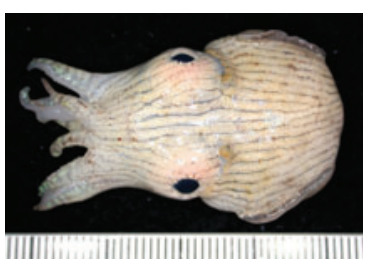

2360800 I-Sepioloidea-lineolata

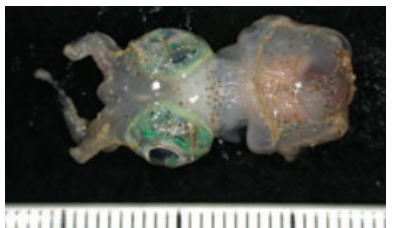

2360990 I-Austrorossia-spp.

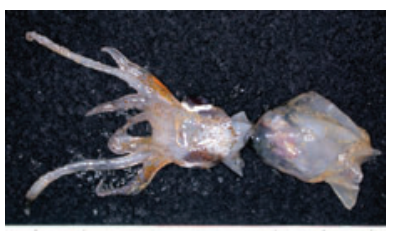

23609000-Sepiolidae-undifferentiated 

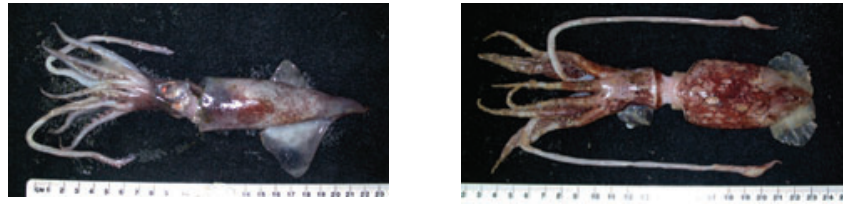

23621904-Enoploteuthis-spp.

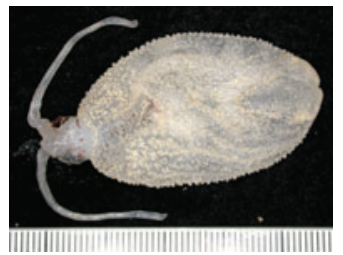

23643002-Cranchia-scabra

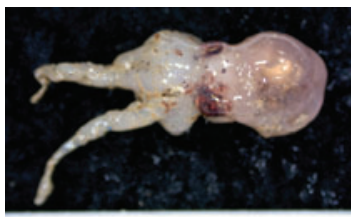

| | | | || || |||||||||||

23659000-Octopodidae-undifferentiated

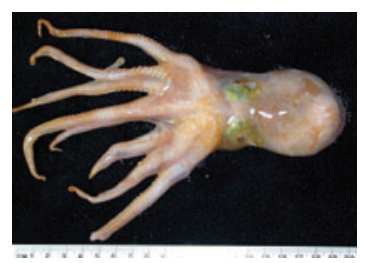

99230234-Pteroctopus-sp.I

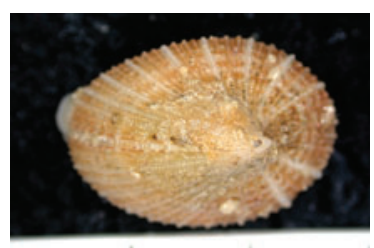

24040004-Emarginula-patula

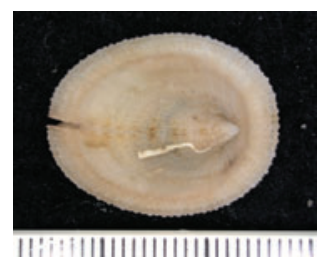

99240159-Emarginula-sp.4
23630003-Histioteuthisceletaria-pacifica

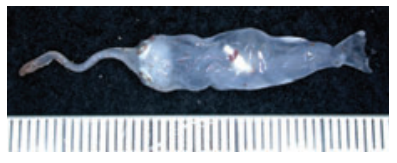

99230226-Liocranchia-sp.I

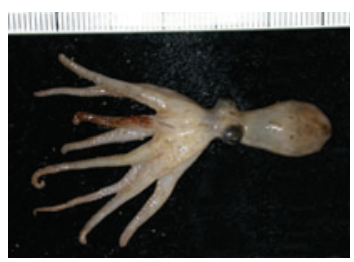

99230228-Octopus-sp.2

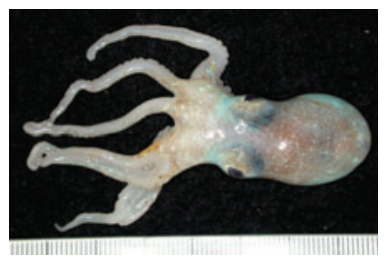

99230232-Callistoctopus-sp. I

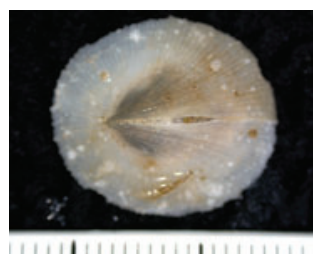

24040049-Cranopsis-corolla

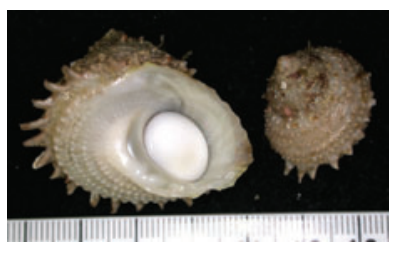

99240 I63-Bolma-sp.I

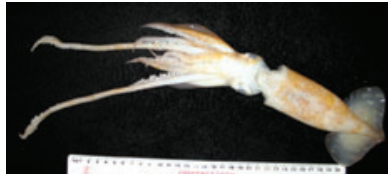

236360|3-Todaropsis-eblanae

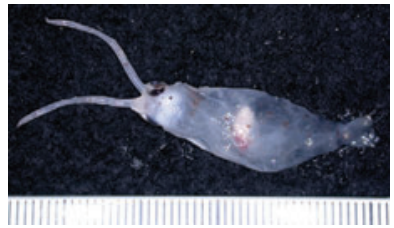

99230227-Liocranchia-sp.2

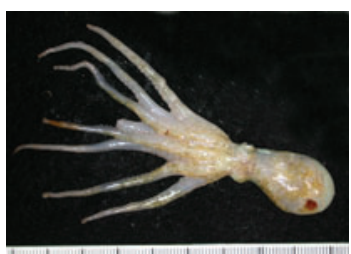

99230229-Octopus-sp.3

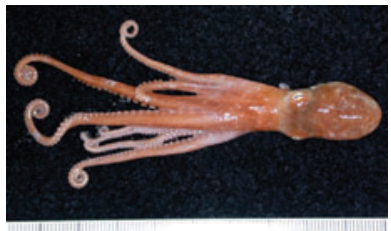

99230233-Callistoctopus-sp.2
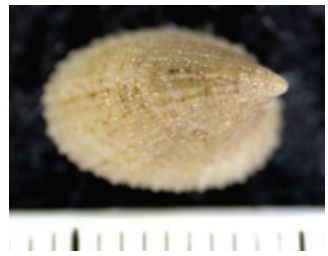

99240 I58-Emarginula-sp.I

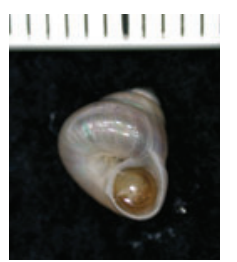

99240 I73-Periaulax-sp.3

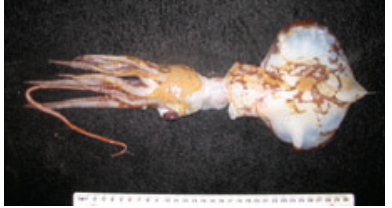

23639002-Mastigoteuthis-cordiformis

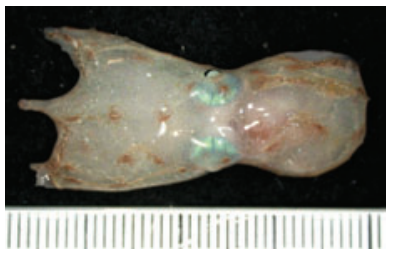

23659009-Eledone-palari

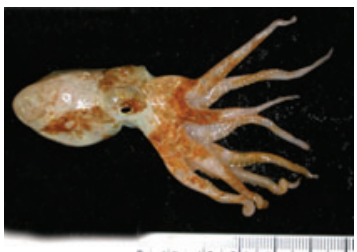

99230230-Octopus-sp.4
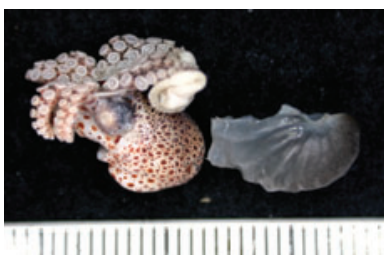

23662003-Argonauta-hians

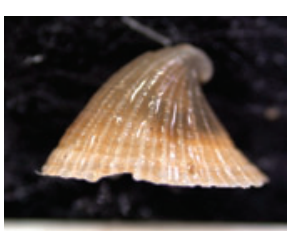

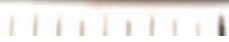

99240162-Puncturella-sp.I

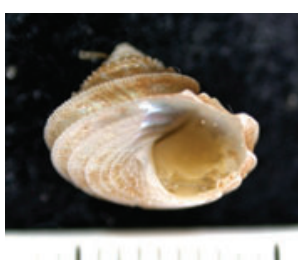

99240 I64-Calliotropis-sp. I 


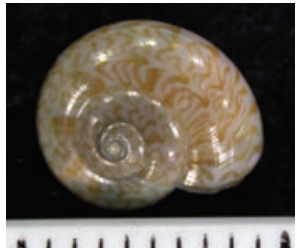

99240I75-Minoleini-sp.3

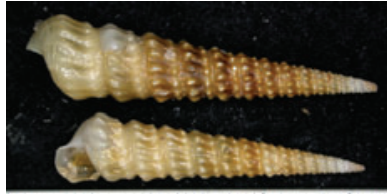

||||||||||||||||||||||||||||||||||||||||'||||

99240 I 82-Turritella-sp.I

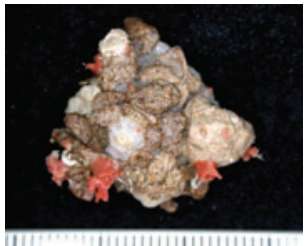

99240 I87-Xenophora-cf.-cerea

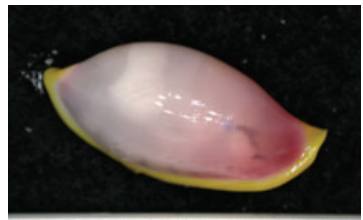

||||||||i||||||||||i||

99240 I90-Xandraovula-cf.-pagoda
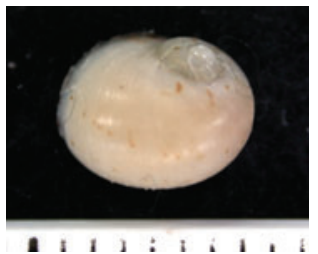

99240 I 97-Notocochlis-sp. I

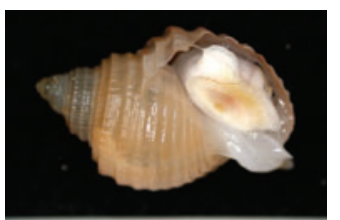

|||||||||||||||||||||||||||||||||

24I71025-Semicassis-whitworthi

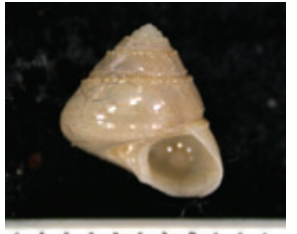

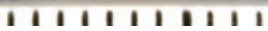

99240I72-Periaulax-sp.2

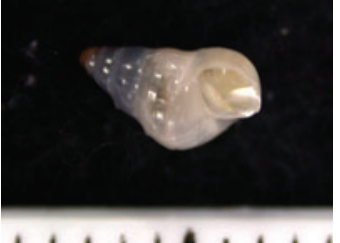

99240 I85-Rissoidae-sp. I

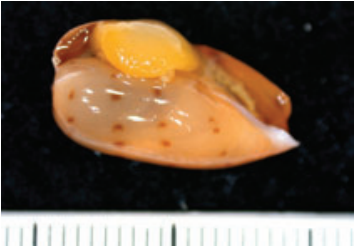

24I55030-Notocypraea-pulicaria

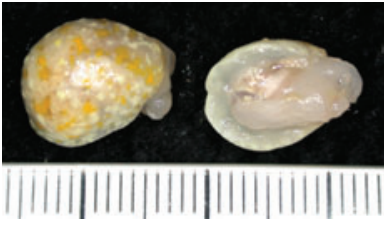

99240 I 92-Lamellaria-sp.I

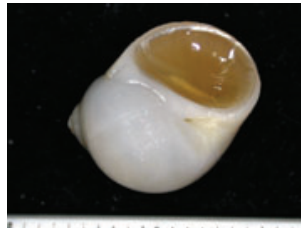

|1111111111111111111

9924020 I-Polinices-sp. I

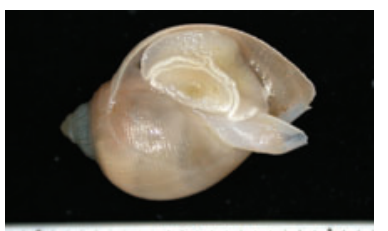

|| I|||||||||||||||||||||||

99240207-Semicassis-sp.I

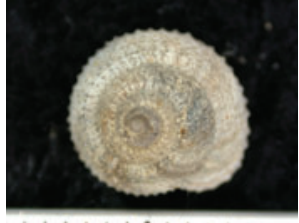

| | | | | | | | i i | | |

99240I70-Sequenziinae-sp.4

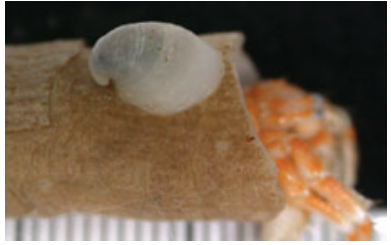

99240 I86-Capulus-sp.I

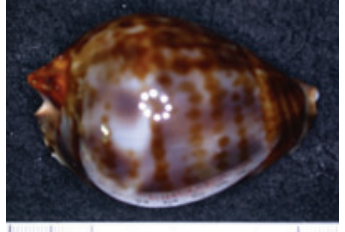

24I 55034-Zoila-friendii-vercoi

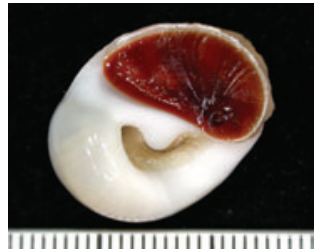

24I65052-Polinices-peselephanti
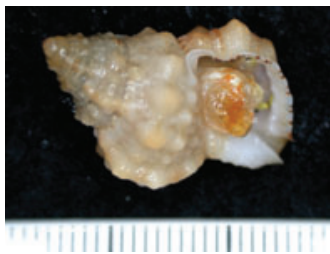

24I700 I I-Bursa-humilis

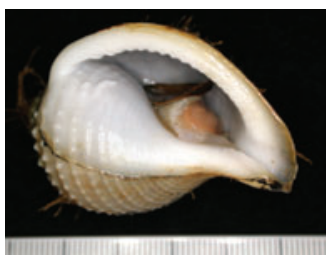

99240204-Galeodea-cf.-leucodoma

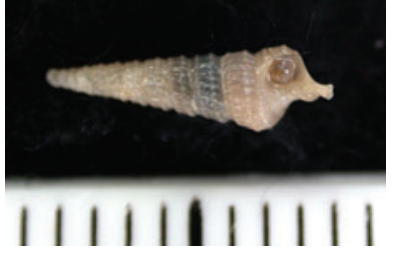

99240180-Cerithium-sp.2

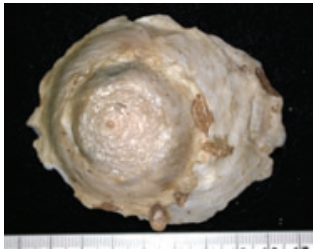

24|450| 0-Xenophora-gigantea

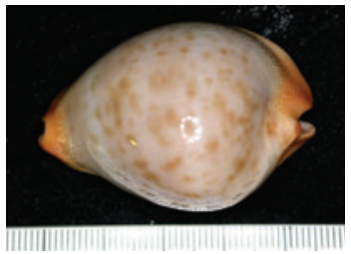

24I55036-Zoila-friendii-jeaniana

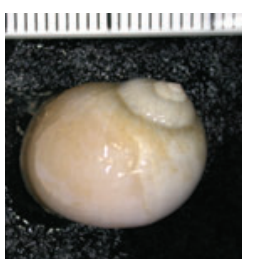

99240 195-Natica-cf.-phytelephas

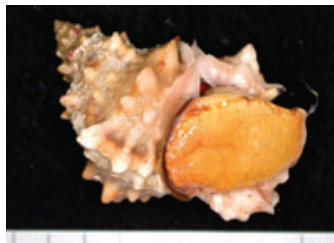

24|700|8-Tutufa-bufo

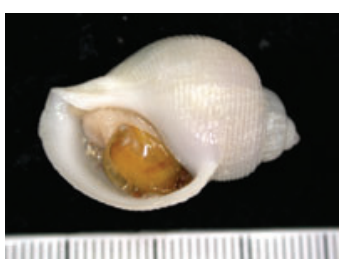

99240206-Oocorys-n-sp. I 


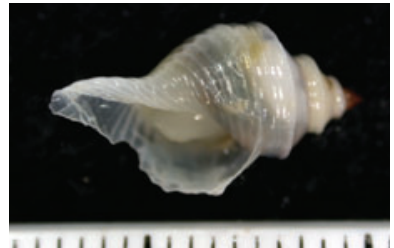

99240205-Galeodea-sp. I

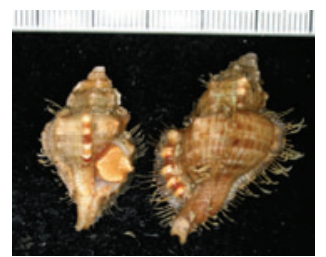

24I76044-Cymatium-vespaceum

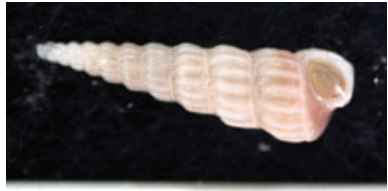

||||$i|||||||||| \mid$

24191048-Opalia-australis

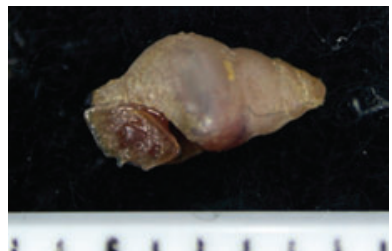

992402 I 7-Eulimidae-sp.3

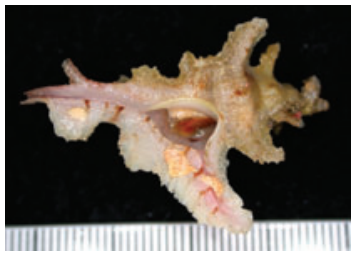

24200032-Pterynotus-undosus

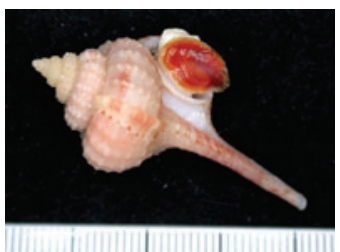

99240227-Haustellum-cf.-multiplicatus

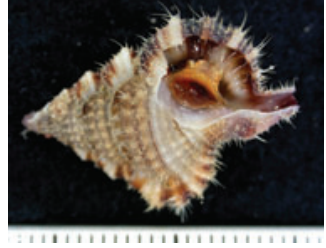

24|76010-Gyrineum-lacunatum

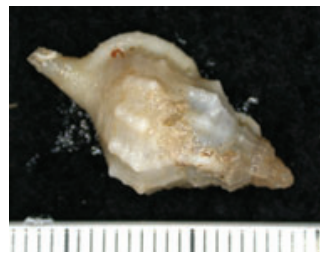

24I76046-Sassia-apenninica

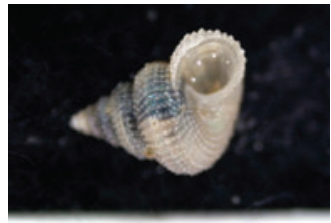

I I I 1 1 1 1 1 i ,

992402 I I-Epitonium-sp.3

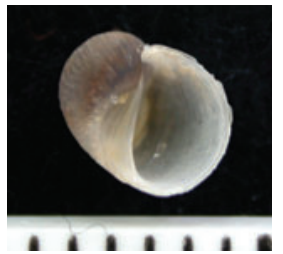

992402 I 8-Eulimidae-sp.4

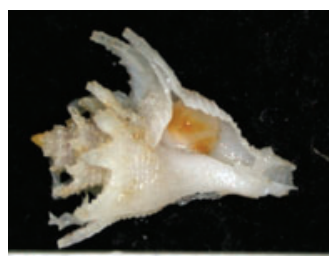

2420007I-Babelomurex-kawamurai

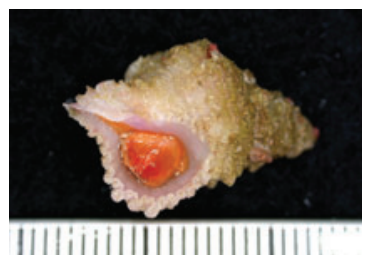

99240223-Coralliophila-cf.-inflato

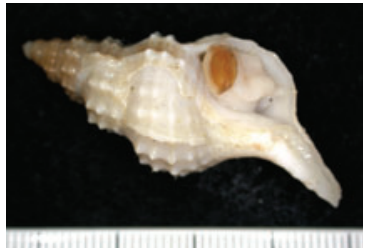

241760I2-Sassia-kampyla

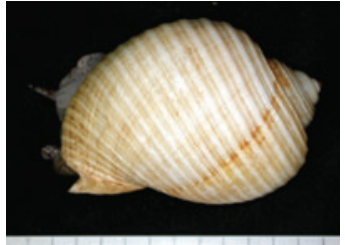

24|7700I-Tonna-variegata

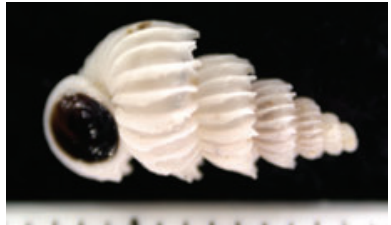

992402 I 0-Epitonium-sp. I

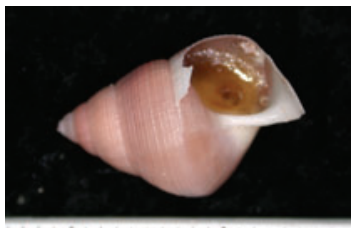

||||||||||||||||||||

992402I4-Alora-sp.2

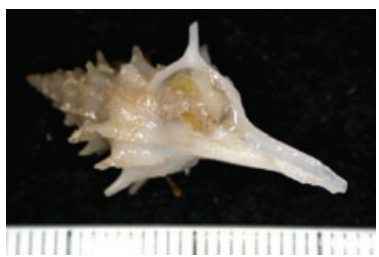

99240219-Pagodula-cf.-carduelis

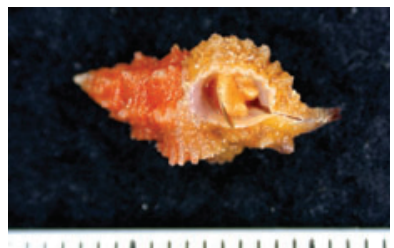

99240230-Muricopsis-sp. I

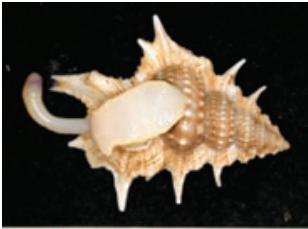

24I76022-Biplex-pulchrum

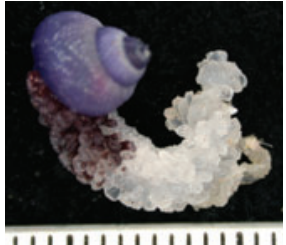

24I9000I-Janthina-exigua

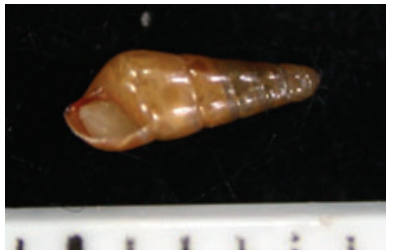

992402 I6-Eulimidae-sp.2

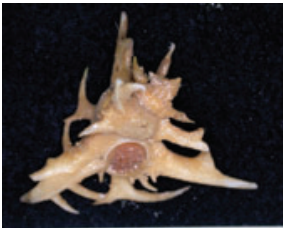

24200020-Chicoreus-cervicornis

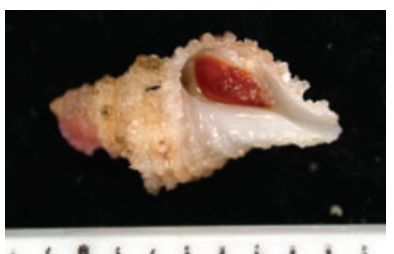

99240222-Coralliophila-sp.4

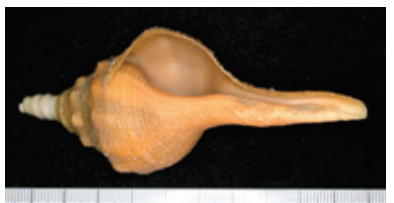

24201002-Syrinx-aruanus 


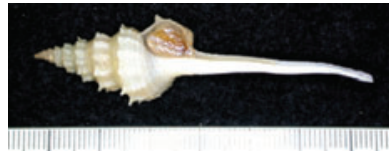

99240234-Coluzea-sp.I

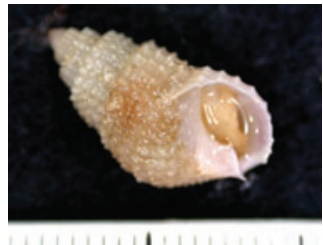

24202 I28-Nassarius-ephamillus

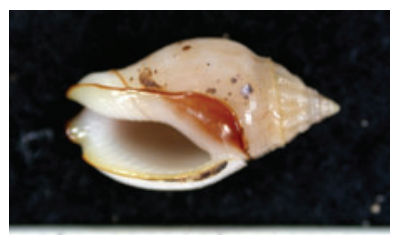

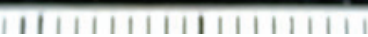

242022 I6-Cyllene-rubrolineata

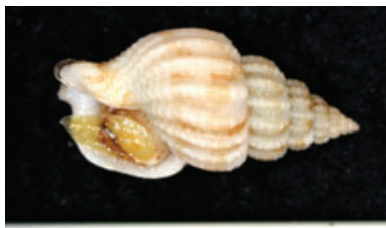

||1|||||||||||||||||||||||||||||| 99240238-Phos-sp.I

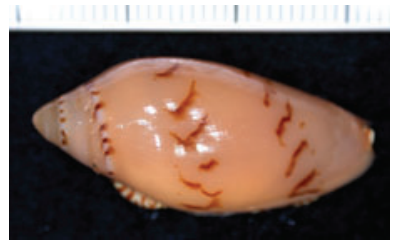

24207023-Amoria-diamantina

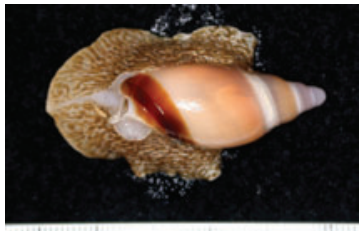

24208003-Ancillista-cingulata

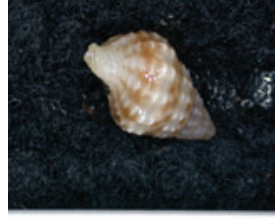

| | | | | | | | | | | | | |

24202065-Nassaria-solida

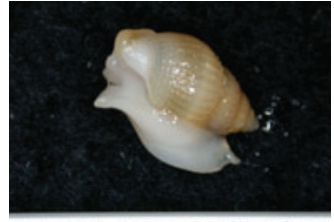

| || || || || | || | ||

24202169-Nassarius-crebricostatus

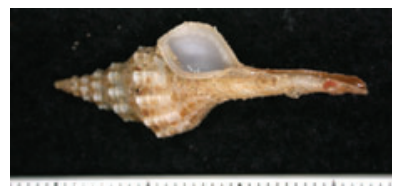

|||||||||||||||||||||||||||||||||||||||||

99240242-Fusinus-sp. I

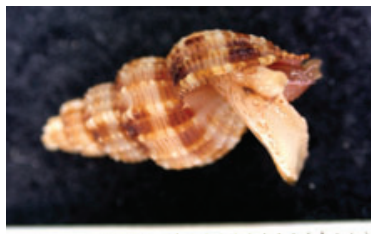

1 11111111111

99240240-Phos-cf.-roseatus

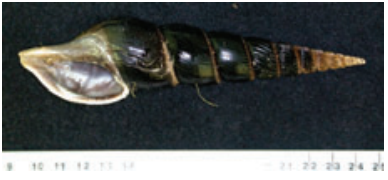

24207057-Teramachia-dalli

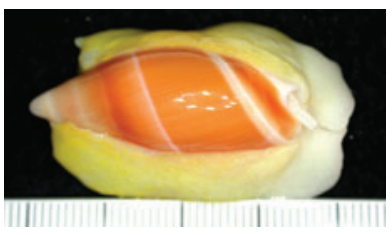

24208023-Amalda-coccinato

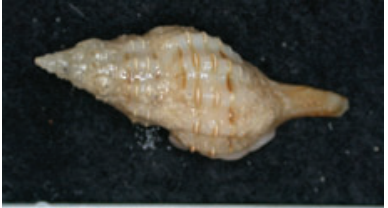

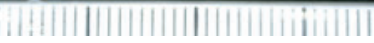

24202089-Granulifusus-kiranus

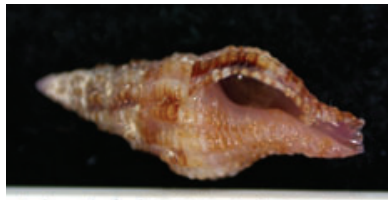

iiiillIIIIIIIII

24202 I85-Cantharus-iostomus

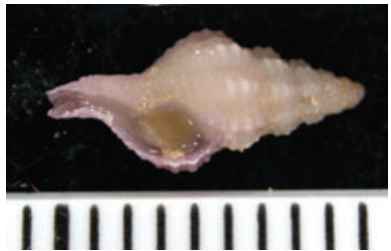

99240246-Pseudolatirus-cf.-pallidus
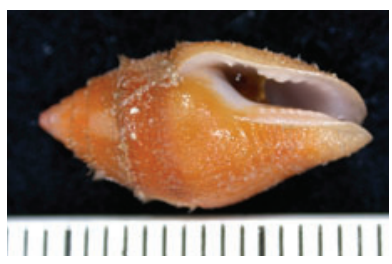

24203047-Pyrene-flava

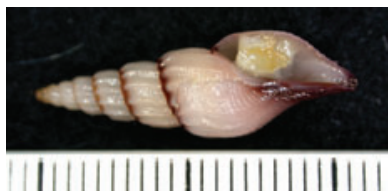

24207058-Teramachia-johnsoni

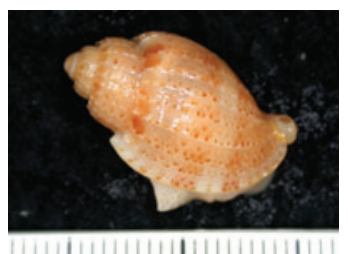

24209008-Austroharpa-wilsoni

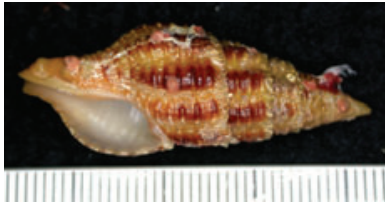

24202094-Latirus-craticulatus

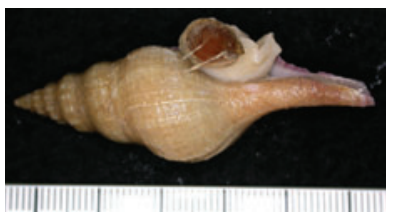

242022 I4-Fusinus-westralis

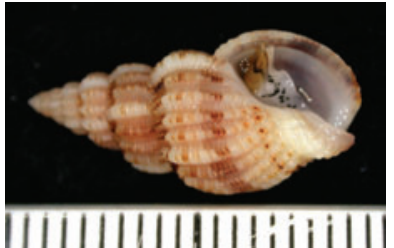

99240239-Phos-cf.-sculptilis

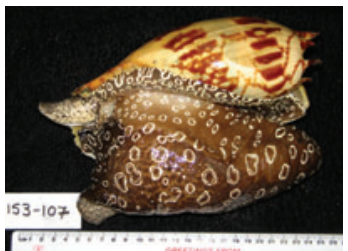

24207002-Melo-amphora

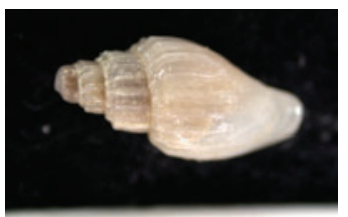

| | | | | | | | | | | |

9924025I-Volutidae-sp. I

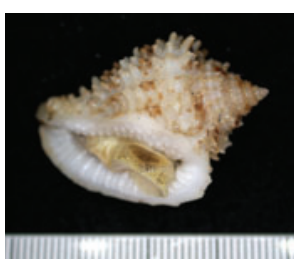

99240252-Morum-sp.I 


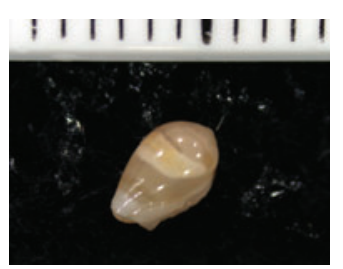

99240253-Mesoginella-sp.2

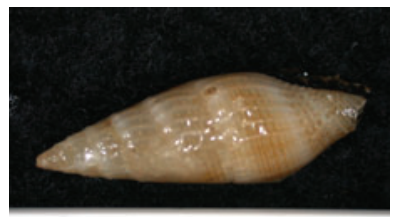

| | | | | | || || || || || |

99240258-Vexillum-cf.-obeliscus

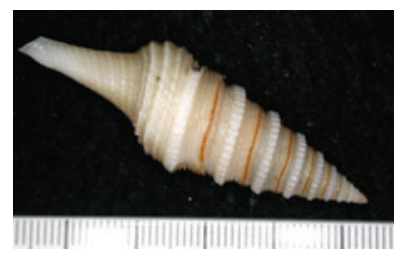

24220I25-Gemmula-westaustralis

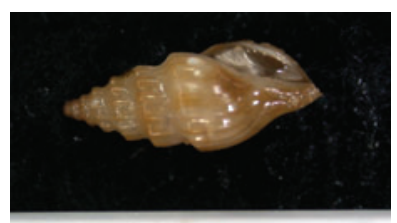

I I I I i I I I I I I

99240297-Turridae-sp.8
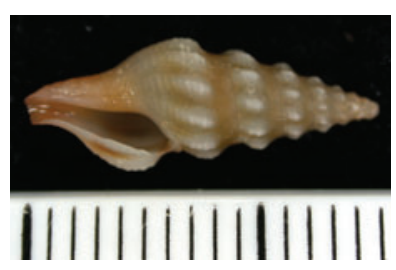

99240293-Turridae-sp.3

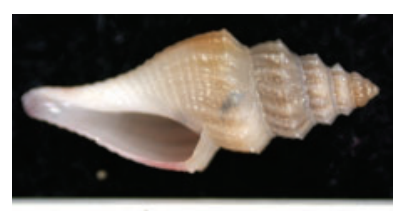

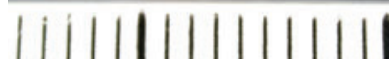

99240287-Marshallena-cf.-gracilispira

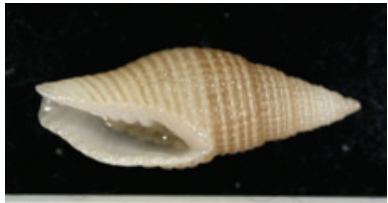

111111111111111

99240256-Cancilla-sp.2

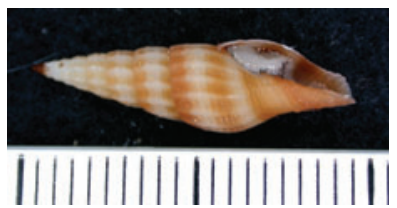

99240259-Vexillum-cf.-radius

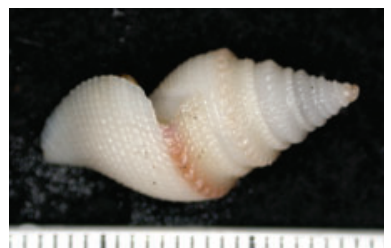

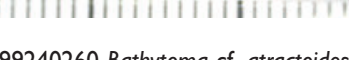

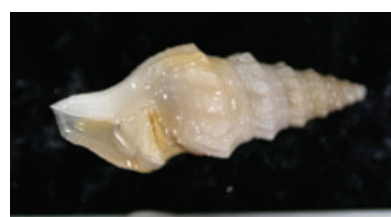

I I III I I I I I III

99240298-Turridae-sp.9

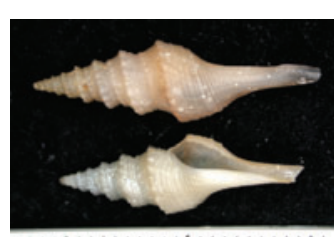

|| |II|||||||||I||||||||||I|

99240264-Cochlespira-sp.3

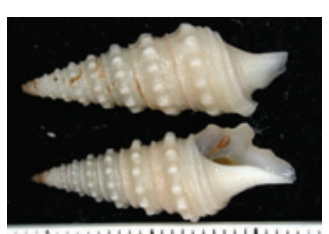

|i|i|||||||||||||||||||||||||||

99240289-Ptychosyrinx-cf.-bisinuata

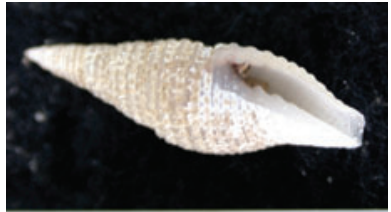

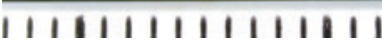

99240255-Cancilla-sp. I

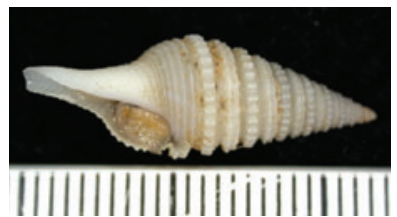

24220096-Gemmula-congener

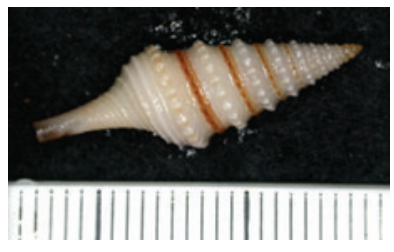

99240275-Gemmula-sp.6

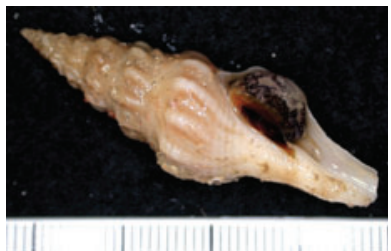

99240266-Comitas-cf.-galatheae

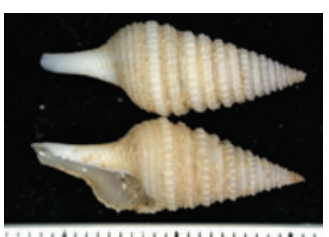

|| II|||||||||||||||||||||||

99240280-Gemmula-sp. 19

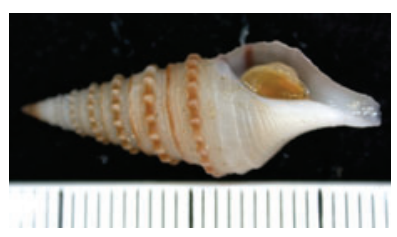

99240276-Gemmula-sp. 12

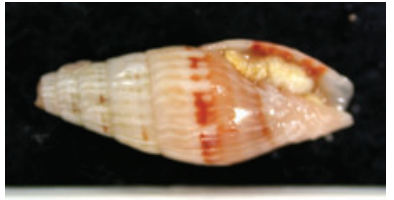

| | | | || | ||||||

242I3054-Vexillum-hansenae

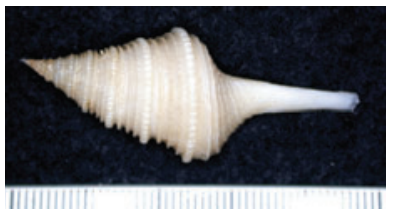

24220 I07-Pinguigemmula-philippinensis

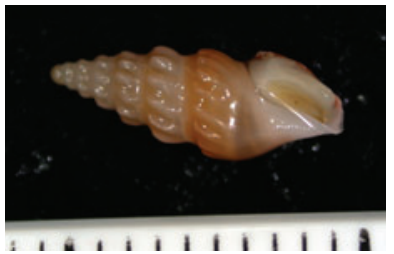

99240296-Turridae-sp.7

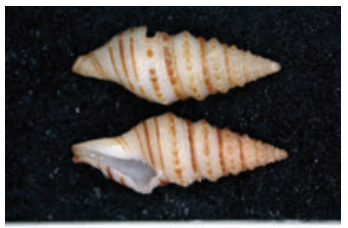

1111111111111111111111111

99240292-Turridae-sp.2

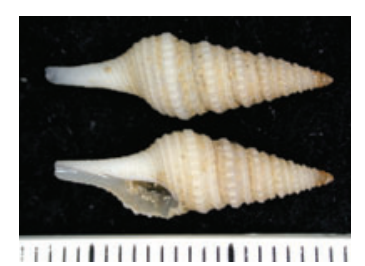

99240274-Gemmula-sp.5

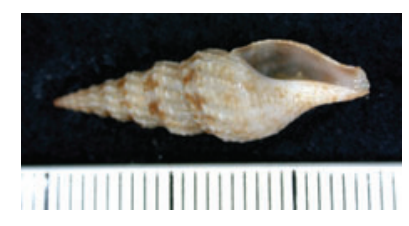

99240284-Inquisitor-sp.3 


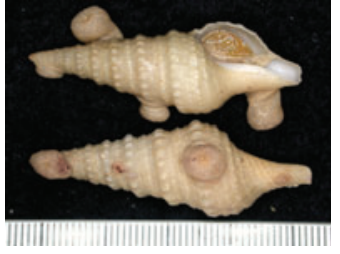

99240273-Gemmula-sp.4

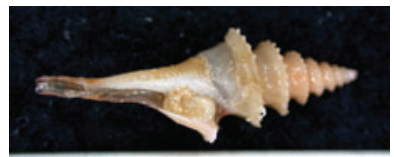

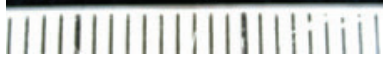

99240262-Cochlespira-sp.I

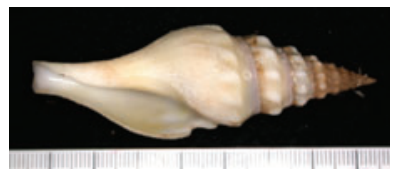

99240285-Inquisitor-sp.4

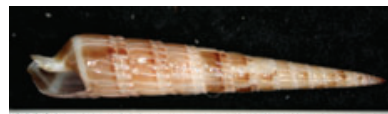

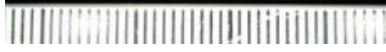

99240305-Terebra-cf.-pertusa

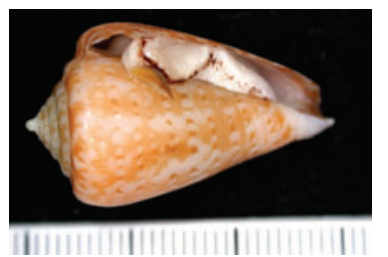

24222056-Conus-gabelishi

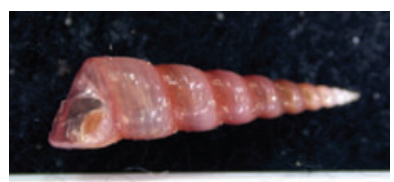

|IIIIIIIIIIIIII

992403 I2-Pyramidellidae-sp. I

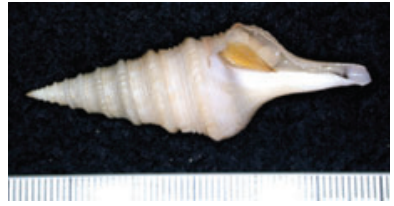

99240269-Gemmula-cf.-sibogae

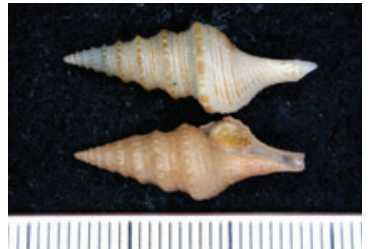

99240272-Gemmula-sp.3

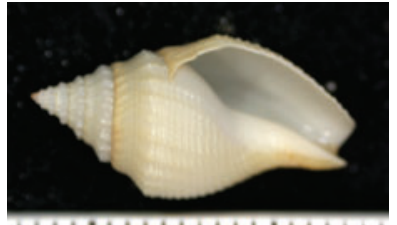

iIIIIIIIIIIIIIIIII

9924026I-Buccinaria-sp. I

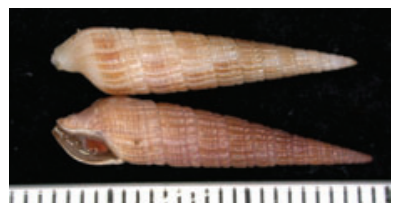

||||||||||||i|i|i||||||||||||||

99240308-Terebra-sp.7

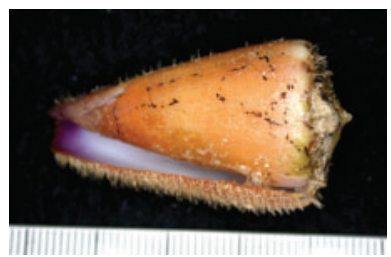

24222098-Conus-planorbis

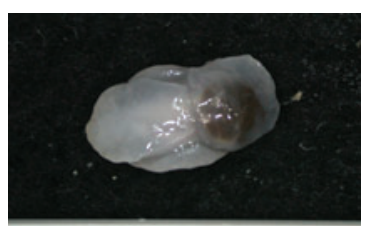

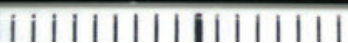

992403 |4-Philine-sp. I

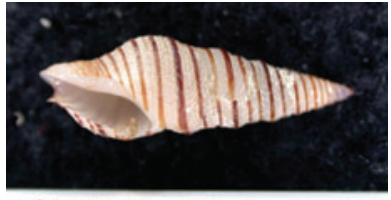

|||||||||||||||||||| $\mid$

99240292-Turridae-sp.2

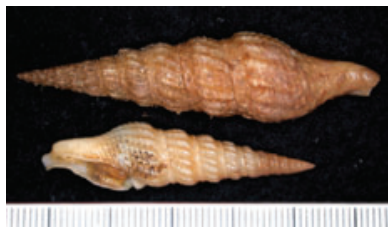

99240288-Ptychobela-cf.-flavidula

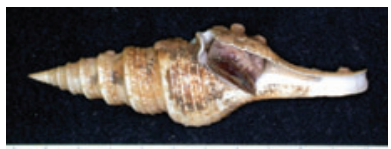

9924027I-Gemmula-sp.I

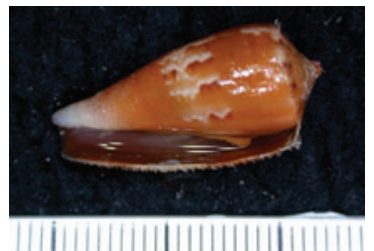

242220I 0-Conus-anemone

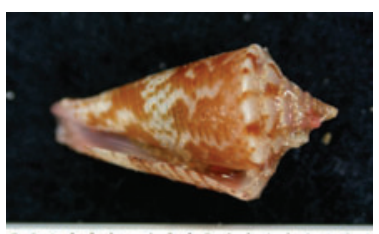

1111!i!il1111111111

99240310-Conus-sp.2

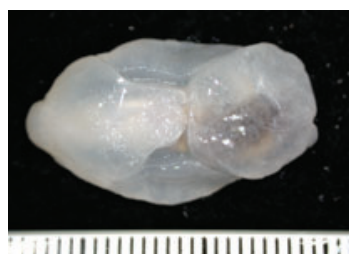

992403|5-Philine-n.sp.2

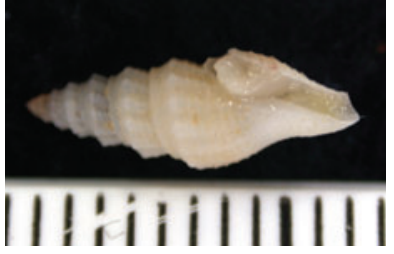

99240295-Turridae-sp.6

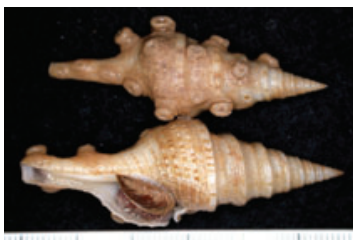

99240270-Gemmula-cf.-unedo

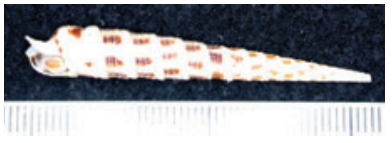

2422100I-Terebra-commaculata

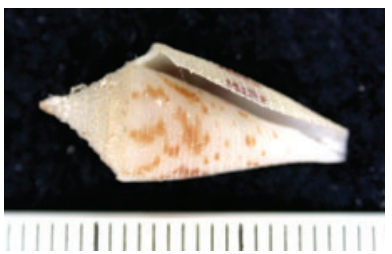

24222025-Conus-boholensis

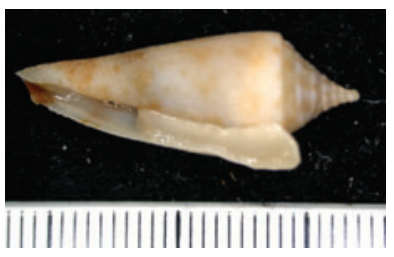

992403 I I-Conus-sp.4

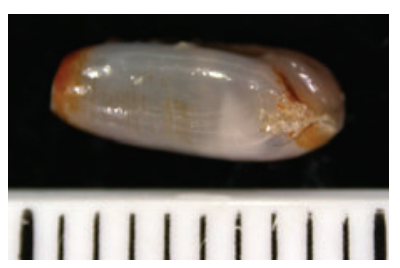

9924032 I-Liloa-cf.-curta 


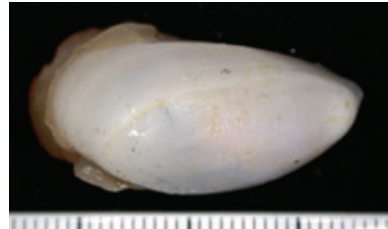

||

992403 I9-Atys-sp.I

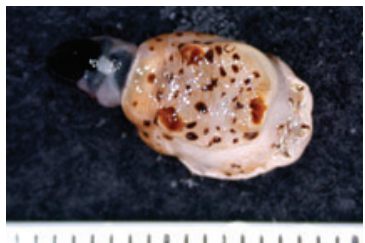

240325 -Pleurobranchidae-sp.

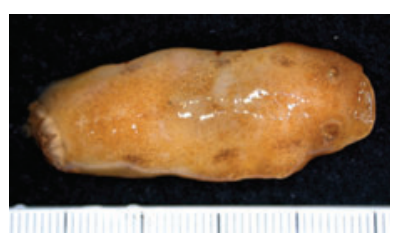

99240336-Dorididae-n.sp. I

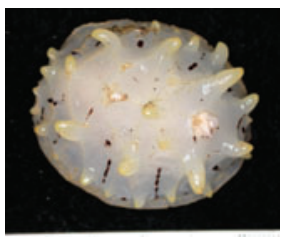

99240334-Halgerda-sp.2

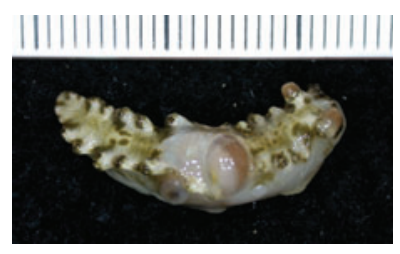

99240338-Marionia-sp.I

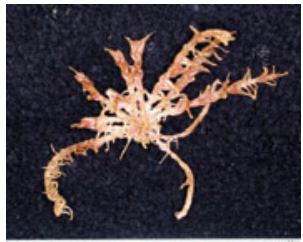

2503002I-Comanthus-parvicirrus

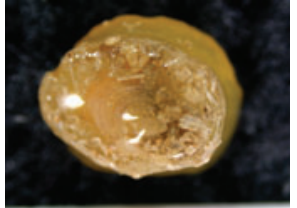

I 1111111111

24391001-Tylodina-corticalis

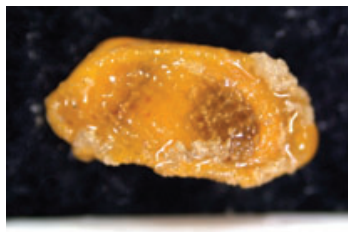

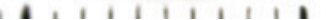

99240326-Eudoridoidea-sp. I

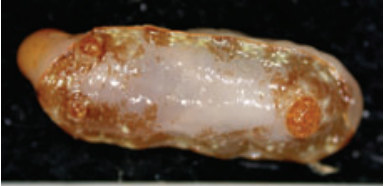

IIIIIIIIIIIIIIIIIIIIII

9924033 I-Aphelodoris-sp. I
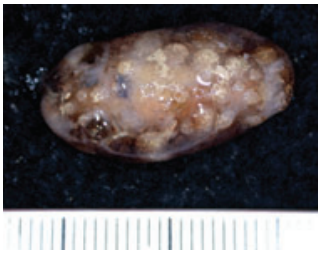

99240335-Platydoris-n.sp.I

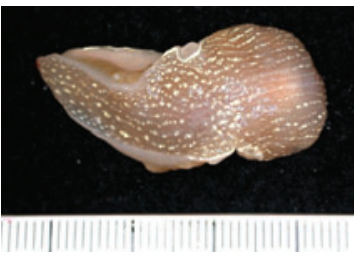

99240339-Armina-sp.I

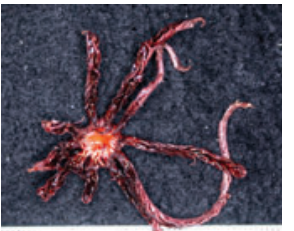

25030030-Comatula-pectinato

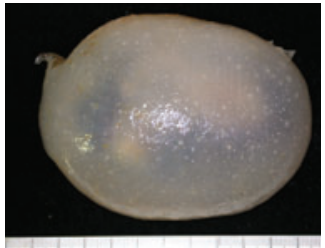

99240323-Pleurobranchus-sp.2

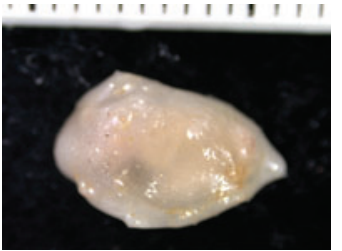

99240328-Alliodoris-sp. I

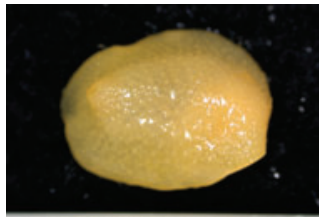

|I||||||||||||||||||

99240330-Aldisa-sp.I

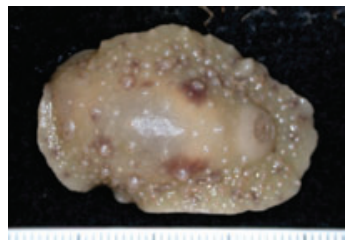

99240332-Doris-sp. I

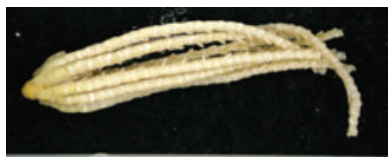

|i||||||||||||||||||||||||||||||||||||||

99250309-Bathycrinidae-sp.I

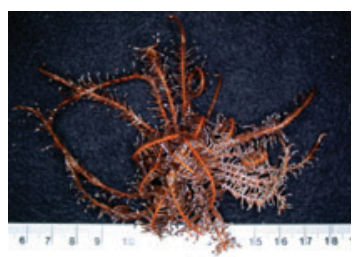

25030043-Phanogenia-brevicirra

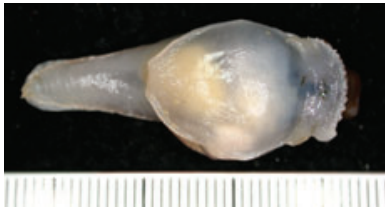

99240324-Pleurobranchaea-sp.I

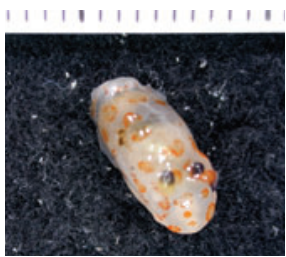

99240329-Gymnodoris-cf.rubropapulosa

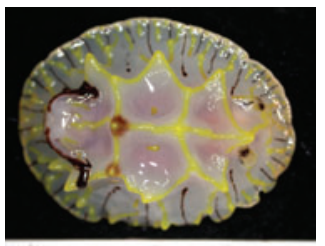

99240333-Halgerda-sp.I

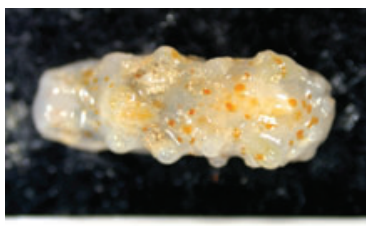

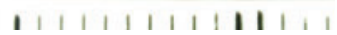

99240337-Glossodoris-sp.I

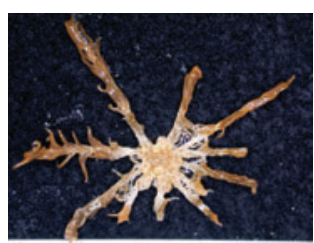

250300 I I-Clarkcomanthusluteofuscum

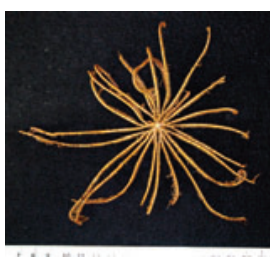

25030903-Cenolia-sp-indet. 


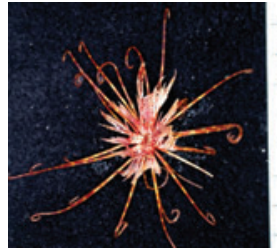

2504700 I-Ptilometra-macronema

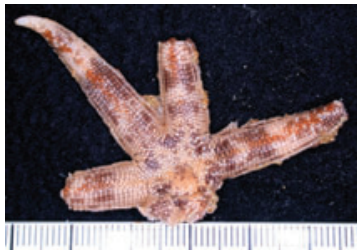

992503 | 3-Luidia-sp. I

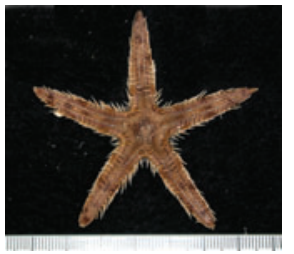

25 I I 009-Astropecten-polyacanthus

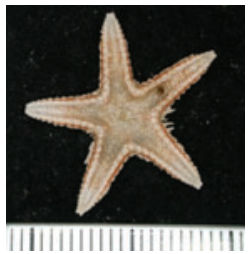

25I I 90 I-Astropecten-spp.

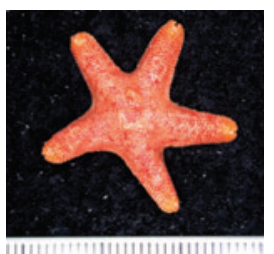

25I I 1000-Astropectinidae undifferentiated

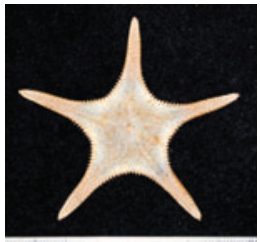

25 ||400 |-Ctenodiscus-orientalis

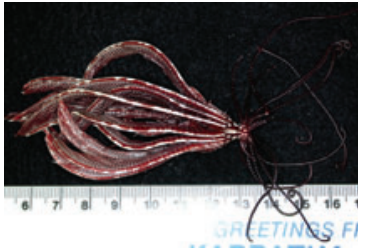

25048004-Pterometra-trichopoda

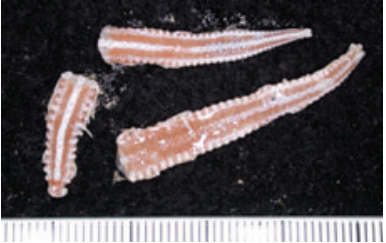

992503 I 6-Luidia-cf.-harwicki

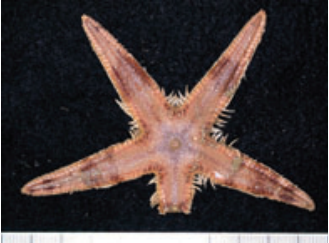

25 I I $015-$ Astropecten-velitaris

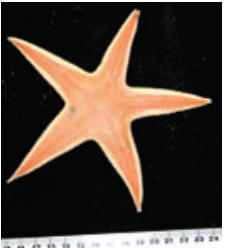

99250322-Proserpinaster-cf.-euryactis

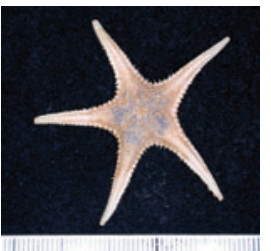

99250327-Plutonaster-sp.2

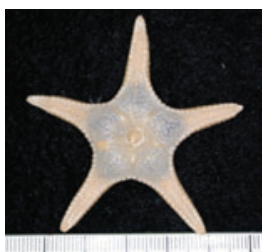

99250329-Ctenodiscus-cf.-orientalis

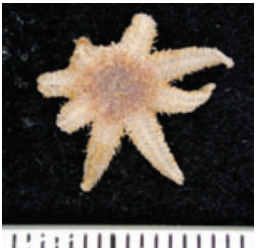

25I05002-Luidia-avicularia

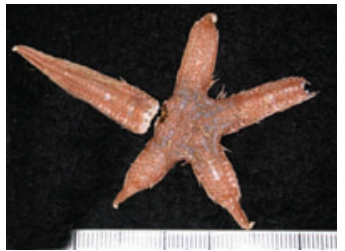

992503 |4-Luidia-sp.2

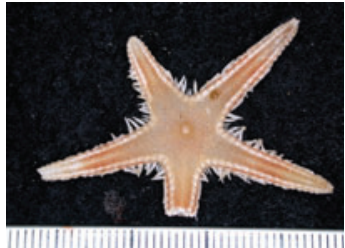

99250304-Astropecten-cf.-eremicus

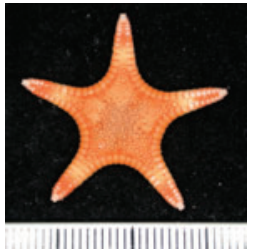

99250323-Patagiaster-sp. I

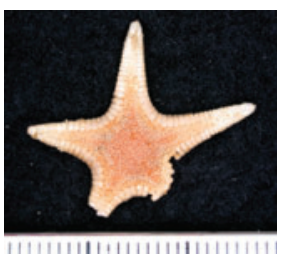

9925032I-Dipsacaster-sp.3

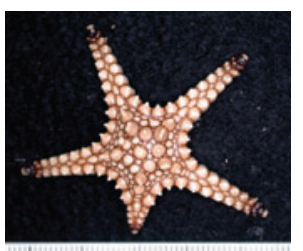

25 | 220 |4-Milteliphaster-regenerator

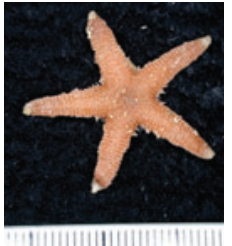

25I05003-Luidia-hardwicki

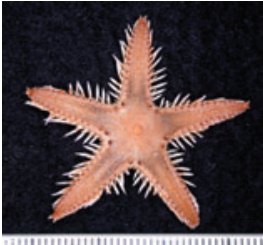

25 I I I008-Astropecten-phragmorus

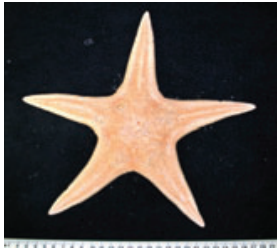

992503 I9-Dipsacaster-sp. I

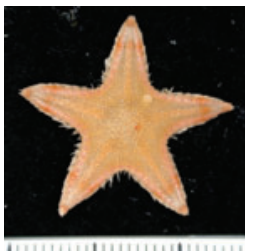

992503 I 7-cf.-Astromesites-sp. I

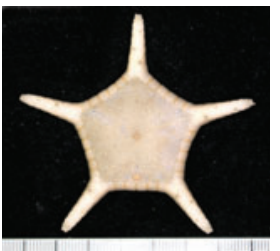

99250328-Styracaster-n.sp. I

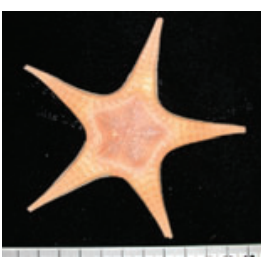

25 | 220 I7-Nymphaster-moebii 


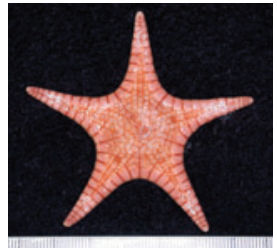

25|220|8-Ogmaster-capella

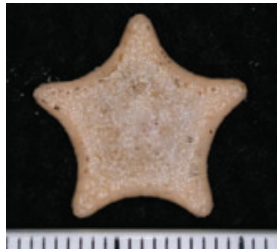

99250335-cf.-Cladaster-sp.I

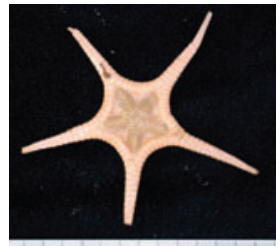

99250339-Rosaster-n.sp.I

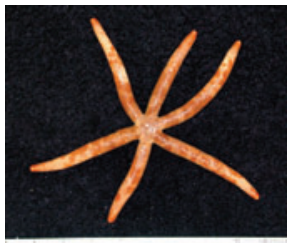

25 I 25027-Linckia-guildingi

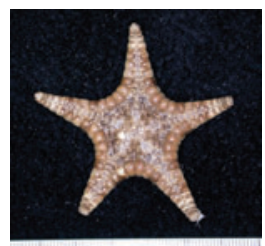

99250346-Goniodiscaster-sp.I

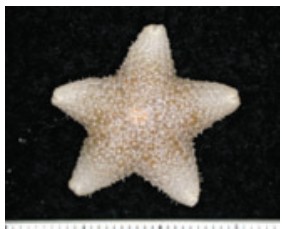

99250352-Pteraster-(Retaster) cf. corynetes

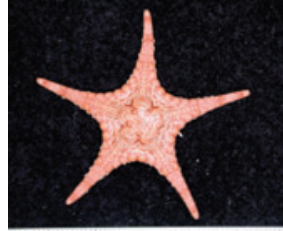

25|22026-Stellaster-equestris

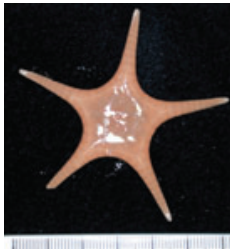

99250333-Lithosoma-cf.-penichra

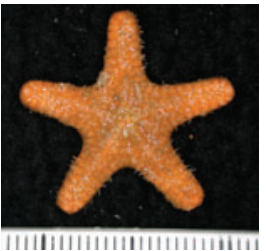

99250343-Hoplaster-sp.I

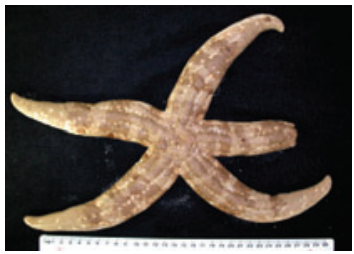

25|25043-Pseudophidiaster-rhysus

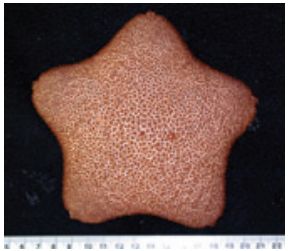

25|28004-Asterodiscides-macroplax

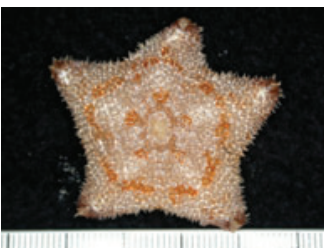

99250293-Pteraster-(Pteraster)-sp. I
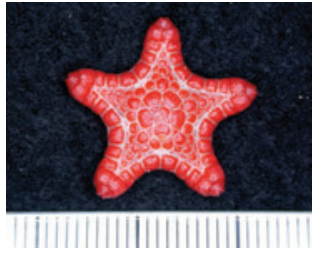

99250340-Goniasteridae-sp. I

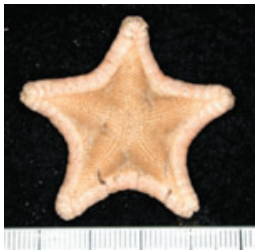

99250306-Ceramaster-sp.I

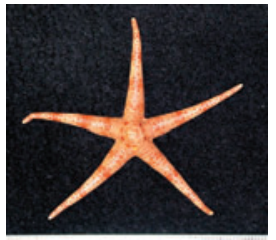

25|25023-Heteronardoa-diamantinae

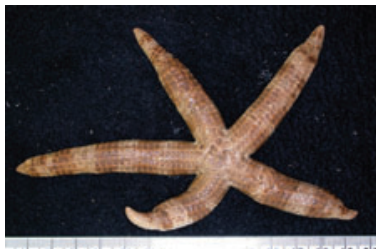

99250344-Ophidiaster-n.sp. I

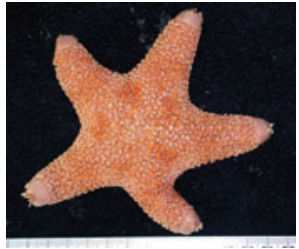

99250347-Asterodiscides-cf.-tessellatus

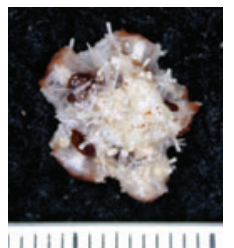

99250292-Diplopteraster-sp. I

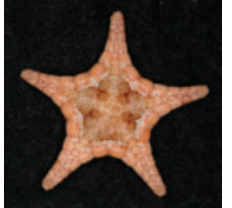

| |||||||||||||||||||| $\mid$

9925034I-Goniasteridae-sp.2

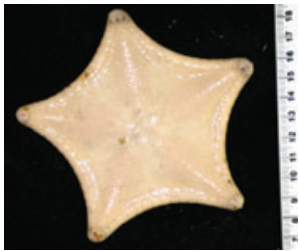

99250332-Ceramaster-cf.-patagonicus

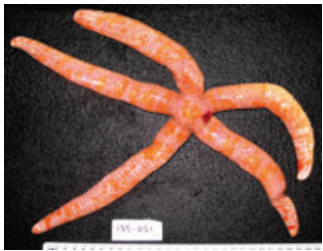

25|25025-Leiaster-leachii

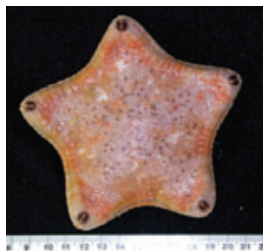

99250345-Anthenea-sp.I

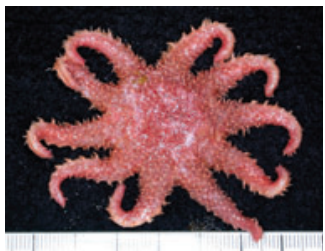

25|3600|-Crossaster-multispinus

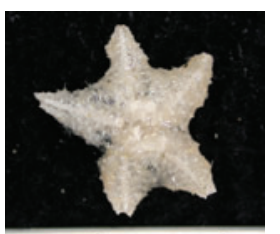

|1]|||||||||||||||||||

9925035 I-Pteraster-(Retaster)-n.sp.I 


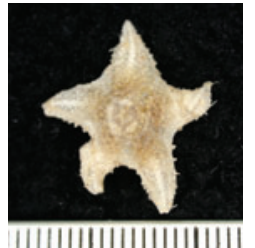

99250350-Hymenaster-sp.I

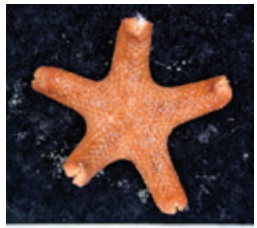

41111111111111111111

99250355-Pseudonepanthia-n.sp.I

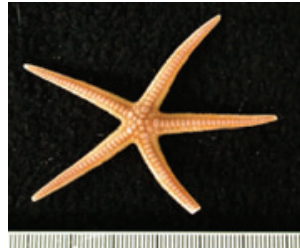

99250357-Pholidaster-n.sp. I

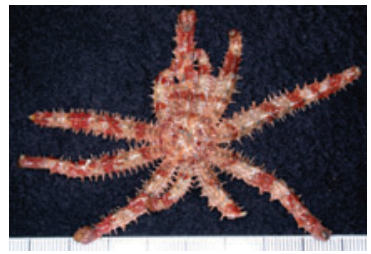

25I540 I I-Coscinasterias-muricata

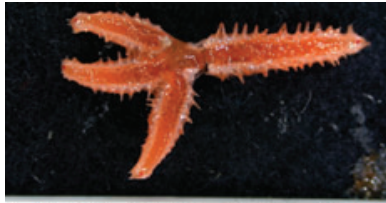

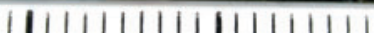

99250363-Anasterias-n.sp.I

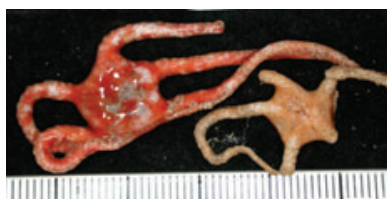

25I66007-Ophiomyxa-sp.MOV5486

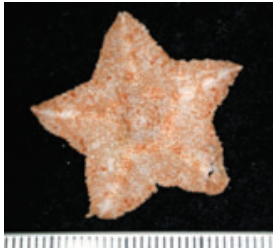

25|40002-Anseropoda-macropora

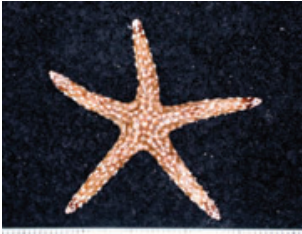

25| 43008-Echinaster-stereosomus

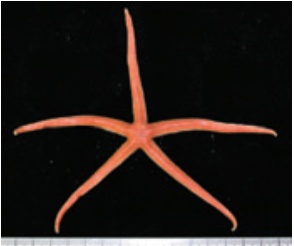

99250358-Zoroaster-n.sp.I

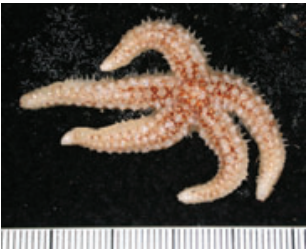

99250364-Sclerasterias-n.sp.I

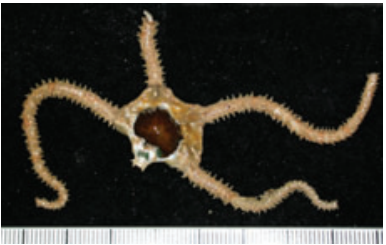

25I6600I-Ophiomyxa-australis

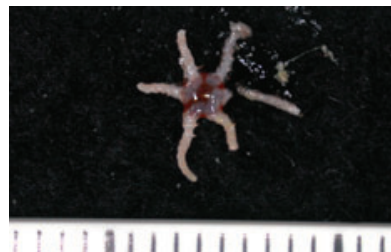

25|85078-Ophiurothamnus-sp.MOV5489

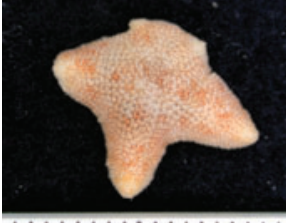

1111111111111111111

25|40047-Aquilonastra-shirleyae

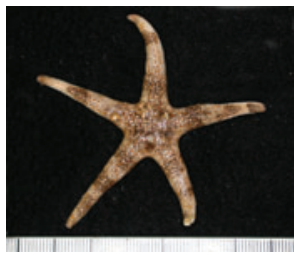

25|430|3-Metrodira-subulata

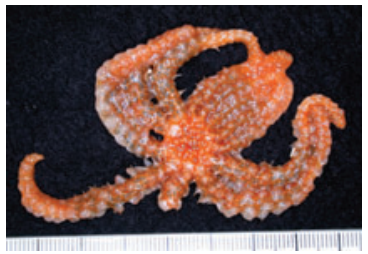

99250360-Coronaster-cf.-volsellatus

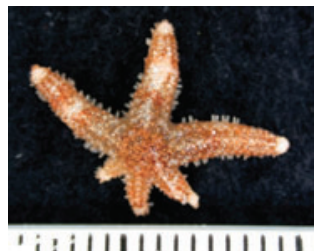

99250362-Allostichaster-n.sp.2

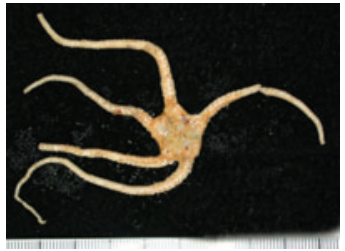

25I66006-Ophiomyxa-crinita

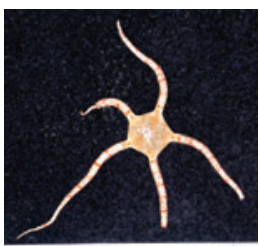

25|66009-Ophiomyxa-MoV550|

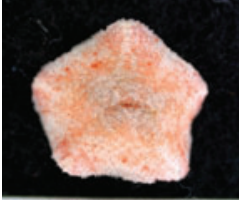

||||||||||||||

99250354-Parvulastra-n.sp.I

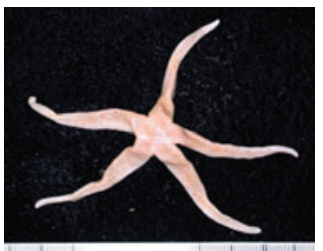

99250356-Henricia-cf.-compacto

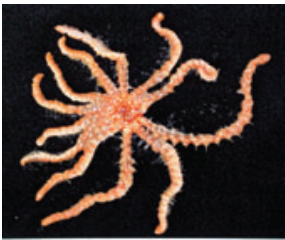

99250359-Coronaster-cf.-halicepus
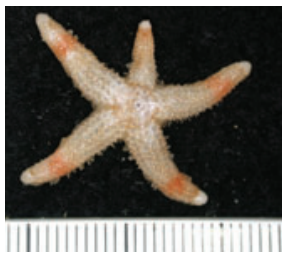

9925036 I-Allostichaster-n.sp.I

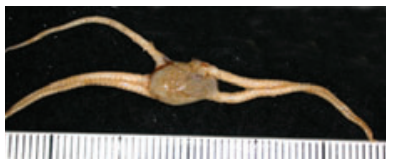

99250368-Ophiurothamnus-sp.I

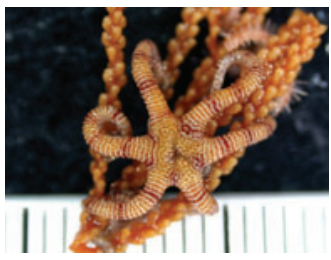

25I70007-Asteromorpha-koehleri 


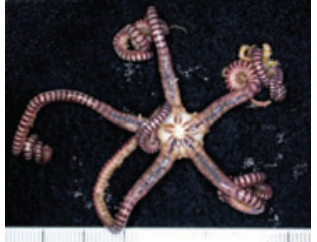

25170008-Astroceras-pergamena

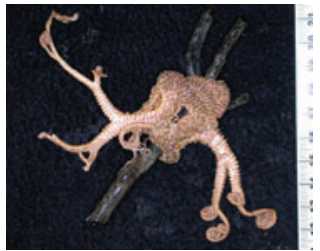

25I7|0I9-Astrosierra-microconus

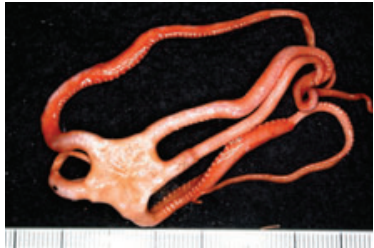

25I7300I-Asteronyx-Ioveni

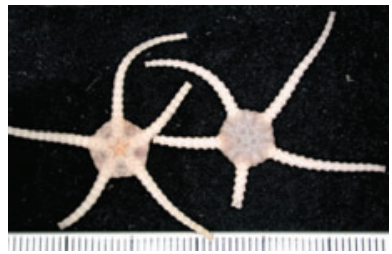

25I76007-Ophiomusium-lymani

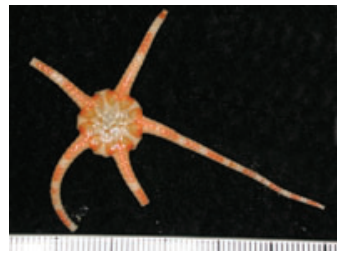

25I76025-Ophiomusium-australe

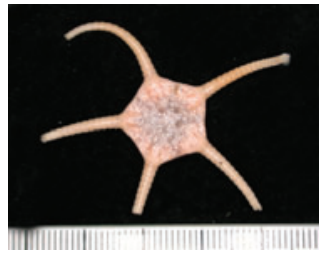

25I7604I-Ophiura-rugosa

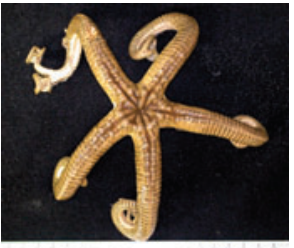

25|70009-Trichaster-flagellifer

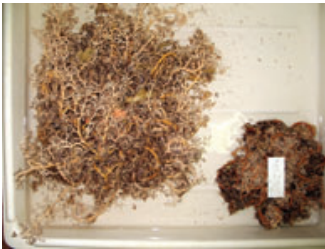

99250365-Astroboa-sp.I

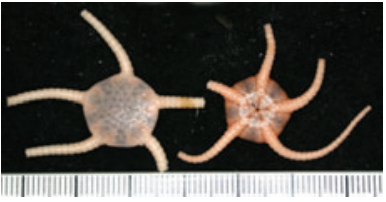

25176002-Ophiura-jejuna

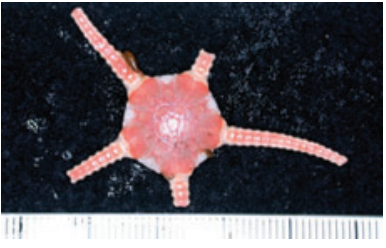

25I760 I0-Amphiophiura-urbana

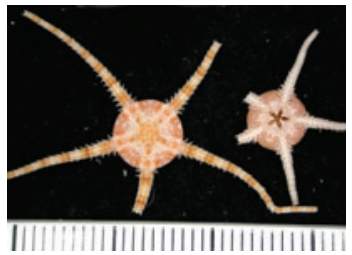

25I76037-Ophiura-kinbergi

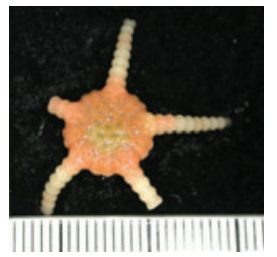

25I76044-Ophiomusium-relictum

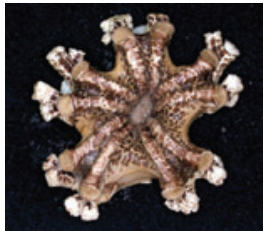

25171009-Astroboa-nigrofurcata

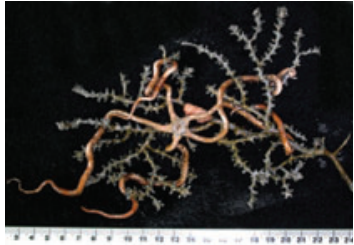

25|7200|-Ophiocreas-sibogae

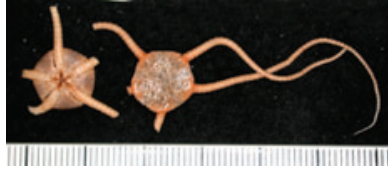

25I76003-Ophiura-irrorata

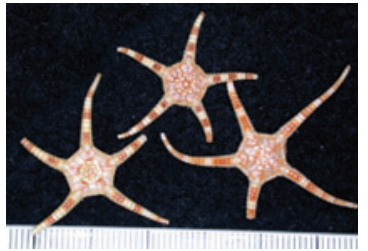

25I760|6-Ophiocrossota-multispina

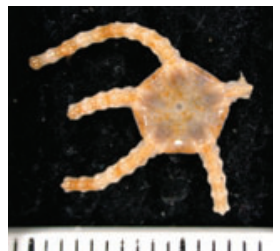

25I76039-Ophiura-ooplax

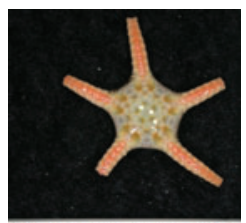

|||||||||||||||||||| $\mid$

25I7606I-Amphiophiura-distincto

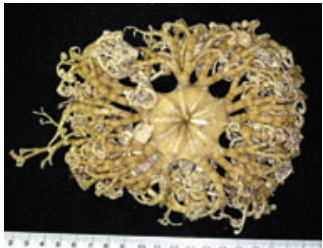

25I7I0I3-Astrocladus-exiguus

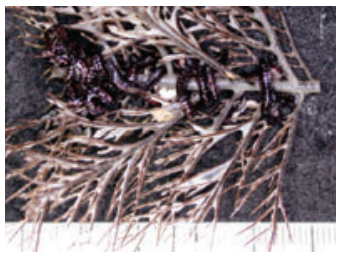

25I72004-Astrobrachion-adhaerens

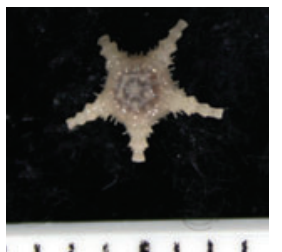

25I76005-Ophiomisidium-irene

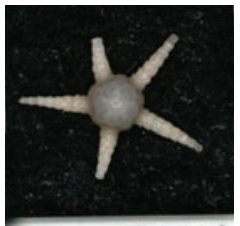

| | | | | | | | | | |

25I76022-Ophiomastus-tegulitius

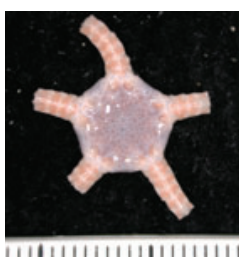

25I76040-Ophiura-palliata

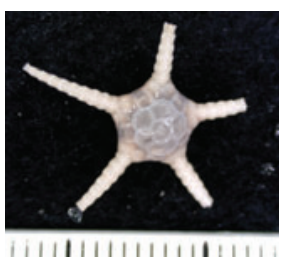

25I76062-Ophiopyrgus-trispinosus 


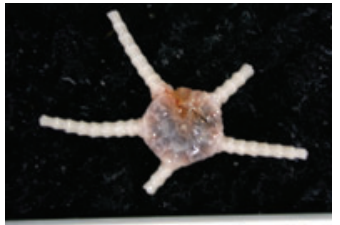

1111111111111111111 25176084-Ophiura-sp.MOV2734

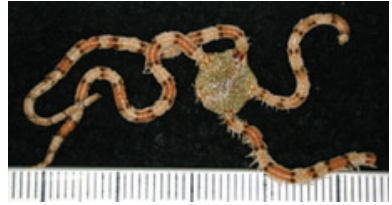

25179009-Ophionereis-schayeri

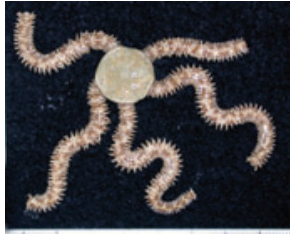

25I7902I-Ophiochiton-sp.MOV5498

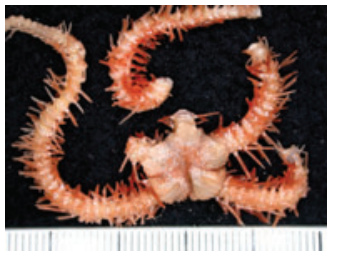

25|85007-Ophioplinthaca-plicato

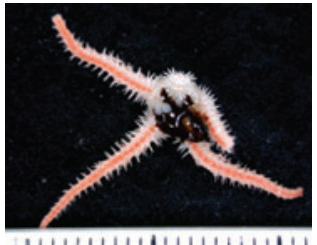

25|85027-Ophiomyces-delato

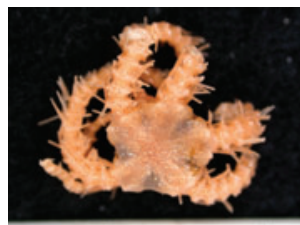

25185056-Ophiacantha-sp.MOV273

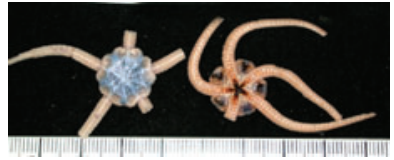

25 I7700I-Ophiernus-vallincola

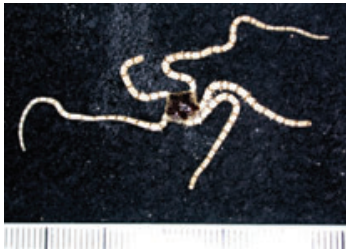

25179010-Ophionereis-semoni

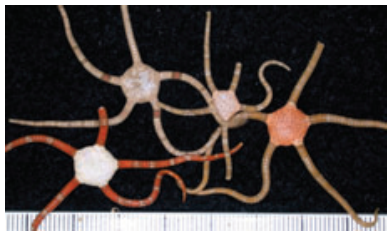

25|80024-Ophiopeza-cylindrica

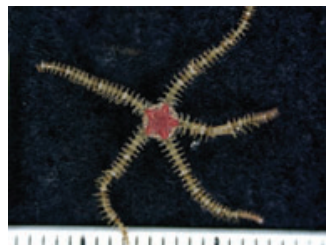

1711111101111111111117n

251850 II-Ophiacantha-alternata

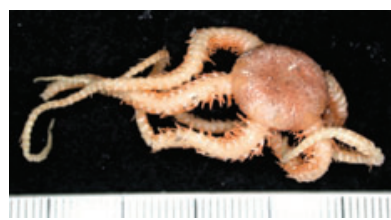

25185029-Ophiophthalmus-relictus

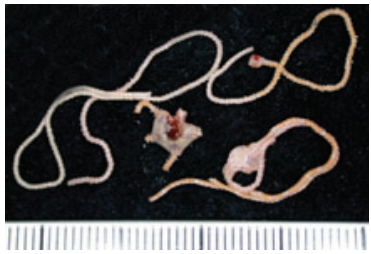

25|85063-Amphlimna-transacta

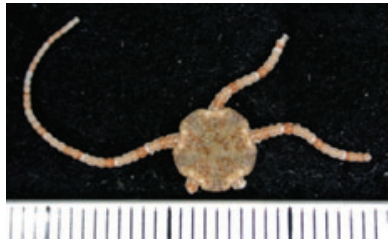

25I77003-Ophioleuce-seminudum

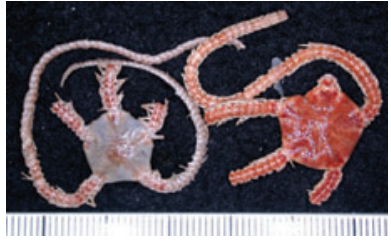

25|790|6-Ophioplax-lamellosa

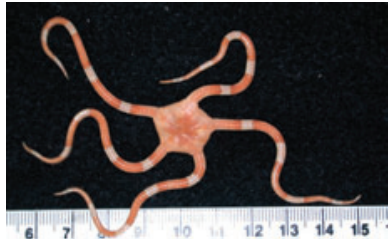

25|80028-Ophiopsammus-assimilis

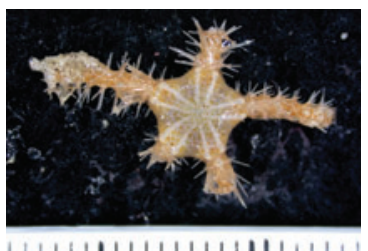

251850 I2-Ophiacantha-brachygnatha

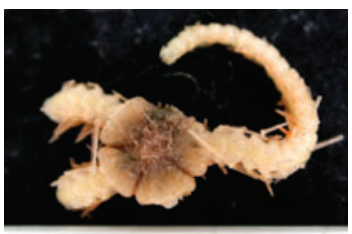

$11111111111111111 \mathrm{i}$

25 |85031-Ophioplinthaca-rudis

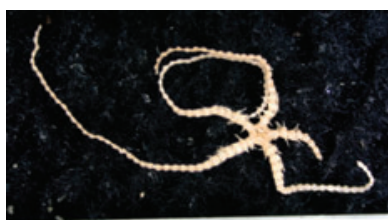

| | | | | | |||| |||| || || 25185064-Ophiothamnus-biocal

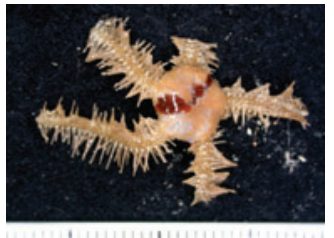

25I7800 I-Clarkcoma-bollonsi

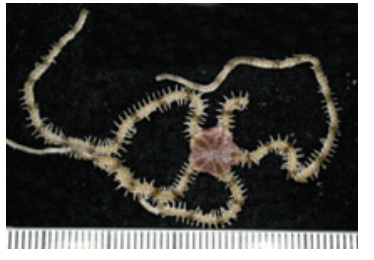

25179020-Ophiodoris-malignus

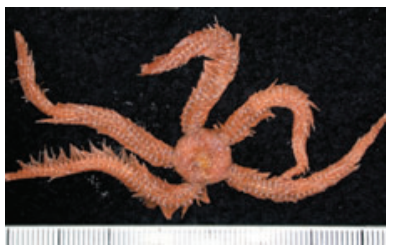

25|85004-Ophiacantha-spectabilis

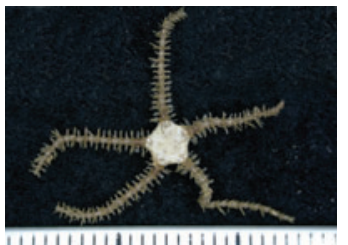

25185013-Ophiacantha-clavigera

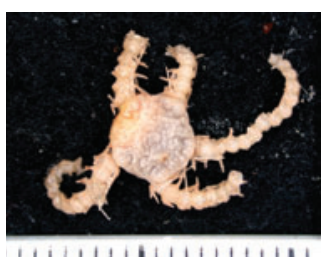

25185045-Ophiurothamnus-clausa

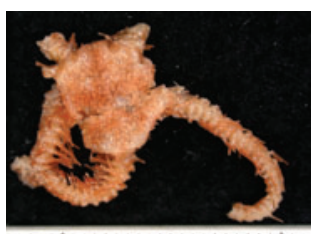

|| ||||||||||||||||||||||||||||

25185065-Ophiacantha-pacata 


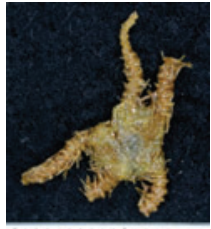

111111111111111111

25|85079-Ophiopristis-sp.MOV5490

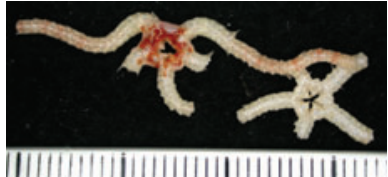

25 I8507|-Ophiotoma-sp.MOV5504

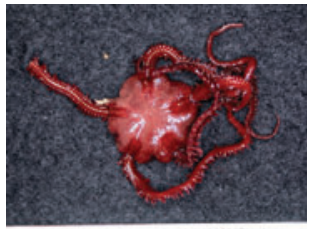

25190017-Ophiodaphne-formatus

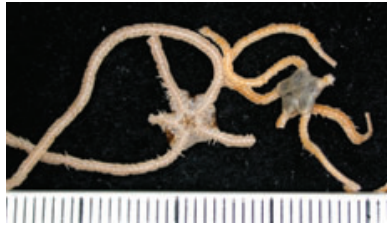

25191062-Amphiura-sp.MOV3579

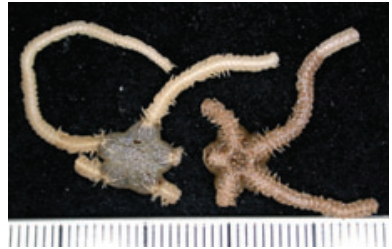

25191068-Amphiura-sp.MOV5508

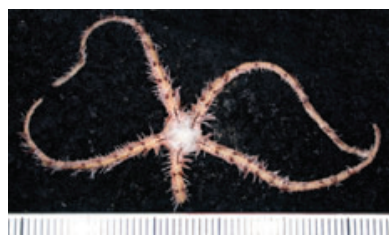

25192027-Ophiogymna-pulchella

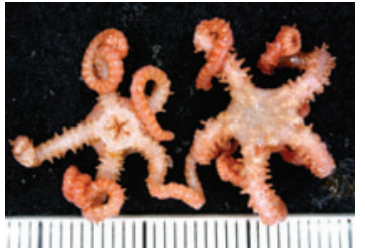

25|85080-Ophiolebes-sp.MOV358|
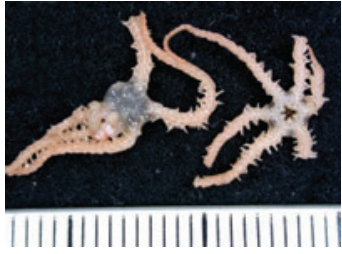

25190003-Ophiactis-hirta

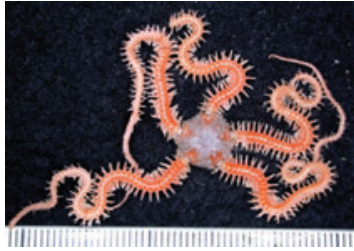

25190022-Ophiactis-sp.MOV5496

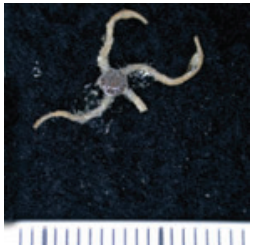

25|91073-Amphiura-sp.MOV5519

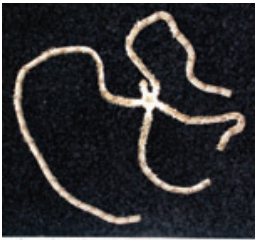

25191075-Amphioplus-sp.MOV5509

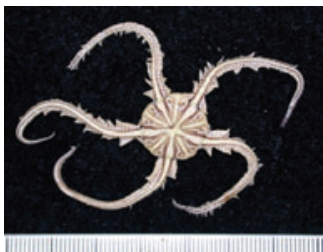

25192028-Ophiomaza-cacaotica

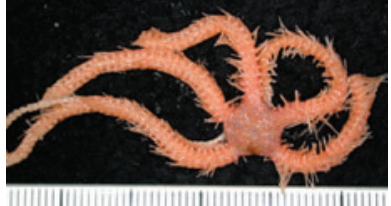

25|85082-Ophiacantha-sp.MOV4537

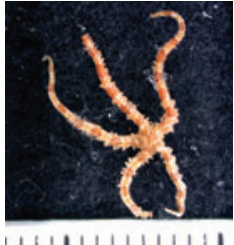

25|900|0-Ophiactis-modesta

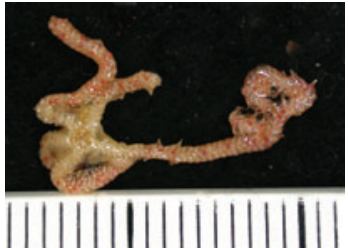

25191046-Amphiura-octacantha

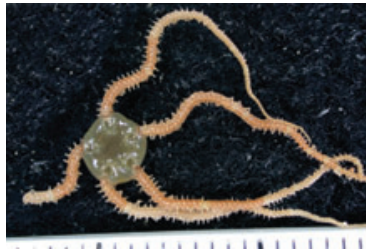

25|91076-Amphioplus-sp.MOV5510

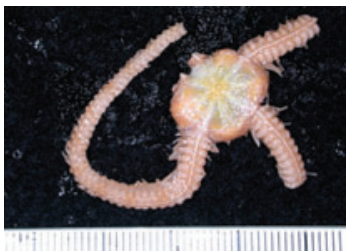

2519200|-Ophiothrix-aristulato

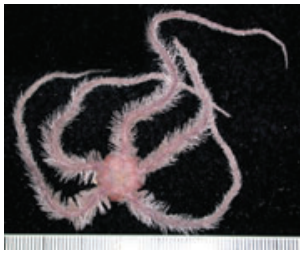

25192036-Ophiothrix-ciliaris

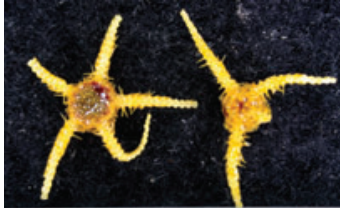

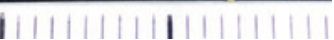

99250370-Ophiocymbium-sp.I

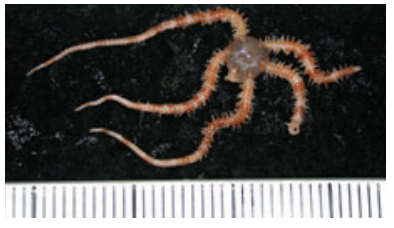

25190012-Ophiactis-profundi

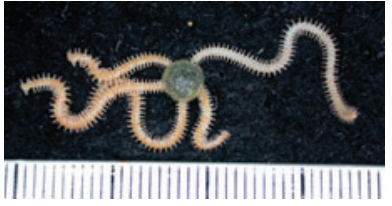

25191050-Ophiocentrus-aspera

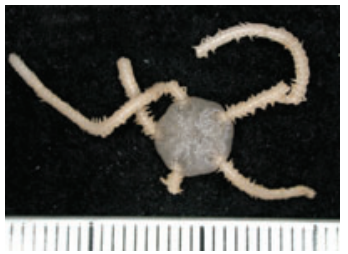

25|91078-Amphioplus-sp.MOV5524

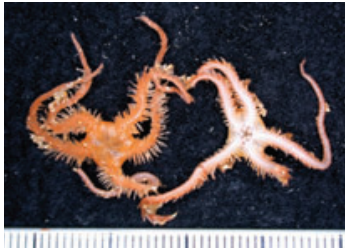

25192002-Ophiothrix-caespitosa

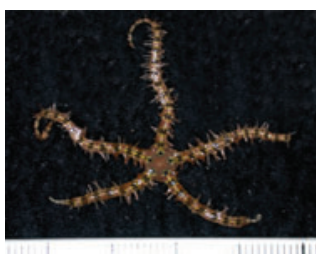

25|92063-Ophiothrix-spongicola 


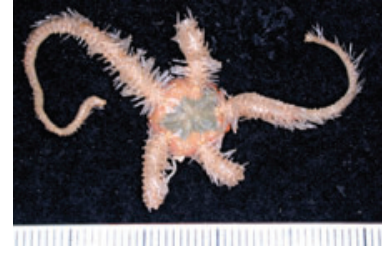

25I9207I-Ophiothrix-sp.MOV5502

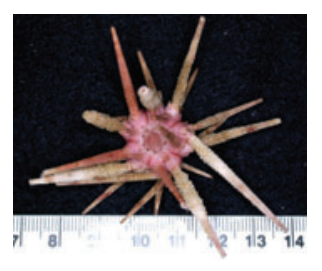

25202024-Stylocidaris-bracteata

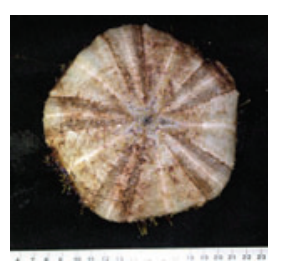

25205007-Asthenosoma-varium

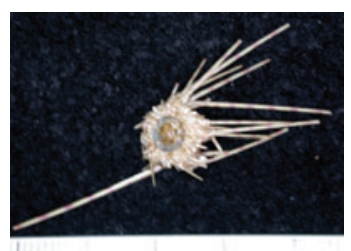

25235002-Coelopleurus-maculatus

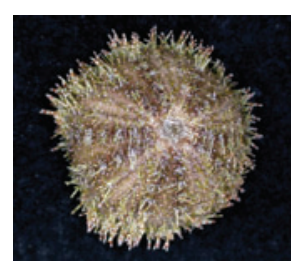

2524I035-Microcyphus-keiensis

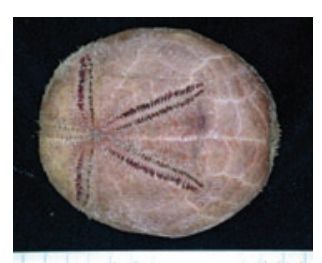

2530600 I-Anametalia-regularis

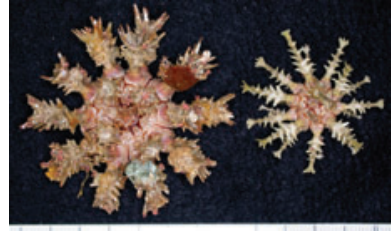

25202007-Goniocidaris-tubaria

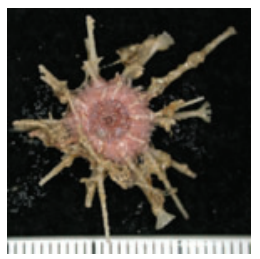

99250374-Rhopalocidaris-sp.I

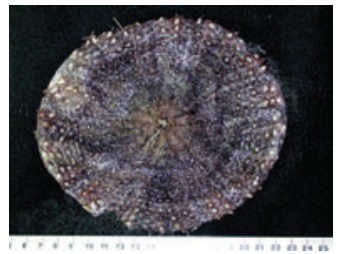

25206002-Phormosoma-rigidum

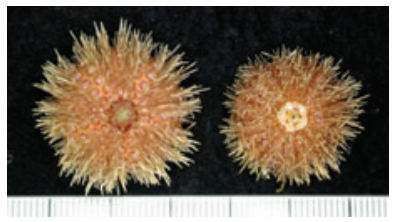

2524I0I4-Microcyphus-annulatus

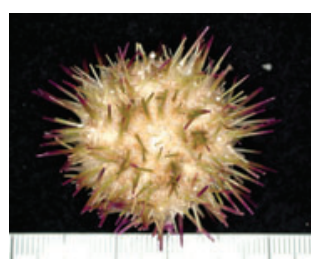

25242008-Pseudoboletia-indiana

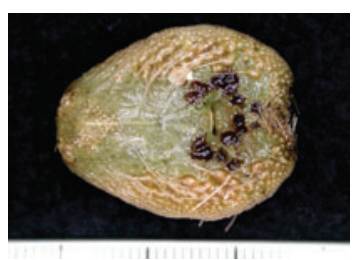

99250376-Lovenia-n.sp.I

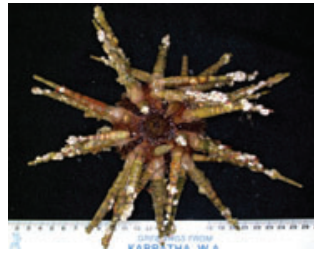

252020I7-Prionocidaris-baculosa

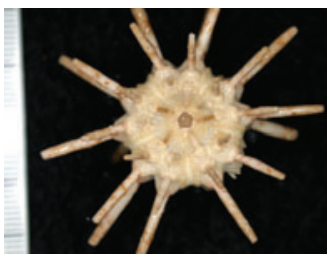

99250373-Phyllacanthus-sp.I

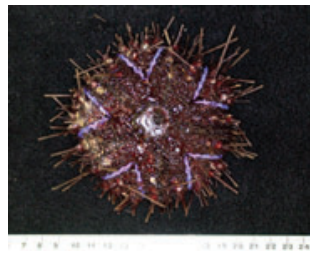

252II0I2-Eremopyga-denudata

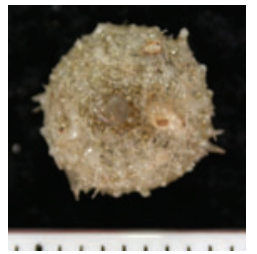

2524I030-Temnotrema-maculatum

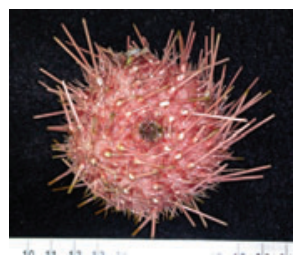

25246002-Gracilechinus-multidentatus

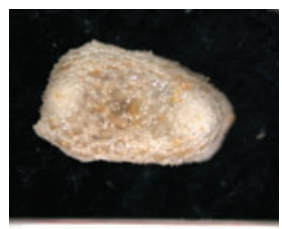

| | | | | | | | | | | | | |

254040 I I-Psolus-parmatus

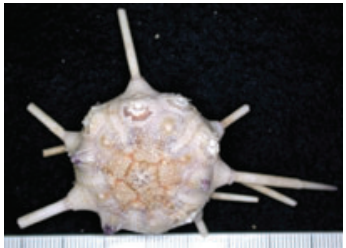

25202023-Stereocidaris-sceptriferoides

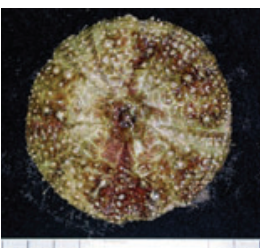

25205002-Araeosoma-owstoni

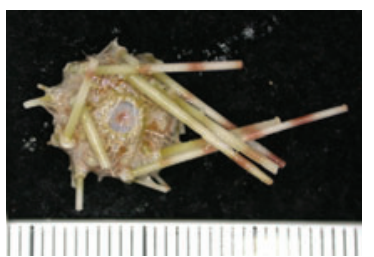

25220003-Caenopedina-mirabilis

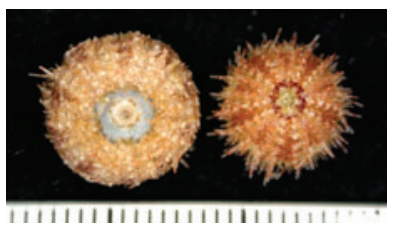

2524I032-Temnotrema-siamense

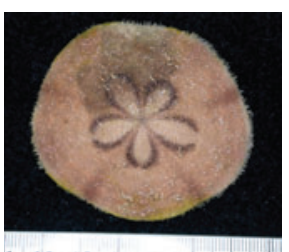

25262007-Clypeaster-virescens

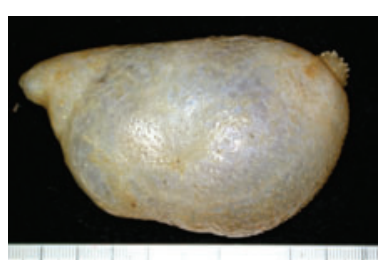

25406027-Neothyonidium-inflatum 


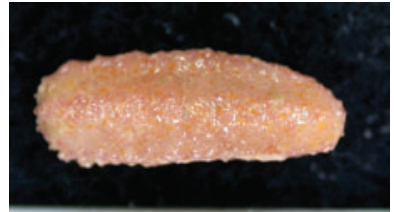

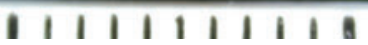

25408025-Plesiocolochirus-australis

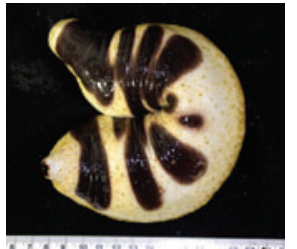

25416063-Holothuria-nigralutea

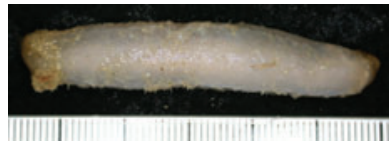

99250386-Pseudostichopus-n.sp.I

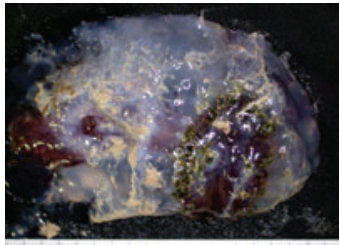

2542500I-Enypniastes-eximia

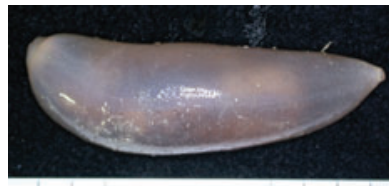

25437008-Aphelodactyla-bacilla

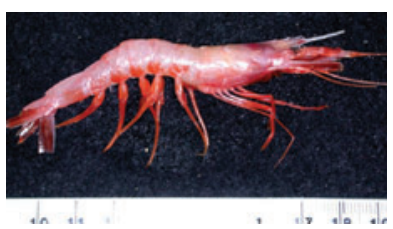

287|2003-Aristeus-virilis

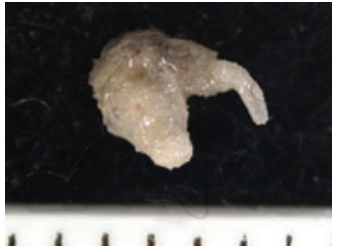

99250383-Echinocucumis-n.sp.I
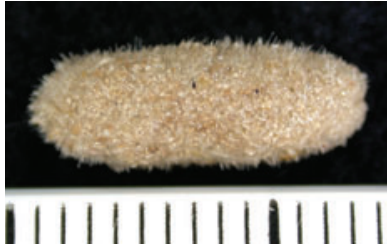

254|80|2-Pseudostichopus-hyalegerus

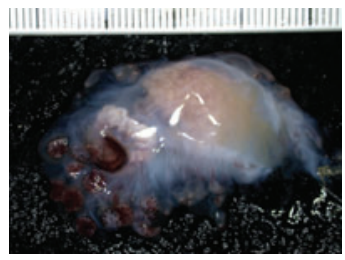

2542200I-Laetmogone-fimbriato

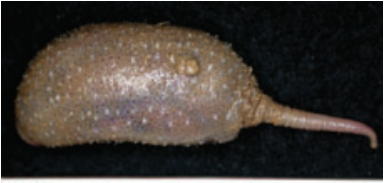

25436004-Molpadia-musculus

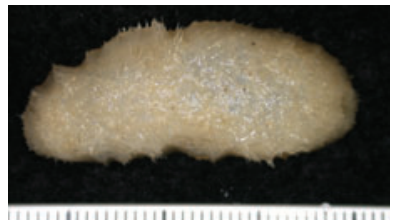

||||||||||||||||||||||||||||||||||||||

25437009-Aphelodactyla-suspecta

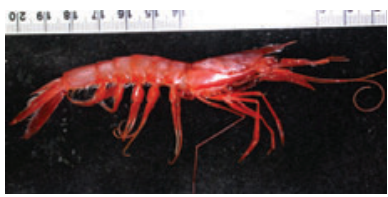

287|20| |-Aristeus-semidentata

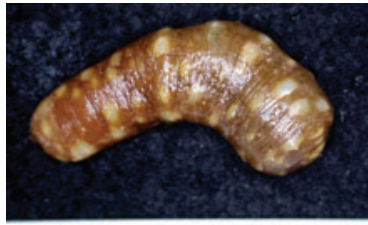

254|6054-Holothuria-hilla

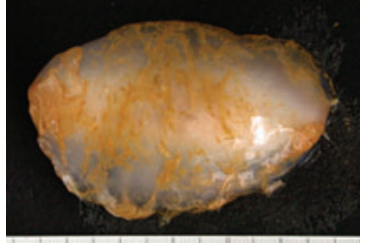

254|8023-Mesothuria-marginata

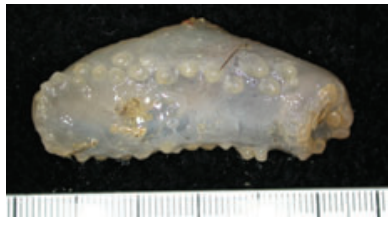

25422002-Laetmogone-maculata

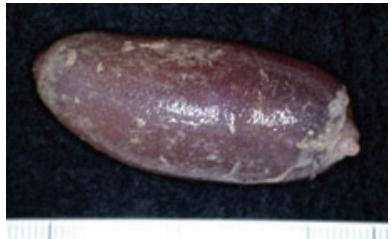

25436005-Trochostoma-parvulum

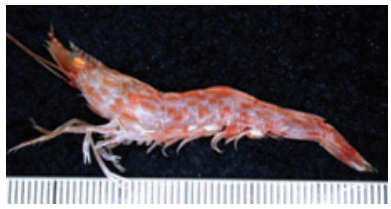

287| I073-Metapenaeopsis-velutina

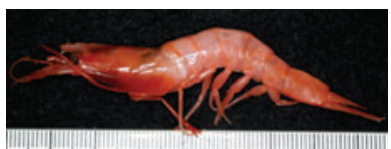

287| 300 I-Benthesicymus-investigatoris

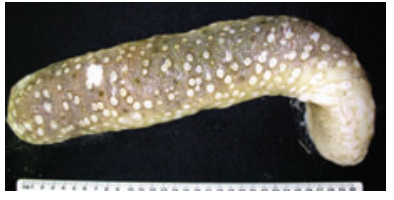

254|6062-Holothuria-austrinabassa

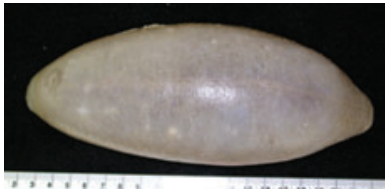

99250285-Mesothuria-n.sp.2

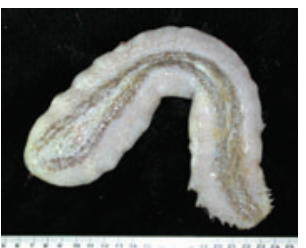

25422006-Benthogone-abstrusa

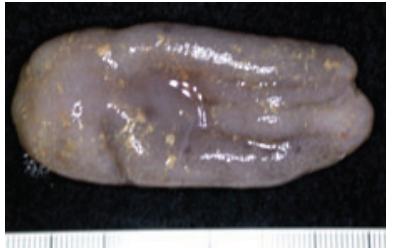

25437002-Aphelodactyla-molpadioides

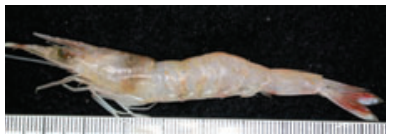

287| I09|-Metapenaeopsis-sp.MOV5458

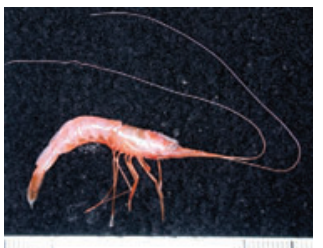

287| 4003-Hadropenaeus-lucasii 

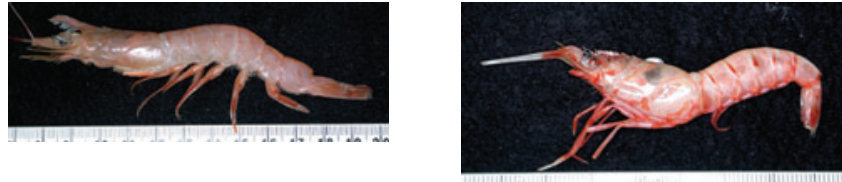

287|4005-Haliporoides-sibogae

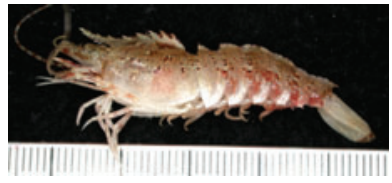

287| 50 I0-Sicyonia-japonica

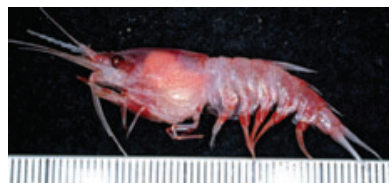

28735030-Oplophorus-gracilirostris

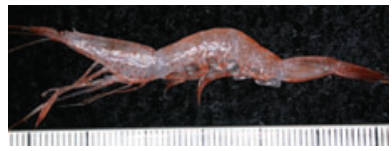

287450 I9-Alainopasiphaea-australis

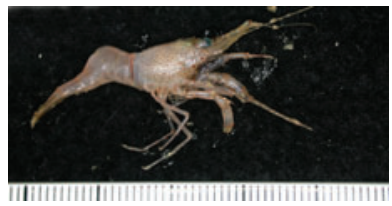

287520 I I-Rhynchocinetes-enigma

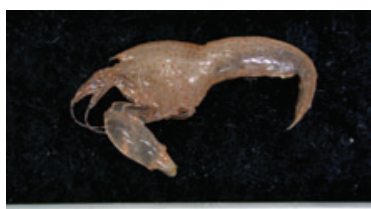

||||||||||||||||||

28765 |40-Synalpheus-neptunus
287|4029-Solenocera-rathbuni

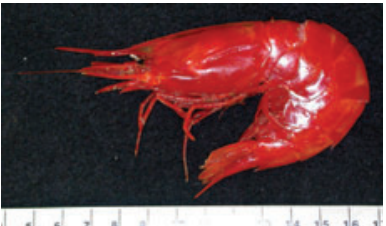

28735002-Acanthephyra-armata

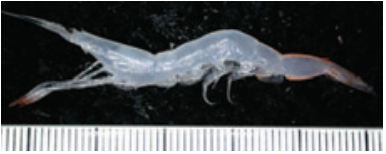

28745005-Pasiphaea-kapala

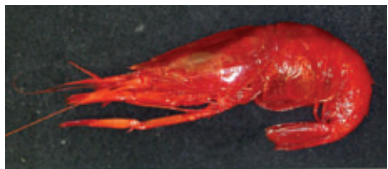

28745030-Eupasiphae-sp.MOV5427

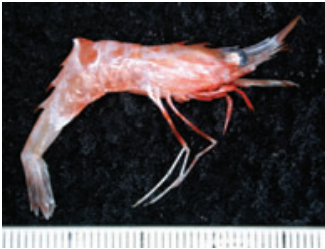

2875500 I-Campylonotus-rathbunae
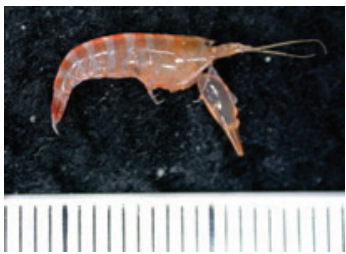

99280437-Alpheopsis-cf.-trispinosa

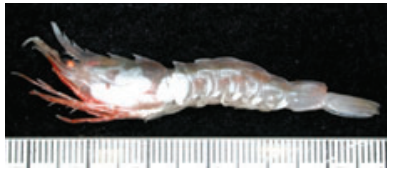

287|5002-Sicyonia-inflexa

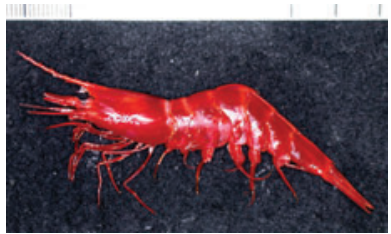

28735007-Acanthephyra-quadrispinosa

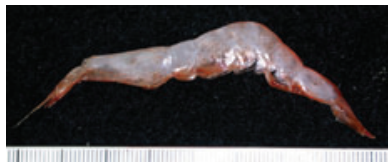

28745006-Pasiphaea-longitaenia

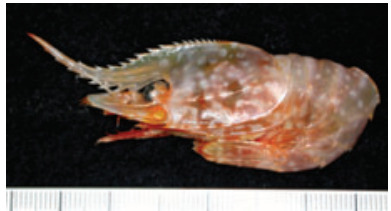

2875 I00 I-Eugonatonotus-chacei

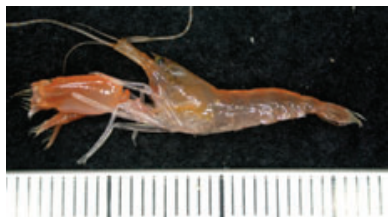

28765048-Alpheus-hailstonei

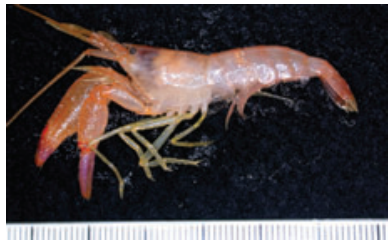

28765 I69-Alpheus-sp.MOV5409

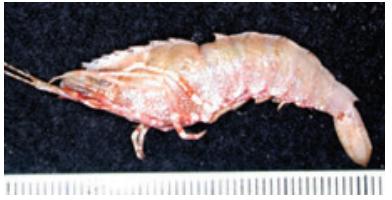

287|5004-Sicyonia-vitulans

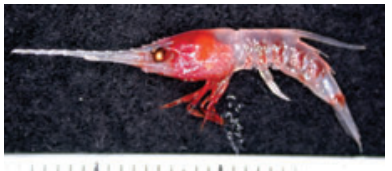

287350 |4-Oplophorus-novaezelandiae

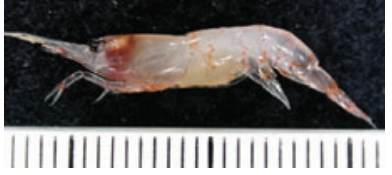

287450 I6-Leptochela-sydniensis

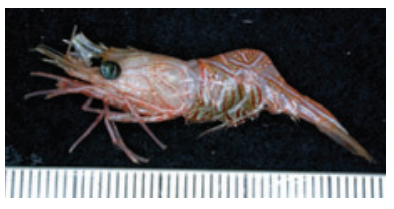

28752002-Rhynchocinetes-brucei

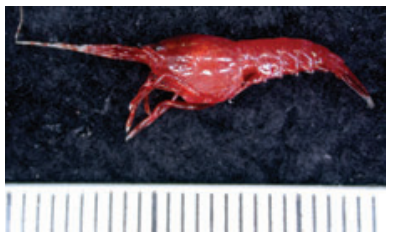

28765 I26-Synalpheus-comatularum

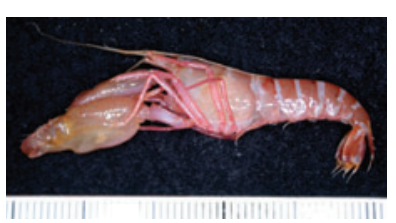

28765I66-Alpheus-sp.MOV5403 

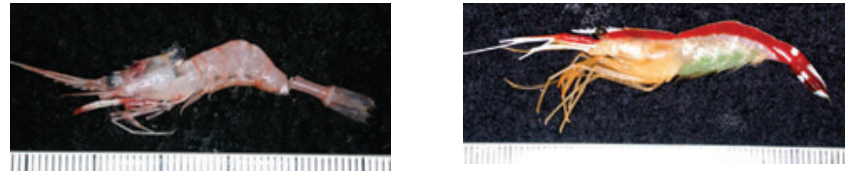

28767004-Merhippolyte-chacei

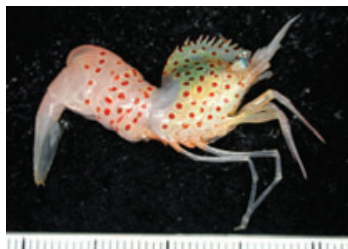

28767048-Lebbeus-clarehannah

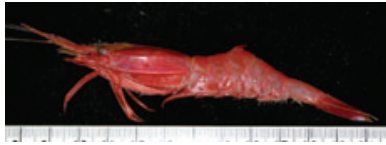

28770007-Heterocarpus-woodmasoni

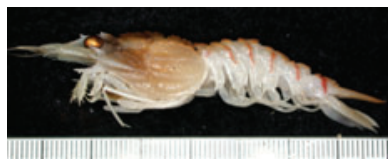

287800 I 0-Glyphocrangon-confusa

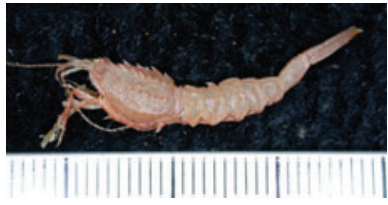

2878I004-Aegaeon-lacazei

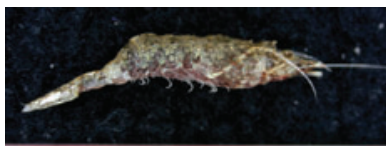

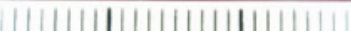

28781032-Philoceras-incisus

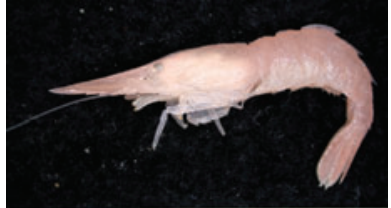

[1,1]IIIIIIIIIIIIIIIIIIIIIII

28767039-Tozeuma-tomentosum

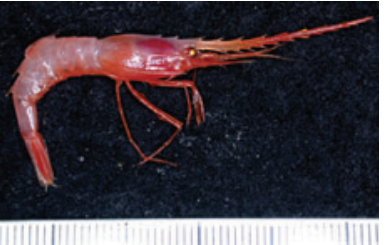

28770002-Heterocarpus-dorsalis

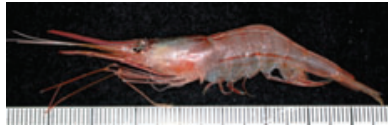

28770050-Plesionika-serratifrons

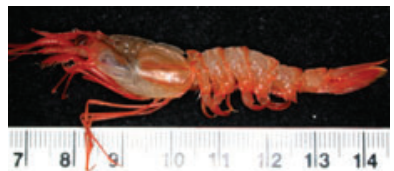

287800 I6-Glyphocrangon-sibogae

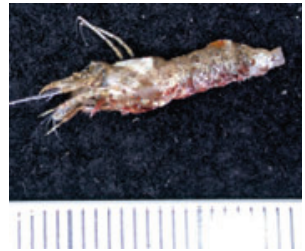

28781024-Pontocaris-pennata

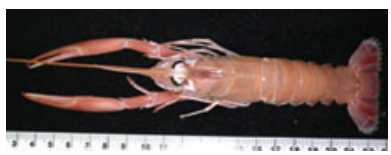

28786002-Metanephrops-boschmai

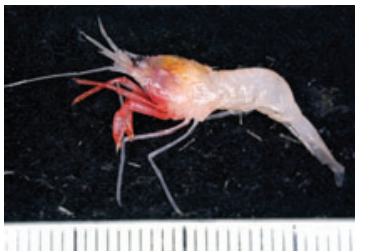

28767040-Eualus-sp.MOV268I

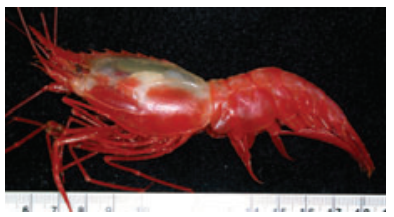

28770006-Heterocarpus-tricarinatus

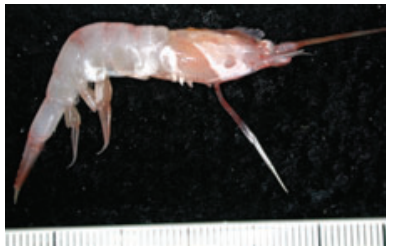

99280438-Plesionika-cf.-kensleyi

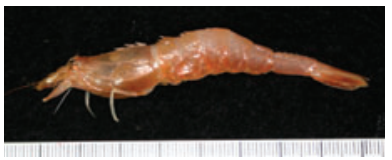

2878 I00 I-Parapontocaris-laevigata

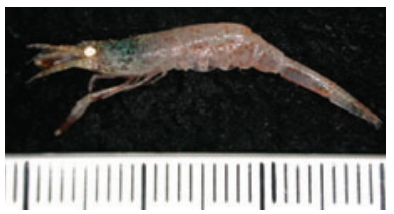

2878I025-Parapontophilus-junceus

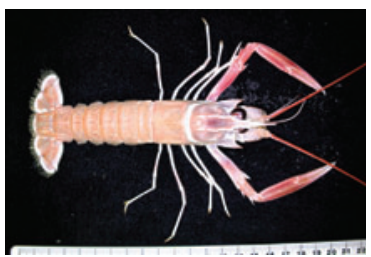

28786005-Metanephrops-velutinus 


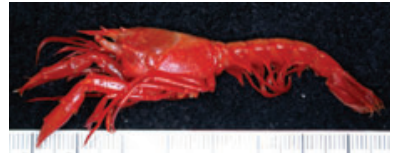

28786006-Nephropsis-acanthura

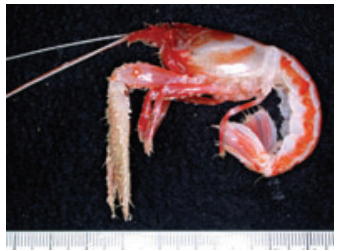

2880 I024-Acanthaxius-gathaagudu

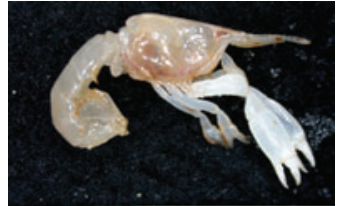

||||||||||||||||||||||||||

288040I 0-Tethisea-sp.MOV5472

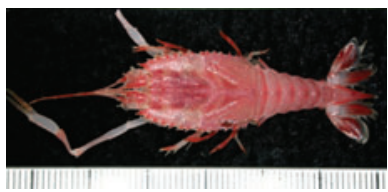

288I50 I0-Polycheles-suhmi

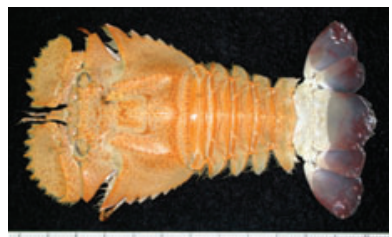

2882 I00 I-Ibacus-alticrenatus

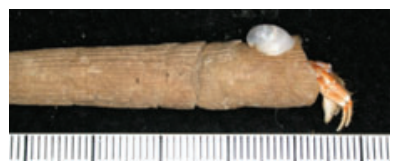

28827077-Paguristes-aciculus
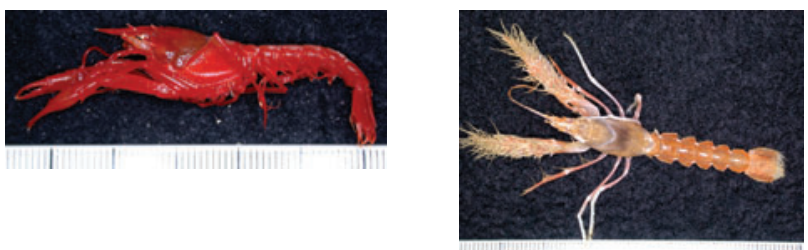

2880 I0I 4-Calaxius-acutirostris

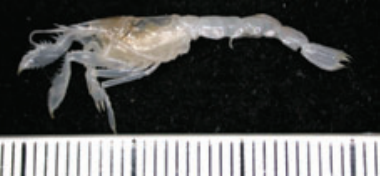

2880 I027-Australocaris-pinjarup

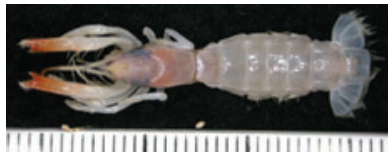

2880502 I-Upogebia-ancylodactyla

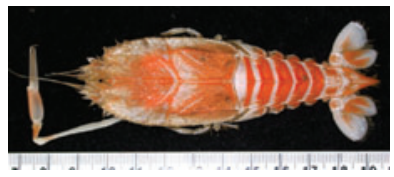

288I50|9-Polycheles-coccifer

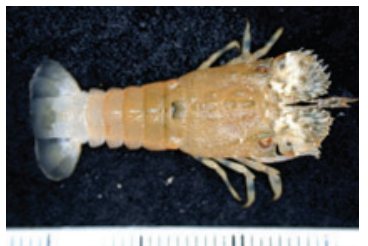

2882I02I-Crenarctus-crenatus

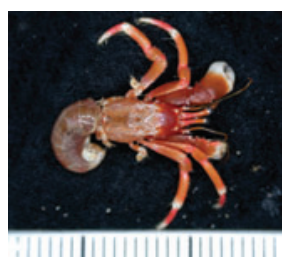

28827I09-Calcinus-sp.MOV5389

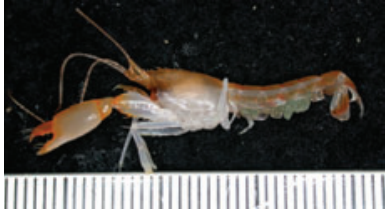

2880 I0I3-Bouvieraxius-keiensis

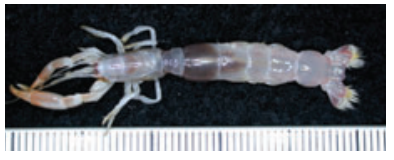

28803035-Corallianassa-sp.MOV4965

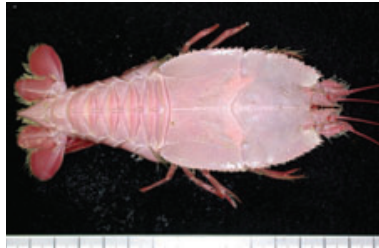

288I5005-Pentacheles-laevis

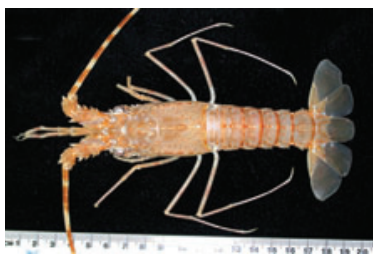

28820007-Puerulus-angulatus

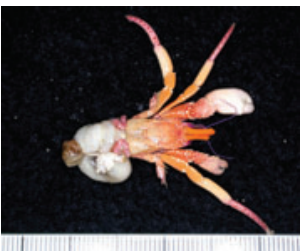

288270 I7-Paguristes-purpureantennatus

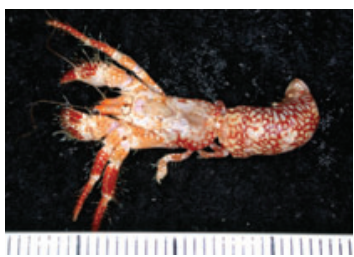

28827| | 3-Dardanus-sp.MOV5266 


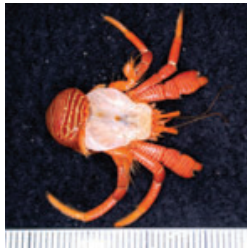

28827 I 17-Ciliopagurus-shebae

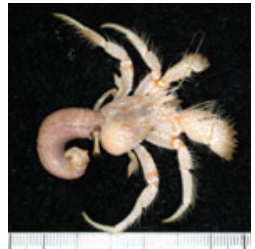

28835032-Propagurus-haigae

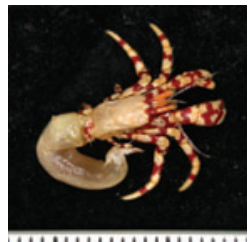

28835062-Paguridae-sp.MOV526

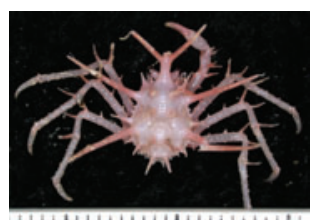

|||||||||||||||||||||||||| $\mid$

288360 I4-Lithodes-rachelae

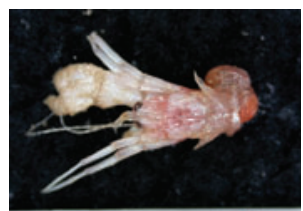

|||||||||||||||||| $\mid$

28837010-Oncopagurus-monstrosus

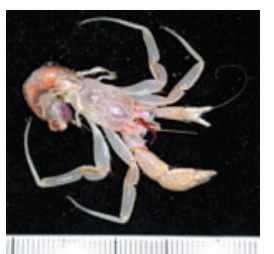

28837029-Paragiopagurus-sp.MOV5272

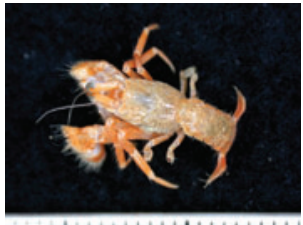

|||||||||||||||||| $\mid$

2883000 I-Pylocheles-mortensenii

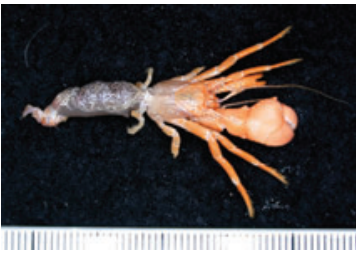

28835049-Bathypaguropsis-yaldwyni

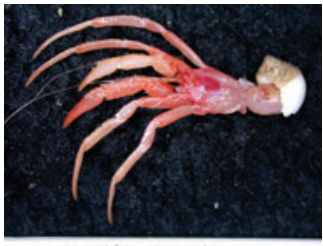

2883507I-Michelopagurus-sp.MOV5280

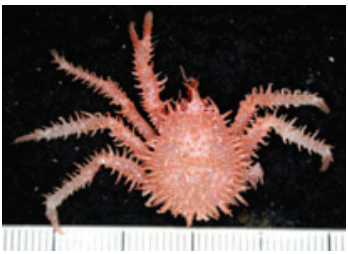

288360 I0-Paralomis-poorei

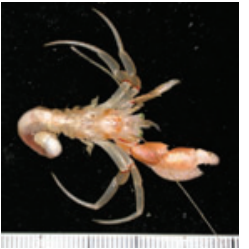

288370 I3-Paragiopagurus-diogenes

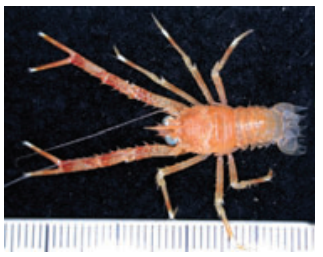

28840003-Munida-haswelli

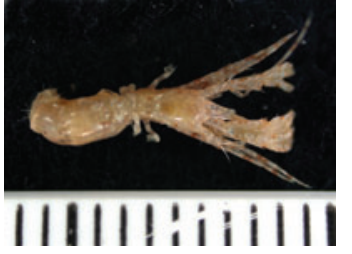

288350 I0-Lophopagurus-triserratus

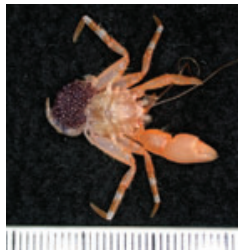

28835050-Porcellanopagurus-filholi

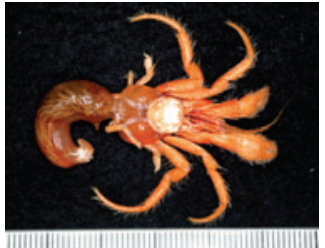

28835075-Paguridae-sp.MOV5270

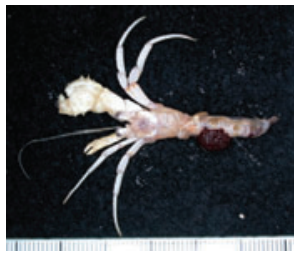

28837002-Sympagurus-dimorphus

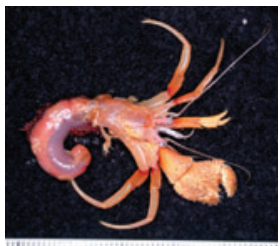

288370 I7-Paragiopagurus-boletifer

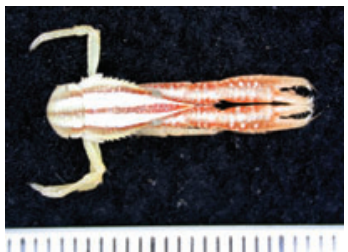

28840004-Allogalathea-elegans

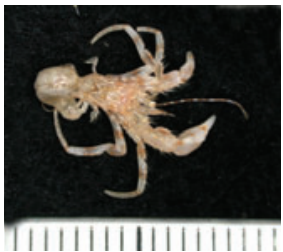

288350 I I-Lophopagurus-nanus

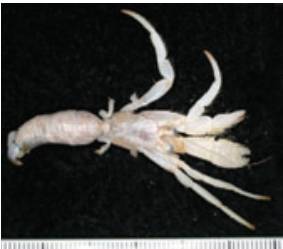

28835069-Cestopagurus-sp.MOV5269

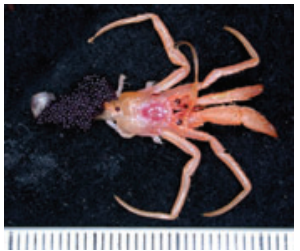

28835076-Paguridae-sp.MOV527I

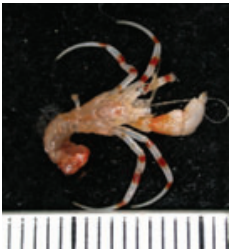

28837009-Oncopagurus-indicus

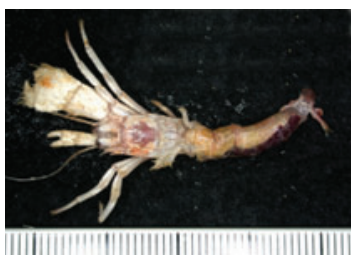

2883702 I-Parapagurus-latimanus

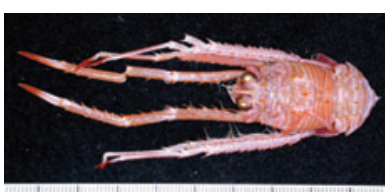

28840005-Agononida-eminens 


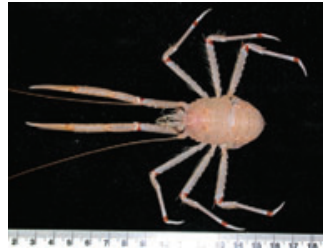

28840006-Agononida-incerta

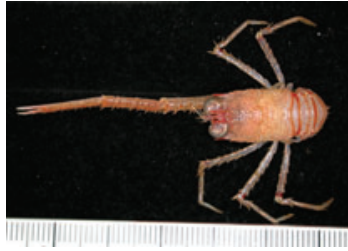

2884003 I-Enriquea-leviantennata

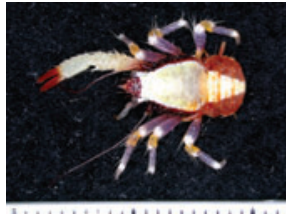

||||||||||||||||||||||||

28840097-Galathea-consobrina

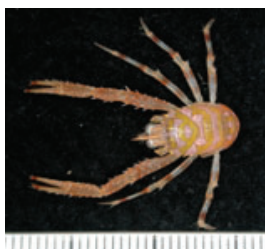

28840 I 25-Munida-gordoae

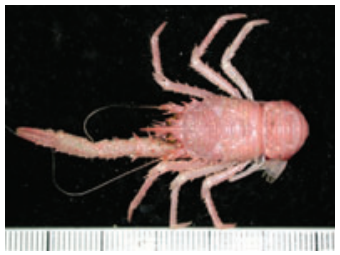

28840I52-Munida-sp.MOV5I99

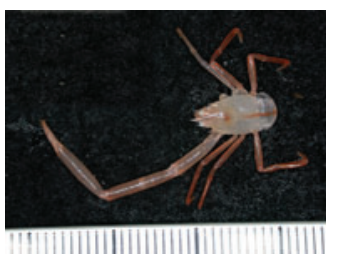

28842008-Uroptychus-gracilimanus

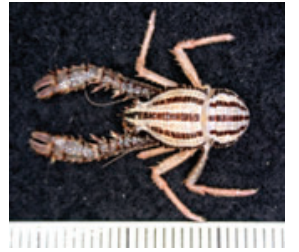

288400 I 2-Galathea-amboinensis

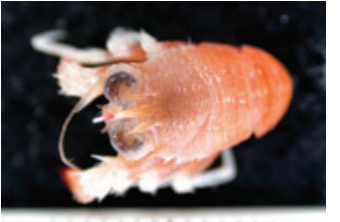

28840034-Munida-rubridigitalis

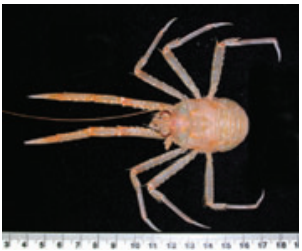

28840098-Paramunida-stichas

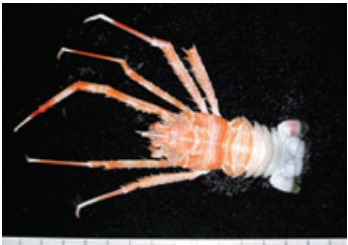

28840 I 58-Agononida-sp.MOV52 I8

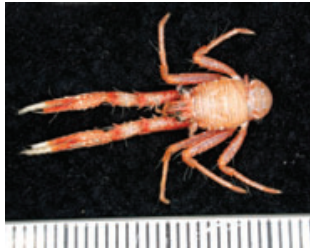

28840 I57-Raymunida-sp.MOV5I98

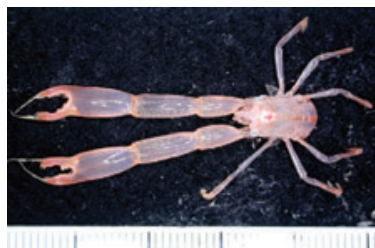

28842020-Uroptychus-flindersi

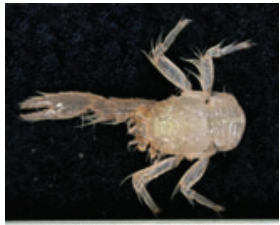

IIIIIIIIIIIIIIII!i

28840024-Lauriea-gardineri

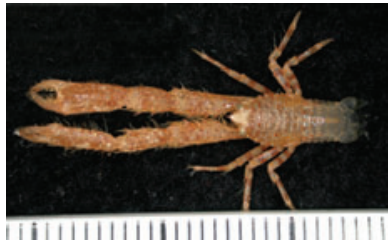

28840042-Phylladiorhynchus-pusillus

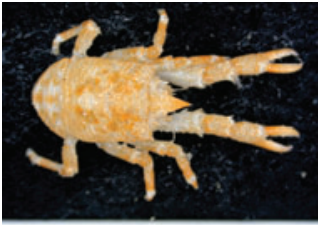

|||||||||||||||||||||||

28840 I 02-Munidopsis-crenatorostris

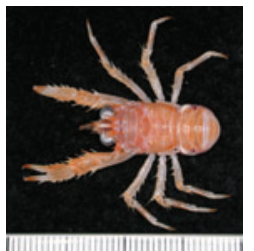

28840 I60-Munida-sp.MOV5I83

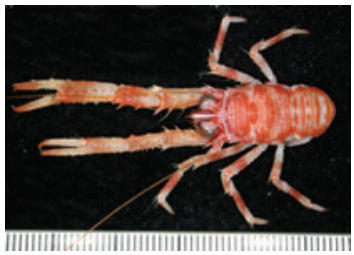

28840 I6I-Munida-sp.MOV5203

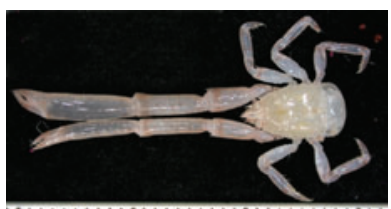

|IIIIIIIIIIIIIIIIIIIIIIIIIIIII

28842054-Uroptychus-sp.MOV5 I 8

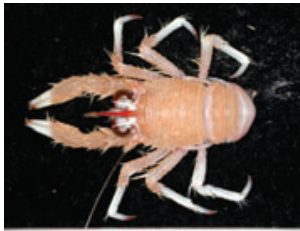

28840026-Munida-andamanica

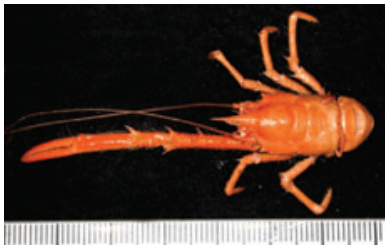

28840056-Munidopsis-dasypus

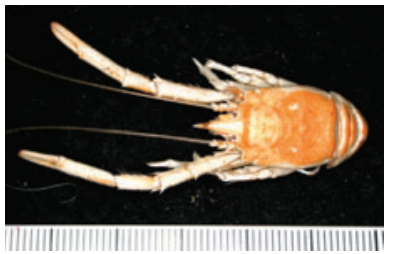

28840076-Munidopsis-andamanica

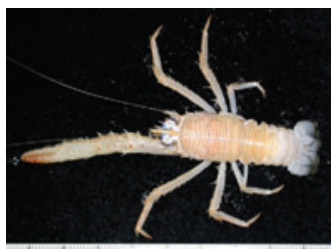

28840I53-Munida-sp.MOV5200

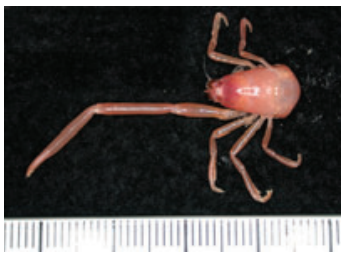

28842002-Uroptychus-australis

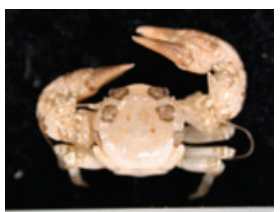

!i i IIIIIIII

288430 I7-Platycheles-sculptus 


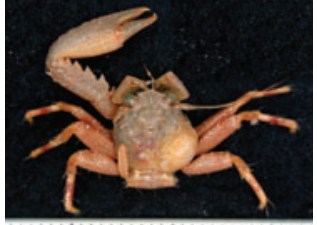

IIIIIIIIIIIIIIIIIIIIIIIIIIIII

28843028-Petrolisthes-militaris

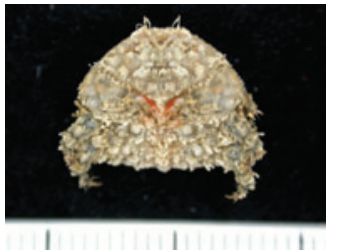

28852003-Austrodromidia-insignis

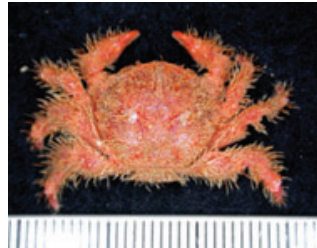

28853004-Hirsutodynomene-spinosa

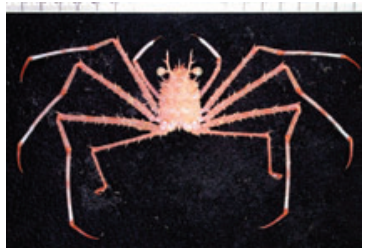

2886000I-Dagnaudus-petterdi

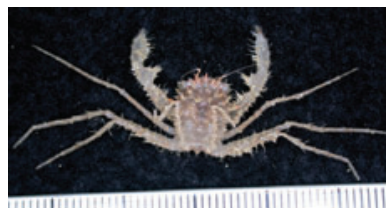

288600 I4-Yaldwynopsis-sp.MOV5004

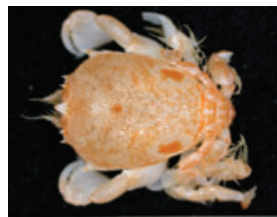

|| || ||||||||||||||||||||

28865005-Umalia-trirufomaculata

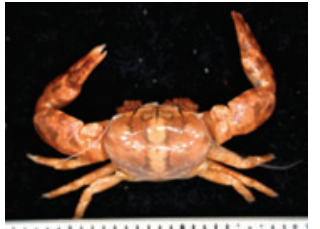

IIIIIIIIIIIIIIIIIIiiillI

28843038-Polyonyx-biunguiculatus

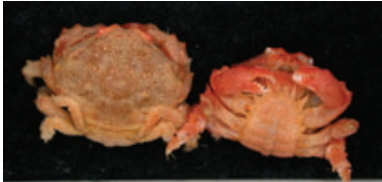

|||||||||||||||||||||||||||||||

28852004-Metadromia-wilsoni

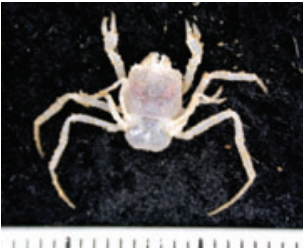

28854008-Cymonomus-sp.MOV500 I

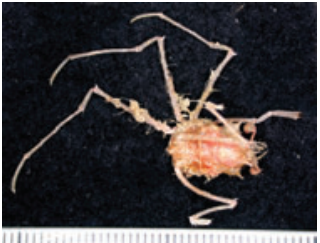

288600 I I-Latreillopsis-tetraspinosa

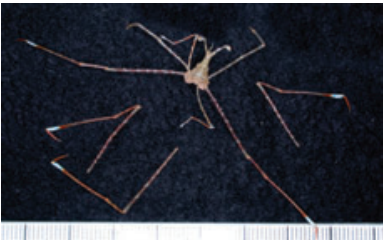

2886100I-Eplumula-australiensis

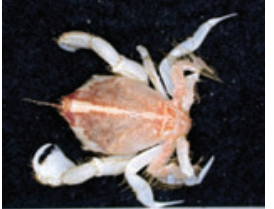

28865006-Cosmonotus-grayi

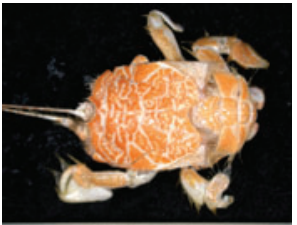

"IIIIIIIIIIIIIIIIIIIIiiil|I

28845009-Stemonopa-insignis

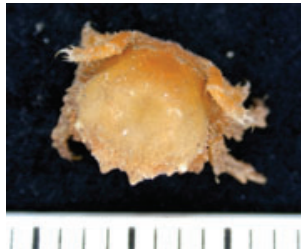

28852026-Fultodromia-nodipes

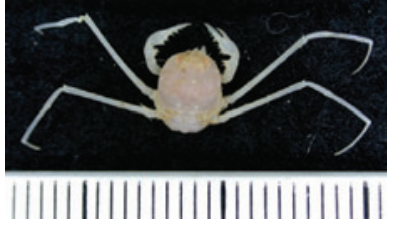

28855002-Tymolus-similis

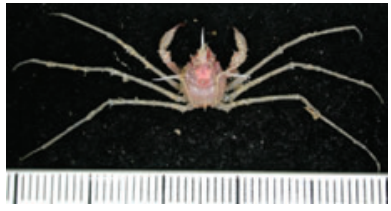

288600 I 2-Homologenus-malayensis

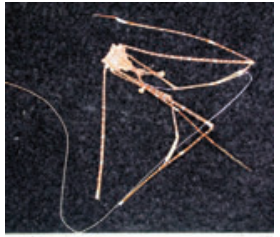

2886 | 002-Latreillia-pennifera

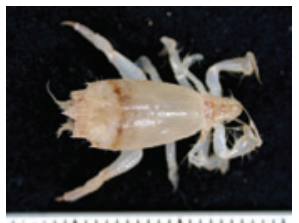

ili||||||||||||||||||||||||||

28865008-Notosceles-serratifrons

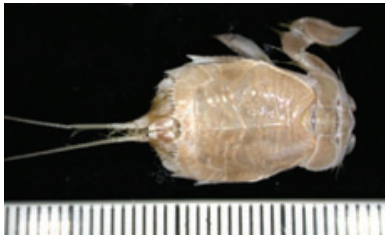

288450 I0-Albunea-occultus

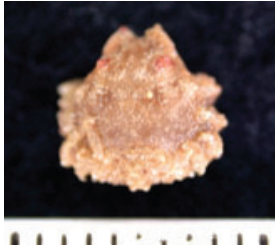

28852036-Takedromia-sp.MOV5003

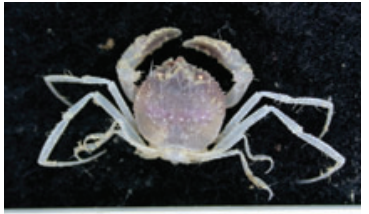

|II||i||||||||||||||||||||

28855003-Krangalangia-spinosa

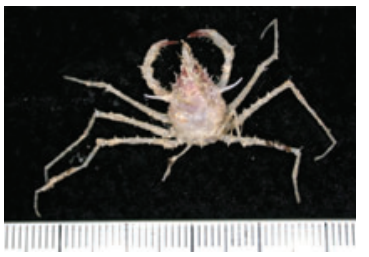

288600 I3-Homologenus-braueri

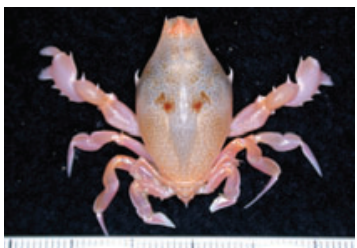

28865002-Lyreidus-tridentatus

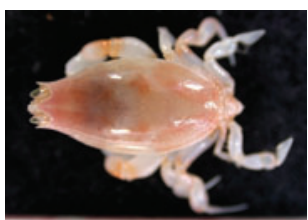

11111111111111111

28865009-Lyreidus-stenops 


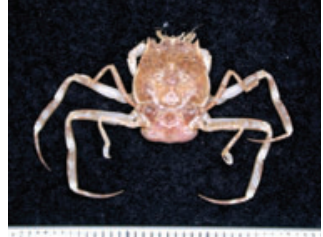

28870002-Paradorippe-australiensis

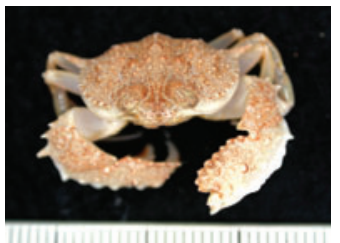

28875004-Mursia-microspina

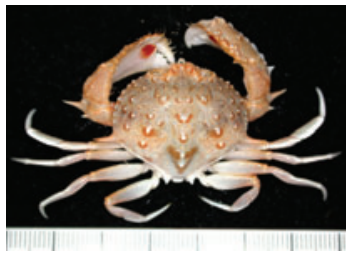

28875019-Mursia-sp.MOV4988

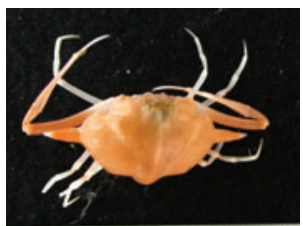

||||||||||||||||||||||||||||||||||||||||||||||||||||| 288760 I5-Arcania-cornuta

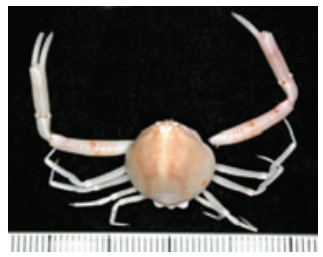

28876080-Randallia-eburnea

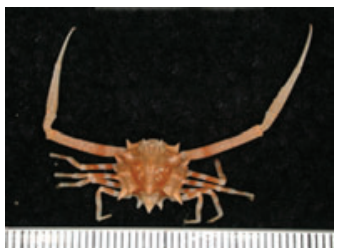

28876096-Arcania-sp.MOV4980

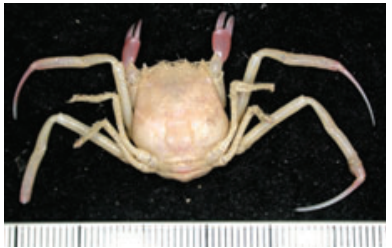

288700 I4-Ethusina-sp.MOV5005

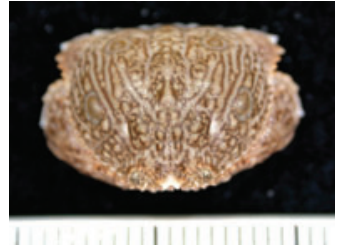

28875005-Calappa-lophos

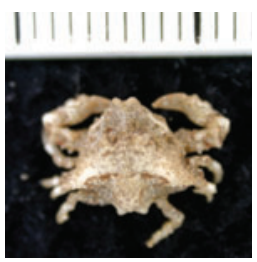

2887600 I-Merocryptus-lambriformis

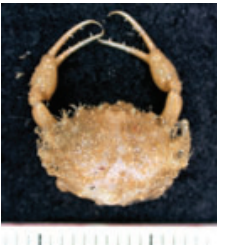

288760 I7-Iphiculus-spongiosus

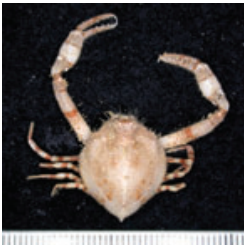

28876082-Myra-curtimana

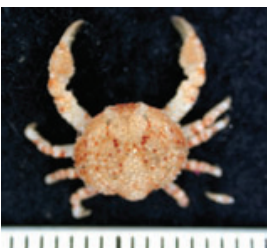

28876 I03-Ebalia-sp.MOV4990

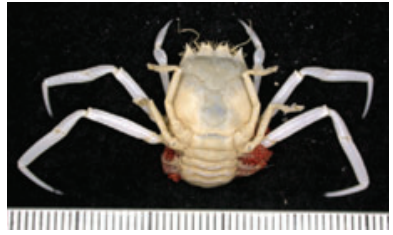

288700 I I-Ethusa-sp.MOV5006

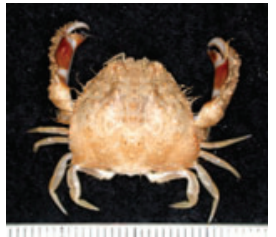

288750 I5-Calappa-depressa

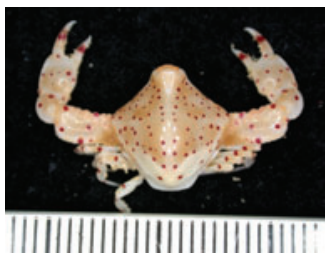

288760 I0-Leucosia-haematosticta

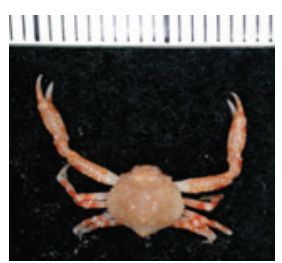

28876046-Ebalia-tuberculosa

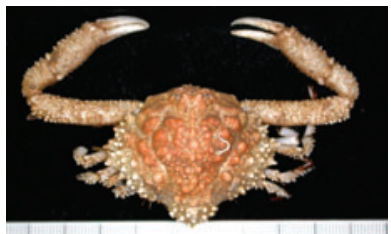

28876084-Randallia-pustuloides

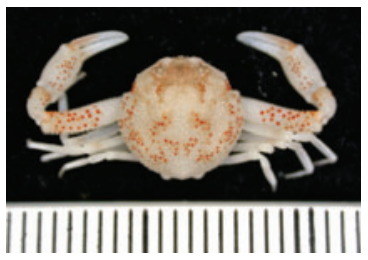

28876095-Randallia-sp.MOV4978

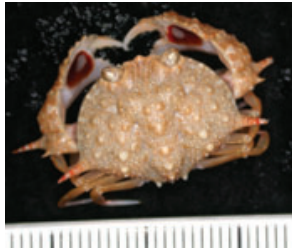

28875002-Mursia-australiensis

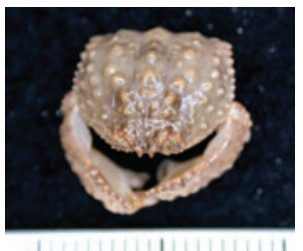

288750 I8-Calappa-pustulosa

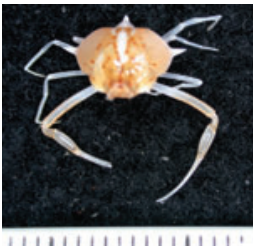

288760 I 2-Arcania-septemspinosa

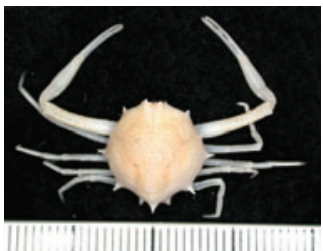

2887606 I-Arcania-elongata

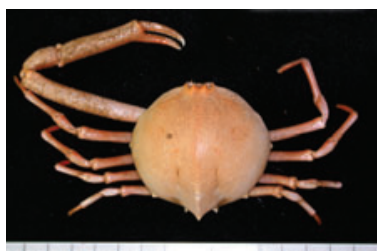

28876085-Parilia-major

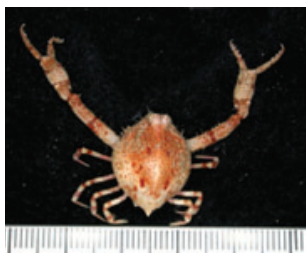

28876I05-Myra-sp.MOV4982 


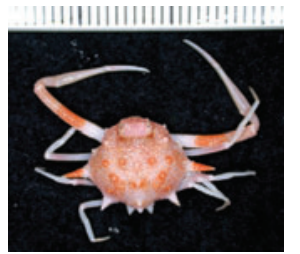

28876098-Arcania-sp.MOV4987

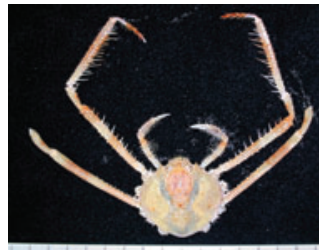

2888000 I-Platymaia-wyvillethomsoni

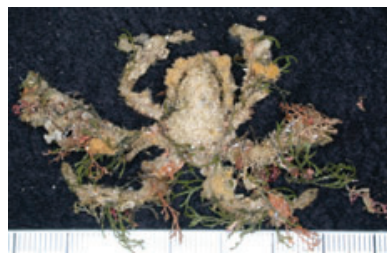

28880032-Camposcia-retusa

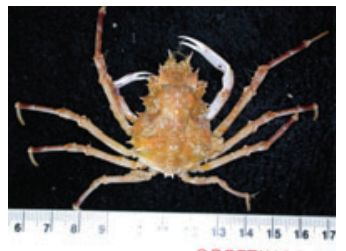

28880086-Leptomithrax-globifer

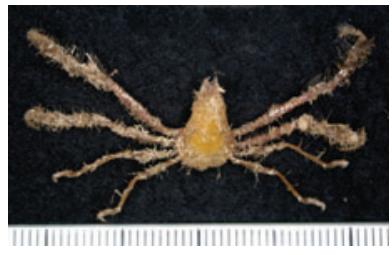

28880 I6I-Oncinopus-cf.-angustifrons

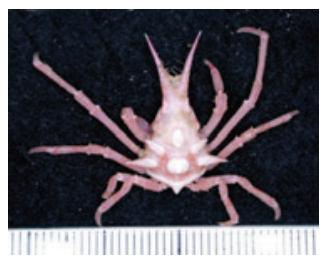

28880 I 72-Oxypleurodon-wilsoni

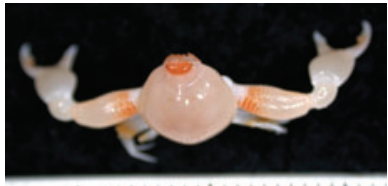

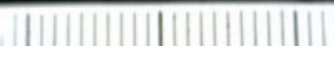

28876 I 07-Philyra-sp.MOV4984

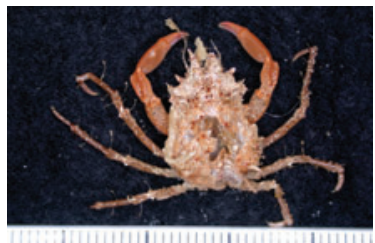

28880005-Leptomithraxsternocostulatus

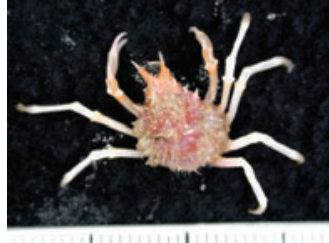

28880047-Griffinia-Iappacea

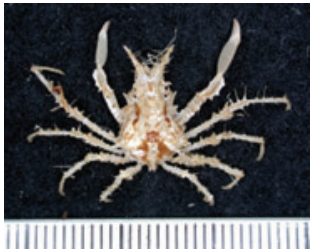

28880097-Prismatopus-occidentalis

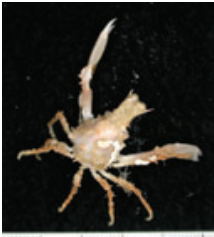

28880 I63-Prismatopus-brevispinosus

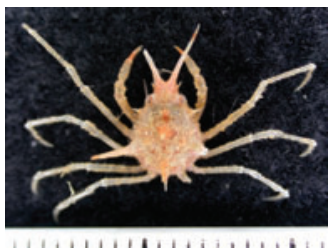

28880 I73-Rochinia-annae

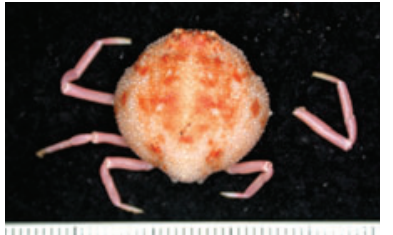

28876 I08-Randallia-sp.MOV4977

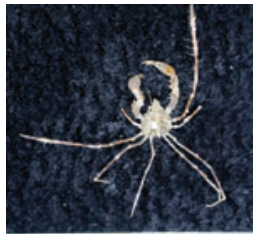

288800 I 3-Dorhynchus-ramusculus

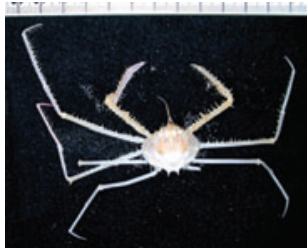

28880068-Cyrtomaia-maccullochi

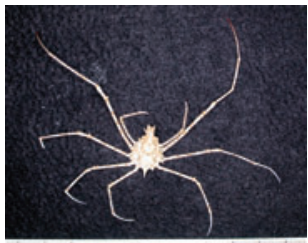

28880 I48-Phalangipus-hystrix

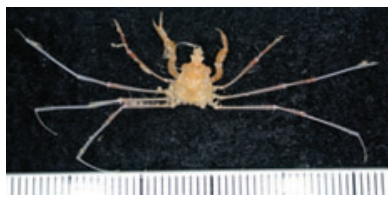

28880 I 64-Physachaeus-ctenurus

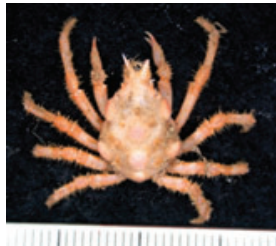

28880 I74-Rochinia-carinato

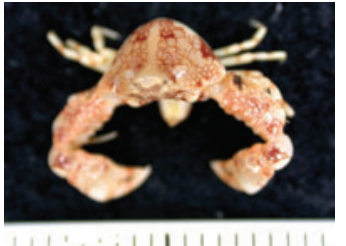

28876I 04-Leucosia-sp.MOV4985

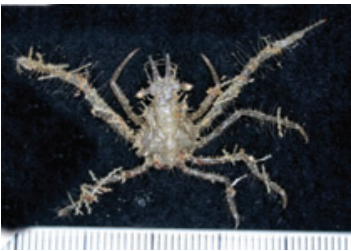

28880022-Achaeus-curvirostris

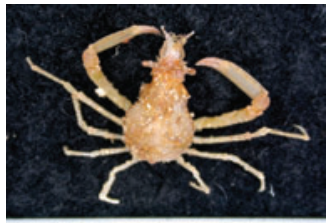

|||||||||||||||||||!|i

28880079-Sunipea-indicus
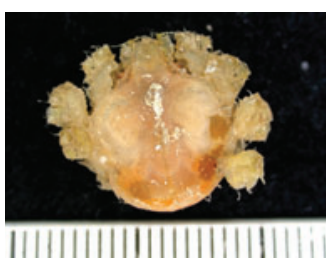

28880 I55-Planotergum-mirabile

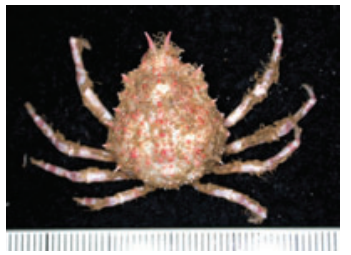

28880 I65-Maja-confragosa

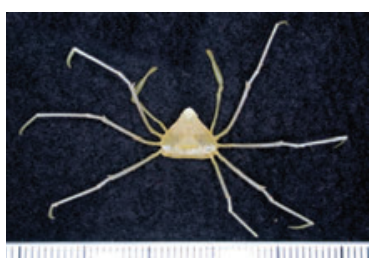

288850 |4-Trigonoplax-longirostris 


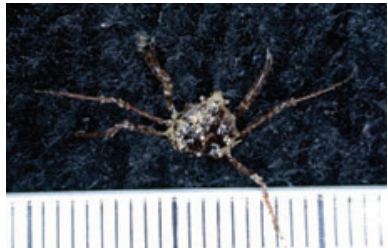

2888503 I-Halicarcinus-sp.MOV5002

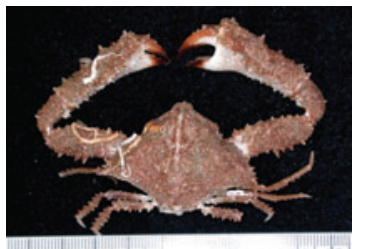

28895039-Garthambrus-sp.MOV5063

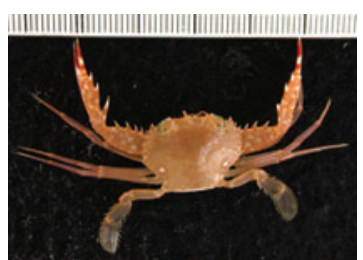

289| I019-Charybdis-miles

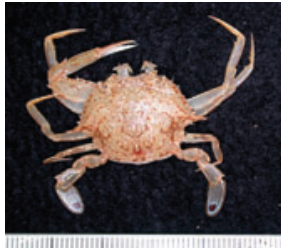

289| I033-Portunus-haanii

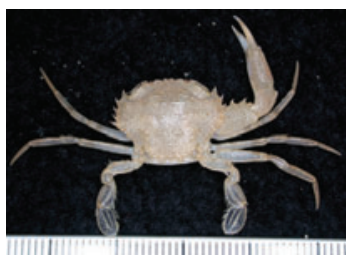

289| I094-Thalamita-macropus

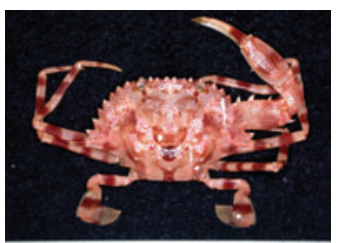

289| I I07-Portunus-nipponensis

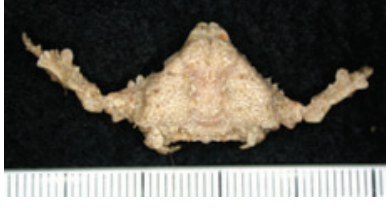

288950 I4-Daldorfia-excavata

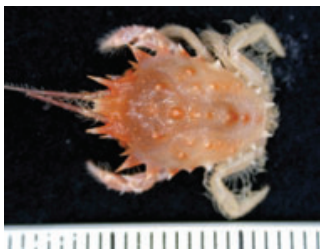

28900004-Jonas-sp.MOV502I

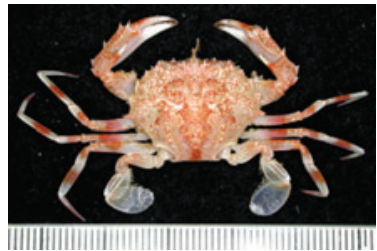

289| I02I-Parathranites-orientalis

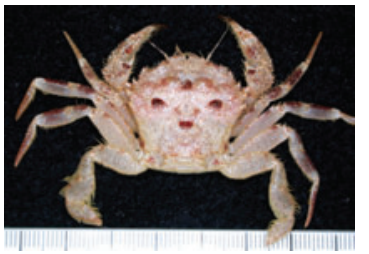

289| I05 I-Nectocarcinus-spinifrons

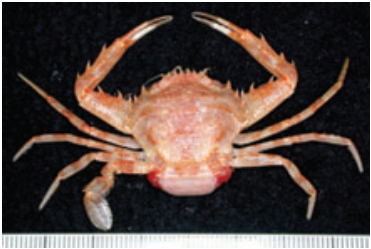

289 I I 103-Lupocyclus-quinquedentatus

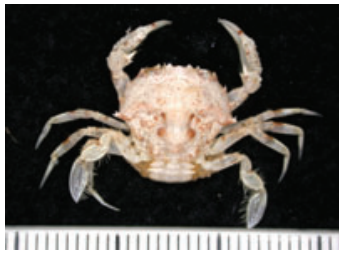

289| I I09-Echinolatus-poorei

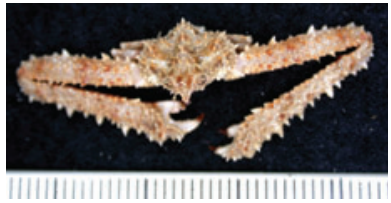

288950 I8-Parthenope-chondrodes

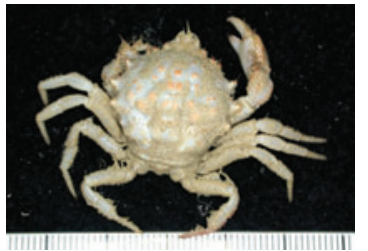

2890 I006-Trichopeltarion-sp.MOV5I35

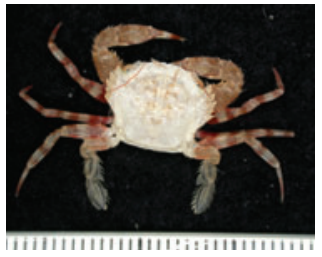

289| 1023-Thalamita-sexlobato

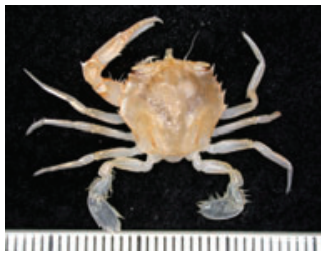

289| 1059-Ovalipes-iridescens

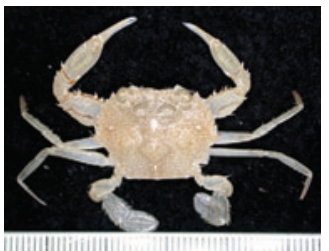

289| I I05-Charybdis-hongkongensis

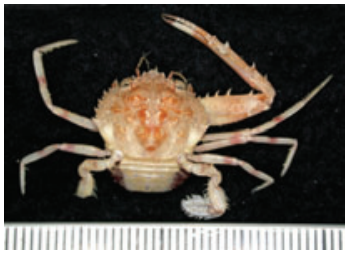

289 I I I 5-Lupocyclus-sp.MOV5 I 85

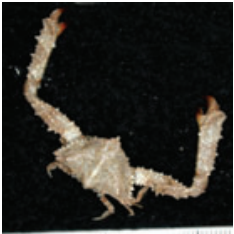

28895038-Garthambrus-sp.MOV50I I

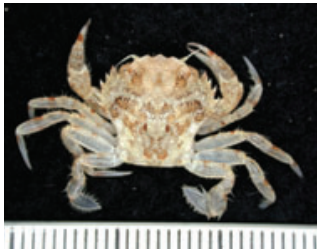

289 | I0 |6-Liocarcinus-corrugatus

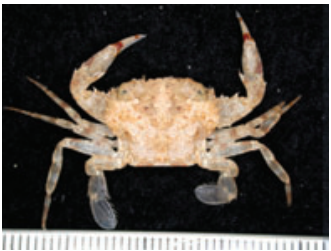

289| I025-Thalamita-spinifera

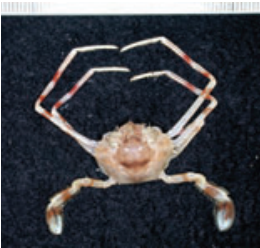

289 I I06 I-Lupocyclus-philippinensis

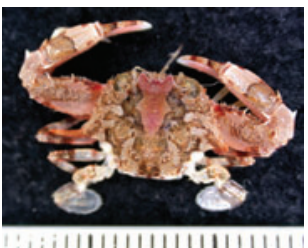

289| | | 06-Portunus-longispinosus

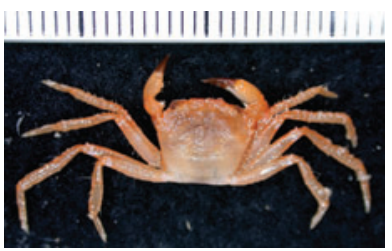

289|700 I-Mathildella-serrata 


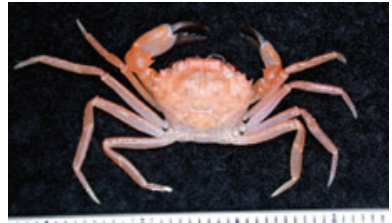

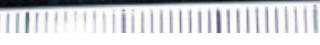

28917007-Neopilumnoplax-nieli

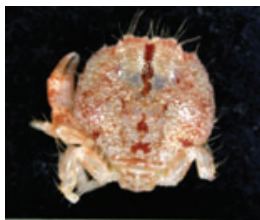

1111111111111

28920 I76-Palapedia-valentini

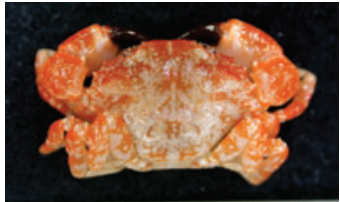

||||||||||||||||||||||||||||

28920195-Xanthias-sp.MOV5076

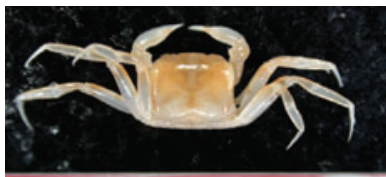

1||||||||||||||||||||

28922022-Notonyx-nitidus

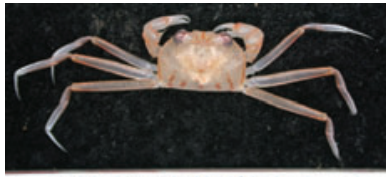

111111111111111111111111111111

28923003-Hexaplax-megalops

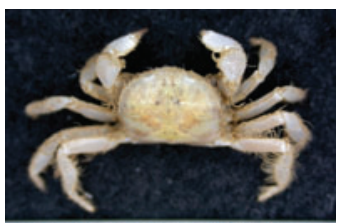

|l||l||liiil|||||||||

28926098-Caecopilumnus-piroculatus

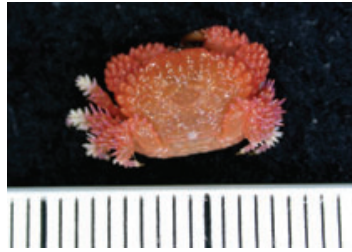

2892000 I-Actaea-peronii-peronii

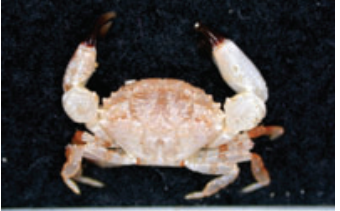

||||||||||||||||||||||||

28920189-Nanocassiope-sp.MOV5087

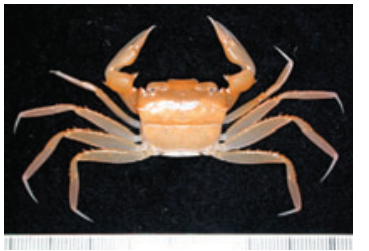

2892200 I-Psopheticus-stridulans

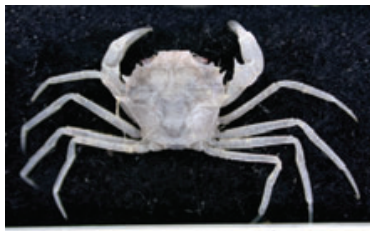

1111111111111111111111i1

289|7008-Goneplacidae-sp.MOV4997

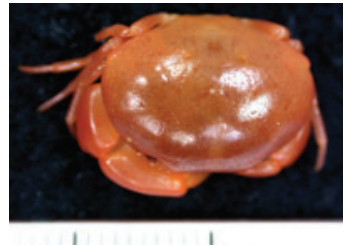

2892400 I-Carpilius-convexus

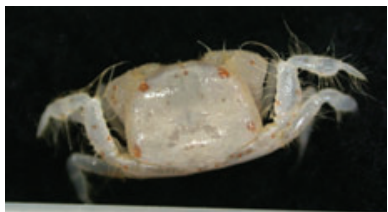

I I 1 I I I I I I I I I

28926100-Mertonia-lanka

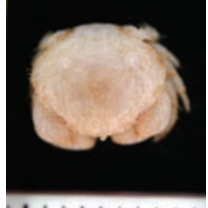

|I|||||||||

28920026-Calvactaea-tumida

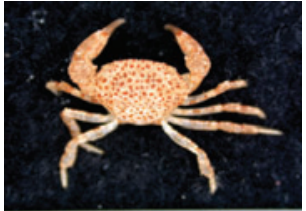

1111111!ा1111!iiii

28920190-Nanocassiope-sp.MOV5299

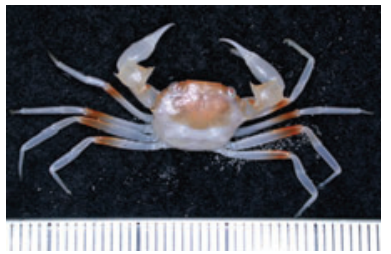

28922002-Pycnoplax-meridionalis

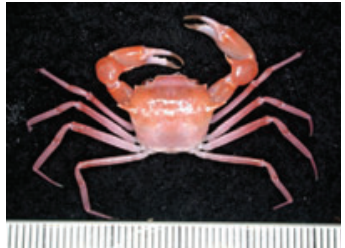

2892203 I-Carcinoplax-sp.MOV4992

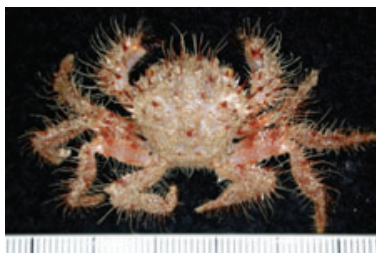

28925009-Hypothalassia-acerba

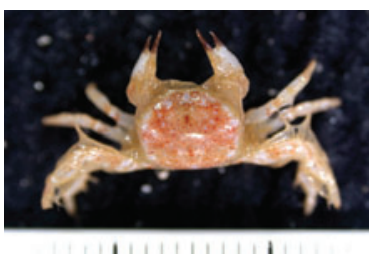

28926 | I5-Paraselwynia-sp.MOV5089

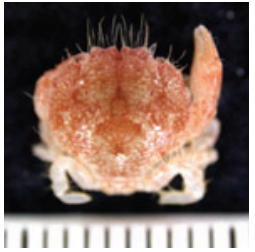

28920102-Palapedia-pelsartensis

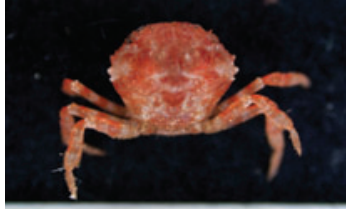

111111111111111

28920193-Paraxanthodes-sp.MOV5072

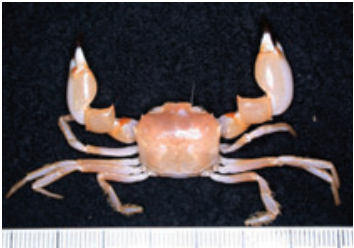

2892202 I-Pycnoplax-bispinosa

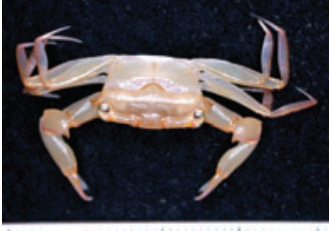

28922035-Paragoneplax-serene

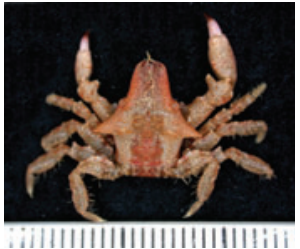

28926020-Eumedonus-niger

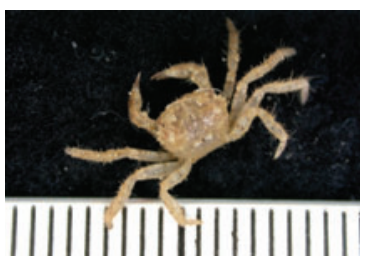

99280440-Pilumnus-cf.-spinicarpus 


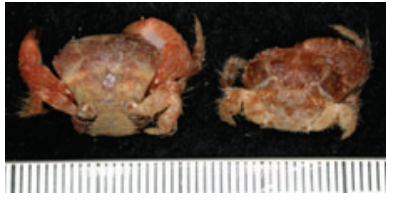

28926 I I9-Pilumnus-sp.MOV5099

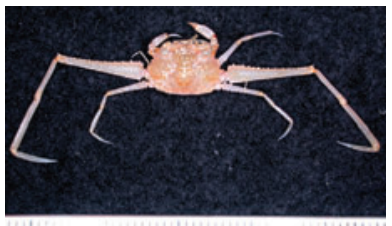

289620 I4-Micropalicus-vietnamensis

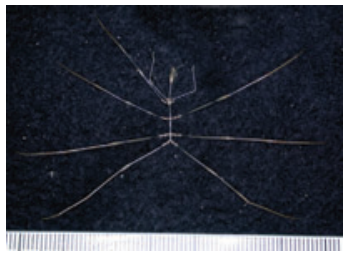

330I40 I7-Rhopalorhynchus-sibogae

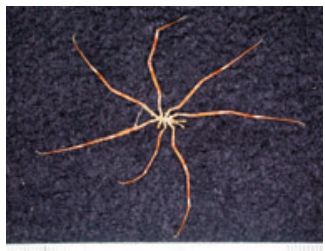

330200 I 5-Pallenopsis-temperans

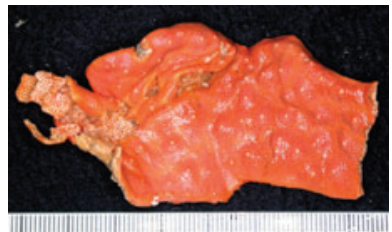

350 |3 | 57-Didemnum-ossium

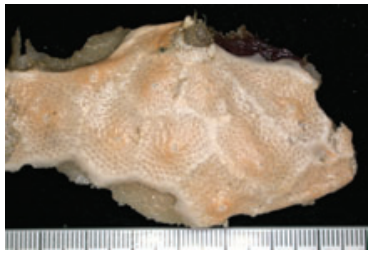

350 I328I-Polysyncraton-pavimentum

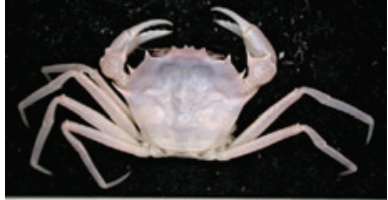

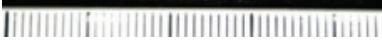

28926 | 26-Pilumnidae-sp.MOV4995

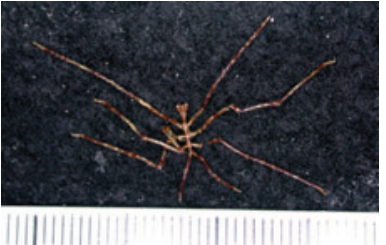

330I I0I6-Parapallene-australiensis

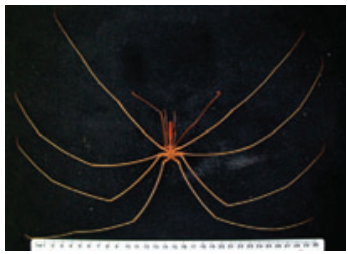

33014019-Colossendeis-colossea

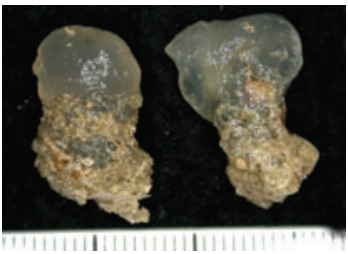

350 I 2002-Rhopalaea-crassa

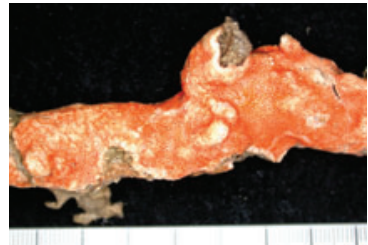

350 I3 162-Didemnum-perplexum

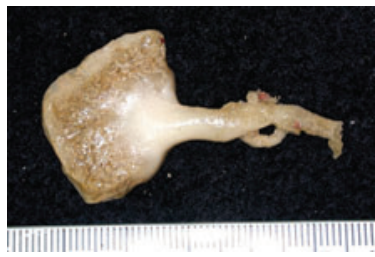

350I 5035-Sycozoa-seiziwadai

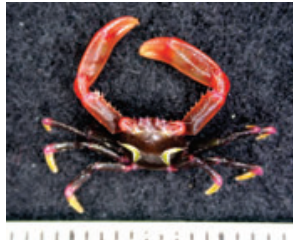

1711111! is i I11114y

28927022-Quadrella-reticulata

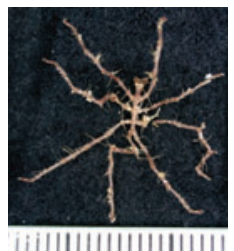

330I 1020-Parapallene-haddoni

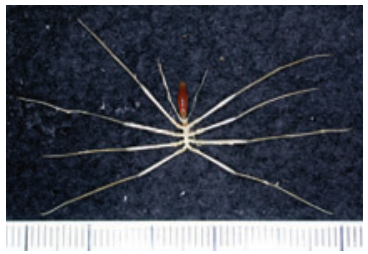

330I4024-Hedgpethia-calva

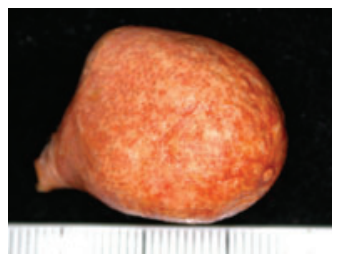

350 I 307 I-Leptoclinides-durus

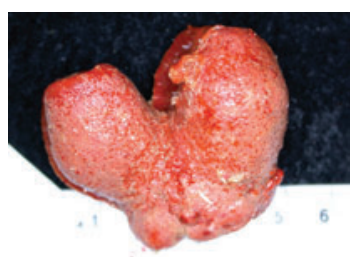

350 I32 I I-Atriolum-bucinum

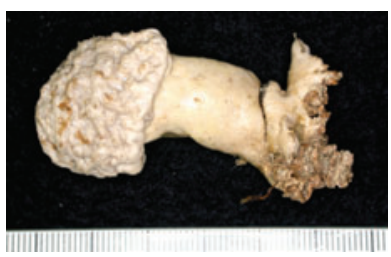

35018004-Eucoelium-coronaria

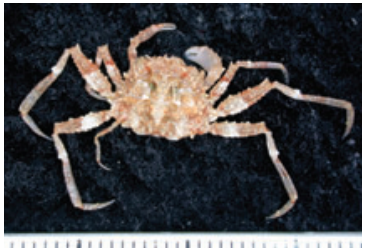

28962007-Pseudopalicus-macromeles

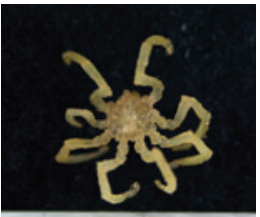

| 1011111

330 I 2054-Tanystylum-zuytdorpi

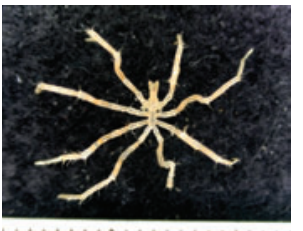

|1||||||1||||||||1||

$33020010-$ Pallenopsis-cribaribatus

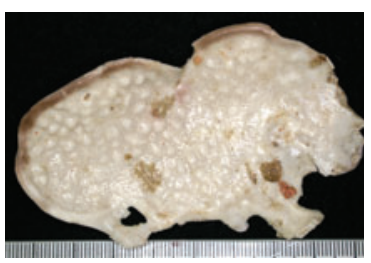

350 I 3094-Polysyncraton-millepore

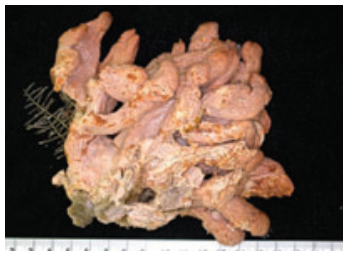

350 I 3275-Polysyncraton-fistulum

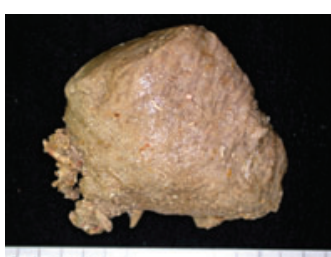

35019010-Aplidium-caelestis 


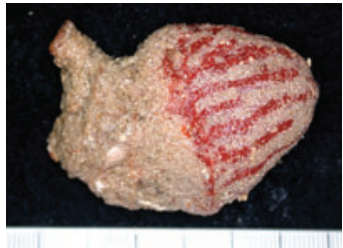

350 I9034-Aplidium-magnilarvum

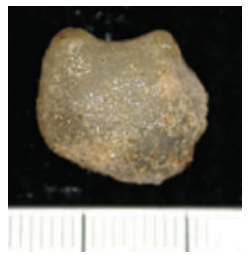

35032008-Herdmania-momus

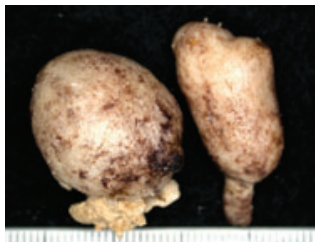

35033098-Polycarpa-viridis

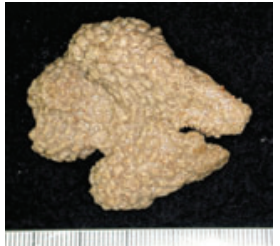

35019047-Aplidium-solidum

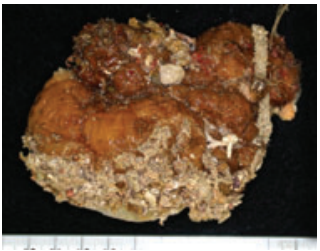

35032046-Herdmania-grandis

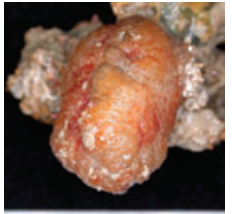

35033101-Styela-plicata

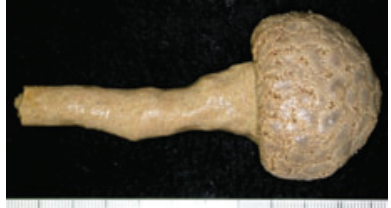

35019072-Synoicum-chrysanthemum

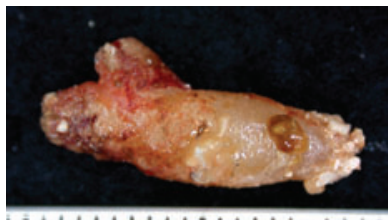

|IIIIIIIIIII!iiiillI 35033048-Cnemidocarpa-completa

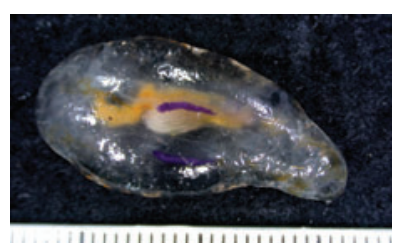

|||||||||||||||||||||||||||||||||

3510200 I-Dolioletta-gegenbauri

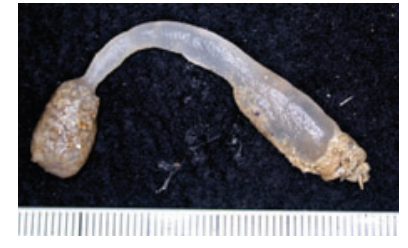

3502 I 0 I I-Sigillina-australis

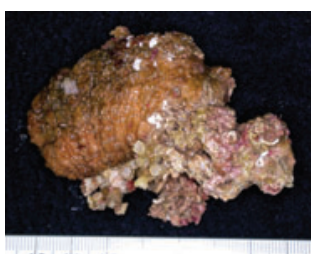

35033065-Polycarpa-aurita

35033101 -Styela-plicata 
APPENDIX 3 Example of sponge mudmaps used for identification purposes, for the species Axinella sp. SS1 (Fromont and Gomez 2007).

\section{Western Australian Sponges}

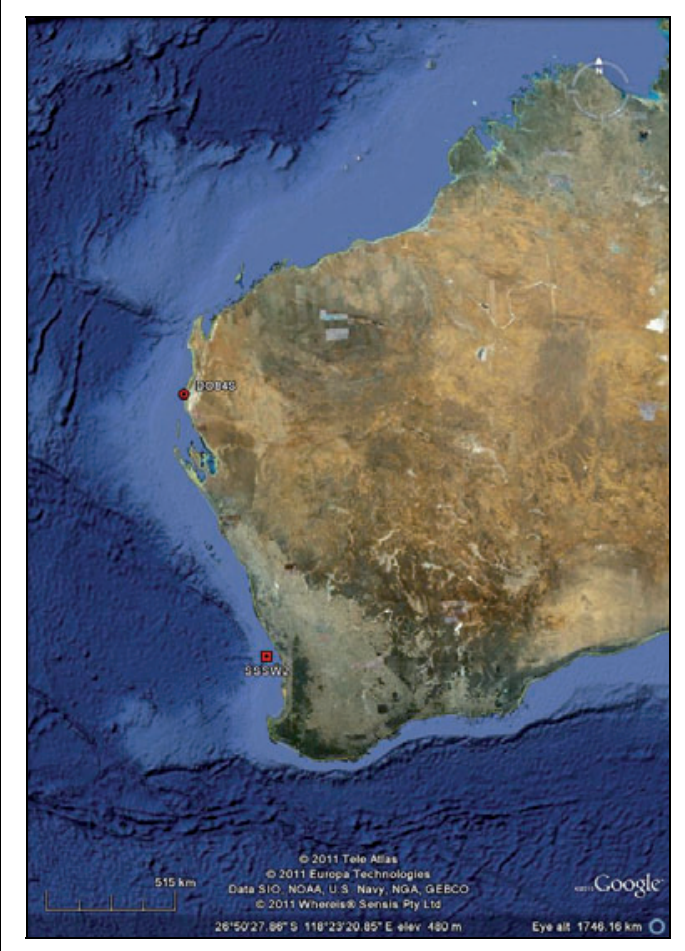

FIELD No: SS1005/002-027, 038 RVS4545/D084-070

REG. No: WAM Z35012, Z45169
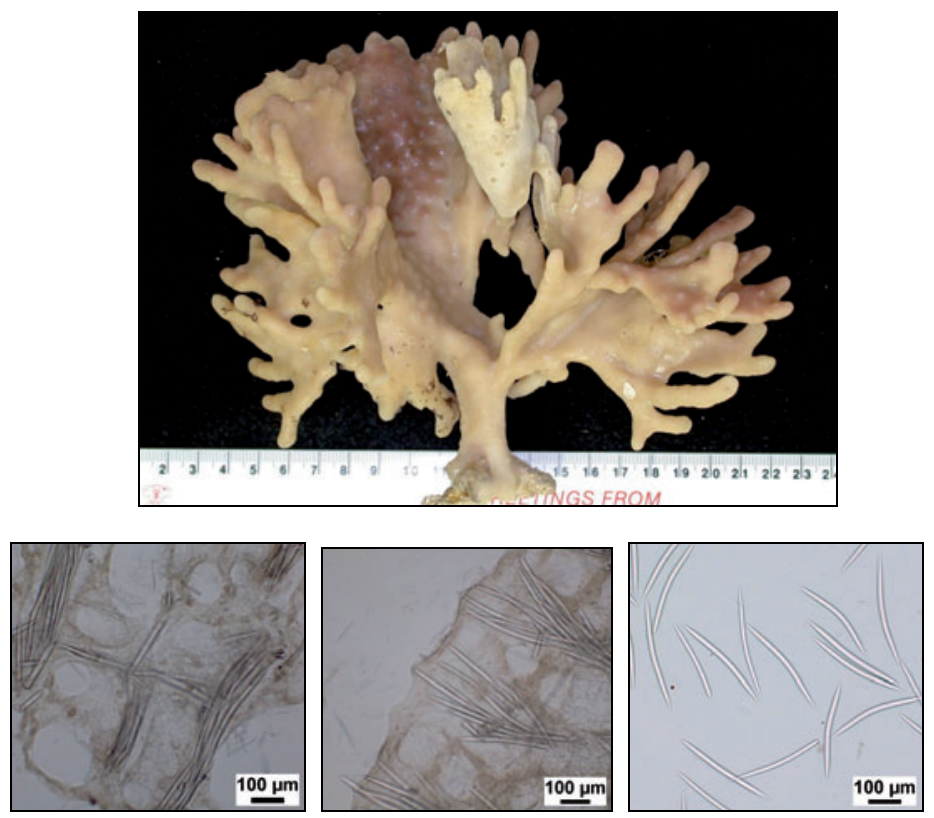

ORDER: Halichondrida

FAMILY: Axinellidae

GENUS: Axinella

SPECIES: $s p$. SS1

AUTHORITY:

Growth Form: Erect branching lobes with basal stem. Dimensions: $16 \mathrm{~cm}(\mathrm{H}) \times 18 \mathrm{~cm}(\mathrm{~W}) \times 0.5 \mathrm{~cm}(\mathrm{~T})$.

Colour: Fawn throughout, pale maroon colouration on parts of surface. Fawn in ethanol.

Oscules: On one side of lobes. Approximately $1 \mathrm{~mm}$ wide and slightly raised.

Texture: Firm, compressible, springy.

Surface Ornamentation: Fine adherent surface membrane, finely porous.

Ectosomal Skeleton: Spicules brushes at right angles to surface, extending slightly beyond. Slightly thickened mesohyl at surface.

Choanosomal Skeleton: Axial condensation in the stem with fibres around spicules. Spicule tracts radiate to surface. Width of primary fibres: $82 \mu \mathrm{m}$ with 4-6 spicules across. Width of secondary fibres: $35 \mu \mathrm{m}$ with $1-2$ spicules across. 


\section{$\underline{\text { Western Australian Sponges }}$}

Megascleres: Oxeas with sharp pencil points, usually centrally bent, some thin forms occur. Occasional styles present. Oxea length: 281-337-378 $\mu \mathrm{m}$. Width: 14-17-20 $\mu \mathrm{m}$. Specimen SS1005/002-038 (Lot 100-3): Oxea length: 312-352-378 $\mu \mathrm{m}$. Width: 11-14-17 $\mu \mathrm{m}$.

Microscleres: None.

Symbionts: None observed.

Associated Fauna: None observed.

Remarks: WAM Z35012 and specimen SS1005/002-038 (Lot 100-3) have oocytes.

Specimens: WAM Z35012 (S1005/002-027 (Lot 100-2)), WAM Z45169 (RVS4545/D084-070, not processed).

Other Material: SS1005/002-038 (Fragment). 